NIST Special Publication 2100-03

\title{
Proceedings of the International Conference on Atom-Probe Tomography \& Microscopy (APT\&M 2018)
}

\author{
Keith E. Knipling \\ Editor \\ Fred Meisenkothen \\ Assistant Editor and Co-Chair, Program and Planning Committee \\ Eric B. Steel \\ Co-Chair, Program and Planning Committee
}

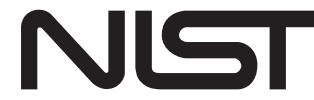

National Institute of Standards and Technology U.S. Department of Commerce 


\section{Proceedings of the International Conference on Atom-Probe Tomography \& Microscopy (APT\&M 2018)}

Fred Meisenkothen Materials Measurement Science Division Material Measurement Laboratory

Eric B. Steel Materials Measurement Science Division Material Measurement Laboratory

Keith E. Knipling U.S. Naval Research Laboratory

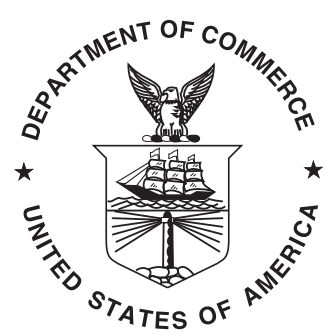

U.S. Department of Commerce Wilbur L. Ross, Jr., Secretary 
Certain commercial entities, equipment, or materials may be identified in this document in order to describe an experimental procedure or concept adequately. Such identification is not intended to imply recommendation or endorsement by the National Institute of Standards and Technology, nor is it intended to imply that the entities, materials, or equipment are necessarily the best available for the purpose.

Publications in the SP 2100 subseries are proceedings from conferences organized predominately by NIST scientific and technical staff. These proceedings are published as a single document that includes all abstracts or extended abstracts accepted by the conference organizers. This publication may include external perspectives from industry, academia, government, and others. The opinions, recommendations, findings, and conclusions in this publication do not necessarily reflect the views or policies of NIST or the United States Government.

National Institute of Standards and Technology Special Publication 2100-03

Natl. Inst. Stand. Technol. Spec. Publ. 2100-03, 355 pages (December 2019)

CODEN: NSPUE2

This publication is available free of charge from: https://doi.org/10.6028/NIST.SP.2100-03 


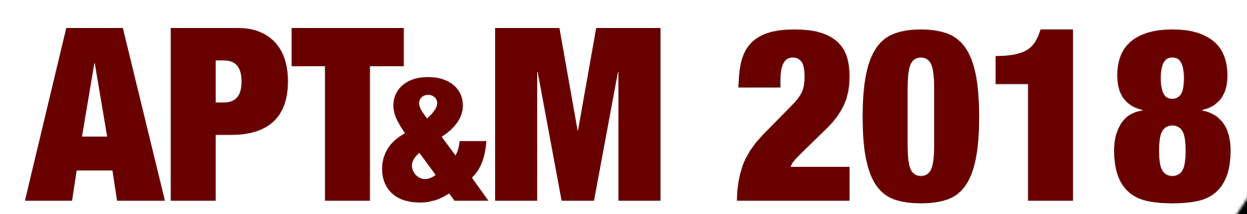

Atom Probe Tomography and Microscopy

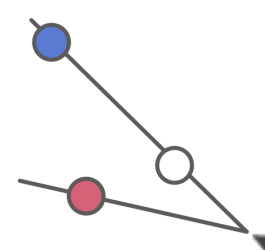

\section{June 10-15, 2018 \\ Washington, DC}

\section{International Conference on Atom-Probe Tomography \& Microscopy}

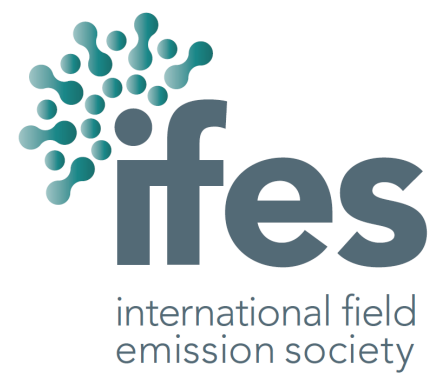

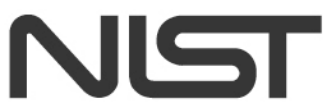

National Institute of Standards and Technology U.S. Department of Commerce 


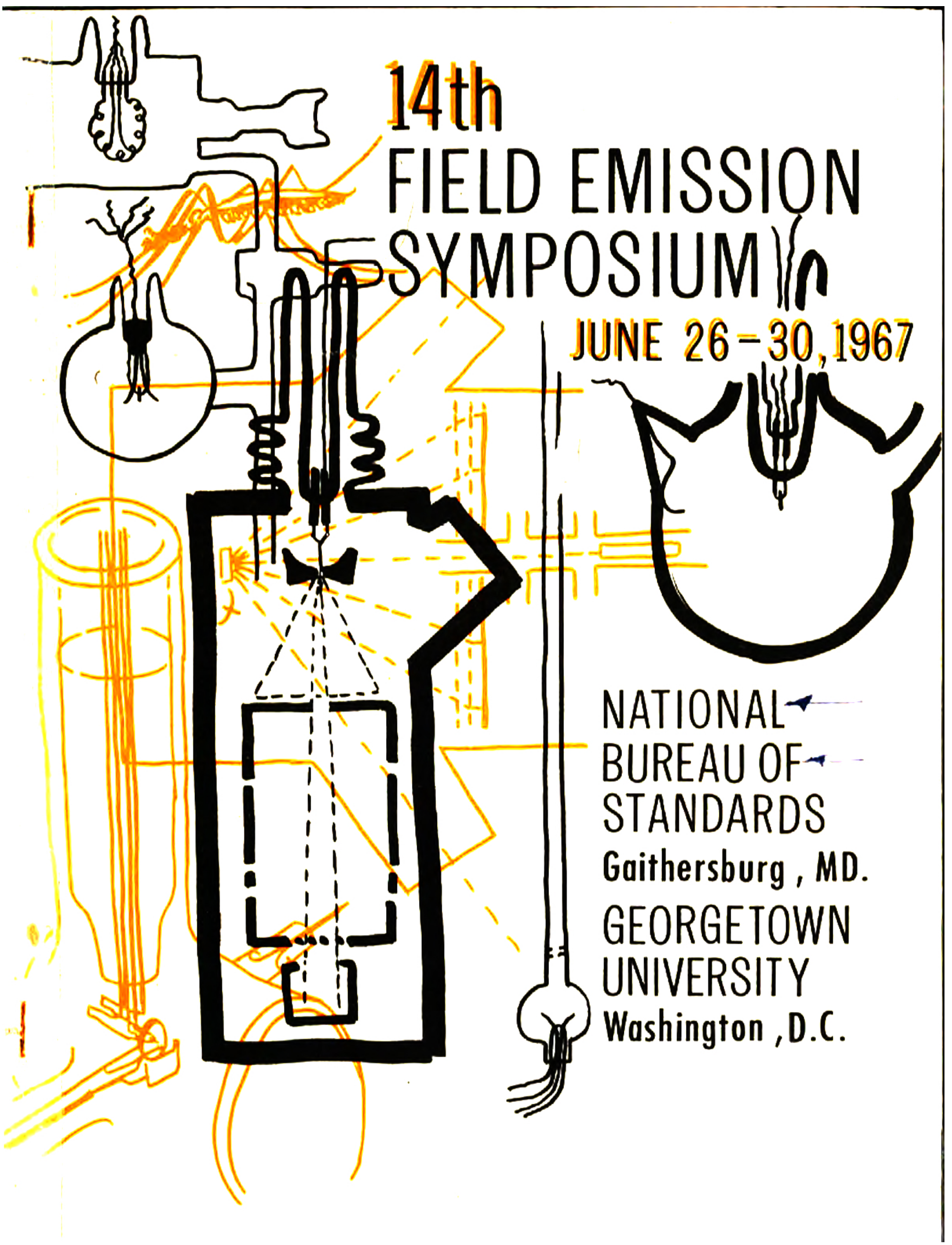




\section{Contents}

\section{Welcome to APT\&M 2018}

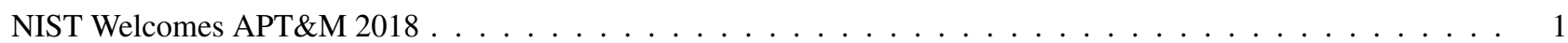

\section{Distinguished Speakers}

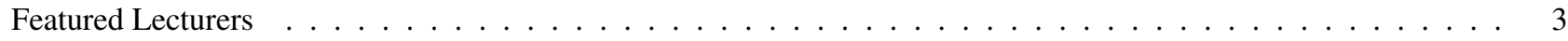

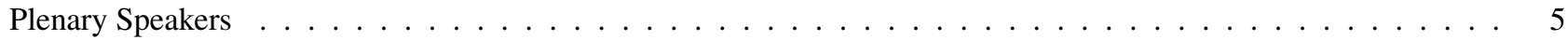

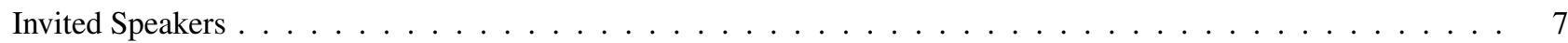

\section{Distinguished Papers}

Allan Melmed

Recollections Revisited: The Mundane and the Magnificent . . . . . . . . . . . . . . . . . . . . 15

Erwin Müller, John Panitz.

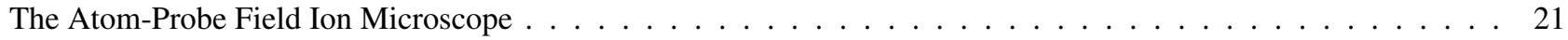

\section{Sunday Afternoon}

\section{Tutorials}

Location: Hilton Hotel, Darnestown/Gaithersburg Rooms

Tomas Silva Santisteban

Avizo $^{\mathrm{TM}}$ Software Tutorial . . . . . . . . . . . . . . . . . . . . . . . . . . . 27

Brian Geiser

Spatial Reconstruction Tutorial . . . . . . . . . . . . . . . . . . . . . . . . 28

\section{Reception}

Location: Hilton Hotel, Montgomery Ballroom

Reception . . . . . . . . . . . . . . . . . . . . . . . . . . . . . . 29 


\section{Monday Morning}

\section{Plenary 1}

Location: Green Auditorium

Chairs: Frederick Meisenkothen (National Institute of Standards and Technology)

Peter Ercius, Yongsoo Yang, Chien-Chun Chen, Colin Ophus, Mary Scott, Rui Xu, Alan Pryor, Li Wu, Fan Sun, Wolfgang Theis, Jihan Zhou, Markus Eisenbach, Paul Kent, Renat Sabirianov, Hao Zeng, Jianwei Miao

Determining Atomic Coordinates in 3D by Atomic Electron Tomography . . . . . . . . . . . . . . . . 35

\section{J. H. Block Lecture}

\section{Location: Green Auditorium}

Chairs: David Larson (CAMECA Instruments Inc.)

David Saxey, Steven Reddy, Denis Fougerouse, William Rickard

Contributions of Atom Probe Microscopy within the Geosciences . . . . . . . . . . . . . . . . . . . 36

\section{Monday Afternoon}

\section{Erwin W. Müller Memorial Lecture \\ Location: Green Auditorium \\ Chairs: Thomas Kelly (CAMECA Instruments Inc.)}

John Panitz.

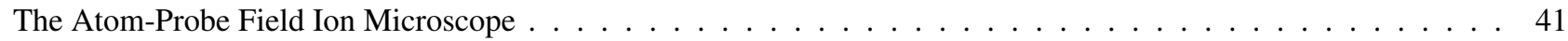

\section{Erwin W. Müller Award Competition}

Location: Green Auditorium

Chairs: François Vurpillot (Université de Rouen)

Enrico Di Russo, Jonathan Houard, Simona Moldovan, Gérald Da Costa, Ivan Blum, François Henry Julien, Maria Tchernycheva, Jean-Michel Chauveau, Maxime Hugues, Didier Blavette, Lorenzo Rigutti

Atom Probe-Based Correlative Multi-Microscopy: New Perspectives for the Study of Micro-Photoluminescence

Emission From Semiconductors Nano-Tips ． . . . . . . . . . . . . . . . . . . . . . 42

Zirong Peng, Yifeng Lu, Constantinos Hatzoglou, Alisson Kwiatkowski da Silva, François Vurpillot, Dirk Ponge, Dierk Raabe, Baptiste Gault

An Automated Computational Approach for Complete In-Plane Compositional Interface Analysis by Atom Probe

Tomography . . . . . . . . . . . . . . . . . . . . . . . . . . . . 4

Jing Xue, Shenbao Jin, Xianghai An, Xiaozhou Liao, Jiehua Li, Gang Sha

The Non-Uniform Distribution of Mg in Al-Mg Alloy Under High Pressure Torsion . . . . . . . . . . . . . . 44

Sten Lambeets, Daniel Perea, Thierry Visart de Bocarmé, Norbert Kruse

$\mathrm{CO}_{2}$ Hydrogenation Over Rhodium Catalysts: Field Emission Techniques Reveal $\mathrm{CO}_{2}$-induced Metal-Surface and

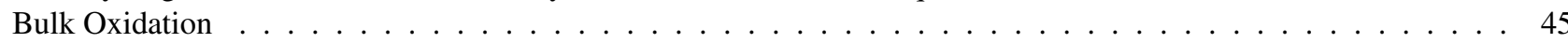

Pritesh Parikh, Arun Devaraj, Ying Shirley Meng

3D Nanoscale Compositional Mapping of Li-ion Battery Cathodes Using Atom Probe Tomography . . . . . . . . . 46 


\section{Tuesday Morning}

\section{Plenary 2}

Location: Green Auditorium

Chairs: Ann Chiaramonti (National Institute of Standards and Technology)

Henry Kapteyn, Margaret Murnane

New Opportunities at the Nanoscale using New VUV-EUV Laser Sources . . . . . . . . . . . . . . . . . 5

\section{Ceramics, Geological, and Glass Materials}

\section{Location: Green Auditorium}

Chairs: Daniel Schreiber (Pacific Northwest National Laboratory), Brian Langelier (McMaster University), and David Saxey (Curtin University)

Steven Reddy, Denis Fougerouse, David Saxey, William Rickard, Chris Kirkland, Mark Pearce, Louise Fisher, Matt Kilburn, Rob Hough, Gretchen Benedix

Atom Probe Characterization of $\mathrm{Pb}$ Isotopic Variations in Terrestrial and Meteoritic Fe Sulfides (Invited) _ . . . . . 54

Lee White, Desmond Moser, Kim Tait, James Darling, David Reinhard, Brian Langelier

Dating Planetary Events Using APT Isotopic Analysis of Natural $\mathrm{ZrO}_{2} \ldots \ldots \ldots \ldots$

Josiah Lewis, Pranav Bhadharla, Christine Floss, Dieter Isheim, David Seidman

Preparation of $\sim 100 \mathrm{~nm}$-Diameter Presolar SiC in Meteoritic Matrix for Atom-Probe Tomography (APT) . . . . 56

Sandra Taylor, Jia Liu, Bruce Arey, John Cliff, Daniel Schreiber, Daniel Perea, Kevin Rosso

Resolving the Sorption Behavior of Fe(II) on Hematite at the Atomic Level . . . . . . . . . . . . . . . . . . 57

Juliane Weber, Jonathan Poplawsky, Karren More, Anton Ievlev, Matthias Lorenz, Steven Higgins, Angela Bertagni, Sarah Jindra, Jacquelyn Bracco, Andrew Stack

Unraveling the Influence of Impurities on Mineral Growth by combining APT, AFM-ToF-SIMS and TEM . . . . . 58

\section{Correlative and Combined Methods}

\section{Location: Heritage Room}

Chairs: Michael Herbig (Max-Planck-Institut für Eisenforschung GmbH), Arun Deveraj (Pacific Northwest National Laboratory), and Joe Bennett (National Institute of Standards and Technology)

Williams Lefebvre, Ivan Blum, Celia Castro, Lorenzo Rigutti, Florian Chabanais, Antoine Normand, François Vurpillot

Opportunities Offered by the Serial and or in Situ Combination of Atom Probe and (Scanning) Transmission Elec-

tron Microscopy $($ Invited $) \ldots \ldots \ldots \ldots \ldots \ldots \ldots \ldots \ldots$

Alain Portavoce, Khalid Hoummada, Lee Chow

Atom Probe Tomography and Secondary Ion Mass Spectrometry: An Interesting Combination for Atomic Diffusion and Segregation Measurements $($ Invited $) \ldots \ldots \ldots \ldots \ldots \ldots$

Hugues Francois-Saint-Cyr, Isabelle Martin, Ty Prosa, David Larson, Xavier Bourrat, Guillaume Wille, Tara Nylese, Matthew Nowell, René de Kloe, David Gibouin, Christelle Guillermier, Matthew Steinhauser

Expensive Jewelry or Casual Decoration? Aragonite to Vaterite Polymorphisms in Nacre Explained by Correlative

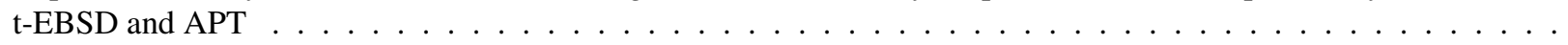


Paul Bagot, John Halpin, Luke Daly, Michael Moody, Jan-Willem Bos, Donald MacLaren

Interfacial Segregation in a Novel Cu-Doped TiNiSn Thermoelectric Generator: An APT and STEM-EDX Study . 62

\section{Laser/Matter Interactions}

\section{Location: Portrait Room}

Chairs: Angela Vella (Université de Rouen), Yu Xia (Sun Yat-sen University), and Janusz Bogdanowicz (IMEC)

Ann Chiaramonti, Luis Miaja-Avila, Paul Blanchard, David Diercks, Brian Gorman, Norman Sanford

Extreme Ultraviolet-Assisted Field Ion Evaporation ． . . . . . . . . . . . . . . . . . . . . . . . . . . . . . .

Chang-Min Kwak, Byeong-Gyu Chae, Jae-Bok Seol, Chan-Gyung Park

Estimation of Relative Evaporation Field Strength of Bulk Oxide-Composite and Comprehension of Evaporation

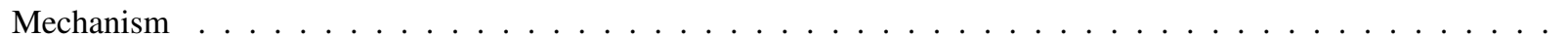

Oana Cojocaru-Mirédin, Min Zhu, Antonio Mio, Jens Keutgen, Michael Küpers, Yuan Yu, Ju-Young Cho, Richard Dronskowski, Matthias Wuttig

Distinctive Bond Breaking in Crystalline Phase-Change Materials . . . . . . . . . . . . . . . . .

Jonathan Houard, Laurent Arnoldi, Maria Spies, Ivan Blum, Auriane Etienne, Rinat Ismagilov, Alexander Obraztsov, Angela Vella

Study of Optical and Thermal Properties of Diamond Nanowires by Laser Assisted Atom Probe Tomography . . . 66

Linda Venturi, Lorenzo Rigutti, Jonathan Houard, Antoine Normand, Elena Silaeva, Mario Borz, S. A. Malykhin, Alexander Obraztsov, Angela Vella

Optical Measurements of the Tensile Stress Induced by a High Electric Field in Diamond Nanoscale Needles . . .

Laurent Arnoldi, Mario Borz, Ivan Blum, Alexander Obraztsov, Angela Vella

Study of Electrical Conduction Properties of Diamond Nanoneedles, by Ion Spectroscopy in Field Ion Microscopy

\section{Tuesday Afternoon}

\section{Ceramics, Geological, and Glass Materials}

Location: Green Auditorium

Chairs: Daniel Schreiber (Pacific Northwest National Laboratory), Brian Langelier (McMaster University), and David Saxey (Curtin University)

Qi Zhou, Rong Hu, Jizi Liu, Gang Sha

Atom Probe Study of Oxidation Behavior of an Amorphous Al-Ga Metal . . . . . . . . . . . . . . 73

Torben Boll, Olof Bäcke, Bruce Pint, Kinga Unocic, Krystyna Stiller

Outward Diffusion Through Protective Alumina on NiAl-alloys . . . . . . . . . . . . . . . . . . . . . 7

George Burton, David Diercks, Brian Gorman

Atom Probe Tomography Study of Space Charge Regions in $\mathrm{BaCe}_{0.8} \mathrm{Y}_{0.2} \mathrm{O}_{3-\text { delta }}-\mathrm{Ce}_{0.8} \mathrm{Y}_{0.2} \mathrm{O}_{2-\text { delta }} \mathrm{Hydrogen}$ Separation Membrane . . . . . . . . . . . . . . . . . . . . . . . . 75

Byeong-Gyu Chae, Seong Yong Park, Jay Hyok Song, Chang-Min Kwak, Jae-Bok Seol, Chan-Gyung Park

Li Concentration Gradient in Cycled $\mathrm{Li}\left(\mathrm{Ni}_{0.8} \mathrm{Co}_{0.15} \mathrm{Mn}_{0.05}\right) \mathrm{O}_{2}$ Cathode Materials . . . . . . . . . . . . . . . . . . 76 


\section{Interlaboratory Studies Tutorial}

Location: Green Auditorium

Chairs: Austin Akey (Harvard University)

David Duewer

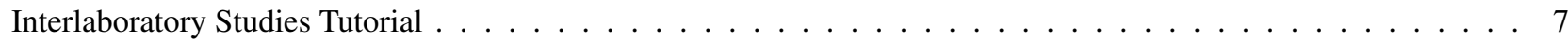

\section{Measurement Science Workshop}

\section{Location: Green Auditorium}

Chairs: Austin Akey (Harvard University)

Clustering Interlaboratory Study ～. . . . . . . . . . . . . . . . . . . . . . . . . . . . 79

Zircon Interlaboratory Study and Geology Perspective on Reporting APT Data . . . . . . . . . . . . . . . . . 79

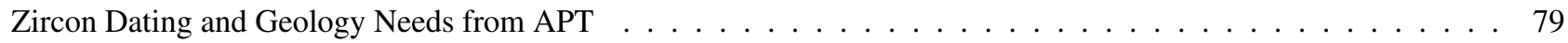

Industry Perspective on Quality Assurance and Measurement Standards ～. . . . . . . . . . . . . . . . 79

Open Forum Discussion . . . . . . . . . . . . . . . . . . . . . . . . . . . . . . . . . . . . 79

\section{Correlative and Combined Methods}

\section{Location: Heritage Room}

Chairs: Michael Herbig (Max-Planck-Institut für Eisenforschung GmbH), Arun Deveraj (Pacific Northwest National Laboratory), and Joe Bennett (National Institute of Standards and Technology)

Tong Li, Olga Kasian, Serhiy Cherevko, Siyuan Zhang, Simon Geiger, Christina Scheu, Peter Felfer, Dierk Raabe, Baptiste Gault, Karl Mayrhofer

Probing Surface Species of Electrocatalyst at Atomic Scale . . . . . . . . . . . . . . . . . . . . . .

Torsten Schwarz, Guillaume Stechmann, Baptiste Gault, Oana Cojocaru-Mirédin, Roland Wuerz, Alberto Lomuscio, Susanne Siebentritt, Dierk Raabe

Correlative Transmission Kikuchi Diffraction and Atom Probe Tomography Analysis of Grain Boundaries in $\mathrm{Cu}(\mathrm{In}, \mathrm{Ga}) \mathrm{S}(\mathrm{e})_{2}$ Based Thin Film Solar Cells $\ldots \ldots \ldots \ldots \ldots \ldots$

Leifeng Zhang, Bertrand Radiguet, Christophe Domain, Yang Shen, Patrick Todeschini, Philippe Pareige Investigation of Grain Boundary Segregation by a Correlative EBSD/TKD/APT Methodology for a 16MND5 Weld

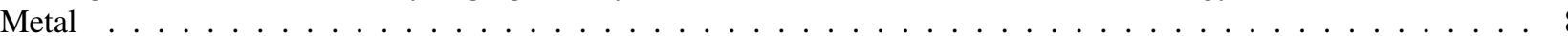

Michael Herbig, Wenjun Lu, Christian Liebscher, Lutz Morsdorf, Ross Marceau, Gerhard Dehm, Dierk Raabe Formation of Nanoscale eta Carbides in Fe-Ni-C Martensite at Room Temperature . . . . . . . . . . . . . . . . . . 83

Xuyang Zhou, Brad Boyce, Blythe Clarke, Gregory Thompson

Elucidating Grain Boundary Segregation Behavior in Nanocrystalline Stabilized $\mathrm{Pt}(\mathrm{Au}) \ldots \ldots$. . . . . . . .

James Douglas, Alexander Carruthers, Paul Bagot, Michael Moody, M. Grace Burke

Comparison of Irradiation-Induced Clustering in Proton Irradiated A508 Gr 4N Steel via Analytical STEM EDX

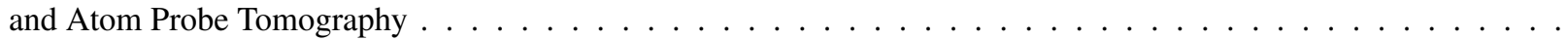

Avinash Hariharan, Jeroen Risse, Eric Jägle, Dierk Raabe

Solidification Cracking During Selective Laser Melting (SLM) of Nickel-Base Superalloy Inconel-738LC . . . . .

Daniel Schreiber, Matthew Olszta, Karen Kruska, John Scully, Angela Gerard, Kathleen Quiambao, Stephen Bruemmer

Combined TEM and APT Characterization of Multi-Phase Oxides Formed During Metal Oxidation and Corrosion .

Ming-Zhe Bian, Taisuke Sasaki, Taiki Nakata, Yu Yoshida, Nozomu Kawabe, Shigeharu Kamado, Kazuhiro Hono APT/TEM Analyses of Solute Segregations Along Dislocations and Solute Clustering in Bake-Hardenable Magne-

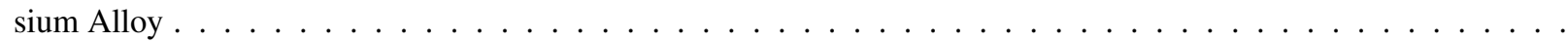


Paraskevas Kontis, Surendra Makineni, Jonathan Cormier, Dierk Raabe, Baptiste Gault

The Role of Atom Probe Tomography on the Development of New High Performance Materials . . . . . . . . . .

\section{Sparse Data Sampling Overview}

Location: Heritage Room

Chairs: Scott Wight (National Institute of Standards and Technology)

John Henry Scott

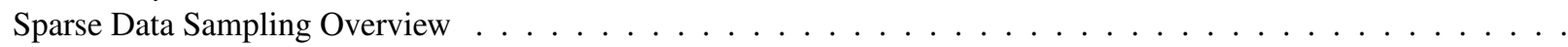

\section{Semiconductors and Devices Location: Portrait Room \\ Chairs: Baishakhi Mazumder (The State University of New York - Buffalo), Jonathan Poplawsky (Oak Ridge National Labora- tory), and Bastien Bonef (University of California Santa Barbara)}

Yasuo Shimizu, Bin Han, Yuan Tu, Koji Inoue, Yasuyoshi Nagai

Atom Probe Study of Silicon-Based Device Structures (Invited) ～. . . . . . . . . . . . . . . . . . . . . . . 91

James Douglas, George Martins, Abu Bakr, John Murphy, Paul Bagot, Michael Moody

Atom Probe Analysis of Surface Regions of Iron Contaminated Silicon Subjected to Phosphorus Diffusion Gettering 92

Eric Jones, Keith Chung, Jonathan Poplawsky, Donavan Leonard, Kevin Mercurio, Paul Brabant, Thomas Adams,

Patrick Shea, Thomas Knight

Quantification of Boron Dopant Profiles in SiGe-based HBT Devices . . . . . . . . . . . . . . . . . . 93

Maria Auger, Pankaj Sharma, Michael Moody, Jan Seidel

Atom Probe Tomography of Ferroelectric $\left(\mathrm{BiFeO}_{3}\right)$ Domain Walls . . . . . . . . . . . . . . . . . . . . . . . . . 94

Jiangtao Qu, Sichao Du, Tim Burgess, Changhong Wang, Xiangyuan Cui, Qiang Gao, Weichao Wang, Hark Hoe

Tan, Hui Liu, Chennupati Jagadish, Hansheng Chen, Mansoor Khan, Simon Ringer, Rongkun Zheng

Growth Mechanism of III-V Group Core-Shell Structured Nanowires (Invited) . . . . . . . . . . . . . . . . . . . .

Matthew Jaskot, Andrew Proudian, Helena-Nikolai Fujishin, Galen Vincent, David Diercks, Brian Gorman, Jeramy

Zimmerman

Atom Probe Tomography of Molecular Organic Electronic Materials . . . . . . . . . . . . . . . . . . . 96

Lynda Amichi, David Cooper, Isabelle Mouton, Philippe Vennéguès, Philippe De Mierry, Pierre-Henri Jouneau,

Catherine Bougerol, Adeline Grenier

Correlative TEM and APT Microscopies to Investigate Mg Distribution in p-Doped GaN Nanostructures GaN

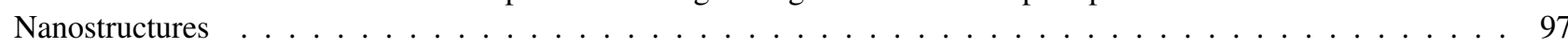

Baishakhi Mazumder, Jung-Hun Seo, Jonathan Poplawsky

Application of Atom Probe Tomography for Engineering $\left(\beta-\mathrm{Al}_{x} \mathrm{Ga}_{1-x}\right)_{2} \mathrm{O}_{3}$, a Wide Band Gap Semiconductor . . . 98

Paul van der Heide, Claudia Fleischmann, Davit Melkonyan, Kristof Paredis, Richard Morris, Janusz Bogdanow-

icz, Wilfried Vandervorst

APT and Its Future in the Semiconductor Industry (Invited $) \ldots \ldots . \ldots$

Hisham Aboulfadl, Jan Keller, Jes Larsen, Mohammad Sattari, Marika Edoff, Charlotte Platzer-Björkman

Microstructural Characterization of Sulfurization Effects in $\mathrm{Cu}(\mathrm{In}, \mathrm{Ga}) \mathrm{Se}_{2}$ Thin Film Solar Cells . . . . . . . . . . 100

Oana Cojocaru-Mirédin, Henning Hollermann, Antonio Mio, Matthias Wuttig

Nanoscale Investigation of GeTe-Sb $\mathrm{Te}_{3}$ Superlattices . . . . . . . . . . . . . . . . . . . . . . . 101

Matthew Jaskot, Andrew Proudian, Helena-Nikolai Fujishin, Galen Vincent, David Diercks, Brian Gorman, Jeramy

Zimmerman

Spatial Statistics as a Quantitative Tool to Study Morphology in Organic Light-Emitting Diodes . . . . . . . . . . 102 


\section{IVAS Tutorials}

Location: Computer Lab

Chairs: Hugues Francois-Saint-Cyr

Hugues Francois-Saint-Cyr

IVAS 101 or How to Make the Most Out of Your Dataset Using Your Favorite 3D Reconstruction Wizard? . . . . . 103

Hugues Francois-Saint-Cyr, Andy Martin

3D Reconstruction of Semiconductor Devices: Can IVAS Give Users What They Really Want? . . . . . . . . . . 104

\section{Wednesday Morning}

\section{Plenary 3}

Location: Green Auditorium

Chairs: Eric Steel (National Institute of Standards and Technology)

Christoph Freysoldt, Arpit Mishra, Michael Ashton, Jörg Neugebauer

Density Functional Modelling of Field Evaporation . . . . . . . . . . . . . . . . . . . . . . . 109

\section{Instrument and Technique Development}

Location: Green Auditorium

Chairs: Tom Kelly (CAMECA, Inc.) and Stephan Gerstl (ETH Zürich/ScopeM)

Anton Lukyanchuk, Sergey Rogozhkin, Andrey Aleev, Anton Shutov, Oleg Raznitsyn

Atom Probe Tomography Development in ITEP . . . . . . . . . . . . . . . . . . . . . . 110

Patrick Stender, Jonas Ott, Irdi Balla, Guido Schmitz

M-TAP_The Modular Atom Probe . . . . . . . . . . . . . . . . . . . . . . . . . . 111

Saba Maeda, Yasuhito Gotoh

Evaluation of Magnification and Spatial Resolution in Electrostatic Divergent Lens with Extremely Short Focal

Length . . . . . . . . . . . . . . . . . . . . . . . . . . . 112

Claudia Fleischmann, Kristof Paredis, Davit Melkonyan, Jonathan Op de Beeck, Janusz Bogdanowicz, Richard Morris, Ramya Cuduvally, Wilfried Vandervorst, Wilfried Vandervorst

3D Imaging of Atom Probe Tip Shapes with Atomic Force Microscopy . . . . . . . . . . . . . . . . . . 113

Joseph Suttle, Thomas Kelly, Robert McDermott

Development of a Superconducting Detector for Atom Probe Tomography . . . . . . . . . . . . . . . . 114

Leigh Stephenson, Andrew Breen, Isabelle Mouton, Yanhong Chang, Ann-Katrin Rusitzka, Agnieszka Szczepaniak, Uwe Tezins, Andreas Sturm, Thomas Kelly, Dierk Raabe, Baptiste Gault

The Laplace Project at MPIE: Modular UHV Cryogenic Protocols for Environmentally-Fragile Nanostructures . . 115

\section{High Entropy Alloys and High Cr Steels}

Location: Heritage Room

Chairs: Goro Miyamoto (Tohoku University), Yongjie Zhang (Tohoku University), Rajarshi Banerjee (University of North Texas), and Talukder Alam (University of North Texas)

Konda Gokuldoss Pradeep, Marshal Amalraj, Mayur Vaidya, Budaraju Srinivasa Murty

High Entropy Alloys to Compositionally Complex Alloys: Relevance of Atom Probe Tomography as a High-

Throughput Characterization Tool (Invited) . . . . . . . . . . . . . . . . . . 116 
Talukder Alam, Bharat Gwalani, Rajarshi Banerjee

Investigation of Clusters in AlxCoCrFeNi High Entropy Alloys . . . . . . . . . . . . . . . . . . . . . . . . . 117

Sascha Seils, Hans Chen, Dorothée Vinga Szabó, Ian Harding, Sharvan Kumar, Sandra Kauffmann-Weiss, Torben Boll, Alexander Kauffmann, Martin Heilmaier

Ordering in Al-Containing Refractory High Entropy Alloys Analyzed by Complemental Microstructure Characterization Techniques . . . . . . . . . . . . . . . . . . . . . . . . . 118

Bharat Gwalani, Stephane Gorsse, Deep Choudhuri, Rajarshi Banerjee

Modifying Transformation Pathways in High Entropy Alloys or Complex Concentrated Alloys via Thermo-Mechanical

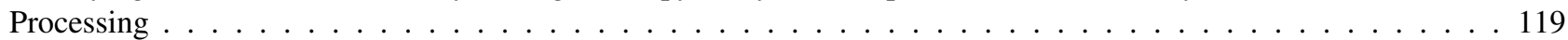

Deodatta Shinde, Mattias Thuvander, Stefan Fritze, Paulius Malinovskis, Erik Lewin, Ulf Jansson, Krystyna Stiller Effect of Carbon Addition in CrNbTaTiW High Entropy Alloy: An Atom Probe Study . . . . . . . . . . . . . . . 120

\section{Theory, Simulations, and Modeling}

\section{Location: Portrait Room}

Chairs: Christian Oberdorfer (The Ohio State University) and Stefan Parviainen (Université et INSA de Rouen)

David Zanuttini, Ivan Blum, Lorenzo Rigutti, Enrico Di Russo, François Vurpillot, Julie Douady, Emmanuelle Jacquet, Pierre-Matthieu Anglade, Benoit Gervais

Theoretical Analysis of Dissociation Tracks Observed in TAP (Invited) . . . . . . . . . . . . . . . . . . . . 121

Christian Bacchi, Gérald Da Costa, François Vurpillot

Spatial and Compositional Biases Introduced by Position Sensitive Detection Systems in APT: A Simulation Ap-

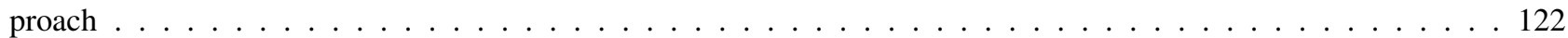

Toshiharu Ohnuma

Evaporation Field and Surface Diffusion on BCC Fe (001) and $\mathrm{Cu}(001)$ Under Electric Field Using First-Principles

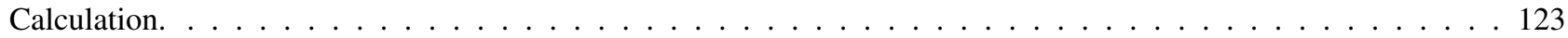

Charles Fletcher, Michael Moody, Daniel Haley

Accurate and Efficient Field Evaporation Modelling Using Level-Set Methods . . . . . . . . . . . . . . . . . . 124

Wolfgang Windl, Christian Oberdorfer, Travis Withrow, Iman Ghamarian, Emmanuelle Marquis

Atomistic-Modeling Based Simulation of Field Evaporation Processes . . . . . . . . . . . . . . . . . . . . . 125

\section{Wednesday Afternoon}

\section{Quantification in Atom Probe}

Location: Green Auditorium

Chairs: Daniel Haley (University of Oxford), Ivan Povstugar (Forschungszentrum Jülich), and Andrew London (United Kingdom Atomic Energy Authority)

Baptiste Gault, Isabelle Mouton, Yanhong Chang, Andrew Breen, Michael Ashton, Siyang Wang, Paraskevas Kontis, Agnieszka Szczepaniak, Michael Herbig, Abigail Ackerman, Felicity Dear, David Dye, Christoph Freysoldt, Jörg Neugebauer, Dierk Raabe, T. Ben Britton, Leigh Stephenson

Quantifying Hydrogen by Atom Probe Tomography (Invited) .

Yanhong Chang, Isabelle Mouton, Leigh Stephenson, Agnieszka Szczepaniak, Guikai Zhang, Dirk Ponge, Dierk Raabe, Baptiste Gault

Influence of the Field Evaporation Conditions on Compositional Analysis of Stable Titanium Deuterides . . . . . . 132

Elizabeth Kautz, Bradley Johnson, John Hardy, Arun Devaraj

Investigation of Hydrogen Isotope Concentration and Distribution in Zircaloy-4 via Atom Probe Tomography . . . 133 
Bastien Bonef, Abdullah Alhassan, Cheyenne Lynsky, Guillaume Lheureux, James Speck

Nanometer Scale Investigation of Alloy Distributions in Quaternary Nitride (In,Al,Ga)N

Davit Melkonyan, Claudia Fleischmann, Janusz Bogdanowicz, Richard Morris, Ramya Cuduvally, Wilfried Van-

dervorst

A Critical View on the Accuracy of Dopant Profiling in Atom Probe Tomography: The Case of Boron in Silicon . . 135

Richard Morris, Ramya Cuduvally, Davit Melkonyan, Ming Zhou, Paul van der Heide, Wilfried Vandervorst

Influence of the Tip Field, Laser, Pole and Zone Lines on the Quantification of GaN/AlGaN Heterostructures . . . 136

Ramya Cuduvally, Richard Morris, Janusz Bogdanowicz, Davit Melkonyan, Laurent Arnoldi, Claudia Fleischmann, Wilfried Vandervorst

Quantitative Compositional Analysis of Compound Semiconductors by Atom Probe Tomography . . . . . . . . . . 137

Leigh Stephenson, Isabelle Mouton, Zirong Peng, Shyam Katnagallu, Mozhdeh Fathidoost, Yanhong Chang, Huan

Zhao, Ann-Katrin Rusitzka, Dierk Raabe, Baptiste Gault

Deconvolving and Interpreting Mass-To-Charge Correlations . . . . . . . . . . . . . . . . . . 138

Andrew London

Quantifying Compositional Uncertainty Arising From Solving Peak Overlaps . . . . . . . . . . . . . . . 139

Jing Wang, Nathan Bailey, Peter Hosemann, Daniel Schreiber, Mychailo Toloczko

A New Density-Based Approach for Clusters Analysis in Atom Probe Tomography Data . . . . . . . . . . . . . . 140

Paul Styman, James Douglas

Using Atom Probe to Characterise Gas Bubbles in Zirconium . . . . . . . . . . . . . . . . . . . . . . . 141

Goro Miyamoto, Tadashi Furuhara

Quantitative Measurement of Compositions in Steels by Atom Probe Tomography . . . . . . . . . . . . . . . . 142

Mattias Thuvander, Deodatta Shinde, Krystyna Stiller

Improving Compositional Accuracy in APT Analysis of Carbides Using a Decreased Detection Efficiency . . . . . 143

\section{High Entropy Alloys and High Cr Steels \\ Location: Heritage Room}

Chairs: Goro Miyamoto (Tohoku University), Yongjie Zhang (Tohoku University), Rajarshi Banerjee (University of North Texas), and Talukder Alam (University of North Texas)

Jonathan Poplawsky, Xing Wang, Wei Guo, Tengfei Yang, Wei-Ying Chen

Understanding Irradiation Induced Defects in Multicomponent Alloys (Invited) . . . . . . . . . . . . . . . . . . . 144

Yujiao Li, Alan Savan, Aleksander Kostka, Helge Stein, Alfred Ludwig

Accelerated Atomic-Scale Exploration of Phase Evolution in Compositionally Complex Materials . . . . . . . . . 145

Alexander Dahlström, Frédéric Danoix, Peter Hedström, Joakim Odquvist, Helena Zpolsky

The Influence of an External Load and Grain Orientation on Spinodal Decomposition in Fe-Cr . . . . . . . . . 146

Timothy Lach, Thak-Sang Byun, Arun Devaraj

Mechanistic Study of Microstructural Evolution of Ferrite/Austenite Phase Boundary in Cast Duplex Stainless

Steels After 10000 Hours Thermal Aging . . . . . . . . . . . . . . . . . . . . . . . . . . 147

Gang Sha, Bo Zhang, Shenbao Jin, Shilei Li, Xitao Wang, Fei Xue

Phase Separation Near the Ferrite/Austenite Interface of a Duplex Stainless Steel . . . . . . . . . . . . . . . . 148

Kristina Lindgren, Martin Bjurman, Pål Efsing, Peter Ekström, Mattias Thuvander

Effects of Thermal Ageing and Neutron Irradiation on a Stainless Steel Weld . . . . . . . . . . . . . . . . . . . . . 449 


\section{Instrument and Technique Development}

Location: Heritage Room

Chairs: Tom Kelly (CAMECA, Inc.) and Stephan Gerstl (ETH Zürich/ScopeM)

Daniel Perea, Daniel Schreiber

Advanced Approaches to FIB-based Specimen Preparation of Cryogenically-Prepared Specimens ～. . . . . . 150

Daniel Haley, Paul Bagot, Michael Moody

Atom Probe of Gas Exposed Metallic Surfaces: Isotopic and Surface Studies . . . . . . . . . . . . . . . . . . 151

Yi-Sheng Chen, Daniel Haley, Paul Bagot, Michael Moody

Simplifying Observation of Hydrogen Trapping in Atom Probe Tomography . . . . . . . . . . . . . . . . . . . 152

Ty Prosa, Isabelle Martin, David Larson

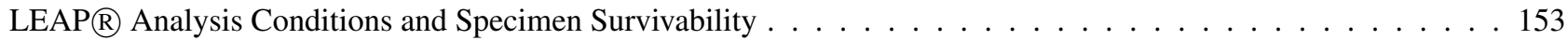

Masahiro Taniguchi, Osamu Nishikawa

Evaluation of the Atomic Level Mass Analysis of Biomolecules by the Scanning Atom Probe Alternately Triggered

by Voltage and Laser Pulses (Invited) … . . . . . . . . . . . . . . . . . . . . . . . 154

\section{Theory, Simulations, and Modeling}

\section{Location: Portrait Room}

Chairs: Christian Oberdorfer (The Ohio State University) and Stefan Parviainen (Université et INSA de Rouen)

Christian Oberdorfer, Travis Withrow, Iman Ghamarian, Li-Jen Yu, Kevin Fisher, Emmanuelle Marquis, Wolfgang Windl

Biased Solute Reconstruction Due to Athermal Surface Drag . . . . . . . . . . . . . . . . . . . . 155

Matthew Koppa, Paul Schwoebel, David Dunlap

Atomic Level Studies of Step Dynamics in Homogeneous Systems ～. . . . . . . . . . . . . . . 156

Stefan Parviainen, François Vurpillot

Molecular Dynamics Modeling of Surface and Subsurface Processes in Metals Under the Influence of High Electric

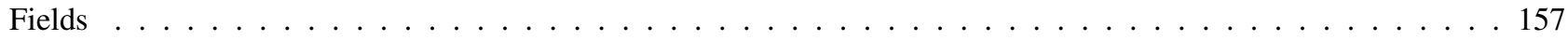

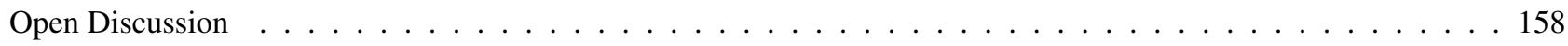

\section{Organic, Biological, Liquid, and Composite Materials}

\section{Location: Portrait Room}

Chairs: Derk Joester, Karen DeRocher, and Robert Free (Northwestern University)

Sebastian Koelling, Tomas Martin, Marcel Verheijen, Shweta Agarwal, Sergio Bertazzo, Remco Fijneman, Helen King, Anat Akiva, Paul Bagot, Michael Moody, Nico Sommerdijk, Molly Stevens, Paul Koenraad

Towards Mapping of Trace Elements in Bio-Minerals (Invited $) \ldots \ldots \ldots$. . . . . . . . . . 159

Stephan Gerstl, Robin Schaeublin

Experiences in APT Analyses of Fluids _ . . . . . . . . . . . . . . . . . . . . . . . 160

Gustav Sundell, Mats Hulander, Astrid Pihl, Martin Andersson

Atom Probe Tomography of Silica-Encapsulated Proteins . . . . . . . . . . . . . . . . . . . . . . . . 161

Xiaoyue Wang, Bryan Lee, Brian Langelier, Kathryn Grandfield

Atom Probe Tomography and Correlative Microscopy for Biological Materials (Invited) . . . . . . . . . . . . . 162

Karen DeRocher, Paul Smeets, Michael Cohen, Lyle Gordon, Derk Joester

Investigation of the Ion Distribution in Human Dental Enamel f . . . . . . . . . . . . . . . . . 163

Robert Free, Derk Joester

Atom Probe Tomography of Sound and Carious Rodent Tooth Enamel ～. . . . . . . . . . . . . . . . . 164 
Florant Exertier, Alexandre La Fontaine, Alexander Zavgorodniy, Julie Cairney

Investigation of Human Tooth Enamel Down to the Atomic Scale . . . . . . . . . . . . . . . . . . . 165

Upoma Guha, Baishakhi Mazumder, Nirupam Aich

Nanoscale Comparative Analysis of Human and Bovine Dental Enamel Composition Using Atom Probe Tomography 166

\section{IVAS Tutorials}

Location: Computer Lab

Chairs: Hugues Francois-Saint-Cyr

Hugues Francois-Saint-Cyr

IVAS 101 or How to Make the Most Out of Your Dataset Using Your Favorite 3D Reconstruction Wizard? . . . . . 167

Alec Day, Hugues Francois-Saint-Cyr

Approaching Perfection in Atomic Positioning: From Image Compression and $k$ Factors to Dynamic Considerations 168

\section{IFES Business Meeting}

Location: Green Auditorium

IFES Business Meeting . . . . . . . . . . . . . . . . . . . . . . . . . . . . . . . . . . . . . . . . . .

\section{Thursday Morning}

\section{Plenary 4}

\section{Location: Red Auditorium}

Chairs: Keith Knipling (U.S. Naval Research Laboratory)

Weng-Keen Wong

Artificial Intelligence and Machine Learning: An Overview from a National Science Foundation Perspective . . . . 175

\section{Metallurgy and Phase Transformations}

\section{Location: Red Auditorium}

Chairs: Goro Miyamoto (Tohoku University), Yongjie Zhang (Tohoku University), Rajarshi Banerjee (University of North Texas), and Talukder Alam (University of North Texas)

Frédéric Danoix

Nitrogen and Nitrides in Steels as Seen by Atom Probe Tomography (Invited) . . . . . . . . . . . . . . . . . . 176

Sharmistha Dhara, Ross Marceau, Kathleen Wood, Thomas Dorin, Ilana Timokhina, Peter Hodgson

Evolution of Carbide Precipitates in Ti-Mo Microalloyed Steel . . . . . . . . . . . . . . . . . . . . . 177

Yongjie Zhang, Goro Miyamoto, Tadashi Furuhara

Atom Probe Compositional Analysis of Nano-Sized Alloy Carbide in Multiple Microalloyed Low-Carbon Steels . 178

Keith Knipling

Core/Triple Shell Precipitates in Al-Er-Sc-Zr-(V,Nb,Ta) Alloys . . . . . . . . . . . . . . . . . . . . . . . . . . . 179

Felix Theska, Simon Ringer, Sophie Primig

Atom Probe Characterization of Strengthening Effects in the Superalloy 718 . . . . . . . . . . . . . . . . . 180 


\section{High-Field Nanoscience, Surface Science, and Field Ion Microscopy}

Location: Heritage Room

Chairs: Thierry Visart de Bocarmé (Universite Libre De Bruxelles)

Flyura Djurabekova, Andreas Kyritsakis, Mihkel Veske, Ekaterina Baibuz, Ville Jansson

Atomistic Simulations of Metal Surface Behavior Under High Electric Field (Invited) . . . . . . . . . . . . . . . . 181

Ekaterina Baibuz, Andreas Kyritsakis, Ville Jansson, Flyura Djurabekova

Electronic Properties of Adatoms Self-Diffusing on Metal Surfaces Under Electric Field . . . . . . . . . . . . . . 182

Shyam Katnagallu, Ali Nematollahi, Blazej Grabowski, Jörg Neugebauer, Dierk Raabe, Baptiste Gault

Applied Machine Learning in Field Ion Microscopy Aided by Field Ion Image Simulation . . . . . . . . . . . . . . 183

Norbert Kruse

Mimicking Electrochemical and Electrocatalytic Reactions With Atom-Probe Devices . . . . . . . . . . . . . 184

Richard Forbes

A Proposal That Constants Used Widely in Electron and Ion Emission Theory Should Be Included in International

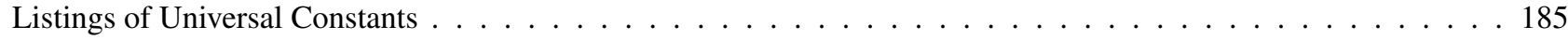

\section{Open Software Workshop}

Location: Portrait Room

Chairs: Karen Henry (Bechtel Marine Propulsion Corporation)

Current Status Of Available Software and Use Cases . . . . . . . . . . . . . . . . . . . . . 186

Practical Open-Source Software for APT . . . . . . . . . . . . . . . . . . . . 186

Characterization Virtual Laboratory: Sharing Closed-Source Software via a Cloud-Based Virtual Machine Acces-

sible with a Web Browser . . . . . . . . . . . . . . . . . . . . . . . . . . . . . . . . . . . . . . .

Atom-Blend: Using Rendering Engines in a Common Computer Graphics Package for High Quality Data Visualization and Animation . . . . . . . . . . . . . . . . . . . . . . . . 186

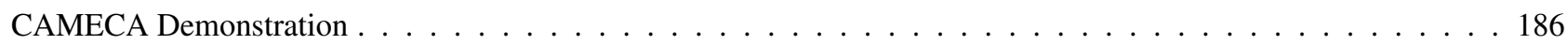

Flash Talks . . . . . . . . . . . . . . . . . . . . . . . . . . . . . 186

\section{Thursday Afternoon}

\section{Metallurgy and Phase Transformations}

\section{Location: Red Auditorium}

Chairs: Goro Miyamoto (Tohoku University), Yongjie Zhang (Tohoku University), Rajarshi Banerjee (University of North Texas), and Talukder Alam (University of North Texas)

Jun Takahashi, Kazuto Kawakami, Jun-ichi Hamada, Ken Kimura

Direct Observation of Niobium Segregation to Dislocations in Steel

Yukiko Kobayashi, Manabu Kubota, Jun Takahashi, Kazuto Kawakami

Atomic Scale Analysis on Retardation of Recrystallization of Work-Hardened Austenite in V-Added Low Alloy

High Carbon Steel . . . . . . . . . . . . . . . . . . . . . . . . . . . . . . 192

Irina Fedorova, Flemming Bjerg Grumsen, John Hald, Hans-Olof Andrén, Fang Liu

Analyzing Boron in 9-12\% Chromium Steels Using Atom Probe Tomography . . . . . . . . . . . . . . . . . . 
Hui Li, Wenqing Liu

The Segregation Features at Grain Boundaries With Various Characters in Highly Twinned Alloy 690 . . . . . . . 194

Arun Devaraj, Elizabeth Kautz, Libor Kovarik, Saumyadeep Jana, Curt Lavender, Vineet Joshi

APT and STEM Analysis of a Metallic Nuclear Fuel to Reveal the Influence of Grain Boundary Segregation on

Kinetics of Discontinuous Precipitation . . . . . . . . . . . . . . . . . . . . . . . . . . . . . .

Maria Auger, David Hoelzer, Kevin Field, Michael Moody

Nanoscale Analysis of Ion Irradiated 14YWTi ODS Steel . . . . . . . . . . . . . . . . . . . . . . . . 196

Mukesh Bachhav, Jian Gan, Brandon Miller, Lingfeng He, Dennis Keiser

Understanding Behavior and Performance of Nuclear Fuels via Atom Probe Tomography . . . . . . . . . . . . . . 197

Tomas Martin, Peter Martin, Anya Keatley, Paul Bagot, Yukihiko Satou, Michael Moody, Thomas Scott

Atom Probe Tomography as a Nuclear Forensics Tool - Analysing Nanoparticulate Material From the Fukushima

Region of Japan . . . . . . . . . . . . . . . . . . . . . . . . . . . . 198

Hyeji Im, Surendra Makineni, Baptiste Gault, Dierk Raabe, Pyuck-Pa Choi

Elemental Partitioning and Site Occupation of Mo and Cr in Co-Ti Based Superalloys . . . . . . . . . . . . . . . . 199

Rüya Duran, Patrick Stender, Guido Schmitz

Atom Probe Investigations of the Miscibility of $\mathrm{Cu}$ and $\mathrm{Ni} \ldots \ldots$. . . . . . . . . . . . . . . . . . . . . .

Natalia Gilis, Luc Jacobs, Sten Lambeets, Eric Genty, Cédric Barroo, Thierry Visart de Bocarmé

Superficial Enrichment in Gold/Silver Alloys: Study of the Physicochemical Influences Using Atom Probe Tomog-

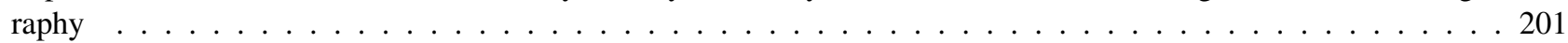

Andrew Breen, Isabelle Mouton, Agnieszka Szczepaniak, Wenjun Lu, Siyang Wang, Paraskevas Kontis, Leigh Stephenson, Yanhong Chang, Christian Liebscher, T. Ben Britton, Baptiste Gault, Michael Herbig, Dierk Raabe

Direct Observations of Atomic Hydrogen and Deuterium Interactions in Zircaloy-4 . . . . . . . . . . . . . . . 202

Hazel Gardner, Anna Radecka, David Rugg, David Armstrong, Michael Moody, Paul Bagot

Investigating the Effects of Oxygen and Nitrogen on Titanium Alloys for Turbine Engine Applications . . . . . . . 203

Mark Lapington, David Crudden, Roger Reed, Michael Moody, Paul Bagot

Atom Probe Characterization of Thermally-Grown Oxides Formed on Nickel Based Superalloys . . . . . . . . . . 204

\section{High-Field Nanoscience, Surface Science, and Field Ion Microscopy}

Location: Heritage Room

Chairs: Thierry Visart de Bocarmé (Universite Libre De Bruxelles)

Marwan Mousa, M-Ali AL-Akhras, Samer Daradkeh, Ala'a Al-Qudah, Hatem Braikat

Behaviour and Characterisation of Various Types of Carbon Nanotubes as Field Emitting Electron Sources . . . . . 205

Ivan Blum, Mario Borz, Olivier Torresin, Julien Mauchain, Benoit Chalopin, Adil Haboucha, Said Idlahcen, Ammar

Hideur, Alexander Obraztsov, Angela Vella

Electron Field Emission and Spectroscopy of Diamond Nano-Needles . . . . . . . . . . . . . . . . . 206

\section{Reconstruction Methods and Data Treatment \\ Location: Heritage Room}

Chairs: Anna Cegeurra (The University of Sydney), Peter Felfer (Friedrich-Alexander Universität), and Andrew Breen (MaxPlanck-Institut für Eisenforschung)

Isabelle Mouton, Shyam Katnagallu, Torsten Schwarz, Surendra Makineni, Tony Printemps, Adeline Grenier, Jean Paul Barnes, Oana Cojocaru-Mirédin, Dierk Raabe, Baptiste Gault

Calibration of Atom Probe Tomography Reconstructions From Correlation With Electron Tomograms or Micro-

graphs $($ Invited $) \ldots \ldots \ldots \ldots$ 
Daniel Beinke, Guido Schmitz

Trajectory-Based Atom Probe Reconstruction With Locally Varying Tip Shape . . . . . . . . . . . . . . . . 208

Richard Forbes

Making Atom-Probe Reconstruction Compatible With Newton's Laws . . . . . . . . . . . . . . . . . . . . . . . . 209

Constantinos Hatzoglou, Gérald Da Costa, François Vurpillot

Enhanced Dynamic Reconstruction for Atom Probe Tomography: A Post Bas Algorithm . . . . . . . . . . . . . 210

Brian Gorman, Anna Ceguerra, Andrew Breen, Julie Cairney, Simon Ringer, David Diercks

Methodologies for APT Data Reconstruction Based Upon Correlative Real Space Imaging and Diffraction With

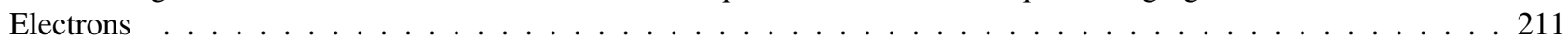

Alec Day, Anna Ceguerra, Simon Ringer

Novel Crystallographic Framework for Reconstruction in APT . . . . . . . . . . . . . . . . . . . . . . . 212

Anna Ceguerra, Simon Ringer

The Atomic Structure of Quasicrystals via Atom Probe Microscopy . . . . . . . . . . . . . . . . . . 213

Tammy Milillo, Scott Broderick, Krishna Rajan, Joseph Gardella

Applying Principles of Multivariate Analysis and Geospatial Interpolation Methods to Atom Probe Tomography

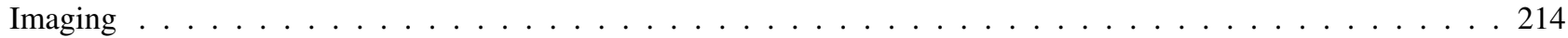

François Vurpillot, Constantinos Hatzoglou, Frédéric Danoix

From the Mass Spectrum to the Atom Map: An Alternative Approach to the Chemical Identification of Elements . 215

Enrico Di Russo, Ivan Blum, Jonathan Houard, Gérald Da Costa, Didier Blavette, Lorenzo Rigutti

Field-Dissociation Dynamics of $P_{m}^{i+}$ Molecular Ions in High DC Electric Field . . . . . . . . . . . . . . . . . . . 216

Olivia Licata, Scott Broderick, Christophe Hurni, James Speck, Krishna Rajan, Baishakhi Mazumder

Measuring Bond Strength Across Heterostructure Interfaces Using Atom Probe Data . . . . . . . . . . . . . . . . 217

\section{Open Software Workshop}

Location: Portrait Room

Chairs: Karen Henry (Bechtel Marine Propulsion Corporation)

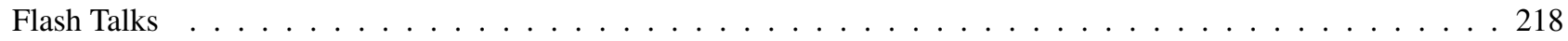

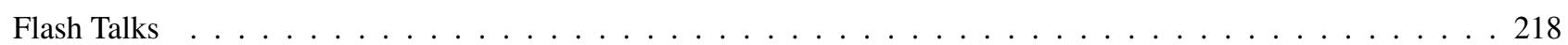

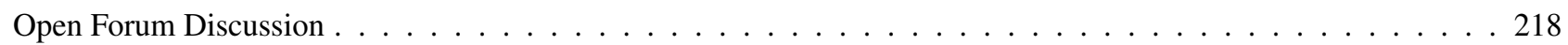

\section{Specimen Preparation, In-situ Specimen Treatment, and Novel Applications}

Location: Portrait Room

Chairs: Daniel Perea (Pacific Northwest National Laboratory) and Paul Bagot (University of Oxford)

Dieter Isheim

Advanced Preparation Methods for Challenging APT Specimens (Invited) . . . . . . . . . . . . . . . . . . . 219

Christina Hofer, Sandra Ebner, Thomas Kurz, Dominik Brandl, Ronald Schnitzer

Atom Probe Specimen Preparation of Steels by Transmission Kikuchi Diffraction . . . . . . . . . . . . . . . 220

Robert Morris, Karen Henry

Advanced Sample Preparation Techniques Using a Laser Ablation Instrument . . . . . . . . . . . . . . . . . . 221

Michael Schmidt, Nicholas Croy, Sang Hoon Lee, Katherine Rice

Semi-Automated Specimen Preparation for Atom Probe Tomography . . . . . . . . . . . . . . . . . . . . . . . . 222

Se-Ho Kim, Phil Woong Kang, O Ok Park, Jae-Bok Seol, Jae-Pyoung Ahn, Ji Yeong Lee, Pyuck-Pa Choi

A New Method for Mapping the Three-Dimensional Atomic Distribution Within Nanoparticles by Atom Probe

Tomography $(\mathrm{APT}) \ldots \ldots \ldots \ldots \ldots \ldots$ 
Brian Langelier, Ayman El-Zoka, Roger Newman, Gianluigi Botton

APT Analysis of Pt-Modified Nanoporous Gold ～. . . . . . . . . . . . . . . . . . . . . . . . . . . . . . . . 224

David Diercks, Rajesh Jha, Cristian Ciobanu, Aaron Stebner

In-Situ Crystallization of Metallic Glass in the Atom Probe ～. . . . . . . . . . . . . . . . . . 225

Ingrid McCarroll, Daniel Haley, Paul Bagot, Michael Moody, Sebastian Thomas, Nick Birbilis, Julie Cairney

Atomic Scale Analysis of Magnesium Oxide Scale ． . . . . . . . . . . . . . . . . . . . . . . . . 226

\section{Awards Banquet}

Location: Hilton Hotel, Grand Ballroom

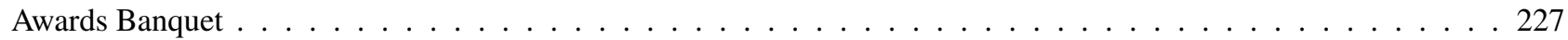

Sarah Hörst

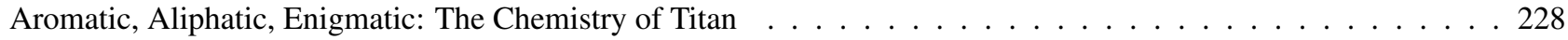

\section{Monday Posters}

\section{Ceramics, Geological, and Glass Materials}

Gabriel Arcuri, Desmond Moser, David Reinhard, Brian Langelier

Dating Large Impacts With Nanoscale Pb Isotope Clusters in Zircon: A Proof of Concept From the Vredefort

Central Uplift . . . . . . . . . . . . . . . . . . . . . . . . . . . . . . . . . . 231

James Douglas, Eleanor Jennings, Michael Moody, Jon Wade

Atom Probe Analysis of Silica Nanospheres in Diamond Anvil Experiments . . . . . . . . . . . . . . . 232

Daniel Schreiber, Daniel Perea, Joseph Ryan

Comparison of APT Measurements at Glass Corrosion Fronts From Wet Cryogenically Frozen and Supercritically

Dried Samples . . . . . . . . . . . . . . . . . . . . . . . . . . . . 233

Rick Verberne, David Saxey, Steven Reddy, William Rickard, Denis Fougerouse

Analysis of Natural Rutile by Laser-Assisted Atom Probe Microscopy . . . . . . . . . . . . . . . . . . . . 234

Daniel Perea, Daniel Gregory

Mapping Water-Filled Inclusions in an Archean Framboidal Pyrite . . . . . . . . . . . . . . . 235

\section{Semiconductors, Micro- and Opto-Electronic Devices}

Sung Ho Lee, Seong Pyo Hong, Yong Bum Kim, Eun Kyung Jeong, Sang Ick Lee, Joong Jung Kim

Quantitative Analysis of Dopant in Semiconductor Device Using Atom Probe Tomography . . . . . . . . . . 236

Rong Hu, Jing Xue, Yanbo Zhang, Huilong Zhu, Gang Sha

Characterization of a Si FinFET Structure and Dopants Distribution by Atom Probe Tomography . . . . . . . . . 237

Torsten Schwarz, Alex Redinger, Susanne Siebentritt, Baptiste Gault, Dierk Raabe, Pyuck-Pa Choi

Increased Detector Efficiency Helps to Reveal New Insights Into Compositional Fluctuations at Defects in $\mathrm{Cu}_{2} \mathrm{ZnSnSe}_{4}$

Thin Films . . . . . . . . . . . . . . . . . . . . . . . . . . . . . 238

A. Devin Giddings, Yong Yang, Ming-Hong Kao, Yi Ching Ong, Wei-Shan Hu, Ruey-Lian Hwang, Jang Jung Lee

Semiconductor Device Failure Analysis with Atom Probe Tomography . . . . . . . . . . . . . . . . . 239 
Mohit Raghuwanshi, Oana Cojocaru-Mirédin

Using Atom Probe to Identify Limitations in Photovoltaic Cells . . . . . . . . . . . . . . . . . . . . . . . . . . . . 240

Alexander Senichev, Jonathan Poplawsky, Mohammad Ali Shirazi, Brandon Dzuba, Oana Malis, Michael Manfra

Quantification of Indium Segregation in InAlN Thin-Films Grown by Plasma-Assisted Molecular Beam Epitaxy . . 241

\section{Specimen Preparation, In-situ Specimen Treatment, and Novel Applications}

Peter Felfer, Kayla Johnson, Jan Joosten

New Experimental Techniques for Nanoparticle Analysis . . . . . . . . . . . . . . . . . . . . . . . . . 242

Yimeng Chen, Katherine Rice, Isabelle Martin, Ty Prosa

New Approaches in Atom Probe Sample Preparation: STEM Imaging and Plasma FIB Milling . . . . . . . . . . 243

Robert Ulfig, Peter Clifton, Ty Prosa, David Reinhard, Jeffrey Shepard, David von Gunten, Urs Maier, Sigrun

Köster

Implementation of a Commercially Available UHV/Cryogenic Specimen Transfer System for LEAP Microscopes . 244

\section{Metals and Alloys}

Felicity Dear, Paul Bagot, Hazel Gardner, Anna Radecka, Michael Moody, David Rugg, David Dye

Crystallographic Ordering of Ti-Al Alloys in the Presence of Beta-Stabilisers and Interstitial Oxygen . . . . . . 245

Qin Shen, Zemin Wang, Hui Li, Wenqing Liu

The Determining Role of $\mathrm{Cu}$ on Precipitation Evolution and Mechanical Properties in Fe-CuNiAlMn Alloy . . . . 246

Na Li, Wei Wang, Shijuan Zhu, Bo Wang, Ziying Zhang

Concurrent $(\mathrm{Fe}, \mathrm{Mo})_{2} \mathrm{C}$ Carbides and $\mathrm{Cu}$-rich Clusters Precipitation in Ferritic Steel Containing Copper During Aging 247

R. Badyka, Cristelle Pareige, Christophe Domain, G. Monnet, S. Saillet

Quantification of Spinodal Decomposition and G-Phase Precipitation Contributions on Micro-Hardening of Ferrite of Aged Duplex Stainless Steel Owing to Microstructural Characterization Using APT Characterization . . . . . . 248

Seon-Hyeong Na, Jae-Bok Seol, Chan-Gyung Park

Effects of Ni Addition on the Grain Boundary Strengthening of High Mn Steel Weldments . . . . . . . . . . . 249

Jong Chan Han, Jae-Bok Seol, Chan-Gyung Park

APT Analysis of the Passivation Layer Formed on Austenitic Stainless Steels . . . . . . . . . . . . . . . . . . . . 250

Yanhong Chang, Andrew Breen, Zahra Tarzimoghadam, Leigh Stephenson, Wenjun Lu, Dirk Ponge, Dierk Raabe, Baptiste Gault

Characterizing Solute Hydrogen and Hydrides in Pure and Alloyed Titanium at the Atomic Scale . . . . . . . . . . 251

Masaya Kozuka, Yasuhiro Aruga, Shigenobu Nanba, Yimeng Chen, Katherine Rice, Ty Prosa

High-Detection-Efficiency APT Analyses of 100 at. ppm Ce Distribution in Heat-Resistant 18-8 Stainless Steel . . 252

Jennifer Zelenty, Nathan Almirall, Peter Wells, G. Robert Odette, James Douglas, Jonathan Hyde, Michael Moody,

George Smith, Nick Riddle, Keith Wilford

The Effect of Ni and Mn Content on Precipitation in Ion Irradiated Split Melt Test Steels . . . . . . . . . . . 253

Kyo Jin Hwang, Yimeng Chen, Yoon Jun Kim

Cluster Analysis of a Cast Aluminum Alloy Utilizing an Atom-Probe Tomography . . . . . . . . . . . . . . . . . 254

Mingxing Guo, Gaojie Li, Yidong Zhang, Gang Sha, Jishan Zhang, Linzhong Zhuang

Effect of Pre-Aging on the Bake Hardening Response of Al-Mg-Si-Cu-Zn Alloy . . . . . . . . . . . . . . . . . . 255

Yuan Wu, Huihui Zhu, Zhifeng Lei, Suihe Jiang, Zhaoping Lu

Characterization of Nano-Precipitation in Multiple Component Alloys . . . . . . . . . . . . . . . . . 256 
Bastien Bonef, Sean Harrington, Dan Pennachio, Chris Palmstrøm, James Speck

Nanometer Scale Investigation of Fe Clustering in Half-Heusler Half Metal Candidate CoTi1-xFexSb ～. . . . . 257

Johannes Webel, Dominik Britz, Jenifer Barrirero, Hardy Mohrbacher, Volker Flaxa, Frank Mücklich

Atomistic Investigation of Niobium Precipitation and Its Effect on Recrystallization in Steel . . . . . . . . 258

Sascha Seils, Daniel Schliephake, Torben Boll, Alexander Kauffmann, Martin Heilmaier

Austenitic Nanoclustered ODS Steels for High Temperature Applications ～. . . . . . . . . . . . . . . . . . . 259

Katja Eder, Xiaopeng Li, Gang Ji, Limei Yang, Sasan Dadbakhsh, Jozef Vleugels, Jan Van Humbeeck, Jean-Pierre Kruth, Julie Cairney

Atom Probe Investigation of a 3D Printed $\alpha / \beta$ Ti Alloy . . . . . . . . . . . . . . . . . . . . . 260

Yu-Chen Lin, Ingrid McCarroll, Julie Cairney, Hung-Wei Yen

Comparing the Effects of Micro-Alloy Carbides and Copper Clusters on Hydrogen Trapping in Martensitic Steels . 261

Yukiko Kobayashi, Jun Takahashi, Kazuto Kawakami, Kazuhiro Hono

Atom Probe Analysis on Chemical Composition of TiC Precipitates in Isothermally-Aged Ferritic Steel . . . . . 262

Shenbao Jin, Yafeng Yang, Qinglong Zhao, Gang Sha

Interface Chemistry Between Titanium Carbide and Metal Matrix in Al-Based Composite Revealed by Atom Probe

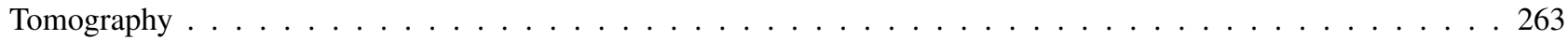

B. Chad Hornbuckle, Chris Marvel, Anthony Roberts, Tom Luckenbaugh, Martin Harmer, Kristopher Darling

Using Atom Probe to Study the Role Impurities Play on the Evolution of the Microstructure of Nanocrystalline

Alloys . . . . . . . . . . . . . . . . . . . . . . . . . . . . . . . . . . . . 264

Alex Costa, Julian Escobar, Joao Oliveira, Jonathan Poplawsky, Antonio Ramirez, André Tschiptschin

The Elemental Partition to the Secondary Phases Found in Co-Ni Based Superalloys After Solution and Ageing

Heat Treatments . . . . . . . . . . . . . . . . . . . . . . . . . . . 265

Keith Knipling, Joshua Tharpe, Peter Liaw

Nanoscale Phase Separation in $\mathrm{Al}_{0.5} \mathrm{CoCrFeNi}(\mathrm{Cu})$ High Entropy Alloys as Studied by Atom Probe Tomography . 266

Julian Escobar, Guilherme Faria, Camilo Salvador, Joao Oliveira, Torben Boll, Sascha Seils, Paulo Mei, Antonio

Ramirez

Kinetic Simulations of the Austenite Isothermal Growth During Tempering of 12Cr-6Ni-2Mo-0.02C-0.13Ti Super-

martensitic Stainless Steel . . . . . . . . . . . . . . . . . . . . . . . . . . . . . . . . . . . . . . . 267

Jenny Voss, Daniel Perea, Libor Kovarik, Mark Bowden, Norbert Kruse

Structural Promotion of Potassium in $\mathrm{CoCu}$ Catalysts for $\mathrm{CO}_{2}$ Hydrogenation ～. . . . . . . . . . . . . 268

Tim Lehmann, Patrick Stender, Guido Schmitz, Wolfgang Engelhart

Deviation of Ti-Al-N Composition During APT Analysis of $\mathrm{Ti}_{0.4} \mathrm{Al}_{0.6} \mathrm{~N} \ldots \ldots \ldots$. . . . . . . . . . . . . 269

Jae-Bok Seol, Jae Wung Bae, Jong Chan Han, Zhiming Li, Dierk Raabe, Hyoung Seop Kim

APT Study of Boron Doping in FCC Structured High-Entropy Alloys ～. . . . . . . . . . . . . . . . . . . 270

Huma Bilal, Bosong Liu, Keita Nomoto, Bernd Gludovatz, Simon Ringer, Jamie Kruzic, Anna Ceguerra

Bulk Metallic Glasses Under Atom Probe Microscopy Analysis . . . . . . . . . . . . . . . . . . . . . . . 271

\section{Organic, Biological, Liquid, and Composite Materials}

Utthara Rameshbabu, Alix Deymier, Jill Pasteris, Derk Joester

Mapping Carbonates in Enamel and Enamel-Like Carbonated-Hydroxylapatite . . . . . . . . . . . . . . . . 272

Torben Boll, Martin Heilmaier, James Earthman

Diamantane Particles in Cryomilled Nanocrystalline Aluminium . . . . . . . . . . . . . . . . . . . . . . . 273

Michelle Friedman, Baishakhi Mazumder, Krishna Rajan, Alan Friedman

An Analysis of a Unique Prostate Cancer Cell Line Model using 3D Imaging using Atom Probe Tomography . . . 274 
Jonas Ott, Patrick Stender, Guido Schmitz

APT Measurements of Glucose Solution Tips 275

\section{Tuesday Posters}

\section{Correlative and Combined Methods}

Jun Uzuhashi, Ken Pradel, Tadakatsu Ohkubo, Naoki Fukata, Kazuhiro Hono

Atom Probe Analysis of Nanoscale Voids in p-Type ZnO Nanowires . . . . . . . . . . . . . . . . . . . . . . 276

Joshua Einsle, Alex Eggeman, Benjamin Martineau, Zineb Saghi, Sean Collins, Roberts Blukis, Paul Bagot, Paul Midgley, Richard Harrison

Microstructural Investigation of an Fe-Ni Meteorite by Complementary APT and 3D Energy Dispersive Spectroscopy 277

Kevin Schweinar, Sebastian Beeg, Philipp Kerger, Caue Correa da Silva, Jing Cao, Matteo Amati, Luca Gregoratti, Patrick Zeller, Dierk Raabe, Robert Schlögl, Mark Greiner, Baptiste Gault

Correlative Atom Probe Tomography and In-Situ Scanning Photoelectron Emission Microscopy Applied to Catalytically Active Materials . . . . . . . . . . . . . . . . . . . . . . . . . . . . . . . 278

Robert Morris, Karen Henry

Complementary Studies of Additively Manufactured Stainless Steel Using Transmission Electron Microscopy and Atom Probe Microscopy . . . . . . . . . . . . . . . . . . . . . . . . . . . . . . . 279

Katherine Rice, David Larson, Yimeng Chen, Niyanth Sridharan, Suresh Babu Grain Boundary Segregation in Inconel 718 Superalloy . . . . . . . . . . . . . . . . . . . . . . . 280

Francisca Mendez-Martin, Georg Haberfehlner, Mihaela Albu

Materials Characterization With Atom Probe Tomography and High Resolution Transmission Electron Microscopy 281

Enrico Di Russo, Jonathan Houard, Vivien Langolff, Simona Moldovan, Lorenzo Rigutti, Bernard Deconihout, Didier Blavette, Janusz Bogdanowicz, Angela Vella

Correlative Study of the Optical Shaping of a Nano-Scale Tip by Femtosecond Laser Assisted Field Evaporation . . 282

Trevor Clark, Nicole Overman, Xiujuan Jiang, Suveen Mathaudhu, Arun Devaraj

High Resolution Multimodal Compositional Characterization of Nanocrystalline Soft Magnetic Materials . . . . . 283

\section{High-Field Nanoscience, Surface Science, and Field Ion Microscopy}

Stefanie Haugg, Christian Henkel, Chris Thomason, Robert Zierold, Robert Blick

Work Function Reduction Due to Water Adsorption on Nanostructured Materials Studied by Electron Field Emission284

Marwan Mousa, Emad Bani Ali, Mark Hagmann

Characterization of Multiwall Carbon Nanotubes as a Potential Electron Source . . . . . . . . . . . . . . . . 285

Marwan Mousa, Ala'a Al-Qudah, Samer Daradkeh, Hatem Braikat, M-Ali AL-Akhras

Field Emission Differences Between Carbon-Black Nanotubes and Single-Walled Carbon Nanotubes, Both Embedded in Glass Tubes . . . . . . . . . . . . . . . . . . . . . . . . . . . . . . . . . 286

Richard Forbes

A Proposal That Constants Used Widely in Electron and Ion Emission Theory Should Be Included in International Listings of Universal Constants . . . . . . . . . . . . . . . . . . . . . . . . . . . . . . . 287

Samer Daradkesh, Marwan Mousa, Richard Forbes

Fowler-Nordheim Plot Shape Associated With Large Series Resistance . . . . . . . . . . . . . . . . . . . . . . . . 288 


\section{Instrument and Technique Development}

Soeren Eyhusen, Fouzia Khanom, David Dowsett, Tom Wirtz

Secondary Ion Mass Spectroscopy with ZEISS ORION NanoFab . . . . . . . . . . . . . . . . . . . . . . . . 289

Jonathan Op de Beeck, Claudia Fleischmann, Kristof Paredis, Wilfried Vandervorst

Improving APT-AFM Technology: Towards High Resolution 3D APT Tip Shapes . . . . . . . . . . . . . . . . 290

Yanhong Chang, Leigh Stephenson, Agnieszka Szczepaniak, Wenjun Lu, Isabelle Mouton, Paraskevas Kontis, Abigail Ackerman, David Dye, Dirk Ponge, Christian Liebscher, Dierk Raabe, Baptiste Gault

Cryo-FIB — A Promising Technique to Prevent Undesired Hydrogen Pick-Up During FIB Milling . . . . . . . . 291

Jonathan Poplawsky, Nickolay Lavrik

A New Grid Design for Fast Correlative APT/TEM Sample Preparation . . . . . . . . . . . . . . . . . . . . . . . 292

Robert Ulfig, Ty Prosa, Dan Lenz, Joseph Bunton, Katherine Rice, Yimeng Chen, David Larson

Performance of the EIKOS Atom Probe Microscope . . . . . . . . . . . . . . . . . . . . . . . . . 293

Mark Hagmann, Marwan Mousa

Virtual Scanning Tunneling Microscope for Training and Teaching . . . . . . . . . . . . . . . . . . . . . . . . 294

David Reinhard, Tim Payne, Brian Geiser, Geoff Sobering, Ed Oltman, Jerry Mandt, Eric Strennen, David Larson,

Robert Ulfig, Ty Prosa, Isabelle Martin, Katherine Rice, Yimeng Chen, Hugues Francois-Saint-Cyr

A New Platform for Atom Probe Analysis: IVAS $4 \ldots \ldots$. . . . . . . . . . . . . . . . . . 295

Ann Chiaramonti, Luis Miaja-Avila, Paul Blanchard, David Diercks, Brian Gorman, Norman Sanford

Extreme Ultraviolet-Assisted Field Ion Evaporation . . . . . . . . . . . . . . . . . . . . . . . . . . . 296

\section{Laser/Matter Interactions}

Deodatta Shinde, Laurent Arnoldi, Arun Devaraj, Angela Vella

Laser-Material Interaction During Atom Probe Tomography of Oxides With Embedded Metal Nanoparticles . . . . 297

\section{Quantification in Atom Probe}

Masoud Dialameh, Claudia Fleischmann, Janusz Bogdanowicz, Natascia De Leo, Luca Boarino, Wilfried Vander-

vorst

Standardized APT Tips for Interlaboratory Studies ～. . . . . . . . . . . . . . . . . . . . . . . . . 298

Cristelle Pareige, Constantinos Hatzoglou, Gérald Da Costa, Philippe Pareige, M Roussel, Bertrand Radiguet Quantification of APT Physical Limitations on Chemical Composition of Precipitates in Fe-Cr Alloys . . . . . . 299

Tomokazu Sasaki, Kazuya Toda, Naomi Arai, Yutaro Hori, Norihito Mayama, Yutaka Fukushima Quantification of Halogen in Si-Substrate for Laser-Assisted APT ～. . . . . . . . . . . . . . . . 300

Davit Melkonyan, Claudia Fleischmann, Janusz Bogdanowicz, Richard Morris, Ramya Cuduvally, Wilfried Vandervorst

Correcting the Boron Concentration for the Detection Losses Through Multi Hit Events . . . . . . . . . . . . 301

Jun Takahashi, Kazuto Kawakami, Yukiko Kobayashi

Study on Quantitative Analysis of Carbon and Nitrogen in Stoichiometric Cementite and Roaldite by Atom Probe

Tomography . . . . . . . . . . . . . . . . . . . . . . . . . 302

Enrico Di Russo, Ivan Blum, Jonathan Houard, Gérald Da Costa, Didier Blavette, Lorenzo Rigutti

Field-Dependent Measurement of GaAs Composition by Atom Probe Tomography . . . . . . . . . . . . . 303

Chikasa Nishimura, Yuki Haneji, Hiroshi Tsuji, Yasuhito Gotoh

Analysis of Field Evaporated Ions From Transition Metal Nitrides . . . . . . . . . . . . . . . . . . . 304 
Constantinos Hatzoglou, Bertrand Radiguet, Philippe Pareige

Correction of APT Chemical Composition Measurements Biases in Nano-Particles: A Chemical Composition

Correction Model . . . . . . . . . . . . . . . . . . . . . . . . . . 305

Scott Broderick, Bhargavea Urala Kota, Tianmu Zhang, Ramachandran Subramanian, Srirangaraj Setlur, Venu Govindaraju, Krishna Rajan

Discrimination of Ions Through MacHine Learning of Atom Probe Data . . . . . . . . . . . . . . . . . 306

David Larson, Ty Prosa, Ed Oltman, David Reinhard, Brian Geiser, Robert Ulfig

A High Multiple Hits Correction Factor for Atom Probe Tomography . . . . . . . . . . . . . . . . . . . . . . 307

Dimitry Kouzminov, James Cournoyer, Harish Muthuraman, Somchintana Norasetthekul, Qi Gao

Quantitative Aspects of PLAD Sidewall Doping Characterization by SIMS and APT . . . . . . . . . . . . . 308

\section{Reconstruction Methods and Data Treatment}

Anna Ceguerra, Alec Day, Simon Ringer

Measuring the Spatial Accuracy of an Atom Probe Tomography Reconstruction . . . . . . . . . . . . . . . . . . 309

Shyam Katnagallu, Giuseppe Di Bernardo, Leigh Stephenson, Markus Rampp, Frédéric De Geuser, Dierk Raabe, Baptiste Gault

Automated Calibration of Atom Probe Reconstructions Using GPU Based Fourier Analysis in a Python Workbench 310

Jens Keutgen, Oana Cojocaru-Mirédin, Matthias Wuttig

EPOSA - User-Friendly Software for Expanded Atom Probe Analysis . . . . . . . . . . . . . . . . . . . 311

David Diercks, Brian Gorman

Self-Consistent Reconstructions Utilizing Electron Microscopy . . . . . . . . . . . . . . . . . . . 312

Takumi Kitayama, Masaya Kozuka, Yasuhiro Aruga, Chikara Ichihara

A Precise Method for Analysis of Elemental Distribution Inside Solute Clusters . . . . . . . . . . . . . . . . . . 313

Andrew Proudian, Matthew Jaskot, David Diercks, Brian Gorman, Jeramy Zimmerman

Reconstruction Solution Envelopes for Improved Morphological Confidence . . . . . . . . . . . . . . . . . . . . 314

Dallin Barton, B. Chad Hornbuckle, Kristopher Darling, Gregory Thompson

Seeing Is Not Always Believing: The Influence of Isosurface Selection in Quantitative Outputs From Proximity

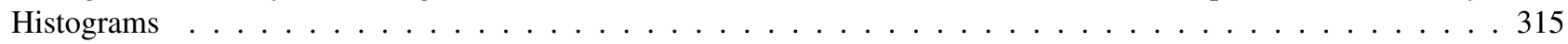

Paul Blanchard, Ann Chiaramonti, Norman Sanford, Sarah Barili

An Open-Source, Graphical Software Package for Computer-Assisted Species Assignment in Atom Probe Tomog-

raphy Compositional Analysis . . . . . . . . . . . . . . . . . . . . . . . . . . . . . . . . .

Sean Jones, Brian Gorman

Detector Space Fourier Analysis for Atom Probe . . . . . . . . . . . . . . . . . . . . . . . . . 317

Leigh Stephenson, Shyam Katnagallu, Huan Zhao, Anna Ceguerra, Andrew Breen, Simon Ringer, Dierk Raabe,

Baptiste Gault

Direct Determination of Misorientation and Grain Boundary Parameters From APT Projections . . . . . . . . . . . 318

Anna Ceguerra, Ingrid McCarroll, Alexandre La Fontaine, Julie Cairney

Atom Probe Learning Module on MyScope . . . . . . . . . . . . . . . . . . . . . . . . . . . . . . . 319

\section{Theory, Simulations, and Modeling}

Charlotte Lynch, Michael Moody, Daniel Haley

Density Functional Theory for Ion Identity in Mass Spectra . . . . . . . . . . . . . . . . . . . . . . . . . . 320

Yu-Ting Ling, Janusz Bogdanowicz, Claudia Fleischmann, Wilfried Vandervorst

A Layer-By-Layer Reconstruction Method Including Field of View Effects, Missing Atoms and Laser Effects . . . 321 
Proceedings of Atom Probe Tomography \& Microscopy (APT\&M) 2018, Washington, DC, June 10-15, 2018

NIST SP 2100-03

Markus Kühbach, Andrew Breen, Michael Herbig, Baptiste Gault, Dierk Raabe

Building a Library of Simulated Atom Probe Data for Different Crystal Structures and Pillar Orientations Using

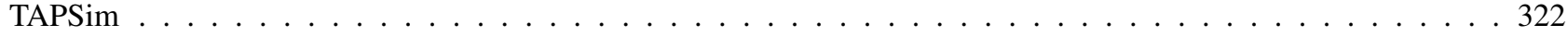

Richard Forbes

Comment on the Competing Influences of Field-Energy and PPI Terms on the Activation Energy for Partial-Ion

Migration at Highly Charged Surfaces . . . . . . . . . . . . . . . . . . . . . . . 323

\section{Author Index}





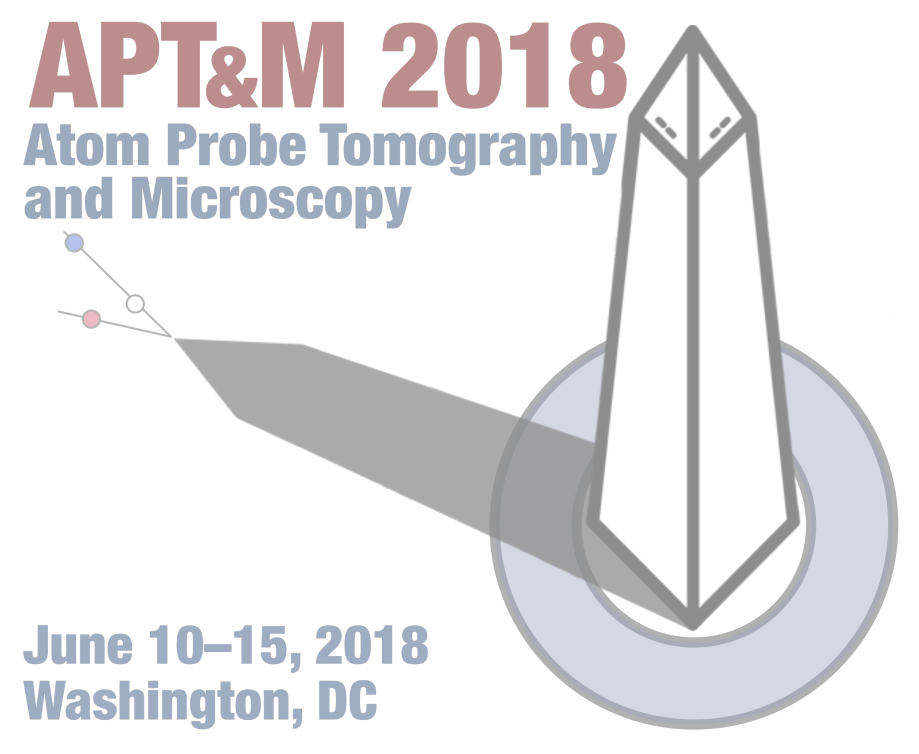

Welcome to APT\&M 2018 



\section{NIST Welcomes APT\&M 2018}

The biennial International Field Emission Symposium, recently renamed Atom Probe Tomography and Microscopy (APT\&M), has been in existence since 1952. It was at the $14^{\text {th }}$ Field Emission Symposium (1967), held at the NBS, in the Green Auditorium, that Erwin W. Müller and John A. Panitz first introduced the atom probe field ion microscope. (A portion of the program is on pages 21-22.) Now, a half-century later, APT\&M returns to the NIST-Gaithersburg campus.

The National Institute of Standards and Technology (NIST), formerly known as the National Bureau of Standards (NBS) was founded in 1901. NIST is the national metrology institute of the United States of America. NIST has two main campuses at Gaithersburg, Maryland and Boulder, Colorado. NIST conducts world-class research and creates critical measurement solutions (e.g. timekeeping, manufacturing, forensic science, computer security, power grid, nanotechnology, quantum science, and many more applications), often in close collaboration with industry, to advance measurement science, standards,

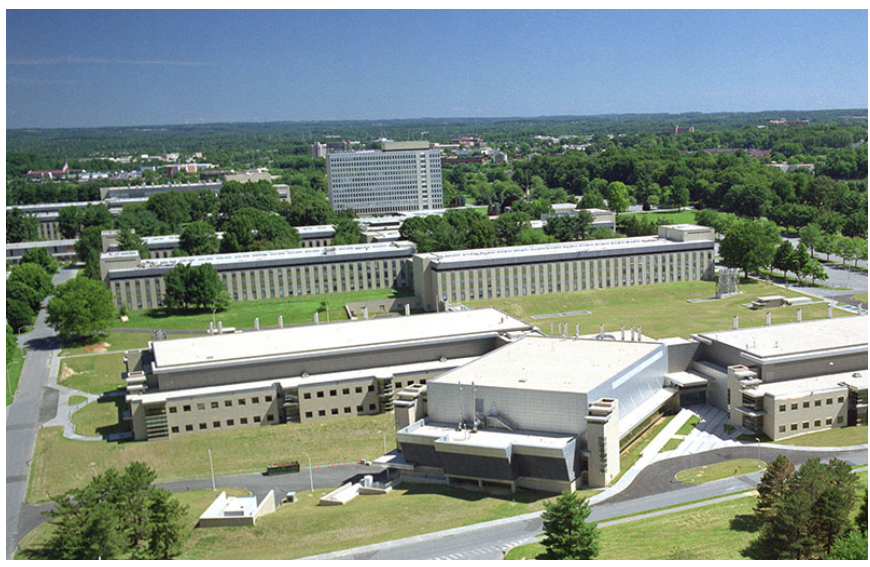

NIST campus in Gaithersburg, Maryland. and technology and help U.S. companies continually improve products and services. The 234-hectare Gaithersburg campus serves as the headquarters for NIST. NIST is home to approximately 3000 staff members and more than 1500 associates organized into five different laboratories - Communications Technology Laboratory, Engineering Laboratory, Information Technology Laboratory, Material Measurement Laboratory, and Physical Measurement Laboratory. The NIST core competencies are measurement science, rigorous traceability, and the development and use of standards.

\section{IFES Steering Committee}

David Larson, President (CAMECA Instruments, Inc.)

François Vurpillot, Vice-President (University of Rouen)

Gregory Thompson, Secretary (University of Alabama)

Michael Moody, Treasurer (University of Oxford)

Julie Cairney (University of Sydney)

Mattias Thuvander (Chalmers University of Technology)

Stephan Gerstl (EMEZ, ETH)

Gang Sha (China Academy of Engineering Physics)

Ross Marceau (Deakin University)

Baptiste Gault (MPIE, Düsseldorf)

\author{
Program and Planning Committee \\ Fred Meisenkothen, Chair (NIST) \\ Eric Steel, Co-Chair (NIST) \\ Keana Scott (NIST) \\ Scott Wight (NIST) \\ Gladys Arrisueno (NIST) \\ Ann Chiaramonti (NIST) \\ Keith Knipling (NRL) \\ Karen Henry (BMPC)
}





\section{Featured Lecturers}

\section{David Saxey}

Dr. David Saxey received his Ph.D. in experimental physics from the University of Western Australia in 2004 and has worked in high-precision instrumentation and materials characterization, with more than 10 years experience in research and technique development in atom probe microscopy. His current research interests are in the application of atom probe techniques to geological research, and the development of atom probe data analysis methods.

David has managed atom probe facilities at the University of Sydney (Australian Centre for Microscopy and Microanalysis, 2004-2006) and the Uni-

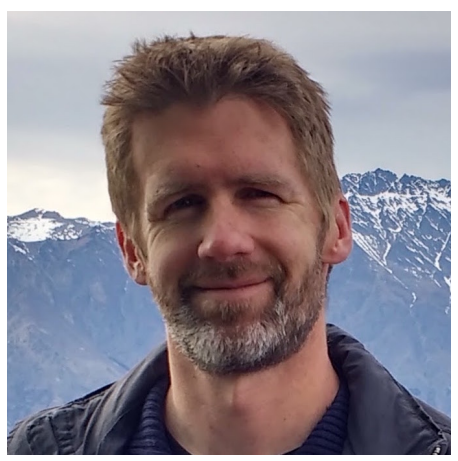
versity of Oxford (UK National Atom Probe Facility, 2007-2010) and has applied APM methods to a wide range of materials, within both academic and industrial collaborations. Prior to his current position, David was lead scientist on a commercial project developing the next generation of airborne gravity survey systems for resources exploration. In August 2015, David joined Curtin University and now manages the Geoscience Atom Probe facility within the John de Laeter Centre. The facility was established in 2015 with the purpose of applying atom probe microscopy to geoscience research.

\section{Allan Melmed}

Dr. Allan Melmed received his Ph.D. in physics from Penn State University in 1958, advised by Erwin W. Müller. After graduation, Dr. Melmed took a post-doctoral position at the University of Chicago from 1958-1960. Other professional positions include Research Physicist at DuPont deNemours Co. (1958-1964); Physicist at NBS/NIST (1964-1989); Proprietor at Custom Probes Unlimited (1987-2011): Visiting Professor at the University of Wisconsin (1989-1990); Research Professor at Johns Hopkins University (1990-2000); Director of Alpine Research and Development Laboratory (2001-2008).

Dr. Melmed's research interests include studying atomic structure by field

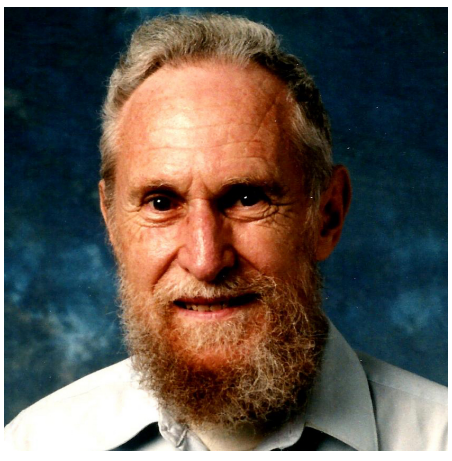
ion microscopy (FIM), especially defects in metals, semiconductors and high-temperature superconductors, epitaxy of metals, structure of icosahedral materials, improving atom probe mass resolution, developing techniques for FIM imaging of new materials, developing ways to consistently make very sharp metal and semiconductor tips for FIM, scanning tunneling microscope (STM), electron and other microscopes, and measuring field electron emission in poor vacuum. 


\section{John Panitz}

Dr. John Panitz is Emeritus Professor of Physics at the University of New Mexico (UNM). During his tenure at UNM he was Professor of Physics, Professor of High Technology Materials, and Professor of Cell Biology and Physiology in the School of Medicine. At UNM he developed the first undergraduate laboratory courseware that encouraged both critical thinking and role playing in the structured environment of cooperative learning groups. In 1967 he introduced, with his thesis advisor Erwin Müller, the Atom-Probe Field Ion Microscope. In 1970 he joined the Surface Science division at Sandia National Laboratories in Albuquerque where he invented and patented the LiFE Detector (a field emission chemical sensor) and the

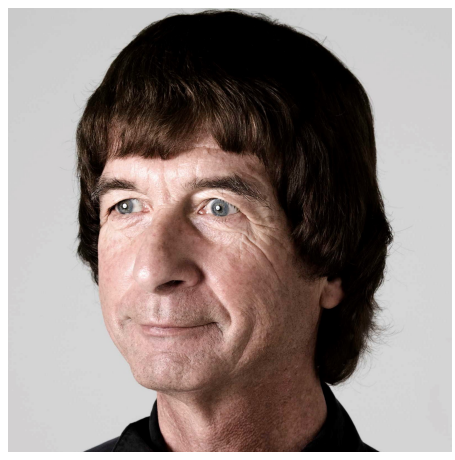
Field Desorption Spectrometer or "Imaging Atom-Probe" that became the progenitor of atom-probe tomography. In 1993 he founded High-Field Consultants. 


\section{Plenary Speakers}

\section{Peter Ercius}

Dr. Peter Ercius graduated from Cornell University with a B.S. in applied and engineering physics in 2003. He remained at Cornell and completed a Ph.D. in applied and engineering physics

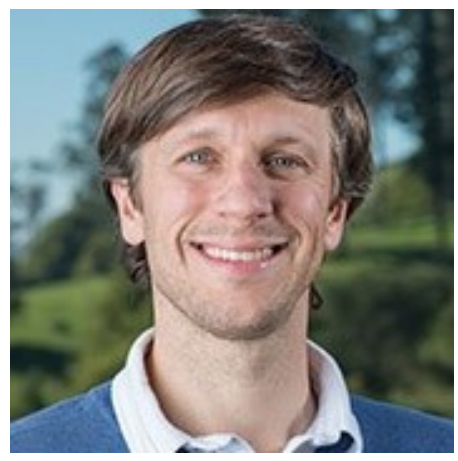
with Professor David A. Muller in 2009. His dissertation project focused on three-dimensional (3D) electron tomography of semiconductor devices using scanning transmission electron microscopy (STEM). He then joined the NCEM facility as a collaborative post-doctoral researcher for two years before being hired as a permanent Staff Scientist of the Molecular Foundry. Peter is currently in charge of the electron tomography program at NCEM and the dual aberration-corrected TEAM 0.5 microscope. Dr. Ercius is a leading expert in electron tomography and collaborates with users of the Molecular Foundry on a wide range of projects including S/TEM atomic resolution imaging, electron tomography, 4D-STEM scanning diffraction, in situ liquid TEM, and electron energy loss spectroscopy (EELS).

\section{Henry Kapteyn}

Dr. Henry C.
Kapteyn is CEO of
Kapteyn-Murnane
Laboratories Inc.
(KMLabs), and a
Professor of Physics
and ECE at the Uni-
versity of Colorado
at Boulder, and a
Fellow of JILA, a

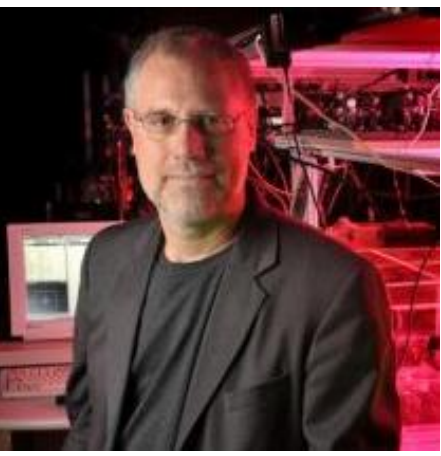

Research Institute joint between the University of Colorado and NIST. He and his wife and long-term collaborator, Margaret Murnane, are well known for their research in femtosecond lasers, and for understanding how to coherently upconvert this light to make a "tabletop X-ray laser" that generates ultrashort bursts of short-wavelength light. In recent years, they have applied this source to pioneering studies of atomic, molecular, and material studies at short length- and time-scales. He has published more than 200 papers in topics ranging from laser science and engineering to materials to nanoimaging. He was elected to the U.S. National Academy of Sciences in 2013, and is a fellow of the American Physical Society, the Optical Society of America, and the American Association for the Advancement of Science. His awards include the Adolph Lomb Medal of the OSA in 1993, the Ahmed Zewail Award of the ACS in 2009, the R.W. Wood Prize of the OSA in 2010, the Arthur Schawlow Prize of the APS in 2010, and the Willis Lamb Award in Quantum Electronics in 2012. Since 2015, his time has been primarily at KMLabs, spearheading the efforts to commercialize coherent EUV laser technologies and novel ultrafast lasers.

\section{Christoph Freysoldt}

Christoph Freysoldt is group leader for Defect Chemistry and Spectroscopy in the Computational Materials Design Department of the Max-Planck-Institut für Eisenforschung $\mathrm{GmbH}$. His main fo-

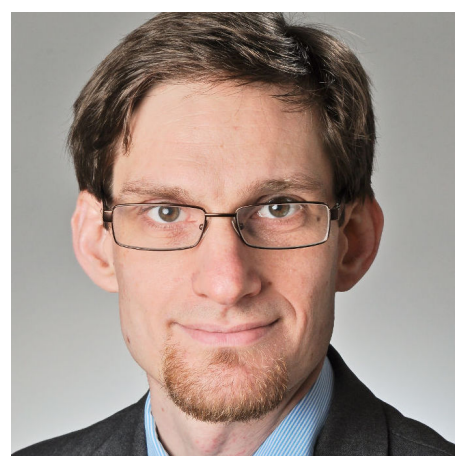
cus is to develop multi-scale simulation techniques for point defects and related topics, and to apply them to problems in functional materials de- 
sign. He also maintains and actively develops the SPHInX density-functional theory package. His work on charged supercell calculations has recently drawn his attention to the simulation of field evaporation and atom probe tomography based on density functional theory.

\section{Weng-Keen Wong}

Dr. Weng-Keen Wong is currently serving as a rotator at the National Science Foundation. He is a Program Director in the Robust Intelligence Program in the Division of Information and Intel-

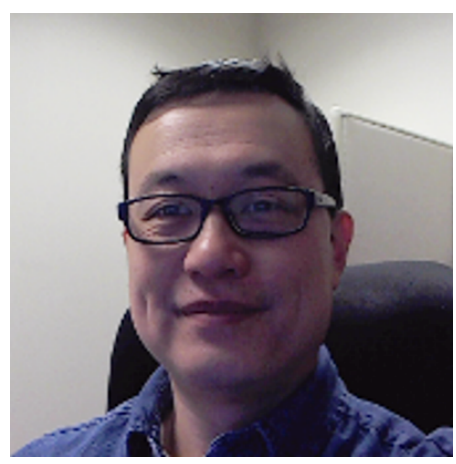
ligent Systems. His home institution is Oregon State University where he is an Associate Professor of Computer Science. He received his Ph.D. (2004) and M.S. (2001) in Computer Science at Carnegie Mellon University. He received his B.Sc. (1997) from the University of British Columbia. His research areas are in data mining and machine learning, with specific interests in anomaly detection, probabilistic graphical models, computational sustainability, and human-in-the-loop learning. 


\section{Invited Speakers}

\section{Steve Reddy}

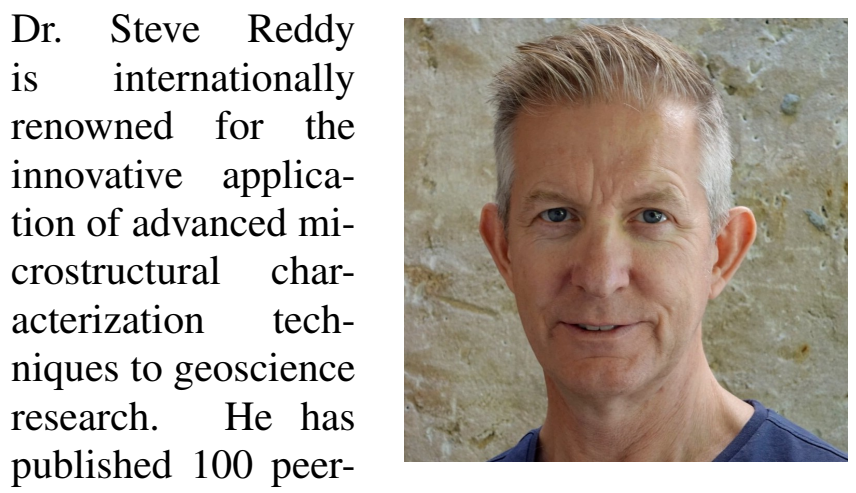
reviewed journal articles. He is a structural geologist, geochemist, and geochronologist with research interests spanning large-scale tectonic processes to nanogeoscience. His expertise lies in the advanced characterization of geological materials, particularly innovative integration of high spatial resolution microstructural and microgeochemical techniques. In recent years, his research has focused on the development of geological applications of atom probe microscopy and he is currently the Science Leader of the Geoscience Atom Probe Facility at Curtin University - the world's first geoscience-focused atom probe facility. He has been at Curtin University for the past 19 years and, in addition to standard academic research and teaching positions, has held the position of Dean of Research for Curtin's Faculty of Science and Engineering.

\section{Williams Lefebvre}

Dr. Williams Lefebvre is an associate professor at the University of Rouen. He received a Ph.D. degree in Materials Science in Rouen in 2001 on the correlative analysis by transmission elec- tron microscopy and atom probe tomography (APT) of phase transformations in titanium aluminides. He earned a fellowship of the Japan Society for the promotion of science in 2002, when he visited the NIMS of Tsukuba. He also worked as a post-doctoral research associate with Chalmers University in 2002. Dr. Lefebvre became an associate professor in the Materials Physics Group in Rouen in 2002 and, since 2014, is a visiting adjunct research associate professor at the University of Nebraska Lincoln (USA). He has been leading research activities in the field of physical metallurgy, focusing on light alloys systems, aiming at improving the methodology associated with the investigation of early stages of precipitation by APT and scanning transmission electron microscopy (STEM). Since 2011, he has lead research projects aiming at combining APT and STEM for the characterization of single nano-objects, taking advantage of the quantification of high-angle annular dark-field imaging (HAADF) STEM images and, more recently, he and his collaborators are working on the combination of an APT and a TEM in a single instrument.

\section{Alain Portavoce}

\section{Dr. Alain Portavoce} received his Ph.D. in Materials Science in 2002 from AixMarseille University. After a postdoctoral position at the Materials Research Science and Engineering Center

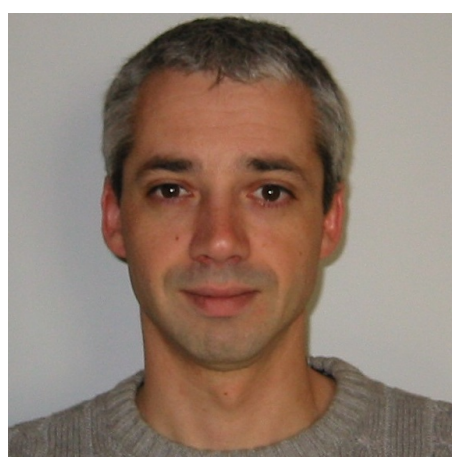
(MRSEC) of the University of Virginia (Charlottesville, USA), he was offered a permanent position by the French National Center of Scientific Research (CNRS) in 2005, for joining the Institute of Materials Microelectronics and Nanoscience 
of Provence (IM2NP, Marseille), where he leads the Reactivity and Diffusion at Interfaces research team. His expertise includes elaboration (molecular beam epitaxy, sputtering, etc.) and characterization (Auger electron spectroscopy, atomic force microscopy, secondary ion mass spectrometry, atom probe tomography, etc.) of thin films and nanostructures. His work mainly concerns nanometer scale solid state diffusion, segregation and reaction in materials (semiconductors, silicides, germanides, etc.) involved in the fabrication of micro-/nano-electronic devices.

\section{Yasuo Shimizu}

\section{Dr. Yasuo Shimizu} received a Ph.D. in engineering with experimental studies of dopant and host atom behavior in $\mathrm{Si}$ using isotopic heterostructures in Prof. Kohei M. Itoh's Group at Keio Uni-

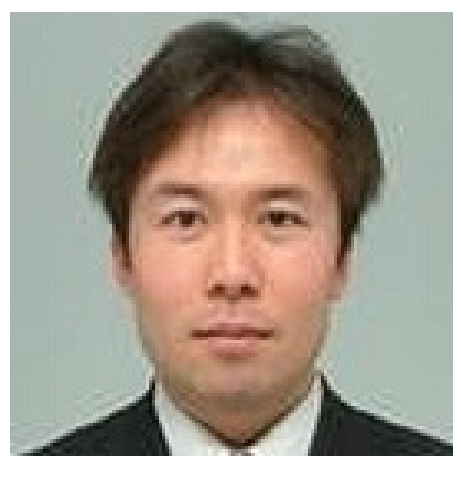
versity (Japan) in March 2009. He worked as Research Fellow of the Japan Society for the Promotion of Science (JSPS) in 2008 and 2009. After his study of a conformal doping technique with vapor phase doping and atomic layer epitaxy on GroupIV semiconductors at IMEC vzw (Belgium) as a post-doctoral researcher at Katholieke Universiteit Leuven, he joined the Oarai Center, Institute for Materials Research at Tohoku University (Japan) in April 2010 as Assistant Professor in Prof. Yasuyoshi Nagai's Group. His research is focused on elemental distribution analysis in semiconductor ( $\mathrm{Si}, \mathrm{Ge}$, etc.)-based nanostructures, and biomaterials by atom probe tomography.

\section{Rongkun Zheng}

Dr. Rongkun Zheng obtained his B.Sc. in Physics from Shandong University in China in 1999 and his Ph.D. in Physics from the Hong Kong University of Science and Technology in 2004. He

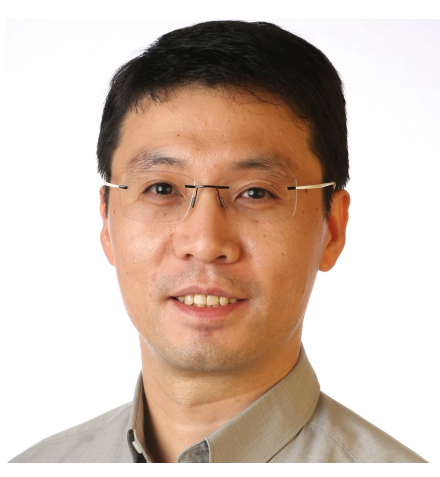
joined the University of Sydney in late 2004, and currently is an associate professor at the School of Physics. His research interest span from condensed matter and materials physics to microscopy and microanalysis, with focus on the growthstructure-property relationships in functional materials and devices using sophisticated microscopy and microanalysis, particularly atom probe tomography (APT) and transmission electron microscopy (TEM). He has published more than 150 papers and has received over 5700 citations. He has received a number of awards, including a prestigious fellowship from the Australian Research Council, and has been regularly invited to national and international conferences in his field.

\section{Paul van der Heide}

Dr. Paul van der Heide is the Director of MCA (Materials and Component Analysis) at imec, in Leuven, Belgium. The scope of MCA is a) to support the materials characterization needs of the

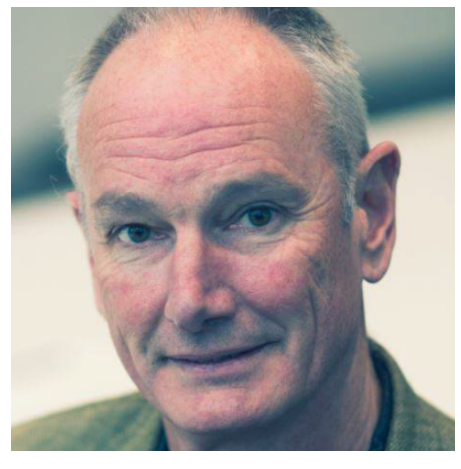
imec R\&D facilities (characterization capabilities include APT, Raman, RBS, SIMS, SPM, TEM, XPS, etc.), and b) to explore, develop and implement the characterization capabilities required for tomorrow's electronics industry. Prior to mov- 
ing to imec, Paul held positions at GLOBALFOUNDRIES, Malta, NY, USA (where he headed the analytical labs support for CMOS manufacturing and R\&D, with APT again being part of the characterization cache), Samsung, Austin, TX, USA (where he established and managed the surface analysis lab for supporting high volume CMOS manufacturing), and the University of Houston, (where he lectured courses in physical chemistry and surface analysis, while also managing the MRSEC SIMS-XPS facility). Paul earned a Ph.D. in Physical Chemistry from the University of Auckland, New Zealand (topic concerned the design and construction of a magnetic sector SIMS instrument), has authored over 100 publications in international peer reviewed journals, and is sole author of two books published through Wiley.

\section{Konda Gokuldoss Pradeep}

\section{Dr. Konda Gokul- doss Pradeep is the Group Leader for Atom Probe Tomog- raphy at the Mate- rials Chemistry In- stitute of RWTH Aachen University in Germany. $\mathrm{He}$ earned his Ph.D. in}

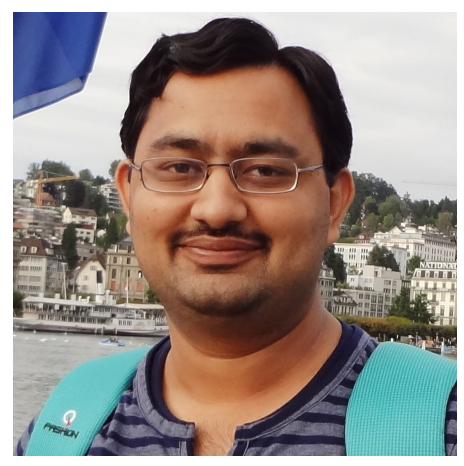
Materials Engineering from RWTH Aachen University for the research performed at Max-PlanckInstitut für Eisenforschung $\mathrm{GmbH}$. His main research focus is on alloy design including the use of combinatorial approaches in order to identify, stabilize specific phases, structures towards developing materials exhibiting outstanding structural and functional properties. Some of the materials of interest include multi-component high-entropy alloys, soft magnetic materials, steels, and magnesium alloys. A significant part of his research activity also includes multi-scale micro/nano characterization of metallic materials and development of correlative microscopic techniques.

\section{Benoit Gervais}

After studying physics as the University of Caen (France), Dr. Benoit Gervais received his Ph.D. for his work about swift heavy ions interaction with condensed matter. This was followed

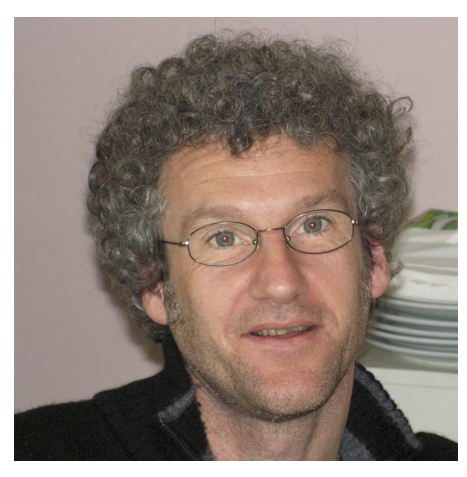
by a past-doctoral placement in the team of Professor J. Burgdörfer at the University of Tennessee, Knoxville (USA). Dr. Gervais returned to France in 1995 with a permanent position at the Commissariat à l'Energie Atomique (CEA) in Caen. The first part of his research career was dedicated to numerical simulation of ion and electron transport through solids. Dr. Gervais has investigated three main topics in this field: the electron emission from solids under swift ion impact, the transport of swift ionic projectiles and the formation of their excited states during transport, and finally the water radiolysis with a particular emphasis on the formation of $\mathrm{HO}_{2}$ radicals and $\mathrm{O}_{2}$ molecules in radio-chemistry.

In the early 2000s, Dr. Gervais moved to chemical physics and he investigated model systems made of alkali atoms and molecules trapped in rare gas matrix, either as solids or clusters. In this field Dr. Gervais has developed non-adiabatic molecular dynamics simulation codes to investigate the properties and the relaxation dynamics of such molecular systems in electronic excited states populated by photon absorption. Dr. Gervais has directed several Ph.D. students in this research field. This work was further extended to study halides and hydroxide anion solvated in small clusters made of a few water molecules. This is one of his current research topics.

Since 2015, Dr. Gervais has been involved in the AQURATE project dedicated to the investigation of molecular ion production and relaxation in the 
field of atom probe tomography (APT). Dr. Gervais is working on the stability of dicationic dimers emitted in the high electric field generated by the tips used in APT experiment to analyze the socalled "dissociation tracks" observed in correlated time-of-flight maps. MHis current interest in this field covers all aspects related to ion emission mechanisms from the tip surface in APT.

\section{Baptiste Gault}

Dr. Baptiste Gault is the Group Leader for Atom Probe Tomography at the MaxPlanck-Institute für Eisenforschung in Düsseldorf. He is a member of the steering committee of the International Field

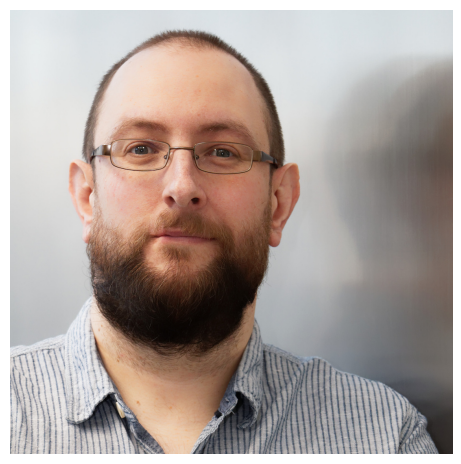
Emission. Dr Gault has a deep knowledge of the fundamental aspects of the technique, with a particular emphasis on data reconstruction and extraction. His group has a broad activity in the field of APT, with projects aiming to link properties to the nanoscale structure and composition of engineering materials finding application in e.g. power generation (Ni-based superalloys), energy conversion (solar cells, thermoelectrics), aerospace ( $\mathrm{Ti}$, Co-based superalloys), automotive (high-strength steel, Al-alloys), glass moulding, etc.

\section{Osamu Nishikawa}

Dr. Osamu Nishikawa
joined the Field
Emission Labora-
tory of Physics De-
partment of Penn
State in 1959 as a re-
search graduate stu-
dent. He received
his Ph.D. in physics
in 1964, advised by

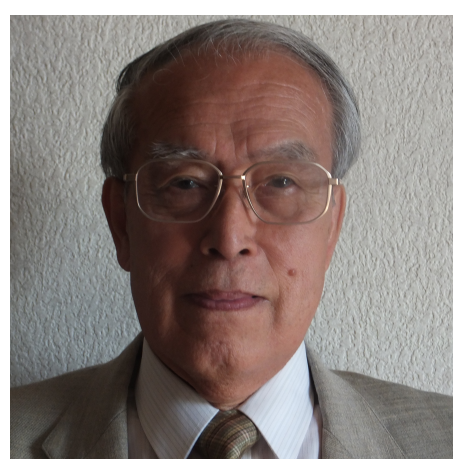

Prof. Erwin Müller. In 1976 he became at Professor at the Tokyo Institute of Technology and in 1992 and shortly thereafter presented the idea of scanning atom probe (SAP) at IFES in Nagoya, Japan. Since the SAP does not require a sharp tip, it allows the analysis of soft materials such as polymers and biomolecules. The novel electrode used in SAP was ultimately developed by Imago as the local electrode atom probe (LEAP).

\section{Jonathan Poplawsky}

Dr. Jonathan Poplawsky currently leads the atom probe tomography (APT) efforts within the Center for Nanophase Materials Sciences (CNMS) at Oak Ridge National Lab-

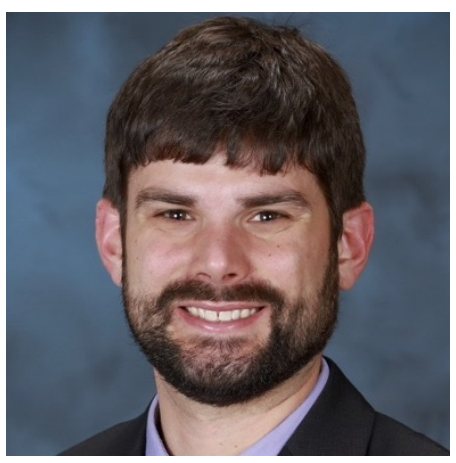
oratory (ORNL). Jonathan received his Bachelor's degree from The University of Scranton in 2007 and his Ph.D. in Physics from Lehigh University in 2012, where he built an apparatus capable of simultaneous cathodoluminescence and photoluminescence experiments. Following his graduate studies, Jonathan worked as a post-doctoral research associate in the Scanning Transmission Electron Microscopy (STEM) Group at ORNL, where he designed a STEM-electron beam induced current (EBIC) holder. Following his post-doctoral appointment, Jonathan received a strategic hire award from ORNL to implement new capabilities within the CNMS APT laboratory. His main research interests have been focused on discovering relationships between nanoscale structure, composition, and function in numerous energy materials using complementary microscopy techniques with a particular focus on atom-probe tomography. 


\section{Sebastian Koelling}

Dr.

Sebastian

Koelling graduated from the Technical University of Dresden, did his research for his Ph.D. thesis on the application of atom probe tomography to semiconductor devices at

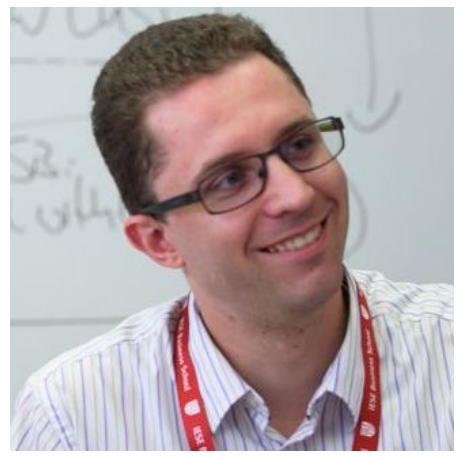
IMEC and got his Ph.D. from the Catholic University of Leuven. He worked at Xerox PARC and Fraunhofer CNT on the development and physical analysis of semiconductor devices before starting the National Atom Probe Facility of the Netherlands at the Eindhoven University of Technology. In Eindhoven, his research is focused on enabling the atomic scale analysis of semiconductor and biological nanostructure using atom probe tomography.

\section{Kathryn Grandfield}

Dr. Kathryn Grandfield is an Assistant Professor in the Department of Materials Science \& Engineering and School of Biomedical Engineering at McMaster University, Canada where her research

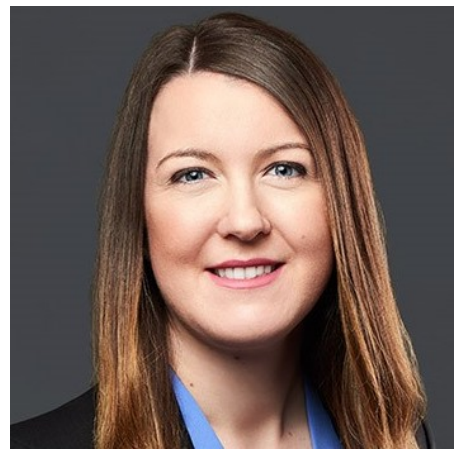
interests include developing correlative multi-scale microscopies to investigate biointerfaces and mineralized tissues. She received her Ph.D. from Uppsala University, Sweden and completed a postdoctoral fellowship at the University of California, San Francisco before joining McMaster University in 2013. She is presently second Vice-President of the Microscopical Society of Canada, board member for the Canadian Biomaterials Society, and Director of User Operations at the Canadian Centre for Electron Microscopy.

\section{Frédéric Danoix}

Dr. Frédéric Danoix is a CNRS senior scientist at the Groupe de Physique des Matériaux, Normandie University in Rouen (France). After undergraduate studies at the University of Rouen, he

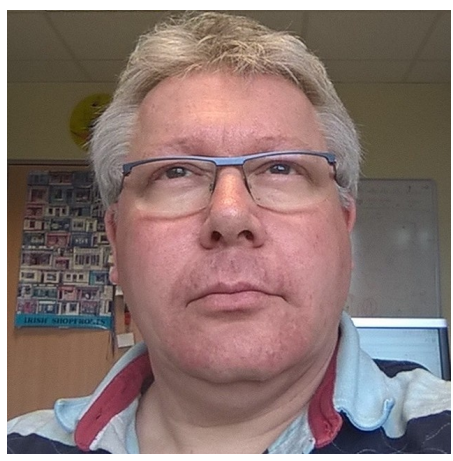
completed a Ph.D. in materials science in 1991, applying the atom probe field ion microscopy to the study of duplex stainless steels embrittlement. Following a post-doctoral stay in the FIM group at the University of Oxford, he joined CNRS to participate to the development of the three dimensional atom probe. He is currently mostly working on the investigation of phase transformations in steels at the atomic scale, both on an academic perspective, and on industrial issues. One of his major topics is the behaviour of interstitial elements, such as $\mathrm{C}$ and $\mathrm{N}$.

\section{Flyura Djurabekova}

Dr. Flyura Djurabekova is an adjunct professor in the Department of Physics and Helsinki Institute of Physics. She got her Ph.D. in 1999 from Arifov Institute of Electronics, Tashkent, Uzbek-

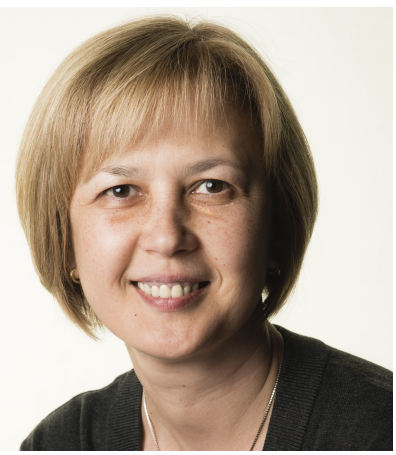
istan. Prior to coming to Finland, she worked for extended research stays at NIRIN, Nagoya, Japan and at SCK-CEN, Mol, Belgium. At the Helsinki Institute of Physics, she is leading a group of 15 scientists, who develop a multiscale model to describe various processes which take place under high electric fields. The main objective - vacuum arcing — is tackled by using different simu- 
lation techniques ranging from density functional theory (DFT) to finite element analysis (FEM) across femtosecond to second time spans. This activity led to the annual international workshop "MeVARC" permanently co-chaired by her, which attracts the scientists working with materials under high electric fields from all over the world. Her areas of interests includes also swift heavy ion interaction with materials, nanomaterials, and complex metal alloys. She has published more than 130 refereed international publications, supervised nine Ph.D. theses, and is currently supervising eight Ph.D. students and mentoring three post-doctoral researchers.

\section{Isabelle Mouton}

Dr. Isabelle Mouton is a post-doctoral researcher at the Max-Planck-Institut for Iron and Steel Research in Düsseldorf, Germany where she applies atom probe tomography in the area of

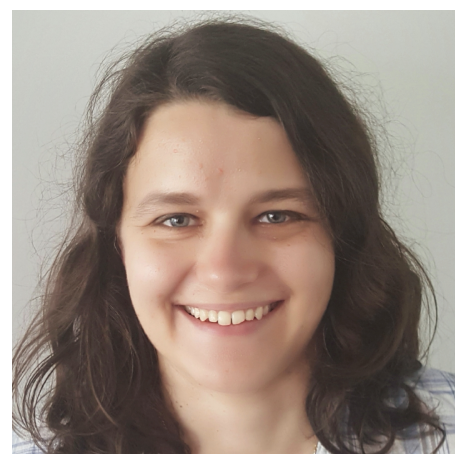
hydrogen in materials, with an emphasis on stable hydrides for energy storage. She earned her Ph.D. at the University of Rouen, France and completed a post-doctoral position at the CEA-LETI in Grenoble, France. Her research interests include the development of new data analysis methods and particularly reconstruction based on correlative approach with electron microscopy/tomography.

\section{Dieter Isheim}

Dr. Dieter Isheim is a research associate professor of materials science and engineering at Northwestern University and manages the Northwestern University Center for Atom-Probe Tomog-

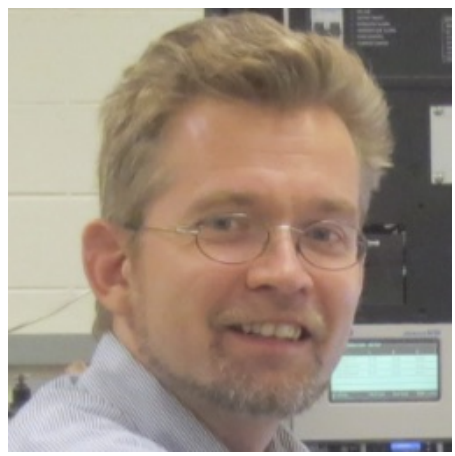
raphy (NUCAPT). After receiving a doctorate in physics and physical metallurgy from the University of Göttingen, Germany, in 1995, he joined Northwestern University for post-doctoral studies in 1996 and was reappointed at Northwestern as research faculty in 2002. His research interests include phase transformations in solids and multiphase nanometer-scale structures and the effects of the nanostructures on the properties of structural and functional materials. He has published more than 100 research papers in these areas. He specializes in high-resolution atomic-scale materials characterization with a focus on atom-probe tomography.

\section{David Duewer}

Dr. David Duewer earned his Ph.D. in Chemistry from the University of Washington in 1976, defining and developing the field of "chemometrics" with Drs. Bruce Kowalski and Svante

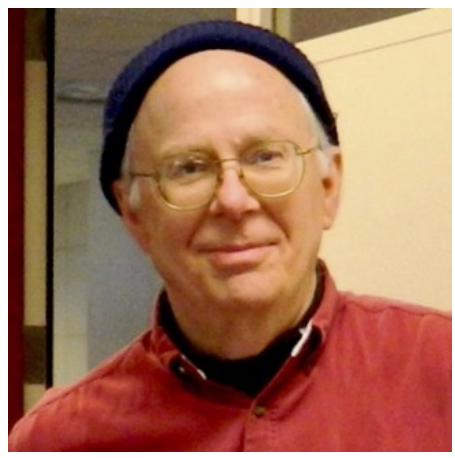

Wold. He explored several different applications of computer-assisted chemistry in post-doctoral research with Dr. Henry Freiser at the University of Arizona and Dr. Gary Christian at the University of Washington. Joining the Monsanto Agricultural Chemistry Company in 1979, he there cham- 
pioned the development and application of computational tools for process chemistry, molecular synthesis, and bio-activity discovery. He joined the NIST in 1991, working on numerous aspects of chemical metrology including experimental design, exploratory data analysis, graphical communications, interlaboratory comparisons, measurement uncertainty, and quality assurance/control. $\mathrm{He}$ is an advocate for collaborative $\mathrm{R} \& \mathrm{D}$ at the interfaces of chemistry, biology, and measurement science. His current interests include better approaches to the: evaluation of chemical purity, demonstration of comparability among reference materials that nominally deliver the same measurands, summarization of chemical data that have defined measurement uncertainties, and evaluating the metrological traceability of DNA quantification results provide by digital polymerase chain reaction (dPCR) measurement platforms.

\section{John Henry Scott}

John Henry works at NIST in Gaithersburg, MD, as a Physicist in the Materials Measurement Science Division and as Senior Advisor to the Office of Data and Informatics. He holds a B.S.

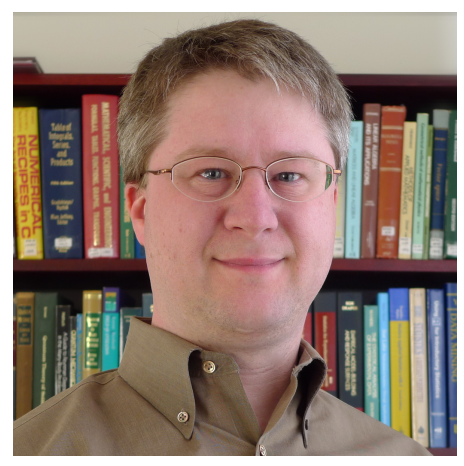
and Ph.D. in Physics from Caltech and Carnegie Mellon University, as well as an M.S. in Biotechnology from Johns Hopkins. His physical science research interests include chemical characterization at the nanoscale using X-ray and electron beam methods as well as focused ion beams, and his data science interests include multivariate statistical analysis, sparse representations, and machine learning. His microanalysis work has focused on applications in semiconductors, metals, inorganic nanoparticles, and the life sciences. $\mathrm{He}$ has served as MAS President, MAS Director, and MSA Program Chair, and has been an instructor at the Lehigh Microscopy School since 2000. John Henry has co-organized M\&M symposia on various topics including advanced X-ray spectrometry, tomography in the physical and biological sciences, and chemical imaging, and coorganized the 2003 MAS Topical Conference on Hyperspectral Imaging. John Henry received the Dept of Commerce Bronze Medal in 2007 for exceptional innovation in chemical and dimensional metrology at the nanoscale, and the Deaprtment of Commerce Gold Medal in 2010 for distinguished achievement in the measurement science of nuclear non-proliferation. From late 2007 until 2009 John Henry served as Senior Policy Analyst in the White House Executive Office of the President during both the George W. Bush and Barack Obama administrations. 



\title{
Recollections Revisited: the Magnificent and the Mundane \\ Allan J. Melmed \\ Williamsport, MD, USA \\ asm32@myactv.net
}

\begin{abstract}
On the $40^{\text {th }}$ anniversary of the achievement of surface-lattice atomic resolution by FIM (October 11, 1955), the author wrote about his witnessing of the event in Erwin W. Mueller's laboratory. The present paper describes the author's further thinking and perspective of the event and its consequences on the occasion of the $50^{\text {th }}$ anniversary of the introduction of the Atom Probe FIM. That event, many years ago, was hugely significant in view of the later developments of related techniques that led to today's Atom Probe Tomography and Microscopy meeting.
\end{abstract}

Twenty-two years have elapsed since the "Recollections of Erwin Mueller's Laboratory: (1951-1956)" (1) was published describing the excitement at the happening of lattice atomic resolution by FIM. The author clarified some details of that paper in a subsequent publication (2), and now has revisited the original paper, mostly with the eyes of a reader, so to speak, rather than the eyes of the author.

\section{1: Comments on the Original Text}

At the time of the magnificent event discussed in (1), when FIM provided the first atomically resolved map of the specimen surface atoms, no other microscopy existed that could do that or even "image" single atoms. Some 28 years would pass before another microscopy, STM, claimed to image a surface atomic array (3).

In 1970, Albert Crewe developed a High-Resolution Scanning Electron Microscope with which he could "see" single atoms of uranium and thorium on a carbon film (4). However, no imaging of an atomic lattice was demonstrated. A human analogy is: I can see stars in the night sky. Does that mean that I have telescopic eyesight? No, for example among other things I cannot distinguish between a single star and a binary pair.

It may seem mundane, but the terms "imaging atoms" and "seeing atoms" are anthropomorphic exaggerations. No microscope, arguably other than the reflection optical microscope actually images or sees objects in human terms. Either directly or indirectly, they produce images interpreted as positions of atoms relative to other atoms. Perhaps "mapping”, Field Ion Atomic Mapping would be a more accurate, though a more mundane description.

In the original Recollections paper (1) the author described the mood in the laboratory immediately after E.W. Mueller's first viewing the FIM image of a fully resolved surface atom array, as one of "unprecedented awe and joy". We all understood that no person had ever established the reality of atomicity of solid matter. Atomicity had been indirectly surmised for centuries, ever since the thinking of Democritus and the x-ray work of Sir Lawrence Bragg. Now the magnificent was unexpectedly upon us! Unbounded joy prevailed at the supposed certainty that our professor would soon win a Nobel Prize.

FIM actually directly projects a map of the ionization probability of the specimen surface, which looks like an array of atoms. STM and HRTEM, produce so-called atomically 
resolved images only with the aid of computers or other kinds of "image processing", such as hand-drawn embellishments (3). Raw STM data, for example, is a series of individual lines, which are the recorded response of the measurement of voltage needed to maintain constant tunneling-electron current $(3,5)$. And HRTEM actually images columns of atoms, not just surface atoms.

A section in the original recollections paper (1) discussed "The paramount importance of field evaporation”. In perspective now, field evaporation was indeed extremely important, not only for FIM per se but also was crucial for the later development of atom probes. In contrast to thermal evaporation, field evaporation can be implemented at all temperatures, and is local to the part of the specimen that is subjected to a high electric field. This capability is unique to FIM and Atom Probes.

The other student present with the author (1) just outside the room where Kanwar Bahadur showed Erwin W. Mueller the first image of a surface array of atoms was Russell D. Young. In retrospect, Russell Young should be recognized much more than he has been. He did the research on field electron energy distribution that later led to the use of field-emission point sources for various forms of atomic-resolution microscopy and he developed the first instrument similar to the later STM (5). This was recognized by the Nobel Prize committee, and in now-mundane fact, Russell D. Young was cited in the background information for the official 1986 Nobel Prize awarded (6) to Binnig, Rohrer and Ruska, for his closely related work (1970) developing the instrument he called the Topografiner (5)! The author was a colleague of Russell Young at NBS (NIST) during the time the Topografiner was developed. All of us in the surface science lunch group were in awe when we learned of the new instrument - it was truly magnificent! We were amazed that a field-electron tip was being used in a completely new way. Then, when the 1986 Nobel Prizes were announced, we were astonished that Russell Young was not included in the Nobel Prize recipients! Later it was leaked that Young was not included because he did not envision his Topografiner achieving atomic resolution. Clearly this was suspect, so suspect. After all, Ernst Ruska did not envision his electron microscope achieving atomic resolution!

\section{2: Comments on the original micrographs.}

The room temperature helium and neon FIM micrographs (figs. 4 and 5) in the original Recollections paper (1) were presented to clearly establish the fact that Mueller's 1951 microscope inherently had the magnificent capability of surface atomic lattice resolution. Importantly, it should be noted that these microscope images and micrographs were recorded with the aid of image intensification, not available in 1951. They were obtained using very sharp specimen tips, due to the fact that room temperature resolution degrades with increasing tip radius, more noticeably than at lower temperatures.

These images, as all FIM images (7), are projections from a curved surface (the specimen) onto a flat screen or detector. In order to understand them, one should first recognize that an FIM image is a projection from a small curved surface, a point specimen. Then recognize that a single FIM image shows an atomic arrangement representing the atoms located in an outermost spherical envelope of surface atoms and that this envelope is very thin, only about $0.3 \mathrm{~nm}$ thick. Due to the shape of FIM specimen (7), the projection arises from about a 60 degree solid angle. To help visualize 
this, Erwin Mueller had a cork ball model of an FIM specimen apex with phosphor paint used to indicate the surface "atoms" that would be seen (in a darkened room) as a simulated FIM image.

It is also important to realize that the dark regions may or may not be populated by atoms (most of them are, for crystalline specimens). By very carefully controlled field evaporation (usually using pulsed voltage), dark regions can be explored for atomic content (7).

\section{3: Some Overall Perspectives}

Revisiting the Recollections paper, an interesting perspective appears. It is ironic that a major feature of FIM is the fact that imaging happens without a lens, that is there is direct projection from a sharp point. It is ironic that this feature also was responsible for the very limited appeal of FIM to researchers around the world! It became clear that FIM studies would always involve the need for the investigator to make a sharp point for every investigation. In FIM, the specimen and the illumination source are inexorably combined. Once the specimen is made, the die is cast, so to speak.

Another perspective is the exquisite simplicity of the original (and many later) FIM instruments compared to various other microscopes. The original FIM was a glass vessel, with a conductive coated on the inside, with the illumination source (positive ions) the specimen itself, and a phosphor screen (or later an image intensifier) to display the image.

The author has conducted and published research in FEM, FIM and APFIM through the years and interacted with the scanning probe community, manufacturing sharp points of many materials (8) for almost 25 years. This experience provides a broad perch from which to look back at the history of it all, leading to Atom Probe Tomography (APT). The author had an especially deep ponder reflecting on the question of evolution vs creation in the historical path from FEM to APT (a question often asked in another context!) and was profoundly affected by Isaac Newton's famous statement. Newton stated that "If I have seen further than others, it is by standing on the shoulders of giants", at first slightly modified by the author to "If I have seen further than others, it is by standing on the shoulders of others". Upon further contemplation, the author modified it to "If I have seen further that others, it is by thinking about the work of others".

The historic path from FEM to APT, was a series of significant developments with magnificent milestones along the way, some very creative and some evolutionary. In the 1930's several researchers were studying the field electron emission from tungsten tips, related to vacuum tubes. R.P, Johnson and W. Shockley (9) were studying field electron emission from thin wires, projecting the emission pattern onto a cylindrical phosphor screen. Immediately after this, Erwin Mueller published his first FEM paper (10), a significant, if incremental advance in microscopy, with a sharp point instead of a thin wire as the electron emission source. Definitely evolution!

Then there was the magnificent discovery of room temperature FIM, introduced by Erwin Mueller in 1951 (11). This arose from change in the polarity of the specimen tip (12), a mundane event, and the capability to introduce hydrogen, a very creative experimental 
feat. Then, as recollected by the author (1), atomic lattice-resolution happened in 1955. An evolutionary offshoot in the direction of 3D FIM occurred in 1974 (13), but it wasn't considered further until much more recent attempts (see ref. 19).

Next, the conventional Atom Probe was developed in a time when multiple laboratories were researching mass spectrometry, some using magnetic sectors, other using electrostatic configurations with tungsten tips similar to the later conventional atom probe. Mark Inghram and Robert Gomer, were, in fact, very close (14) to inventing the conventional atom probe, lacking a single-ion detector and time-of-flight electronics. Also, Jochen H. Block reported (15) experiments on field-ion mass spectrometry, using pulsed field desorption, bringing him even closer to an AP. John A. Panitz, then a student in the laboratory of Erwin W. Mueller, has written about the development of the first atom probe and his involvement (16), relating that he was tasked with making a single-ion detector, essential for the then-planned AP.

The author's perspective on the development of conventional Atom Probe - evolution indeed. Several iterations of atom probes followed the introduction of the conventional AP, most notably John A. Panitz's 10-cm AP (also called the Imaging Atom Probe), leading to TAP, and these have been well-reviewed (17). They all used single sharp points as the specimen.

Then, in 1993, Osamu Nishikawa had a "magnificently" creative idea. Nishikawa, a student of Erwin W. Mueller, at PSU in the late 1960's was among many whom were dismayed that the small point that was the APFIM specimen severely limited the amount of data one could get before replacing the specimen. Later, when he returned to his native Japan, he reacted by getting a completely novel idea. He built a magnificent atom probe, the Scanning Atom Probe, SAP (18). In this instrument, the original point specimen was replaced by a macroscopic flat specimen, and a small counter-electrode probe that could be moved (scanned) to various positions, all very close to the flat surface. This probe applied a local electric field that enabled controlled field evaporation of surface atoms, that were then analyzed as in a conventional AP. Following this development, Tom Kelly at IMAGO (now CAMECA) produced the commercial local electrode atom probe (LEAP), now extensively used.

\section{4: A Closing Perspective}

The author suggested earlier (1) that absent the contribution of Kanwar Bahadur the ultimate success of FIM would be questionable as well as the length of time it might take. However, the author's perspective now, is very optimistic that FIM would definitely have achieved surface-array atomic resolution sooner rather than later; perhaps elsewhere, if it didn't happen in 1955 in EWM's laboratory. So, independent of the mundane activity of October 11, 1955, the world would still have Atom Probe Tomography and Microscopy today.

With the magnificent development of local electrode atom probes the author's perspective was that the evolution of point projection instruments was at its end, that they had become extinct, so to speak. However, the recent introduction of so-called 3D FIM (19) seems to extend the point's life. It also seems to me that the name "3D FIM" is not 
correct. I believe it should be called "Tomographic FIM" and the in the author's opinion, with the perspective of earlier work (13), this work (19) is quantitatively futile!

It seems that history always heaps praise on the first violin player, but often the second fiddler and the seemingly mundane other fiddlers also play great music! They all make up the magnificent sound of the orchestra.

Finally, it is important to recognize that the classifications of "magnificent" and "mundane" are very much subjective, in the eyes of the beholder.

\section{.REFERENCES:}

1. Recollections original paper. "Recollections of Erwin Mueller’s laboratory: the development of FIM (1951-1956), Appl. Surf. Sci. 94/95 (1996) 17-25

2. The Day Atomic Resolution Microscopy Happened, Allan J. Melmed, Microscopy Today, March 2006.

3. 7x7 Reconstruction on Si(111) Resolved in Real Space, G. Binnig, H. Rohrer, Ch. Gerber and E. Weibel, Phys. Rev. Lett. 50, 2 (1983) 214.

4. A.V. Crewe, J. Wall, J. Lanomore, "Visibility of Single Atoms”, Science 168 (1970) 1338-1340.

5. The Topografiner: An Instrument for Measuring Surface Microtopography, Russell Young, John Ward and Frederic Scire, Rev. Sci. Instr. 43 (1972) 999.

6. Nobel Prize Press Release- October 15, 1986.

7. Field Ion Microscopy: Principles and Applications, E. W, Mueller and T. T. Tsong, (Elsevier, New York, 1969).

8. Custom Probes Unlimited, see The Art and Science and Other Aspects of Making Sharp Tips, J. Vac. Sci. Technol. B $\underline{9}, 601$ (1991).

9. R.P, Johnson and W. Shockley, Phys. Rev. 49 436-440 (1936).

10. Erwin Mueller, "Beobachtungen ueber die Field Emission und die Kathodenzerstaeubung an thoriertem Wolfram”, Z. Phys. 106 (1937) 132-40.

11. E,W, Mueller, Z. Phys. 131 (1951) 136.

12. Related by G. Pankow, Described in “Erwin W. Mueller 1911-1977” Biographical Memoirs, Vol. 42, National Academy Press, Washington, D.C. (2002).

13. D.G. Brandon and A.J. Melmed, "FIM Analysis of Dislocation Core Structure”, J. Microscopy, 100 (1974) 75-80.

14. Inghram and Gomer, "Mass Spectrometric Analysis of Ions From the Field Ion Microscope, J. Chem. Phys 22 (1954) 1279-80.

15. J.H, Block, Z. Phys. Chemie N.F. 39 (1963) 169.

16. J.A. Panitz $50^{\text {th }}$ Anniversary at Penn State Imago June 16, 2005- J. A. Panitz 1974. and private communication.

17. David N. Seidman Annu, Rev. Mater. Res., 2007, 37;127-158.

18. O. Nishikawa, Materials Characterization, Elsevier, vol. 44, Jan.-Feb. 2000 and Jap. J. Appl. Phys. July 1, 2003.

19. F. Vurpill, M. Gilbert and B. Deconihout, Surf. and Interface Anal. 39 (2007) 273277. 

Proceedings of Atom Probe Tomography \& Microscopy (APT\&M) 2018, Washington, DC, June 10-15, 2018

NIST SP $2100-032,55-3: 10$

3815

P.16 3845-4:05

p.17 $4: 10-4840$

p.19 4:45 -5:00

$5: 05-5: 20$

$5 \$ 30$

TUESDAY, JUNB 27, 1967 - GREEN AUDITORTUM, NBS

$8: 30 \mathrm{~A} \cdot \mathrm{M}$.

Busea leave Harbin Hall, Georgetown

Session 3. FEM and Technigues

$$
\text { Chalrman: R. Gomer }
$$

p.22 9:30-9:50 Glen N. Bates and George Barne: The $\alpha \rightarrow B$ Phase Transition in Hafalum.

P.24 9:55-10:10 J. A. Clum and R. D. Gretz: Surface SeIfaDiffusion 8 Impurity gffect.

P.26 10815-10:35 T. HLb1 and F. Okuryama: A New Method for Preparing Urusully Oriented Tungsten T1ps and their Growth Mechanism.

10840 Coffee break.

P.28 11800-11:15 Takao Utsum18 A Proposed H1gh Frequency Negative Resigtance Device by Dsing Pre-Breakdown Fleld Emisgion.

p.29 II\&20-11:30 R。D。Gretz \& FEM with a Reverged Screen.

p.30 11830-11840 R, D. Gretz: A New Phosphor for FEM.

p.31 11845-12:00 Erwin W. Mưller and John Panltz: The Atom-Probe Fleld Ion Microscope. 


\section{The Atom-Probe Field Ion Microscope \\ Erwin W. Muiller and John Panitz \\ Physics Department, The Pennsylvania State University University Park, Pa。}

A serious limitation of the field ion microscope has been its inability to identify individually imaged atoms. A newly conceived Atom-Probe FIM, consisting of a combination probe hole FIM and mass spectrometer having single particle sensitivity, will be described. During operation, the observer selects an atomic site of interest and places it over the probe hole. Pulsed field evaporation sends the chosen particle through the hole and into the spectrometer section which may be of the magnetic sector or time-of-flight type. Each has its own special advantages depending upon the particular application. These might include: identification of bright atom spots in the controversial adsorption experiments, investigation of the atomic nature of impurity and interstitial atom spots, analysis of segregations and precipitations, or the investigation of short range order in alloys. In some cases an adjustable probe hole covering an area of several atomic sites is advantageous. Experiences with a prototype time-of-flight instrument will be reported. 


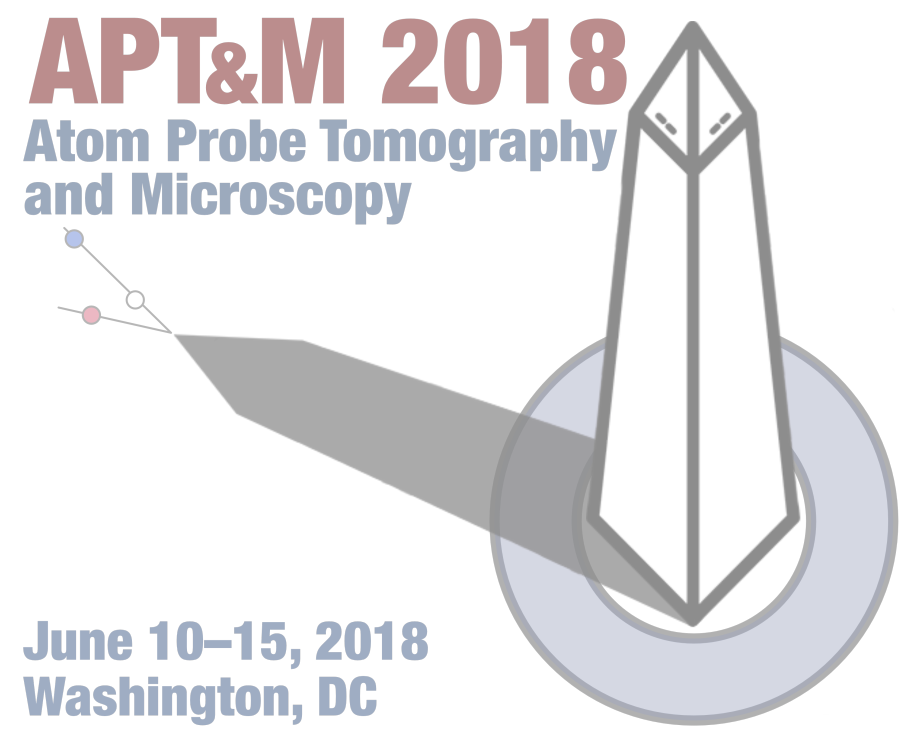

Sunday Afternoon 



\section{Sunday Afternoon}

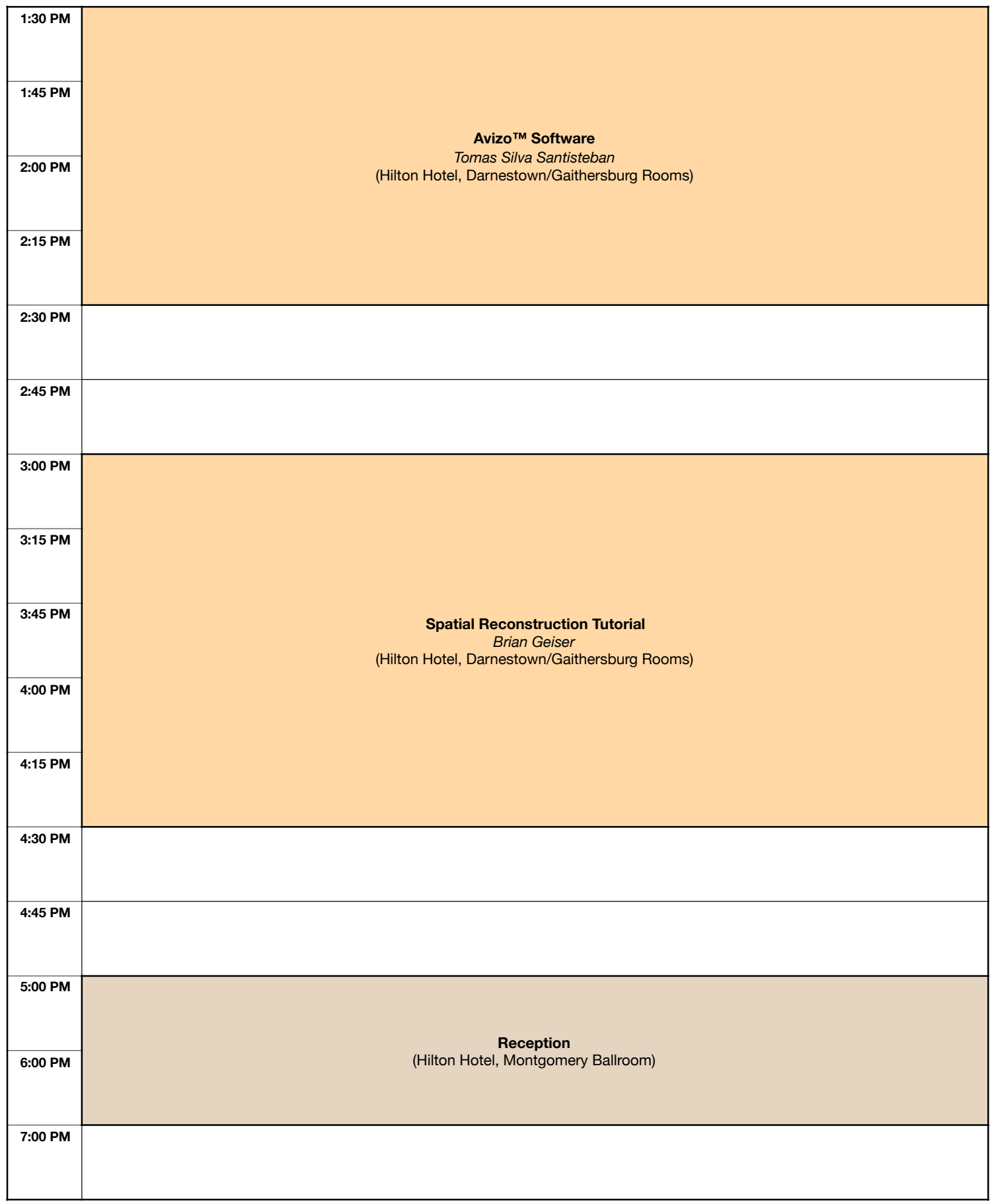





\section{Avizo $^{\text {TM }}$ Software Tutorial}

Tomas Silva Santisteban ${ }^{1}$

Corresponding Author: tomas.silvasantisteban@fei.com

1. Thermo Fisher Scientific, Materials \& Structural Analysis, Houston, TX 77084.

This technical session will introduce the Avizo software and show how it can be used with atom probe related data sets. An introduction and overview of APT applications is followed by a live demonstration of a complete workflow of an atom probe data set within the software. The demonstration will show how to load the data, visualize it and perform an analysis, like filtering data by atomic numbers amongst others. 


\section{Spatial Reconstruction Tutorial}

Brian Geiser $^{1}$

Corresponding Author: brian.geiser@ametek.com

1. CAMECA Instruments, Inc., 5470 Nobel Drive, Madison, Wisconsin 53711, USA.

To obtain useful structural composition information from atom probe data, it must be spatially reconstructed as accurately as possible. This discussion will present the basic ideas behind current reconstruction techniques. The strengths and weaknesses of some of the methods will be discussed, including the situations in which the techniques start to fail and some of the artifacts that result. Further techniques for improving the results of reconstructions and quantifying their accuracy will also be reviewed. 
Proceedings of Atom Probe Tomography \& Microscopy (APT\&M) 2018, Washington, DC, June 10-15, 2018 NIST SP 2100-03

\section{Reception}

Hilton Hotel, Montgomery Ballroom

5:00 to 7:00 PM 



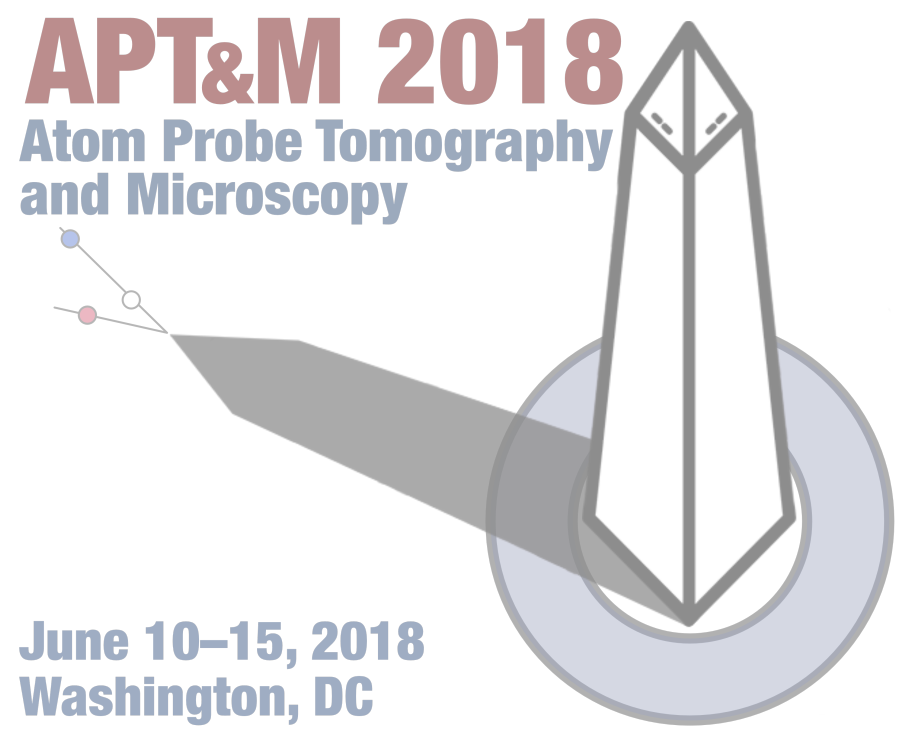

\section{Monday Morning}





\section{Monday Morning}

\begin{tabular}{|c|c|}
\hline 9:00 AM & \\
\hline 9:15 AM & $\begin{array}{l}\text { Opening Remarks } \\
\text { (Green Auditorium) }\end{array}$ \\
\hline 9:30 AM & \\
\hline 9:45 AM & $\begin{array}{l}\text { Plenary 1: Determining Atomic Coordinates in 3D by Atomic Electron Tomography } \\
\text { Peter Ercius } \\
\text { (Green Auditorium) }\end{array}$ \\
\hline 10:00 AM & \\
\hline 10:15 AM & Break (10:15 to $10: 45 \mathrm{AM})$ \\
\hline 10:45 AM & \\
\hline 11:00 AM & $\begin{array}{l}\text { J. H. Block Lecture: Contributions of Atom Probe Microscopy Within the Geosciences } \\
\text { David Saxey } \\
\text { (Green Auditorium) }\end{array}$ \\
\hline 11:15 AM & \\
\hline 11:30 AM & Lunch / Poster Viewing (11:30 AM to 1:00 PM) \\
\hline
\end{tabular}





\section{Determining Atomic Coordinates in 3D by Atomic Electron Tomography}

Peter Ercius ${ }^{1}$, Yongsoo Yang ${ }^{2}$, Chien-Chun Chen ${ }^{2,3}$, Colin Ophus ${ }^{1}$, Mary C. Scott ${ }^{1,4}, \mathrm{Rui} \mathrm{Xu}^{2}$, Alan Pryor $\mathrm{Jr}^{2}$, Li Wu ${ }^{2}$, Fan Sun ${ }^{5}$, Wolfgang Theis ${ }^{6}$, Jihan Zhou ${ }^{2}$, Markus Eisenbach ${ }^{7}$, Paul R. C. Kent $^{8,9}$, Renat F. Sabirianov ${ }^{10}$, Hao Zeng ${ }^{5}$, Jianwei Miao ${ }^{2}$

Corresponding Author: percius@lbl.gov

1. Molecular Foundry, Lawrence Berkeley National Laboratory, Berkeley, CA, USA.

2. Dept. Physics and Astronomy and California NanoSystems Institute, U. California, Los Angeles, CA, USA.

3. Dept. Physics, National Sun Yat-sen University, Kaohsiung, Taiwan.

4. Dept. Materials Science and Engineering, U. California Berkeley, Berkeley, CA, USA.

5. Dept. Physics, University at Buffalo, Buffalo, NY, USA.

6. Nanoscale Physics Research Laboratory, School of Physics and Astronomy, U. Birmingham, Birmingham, UK.

7. National Center for Computational Sciences, Oak Ridge N.L., Oak Ridge, Tennessee, USA.

8. Computer Science and Mathematics Division, Oak Ridge N.L., Oak Ridge, Tennessee, USA.

9 . Center for Nanophase Materials Sciences, Oak Ridge N.L., Oak Ridge, Tennessee, USA.

${ }^{10}$. Dept. Physics, U. Nebraska at Omaha, Omaha, Nebraska, USA.

Scanning transmission electron microscopy is an important technique in materials science to examine the atomic structure of materials with sub- $\AA$ resolution and single-atom sensitivity [1]. Combining this imaging tool with powerful iterative 3D reconstruction algorithms for electron tomography is opening a new field called atomic electron tomography (AET) with the ability to determine the coordinates of atoms in a structure without the assumption of crystallinity [2]. This talk will cover recent develops in AET, which will be critical to our understanding of the atomic structure of complex materials systems. The details of the AET acquisition and reconstruction method, atom tracing procedure and atomic scale quantitative analysis will be presented using a recent 3D atomic resolution reconstruction of a FePt nanoparticle [3].

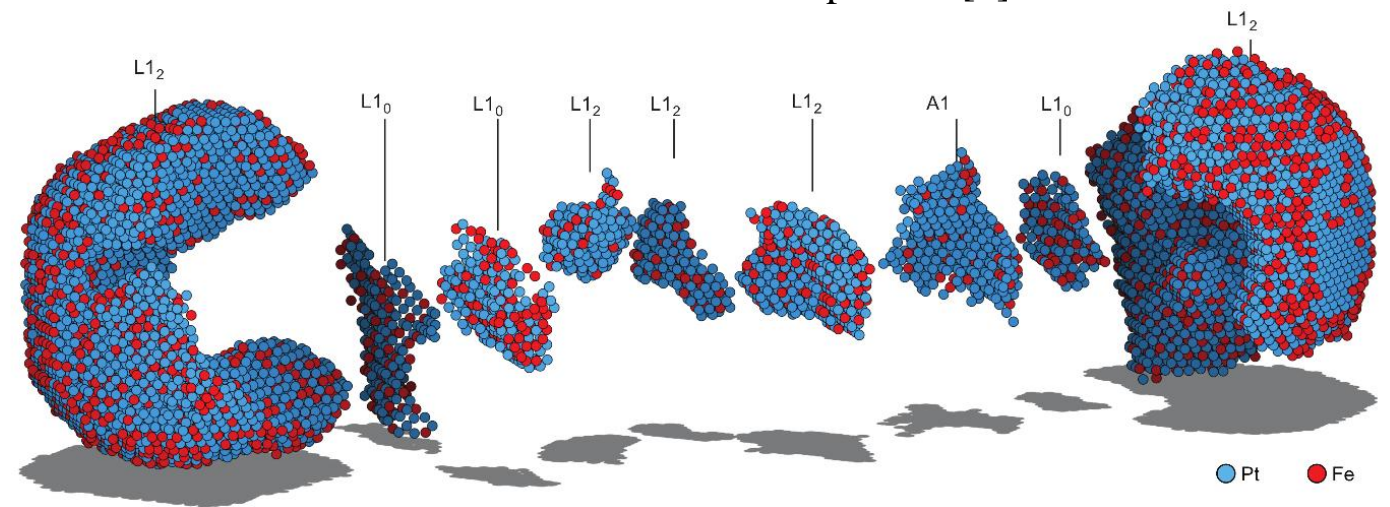

Figure 1. The nanoparticle consists of multiple FePt grains (type $\mathrm{L} 1_{0}$ and $\mathrm{L} 1_{2}$ ) and a Pt-rich $\mathrm{A} 1$ grain.

\section{$\underline{\text { References }}$}

[1] S.J. Pennycook and P.D. Nellist. Scanning Transmission Electron Microscopy: Imaging and Analysis, Springer, NY, 2011.

[2] J. Miao, et al., Atomic electron tomography: 3D structures without crystals, Science 353 (2016), aaf2157.

[3] Y. Yang, et al., Deciphering chemical order/disorder and material properties at the single-atom level, Nature 542 (2017), 75-79. 


\title{
Contributions of Atom Probe Microscopy within the Geosciences
}

\author{
David W. Saxey ${ }^{1,2}$, Steven M. Reddy ${ }^{1,2}$, Denis Fougerouse ${ }^{1,2}$, and William D. A. Rickard ${ }^{1,2}$ \\ Corresponding Author: david.saxey@curtin.edu.au \\ 1. Geoscience Atom Probe, Advanced Resource Characterisation Facility, John de Laeter Centre, \\ Curtin University, Perth, Australia. \\ 2. School of Earth and Planetary Sciences, Curtin University, Perth, Australia.
}

In recent years atom probe microscopy (APM) has made inroads into the chemical and isotopic analysis of geological samples. Steady progress has been made in applying atom probe techniques across a growing collection of minerals, with several research groups active in the field, addressing long-standing questions in planetary science, geochronology, and fundamental processes within geological materials [1-7].

Conventional geoanalytical techniques have remarkable chemical and isotopic sensitivity, down to parts-per-trillion or lower. However, their spatial resolution is typically at micron lengthscales, with a few secondary ion mass spectrometry techniques capable of sub-micron resolution (e.g. NanoSIMS). The chemical and isotopic landscape at $\mathrm{nm}$ length scales is therefore largely unexplored within geological materials - though it is expected to contain a wealth of information on past geological, biochemical and planetary processes. APM allows these variations to be studied at much smaller scales, providing access to new information of geological significance.

This presentation will show-case a selection of work across several geoscience applications, highlighting the significant role that APM is playing within the nascent field of nanoscale geochemistry. Examples include the analysis of silicates, oxides, sulphides, and phosphates, with applications in mineral processing, waste management, extra-terrestrial materials, isotope geochemistry, and geochronology. Future prospects for geoscience applications will also be discussed [8].

\section{$\underline{\text { References: }}$}

[1] J.W. Valley, et al., Nano- and micro-geochronology in Hadean and Archean zircons by atom-probe tomography and SIMS: New tools for old minerals. American Mineralogist 100 (2015), 1355-1377.

[2] A.R. Felmy, et al., Enhancing Magnesite Formation at Low Temperature and High CO2 Pressure: The Impact of Seed Crystals and Minor Components, Chemical Geology, 395 (2015), 119-125.

[3] J.B. Lewis, et al., ${ }^{12} \mathrm{C} /{ }^{13} \mathrm{C}$ - Ratio Determination in Nanodiamonds by Atom-Probe Tomography, Ultramicroscopy, 159 (2015), 248-254.

[4] S.M. Reddy, et al., Mechanisms of deformation-induced trace element migration in zircon resolved by atom probe and correlative microscopy, Geochimica et Cosmochimica Acta, 195 (2016), 158-170.

[5] S. Piazolo, et al., Deformation-Induced Trace Element Redistribution in Zircon Revealed Using Atom Probe Tomography, Nature Communications, 7 (2016), 10490.

[6] A. Pérez-Huerta, et al., Atom Probe Tomography (APT) of Carbonate Minerals, Micron, 80 (2016), 83-89.

[7] L.F. White, et al. Atomic-Scale Age Resolution of Planetary Events, Nature Communications, 8 (2017), 15597.

[8] D.W. Saxey, et al. Atomic Worlds: Current State and Future of Atom Probe Tomography in Geoscience, Scripta Materialia, (2017), In-press. 


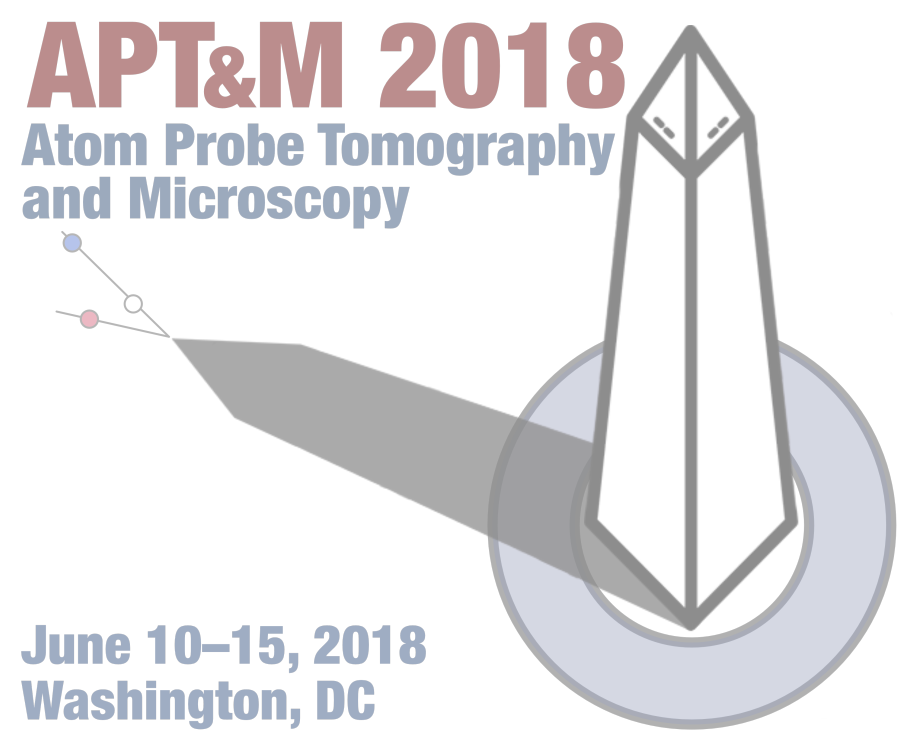

Monday Afternoon 



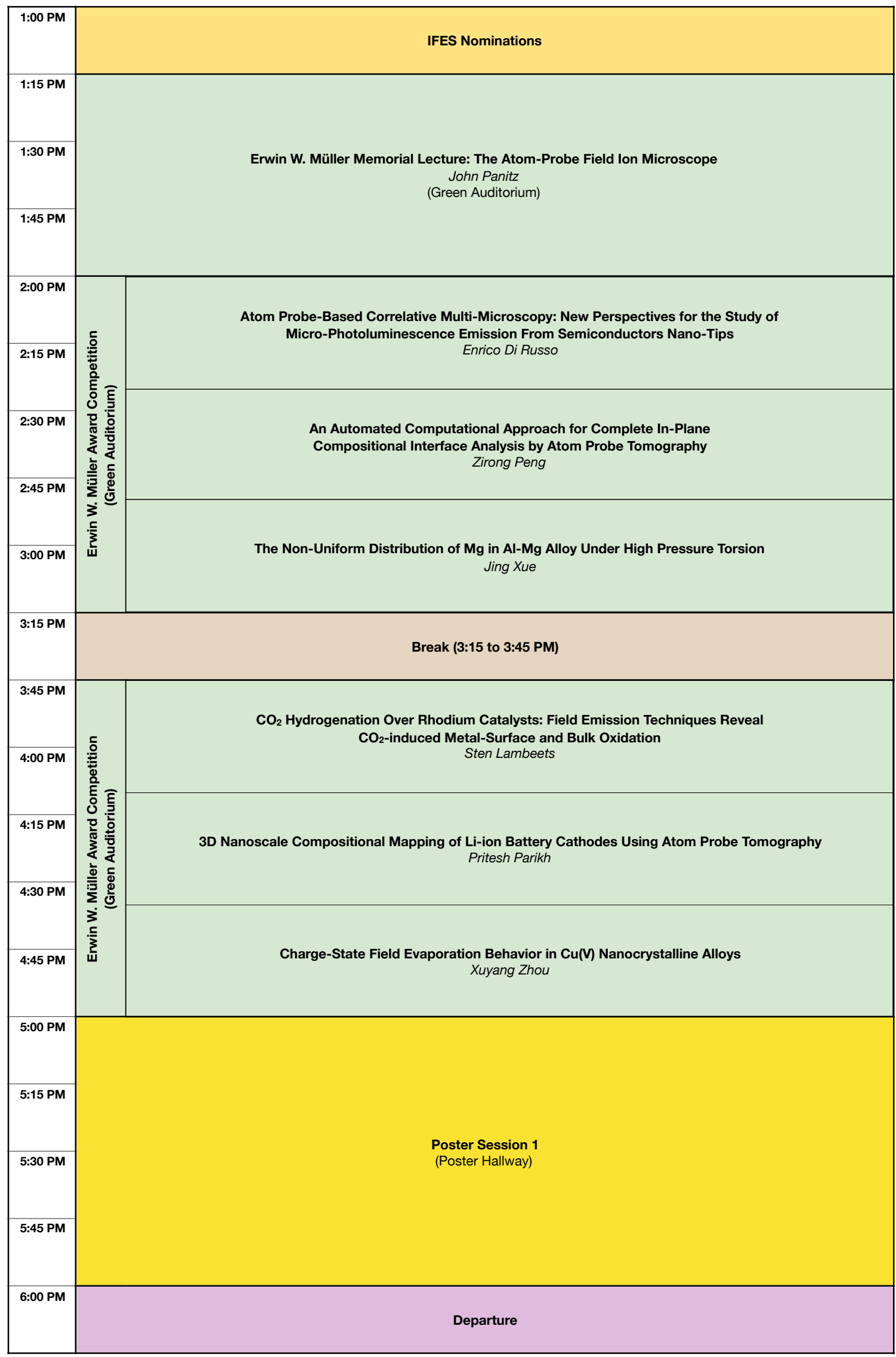





\section{The Atom-Probe Field Ion Microscope}

John A. Panitz ${ }^{1}$

Corresponding Author: panitz.unm.edu

1. The University of New Mexico, Department of Physics and Astronomy, Albuquerque, NM 87131.

A serious limitation of the field ion microscope has been its inability to identify individually imaged atoms. An Atom-Probe FIM, consisting of a combination probe hole FIM and mass spectrometer having single particle sensitivity, will be described. During operation, the observer selects an atomic site of interest and places it over the probe hole. Pulsed field evaporation sends the chosen particle through the hole and into the spectrometer section which may .be of the magnetic sector or time-of-flight type. Each has its own special advantages depending upon the particular application. These might include: identification of bright atom spots in the controversial adsorption experiments, investigation of the atomic nature of impurity and interstitial atom spots, analysis of segregations and precipitations, or the investigation of short range order in alloys. In some cases an adjustable probe hole covering an area of several atomic sites is advantageous. Experiences with a prototype time-of-flight instrument and its progeny will be reported. Memories of Erwin W. Müller who died on May 17, 1977 will be shared. 


\section{Atom probe-based correlative multi-microscopy: new perspectives for the study of micro-photoluminescence emission from semiconductors nano-tips.}

Enrico Di Russo ${ }^{1}$, Jonathan Houard ${ }^{1}$, Simona Moldovan ${ }^{1}$, Gérald Da Costa ${ }^{1}$, Ivan Blum ${ }^{1}$, François Henry Julien², Maria Tchernycheva², Jean-Michel Chauveau ${ }^{3}$, Maxime Hugues ${ }^{3}$, Didier Blavette ${ }^{1}$, Lorenzo Rigutti ${ }^{1}$.

Corresponding Author: enrico.di-russo1@etu.univ-rouen.fr

1. Normandie Univ., UNIROUEN, Groupe de Physique des Matériaux, 76000 Rouen, France.

2. Centre de Nanosciences et de Nanotechnologies, CNRS UMR 9001, Univ. Paris-Sud, Université Paris-Saclay, C2N - Orsay, 91405 Orsay Cedex, France.

3. Université Côte d'Azur, CNRS, CRHEA, 06560 Valbonne, France.

The $\mathrm{ZnO} / \mathrm{Mg}_{\mathrm{x}} \mathrm{Zn}_{1-\mathrm{x}} \mathrm{O}$ non-polar Multiple Quantum Well (MQW) system has been studied adopting an advanced correlative microscopy approach [1]. Atom probe tips were analyzed using Laserassisted Atom Probe Tomography (La-APT), Scanning Transmission Electron Microscopy (STEM) and micro-PhotoLuminescence $(\mu-\mathrm{PL})$. Complementary La-APT and STEM structural investigations make it possible to get a clear picture of both morphology and composition of the studied system. Surprisingly, the $\mathrm{ZnO} / \mathrm{Mg}_{\mathrm{x}} \mathrm{Zn}_{1-\mathrm{x}} \mathrm{O} \mathrm{MQWs}$ were found not planar, but exhibit a Vgroove grating profile when observed along the c-axis crystal direction (fig. a). Furthermore, STEM-EDS and La-APT analyses reveal that Zn-rich and -depleted layers are alternatively formed in $\mathrm{Mg}_{\mathrm{x}} \mathrm{Zn}_{1-\mathrm{x}} \mathrm{O}$ barriers in correspondence with the grating edges (fig. b). Both systematic $\mu$-PL and La-APT investigations allow to successfully determine the composition of $\mathrm{Mg}_{\mathrm{x}} \mathrm{Zn}_{1-\mathrm{x}} \mathrm{O}$ barriers (fig. b,c). Effective mass calculations based on structural information allow to calculate the radiative transition energy of $\mathrm{ZnO}$ QWs. The results are in excellent agreement with the experimental $\mu$-PL spectra. The innovative approach adopted can be generalized to a wide range of low-dimensional systems, opening new perspectives for correlatives studies of atom probe tips [2].

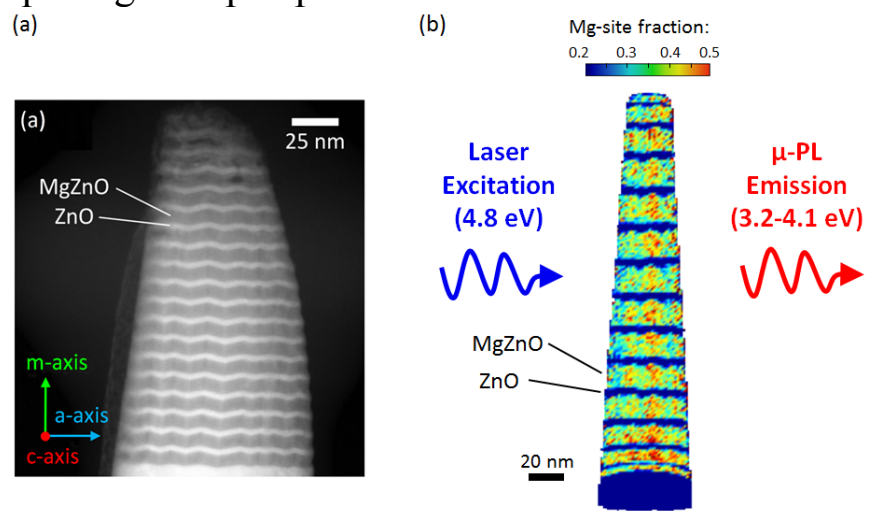

(c)

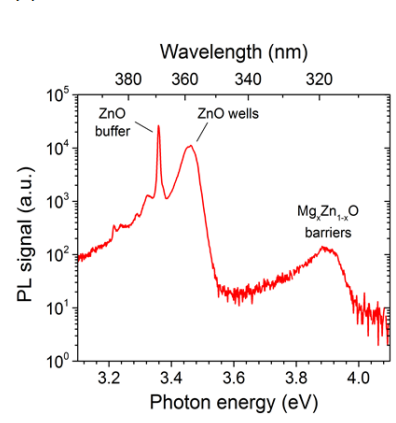

Figure: (a) STEM-HAADF image of $\mathrm{ZnO} / \mathrm{Mg}_{\mathrm{x}} \mathrm{Zn}_{1-\mathrm{x}} \mathrm{O} \mathrm{MQWs}$. (b) $\mathrm{Mg}$-site fraction map calculated from an atom probe tomographic $3 \mathrm{D}$ reconstruction. (c) $\mu$-PL signal of a $\mathrm{ZnO} / \mathrm{Mg}_{\mathrm{x}} \mathrm{Zn}_{1-\mathrm{x}} \mathrm{O} \mathrm{MQW}$ s system measured from an atom probe tip.

$\underline{\text { References: }}$

[1] E. Di Russo et al., Appl. Phys. Lett. 111 (2017), 032108.

[2] L. Mancini et al., Nano Lett. 17.7 (2017), 4261. 


\section{An Automated Computational Approach for Complete In-plane Compositional Interface Analysis by Atom Probe Tomography}

Zirong Peng ${ }^{1}$, Yifeng $\mathrm{Lu}^{2}$, Constantinos Hatzoglou ${ }^{3}$, Alisson Kwiatkowski da Silva ${ }^{1}$, Francois Vurpillot $^{3}$, Dirk Ponge ${ }^{1}$, Dierk Raabe ${ }^{1}$, and Baptiste Gault ${ }^{1}$

Corresponding Author: z.peng@mpie.de, b.gault@mpie.de

1. Max-Planck-Institut für Eisenforschung, Max-Planck-Straße 1, 40237 Düsseldorf, Germany.

2. Database Systems and Data Mining Group, Ludwig-Maximilians-Universität München, Oettingenstraße 67, 80538 München, Germany.

3. Normandie Univ, UNIROUEN, INSA Rouen, CNRS, GPM, 76000 Rouen, France.

Interfaces are ubiquitous microstructural features in multiphase, polycrystalline and nanocrystalline materials with a strong influence on their macroscopic properties. Manipulating the chemistry of interfaces and ultimately tailoring the performance of materials is an emerging concept for material and process design. Atom probe tomography (APT) is the only technique providing three-dimensional distribution of atoms with a near-atomic resolution. However, robust approaches for efficient and precise extraction of the local composition of solutes at and inside complex interfaces are still lacking.

Building upon the preliminary work by Felfer et al. [1,2], we introduce a framework to accurately define the position of the interface, estimate its width, characterize the local compositions and map the Gibbsian interfacial excesses of solutes. The effectiveness of the approach and possible impacts of ion trajectory aberrations are demonstrated in the analysis of compositional fluctuations inside grain boundary (GB) planes in Fe-9 wt. \% Mn alloys and corresponding electrostatic simulations. This method is also applied in the study of $\mathrm{PtIr} / \mathrm{Cr}$ bimetallic thin films. As Fig. 1 shows, here, we produced a $\mathrm{Cr}$ compositional gradient along GBs of PtIr by controlled annealing. Combined with electron energy loss spectroscopy (EELS) in scanning transmission electron microscopy (STEM), the GB segregation and phase formation behavior as a function of the chemical potential are accessed, which largely facilitates our highthroughput investigation of interfacial composition-phase-property relationships.
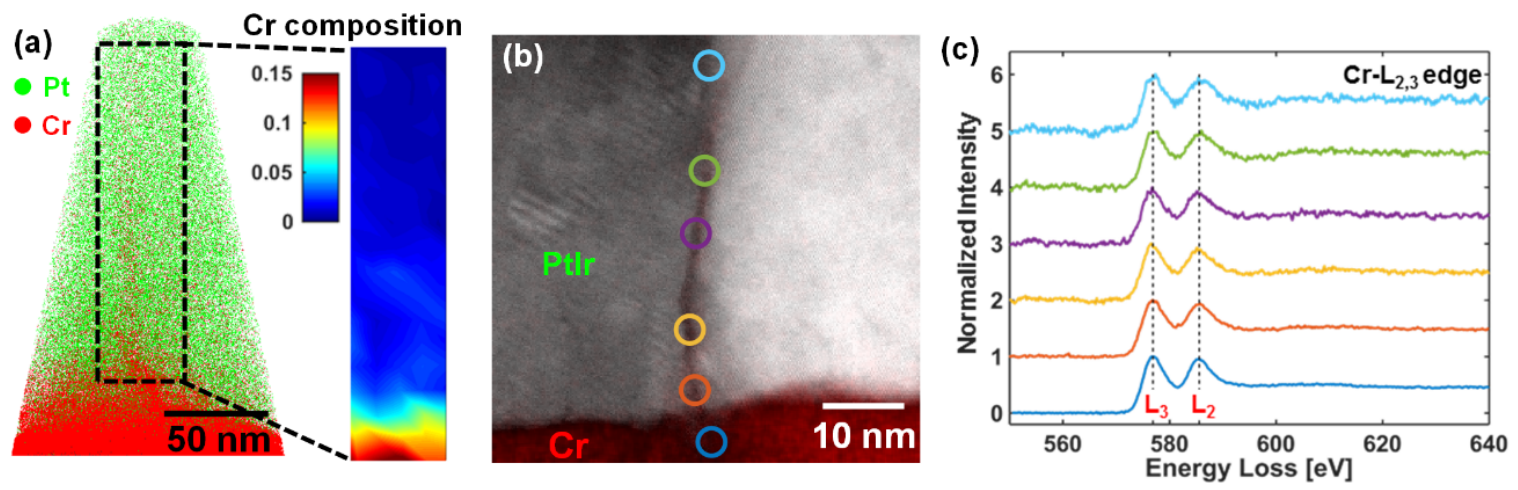

Figure 1 (a) Atom map with an in-plane Cr composition map of a GB. (b) STEM-HAADF image overlapped with a Cr EDX map. (c) Serial EEL spectra of the $\mathrm{Cr}-\mathrm{L}_{2,3}$ edge along a GB.

References:

[1] P. Felfer et al., Ultramicroscopy. 132 (2013) 100-6.

[2] P. Felfer et al., Ultramicroscopy. 159 (2015) 438-444. 


\section{The Non-Uniform Distribution of Mg in Al-Mg Alloy Under High Pressure Torsion}

Jing Xue ${ }^{1}$, Shenbao Jin ${ }^{1}$, Xianghai An², Xiaozhou Liao ${ }^{2}$, Jiehua Li $^{3}$, Gang Sha ${ }^{1}$

Corresponding Author: gang.sha@,njust.edu.cn

1. Herbert Gleiter Institute of Nanoscience, School of Materials Science and Engineering, Nanjing University of Science and Technology, Nanjing, 210094, China

2. School of Aerospace, Mechanical and Mechatronic Engineering, The University of Sydney, Sydney, NSW 2006, Australia

3. Institute of Casting Research, Montanuniversität Leoben, A-8700, Leoben, Austria

Severe plastic deformation (SPD) techniques have been widely used to produce nano-structured and ultrafine-grained alloys. Besides grain refinement, a significant redistribution of elements could take place during SPD [1], which may significantly alter the mechanical properties of the alloys. Therefore, a basic knowledge between deformation and redistribution of different elements must be established. In the present paper, the Al-Mg alloys were subjected to different turns of HPT processing. The distribution of $\mathrm{Mg}$ before and after deformation was examined using atom probe tomography (APT), as shown in Fig.1. Meanwhile, the crystallographic information was also extracted from APT datasets. The results indicate that a highly non-uniform distribution of $\mathrm{Mg}$ forms in the deformed microstructure, and the movements of the dislocations play an important role in the redistribution of $\mathrm{Mg}$.
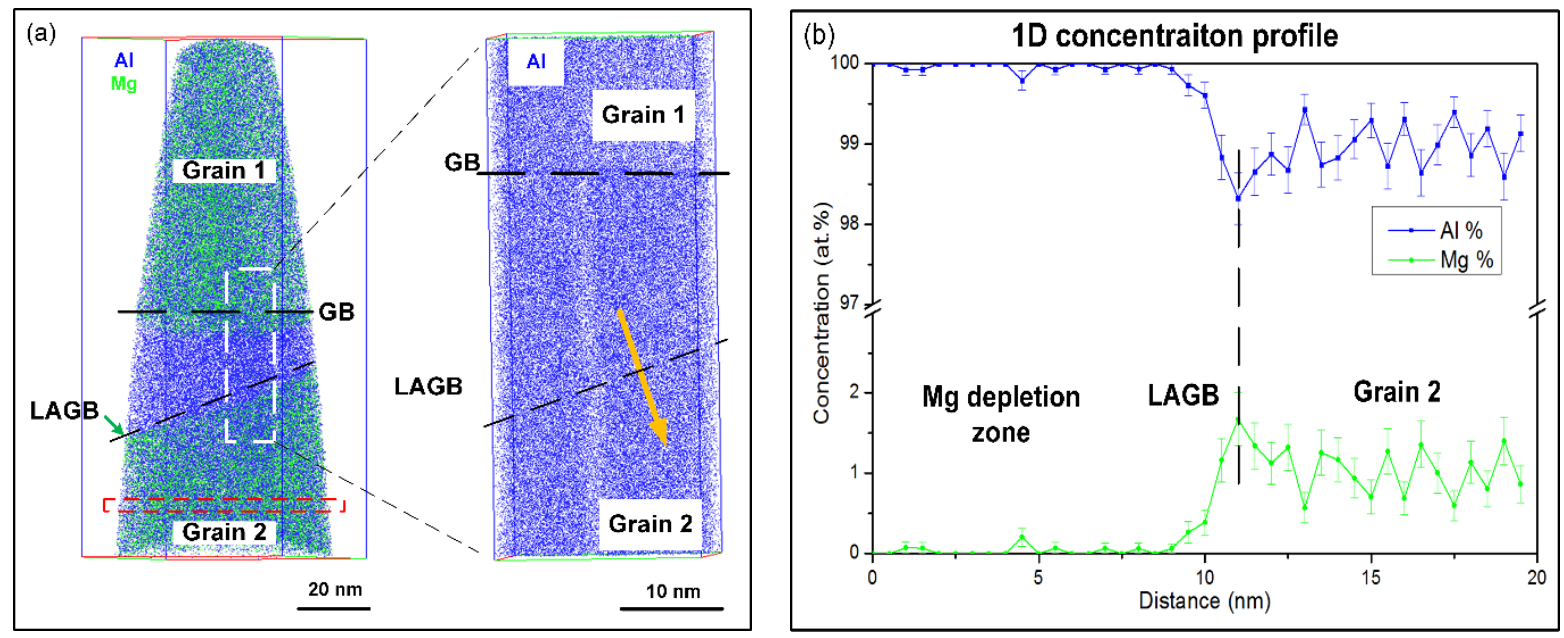

Figure 1 (a) Atom map of reconstructed volume (Al atoms are blue and $\mathrm{Mg}$ atoms are green) (b) 1D concentration profile alone the orange arrow across the low angle grain boundary

References:

[1] G Sha, YB Wang, XZ Liao, ZC Duan, SP Ringer, TG Langdon, Acta Materialia 57 (10), 3123-3132. 


\title{
$\mathrm{CO}_{2}$ Hydrogenation over Rhodium Catalysts: Field Emission Techniques Reveal $\mathrm{CO}_{2}$-Induced Metal-Surface and Bulk Oxidation
}

\author{
Sten V. Lambeets ${ }^{1}$, Daniel E. Perea ${ }^{2}$, Thierry Visart de Bocarmé ${ }^{1}$, Norbert Kruse ${ }^{2,3}$ \\ Corresponding Author: Sten8711@gmail.com \\ 1. Chemical Physics of Materials and Catalysis, ULB, CP243, 1050 Brussels, Belgium. \\ 2. PNNL, Richland, WA 99352, USA. \\ 3. Voiland School of Chemical Engineering and Bioengineering, WSU, Pullman, WA 99164, USA.
}

Field Emission Techniques (FET), such as Field Ion Microscopy (FIM) and Field Emission Microscopy (FEM), can be used to study the dynamics of a chemical reaction on a complex surface of a model nanoparticle conditioned as a tip and presenting a rich diversity of crystallographic planes. In this work we studied the $\mathrm{CO}_{2}$ hydrogenation over $\mathrm{Rh}$. FEM revealed reaction-induced patterns which can be associated with oxygen atom penetration through the $\mathrm{Rh}$ surface testifying a possible inhomogeneous subsurface oxidation process via vicinal facets. The $\{012\}$ facets act as dissociation sites for $\mathrm{CO}_{2}$ (or $\left.\mathrm{O}_{2}\right)$, producing $\mathrm{O}(\mathrm{ads})$ on the surface, as confirmed in 1-D atom probe (1DAP). Subsequently, these O(ads) diffuse beneath the surface through the structures between $\{012\}$ and the $\{113\}$ facets, forming $O($ sub) under $\{113\}$ facets. This hypothesis is supported by APT analysis of Rh specimens which were reacted in an oxygenrich high temperature environment within an in-situ reactor directly attached to LEAP. Prior to any treatment, $\mathrm{Rh}$ specimens were cleaned by field evaporation, leaving a clean surface and revealing the (111) surface geometry after evaporation of the native oxide with a distinguishing 2 x 3-fold symmetric zone line pattern. APT analysis reveals that rhodium oxides preferentially gather only along three zone lines and beneath the central Rh(111) pole. Considering our FIM and FEM observations, we assert that the three corresponding poles correspond to vicinal facets of $\{012\}$ facets. The oxygen atoms diffuse through these facets and nucleate to oxidize the bulk beneath the central (111) pole. This work illustrates the role of bulk oxide formation in evolution of a catalytic particle morphology and the importance of FET in studies of catalytic reactions studies since both surface and subsurface regions may undergo structural and chemical modifications.

Figure 1. (a) FEM pattern transformations of $\mathrm{Rh}$ specimen at $700 \mathrm{~K}$ during $\mathrm{CO}_{2} / \mathrm{N}_{2} \mathrm{O} / \mathrm{O}_{2}$ exposure and subsequent $\mathrm{H}_{2}$ addition. (b) $2 \mathrm{D}$ projection of the $\mathrm{Rh}$ isodensity and APT/FIM overlay of a pure $\mathrm{Rh}$ sample. $\mathrm{RhO}_{\mathrm{x}^{-}}$isodensity profile and APT/FIM overlay of a pure $\mathrm{Rh}$ sample after exposure to $20 \mathrm{sccm}$ of pure $\mathrm{O}_{2}$ during $600 \mathrm{~s}$ at $700 \mathrm{~K}$. a)

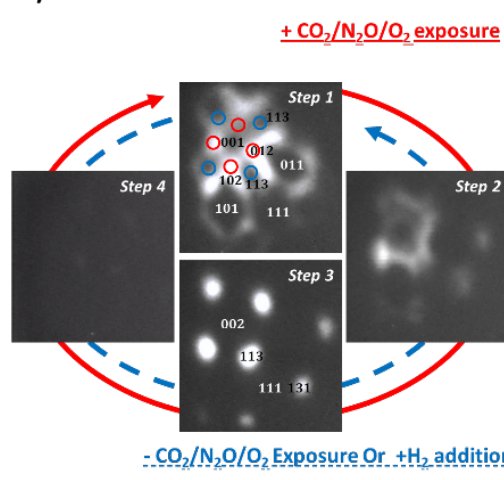

b)

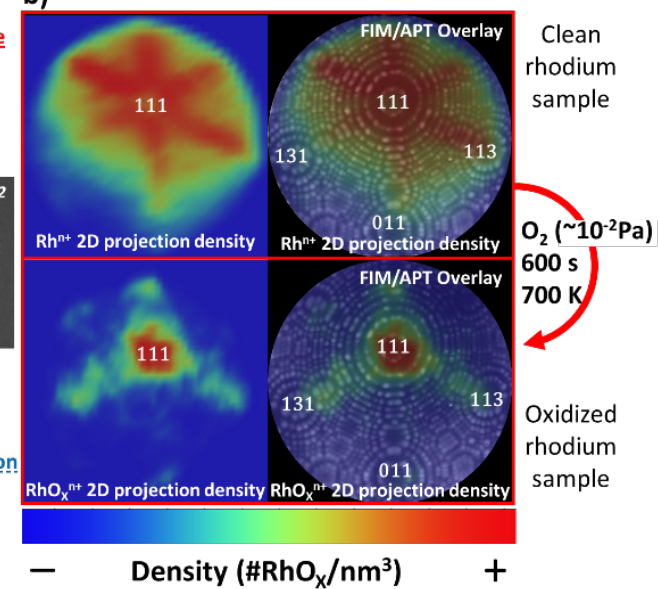




\section{D Nanoscale Compositional Mapping of Li-ion Battery Cathodes Using Atom Probe Tomography}

Pritesh Parikh ${ }^{1}$, Arun Devaraj ${ }^{2}$, and Ying Shirley Meng ${ }^{3}$

Corresponding Author: shmeng@ucsd.edu

1,3. Department of NanoEngineering, University of California San Diego, 9500 Gilman Drive, La Jolla, CA 92093, USA.

2. Physical and Computational Sciences Directorate, Pacific Northwest National Laboratory, P.O. Box 999, Richland, Washington 99352, USA.

Li-ion batteries form the crux of modern technology with every phone, laptop, and now cars utilize them to provide energy. The use of lithium cobalt oxide (LCO) and later other transition metal configurations utilizing $\mathrm{Mn}, \mathrm{Ni}$, and $\mathrm{Co}$ in tandem provide structural stability, good cycle life and high energy density $[1,2]$. Improvements using ALD (atomic layer deposition) based coating as well as doping cathode materials (at less than $1 \%$ of the total stoichiometry) with $\mathrm{Al}$ and $\mathrm{Mg}$ have shown enhanced electrochemical performance [3 4], yet a thorough understanding of the atomic scale mechanisms is still lacking. Specifically certain questions remain unanswered such as how can you characterize nanoscale films and coatings used? How do understand, visualize, and interpret low concentration (<1\%) doping profiles used for improved electrochemical performance and how would you characterize interfaces for next generation of batteries?

In this respect, atom probe tomography (APT) with its ppm (parts per million) sensitivity to all elements and nanoscale resolution forms an ideal method to explore battery materials for the next generation devices. Moreover the nanoscale nature of the coating and low doping levels makes it difficult to analyze these materials effectively using other techniques as SIMS (secondary ion mass spectrometry), STEM (scanning transmission electron microscopy), EELS (electron energy loss spectroscopy), and EDX (energy dispersive x-ray spectroscopy). But the small size of primary particles $(200-500 \mathrm{~nm})$ and the compact nature of them, attached to each other to make micron sized secondary particles, hinder it from using conventional lift-out techniques in the FIB for APT analysis.

Here we developed a direct electrostatic manipulation method, which would allow analysis of single primary particles. We make use of electrostatic forces between particles dispersed on a silicon substrate and the nanomanipulator to transfer selected primary particles of size 200-500 nm onto Si microtip arrays. Our APT analysis shows that different cathode materials behave differently to conventional liftout techniques but the direct electrostatic manipulation method works ubiquitously for all nanoparticle systems with sizes greater than $100 \mathrm{~nm}$, providing a novel way to prepare needle specimens of nanoparticles and analyze them using atom probe tomography.

\section{References:}

[1] Kim, T.-H. et al. The Current Move of Lithium Ion Batteries Towards the Next Phase. Adv. Energy Mater. 2, 860-872 (2012).

[2] Tarascon, J.-M. \& Armand, M. Issues and challenges facing rechargeable lithium batteries. Nature 414, 359-367 (2001).

[3] Cao, Z. et al. Improvement of the Cycling Performance and Thermal Stability of Lithium-Ion Batteries by Coating Cathode Materials with Al2O3 Nano Layer. J. Electrochem. Soc. 164, A475-A481 (2017).

[4] Takanashi, S. \& Abe, Y. Improvement of the electrochemical performance of an NCA positive-electrode material of lithium ion battery by forming an Al-rich surface layer. Ceramics International 43, 9246-9252 (2017). 


\section{Charge-State Field Evaporation Behavior in $\mathrm{Cu}(\mathrm{V})$ Nanocrystalline Alloys}

Xuyang Zhou ${ }^{1}$, and Gregory B. Thompson ${ }^{1}$

Corresponding Author: gthompson@eng.ua.edu

1. The University of Alabama, Department of Metallurgical \& Materials Engineering, Tuscaloosa, USA.

A series of nanocrystalline $\mathrm{Cu}-7 \mathrm{at} . \% \mathrm{~V}$ and $\mathrm{Cu}-27 \mathrm{at} . \% \mathrm{~V}$ films were annealed at $400^{\circ} \mathrm{C}$ and $800^{\circ} \mathrm{C}$ for one hour to study the chemical partitioning effects of solute segregation to grain boundaries as well as their clustering and precipitation behavior. Using cross-correlative precession electron diffraction, the grain boundary's character was determined. Significant clustering of V was noted within high angle grain boundaries and at triple junctions. Upon field evaporation for atom probe tomography analysis, the $\mathrm{V}^{+2} / \mathrm{V}^{+1}$ ratio from the decomposed ions was approximately $\sim 3$ within the grains and $\sim 10$ within the $\mathrm{V}$ clusters and at the grain boundaries. For the $\mathrm{Cu}-27 \mathrm{at} . \% \mathrm{~V} / 800^{\circ} \mathrm{C}$, the onset of oxidation was noted, revealing $\mathrm{VO}^{+1}$ and $\mathrm{VO}^{+2}$ charge states in the $\mathrm{V}$ clusters and a precipitous loss of elemental $\mathrm{V}^{+1}$ charge states. The change in $\mathrm{V}^{+2} / \mathrm{V}^{+1}$ charge with phase is discussed in terms of the chemical coordination of $\mathrm{V}$ with $\mathrm{Cu}$ and with itself. The results reveal how charge state distribution maps can lend insights into the binding energy of solutes in alloys.
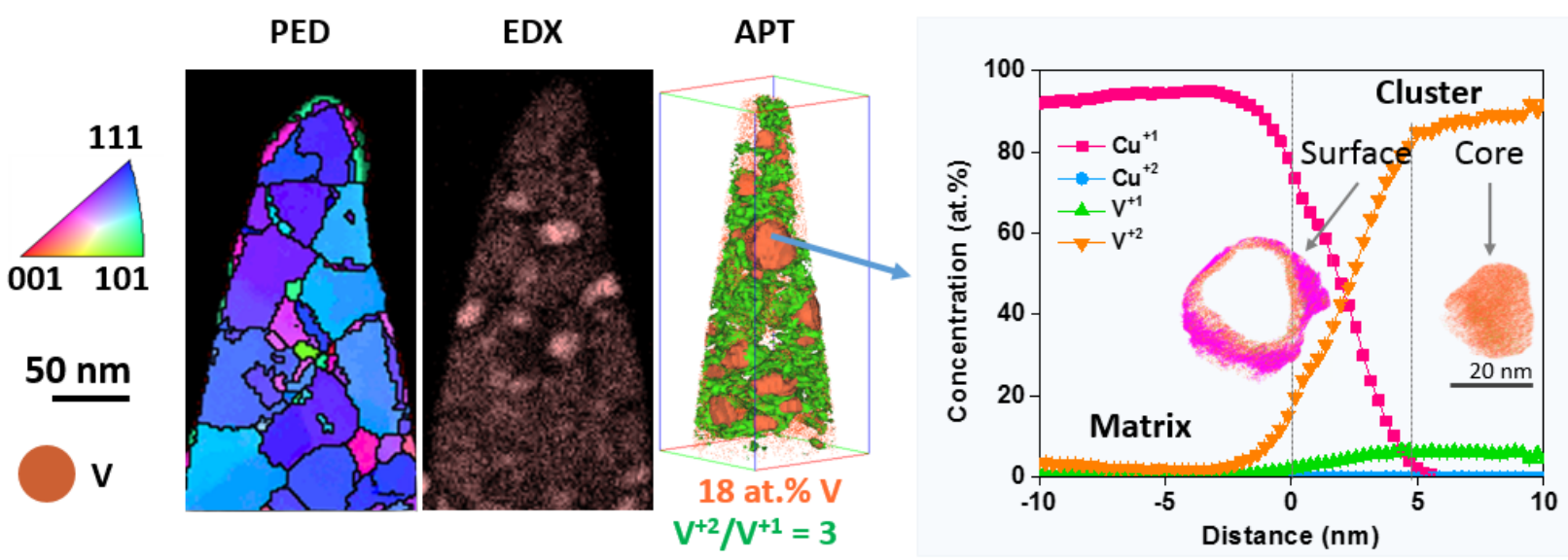

Figure 1 Cross-correlative PED, energy dispersive X-ray spectroscopy (EDX) and APT study of the $400^{\circ} \mathrm{C} 1$ hour annealed $\mathrm{Cu}-7 \mathrm{~V}$ film. The proxigram from one large cluster was also included.

\section{References:}

[1] The authors gratefully acknowledge ARO W911NF-17-1-0528, Dr, Micheal Bakas Program Manager, as well as the University of Alabama’s Central Analytical Facility for support of this research. 



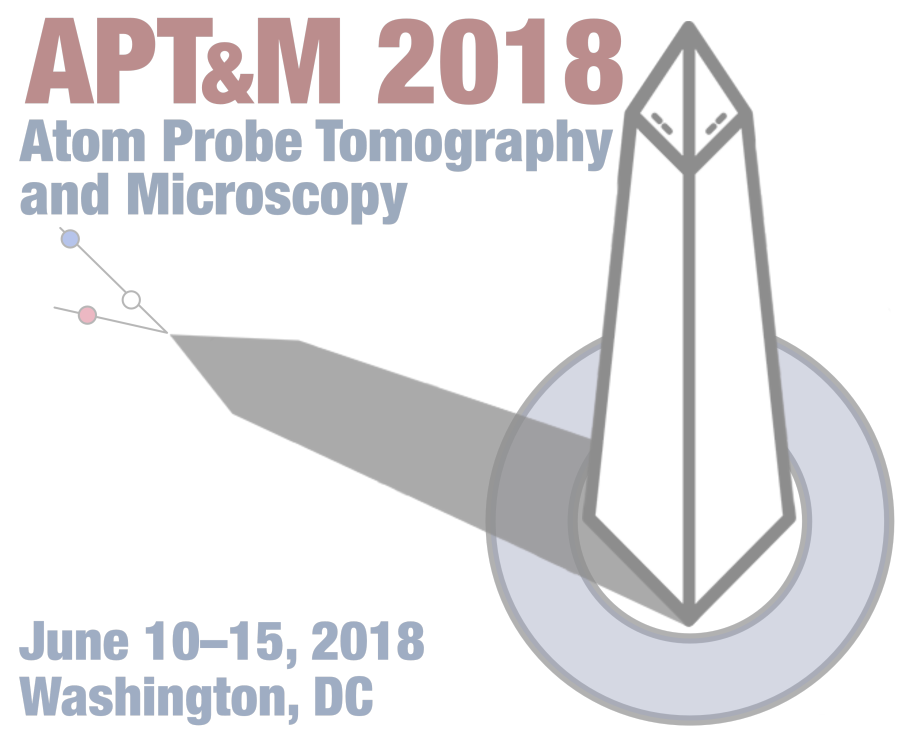

\section{Tuesday Morning}





\section{Tuesday Morning}

\begin{tabular}{|c|c|c|c|c|c|c|}
\hline 8:45 AM & & & & & & \\
\hline 9:00 AM & \multirow{2}{*}{\multicolumn{6}{|c|}{$\begin{array}{c}\text { Plenary 2: New Opportunities at the Nanoscale Using New VUV-EUV Laser Sources } \\
\text { Henry Kapteyn } \\
\text { (Green Auditorium) }\end{array}$}} \\
\hline 9:15 AM & & & & & & \\
\hline 9:30 AM & \multicolumn{6}{|c|}{ Break (9:30 to $10: 00 \mathrm{AM})$} \\
\hline 10:00 AM & \multirow{6}{*}{ 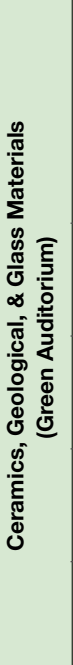 } & \multirow{2}{*}{$\begin{array}{l}\text { Atom Probe Characterization of } \mathrm{Pb} \\
\text { Isotopic Variations in Terrestrial and } \\
\text { Meteoritic Fe Sulfides (Invited) } \\
\text { Steven Reddy }\end{array}$} & \multirow{6}{*}{ 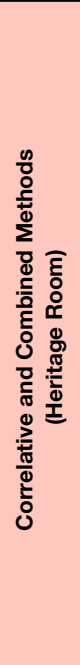 } & \multirow{4}{*}{$\begin{array}{l}\text { Atom Probe Tomography and Secondary } \\
\text { lon Mass Spectrometry: An Interesting } \\
\text { Combination for Atomic Diffusion and } \\
\text { Segregation Measurements (Invited) } \\
\text { Alain Portavoce }\end{array}$} & \multirow{6}{*}{ 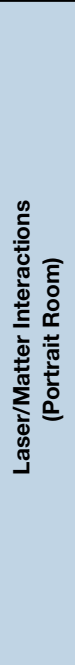 } & $\begin{array}{l}\text { Extreme Ultraviolet-Assisted } \\
\text { Field lon Evaporation } \\
\text { Ann Chiaramonti }\end{array}$ \\
\hline 10:15 AM & & & & & & $\begin{array}{l}\text { Estimation of Relative Evaporation Field } \\
\text { Strength of Bulk Oxide-Composite and } \\
\text { Comprehension of Evaporation } \\
\text { Mechanism } \\
\text { Chang-Min Kwak }\end{array}$ \\
\hline 10:30 AM & & $\begin{array}{l}\text { Dating Planetary Events Using APT } \\
\text { Isotopic Analysis of Natural ZrO } \\
\text { Lee White }\end{array}$ & & & & $\begin{array}{l}\text { Distinctive Bond Breaking in Crystalline } \\
\text { Phase-Change Materials } \\
\text { Oana Cojocaru-Mirédin }\end{array}$ \\
\hline 10:45 AM & & $\begin{array}{l}\text { Preparation of } \sim 100 \mathrm{~nm} \text {-diameter } \\
\text { Presolar SiC in Meteoritic Matrix for } \\
\text { Atom-Probe Tomography (APT) } \\
\text { Josiah Lewis }\end{array}$ & & & & $\begin{array}{l}\text { Study of Optical and Thermal Properties } \\
\text { of Diamond Nanowires by Laser Assisted } \\
\text { Atom Probe Tomography } \\
\text { Jonathan Houard }\end{array}$ \\
\hline 11:00 AM & & $\begin{array}{c}\text { Resolving the Sorption Behavior of Fe(II) } \\
\text { on Hematite at the Atomic Level } \\
\text { Sandra Taylor }\end{array}$ & & $\begin{array}{l}\text { Expensive Jewelry or Casual Decoration? } \\
\text { Aragonite to Vaterite Polymorphisms in } \\
\text { Nacre Explained by Correlative t-EBSD } \\
\text { and APT } \\
\text { Hugues Francois-Saint-Cyr }\end{array}$ & & $\begin{array}{l}\text { Optical Measurements of the Tensile } \\
\text { Stress Induced by a High Electric Field In } \\
\text { Diamond Nanoscale Needles } \\
\text { Linda Venturi }\end{array}$ \\
\hline 11:15 AM & & $\begin{array}{c}\text { Unraveling the Influence of Impurities on } \\
\text { Mineral Growth by combining APT, AFM- } \\
\text { ToF-SIMS and TEM } \\
\text { Juliane Weber }\end{array}$ & & $\begin{array}{c}\text { Interfacial Segregation in a Novel Cu- } \\
\text { Doped TiNiSn Thermoelectric Generator: } \\
\text { An APT and STEM-EDX Study } \\
\text { Paul Bagot }\end{array}$ & & $\begin{array}{c}\text { Study of Electrical Conduction Properties } \\
\text { of Diamond Nanoneedles, by lon } \\
\text { Spectroscopy in Field lon Microscopy } \\
\text { Angela Vella }\end{array}$ \\
\hline 11:30 AM & \multicolumn{6}{|c|}{ Lunch / Poster Viewing (11:30 AM to 1:00 PM) } \\
\hline
\end{tabular}





\title{
New Opportunities at the Nanoscale using New VUV-EUV Laser Sources
}

\author{
Henry C. Kapteyn ${ }^{1,2}$ and Margaret M. Murnane ${ }^{2,1}$ \\ Corresponding Author: hkapteyn@,kmlabs.com \\ 1. Kapteyn-Murnane Laboratories Inc., 4775 Walnut St \#102, Boulder CO 80301. \\ 2. JILA and Department of Physics, University of Colorado, Boulder, CO 80309-0440.
}

Recent years have seen the development and increasing maturation of tabletop-scale coherent laser sources at short-wavelengths spanning from the UV into the x-ray regions of the spectrum. These sources, implemented through strong-field high-order harmonic generation driven by a femtosecond laser, have found increasing use in a variety of scientific applications, including nanoscience and nanotechnology. In this talk, I will describe the state of the art of these sources, as well as our work using them for static and dynamic nanoimaging applications. The capabilities of these sources is continually progressing. In this talk, I will focus on KMLabs' implementation of fully-integrated sources, including the $\mathrm{XUUS}_{4}{ }^{\mathrm{TM}}$ system[1] for generation of coherent light up to $h v>100 \mathrm{eV}$ at $>10 \mathrm{kHz}$ repetition-rates, and the Y-Fi VUV ${ }^{\mathrm{TM}}$, a fiber laserbased source capable of generating coherent ionizing radiation up to $\mathrm{hv} \sim 11 \mathrm{eV}$ at $\mathrm{MHz}$ repetition-rates.

The excellent coherence of these sources make them well-suited for recently-developed coherent diffraction imaging (CDI) techniques, that allow for the first time imaging in this region of the spectrum with diffraction-limited, sub-wavelength resolution. Examples of these imaging capabilities include our recent demonstration of $12.6 \mathrm{~nm}$ resolution using $\lambda=13.5 \mathrm{~nm}$ illumination,[2] EUV nanoimaging in reflection,[3] the first femtosecond-resolution "movies" of surface acoustic wave dynamics,[4] and the use of these sources to characterize self-assembled colloidal crystals. These demonstrations establish EUV imaging as a viable new nanoimaging technique that combines aspects of SEM and AFM imaging with new elemental constrast mechanisms and the ability to access buried structures.[5] The possible use of these sources in APT both as an ionizing source, and for imaging tip shape during APT operation, will also be discussed.

\section{References:}

[1] KMLabs. (2017, 11/12/2017). XUUS ${ }^{\mathrm{TM}}$ Coherent EUV and Soft X-Ray Source. Available: https://kmlabs.com/product/xuus/

[2] D. F. Gardner et al., "Subwavelength coherent imaging of periodic samples using a $13.5 \mathrm{~nm}$ tabletop high-harmonic light source," Nature Photonics, vol. 11, no. 4, pp. 259-+, Apr 2017.

[3] C. L. Porter et al., "General-purpose, wide field-of-view reflection imaging with a tabletop $13 \mathrm{~nm}$ light source," Optica, vol. 4, no. 12, pp. 1552-1557, 2017.

[4] J. R. M. Karl et al., "Stroboscopic Imaging of Acoustic Waves in Nanostructures using Tabletop High Harmonics," in Imaging and Applied Optics 2017 (3D, AIO, COSI, IS, MATH, pcAOP), San Francisco, California, 2017, p. CW1B.2: Optical Society of America.

[5] E. R. Shanblatt et al., "Quantitative Chemically Specific Coherent Diffractive Imaging of Reactions at Buried Interfaces with Few Nanometer Precision," Nano Letters, vol. 16, no. 9, pp. 5444-5450, September 2016. 


\title{
Atom Probe Characterization of Pb Isotopic Variations in Terrestrial and Meteoritic Fe Sulfides
}

\author{
Steven M. Reddy ${ }^{1,2}$, Denis Fougerouse ${ }^{1,2}$, David Saxey ${ }^{1,2}$, William D.A. Rickard ${ }^{1,2}$, Chris \\ Kirkland $^{2}$, Mark Pearce ${ }^{3}$, Louise Fisher ${ }^{3}$, Matt Kilburn ${ }^{4}$, Rob Hough ${ }^{3}$, Gretchen Benedix ${ }^{2}$ \\ Corresponding Author: s.reddy@curtin.edu.au \\ 1. Geoscience Atom Probe, Advanced Resource Characterisation Facility, John de Laeter Centre, \\ Curtin University, Perth, Australia. \\ 2. School of Earth and Planetary Sciences, Curtin University, Perth, Australia. \\ 3. CSIRO Earth Sciences and Resource Engineering, ARRC, Kent Street, Perth, Australia \\ 4. CMCA, University of Western Australia, Perth, Australia
}

Fe sulfide minerals are ubiquitous in terrestrial and extra-terrestrial rock samples and their presence provides valuable constraints on a range of geological conditions and processes, from deep in the Earth's mantle to its atmosphere. One important aspect of Fe sulfides is that they often incorporate $\mathrm{Pb}$ during their growth. Since the parent $\mathrm{U}$ and $\mathrm{Th}$ isotopes responsible for radiogenic $\mathrm{Pb}$ are not partitioned into Fe sulfide, then the measured $\mathrm{Pb}$ composition reflects the local isotopic composition at the time of sulfide growth. This has useful applications in the geosciences, but commonly $\mathrm{Pb}$ isotope systematics of $\mathrm{Fe}$ sulfides are complicated by mixing between multiple isotopic reservoirs. Such mixing cannot be spatially resolved by conventional analytical techniques, indicating that it represents a nanoscale phenomena that could be addressed by atom probe microscopy.

To understand the nature of different $\mathrm{Pb}$ reservoirs and how they form, we have undertaken an atom probe study of two different Fe sulfide systems. The first example is the meteoritic Canyon Diablo troilite, which yields the solar system's most primordial $\mathrm{Pb}$ isotopic composition.

Correlative microscopy techniques (synchrotron-based X-Ray mapping, EBSD \& NanoSIMS) show that the majority of the $\mathrm{Pb}$ does not reside in the troilite but is found in sub-micrometre inclusions. Atom probe analysis of these inclusions provide new constraints on the distribution and modification of primordial $\mathrm{Pb}$ in the early solar system. In a second study, atom probe analysis of Fe sulfides from the world's largest $\mathrm{Au}$ ore system shows heterogeneous $\mathrm{Pb}$ and other trace element distributions related to discrete nanoscale defects. These defects contain varying $\mathrm{Pb}$ isotopic compositions that place new constraints on the mechanisms of $\mathrm{Pb}$ mobility in $\mathrm{Fe}$ sulfide at the nano to $\mathrm{mm}$ scales. These constraints allow resolution of temporally-distinct $\mathrm{Pb}$ mobility events, and provide a new explanation for isotopic mixing that has been previously reported across the whole sedimentary basin.

The results of both of these studies provide new constraints on the incorporation and mobility of $\mathrm{Pb}$ in $\mathrm{Fe}$ sulfides that would not have been possible without the atom probe. The results illustrate the ability of atom probe studies to place constraints on large-scale geological processes, with the potential to be widely applied across the geosciences discipline.

\section{References:}

[1] The Geoscience Atom Probe at Curtin University is part of the Advanced Resource Characterization Facility under the auspices of the National Resource Sciences Precinct - a collaboration between the Commonwealth Scientific and Industrial Research Organization, Curtin University, and the University of Western Australia - and is supported by the Science and Industry Endowment Fund (SIEF RI13-01). 


\section{Dating Planetary Events Using APT Isotopic Analysis of Natural $\mathrm{ZrO}_{2}$}

Lee White ${ }^{1,2}$, Desmond Moser ${ }^{3}$, Kim Tait ${ }^{1,2}$, James Darling ${ }^{4}$, David Reinhard ${ }^{5}$, and Brian Langelier $^{6}$

Corresponding Author: 1white@,rom.on.ca

1. Department of Earth Sciences, University of Toronto, Toronto, Canada

2. Department of Natural History, Royal Ontario Museum, Toronto, Canada

3. Department of Earth Sciences, University of Western Ontario, London, Canada

4. School of Earth and Environmental Sciences, University of Portsmouth, Portsmouth, UK

5. Cameca Instruments Inc., Madison, Wisconsin, USA

6. Canadian Centre for Electron Microscopy (CCEM), McMaster University, Hamilton, Canada

The accessory mineral phase baddeleyite (monoclinic- $\mathrm{ZrO}_{2}$ ) commonly crystallizes in a wide range of silica depleted rock types including many volcanic rocks on Earth, and in Martian, Lunar and asteroidal meteorites [1]. Baddeleyite often incorporates U into the lattice during crystallization, allowing the ratio of $U$ to radiogenic $\mathrm{Pb}$ to be used to calculate the age of the crystal [2]. While this is a relatively straight forward process for undeformed terrestrial grains, many of the baddeleyite grains found in meteorites have been highly altered by shock metamorphism during ejection from their parent planet. This process, in which rocks are often exposed to transient loading in excess of $30 \mathrm{GPa}$, can cause the development of complex nanostructures that are linked to variable disturbance of the U-Pb systematics of the baddeleyite grain and either fully or partially reset the measurable age of the crystal [3]. Recent work using atom probe tomography (APT) has revealed that the technique can accurately measure $\mathrm{U} / \mathrm{Pb}$ ratios from nanoscale volumes, allowing us to differentiate primary age reservoirs in baddeleyite from those reset by impact bombardment and facilitating accurate dating of these large planetshaping events [4]. Here, we use this new approach to date baddeleyite grains within a range of planetary materials, including a terrestrial impact structure and lunar meteorite Northwest Africa (NWA) 3163, to begin to refine our understanding of the timing and mechanisms of impact processes and the formation and evolution of the Moon. Going forward, the application of APT to baddeleyite promises to augment many ongoing studies attempting to constrain the formation and evolution of the Solar System.

\section{References:}

[1] Herd, C. D. K., Moser, D. E., Tait, K., Darling, J. R., Shaulis, B. J., \& McCoy, T. J. (2018). Crystallization of Baddeleyite in Basaltic Rock from Mars, and Comparisons with the Earth, Moon, and Vesta. In: Moser et al., Microstructural Geochronology, John Wiley \& Sons Inc.

[2] Heaman, L. M., \& LeCheminant, A. N. (1993). Paragenesis and U-Pb systematics of baddeleyite $\left(\mathrm{ZrO}_{2}\right)$. Chemical Geology, 110(1-3), 95-126.

[3] Darling, J. R., Moser, D. E., Barker, I. R., Tait, K. T., Chamberlain, K. R., Schmitt, A. K., \& Hyde, B. C. (2016). Variable microstructural response of baddeleyite to shock metamorphism in young basaltic shergottite NWA 5298 and improved U-Pb dating of Solar System events. Earth and Planetary Science Letters, 444, 1-12.

[4] White, L. F., Darling, J. R., Moser, D. E., Reinhard, D. A., Prosa, T. J. Bullen, D., Olson, D., Larson, D. J., Lawrence, D., \& Martin, I. (2017). Nature Communications, 15597. 


\section{Preparation of $\sim 100 \mathrm{~nm}$-diameter Presolar $\mathrm{SiC}$ in Meteoritic Matrix for Atom-Probe Tomography (APT)}

Josiah Lewis ${ }^{1}$, Pranav Bhadharla ${ }^{1}$, Christine Floss ${ }^{1}$, Dieter Isheim ${ }^{2}$, and David N. Seidman ${ }^{2}$

Josiah Lewis: jblewis@go.wustl.edu

${ }^{1 .}$ Laboratory for Space Sciences, Physics Department, Washington University in St. Louis, St. Louis, MO, USA.

2. Northwestern University Center for Atom-Probe Tomography (NUCAPT), and Department of Materials Science and Engineering, Northwestern University, Evanston, IL, USA.

Presolar SiC grains are identified in primitive meteorites based on anomalies in stable isotopic ratios that are inconsistent with solar system processes [1]. The morphologies, compositions, and crystallographies of these grains yield insights into the nuclear synthesis processes occurring in the asymptotic giant branch stars and supernovae, where they formed.

We are using atom-probe tomography (APT) to study the isotopic ratios, elemental concentrations, trace element distributions, subgrains, and surface layers of individual 100 to 200 $\mathrm{nm}$ diameter presolar $\mathrm{SiC}$ grains identified in situ in polished meteorite thin-sections by Auger electron spectroscopy and secondary-ion mass-spectrometry [2, 3]. SiC grains in this size range have not previously been well-characterized, except in bulk. Using FIB liftout and $\mathrm{Ga}^{+}$ionmilling we have prepared SiC grains in APT nanotips (Figure 1) and will utilize a LEAP5000XS for APT analyses.

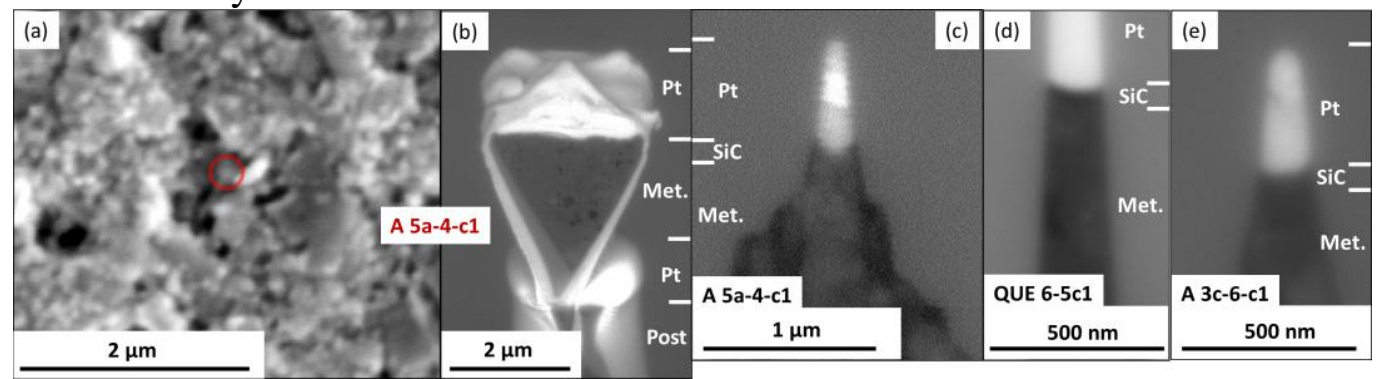

Figure 1 (a) Secondary electron image of a section of the meteorite Adelaide with a SiC grain, 5a-4-c1, circled. (b) Backscatter electron image of the region surrounding the $\mathrm{SiC}$ grain after lift-out (dark contrast, label "Met."), covered with Pt strap and fiduciary ' $\mathrm{x}$ ' (bright contrast, top "Pt" label), attached to a micropost using a Pt deposit (lower "Pt"). Backscatter images of SiC grains (c) Adelaide 5a-4-c1, (d) QUE 99177 6-5c1, and (e) Adelaide 3c-6-c1, prepared in nanotips. "SiC" is the region where the grains are putatively located. The bright material is layers of protective Pt. Darker material is meteoritic.

References:

[1] E. Zinner, Presolar Grains in: A. Davis, Treatise on Geochemistry, $2^{\text {nd }}$ Ed., vol. 1, Elsevier, 2014, pp. $181-213$.

[2] C. Floss and F. J. Stadermann, High abundances of circumstellar and interstellar C-Anomalous phases in the primitive CR3 chondrites QUE 99177 and MET 00426, ApJ 697 (2009), 1242-1255.

[3] C. Floss and F. J. Stadermann, Presolar silicate and oxide abundances and compositions in the ungrouped carbonaceous chondrite Adelaide and the K chondrite Kakangari: The effects of secondary processing, Meteoritics and Planetary Science 47 (2012), 992-1009.

[4] This work is supported by NASA grant NNX16AD26G (C.F.). The LEAP tomograph at NUCAPT was acquired with grants from the NSF (DMR-0420532) and ONR-DURIP (N00014-0400798, -0610539, -0910781, -1712870) programs. NUCAPT received support through the Materials Research Center (NSF DMR-1720139), the SHyNE Resource (NSF ECCS-1542205), and ISEN at Northwestern University. 


\section{Resolving the Sorption Behavior of Fe(II) on Hematite at the Atomic Level}

Sandra D. Taylor*, Jia Liu, Bruce W. Arey, John B. Cliff, Daniel K. Schreiber, Daniel E. Perea, and Kevin M. Rosso

*Corresponding Author: sandra.taylor@pnnl.gov

Pacific Northwest National Laboratory, Richland, WA 99352 USA

The interaction and interconversion between $\mathrm{Fe}(\mathrm{II})_{\mathrm{aq}}$ and dominant $\mathrm{Fe}(\mathrm{III})$ (oxyhydr)oxides minerals is a fundamental redox process in nature, influencing the redox cycling of iron (as well as heavy metals, contaminants, and nutrients) in the subsurface. However, the mechanisms and atom exchange pathways involved in the $\mathrm{Fe}$ (II)-accelerated recrystallization of $\mathrm{Fe}$ (III) (oxyhydr)oxides are poorly understood. Isotopic mapping probes, specifically atom probe tomography (APT) and nanoscale secondary ion mass spectrometry (NanoSIMS), were applied here to explore the recrystallization of individual hematite $\left(\mathrm{Fe}_{2} \mathrm{O}_{3}\right.$, naturally abundant in $\left.{ }^{56} \mathrm{Fe}\right)$ microparticles reacted with ${ }^{57} \mathrm{Fe}(\mathrm{II})_{\mathrm{aq}}$ as a tracer. By providing information on the depth and spatial distribution of ${ }^{57} \mathrm{Fe}$ sorbed onto surfaces, these techniques can aid in establishing the physical location(s) of atom exchange fronts at these dynamic mineral-water interfaces.

${ }^{57} \mathrm{Fe}$-enrichment at the hematite surfaces was resolved with both techniques, where results generally indicate that apparent atom exchange front distributions are heterogeneous with respect to different crystallographic surface terminations and morphological features. For example, NanoSIMS measurements of ${ }^{57} \mathrm{Fe} /{ }^{56} \mathrm{Fe}$ ratios across individual crystallites show $\mathrm{Fe}(\mathrm{II})$ sorption is facet-dependent. Adsorption of ${ }^{57} \mathrm{Fe}$ is highest on the basal surface, and exhibits up to $10 \times \mathrm{s}$ more enrichment relative to edge surfaces (e.g. (012)). APT was used to further analyze the basal surface, enabling spatial and temporal resolution of ${ }^{57} \mathrm{Fe}$-enriched regions at the sub-nanometer level. ${ }^{1}$ Concentration profiles across the ${ }^{57} \mathrm{Fe}$-hematite interface show that at these conditions ${ }^{57} \mathrm{Fe}$ in solution primarily adsorbs to the surface with little recrystallization occurring (i.e., significant atom exchange between ${ }^{57} \mathrm{Fe}$ and ${ }^{56} \mathrm{Fe}$ from the surface could not be found). The average net sorption of ${ }^{57} \mathrm{Fe}$ was measured to be $1.3-1.7$ monolayers. Statistical analyses utilizing grid-based frequency distributions show a heterogeneous, non-random distribution of oxidized Fe on the (001) surface, indicating atomic segregation and formation of a nanostructured surface texture at the reacted ${ }^{57} \mathrm{Fe}$-hematite interface that is consistent with Volmer-Weber-like island growth. The collective findings shed new light on potential electron and atom exchange pathways between $\mathrm{Fe}(\mathrm{II})_{\mathrm{aq}}$ and dominant $\mathrm{Fe}(\mathrm{III})$ (oxyhydr)oxides minerals, with important and broad implications on our understanding biogeochemical cycling of elements in the environment.

\section{References:}

[1] S. D. Taylor, et al., Resolving Fe(II) Sorption and Oxidative Growth on Hematite (001) Using Atom Probe Tomography, JPCC (2018), Accepted.

[2] This research is based upon work supported by the U.S. DOE Office of Science (DOE), Office of Basic Energy Sciences, Division of Chemical Sciences, Geosciences, and Biosciences through its Geosciences program at PNNL. Sample preparation with the FEI Helios NanolabSEM and analyses with LEAP 4000 XHR and Cameca NanoSIMS were performed using EMSL, a national scientific user facility sponsored by DOE's Office of Biological and Environmental Research and located at PNNL. 


\section{Unraveling the Influence of Impurities on Mineral Growth by Combining} APT, AFM-ToF-SIMS and TEM

Juliane Weber ${ }^{1}$, Jonathan Poplawsky ${ }^{2}$, Karren L. More ${ }^{2}$, Anton Ievlev², Matthias Lorenz ${ }^{2}$, Steven R. Higgins ${ }^{3}$, Angela L. Bertagni ${ }^{3}$, Sarah Jindra ${ }^{3}$, Jacquelyn N. Bracco ${ }^{4}$ and Andrew G. Stack ${ }^{1}$

Corresponding Author: weberj@ornl.gov

${ }^{1}$ Geochemistry and Interfacial Sciences Group, Chemical Sciences Division, Oak Ridge National Laboratory, Oak Ridge, USA.

2. Center for Nanophase Materials Science, Oak Ridge National Laboratory, Oak Ridge, USA.

${ }^{3}$ Wright State University, Dayton, USA.

4.Argonne National Laboratory, Chicago, USA.

Various minerals showed useful capabilities in capturing toxic elements, e.g., radionuclides, during nuclear waste disposal, oil/gas production or unconventional gas extraction [1]. These hazardous elements influence the mineral growth rate, but the link between their incorporation and their effects on growth rates and morphology has not been fully established. In our study, we chose the model system of barite $\left(\mathrm{BaSO}_{4}\right)$ and $\mathrm{Sr}$ to elucidate the relationship between the rates of growth and incorporation. Here, we combined in-situ growth observations by atomic force microscopy (AFM) with high-resolution ex-situ chemical imaging. To excavate the Sr-distribution within the grown solid, we employed atom probe tomography (APT), time of flight secondary ion mass spectrometry equipped with AFM (AFM-ToF-SIMS) and scanning transmission electron microscopy energy-dispersive X-ray spectroscopy (STEM-EDX). Barite growth experiments under incrementally increasing Sr-concentration were conducted showing a decrease in growth rate with added Sr. At a threshold concentration of strontium, the growth mechanism transitioned and produced a different rate of growth. APT and AFM-ToF-SIMS characterization showed the spatially uniform incorporation whereas Sr-concentration with depth was correlated with Sr-concentration in the solution during growth. Based on these results, we will discuss the underlying mechanism by which inhibitors decrease mineral growth and implications for our understanding of mineral precipitation kinetics.
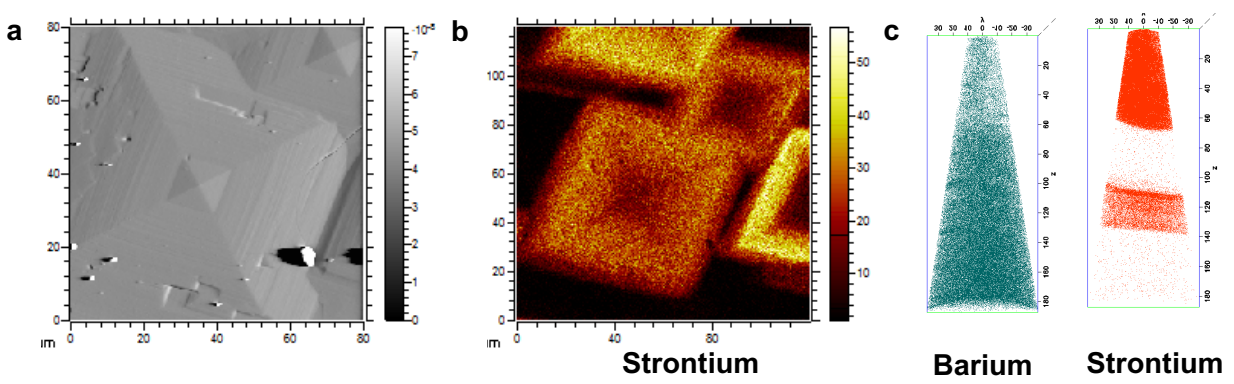

Figure 1 Combined analyses of Sr-concentration in barite showing spatially uniform distribution $(a, b)$ with AFM-ToF-SIMS and with depth by APT (c).

\section{References:}

[1] Prieto, M. Heberling, F. Rodriguez-Galan, R.M., Brandt, F., Crystallization behavior of solid solutions from aqueous solutions: An environmental perspective. Progress in Crystal Growth and Characterization of Materials, 62 (2016), 29-68. 


\section{Opportunities offered by the Serial and or In Situ Combination of Atom Probe and (Scanning) Transmission Electron Microscopy}

Williams Lefebvre ${ }^{1}$, Ivan Blum ${ }^{1}$, Celia Castro, Lorenzo Rigutti ${ }^{1}$, Florian Chabanais ${ }^{1}$, Antoine Normand $^{1}$ and François Vurpillot ${ }^{1}$

Corresponding Author: williams.lefebvre@univ-rouen.fr

1. Normandie Univ., GPM, UNIROUEN, INSA Rouen, CNRS

The ultimate capabilities achieved by electron microscopies and their associated techniques inevitably raise the following question: is there room for conceiving new ways of investigating materials at the nano-scale? Indeed, most recent TEMs and STEMs easily achieve sub-Angström spatial resolution, while allowing elemental mapping at the same scale. Meanwhile, electron tomography has unambiguously demonstrated the possibility to image atomic positions and defects. In these instruments, some physical properties (e.g. optical, magnetic) are now accessible, again with increased resolution. However, as far as an ultimate machine would allow correlating physical properties with a "perfect" determination of atomic species and atomic positions in $3 \mathrm{D}$, one must recognize that such a tool is not yet available. Aside from electron microscopes, Atom Probe Tomography (APT), which is intrinsically a 3D technique, has received increased attention owing to drastic developments during the last decade. Thanks to the improvement of specimen preparation protocols, APT can be applied to much broader areas of materials science (semi-conductors, bio-materials, geo-materials, soft mater and even liquids).

Strong advantages of APT rely in its possibility to detect all types of atoms, independently of their atomic number, in its excellent detection limit and in its intrinsic 3D nature. In order to collect a significant amount of information on a same nano-object, it is relevant to consider a correlative approach combining a TEM/STEM and APT. Motivations for such an approach are numerous. A non-exhaustive list would evoke: i) the possibility to associate structural defects (in TEM) with segregations (in APT); ii) associating the morphology of a particle (in electron tomography) with a 3D field of composition (in APT); iii) improving the quality of APT reconstructions by accessing additional information about the specimen morphology in TEM/STEM.

This presentation will provide some illustrations of correlative investigations on alloys and quantum wells. Then, possibility to combine an atom probe with a transmission electron microscope will be discussed. 


\title{
Atom Probe Tomography and Secondary Ion Mass Spectrometry: An Interesting Combination for Atomic Diffusion and Segregation Measurements
}

\author{
Alain Portavoce ${ }^{1}$, Khalid Hoummada ${ }^{1}$, and Lee $\mathrm{Chow}^{2}$ \\ Corresponding Author: alain.portavoce@im2np.fr \\ 1. IM2NP, CNRS/Aix-Marseille University, Faculté des Sciences de Saint-Jérôme case 142, \\ 13397 Marseille, France \\ 2. Department of Physics, University of Central Florida, Orlando, Florida 32816, USA
}

For a long time, secondary ion mass spectrometry (SIMS) was the only technique allowing impurity concentrations below 1 at $\%$ to be precisely measured in a sample with a depth resolution of few nanometers. For example, SIMS is the classical technique used in microelectronics to study dopant distribution in semiconductors and became, after radiotracers were forsaken, the principal tool used for atomic transport characterization (diffusion coefficient measurements). Due to the lack of equivalent techniques, SIMS could be erroneously used, especially when the analyzed solute atoms formed clusters, or for interfacial concentration measurements (segregation coefficient measurements) for example. Today, concentration profiles measured by atom probe tomography (APT) can be compared to SIMS profiles and allow the accuracy of SIMS measurements to be better evaluated. However, APT measurements can also carry artifacts and limitations that can be investigated by SIMS. After an illustrated summary of SIMS and APT measurement advantages and disadvantages, the complementarity of these two techniques will be discussed, especially in the case of experiments aiming to measure diffusion and segregation coefficients.
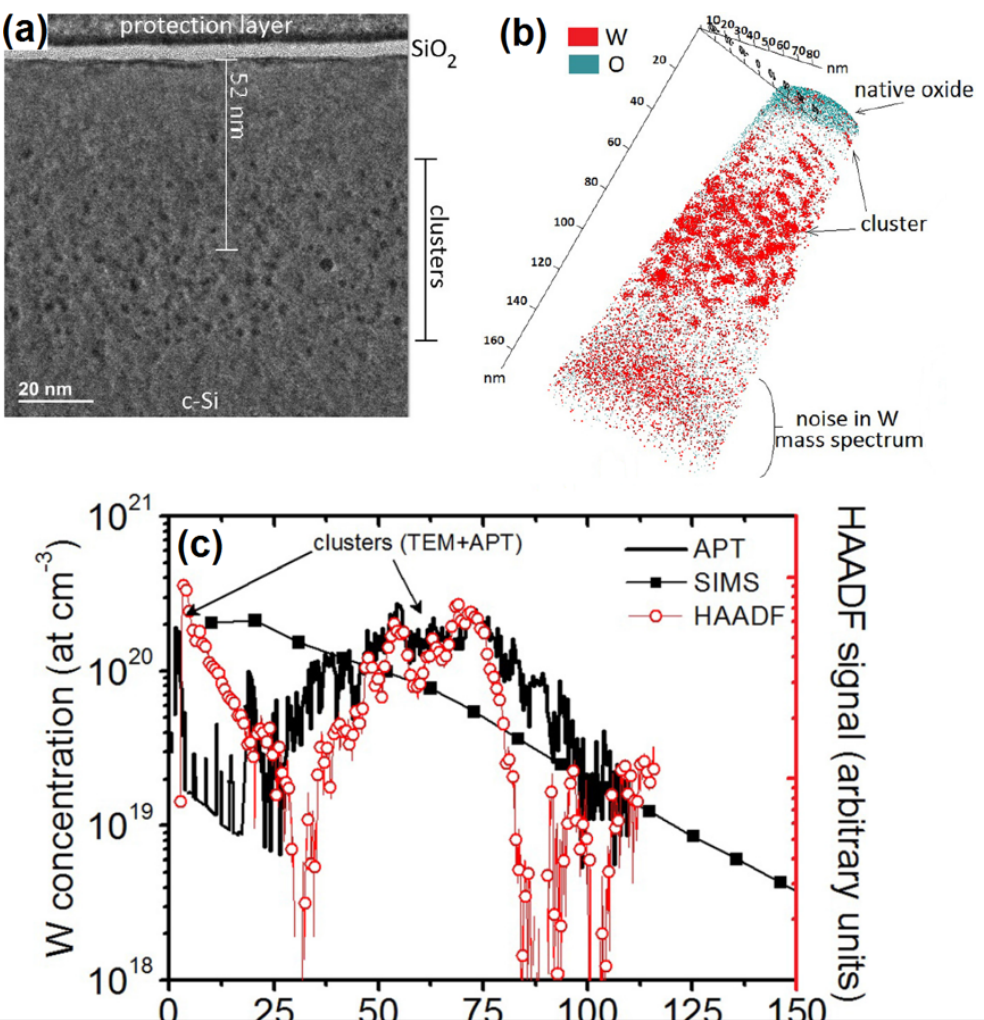

Tuesday Morning 10:30 to $11: 00$ AM

Heritage Room
Figure 1. Comparison between TEM (a), APT (b), and SIMS (c) measurements performed on a same sample consisting of a $\mathrm{Si}(001)$ substrate implanted with a dose of $10^{15} \mathrm{~W}^{+}$ions $\mathrm{cm}^{-2}$ using a beam energy of $165 \mathrm{Kev}$, and annealed at $837^{\circ} \mathrm{C}$ for $48 \mathrm{~h}$. 


\section{Expensive Jewelry or Casual Decoration? Aragonite to Vaterite Polymorphisms in Nacre Explained by Correlative t-EBSD and APT}

Hugues Francois-Saint-Cyr ${ }^{1}$, Isabelle Martin ${ }^{1}$, Ty J. Prosa ${ }^{1}$, D.J. Larson ${ }^{1}$, Xavier Bourrat ${ }^{2}$, Guillaume Wille ${ }^{2}$, Tara Nylese ${ }^{3}$, Matthew Nowell ${ }^{3}$, René de Kloe ${ }^{3}$, David Gibouin ${ }^{4}$, Christelle Guillermier $^{5}$ and Matthew Steinhauser ${ }^{5}$

Corresponding Author: hugues.fsc@ametek.com

1. CAMECA Instruments Inc., 5470 Nobel Drive, Madison WI, 53711, USA.

2. BRGM, French Geological Survey, 3 Av. Guillemin, BP 36009, 45060 Orleans cedex2, France

3. EDAX Inc., 91 McKee Drive, Mahwah NJ, 07430, USA.

4. Faculté des Sciences, U. de Rouen, EA3829, Place E. Blondel, 76131 Mont-St-Aignan, France

5. Harvard Medical School, 65 Landsdowne Street, Room 535, Cambridge MA 02139, USA

The Hyriopsis cumingii Chinese pearl sometimes exhibits synchronous co-growths consisting of Aragonite and Vaterite, two polymorphic phases in direct contact with each other. The highly desirable shiny appearance of pearls is provided by the Aragonite while the Vaterite, full of defects, causing a milky appearance, highly detrimental from an economic point of view.

A comprehensive study [1] involving NanoSIMS (NS), Atom Probe Tomography (APT) and Raman-in-SEM has shown that although similar $\mathrm{Ca}, \mathrm{C}$ and $\mathrm{O}$ contents in both phases were found, the presence of mineral traces (especially $\mathrm{Mn}, \mathrm{Al}$ and $\mathrm{Mg}$ ) more abundant in the Vaterite phase, was suspected to be at the basis of a distortion mechanism of the Aragonite phase. However, the Vaterite structure itself is still the subject of controversy due to its biogenic character and its limited crystal size. A recent $\mathrm{C}_{\mathrm{s}}$ aberration-corrected Transmission Electron Microscopy (TEM) study depicted Vaterite as a mixture of two interspersed crystalline structures, mostly hexagonal [2] and other works involve a micro-twining mechanism [3]. Therefore, using the synergetic efforts achieved over the past years between APT and Transmission Electron Backscatter Diffraction users, we aim at comparing regions extracted from the Aragonite region with respect to the Vaterite area, where the biomineral region of interest is about $20 \mathrm{~nm}$, sandwiched by $\mathrm{CaCO}_{3}$ platelets of 400- to 500-nm long. Some specific specimen preparation issues had to be overcome with the Focused Ion beam due to the onion ring structure of these phases often leading to delamination. In addition, a proper set of analytical conditions was refined for APT through a design of experiments in laser-assisted pulsing mode. Once the practical aspects are overcome, ascertaining which one of the trace elements is the most critical one driving the polymorphism is of the upper importance to the pearl industry, as it could play a major role on achieving pearls with a perfect shine with a higher return on investment.

$\underline{\text { References: }}$

[1] H. Francois-Saint-Cyr et al., Correlative NanoSIMS, Confocal Raman-in-SEM \& Atom Probe Study of Nacre: Toward Understanding Polymorphism Effects in a Chinese Pearl, Microscopy and Microanalysis, 22(3) (2016).

[2] L. Kabalah-Amitai, et al., Vaterite Crystals Contain Two Interspersed Crystal Structures, Science, 340 (2013), 454-457.

[3] A. Le Bail, et al., Microtwinning Hypothesis for a More Ordered Vaterite Model, Powder Diffraction. 26 (2011), 16-21. 


\title{
Interfacial Segregation in a Novel Cu-doped TiNiSn Thermoelectric Generator: An APT and STEM-EDX Study
}

\author{
P.A.J. Bagot ${ }^{1}$, J. Halpin ${ }^{2}$, L. Daly ${ }^{3}$, M.P. Moody ${ }^{1}$, J.-W.G. Bos ${ }^{4}$, D.A. MacLaren ${ }^{2}$
}

Corresponding Author: paul.bagot@materials.ox.ac.uk

1. University of Oxford, Department of Materials, Oxford, OX1 3PH, UK

2. University of Glasgow, School of Physics \& Astronomy, Glasgow, UK

3. University of Glasgow, School of Geographical and Earth Sciences, Glasgow, UK

4. Institute of Chemical Sciences and Centre for Advanced Energy Storage and Recovery, School of Engineering and Physical Sciences, Heriot-Watt University, Edinburgh, UK

Thermoelectric generators (TE) are solid-state devices that can convert waste heat from a variety of sources directly into electricity. This makes them especially attractive materials from an energy efficiency perspective, although their wide-scale adoption is currently impeded by the high cost of the often-scarce materials used, as well as present limiting heat-to-electricity conversion efficiencies. The half-Heusler system TiNiSn has demonstrated considerable promise in terms of potential device performance, particularly when doped with trace $\mathrm{Cu}$ which introduces phonon point scattering to lower lattice thermal conductivity. This system is also inexpensive compared to other candidate TE's, and does not require the current common approach of introducing nano-engineered phases into the microstructure to achieve acceptable performance. However, it is still vital to understand the distribution of introduced trace $\mathrm{Cu}$ atoms at a fundamental level, identifying any preferential site occupancies in the crystal lattice or local differences in elemental composition. In this work, we use Atom Probe Tomography to explore the microstructure of a novel TiNiCu0.02Sn half-Heusler alloy. Complementary STEM analysis has highlighted the formation of $\mathrm{Cu}$ wetting-layers at grain boundaries, along with slight compositional variations in grain chemistries either side of the boundary. The 3D distribution of the $\mathrm{Cu}$-rich features and grain chemistries has been further examined in detail by APT, revealing new and important insights into the segregation behavior in these materials, vital to optimize future device performance.

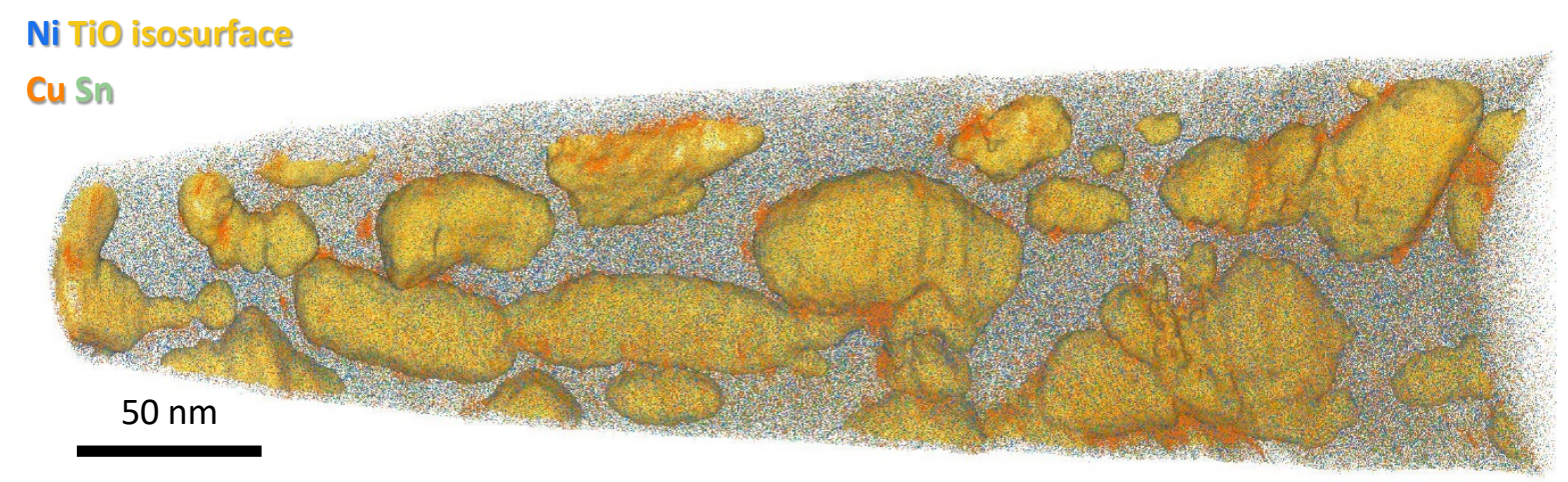

Figure 1 Atom map of TiNiCu0.02Sn half-Heulser alloy, showing nanoscale $\mathrm{Cu}$ segregation along grain boundary decorated by $\mathrm{TiO}$ inclusions ( $2 \% \mathrm{TiO}$ iso-concentration surface shown). 


\section{Extreme Ultraviolet-Assisted Field Ion Evaporation}

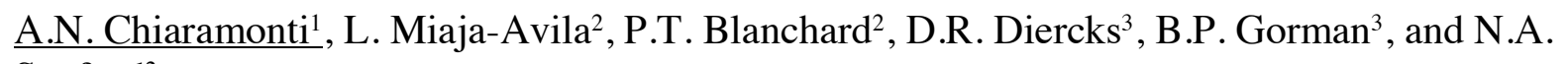
Sanford ${ }^{2}$

Corresponding author: chiaramonti@nist.gov

1. Material Measurement Laboratory, National Institute of Standards and Technology, Boulder, CO USA

2. Physical Measurement Laboratory, National Institute of Standards and Technology, Boulder, CO USA

3. Metallurgical and Materials Engineering, Colorado School of Mines, Golden, CO USA

Laser assisted probe tomography (LAPT) is a powerful tool for materials characterization due to its desirable combination of high spatial resolution and analytical sensitivity. In state of the art LAPT the thermal transient from a near UV laser ( $E \approx 3.5 \mathrm{eV}$ to $3.6 \mathrm{eV} ; \lambda \approx 343 \mathrm{~nm}$ to $355 \mathrm{~nm}$ ) provides the energy to overcome the activation barrier for field ion evaporation. This technique is generally superior to voltage pulsing, which is limited strictly to conductors, and has allowed APT to expand its capability to effectively analyze a wide-range of materials including semiconductors and insulators. However, the thermal process is not without drawbacks. For example, LAPT data quality can be degraded due to thermal tails that limit sensitivity, formation of cluster ions that may have isobaric overlap with elemental species, and undetected neutral species which can adversely influence composition measurements. This is especially true for many ionic and covalent materials and can limit the recovery of bulk stoichiometry or composition to a narrow range of experimental conditions, if at all $[1,2]$.

Ionizing radiation in the extreme ultraviolet (EUV) region of the electromagnetic spectrum $(\approx 10 \mathrm{eV}$ to $\approx 100 \mathrm{eV} ; 124 \mathrm{~nm}$ to $12 \mathrm{~nm})$ may offer a potential athermal field ionization pathway. Dependent on the particular photon energy used, EUV radiation is above the ionization potential of any naturally occurring element and photoionization cross-sections peak in the EUV band across the entire periodic table [3]. EUV is highly absorbed within the first few nm of the sample surface and may also offer a potential in situ method for imaging the evolving specimen shape in real time through simultaneous coherent diffractive imaging or related methods [4].

We will present the instrument design and results from a tunable EUV-APT that uses femtosecond pulsed coherent radiation from phase-matched high harmonic generation in a noble gas cell. Initial experiments using Ar gas $(\mathrm{E}=41.85 \mathrm{eV} ; \lambda=29.6 \mathrm{~nm})$ conclusively demonstrate EUV-assisted field ion evaporation in fused quartz (amorphous $\mathrm{SiO}_{2}$ ). Thermal tails, $\mathrm{Si}^{\mathrm{n}+}, \mathrm{O}^{\mathrm{n}+}$, and $\mathrm{O}_{2}{ }^{\mathrm{n}+}$ peak widths, and the relative number of the cluster ions $\mathrm{SiO}^{\mathrm{n}+}$ and $\mathrm{SiO}_{2}{ }^{\mathrm{n+}}$ are significantly decreased when compared with near UV-LAPT $(\mathrm{E}=3.49 \mathrm{eV} ; \lambda=355 \mathrm{~nm})$ experiments on the same samples and specimens.

[1] Mancini, L. et al. Physical Chemistry C 118 (2014) 24136.

[2] Diercks, D.R. et al. J. Appl. Phys. 114 (2013) 184903.

[3] Yeh, J.-J. and I. Landau. At. Data Nucl. Data Tables 32 (1985) 1.

[4] Gardner, D.F. et al. Nature Photonics 11 (2017) 259. 


\title{
Estimation of Relative Evaporation Field Strength of Bulk Oxide-Composite and Comprehension of Evaporation Mechanism
}

\author{
Chang-Min Kwak ${ }^{1}$ Byeong-Gyu Chae ${ }^{1}$, Jae-Bok Seol${ }^{2}$, and Chan-Gyung Park ${ }^{1,2}$ \\ Corresponding Author: cgpark@postech.ac.kr \\ 1. Department of Materials Science and Engineering, POSTECH, Pohang 36676, South Korea. \\ 2. National Institute for Nanomaterial Technology (NINT), POSTECH, Pohang 36676, South \\ Korea
}

Atom probe tomography (APT) can provide unique information about the $3 \mathrm{D}$ atomic distribution and chemistry of materials. Recently, laser-assisted APT has widened the field of application to non-conductive materials such as thin film or bulk oxides. To improve data reliability, it is necessary to estimate the evaporation field strength of oxides. Our recent study have revealed that the evaporation field of $\mathrm{LaAlO}_{3}$ (LAO) is quite similar to that of $\mathrm{Co}$, but higher than that of Ni metal. We, therefore, have suggested the evaporation field strength of LAO for the first time ${ }^{1}$. This result was observed under the condition of intensity of $6000 \mu \mathrm{W}$ and base temperature of around $40 \mathrm{~K}$.

In this study, we have extended the relative evaporation field strength depending on laser conditions and base temperature. In order to compare the radius of curvature variations between capping layer and core-oxide, correlative transmission electron microscopy (TEM) and atom probe tomography (APT), named 'step-wise APT analysis', have been conducted. In fact, high laser intensity led to a gradual decrease in field evaporation strength with increasing surface curvature of the tips. The same experiments were also carried out by varying base temperature. These two results indicate that the evaporation field strength could be changed by laser intensity or base temperature. In addition, our results suggest the scientific clue to the evaporation mechanism of oxides and a guide for optimized experimental conditions. The reasonable experimental conditions required for analyzing oxides are the high field environment with low laser energy.

\section{References:}

[1] C. M. Kwak, Y. T. Kim, J. B. Seol, and C. G. Park, Understanding of Capping Effects on the Tip Shape Evolution and on the Atom Probe Data of Bulk $\mathrm{LaAlO}_{3}$ Using Transmission Electron Microscopy. Microsc. Microanal. 23 (2) (2017) 329-335.

[2] A. Devaraj, R. Colby, W. P. Hess, D. E. Perea, and S. Thevuthasan, Role of Photoexcitation and Field Ionization in the Measurement of Accurate Oxide Stoichiometry by Laser-Assisted Atom Probe Tomography. J. Phys. Chem. Lett. 4 (6) (2013), 993-998. 


\section{Distinctive Bond Breaking in Crystalline Phase-Change Materials}

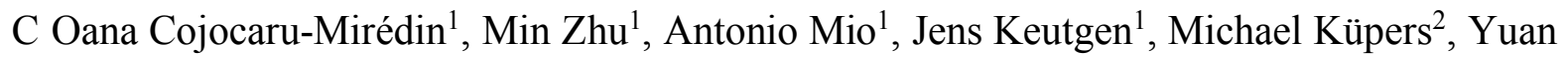
$\mathrm{Yu}^{1}$, Ju-Young Cho ${ }^{1}$, Richard Dronskowski ${ }^{2}$, and Matthias Wuttig ${ }^{1,3}$

Corresponding Author: cojocaru-miredin@physik.rwth-aachen.de

1. I. Institute of Physics (IA), RWTH Aachen University, 52074 Aachen, Germany.

2. Institute of Inorganic Chemistry, RWTH Aachen University, 52056 Aachen, Germany

3. JARA-FIT Institute Green-IT, RWTH Aachen University and Forschungszentrum Jülich, 52056 Aachen, Germany

In the present work a large number of phase change materials (see Figure 1), including amorphous and crystalline states, has been studied by laser-assisted atom probe tomography. Our study reveals significant differences in field evaporation behavior between amorphous and crystalline phase change materials. The crystalline phase change materials show clearly high probabilities for multiple events (i.e. more than one ion detected per laser pulse, see greencolored region in Figure 1). This is unlike any other mechanism shown previously to lead to high probabilities of multiple events. On the contrary, amorphous phase change materials as well as other covalently bonded compounds (red-colored region in Figure 1) possess much lower probabilities for multiple events. Hence, laser-assisted field evaporation in amorphous and crystalline phase change materials reveals striking differences in bond rupture.

These findings imply that the bonding mechanism in crystalline phase change materials differs substantially from conventional bonding mechanisms [1] such as metallic, ionic and covalent bonding. These findings confirm a recently developed conjecture, namely that metavalent bonding is a novel bonding mechanism.

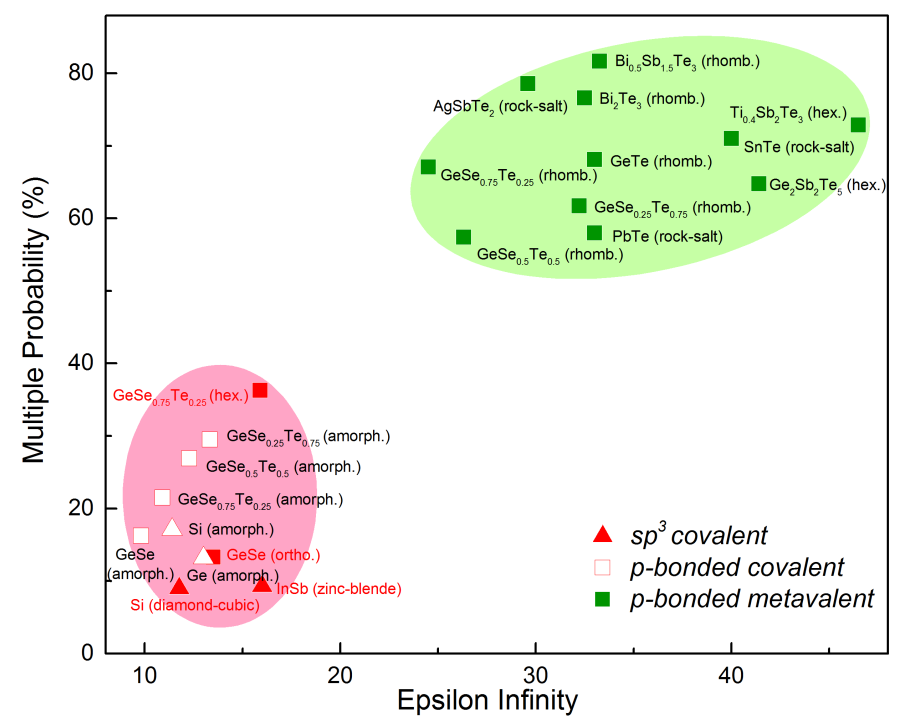

Figure 1 A wide variety of materials which are categorized into two classes based on their bonding mechanism: covalently bonded materials in red and crystalline PCMs in green.

$\underline{\text { References: }}$

[1] K. Shportko, S. Kremers, M. Woda, D. Lencer, J. Robertson and M. Wuttig, Resonant bonding in crystalline phase-change materials, Nat. Mat. (2007), 7, 653-658. 


\title{
Study of optical and Thermal Properties of Diamond Nanowires by Laser Assisted Atom Probe Tomography
}

\author{
J. Houard ${ }^{1}$ L. Arnoldi ${ }^{1}$, M. Spies ${ }^{1}$, I. Blum ${ }^{1}$, A. Etienne ${ }^{1}$, R. Ismagilov ${ }^{2}$ A. Obraztsov ${ }^{2,3}$, and A. \\ Vella ${ }^{1}$ \\ Corresponding Author: jonathan.houard@univ-rouen.fr \\ 1. Groupe de Physique des Matériaux UMR CNRS 6634 - Université et INSA de ROUEN, \\ Université Normandie 76801 SAINT ETIENNE DU ROUVRAY CEDEX France \\ 2. M. V. Lomonosov Moscow State University, Department of Physics, Moscow 119991, Russia \\ 3. University of Eastern Finland, Department of Physics and Mathematics, Joensuu 80101, \\ Finland
}

Diamond is an amazing material with outstanding properties (chemical inertness, record hardness, thermal conductivity, optical transparency and high band-gap). Moreover, in nanostructural shape, like nanowire, this material can be a key element for a wide range of applications such as light detectors and emitters, tensile stress sensors or ultra-bright electron sources. Some of these applications are based on ultra-high thermal conductivity of bulk diamond. However, in the case of nanostructures, ab-initio computation has shown that this property is degraded [1] but no experiment has been done to corroborate this prediction.

Another uncertain physical property of these structures is exhibit by recent Laser-assisted Atom Probe Tomography (La-APT) analyses on diamond layers [2]: how does laser interact with such a transparent structure? How this interaction depends on the sample preparation technique and the samples structure?

In this talk, we will show La-APT analyses on long single crystalline diamond nanowires $(>50 \mu \mathrm{m})$. These samples are obtained by combination of the chemical vapor deposition growth of a diamond polycrystalline film followed by a selective plasma oxidation [3].

For the optical absorption properties, the results show a optical absorption of laser energy in both visible and UV range by diamond nanowires, at the tip apex (surface absorption) and far from the tip apex (volume absorption). Numerical simulations of volume absorption suggest us to use an original illumination condition in La-APT to measure the diamond nanowires thermal conductivity.

\section{References:}

[1] W. Li, N. Mingo, L. Lindsay, D. A. Broido, D. A. Stewart, and N. A. Katcho, Physical Review

B 85, $195436(2012)$

[2] Mukherjee, S., Watanabe, H., Isheim, D., Seidman, D. N., \& Moutanabbir, O. (2016). Nano Letters, 16(2), 1335-1344

[3] A. Zolotukhin, P. G. Kopylov, R. R. Ismagilov, and A. N. Obraztsov, Diamond and Related Materials 19, 1007 (2010) 


\section{Optical Measurements of the Tensile Stress Induced by a High Electric Field in Diamond Nanoscale Needles}

L. Venturi*, L. Rigutti*, J. Houard*, A. Normand", E. P. Silaeva*, M. Borz*, S. A. Malykhin**, A.N. Obraztsov ${ }^{* * *}$ and Angela Vella*

Corresponding Author: linda.venturi@etu.univ-rouen.fr

*Normandie Univ, UNIROUEN, INSA Rouen, CNRS, Groupe de Physique des Matériaux, 76000 Rouen, France

** University of Eastern Finland, Department of Physics and Mathematics, Joensuu 80101, Finland

*** M V Lomonosov Moscow State University, Department of Physics, Moscow 119991, Russia

In this work we report a method to perform piezo-spectroscopy of nanoscale systems by electrostatic field regulation.

The nanoscale systems studied are diamond needles containing color centers, that have been investigated since two decades, e.g., for the implementation of qubits in quantum informatics protocols [1]. The photoemission of these centers is studied using a micro-photoluminescence $(\mu-$ PL) system (figure a). The application of a high electrostatic field at the apex of monocrystalline diamond nanoscale needles induces an energy splitting of the photoluminescence lines of color centers [2]. The splitting of the zero-phonon PL line of the $\mathrm{NV}^{0}$ defect (figure b) has been studied as a function of the voltage applied to the tip. The measured quadratic dependence of the energy splitting on the applied voltage, reported for the $\mathrm{NV}^{0}$ defect (figure $\mathrm{c}$ ), corresponds to the stress generated on the metal-like apex surface by the electrostatic field. Tensile stress up to $7 \mathrm{GPa}$ has thus been measured in the proximity of the needle apex. The intensity of the stress, measured on the diamond nanoneedles, is higher as the apex radius of the diamond nanotips is smaller. These results open up interesting perspective not only in the domain of APT users (it becomes possible to measure the stress induced in field emission tips under typical conditions for APT analysis) but could also yield original analyses of the defect symmetries of color centers in diamond and in other materials.

(a)

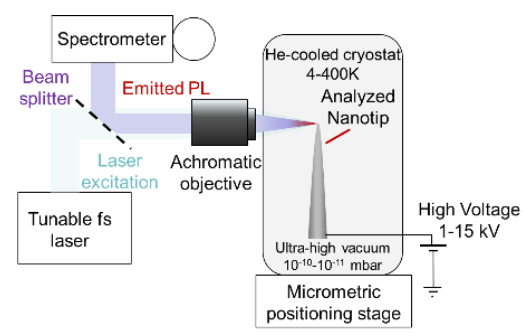

(b)

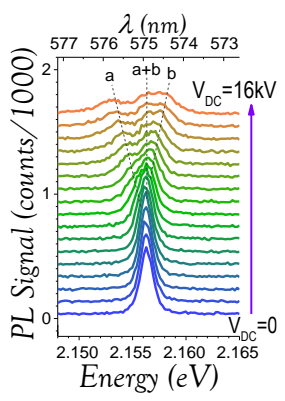

(c)

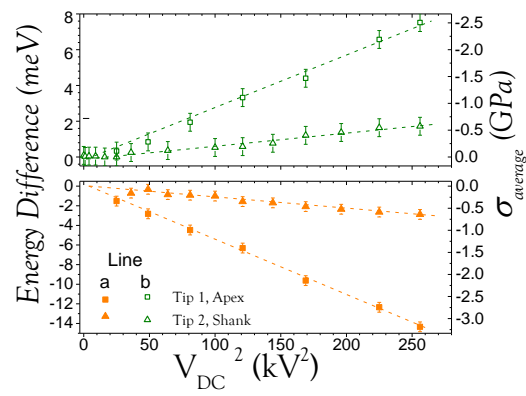

Figure 1 (a) $\mu$-photoluminescence system. (b) Optical study of the $\mathrm{NV}^{0} \mathrm{ZPL}$ as function of the applied bias. (c) Dependence of the energy splitting on the square of the applied voltage.

References:

[1] Kurtsiefer et al., Stable Solid-State Source of Single Photons. Phys. Rev. Lett. 2000, 85 (2), 290-293.

[2] L. Rigutti, et al., Optical Contactless Measurement of Electric Field-Induced Tensile Stress in

Diamond Nanoscale Needles. Nano Lett., ACS Pub., 2017. 


\title{
Study of Electrical Conduction Properties of Diamond Nanoneedles, by Ion Spectroscopy in Field Ion Microscopy
}

\author{
L. Arnoldi ${ }^{1}$, M. Borz ${ }^{1}$, I. Blum ${ }^{1}$, A. Obraztsov ${ }^{2,3}$, and A. Vella ${ }^{1}$
}

Corresponding Author: angela.vella@univ-rouen.fr

1. Groupe de Physique des Matériaux UMR CNRS 6634, Université et INSA de ROUEN, Université Normandie 76801 SAINT ETIENNE DU ROUVRAY CEDEX France

2. University of Eastern Finland, Department of Physics and Mathematics, Joensuu 80101, Finland

3. M. V. Lomonosov Moscow State University Department of Physics, Moscow 119991, Russia

Mono-crystalline diamond needles are quite attractive samples for field emission applications, mainly used as point electron source [1]. Even though the CVD production process of this kind of sample is now quite efficient and reproducible, the performances of these electrons sources can change from one needle to another. These changes could be related to a change in the electrical conduction therefore we used a new experimental setup to study the conduction properties of diamond nano-needles in a large range of emission currents and under femtosecond laser illumination.Strong advantages of APT rely in its possibility to detect all types of atoms, independently of their atomic number, in its excellent detection limit and in its intrinsic 3D nature. In order to collect a significant amount of information on a same nanoobject, it is relevant to consider a correlative approach combining a TEM/STEM and APT. Motivations for such an approach are numerous. A non-exhaustive list would evoke: i) the possibility to associate structural defects (in TEM) with segregations (in APT); ii) associating the morphology of a particle (in electron tomography) with a 3D field of composition (in APT); iii) improving the quality of APT reconstructions by accessing additional information about the specimen morphology in TEM/STEM.

Changing the voltage applied to the nanoneedle, we exhibited two different conduction behaviors: a first regime, at low emission current, which corresponds to the ohmic conduction and a second regime, at high emission current, which corresponds to a Poole-Frenkel (PF) conduction mechanism [2]. The PF effect occurs when a high field is applied inside the material $\left(\sim 10^{5}-10^{6} \mathrm{~V} / \mathrm{m}\right)$ and is strong enough to activate the trapped carriers. As it increases the free carrier density, the resistivity becomes itself a function of the applied field. We discuss the transition between these two conduction mechanisms and its dependence as a function of the emitted current and the needle geometry.

Under femtosecond laser illumination, the resistivity changes and the transition from the ohmic conduction regime to the PF regime is observed at higher emission currents. The study of the changes of the conduction parameters under illuminations allows us to draw conclusion on the optical absorption process of these nanoneedles and on their heating induced by laser illumination.

\section{References:}

[1] V.I. Kleshch et al., "Single Crystal Diamond Needle as Point

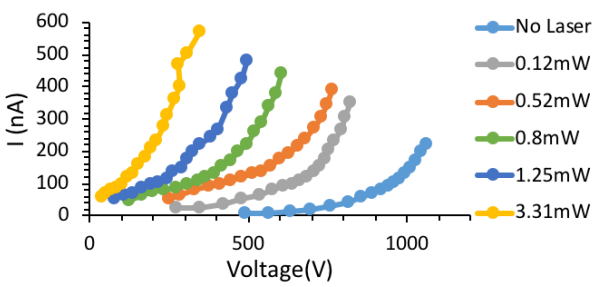

Figure 1 FIM emitted current from a diamond needle as function of the applied voltage at $80 \mathrm{~K}$ for different laser power at $\lambda=515 \mathrm{~nm}$ and Neon pressure of $4.10^{-5}$ mbar.

Electron Source”, Scientific Reports 6, Article number: 35260 (2016).

[2] L. Arnoldi et al., "Role of the resistivity of insulating field emitters on the energy of field-ionized and field-evaporated atoms", Ultramicroscopy, Vol 159, Part 2 (2015). 


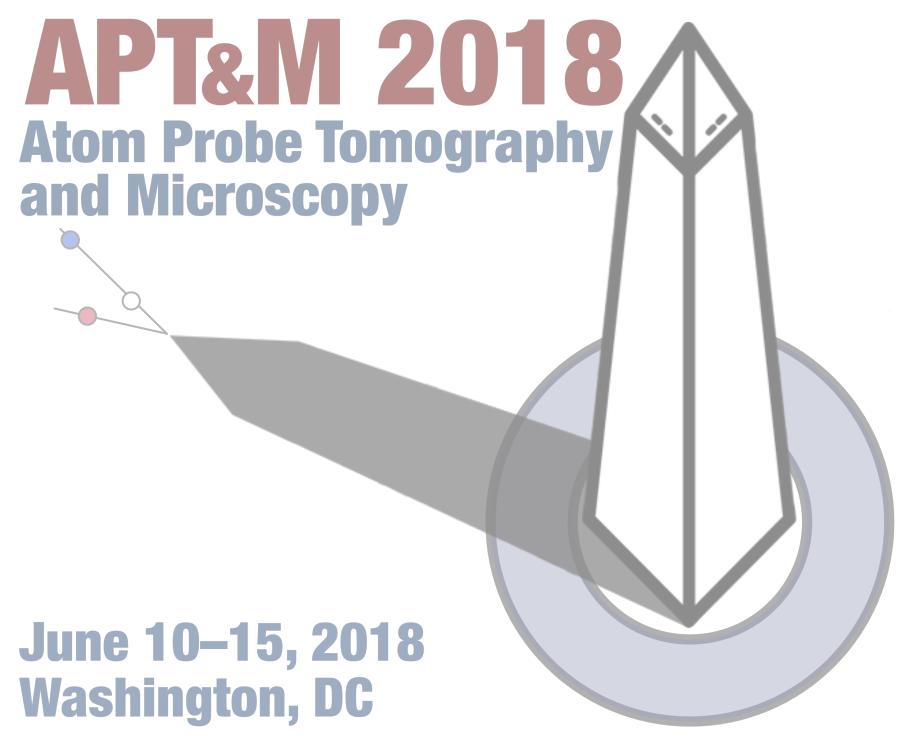

Tuesday Afternoon 



\section{Tuesday Afternoon}

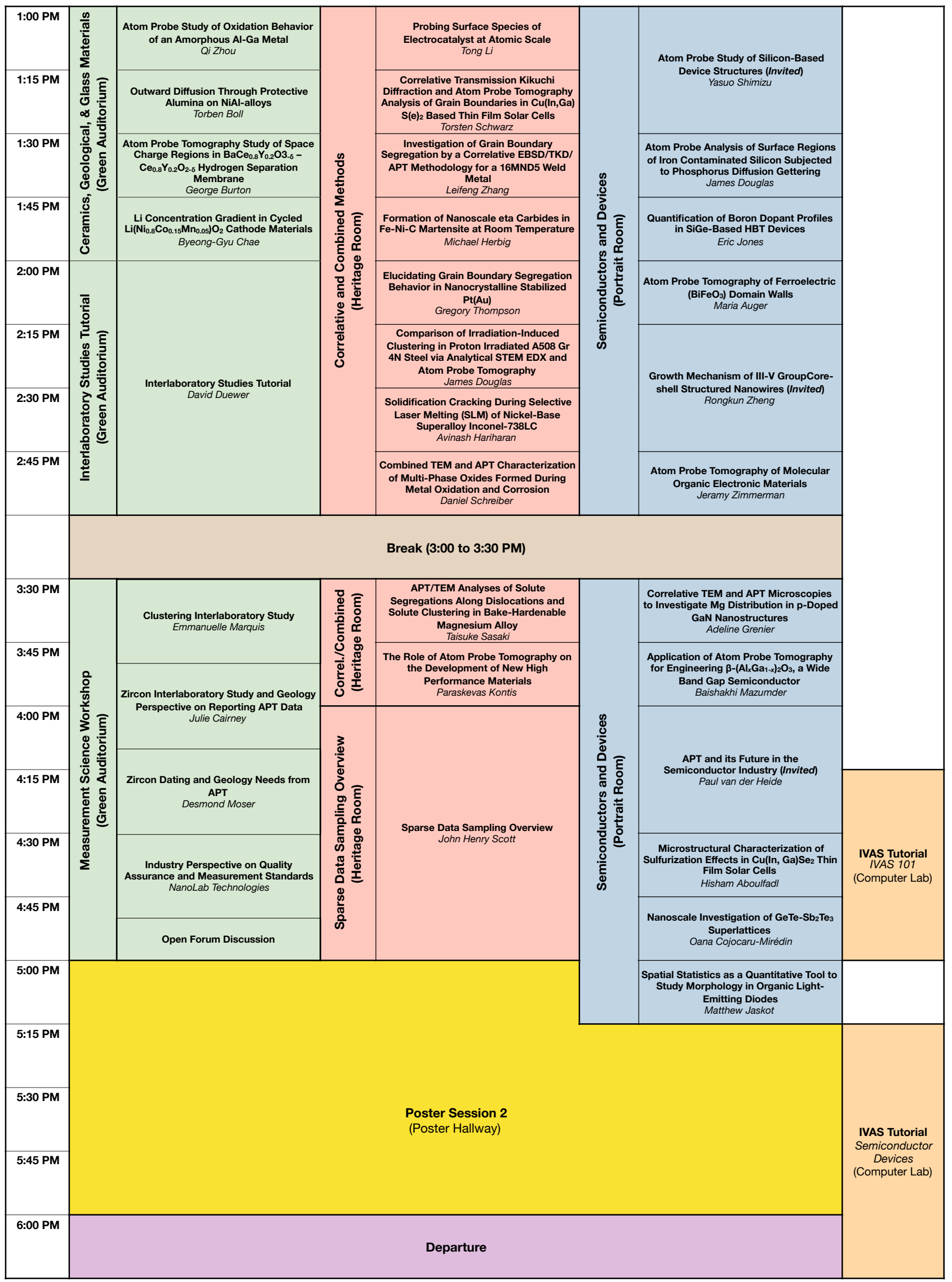





\section{Atom Probe Study of Oxidation Behavior of an Amorphous Al-Ga Metal}

Qi Zhou ${ }^{1}$, Rong $\mathrm{Hu}^{2}$, Jizi Liu ${ }^{2}$, Gang $\mathrm{Sha}^{2}$

Corresponding Author: gang.sha@njust.edu.cn

1. School of Materials Science and Engineering, Nanjing University of Science and Technology, Nanjing, China

2. Herbert Gleiter Institute of Nanoscience, Nanjing University of Science and Technology, Nanjing,210094, China

Amorphous materials exhibit completely different oxidation behaviour comparing with crystals, due to their irregular structure and uncompact arrangement of atoms [1-2]. To date, the oxidation process of amorphous materials has seldom been investigated in detail. The lack of deep understanding of oxidation mechanism of amorphous materials has severely limited the study and application of amorphous materials. In this study, by combining the cutting-edge atom probe tomography (APT) with transmission electron microscopy (TEM), we systematically investigated the oxidation behaviour of amorphous Al-Ga material at room temperature under different oxidation conditions. The results revealed very different oxidation process of amorphous Al-Ga compared with (001) Al single crystals. In amorphous Al-Ga, atom redistribution was found along with the growth of oxidation layer. Non-uniform oxidation first occurred in locally low atomic density regions, then followed with oxygen diffusion and the growth of a thick oxide layer (over $200 \mathrm{~nm}$ with 48 hours oxidation in atmosphere). The effect of oxidation on the redistribution of atoms was discussed in detail, and a new oxidation model of amorphous materials has been established.

\section{$\underline{\text { References: }}$}

[1] K. L. Chang, G. W. Pickrell, D. E. Wohlert, J. H. Epple, Microstructure and wet oxidation of lowtemperature-grown amorphous (Al/Ga,As), Journal of Applied Physics 89(1) (2001), 747-52.

[2] K. Weller, L. P. H. Jeurgens, Z. Wang, E. J. Mittemeijer, Thermal oxidation of amorphous Al0.44Zr0.56 alloys, Acta Materialia. 87 (2015), 187-200. 


\section{Outward Diffusion Through Protective Alumina on NiAl-Alloys}

Torben Boll ${ }^{1}$, Olof Bäcke ${ }^{2}$, Bruce A. Pint ${ }^{3}$, Kinga A. Unocic ${ }^{3}$, Krystyna Stiller ${ }^{2}$

Corresponding Author: Torben.Boll@kit.edu

1. Institute for Applied Materials (IAM), Karlsruhe Institute of Technology (KIT), Karlsruhe, Germany

2. Department of Physics, Chalmers University of Technology, Göteborg, Sweden

3. Materials Science and Technology Division, Oak Ridge National Laboratory, Oakridge TN, USA

The main process of alumina scale growth on NiAl-alloys, after a protective layer has been formed, is the diffusion of oxygen through the scale to the metal. However, there is also a contribution of metal outward diffusion. This diffusion processes along grain boundaries (GBs) depends largely on the presence of alloying reactive elements, which segregate to the GBs. For this study, we applied scanning transmission electron microscopy (STEM) and atom probe tomography (APT) to analyze oxidized NiAl with different dopants. By means of APT it was possible to observe and even quantify GB segregation of $\mathrm{Hf}, \mathrm{Zr}$ and $\mathrm{Ni}$ (fig.1). With careful sitespecific sample preparation and a two-step oxidation process producing small ridges on a polished oxide surface, it was also possible to determine the outward diffusion of $\mathrm{Ni}$, $\mathrm{Hf}$ and $\mathrm{Zr}$. Their contribution is four orders of magnitude smaller than the Al-diffusion, which itself is dwarfed by the six orders of magnitude larger O-inward diffusion [1].

a)
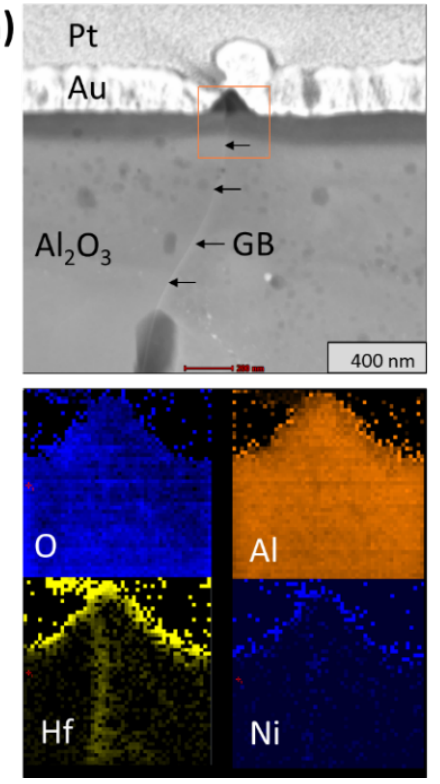

b)

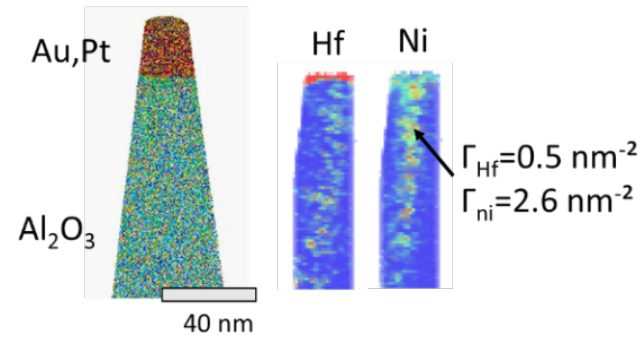

c)

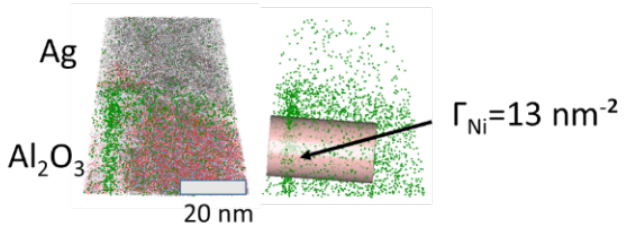

Figure 1 (a) STEM at a GB in a small ridge on Hf-doped $\mathrm{NiAl}$; ;enrichment at the GB shown with EDS. (b) APT of the same material allows to obtain the Gibbsian excess $\Gamma$. (c) For nondoped NiAl alumina a significantly stronger Ni-outward diffusion can be observed.

\section{References:}

[1] T. Boll, K. A. Unocic, B. A. Pint, and K. Stiller, "Interfaces in Oxides Formed on NiAlCr Doped with Y, Hf, Ti, and B," Microsc. Microanal., vol. 23, no. 2, pp. 396-403, Apr. 2017. 


\title{
Atom Probe Tomography Study of Space Charge Regions in $\mathrm{BaCe}_{0.8} \mathrm{Y}_{0.2} \mathrm{O}_{3-\delta}-$ $\mathrm{Ce}_{0.8} \mathrm{Y}_{0.2} \mathrm{O}_{2-\delta}$ Hydrogen Separation Membrane
}

\author{
George L. Burton ${ }^{1}$, David R. Diercks ${ }^{1}$, and Brian P. Gorman ${ }^{1}$ \\ Corresponding Author: gburton@mines.edu \\ 1. Colorado School of Mines, Department of Metallurgical and Materials Engineering, Golden, \\ CO, USA 80401.
}

Ceramic hydrogen separation membranes could offer a low cost, energy efficient alternative to current energy intensive hydrogen separation techniques. The 50/50 wt.\% dense composite ceramic, $\mathrm{BaCe}_{0.8} \mathrm{Y}_{0.2} \mathrm{O}_{3-\delta}-\mathrm{Ce}_{0.8} \mathrm{Y}_{0.2} \mathrm{O}_{2-\delta}(\mathrm{BCY}-\mathrm{YDC})$, can be synthesized via solid state reactive sintering [1] and has functioned at high hydrogen fluxes [2]. Under a reducing atmosphere, YDC acts as an electron conductor and BCY as an ion conductor. Hydrogen molecules dissociate into $\mathrm{H}+$ ions and electrons and the charges travel through their respective phases and recombine on the opposite side of the membrane to form pure hydrogen gas. Like a number of other technologically relevant conducting oxides, electron and ion conductivity in BCY-YDC is impeded by the formation of space charge regions at grain boundaries (GBs) [3]. Characterizing grain and phase boundaries in BCY-YDC is essential in further improving the GB conductivity and therefore the overall hydrogen flux.

Due to challenges related to directly quantifying chemical composition, particularly oxygen, across general three-dimensional boundaries, atom probe tomography (APT) is one of the only techniques available. The technique is particularly useful in identifying compositional changes across GBs, and has even been used recently to quantify the 3-D space charge voltages around GBs of an ionic conducting oxide [4]. The compositional nature of both GBs and phase boundaries in BCY-YDC are explored in this work using APT. In all cases, deviations in concentrations of cations, oxygen, and impurities around the boundary regions are studied with the goal of relating structure and composition to macro-scale performance. Transmission electron microscopy (TEM) images taken before and after APT analysis were used to provide complementary information and assist in APT reconstruction accuracy [5].

\section{$\underline{\text { References: }}$}

[1] S. Ricote, et al., Preparation of dense mixed electron- and proton-conducting ceramic composite materials using solid-state reactive sintering: $\mathrm{BaCe}_{0.8} \mathrm{Y}_{0.1} \mathrm{M}_{0.1} \mathrm{O}_{3-\delta}-\mathrm{Ce}_{0.8} \mathrm{Y}_{0.1} \mathrm{M}_{0.1} \mathrm{O}_{2-\delta}(\mathrm{M}=\mathrm{Y}, \mathrm{Yb}, \mathrm{Er}, \mathrm{Eu}), \mathrm{J}$. Mater. Sci. 49 (2014), 4332-4340.

[2] W. Rosensteel et al., Hydrogen permeation through dense $\mathrm{BaCe}_{0.8} \mathrm{Y}_{0.2} \mathrm{O}_{3-\delta}-\mathrm{Ce}_{0.8} \mathrm{Y}_{0.2} \mathrm{O}_{2-\delta}$ compositeceramic hydrogen separation membranes, J. of Hydrogen Ener. 41 (2016), 2598-2606.

[3] X. Guo and R. Waser, Space charge concept for acceptor-doped zirconia and ceria and experimental evidences, Solid State Ionics 173 (2004), 63-67.

[4] D. Diercks et al., Three-dimensional quantification of composition and electrostatic potential at individual grain boundaries in doped ceria, J. Mater. Chem. 4 (2016), 5167-5175.

[5] B. Gorman et al., Hardware and techniques for cross-correlative TEM and atom probe analysis, Microscopy Today 16 (2008), 42-47. 


\section{Li Concentration Gradient in Cycled $\mathrm{Li}\left(\mathrm{Ni}_{0.8} \mathrm{Co}_{0.15} \mathrm{Mn}_{0.05}\right) \mathrm{O}_{2}$ Cathode Materials}

Byeong-Gyu Chae ${ }^{1}$, Seong Yong Park ${ }^{2}$, Jay Hyok Song ${ }^{3}$, Chang-Min Kwak ${ }^{1}$, Jae-Bok Seol ${ }^{4}$, and Chan-Gyung Park ${ }^{1,4}$

\section{Corresponding Author: cgpark@postech.ac.kr}

1. Department of Materials Science and Engineering, POSTECH, Pohang 36676, South Korea.

2. Analytical Engineering Group, Samsung Advanced Institute of Technology (SAIT), Suwon 16678, South Korea.

3. Energy1 Lab, Samsung SDI, Suwon 16678, South Korea.

4. National Institute for Nanomaterial Technology (NINT), POSTECH, Pohang 36676, South Korea

Li-ion batteries are receiving a great attention due to the possibility of their application to future portable electronics and electric vehicles ${ }^{1}$. Numerous applications of Li-ion batteries are caused by the unique characteristics of Li ions, which allow high cell potential, high energy density and high mobility for Li-ion batteries ${ }^{1,2}$. Therefore, the understanding the behavior of Li ions during charge-discharge cycles is essential in improving the performance of Li-ion batteries. However, observation and quantification of Li ions are very challenging by using conventional analytical techniques.

In the present study, scanning transmission electron microscopy (STEM) and atom probe tomography (APT) have been used to reveal the origin of capacity degradation in a layered $\mathrm{Li}\left(\mathrm{Ni}_{0.8} \mathrm{Co}_{0.15} \mathrm{Mn}_{0.05}\right) \mathrm{O}_{2}(8 \mathrm{NCM})$ cathode materials. Correlating the structural change with the quantitative concentration of constituent element can provide a deeper understanding of the irreversible capacity loss phenomenon. Our results exhibited that cycled $8 \mathrm{NCM}$ had a significant $\mathrm{Li}$ deficiency near the top surface with a fully disordered layered structure. The Li deficiency was gradually alleviated as far away from the particle surface, showing the gradient of Li concentration with a partially disordered layered structure. Li concentration in the bulk of the particle was the same as that in the pristine $8 \mathrm{NCM}$. Therefore, the significant capacity degradation might be attributed to the Li concentration gradient near the several hundred nanometers from the particle surface. In addition, non-uniform spatial distribution of Li ions revealed the direct evidence on the blocking of the Li diffusion pathway after charge-discharge cycles.

\section{References:}

[1] J. M. Tarascon and M. Armand, Issues and challenges facing rechargeable lithium batteries. Nature 414 (2001), 359-367.

[2] N. Nitta, F. Wu, J. T. Lee, and G. Yushin, Li-ion battery materials: present and future. Materials today 18 (2015), 252-264. 
Proceedings of Atom Probe Tomography \& Microscopy (APT\&M) 2018, Washington, DC, June 10-15, 2018 NIST SP 2100-03 


\section{Interlaboratory Studies Tutorial}

David L. Duewer ${ }^{1}$

Corresponding Author: david.duewer@,nist.gov

1. National Institute of Standards and Technology, Chemical Sciences Division, Gaithersburg, MD 20899.

Paraphrasing William Horwitz, late champion and chronicler of Interlaboratory studies (ILS) in analytical chemistry, an ILS is a study in which several laboratories address the same issue under documented conditions, the results of which are compiled into a single report. Participants typically measure some quantity(ies) in some common set of materials, analyze the same set of data, or attack the same well-defined problem using their own approaches and data. There are four basic types of ILS: proficiency testing (PT), method evaluation, material characterization, and community performance. PT studies evaluate the competence of the participants, are by far the most abundant type of ILS, have well-defined codes of conduct, and are mostly provided by for-profit organizations. Method evaluation studies characterize well-defined measurement or data analysis procedures under real-world conditions and are typically sponsored (and/or mandated) by regulatory authorities. Material characterization studies are typically conducted by National Metrology Institutes - like NIST - and others as part of their reference material certification processes. Community performance studies are like PT but without the angst, seeking to understand the strengths and weaknesses of a community rather than those of the individual players within that community; they are often conducted by the community itself. 
The practice of a measurement technique is as important as the design of the instrument, and must be thoroughly studied for accurate measurements and consistent reporting. At present, atom probe tomography (APT) is a well-developed technique with a rapidly growing population of expert practitioners throughout the world; there is however significant variation in both the practice of APT and the reporting of data across this population. Given the ultimate goal in APT development of consistent, standards-less, absolute quantitative measurement of atomic-scale composition in three dimensions, the field requires tools to address these outstanding measurement science challenges. This workshop, hosted by Austin Akey, focuses on the use of interlaboratory studies as a tool to achieve these goals, featuring presentations on the design of these studies, ongoing work in this direction, and the needs of the broader scientific community including our colleagues in industry.

\section{Clustering Interlaboratory Study}

Emmanuelle Marquis

$3: 30$ to $3: 50 \mathrm{PM}$

\section{Zircon Interlaboratory Study and Geology Perspective on Reporting APT Data}

Julie Cairney

3:50 to 4:10 PM

\section{Zircon Dating and Geology Needs from APT}

Desmond Moser

4:10 to $4: 30$ PM

\section{Industry Perspective on Quality Assurance and Measurement Standards} NanoLab Technologies

4:30 to $4: 50 \mathrm{PM}$

\section{Open Forum Discussion}

4:50 to 5:00 PM 


\section{Probing Surface Species of Electrocatalyst at Atomic Scale}

T. Li $^{1,2}$, O. Kasian ${ }^{1}$, S. Cherevko ${ }^{1,3}$, S. Zhang ${ }^{1}$, S. Geiger ${ }^{1}$,C. Scheu ${ }^{1}$, P. Felfer ${ }^{4}$, D. Raabe ${ }^{1}$, B. Gault $^{1} \&$ K.J. J. Mayrhofer ${ }^{1,3,5}$

Corresponding Author: tong.li@ rub.de

1. Max-Planck-Institut für Eisenforschung, Max-Planck-Straße 1, 40237 Düsseldorf, Germany

2. Institute for Materials \& Zentrum für Grenzflächendominierte Höchstleistungswerkstoffe (ZGH), Ruhr-Universität Bochum, Universitätsstraße 150, 44780 Bochum, Germany

${ }^{3}$. Helmholtz-Institute Erlangen-Nürnberg for Renewable Energy (IEK-11), Forschungszentrum Jülich, Egerlandstr. 3, 91058 Erlangen, Germany

4. Institute for General Materials Properties, Department of Materials Science, FriedrichAlexander University Erlangen-Nürnberg, 91058 Erlangen, Germany

${ }^{5 .}$ Department of Chemical and Biological Engineering, Friedrich-Alexander-Universität Erlangen-Nürnberg, Egerlandstr. 3, 91058 Erlangen, Germany

The topmost atomic layers of electrocatalysts determine mechanism and kinetics of the reactions and play a crucial role in many important industrial processes, e.g. water splitting for hydrogen generation, chlor-electrolysis, or carbon dioxide reduction. Optimizing the performance of electrocatalysts requires a detailed understanding of how species react during the catalytic process, and thus the surface state ideally at the atomic scale. Here, we combine atom probe tomography, electron microscopy and spectroscopy to reveal in a model study the three-dimensional, atomic scale surface structure of a thermally and electrochemically grown iridium-based oxides, efficient electrocatalysts for the oxygen evolution reaction. We discovered low stoichiometry Ir-O species on the surfaces of the three oxides, which correlate with their activity and stability. Additionally, electrochemical growth of oxides in deuterated solutions allowed us to detect a monolayer of hydroxy-groups in Ir-O which provides an additional explanation for enhanced activity of electrochemically formed oxides (Fig. 1)

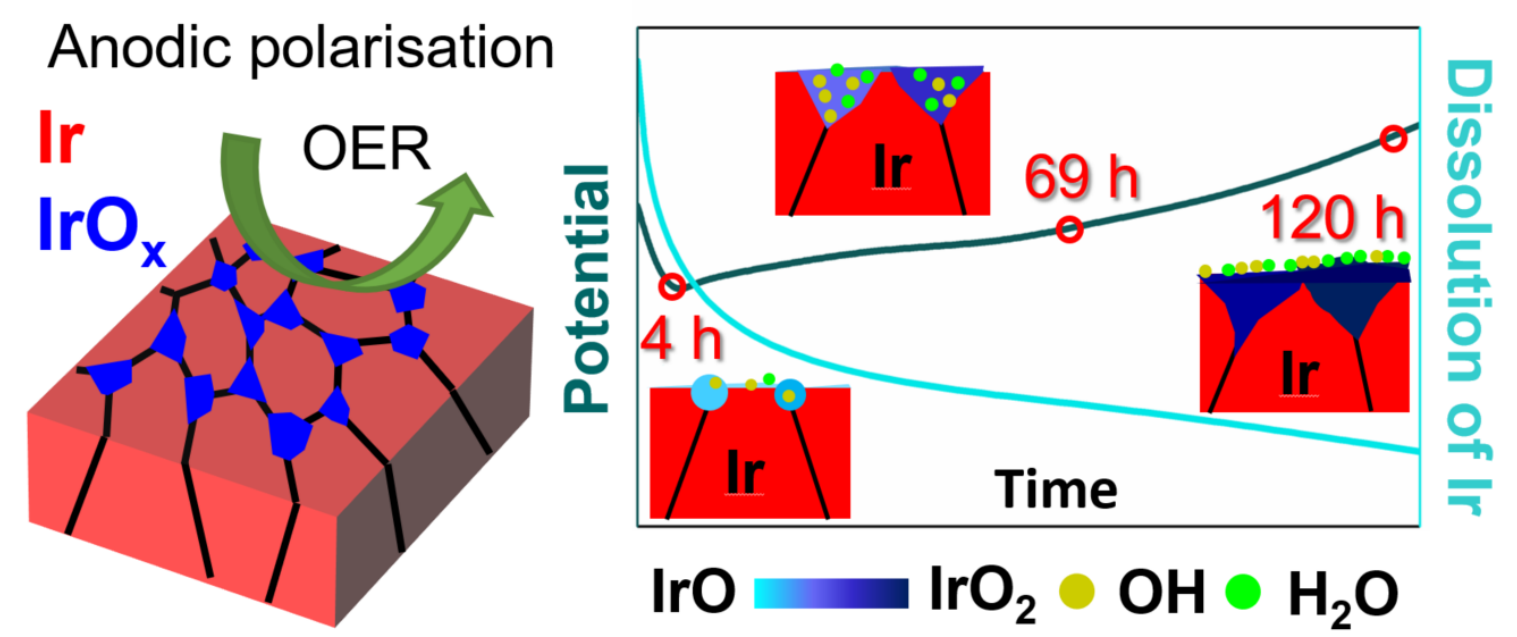

Figure 1. Schematic diagram showing how the surface species influence the activity and stability of iridium during the oxygen evolution reaction (OER). 


\section{Correlative Transmission Kikuchi Diffraction and Atom Probe Tomography} Analysis of Grain Boundaries in Cu(In,Ga) S(e)2 Based Thin Film Solar Cells

Torsten Schwarz ${ }^{1}$, Guillaume Stechmann ${ }^{1}$, Baptiste Gault ${ }^{1}$, Oana Cojocaru-Mirédin ${ }^{1,2}$, Roland Wuerz $^{3}$, Alberto Lomuscio ${ }^{4}$, Susanne Siebentritt ${ }^{4}$, and Dierk Raabe ${ }^{1}$

Corresponding Author: schwarz@mpie.de

1. Max-Planck-Institut für Eisenforschung GmbH, Düsseldorf, Germany.

2. Max-Planck-Institut für Eisenforschung GmbH, Düsseldorf, Germany.

3. Zentrum für Sonnenenergie-und Wasserstoff-Forschung Baden-Württemberg, Stuttgart, Germany.

4. Laboratory for Photovoltaics, University of Luxembourg, Belvaux, Luxembourg.

The compound semiconductor $\mathrm{Cu}(\mathrm{In}, \mathrm{Ga})(\mathrm{S}, \mathrm{Se}) 2$ (CIGSSe) is used as absorber materials in thinfilm solar cells. Currently, a record efficiency of $22.9 \%$ is achieved for CIGSSe based solar cells. When considering the polycrystalline nature of the CIGSSe thin-films, this efficiency is surprisingly high. For further enhancement of the efficiency it is important to understand how the microstructure affects the electrical properties of the absorber films and, hence, the device performance. Therefore, one needs to study the relationship between structural and chemical properties of grain boundaries (GB). Compositional changes at GBs in CIGSe thin-films have been already reported [1]. However, there are only few studies, which correlate structural and chemical properties of GBs in the CIGS system.

Here, we investigate the correlation between crystallographic and chemical information of GBs in CIGSSe by using transmission Kikuchi diffraction (TKD) and atom probe tomography (APT). We address practical issues related to the specimen preparation for TKD and how to resolve them. We detect $\mathrm{Cu}$ depletion and $\mathrm{In}, \mathrm{Se}, \mathrm{Na} \& \mathrm{~K}$ enrichment at random high-angle GBs (RHAGB) as shown in Figure 1, whereas we detect no compositional changes at twin boundaries [2].
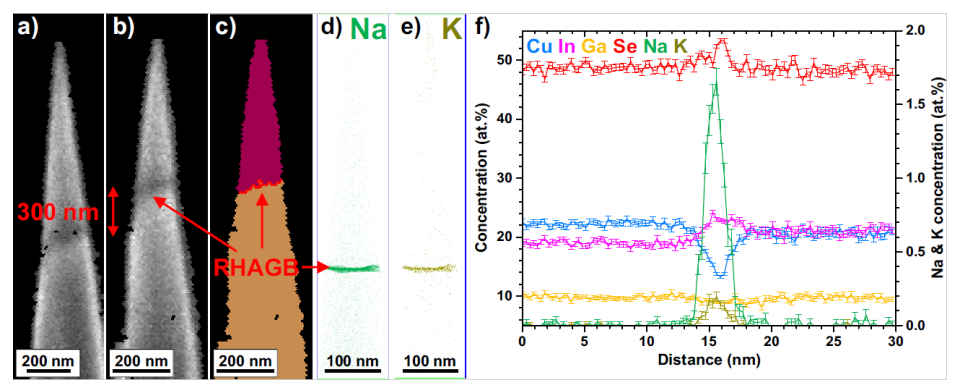

Figure 1 (a)-(b) Image quality maps from a TKD measurement of an APT tip. (c), Corresponding unique color map to (b) showing a RHAGB. (d)-(e) $\mathrm{Na} \& \mathrm{~K}$ co-segregation at the RHAGB shown in (c). (f) Concentration profile across the RHAGB revealing an atomic redistribution.

\section{References:}

[1] O. Cojocaru-Mirédin, et al., Assessment of elemental distributions at line and planar defects in $\mathrm{Cu}(\mathrm{In}, \mathrm{Ga}) \mathrm{Se} 2$ thin films by atom probe tomography, Scripta Materialia (2017), 1-9.

[2] T. Schwarz, et al., Correlative transmission Kikuchi diffraction and atom probe tomography study of $\mathrm{Cu}(\mathrm{In}, \mathrm{Ga}) \mathrm{Se} 2$ grain boundaries, Prog Photovolt Res Appl. (2017), 1-9. 


\title{
Investigation of Grain Boundary Segregation by a Correlative EBSD/TKD/APT Methodology for a 16MND5 Weld Metal
}

\author{
Leifeng Zhang ${ }^{1}$, Bertrand Radiguet ${ }^{1}$, Christophe Domain ${ }^{2}$, Yang Shen ${ }^{3}$, Patrick Todeschini ${ }^{2}$, \\ Philippe Pareige ${ }^{1}$ \\ Corresponding Author: bertrand.radiguet@univ-rouen.fr \\ 1. University of Rouen Normandie, INSA Rouen, CNRS, Groupe de Physique des Matériaux, \\ 76000 Rouen, France \\ 2. EDF Lab, Materials and Mechanics of Component Department, Site des Renardieres-Ecuelles, \\ 77818 Moret-sur-Loing cedex, France \\ 3. EDF DIPNN, Industrial Division, Laboratory Department, 37420 Avoine cedex, France
}

Grain boundary (GB) segregation has long been an attractive issue in material science field [1, 2]. In the present study, the correlative Electron Backscatter Diffraction (EBSD)/ Transmission Kikuchi Diffraction (t-EBSD or TKD)/ Atom Probe Tomography (APT) methodology [3] was used to investigate the intergranular segregation behavior of a 16MND5 weld metal from a quantitative perspective. Both the three dimensional chemical information and 5-parameter crystallographic details of a grain boundary were obtained

The steel has a complex microstructure of acicular ferrite with intergranular carbides, in which $\sim 78 \%$ of high angle grain boundaries (HAGBs, with $\sim 12 \%$ of $\Sigma 3$ special HAGBs) and $\sim 22 \%$ of low angle grain boundaries (LAGBs) were observed. APT analyses revealed that all boundary types show a segregation of several elements $(\mathrm{C}, \mathrm{P}, \mathrm{Mn}$, $\mathrm{Mo}, \mathrm{Cr}, \mathrm{Si}, \mathrm{Ni} . .$.$) . By taking into$ consideration all segregated chemical species, both interstitial and substitutional segregations were discussed with regard to GB types, as exhibited in Fig. 1. Besides, the element segregation at carbide/matrix interfaces was also quantified.

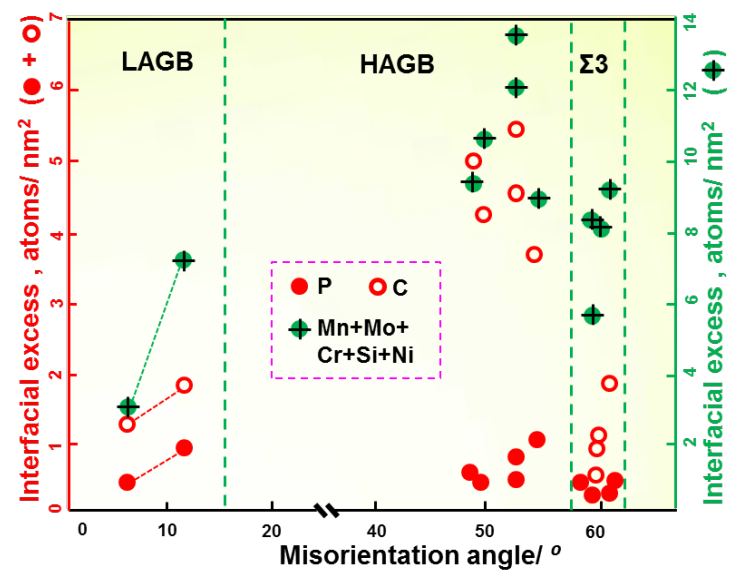

Figure 1 Variation of the total interfacial excess values with misorientation angle in the RPV weld metal for both substitutional elements $(\mathrm{Si}+\mathrm{Cr}+\mathrm{Mo}+\mathrm{Ni}+\mathrm{Mn}) \&$ interstitial elements $(\mathrm{C} \& \mathrm{P})$.

\section{References:}

[1] Lejcek P, Grain boundary segregation in metals, Springer Science \& Business Media, Berlin, 2010. [2] Lejček, Pavel, Mojmír Šob, Vaclav Paidar, Interfacial segregation and grain boundary embrittlement: an overview and critical assessment of experimental data and calculated results. Progress in Materials Science 87 (2017), 83-139.

[3] Babinsky K, De Kloe R, Clemens H, et al., A novel approach for site-specific atom probe specimen preparation by focused ion beam and transmission electron backscatter diffraction. Ultramicroscopy 144 (2014), 9-18. 


\section{Formation of Nanoscale eta Carbides in Fe-Ni-C Martensite at Room Temperature}

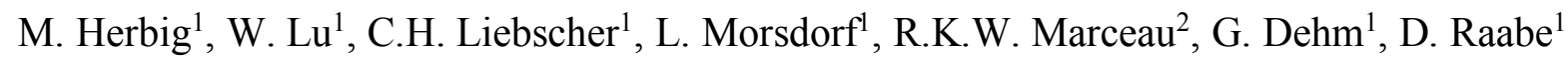

Corresponding Author: m.herbig@mpie.de

1. Max-Planck-Institut für Eisenforschung GmbH, Max-Planck-Straße 1, 40237 Düsseldorf, Germany

2. Institute for Frontier Materials, Deakin University, Waurn Ponds VIC 3216, Australia

The formation of the so called transition carbides with $\mathrm{C}$ contents between 25 and 33.3 at.\% ferrous in martensite is for many years a matter of controversial discussion. Their rapid formation at a broad temperature range during cooling makes it difficult to investigate composition and crystallography of a single type of transition carbides. Here we investigate structure and chemistry of nanoscale carbides that formed in Fe-15Ni-1C (wt.\%) martensite after long term aging of $2-3$ years at $\sim 25^{\circ} \mathrm{C}$ under clean experimental conditions. The low martensite start temperature of $-25^{\circ} \mathrm{C}$ of the well-homogenized alloy enabled to store it in $100 \%$ austenitic condition at room temperature with all carbon in solid solution. Carbon-oversaturated martensite with immobile carbon was formed by quenching in liquid nitrogen. The temperature was increased back to room temperature where $\mathrm{C}$ has enough mobility to form tweed-like structures within hours that further evolve over several months in morphology and composition. Between 2-3 years no further evolution was observed. We identify the carbon-rich features in this condition using combined electron microscopy and atom probe tomography as eta carbides $(\eta$ $\mathrm{Fe}_{2} \mathrm{C}$ ). 


\section{Elucidating Grain Boundary Segregation Behavior in Nanocrystalline Stabilized Pt(Au)}

Xuyang Zhou ${ }^{1}$, Brad Boyce ${ }^{2}$, Blythe Clarke ${ }^{2}$ and Gregory B. Thompson ${ }^{1}$, Corresponding Author: gthompson@eng.ua.edu

1. The University of Alabama, Department of Metallurgical \& Materials Engineering, Tuscaloosa, USA.

2. Sandia National Laboratories, Albuquerque, USA.

Grain boundary (GB) segregation is a key factor in the stabilization of nanocrystalline alloys. The atom probe can provide a tool that can provide unpreceded chemical quantification with high spatial resolution of how solutes partition within the alloy. However, reconstruction assumptions, particularly the use of the single field value, can lead to a loss of lattice rectification making crystallographic determination difficult. Using a cross-correlative technique, precession electron diffraction (PED) has been applied to a series of atom probe tips to identify the grain boundary types in a $\mathrm{Pt}(\mathrm{Au})$ nanocrystalline alloy, after which the tips were field evaporated and the atoms reconstructed. The overlay of the PED map with the atom map enabled the interfacial excess (IE) mapping for each specific grain boundary to be determined, as shown in Figure 1. This talk will discuss how this cross-correlative method between PED-atom probe tomography is enabling insights into both thermodynamic and kinetic factors that can lead to nanocrystalline stability.
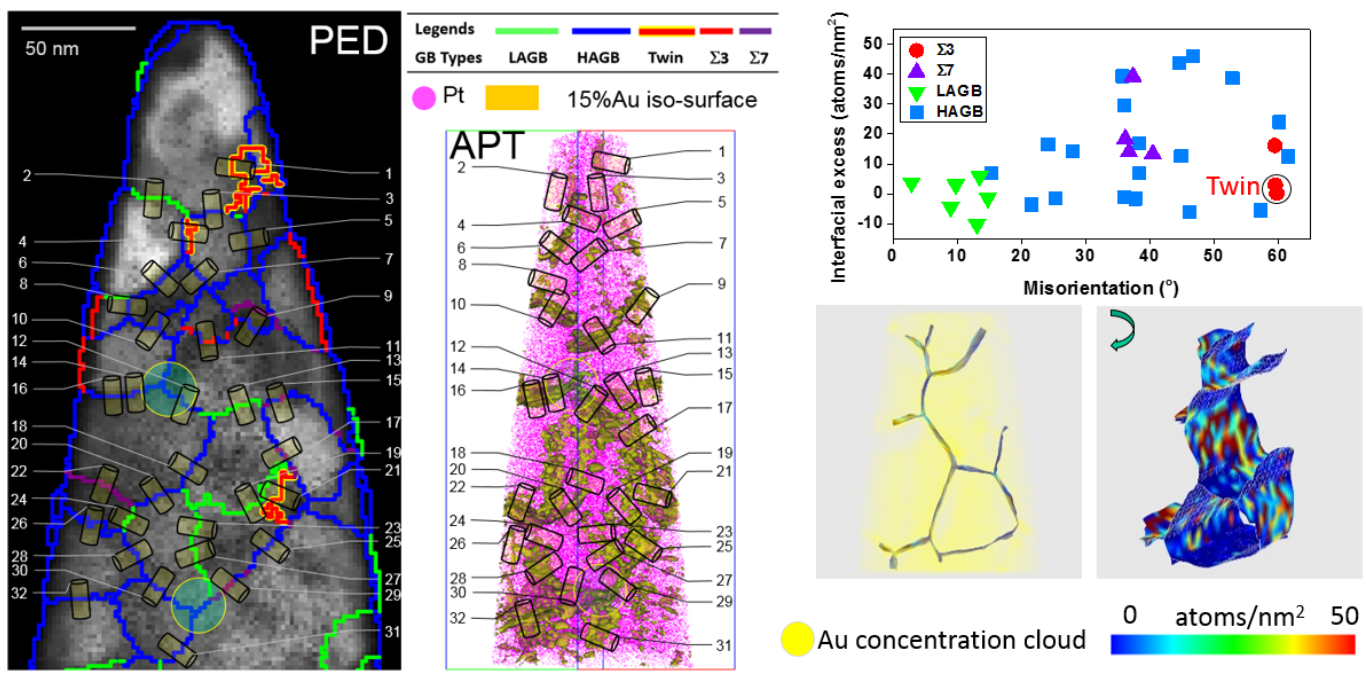

Figure 1 Cross-correlative PED and APT study of a Pt-11Au film. The IE values of different GBs were shown as a function of grain boundary misorientation with a representative IE map included.

\section{$\underline{\text { References: }}$}

[1] XZ and GBT gratefully recognize the National Science Foundation DMR-1709803; BB and BC acknoweldge the Department of Energy - Basic Energy Sciences for their support. 


\title{
Comparison of Irradiation-Induced Clustering in Proton Irradiated A508 Gr 4N Steel via Analytical STEM EDX and Atom Probe Tomography
}

\author{
J. O. Douglas ${ }^{1}$, A. Carruthers 2 , P. A. J. Bagot ${ }^{1}$, M. P. Moody ${ }^{1}$, M. G. Burke ${ }^{2}$
}

Corresponding Author: james.douglas@materials.ox.ac.uk

1. University of Oxford, Atom Probe Research Group, Department of Materials, Parks Road, Oxford, OX1 3PH, UK.

2. University of Manchester, Materials Performance Centre, School of Materials, The Mill, Manchester, M11 9PL, UK.

Reactor Pressure Vessels (RPV) in Pressurised Water Reactors operate at elevated temperatures and may be subjected to a maximum of 0.1 dpa over $60+$ years of operation. Neutron irradiationinduced solute clustering can lead to increases in ductile-to-brittle transition temperature and hardness resulting in a decrease in toughness [1]. Quantitative nanoscale microscopy is required in order to determine the size, number density and composition of these features, and to assess the extent of solute segregation to dislocation loops and grain boundaries [2],[3]. A508 Gr 4N steel is a high Ni, low Mn forging steel that has excellent toughness, and has exhibited good performance after neutron irradiation. Proton-irradiations $\left(2 \mathrm{MeV} \mathrm{p}+\right.$ at $225^{\circ} \mathrm{C}, 90 \mathrm{mdpa}$ and 1.1 dpa) of A508 Gr 4N steel were performed to assess the microstructural changes and examine the nature of the irradiation-induced nanoscale features since proton irradiation can be performed in relatively short times with low resultant activity levels. APT analysis was carried out with a LEAP 5000XR using the maximum separation method to determine cluster size and composition. AEM characterisation was performed using an FEI Talos F200 S/TEM with Super X (4 SDDs) for STEM-EDX analyses and used the parallax method to measure foil thickness and spectrum subtraction to estimate average cluster composition.
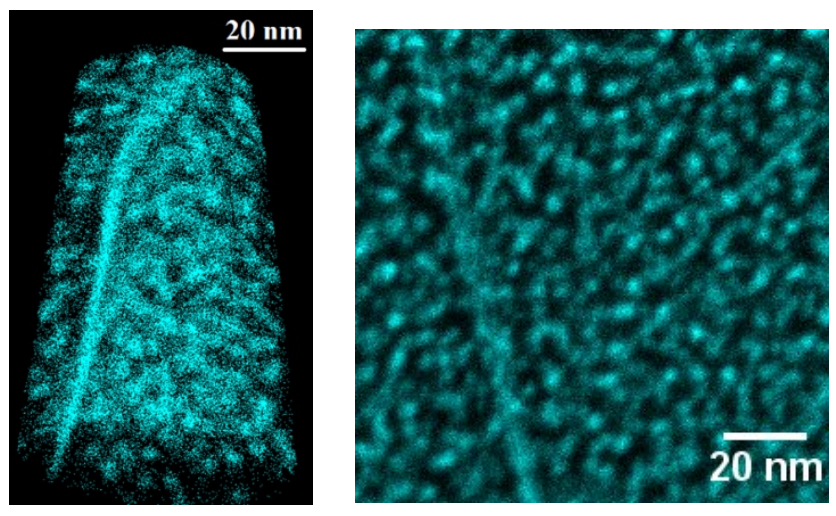

Solute clustering of $\mathrm{Ni}, \mathrm{Mn}$ and $\mathrm{Si}$ in nanoscale spherical clusters along with solute segregation to grain boundaries was observed using both techniques (Figure 1). Cluster composition and extent of solute segregation to grain boundaries as measured by both techniques will be presented and the relative strengths of each will be discussed.

Figure 11.1 dpa $2 \mathrm{MeV} \mathrm{p}^{+}$irradiated A508 Gr 4N. Left: APT atom map of Ni ions. Right: STEMEDX Ni map.

References:

[1] M.G. Burke, R.J. Stofanak, J.M. Hyde, C.A. English, and W.L. Server, Eff. Radiat. Mater. ASTM STP 1447 (2002), 1-14.

[2] M.G. Burke, M. Watanabe, D.B. Williams, and J.M. Hyde, J. Mater. Sci. 41 (2006), 4512-4522.

[3] P.D. Edmondson, C.M. Parish, R.K. Nanstad, Acta Materialia, 134 (2017), 31-39. 


\title{
Solidification Cracking During Selective Laser Melting (SLM) of Nickel-Base Superalloy Inconel-738LC
}

\author{
A. Hariharan ${ }^{1}$, J. Risse ${ }^{2}$, E. A. Jägle ${ }^{1}$, D. Raabe ${ }^{1}$ \\ Corresponding Author: a.hariharan@mpie.de, e.jaegle@mpie.de \\ 1. Department of Microstructure Physics and Alloy Design, Max-Planck-Institut für \\ Eisenforschung GmbH, Max-Planck-Straße 1, 40237, Düsseldorf, Germany \\ 2. Fraunhofer-Institut für Lasertechnik ILT, Steinbachstraße 15, 52074, Aachen, Germany
}

In the additive manufacturing of polycrystalline nickel-base superalloy Inconel-738LC by selective laser melting (SLM), the key issue is the formation of micro-cracks during the process. These are solidification cracks that occur at the last stage of solidification. According to the most widely accepted theory for solidification cracking[1], microsegregation of alloying elements to the interdendritic liquid at this stage stablizes it to low temperatures. The theory also predicts different microsegregation behaviour dependent on the misorientation between the growing dendrites. The remaining liquid film between the solidifying dendrites are vulnerable to the initiation and propagation of these microcracks. Post solidification, all cracks are observed on high angle grain boundaries in accordance with solidification cracking theory. However, our Atom Probe Tomography (APT) results show different solute profiles than predicted by the same theory.

In the current study, we use APT coupled with targeted sample preparation and transmission Kikuchi diffraction (TKD) to analyse the chemical composition profile of the alloying elements across grain boundaries with unambiguously identified misorientation. We characterize the chemical composition profile at high-angle and low-angle grain boundaries with solidification cracks and on those that are devoid of cracks. The APT-TKD study reveals a nearly tenfold increase in the amount of $\mathrm{Zr}, \mathrm{B}, \mathrm{Si}$ and $\mathrm{C}$ at the high angle grain boundaries compared to their bulk nominal composition in the matrix. These results are compared to solidification cracking theory and are used to rationalize the contribution of microsegregation to solidification cracking.

a)

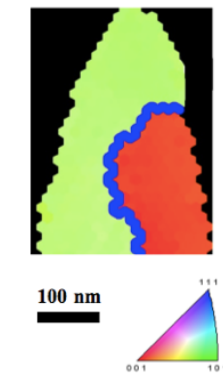

b)

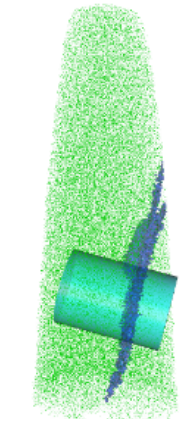

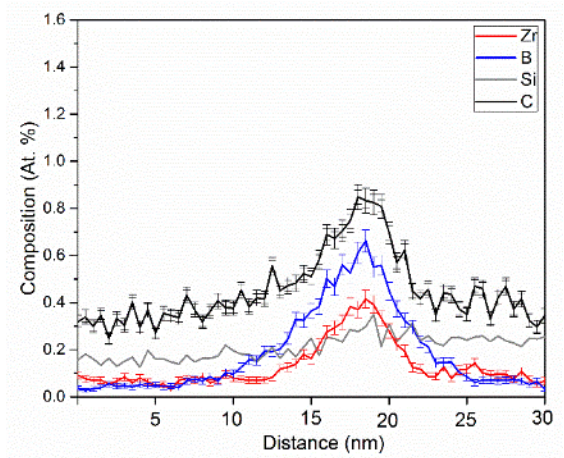

Figure 1 (a) TKD of APT tip (b) 1-D concentration profile of $\mathrm{Zr}, \mathrm{B}$ and Si across a high angle grain boundary measured by Atom Probe Tomography.

References:

[1] Rappaz, M., Jacot, A., \& Boettinger, W. J.: Last-Stage Solidification of Alloys : Theoretical Model of Dendrite-Arm and Grain Coalescence, Metallurgical and Materials Transactions A, Volume 34A, 467479 (2003). 


\section{Combined TEM and APT Characterization of Multi-Phase Oxides Formed During Metal Oxidation and Corrosion}

Daniel K. Schreiber ${ }^{1}$, Matthew J. Olszta ${ }^{1}$, Karen Kruska ${ }^{1}$, John R. Scully ${ }^{2}$, Angela Y. Gerard ${ }^{2}$, Kathleen F. Quiambao' ${ }^{2}$ and Stephen M. Bruemmer ${ }^{1}$

Corresponding Author: daniel.schreiber@pnnl.gov

1. Energy \& Environment Directorate, Pacific Northwest National Laboratory, Richland, WA USA.

${ }^{2}$ Department of Materials Science \& Engineering, University of Virginia, Charlottesville, VA USA

The oxidation and corrosion of metal alloys is a critical concern in many industrial applications. At the same time, a fundamental understanding of these behaviors at the atomistic level is elusive. High resolution microscopy has a critical role to play in unraveling the basic mechanisms that control material degradation. However, even the most advanced analytical techniques cannot fully describe the complex 3D nature of these microstructures.

In this work we describe our efforts combining analytical transmission electron microscopy (TEM) and atom probe tomography (APT) to generate novel insights into the oxidation and corrosion behavior of complex alloys. Multi-phase oxides are a ubiquitous part of these microstructures and are difficult to discern fully by either technique independently. Combining these techniques leads to a more complete understanding of the competition between kinetics and thermodynamics during material degradation. Examples will be discussed from both conventional complex alloys (e.g. alloys 600 and 690) and novel high entropy alloys.
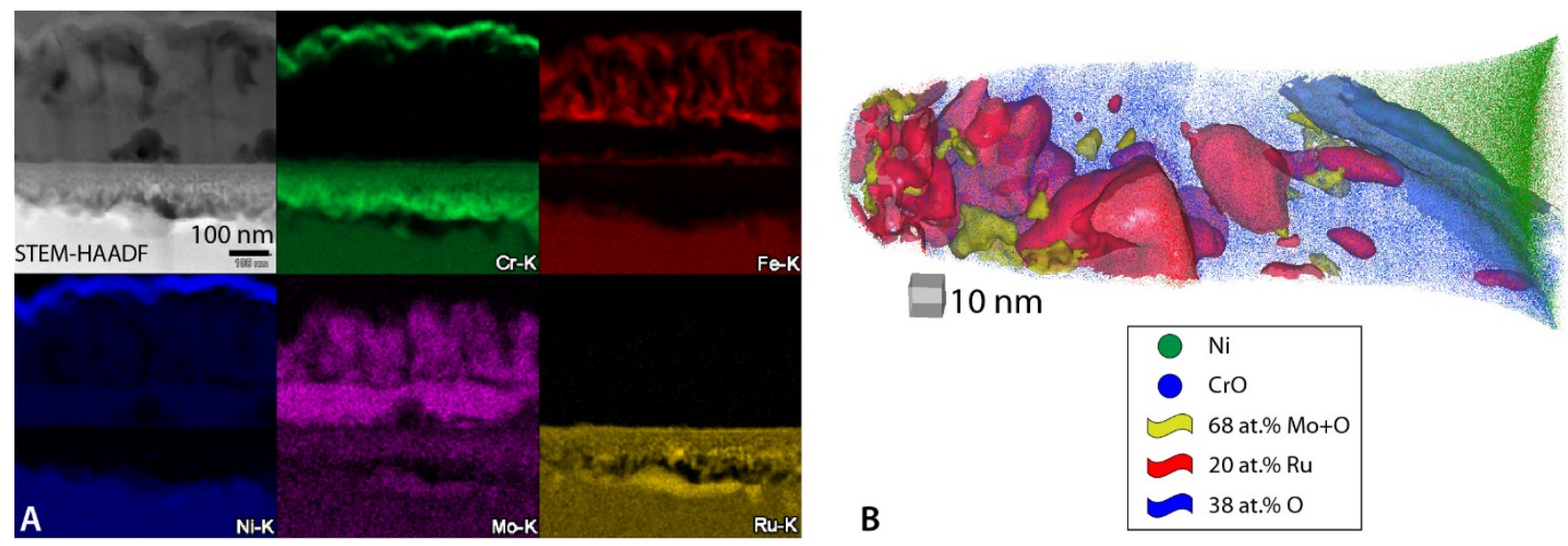

Figure 1 Combined TEM and APT observations of multi-phase oxide formation on the surface of a high entropy Ni-base alloy. (A) STEM HAADF and EDS analysis observations reveal the presence of many spatially separated and compositionally unique layers. (B) APT characterization reveals further 3D distributions of nanoscale oxide phases within the layered structure. 


\title{
APT/TEM Analyses of Solute Segregations Along Dislocations and Solute Clustering in Bake-Hardenable Magnesium Alloy
}

\author{
M.-Z. Bian ${ }^{1}$, T.T. Sasaki ${ }^{1}$, T. Nakata ${ }^{2}$, Y. Yoshida ${ }^{3}$, N. Kawabe ${ }^{3}$, S. Kamado ${ }^{2}$ and K. Hono ${ }^{1}$ \\ Corresponding Author: SASAKI.Taisuke@nims.go.jp
}

1. National Institute for Materials Science (NIMS), Tsukuba, Japan .

2. Nagaoka University of Technology, Nagaoka, Japan.

3. Sumitomo Electric Industries, Ltd., Itami, Japan.

Recent intense interest in developing lighter wrought alloys revived researches on precipitation hardenable magnesium alloys. Since the age-hardening responses of commercial magnesium alloys are poor, the precipitation hardening has not been used in conventional wrought magnesium alloys. However, optimizations of alloy compositions lead to the formation of metastable nanosized precipitates during artificial aging, which substantially enhances the yield strength. In order to understand the mechanism of age-hardening behaviors, three-dimensional atom probe (3DAP) is an ideal tool to analyze the solute clusters and nano-scale precipitates to complement transmission electron microscopy.

In this presentation, we show examples of applications of correlative TEM/3DAP analyses to the first bake hardenable $\mathrm{Mg}-1.2 \mathrm{Al}-0.5 \mathrm{Ca}-0.4 \mathrm{Mn}-0.8 \mathrm{Zn}$ (wt.\%) (AXMZ1000) sheet alloy developed in our group. The AZMX1000 sheet alloy shows excellent room temperature formability comparable with that of 6xxx series aluminum alloys, Fig. 1. More importantly, the yield strength can be significantly increased to about $240 \mathrm{MPa}$ by a bake-hardening $(\mathrm{BH})$ treatment, i.e. $2 \%$ prior stretch and aging for $20 \mathrm{~min}$ at $170{ }^{\circ} \mathrm{C}$. The bake-hardenability of this alloy is comparable to that of low carbon steels and 6\#\#\# series aluminum alloys. A correlative TEM-APT reveals that solute atoms of $\mathrm{Al}, \mathrm{Ca}$ and $\mathrm{Zn}$ are segregated to dislocation cores after the bake-hardening treatment, and this pins the dislocations giving rise to the yield phenomenon, Fig. 1 and 2. The BH treatment also causes to form a number of co-clusters within the magnesium matrix, which contribute to the increase in strength. The role of the solute clusters and solute segregation along dislocations on the strength will be discussed based on the TEM-APT analysis results.

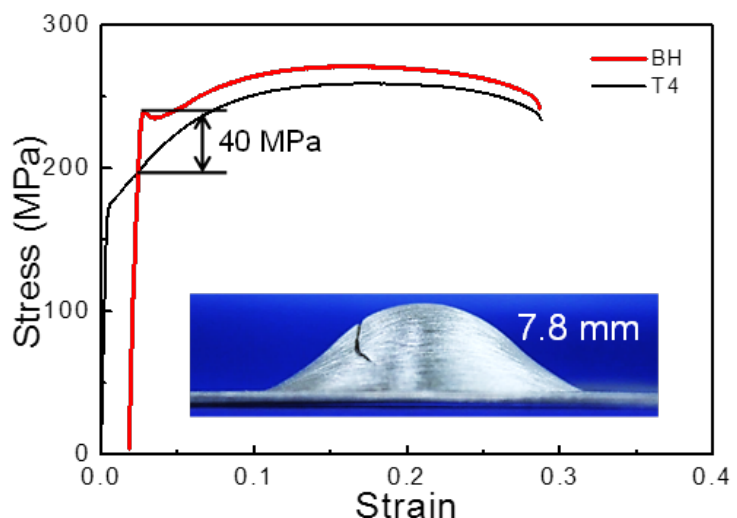

Fig. 1. Mechanical properties of BHed AZMX1110 sheet alloy

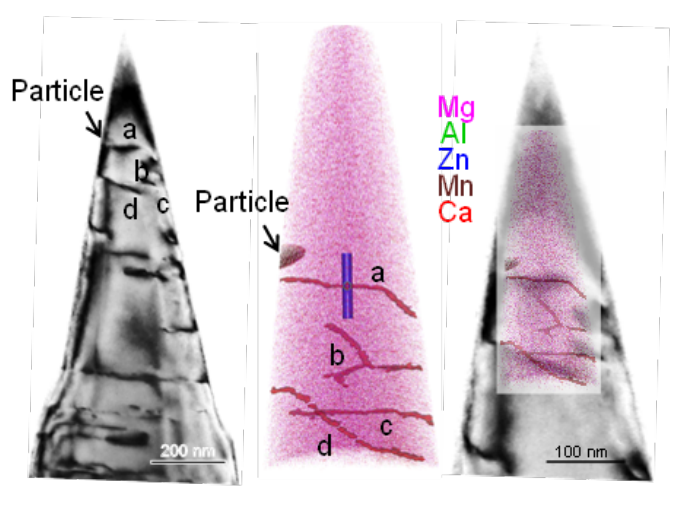

Fig. 2. TEM/APT analysis of BHed AZMX1110 sheet allov 


\section{The Role of Atom Probe Tomography on the Development of New High Performance Materials}

Paraskevas Kontis ${ }^{1}$, Surendra K. Makineni ${ }^{1}$, Jonathan Cormier $^{2}$, Dierk Raabe ${ }^{1}$, Baptiste Gault ${ }^{1}$

Corresponding Author: p.kontis@mpie.de

1. Max-Planck-Institut für Eisenforschung, Department of Microstructure Physics and Alloy

Design, Max-Planck-Str. 1, 40237, Düsseldorf, Germany.

2. Institut Pprime, Physics and Mechanics of Materials Department, UPR CNRS 3346, ISAE-

ENSMA, 1 avenue Clément Ader, BP 40109, 86961 Futuroscope-, Chasseneuil, France

The development of new high performance materials, such as nickel-based superalloys, is critical for the increase of the performance of aero-engines and industrial gas turbines. Systematic highresolution characterization at the near-atomic scale by atom probe tomography (APT) can provide key information to understand deformation mechanisms at elevated temperatures. We present correlative electron channeling contrast images (ECCI) and APT analyses of dislocations in deformed superalloys. The segregation and diffusion of chromium and cobalt along the dislocations allow us to interpret the partial dissolution and rafting of the strengthening $\gamma^{\prime}$ precipitates in a single-crystal superalloy [1]. Besides, the complete dissolution of $\gamma^{\prime}$ precipitates resulting in soft recrystallized regions due to oxidation of $\mathrm{MC}$ carbides in a polycrystalline superalloy was studied. APT revealed substantial compositional variations within the recrystallized regions compared to the original $\gamma / \gamma^{\prime}$ microstructure [2]. These examples show how APT can be utilized for the design and development of new high performance materials for high temperature applications by providing new insights into their microstructural behaviour.
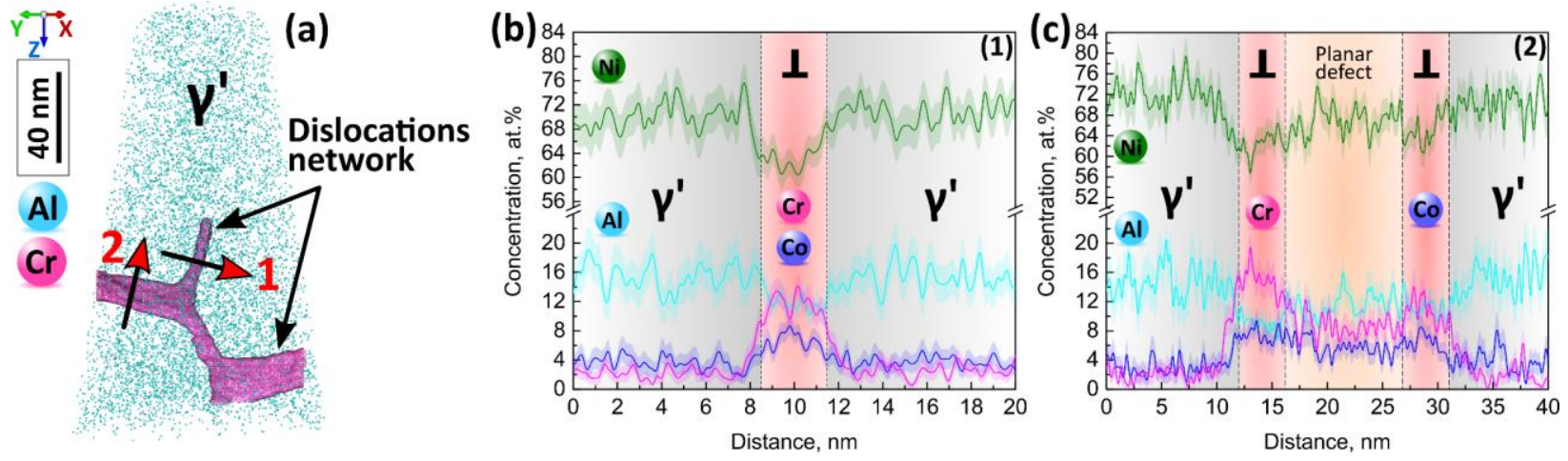

Figure 1 (a) APT reconstruction from a $\gamma^{\prime}$ precipitate containing a network of dislocations showing with an iso-concentration surface at 8.0 at. $\% \mathrm{Cr}$. (b) $1 \mathrm{D}$ concentration profile perpendicular to the dislocation denoted by the arrow \#1. (b) 1D concentration profile perpendicular to the dislocation denoted by the arrow \#2. Error bars are shown as lines filled with colour and correspond to the $2 \sigma$ counting error.

\section{References:}

[1] P. Kontis, Z. Li, D.M. Collins, J. Cormier, D. Raabe and B. Gault, The effect of chromium and cobalt segregation at dislocations on nickel-based superalloys, Scripta Materialia, 145 (2018), 76-80.

[2] P. Kontis, D.M. Collins, A.J. Wilkinson, R.C. Reed, D. Raabe and B. Gault, Microstructural degradation of polycrystalline superalloys from oxidized carbides and implications on crack initiation, Scripta Materialia, 147 (2018), 59-63. 


\section{Sparse Data Sampling Overview}

John Henry Scott ${ }^{1}$

\section{Corresponding Author: johnhenry.scott@nist.gov}

1. National Institute of Standards and Technology, Materials Measurement Science Division, Gaithersburg, MD 20899.

This tutorial will explore the advantages and pitfalls of using sparse data representations and related methods for reconstructing complete datasets from a limited number of measurements or observations. In favorable cases, near-perfect reconstructions of images or volumetric datasets are possible even when more than $90 \%$ of the data are missing or hidden. This result can be exploited to reduce the dose required during data acquisition. Examples using standard test data from the applied mathematics and computer science literature will be supplemented by additional real-world examples drawn from the fields of microscopy and microanalysis. We will also discuss the deep connections between the information content in an image and the constraints, assumptions, or a priori structure imposed by the image representation, and how these constraints can lead to powerful denoising algorithms. Finally, this survey will briefly cover the relatively new concept of compressed sensing, explain its relationship to the above topics, and review results in the field that provide solid, quantitative predictions of information recovery well past the limits of what was thought possible based on the Nyquist-Shannon sampling theorem. While some advanced mathematics will be mentioned and explained as needed during the talk, no prior knowledge of methods or jargon is required, and the surprising power and utility of these approaches can be appreciated based solely on the examples. 


\section{Atom Probe Study of Silicon-Based Device Structures}

Yasuo Shimizu, Bin Han, 'Yuan Tu, Koji Inoue, and Yasuyoshi Nagai

Corresponding Author: yshimizu@imr.tohoku.ac.jp

Institute for Materials Research, Tohoku University, Oarai, Ibaraki 311-1313, Japan

*Present Affiliation: Department of Mechanical and Biomedical Engineering, City University of Hong Kong, Hong Kong, China

An accurate characterization of elemental distributions in semiconductor-based electronic devices is of importance for achieving future device design. At present, metal-oxidesemiconductor field-effect transistors (MOSFETs) device is shifting from planar to complex structures such as fin, trench, and gate-all-around FET [1]. The functional properties of these devices strongly depend on their elemental distribution. Atom probe tomography (APT) has proven useful for obtaining the spatial distributions of dopants, their clusters, and segregation on the order of atomic-scale resolution, thus APT has attracted considerable attention as a technique to meet the challenges in the semiconductor industry [2].

In this conference, the recent achievements on APT studies for Si-based device structures carried out in Tohoku University will be presented; I) dopant diffusion paths in polycrystalline Si for the development of trench-type transistors [3], II) direct observation of dopants in Si/Ge nanowire structure [4], and III) spatial distribution assessment of ion-implanted Ge atoms in Si substrates [5]. The APT method combined with site-specific focused ion beam for visualizing elements in 3D gives us a great impact on future nanostructured device designing in the semiconductor industry.

\section{$\underline{\text { References: }}$}

[1] International Technology Roadmap for Semiconductors; http://www.itrs2.net/.

[2] A. D. Giddings, S. Koelling, Y. Shimizu, R. Estivill, K. Inoue, W. Vandervorst, and W. K. Yeoh, Industrial application of atom probe tomography to semiconductor devices, Scripta Materialia, in press. DOI: 10.1016/j.scriptamat.2017.09.004.

[3] B. Han et al., Appl. Phys. Lett. 107 (2015) 023506.

[4] B. Han et al., Nanoscale 8 (2016) 19811-19815.

[5] Y. Tu et al., Nanotechnology 28 (2017) 385301.

[6] The authors thank F. Yano, A. Nishida, M. Inoue, Y. Kunimune, Y. Shimada, T. Katayama, T. Ide, H. Takamizawa, N. Ebisawa, K. Tomura, and Y. Nozawa for their support. For collaborative work, Y.S. would thank Dr. N. Fukata (Si/Ge nanowires), and Prof. T. Tanii and Prof. T. Shinada (Ge ion implantation). This work was supported in part by JSPS KAKENHI (Grant Nos. 24760246 and 15H05413) and JST/CREST (Grant No. JPMJCR17J1). 


\title{
Atom Probe Analysis of Surface Regions of Iron Contaminated Silicon Subjected to Phosphorus Diffusion Gettering
}

\author{
J. O. Douglas ${ }^{1}$, G. F. Martins ${ }^{1}$, A. S. Bakr ${ }^{1}$, J. D. Murphy ${ }^{2}$, P. A. J. Bagot ${ }^{1}$ and M. P. Moody ${ }^{1}$
}

Corresponding Author: james.douglas@materials.ox.ac.uk

${ }^{1}$ Department of Materials, University of Oxford, Parks Road, OX1 3PH, UK.

${ }^{2}$ School of Engineering, University of Warwick, Coventry, CV4 7AL, UK.

Transition metals, especially iron, are a major centre for carrier recombination in silicon photovoltatics and thus cause a loss of efficiency in solar cells when they are present. Phosphorus Diffusion Gettering (PDG) is a common method of sequestering interstitial iron from the bulk to a phosphorus rich surface region during emitter fabrication of silicon photovoltaic cells but the exact mechanism is not fully understood. It has been suggested that iron decoration of oxide precipitates in the phosphorus rich layer can play a role [1] but it also possible that silicon phosphide precipitates, often formed at the silicon surface during the diffusion process, can be involved due to the ejection of interstitials during their formation [2]. Secondary Ion Mass Spectrometry (SIMS) has been used to obtain high resolution compositional depth profiles of the surface regions but lacks the lateral resolution to determine if nanoscale variations in surface composition are present. SIMS has determined that iron and oxygen segregate to the topmost $\sim 50 \mathrm{~nm}$ of the phosphorus rich layer, which is also where phosphorus concentrations peak [3]. In this work APT analysis was carried out on the surface tens of nm of the phosphorus rich layer of intentionally iron contaminated, PDG processed silicon substrates in order to investigate their nanoscale structure with a long-term aim of furthering understanding into the mechanism of PDG. Ex-situ thermally evaporated capping layers of cobalt and optimised Focused Ion Beam sample preparation was carried out to preserve and delineate the surface regions [4]. The majority of phosphorus in the surface tens of nm was found to be in the form of silicon phosphide precipitates orientated along crystal planes. Oxygen rich regions in direct contact with these precipitates were observed extending from the surface into the substrate (Figure 1), indicating a potential link between oxygen and phosphorus precipitation in the gettering process.
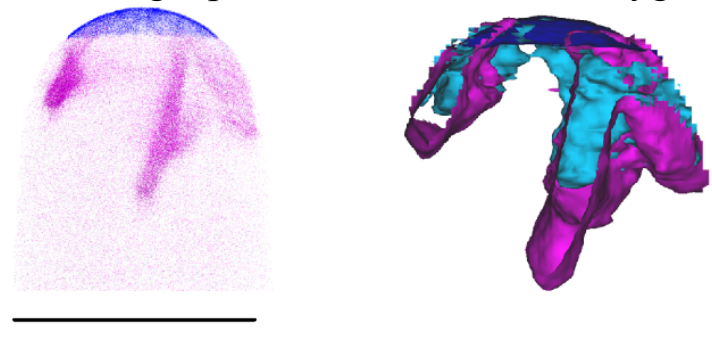

References:
Figure 1 - Atom map of cobalt capped (dark blue) silicon (gray) with linear phosphorus rich regions (purple) extending from the surface. Isosurfaces delineate $10 \%$ phosphorus (purple) and oxygen (light blue) in direct contact. Oxygen rich regions were not observed beneath the surface when silicon phosphide precipitates were absent. Scale bar $120 \mathrm{~nm}$.

[1] M. Syre, et al., Evaluation of possible mechanisms behind P gettering of iron, Journal of Applied Physics 110 (2011), 024912.

[2] A. Ourmazd and W. Schröter, Gettering of Metallic Impurities In Silicon, Mat. Res. Soc. Symp. Proc. 36 (1985), 25-31.

[3] M. B. Shabani et al., Study of gettering mechanisms in silicon: Competitive gettering between phosphorus diffusion gettering and other gettering sites, Solid State Phenomena 131-133 (2008), 399-404.

[4] J. O. Douglas et al., Optimisation of sample preparation and analysis conditions for atom probe tomography characterisation of low concentration surface species, Semiconductor Science and Technology 31 (2016), 084004. 


\section{Quantification of boron dopant profiles in SiGe-based HBT devices}

$\underline{\text { Eric J. Jones }}^{1}$, Keith Chung ${ }^{1}$, Jonathan Poplawsky ${ }^{2}$, Donavan Leonard ${ }^{2}$, Kevin Mercurio ${ }^{1}$, Paul Brabant $^{1}$, Thomas Adams ${ }^{1}$, Patrick Shea ${ }^{1}$, Thomas Knight ${ }^{1}$

Corresponding Author: eric.jones2@ngc.com

1. Northrop Grumman Corporation, Mission Systems, Baltimore, MD, USA.

2. Oakridge National Laboratories, Center for Nanophase Materials Science, Oakridge, TN, USA.

SiGe heterostructure bipolar transistors are one of the crucial technologies in fifth generation (5G) network and other RF technologies. [1] By leveraging volume silicon manufacturing and high speed mixed signal performance, wireless communications at significantly reduced cost are enabled. SiGe HBT device electrical models are typically based on SIMS dopant profiles measured on large pads ranging from 20x20um to 100x100um. These large pads may not be reflective of the actual dopant concentrations inside the device. Therefore, to improve our device modeling and critical understanding of these devices and systems, techniques for dopant characterization with high spatial resolution are needed.

In this study we use atom probe tomography (APT), scanning transmission electron microscopy (STEM), and secondary ion mass spectroscopy (SIMS) to characterize doping profiles in the base region of an HBT device shown in Figure 1a. We compare SIMS profiles obtained from large $\left(400 \mu \mathrm{m}^{2}\right)$ regions of the device wafer to profiles obtained from individual devices of different emitter window width -0.25 and $0.18 \mu \mathrm{m}-$ using APT (Figures $1 \mathrm{~b}$ and 1c). From this comparison we will show how the APT data provides a deeper insight into evaluating the fabrication process and its effects on electrical models of device performance and enabling the building of higher performance systems.

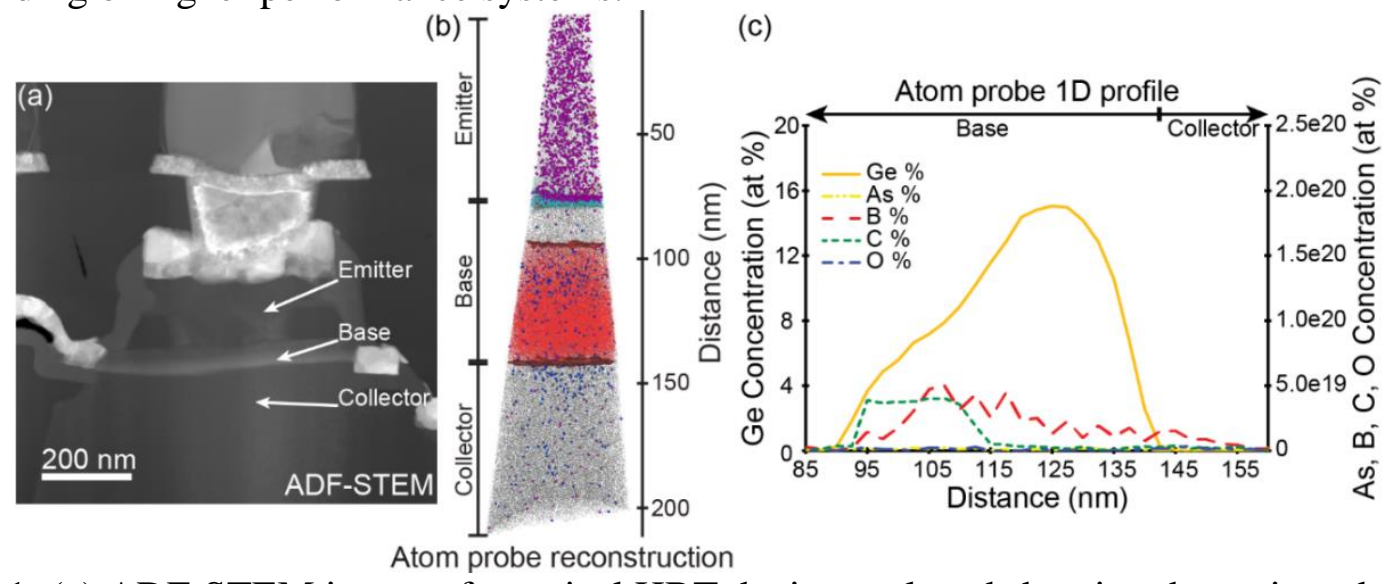

Figure 1: (a) ADF-STEM image of a typical HBT device analyzed showing the emitter, base, and collector regions. (b) Reconstruction of atom probe data obtained from an HBT device having an emitter window width of $0.25 \mu \mathrm{m}$ showing all ions. (c) 1-dimensional profile of atom probe data highlighting Ge and dopant concentrations in the base region.

\section{References:}

[1] Voinigescu, S. P.; et al. Silicon Millimeter-Wave, Terahertz, and High-Speed Fiber-Optic Device and Benchmark Circuit Scaling Through the 2030 ITRS Horizon, Proceedings of the IEEE 105 (2017), 10871104.

[2] APT and STEM was conducted at ORNL's CNMS, which is a U.S. DOE Office of Science User Facility. 


\title{
Atom Probe Tomography of Ferroelectric $\left(\mathrm{BiFeO}_{3}\right)$ Domain Walls
}

\author{
M.A. Auger ${ }^{1}$, P. Sharma², M.P. Moody ${ }^{1}$, J. Seidel ${ }^{2}$ \\ Corresponding Author: maria.auger@materials.ox.ac.uk \\ ${ }^{1}$ Department of Materials, University of Oxford. Parks Road OX1 3PH, Oxford, UK. \\ 2.School of Materials Science and Engineering, University of New South Wales Australia. \\ Sydney, New South Wales 2052, Australia
}

Ferroelectric domain walls (FEDWs) are reconfigurable nanoscale elements which display a variety of exotic physical phenomena including electronic conduction [1] in otherwise insulating oxides. Using an applied electrical stimulus, a rewritable control over the spatial location and nucleation of the FEDWs can be achieved. This offers a viable route to realize high-density, energy efficient nanoelectronic devices based on FEDWs [2]. The exotic properties of the FEDWs arise because of changes in the symmetry and structure across these atomically sharp interfaces necessitating their full 3-dimensional (3D) visualization with ultra-high resolution. In most studies thus far, domain walls have been visualized in a 2-dimensional space.

For this study, we prepared single crystalline high-quality thin films of the multiferroic $\mathrm{BiFeO}_{3}$ (BFO) using pulsed laser deposition. The films were grown on (110)-oriented $\mathrm{DyScO}_{3}$ (DSO) substrates resulting in the presence of well-defined $109^{\circ}$ domain walls as confirmed by the piezoresponse force microscopy (PFM) measurements. Using atom probe tomography (APT), an unprecedented 3D reconstruction of the domain wall structure was obtained.

APT samples were prepared by the FIB lift-out method and the samples were analyzed in a CAMECA LEAP 5000 instrument in laser mode, using a $0.050 \mathrm{~nJ}$ laser energy at $200 \mathrm{kHz}$ frequency and at a temperature of $50 \mathrm{~K}$. Fig. 1-a) shows the $3 \mathrm{D}$ ion maps of the Fe and Bi ions from the domain walls and the Dy and Sc ions from the substrate, with O present in both. Fig. 1b) highlights the 5.6 at.\% Fe isosurfaces in the analyzed volume. These features are measured to be $\sim 5 \mathrm{~nm}$ in diameter and 20-30 $\mathrm{nm}$ long, with the tilting angle respect to the substrate being $95^{\circ}$. The measurements obtained from the APT reconstruction agree with the nominal values for this nanostructure.

a)

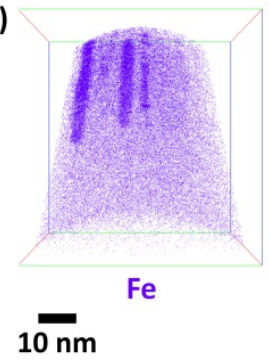

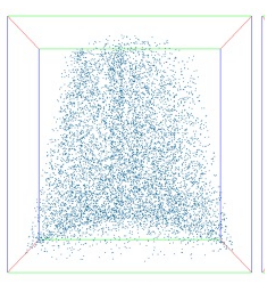

Bi

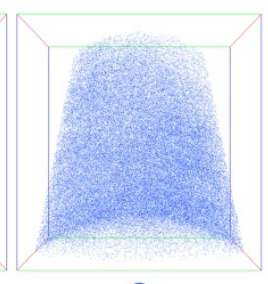

O

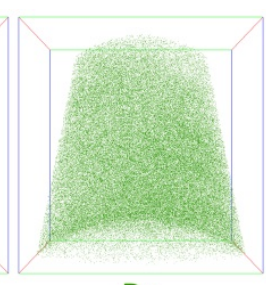

Dy

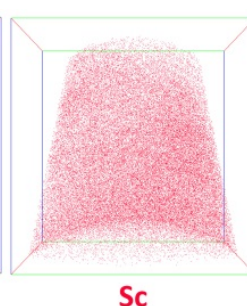

Sc

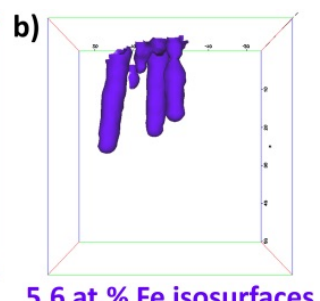

5.6 at.\% Fe isosurfaces

Figure 1 (a) 3D ion maps of the BFO domain walls on a DSO substrate. (b) 5.6 at.\% Fe isosurfaces highlighting the domain wall structure.

\section{References:}

[1] J. Seidel et al., Nat. Mater. 8, 229-234 (2009).

[2] P. Sharma et al., Sci. Adv. 3, e1700512 (2017)

[3] Funding from UK-EPSRC grants EP/P001645/1 and EP/M022803/1 is acknowledged. 


\section{Growth Mechanism of III-V Group Core-shell Structured Nanowires}

Jiangtao Qu ${ }^{1}$, Sichao Du ${ }^{1}$, Tim Burgess ${ }^{2}$, Changhong Wang ${ }^{3}$, Xiangyuan Cui ${ }^{4}$, Qiang Gao ${ }^{2}$, Weichao Wang ${ }^{3}$, Hark Hoe Tan ${ }^{2}$, Hui Liu ${ }^{3}$, Chennupati Jagadish ${ }^{2}$, Hansheng Chen ${ }^{1}$, Mansoor Khan $^{1}$, Simon Ringer ${ }^{4}$, and Rongkun Zheng ${ }^{1 *}$

Corresponding Author: rongkun.zheng@sydney.edu.au

1. School of Physics, The University of Sydney, Sydney, Australia.

2. Department of Electronic Materials Engineering, The Australian National University,Canberra, Australia

3. Department of Electronic Science and Engineering, Nankai University, 300350 Tianjin, China.

4. Aerospace, Mechanical and Mechatronic Engineering, The University of Sydney, Australia

III-V ternary InGaAs nanowires have great potential for electronic and optoelectronic device applications; however, the 3D structure and chemistry at the atomic-scale inside the nanowires remain unclear, which hinders tailoring the nanowires for specific applications. Here, atom probe tomography is used in conjunction with a first-principles simulation to investigate the 3D structure and chemistry of Au-seeded and the self-seeded InGaAs nanowires [1,2].

In the Au-seeded nanowires, we reveals (1) the nanowires form a spontaneous core-shell structure with a Ga-enriched core and an In-enriched shell, due to different growth mechanisms in the axial and lateral directions; (2) the shape of the core evolves from hexagon into Reuleaux triangle and grows larger, which results from In outward and Ga inward interdiffusion occurring at the core-shell interface; and (3) the irregular hexagonal shell manifests an anisotropic growth rate on $\{112\} \mathrm{A}$ and $\{112\} \mathrm{B}$ facets. Accordingly, a model in terms of the core-shell shape and chemistry evolution is proposed.

Furthermore, our experimental results reveal the difference between the Au-seeded and the selfseeded InGaAs nanowires on the following aspects: (1) Different geometries: the pyramidal geometry of the Au-seeded nanowires in contrast to the hexagonal cone shape of the self-seeded ones. (2) Different compositions: the Au-seeded nanowires with a Ga-rich core of 0.9 Ga-mole fraction and an In-rich shell of 0.6 and 0.8 In fraction on polarized $\{112\}$ A and B facets versus the self-seeded wires with a hexagonal core of 0.35 Ga-mole fraction and a hexagonal shell of 0.85 In fraction and six In rich bands of 0.9 In fraction bridging the hexagonal corner between core and shell. (3) Different crystal structures: zinc blend Au-seeded nanowires and wurtzite selfseeded ones.

We discovered a seed-induced crystal phase transition for the first time, and more importantly, our experimental results physically confirm the controversial role of the seeds: the seed can act as catalyst to promote the pyrolysis of ambient precursors in addition to the reservoir effect to collect species from pyrolyzed precursors.

\section{References:}

[1] J. T. Qu, et al; "3D Atomic-Scale Insights into Anisotropic Core-Shell-Structured InGaAs Nanowires Grown by Metal-Organic Chemical Vapor Deposition"; Advanced Materials 29, 1701888, (2017).

[2] J. T. Qu, et al; "3D Atomic-Scale Insights into the InGaAs Nanowires Grown by MOCVD"; unpublished 


\section{Atom Probe Tomography of Molecular Organic Electronic Materials}

Matthew B. Jaskot ${ }^{1}$, Andrew P. Proudian ${ }^{2}$, Helena-Nikolai Fujishin ${ }^{2}$, Galen Vincent ${ }^{2}$, David R. Diercks $^{1}$, Brian P. Gorman ${ }^{1}$, and Jeramy D. Zimmerman ${ }^{1,2}$

Corresponding Author: jdzimmer@mines.edu

1. Materials Science Program, Colorado School of Mines, Golden, CO USA

2. Physics Department, Colorado School of Mines, Golden, CO USA

Organic "small molecules" are used in variety of electronic devices including organic photovoltaics (OPVs), organic thin film transistors (OTFTs), and organic light emitting diodes (OLEDs). The molecules used in these devices range from pure carbon (e.g. $\mathrm{C}_{60}$ ), to polycyclic aromatic hydrocarbons (e.g. pentacene), to chelated metalorganics (e.g. iridium trisphenylpyridine or $\left.\operatorname{Ir}(\mathrm{ppy})_{3}\right)$. Many OPV and OLED devices contain blends of two of more molecules and have interfaces of which morphology is critically important. Research needs include morphological evaluation, impurity analysis, and understanding degradation pathways, which have all proven exceedingly difficult with electron microscopy and spatially resolved chemical analysis techniques. The mass-to-charge based imaging modality of APT allows evaluation of both nanometer-scale morphology and chemistry of molecular organic electronic materials blends and interfaces, providing previously inaccessible information.

Under proper analysis conditions, a broad variety of classes of molecules can be ionized with without indication of fragmentation. Spatial resolutions of several nanometers, sub-Dalton mass discrimination (Fig. 1a), and the ability to identify impurities or degradation products around 0.01 atomic \% have been achieved. We have, for example, used APT to identify a Diels-Alder adduct reaction at the interface between tetracene and $\mathrm{C}_{60}[1]$ and phase segregation in prototypical OLED blends (Fig. 1b). Techniques for creating APT samples of small molecule organic films, typical analysis conditions, and the use of spatial statistics to evaluate datasets will be discussed.

(a)

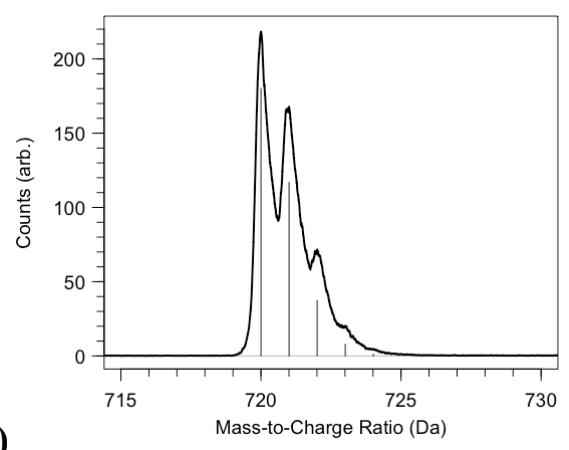

Figure 1 (a) Mass spectrum of $\mathrm{C}_{60}$ showing isotopic peaks (b) heat map displaying inhomogeneity in molecular fraction for a $20 \mathrm{~nm}$-thick section of an OLED film of $6 \% \operatorname{Ir}(\mathrm{ppy})_{3}$ in carbazol-biphenyl.

\section{References:}

[1] A.P. Proudian, M.B. Jaskot, C. Lyiza, D. R. Diercks, B.P. Gorman, and J.D. Zimmerman, Effect of Diels-Alder Reaction in C60-Tetracene Photovoltaic Devices, NanoLetters 16 (2016), 6086-6091.

[2] The work was supported by the grant DE-SC0018021 funded by the U.S. Department of Energy, Office of Science Early Career program. 


\section{Correlative TEM and APT microscopies to investigate Mg distribution in p- doped GaN nanostructures}

Lynda Amichi ${ }^{1,2}$, David Cooper ${ }^{1,3}$, Isabelle Mouton ${ }^{1,3}$,Philippe Vennéguès ${ }^{4}$, Philippe De Mierry ${ }^{4}$, Pierre-Henri Jouneau ${ }^{1,2}$, Catherine Bougerol ${ }^{1,5}$, Adeline Grenier ${ }^{1,3}$

Corresponding Author: adeline.grenier@cea.fr

1. Univ. Grenoble Alpes, F-38000 Grenoble, France

2. CEA-INAC, MINATEC Campus, F-38054 Grenoble, France

3. CEA-LETI, MINATEC Campus, F-38054 Grenoble, France

4. Université Côte d'Azur, CRHEA, Rue Bernard Grégory, 06560 Valbonne, France

5. CNRS, Inst. NEEL, F-38042 Grenoble, France

Gallium nitride $(\mathrm{GaN})$ and its alloys with $\mathrm{Al}$ and In are leading technological materials for a wide range of applications including visible lighting (Nobel Prize 2014), ultraviolet emitters, $\mathrm{THz}$ optoelectronics or power electronics. One major problem which must be solved is the ability to provide highly conductive p-type $\mathrm{GaN}$ and $\mathrm{AlGaN}$ layers. $\mathrm{Mg}$ atoms are commonly used as dopants, however methods that can accurately determine the incorporated concentrations and the spatial distribution of the dopants, as well as the evaluation of their activity are required. In 2Dlayers grown by metalorganic chemical vapor deposition (MOCVD), Mg at concentrations higher than $10^{19} \mathrm{~cm}^{-3}$ has been observed to segregate into a variety of defects such as pyramidal inversion domains ${ }^{[1]}$. The segregation of $\mathrm{Mg}$ atoms causes a drop in free carrier concentration, which is detrimental to the electrical properties of the devices.

In this work, we report the influence of the growth temperature on the $\mathrm{Mg}$ distribution and electrical activity of p-type GaN multilayer stack by coupling atom probe tomography (APT) and off-axis electron holography data. The analyzed sample was a stack of 2D Mg-doped GaN layers separated by non-intentionally doped (NID) layers grown by MOCVD at increasing temperatures between $970^{\circ} \mathrm{C}$ and $1090^{\circ} \mathrm{C}$. The 3D APT reconstruction reveals the presence of several $\mathrm{Mg}$ clusters in the doped layers. The number of $\mathrm{Mg}$ atoms contained in each cluster increases with the growth temperature in agreement with HR-TEM results that reveal the presence of pyramidal inversion domains for the layers grown at temperatures above $1030^{\circ} \mathrm{C}$. The presence of $\mathrm{Mg}$ atoms has been detected also in the NID layers, with an estimated concentration of $9.6 \times 10^{19} \mathrm{~cm}^{-3}$. Offaxis electron holography has been performed on the same sample to investigate the electrical activity of the dopant atoms at RT and $400{ }^{\circ} \mathrm{C}$. The results show a decrease in the step in phase and increase in the width of the depletion regions which is assigned to a reduction of the electrical activity as the $\mathrm{T}_{\text {growth }}$ increases, in agreement with APT results.

\section{References:}

[1] P. Vennéguès et al., Atomic structure of pyramidal defects in Mg-doped GaN, Phy. Rev. B 68 (2003) 235214

[2] This work was performed on the nanocharacterization platform (PFNC) at Minatec Campus with support from the French RTB program. 


\title{
Application of Atom Probe Tomography for Engineering $\beta-\left(\mathrm{Al}_{\mathrm{x}} \mathrm{Ga}_{1-\mathrm{x}}\right)_{2} \mathrm{O}_{3}$, a Wide Band Gap Semiconductor
}

\author{
Baishakhi Mazumder ${ }^{1}$, Jung-Hun Seo ${ }^{1}$, and Jonathan Poplawsky ${ }^{2}$ \\ Corresponding Author: baishakh@buffalo.edu \\ 1. Department of Materials Design and Innovation, University at Buffalo, Buffalo, USA. \\ 2. Center for Nanophase Materials Sciences, Oak Ridge National Laboratory, Oak Ridge, USA.
}

Wide band gap semiconductor devices based on $\mathrm{Ga}_{2} \mathrm{O}_{3}$ are extensively overcoming limitations of Si based technologies, with increased current carrying capabilities, breakdown voltages and switching frequencies [1]. To broaden their application spectrum, it is imperative to widen the

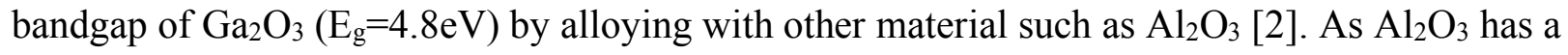
larger bandgap of $\sim 8.8 \mathrm{eV}$, the formation of $\beta-\left(\mathrm{Al}_{\mathrm{x}} \mathrm{Ga}_{1-\mathrm{x}}\right)_{2} \mathrm{O}_{3}$ alloy has a promising future in technology. However, the material properties of $\beta-\left(\mathrm{Al}_{\mathrm{x}} \mathrm{Ga}_{1-\mathrm{x}}\right)_{2} \mathrm{O}_{3}$ are still not well known, which inhibit the widened use and applications of this system. Understanding of crystal structure, phase stability and defects in $\beta-\left(\mathrm{Al}_{\mathrm{x}} \mathrm{Ga}_{1-\mathrm{x}}\right)_{2} \mathrm{O}_{3}$ during the process of formation is very critical from the material science point of view. In order to grow a stable material system, the doping stability, dopant distribution, interface properties, defect formation and $\mathrm{Ga} / \mathrm{O} / \mathrm{Al}$ segregation in this system needs to be better understood. In this study, we have employed Atom Probe Tomography due to its unique capabilities to provide compositional information at atomic scale, while transmission electron microscopy is used for crystal structure analysis.

$\beta-\mathrm{Ga}_{2} \mathrm{O}_{3}$ and $\beta-\left(\mathrm{Al}_{x} \mathrm{Ga}_{1-x}\right)_{2} \mathrm{O}_{3}$ epitaxial films grown on $\beta-\mathrm{Ga}_{2} \mathrm{O}_{3}$ substrates with different $\mathrm{Al}$ contents, and were analyzed via APT. An atom map showing the contacts, $\left(\mathrm{Al}_{x} \mathrm{Ga}_{1-x}\right)_{2} \mathrm{O}_{3}$, and $\mathrm{Ga}_{2} \mathrm{O}_{3}$ substrate is shown in Figure 1. Results show that with certain Al molar concentrations, the $\beta$ - $\left(\mathrm{Al}_{x} \mathrm{Ga}_{1-x}\right)_{2} \mathrm{O}_{3}$ phase is stable. Homogeneity or segregation of various dopants with different dopant concentrations have been verified. Crystal defects were studied in detail with different Al concentrations via TEM. Electrical data from these samples were measured to verify the device quality of the structure. The information found in this study will help in engineering and optimizing $\beta$ - $\left(\mathrm{Al}_{\mathrm{x}} \mathrm{Ga}_{1-\mathrm{x}}\right)_{2} \mathrm{O}_{3}$ with various $\mathrm{Al}$ composition for the next generation power electronics and optoelectronics.

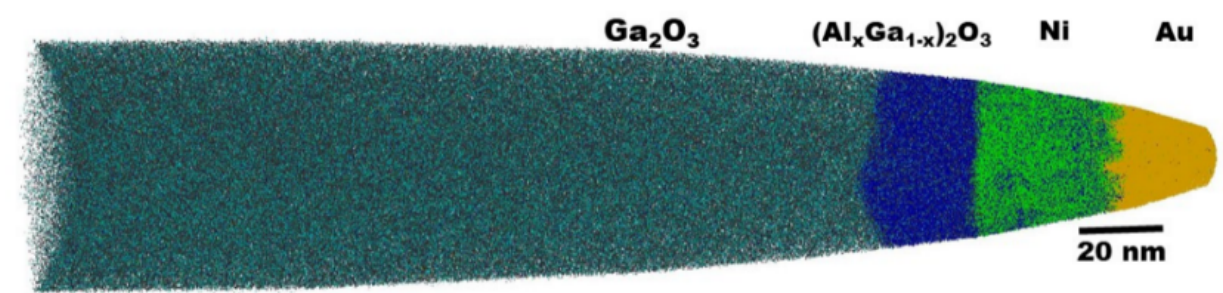

Figure $13 \mathrm{D}$ map of a $(\mathrm{AlxGa} 1-\mathrm{x})_{2} \mathrm{O}_{3} / \mathrm{Ga}_{2} \mathrm{O}_{3}$ field effect transistor showing all individual layers.

References:

[1] W. Mu et al.,RSC Adv. 7, (2017) 21815-21819.

[2] H. Peelaers and C. G. Van de Walle, Phys. Status Solidi B 252, (2015) 828-832.

[3] Authors acknowledge Prof. S. Rajan from Ohio State University for providing the sample shown in the figure. 


\section{APT and its Future in the Semiconductor Industry}

Paul van der Heide ${ }^{1}$, Claudia Fleischmann ${ }^{1}$, Davit Melkonyan ${ }^{1,2}$, Kristof Paredis ${ }^{1}$, Richard Morris $^{1}$, Janusz Bogdanowicz ${ }^{1}$, and Wilfried Vandervorst ${ }^{1,2}$

Corresponding Author: paul.vanderheide@imec.be

1. IMEC, Kapeldreef 75, 3001 Leuven, Belgium

2. Instituut voor Kern- en Stralingsfysica (IKS), KU Leuven, Celestijnenlaan 200D, 3001 Leuven, Belgium.

Since the turn of the $21^{\text {st }}$ century, Atom Probe Tomography (APT) has opened materials characterization possibilities beyond that previously imagined [1]. This is in part due to the introduction of laser induced field evaporation, as this has allowed the applicability of APT to be extended from zero/small bandgap materials to those with larger bandgaps. The semiconductor industry is another field that has experienced progress beyond that previously thought possible [2]. And with $14 \mathrm{~nm}$ logic devices now in production, Research and Development (R\&D) efforts are pushing for the ability to characterize next generation heterogeneous nano-structures in 3D to atomic resolution and with all atoms identifiable, a capability that, in principle, APT can provide.

APT can be thought of as a bridge between Transmission Electron Microscopy (TEM) and Secondary Ion Mass Spectrometry (SIMS). This is realized since the former provides atomic scale imaging capabilities to unparalleled spatial resolution, while the later provides elemental depth profiling capabilities to unparalleled detection limits, albeit over much larger areas. These capabilities along with the maturatization of TEM and SIMS have also resulted in TEM and SIMS being accepted as part of the analytical tool box routinely applied in both High-Volume manufacturing (HVM) and R\&D whereby reproducibility, accuracy, speed of analysis and sampling yield are key expectations. The same cannot be said for APT. This stems from ongoing issues with a) sampling yield, b) quantification, and c) image distortion.

This talk will convene with a quick chronological tour of the semiconductor industry, APT, SIMS and TEM. Financial drivers, as well as, what constitutes an analytical tool box pertinent to the semiconductor industry will then be touched upon. Following this, the primary issues suffered by APT when applied to the analysis of heterogeneous structures will be covered. Some potential solutions to image distortion issues will also be presented. With the resolution of the sampling yield, quantification, and image distortion issues, it is expected that APT will experience a further resurgence within this industry, with one example being the introduction of APT into the HVM failure analysis flows.

\section{References:}

[1] M.K. Miller, R.G. Forbes, Atom-Probe Tomography: The local electrode atom probe, Springer, New York, 2014

[2] http://www.economist.com/technology-quarterly/2016-03-12/after-moores-law 


\title{
Microstructural Characterization of Sulfurization Effects in $\mathrm{Cu}(\mathrm{In}, \mathrm{Ga}) \mathrm{Se}_{2}$ Thin Film Solar Cells
}

\author{
H. Aboulfadl ${ }^{1}$, J. Keller ${ }^{2}$, J. Larsen ${ }^{2}$, M. Sattari ${ }^{1}$, M. Edoff ${ }^{2}$, C. Platzer-Björkman ${ }^{2}$ \\ Corresponding Author: hisham.aboulfadl@chalmers.se \\ ${ }^{1 .}$ Department of Physics, Chalmers University of Technology, Göteborg, Sweden. \\ 2. Department of Engineering Sciences, Uppsala University, Uppsala, Sweden.
}

Photovoltaic thin film devices using copper indium gallium diselenide (CIGS) absorbers exhibit high conversion efficiencies reaching up to $22.6 \%$ [1], while having low cost and possible application to flexible substrates. One approach to improve efficiency is surface sulfurization of the absorber (p-type) before the deposition of the wide band gap layer (n-type). The incorporation of $\mathrm{S}$ atoms into the CIGS surface increases the band gap energy towards the heterojunction. This potentially increases the open-circuit voltage by a gradually lowered valence band maximum $[2,3]$, which acts as a barrier for holes and reduces the recombination at the pn-junction. Optimization of the solar cell performance via sulfurization requires an understanding of the $\mathrm{S}$ distribution and the quaternary phase stability. Changes in the sub-surface microstructure of the absorber after sulfurization treatment have been reported, such as finer grain sizes, as well as formation of mixed chalcopyrite crystals of $\mathrm{CuInSe} 2$ and $\mathrm{CuInS}_{2}$ phases, respectively [4].

In this work, we present high-resolution characterization of the sub-surface region for sulfurized CIGS absorbers via transmission electron microscopy, energy dispersive X-ray spectroscopy and atom probe tomography (APT). The study revealed inhomogeneous elemental concentrations within the sub-surface region showing a Ga-segregated and In-depleted zone. Furthermore, the APT analyses displayed preferential segregation of S along the grain boundaries of the absorber. The distribution of alkali metals, diffusing out from the glass substrate into the grain boundaries, is also investigated. The influence of the processing conditions and the implications of such results on the solar cell performance are discussed.

\section{$\underline{\text { References: }}$}

[1] P. Jackson, R. Wuerz, D. Hariskos, E. Lotter, W. Witte, M. Powalla, Effects of heavy alkali elements in $\mathrm{Cu}(\mathrm{In}, \mathrm{Ga}) \mathrm{Se} 2$ solar cells with efficiencies up to $22.6 \%$, Phys. Status Solidi - Rapid Res. Lett. 10 (2016) 583-586.

[2] T. Kobayashi, H. Yamaguchi, Z. Jehl Li Kao, H. Sugimoto, T. Kato, H. Hakuma, et al., Impacts of surface sulfurization on $\mathrm{Cu}$ (In1-x,Gax)Se2 thin-film solar cells, Prog. Photovolt Res. Appl. 23 (2015) 1367-1374.

[3] J. Keller, R. Schlesiger, I. Riedel, J. Parisi, G. Schmitz, A. Avellan, et al., Grain boundary investigations on sulfurized $\mathrm{Cu}(\mathrm{In}, \mathrm{Ga})(\mathrm{S}, \mathrm{Se}) 2$ solar cells using atom probe tomography, Sol. Energy Mater. Sol. Cells. 117 (2013) 592-598.

[4] P.C. Huang, C.C. Sung, J.H. Chen, R.C. Hsiao, C.Y. Hsu, Effect of selenization and sulfurization on the structure and performance of CIGS solar cell, J. Mater. Sci. Mater. Electron. 29 (2018) $1444-1450$. 


\section{Nanoscale Investigation of GeTe-Sb $2 \mathrm{Te}_{3}$ Superlattices}

Oana Cojocaru-Mirédin ${ }^{1}$, Henning Hollermann ${ }^{1}$, Antonio Mio ${ }^{1}$, and Matthias Wuttig ${ }^{1,2}$ *

Corresponding Author: cojocaru-miredin@physik.rwth-aachen.de

${ }^{1}$ I. Institute of Physics (IA), RWTH Aachen University, 52074 Aachen, Germany

${ }^{2}$ JARA-FIT Institute Green-IT, RWTH Aachen University and Forschungszentrum Jülich, 52056 Aachen, Germany

Interfacial Phase Change Materials (IPCM) based on $\mathrm{GeTe}_{-} \mathrm{Sb}_{2} \mathrm{Te}_{3}$ chalcogenide superlattices (CSL) are promising candidates for non-volatile random-access memory devices. They exhibit improved properties compared to its bulk analogues, such as multi-level switching [1], faster switching time [2], and reduced thermal conductivity [1]. However, their precise local composition at the nanometer scale remains the missing piece in the material design puzzle.

In the present work, correlative atom probe tomography-transmission electron microscopy studies were conducted for the first time to quantify the intermixing between GeTe and $\mathrm{Sb}_{2} \mathrm{Te}_{3}$ in highly-textured $\left[\mathrm{GeTe}-\mathrm{Sb}_{2} \mathrm{Te}_{3}\right]_{18}$ crystalline superlattices. These studies clearly proved the presence of $\mathrm{Sb}$ in $\mathrm{GeTe}$, but also of $\mathrm{Ge}$ in $\mathrm{Sb}_{2} \mathrm{Te}_{3}$. Moreover, a much stronger intermixing takes place at the grain boundaries, where the intermixing degree is strongly dependent on the disorientation angle; i.e. $\mathrm{Ge}_{2} \mathrm{Sb}_{2} \mathrm{Te}_{5}$ metastable phase nucleates at the boundary with a disorientation angle of $34^{\circ}$ (see Figure 1).

Finally, the impact of the chemical intermixing on the physical properties of IPCM will be discussed.

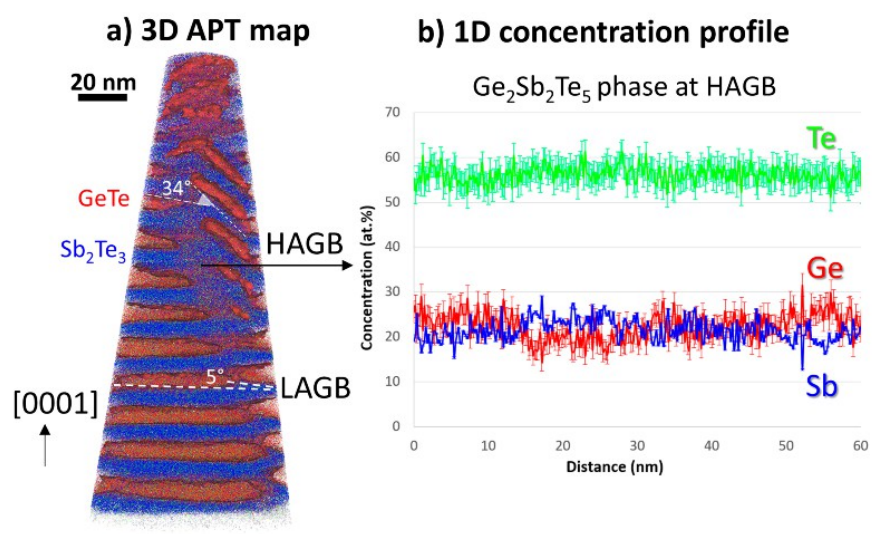

Figure 1: (a) 3D elemental maps showing the redistribution of $\mathrm{Sb}$ (blue), Te (light green), and Ge (red) in Sb2Te3-GeTe superlattices. (b) 1D concentration profile of $\mathrm{Sb}$ (blue), $\mathrm{Te}$ (light green), and Ge (red).

\section{References:}

[1] J. Momand, R. Wang, JE. Boscheker, MA. Verheijen, R. Calarco, B. Kooi, Formation of Two- and Three-Dimensionally Bonded Materials in the Case of Gete-Sb(2)Te(3) Superlattices, Nanoscale (2015), 7, 19136-43.

[2] B. Casarin, A. Caretta, J. Momand, B. Kooi, MA Verheijen, V. Bragalia, R. Calarco, M. Chukalina, X. Yu, J. Robertson, F. Lange, M. Wuttig, A. Redalli, E. VaresiF. Parmiagi, M. Malvestuto, Revisiting the Local Structure in Ge-Sb-Te Based Chalcogenide Superlattices, Sci. Rep. Nature (2016), 6, 22353. 


\section{Spatial Statistics as a Quantitative Tool to Study Morphology in Organic Light-Emitting Diodes}

Matthew B. Jaskot ${ }^{1}$, Andrew P. Proudian ${ }^{2}$, Helena-Nikolai Fujishin ${ }^{2}$, Galen Vincent ${ }^{2}$, David R. Diercks $^{1,3}$, Brian P. Gorman ${ }^{1,3}$, and Jeramy D. Zimmerman ${ }^{1,2}$

Corresponding Author: mjaskot@mymail.mines.edu

1. Materials Science Program, Colorado School of Mines (CSM), Golden, CO USA

2. Physics Department, CSM, Golden, CO USA

3. Department of Metallurgical and Materials Engineering, CSM, Golden, CO USA

The light emissive layer in an Organic Light-Emitting Diode (OLED) is typically composed of a blend of non-emissive host and emissive dopant molecules. The microstructure of this molecular blend can strongly affect properties, but is generally not considered because researchers have lacked methods for gathering relevant morphological information. For example, aggregation of light-emitting dopants in OLED emissive layers is expected to contribute to reductions in lightemission efficiency; however, the degree of molecular aggregation in these films is typically unknown. Atom Probe Tomography (APT) allows for the collection of morphological data with molecular discrimination previously unattainable in organic material systems;[1] however, spatially random data often appears clustered by visual inspection, so quantifying morphological trends in the APT data requires appropriate tools. Spatial statistics can be used to expose and quantify even weak morphological trends in APT data, and is therefore a powerful tool for the analysis of morphology in APT data sets.

Here, we report on the use of spatial statistics, such as Ripley's K-function, to quantify clustering of emissive dopants in OLED emissive layer films (Fig. 1). We find that more spatially random distributions of the emitter molecule in a wide-gap host are correlated with lower device drive voltage and higher brightness. By correlating microstructure with device performance, we can inform the design of more efficient materials, processing conditions, and device architectures.

(a)

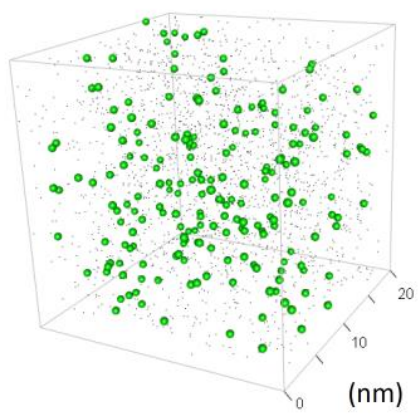

Figure 1 (a) APT pointcloud of emissive dopant molecules (green spheres) in a host matrix. (b) Ripley's $\mathrm{K}$ function for the pointcloud shown, indicating aggregation of dopant molecules.

\section{References:}

[1] A.P. Proudian, M.B. Jaskot, C. Lyiza, D. R. Diercks, B.P. Gorman, and J.D. Zimmerman, Effect of Diels-Alder Reaction in C6-Tetracene Photovoltaic Devices, NanoLetters 16 (2016), 6086-6091.

[2] The work was supported by the grant DE-SC0018021 funded by the U.S. Department of Energy, Office of Science Early Career program. 


\section{IVAS 101 or How to Make the Most Out of Your Dataset Using Your Favorite 3D Reconstruction Wizard?}

Hugues Francois-Saint-Cyr ${ }^{1}$

Corresponding Author: Hugues.FSC@,ametek.com

1. CAMECA Instruments, Inc., 5470 Nobel Drive, Madison, Wisconsin 53711, USA.

Despite the efforts and research deployed, especially for the last 20 years, in terms of specimen preparation and instrumentation to improve Atom Probe Tomography (APT) on materials, the atomic reconstruction in three dimensions (3D) remains the most uncertain step as it relies on many modeling assumptions and individual paths for data processing. This tutorial aims at providing a systematic methodology to treat APT data, hunting down as many clues as possible and exploring many options from the Integrated Visualization and Analysis Software (IVAS), leading to the clearest representation of the reconstruction, even before being able to see the volume in $3 \mathrm{D}$. This tutorial has been specially created for users new to the APT field or willing to accept that their current approach might be either rusty or incomplete. 


\title{
3D Reconstruction of Semiconductor Devices: Can IVAS Give Users What They Really Want?
}

\author{
Hugues Francois-Saint-Cyr ${ }^{1}$, Andy Martin² \\ Corresponding Author: Hugues.FSC@,ametek.com \\ 1. CAMECA Instruments, Inc., 5470 Nobel Drive, Madison, Wisconsin 53711, USA. \\ 2. GLOBALFOUNDRIES 400 Stone Break Rd Ext Malta, NY 12020 USA.
}

The semiconductor industry has historically been known to drive innovation and push manufacturers to reach higher limits in technological milestones. Although Atom Probe Tomography (APT) has become a must-have characterization technique in this field, IVAS, the Integrated Visualization and Analysis Software, has not been used to its fullest by APT users to provide its best outcome. This tutorial should be beneficial to users who want to present their reconstructed volumes under their best angle and explore the data analysis space to their advantages. Dr. Hugues Francois-Saint-Cyr (CAMECA) and Dr. Andy Martin (GLOBALFOUNDRIES) will share their views with users and help set the right expectations and moves on a 14-nm FinFET device which was graciously provided by researchers in the field of semiconductors [1].

[1] Pritesh Parikh et al., Three-Dimensional Nanoscale Mapping of State-of-the-Art Field-Effect Transistors (FinFETs), Microscopy and Microanalysis. 23 (2017), 916-925. 


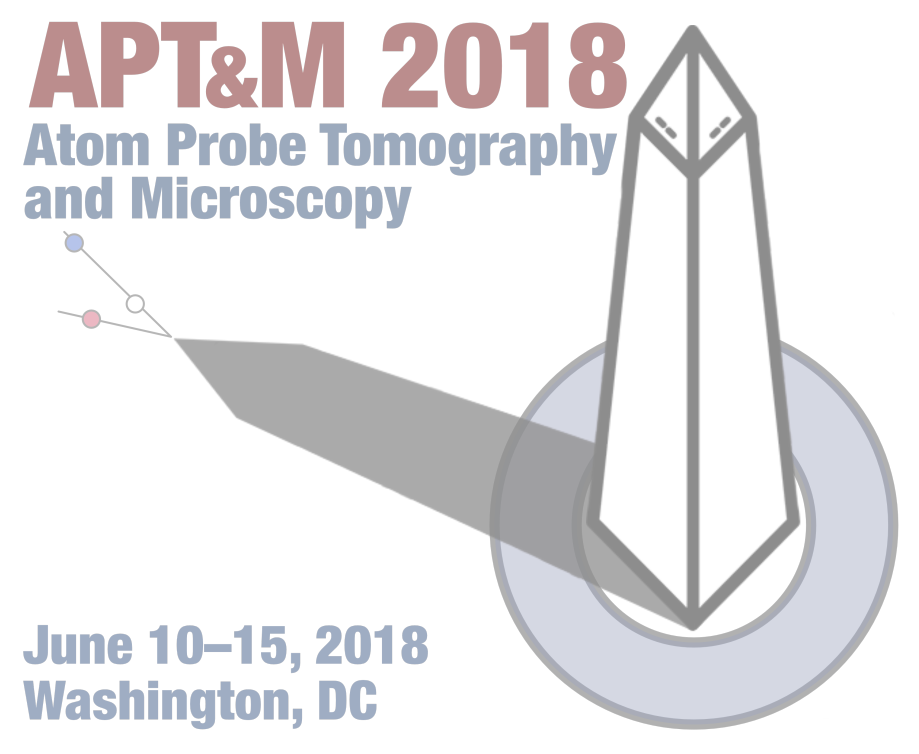

\section{Wednesday Morning}





\section{Wednesday Morning}

\begin{tabular}{|c|c|c|c|c|c|c|}
\hline 8:45 AM & & & & & & \\
\hline 9:00 AM & \multicolumn{6}{|c|}{$\begin{array}{l}\text { Plenary 3: Density Functional Modelling of Field Evaporation } \\
\qquad \begin{array}{l}\text { Christoph Freysoldt } \\
\text { (Green Auditorium) }\end{array}\end{array}$} \\
\hline \multicolumn{7}{|l|}{ 9:15 AM } \\
\hline \multicolumn{7}{|l|}{ 9:30 AM } \\
\hline & \multicolumn{6}{|c|}{ Break (9:30 to $10: 00$ AM) } \\
\hline 10:00 AM & \multirow{6}{*}{ 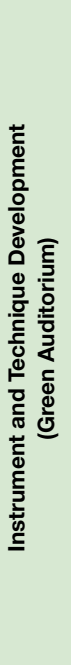 } & $\begin{array}{l}\text { Atom Probe Tomography } \\
\text { Development in ITEP } \\
\text { Anton Lukyanchuk }\end{array}$ & \multirow{6}{*}{ 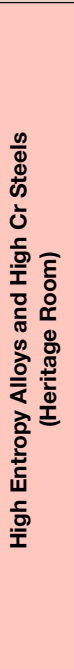 } & \multirow{2}{*}{$\begin{array}{l}\text { High Entropy Alloys to Compositionally } \\
\text { Complex Alloys: Relevance of Atom } \\
\text { Probe Tomography as a High-Throughput } \\
\text { Characterization Tool (Invited) } \\
\text { Konda Gokuldoss Pradeep }\end{array}$} & \multirow{6}{*}{ 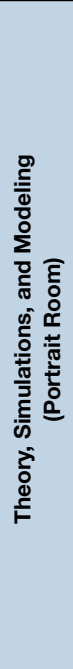 } & \multirow{2}{*}{$\begin{array}{c}\text { Theoretical analysis of dissociation } \\
\text { tracks observed in TAP (Invited) } \\
\text { Benoit Gervais }\end{array}$} \\
\hline 10:15 AM & & $\begin{array}{l}\text { M-TAP - The Modular Atom Probe } \\
\text { Guido Schmitz }\end{array}$ & & & & \\
\hline 10:30 AM & & $\begin{array}{c}\text { Evaluation of Magnification and Spatial } \\
\text { Resolution in Electrostatic Divergent } \\
\text { Lens with Extremely Short Focal Length } \\
\text { Saba Maeda }\end{array}$ & & $\begin{array}{c}\text { Investigation of Clusters in } \mathbf{A l}_{\mathbf{x}} \mathbf{C o C r F e N i} \\
\text { High Entropy Alloys } \\
\text { Talukder Alam }\end{array}$ & & $\begin{array}{c}\begin{array}{c}\text { Spatial and Compositional Biases } \\
\text { Introduced by Position Sensitive } \\
\text { Detection Systems in APT: A Simulation } \\
\text { Approach } \\
\text { Christian Bacchi }\end{array} \\
\end{array}$ \\
\hline 10:45 AM & & $\begin{array}{l}\text { 3D Imaging of Atom Probe Tip Shapes } \\
\text { with Atomic Force Microscopy } \\
\text { Claudia Fleischmann }\end{array}$ & & $\begin{array}{l}\text { Ordering in Al-Containing Refractory } \\
\text { High Entropy Alloys Analyzed by } \\
\text { Complemental Microstructure } \\
\text { Characterization Techniques } \\
\text { Sascha Seils }\end{array}$ & & $\begin{array}{c}\text { Evaporation Field and Surface Diffusion } \\
\text { on BCC Fe (001) and Cu (001) Under } \\
\text { Electric Field Using First-Principles } \\
\text { Calculation } \\
\text { Toshiharu Ohnuma } \\
\end{array}$ \\
\hline 11:00 AM & & $\begin{array}{l}\text { Development of a Superconducting } \\
\text { Detector for Atom Probe Tomography } \\
\text { Joseph Suttle }\end{array}$ & & $\begin{array}{c}\text { Modifying Transformation Pathways in } \\
\text { High Entropy Alloys or Complex } \\
\text { Concentrated Alloys via Thermo- } \\
\text { Mechanical Processing } \\
\text { Bharat Gwalani }\end{array}$ & & $\begin{array}{l}\text { Accurate and Efficient Field Evaporation } \\
\text { Modeling Using Level-Set Methods } \\
\text { Charles Fletcher }\end{array}$ \\
\hline 11:15 AM & & $\begin{array}{l}\text { The Laplace Project at MPIE: Modular } \\
\text { UHV Cryogenic Protocols for } \\
\text { Environmentally-Fragile Nanostructures } \\
\text { Leigh Stephenson }\end{array}$ & & $\begin{array}{c}\text { Effect of Carbon Addition in CrNbTaTiW } \\
\text { High Entropy Alloy: An Atom Probe Study } \\
\text { Deodatta Shinde }\end{array}$ & & $\begin{array}{c}\text { Atomistic-Modeling Based Simulation of } \\
\text { Field Evaporation Processes } \\
\text { Wolfgang Windl }\end{array}$ \\
\hline \multirow[t]{2}{*}{ 11:30 AM } & \multirow{2}{*}{\multicolumn{6}{|c|}{ Lunch / Poster Viewing (11.30 AM to 1.00 PM) }} \\
\hline & & & & & & \\
\hline
\end{tabular}





\title{
Density Functional Modelling of Field Evaporation
}

\author{
Christoph Freysoldt ${ }^{1}$, Arpit Mishra $^{1}$, Michael Ashton ${ }^{1}$, and Jörg Neugebauer ${ }^{1}$ \\ Corresponding Author: freysoldt@mpie.de \\ 1. Max-Planck-Institut für Eisenforschung GmbH, Max-Planck-Str. 1, 40237 Düsseldorf, \\ Germany.
}

At the heart of atom probe tomography (APT) is the evaporation of single atoms from a very sharp tip exposed to huge electric fields $\left(10^{11} \mathrm{~V} / \mathrm{m}\right)$. Field evaporation is a complex process in which not only the atom must break one or more bonds to its chemical environment, but the atom is also ionized at some point in the trajectory (or even multiple times) before it follows the electric-field lines away from the surface. The electric fields, in turn, depend on the local surface morphology. Commonly used geometric reconstruction algorithms for APT fail to take into account any details of the evaporation mechanism, such as differences in evaporation between chemical species or from different sites. Indeed, the atomistic details of field evaporation remain largely obscure, both qualitatively and quantitatively. This significantly hinders the development of improved reconstruction algorithms beyond the state of the art.

Density-functional theory (DFT) may provide important insights. DFT has been shown to reproduce barriers and critical fields in reasonable agreement with experiment for adatoms on $\mathrm{Al}$ surfaces [1]. Unfortunately, this seminal study has not been followed by systematic investigations for other sites or other materials because common computer codes must be adapted to account for large electric fields. We have therefore implemented an efficient approach to incorporate electric fields for slab models of the surface into our DFT code SPHInX [2].

To shed light on the factors influencing field evaporation, we study desorption from various sites (ad-atom, steps, corners) on prototypical metal surfaces (Al, W). From these calculations, we obtain the field-dependent evaporation barriers, which may be used in subsequent APT simulations. We further compare the outcome of explicit-field calculations with the prediction of traditional models based on zero-field work functions and binding energies. We also find evidence for the roll-over effect at steps, that has been proposed to explain orientational bias in evaporation trajectories.

\section{$\underline{\text { References: }}$}

[1] G. Sanchez, A.Y. Lozovoi, A. Alavi, Mol. Phys. 102 (2004), 1045-1055.

[2] S. Boeck et al, Comp. Phys. Commun. 182 (2011), 543-554. 


\section{Atom Probe Tomography Development in ITEP}

Anton A. Lukyanchuk ${ }^{1}$, Sergey V. Rogozhkin ${ }^{1}$, Andrey A. Aleev ${ }^{1}$, Anton S. Shutov ${ }^{1}$, Oleg A. Raznitsyn $^{1}$

\section{Corresponding Author: Anton.Lukyanchuk@itep.ru}

1. Institute for Theoretical and Experimental Physics named by A.I. Alikhanov of National Research Centre "Kurchatov Institute", Moscow, Russia

Atom-probe tomography (APT) has been applying in ITEP (Moscow) to analysis of radiation effects in structural materials more than 10 years [1-3]. This report describes the design of the Atom Probe Prototype with femtosecond Laser Evaporation and a position-sensitive detector based on delay lines for tomographic (3D) analysis of the chemical composition of materials (APPLE-3D) which has been assembled at ITEP [3]. The prototype features were demonstrated on tungsten, titanium and aluminum alloys, Fe-Cr based alloys and oxide dispersion strengthened steels. In these materials were studied oxide nano-particles, Cottrell atmospheres, GuinierPreston zones, carbonitride pre-precipitates. Mass and spatial resolutions, data collection efficiency and data collection characterization of APPLE-3D are presented. In addition, the functional of the developed software for data-mining is described.

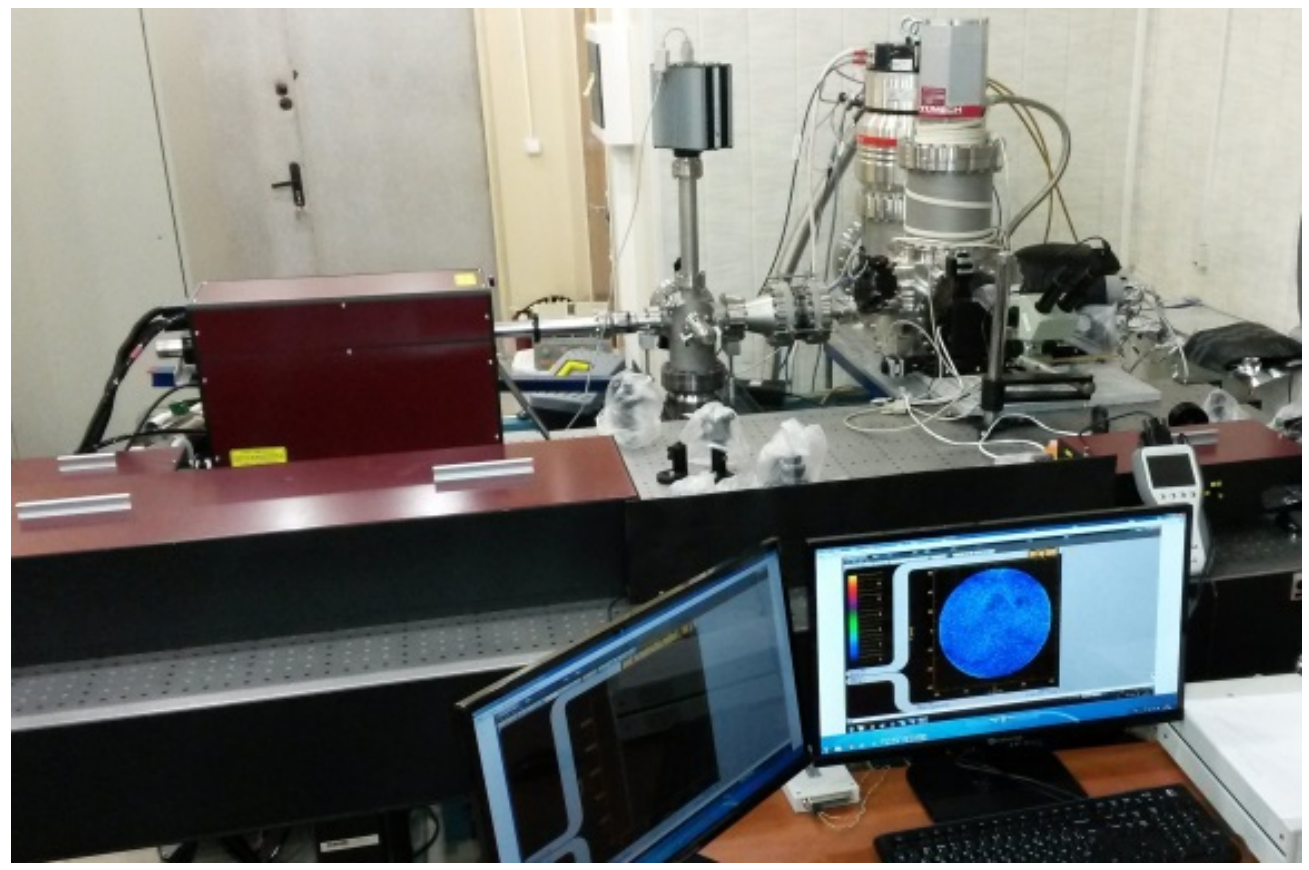

Figure 1 Atom Probe Prototype with femtosecond Laser Evaporation APPLE-3D.

\section{References:}

[1] Rogozhkin S.V. et al. The Physics of Metals and Metallography 108 (2009) 579-585.

[2] Rogozhkin S. et al. J. Nucl. Mat. 409 (2011) 94-99.

[3] Rogozhkin S. et al. MRS Advances 2 (2017) 1143-1155.

[4] Rogozhkin S.V., Aleev A.A., Lukyanchuk A.A., et al. Instrum. Exp. Tech. 60 (2017) 428-433.

[5] The work in the part of Fe-Cr based alloys and oxide dispersion strengthened steels was supported by Russian Science Foundation (grant 17-19-01696) 


\section{M-TAP - The Modular Atom Probe}

Patrick Stender, Jonas Ott, Irdi Balla, Guido Schmitz

Corresponding Author: guido.schmitz@imw.uni-stuttgart.de

Institute of Materials Science, University of Stuttgart, Stuttgart, Germany

A new hybrid instrument joining a dual beam scanning microscope (FIB) and a miniaturized atom probe chamber has been designed and assembled at the University of Stuttgart [1]. The heart of the instrument is a small shuttle that comprises the sample stage with cooling, high voltage supply and a piezo driven extraction electrode (see Fig. 1). The shuttle can easily move within seconds between a cryo-stage inside the FIB and a dedicated piezo-driven mount in the atom probe chamber. A sequence of needles may be cut directly into planar wafer materials. Imaged by the scanning microscope, the extraction electrode is exactly positioned in front of these tips, and the coordinates of the different positions stored in computer memory. After transporting the shuttle into the atom probe, the predefined positions are re-addressed by computer control which allows automated measurement of a serious of samples. Due to the fast transfer, the instrument is especially suited to measure cryo-prepared samples containing liquid or soft matter components.

The talk reports the concept of the new instrument and the used real time measurement and offline data-processing software. Example measurements and performance data are presented. The capability of measuring liquids is demonstrated by needles of frozen honey and for comparison, purified scientific sugar solutions in water. The corresponding mass spectra (see example in fig. 1) and volume reconstruction are presented and compared. As typical for the field desorption of soft matter, the mass spectra are complex containing large molecular fractions. These are characteristically linked to the five and six fold carbon rings of the sugar molecules. The dependence of the observed molecular fractions on the supplied fields are discussed.

[1] Support by the German ministry of education of science is gratefully acknowledged (FZ 03V0756).
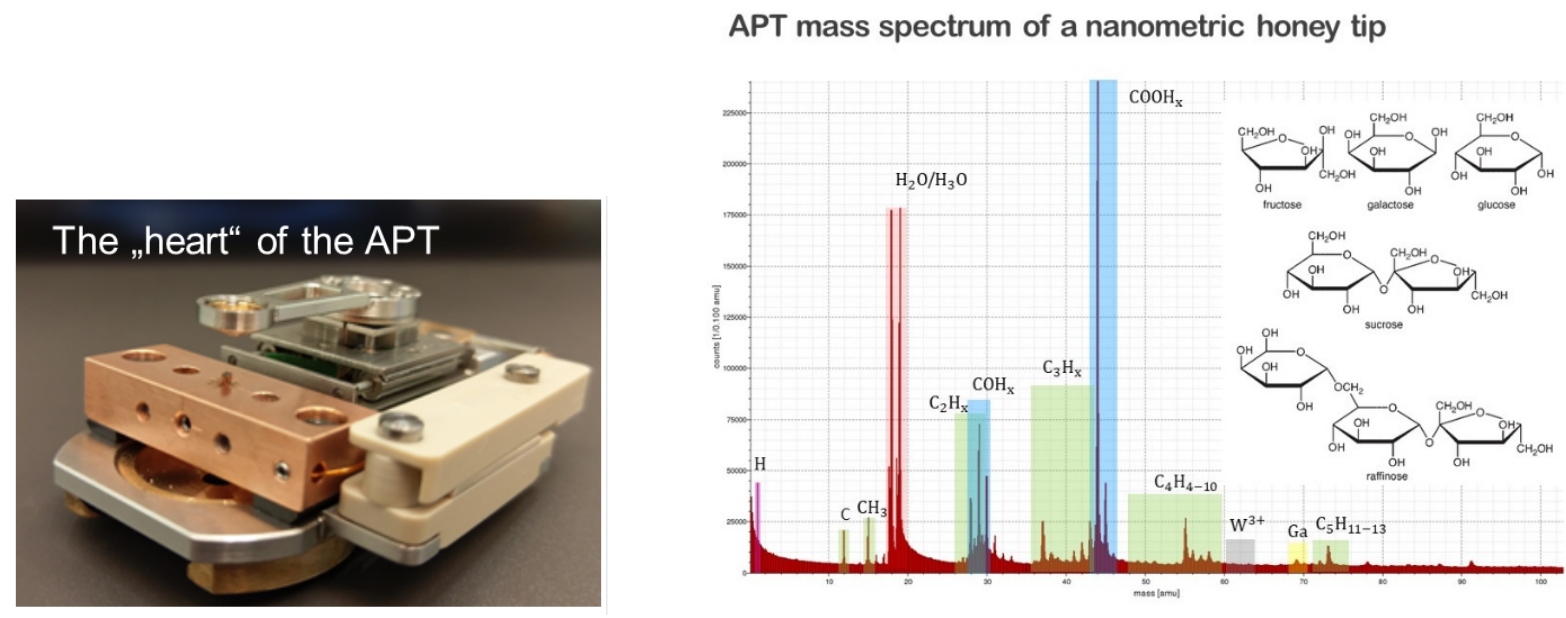

Jonas Ott, Univ. Stuttgart, Aug. 2017

Figure 1 The atom probe shuttle on a footprint of $4 \times 6 \mathrm{~cm}^{2}$ (left) and field desorption spectrum of a natural honey tip (right). 


\section{Evaluation of Magnification and Spatial Resolution in Electrostatic Divergent Lens with Extremely Short Focal Length}

Saba Maeda ${ }^{1}$ and Yasuhito Gotoh ${ }^{1}$

Corresponding Author: maeda.saba.88u@st.kyoto-u.ac.jp

1. Department of Electronic Science and Engineering, Kyoto University, Kyoto, Japan

Atom probe tomography (APT) requires a needle-shaped specimen in order to magnify the surface structure. This limitation is one of the major problems of the APT, especially when the specimen is a large organic molecule. Nishikawa and Kimoto proposed the scanning atom probe for planar samples, but they expected presence of tiny protrusions on the specimen [1]. We have proposed use of a single aperture electrostatic lens with an extremely small electrode spacing $d$, in order to magnify the molecular position on a flat surface with a large magnification [2]. A schematic drawing is shown in Fig. 1; red and blue lines represent the trajectories and equipotential lines. The focal length $f$ of the accelerating single aperture lens is given by $f=-4 d$, which can be obtained by integrating a paraxial ray equation [3], the magnification of the lens $M$ is given by $M=L / 4 d$, where $L$ is the length between the lens and the detector. Therefore, extremely large $M$ will be expected with an extremely small $d$. In this study, properties of the present lens system, $M$ and spatial resolution $\delta$ were numerically evaluated as a function of $d$.

Fig. 2 shows the evaluated $M$ and $\delta$ as a function of $d$. The voltage between the electrodes was kept constant value of $100 \mathrm{~V}$. It was found that the numerically obtained $M_{\mathrm{n}}$ agrees well with the analytically derived value $M_{\mathrm{a}}=L / 4 d$ with $d$ between $70 \mathrm{~nm}$ and $300 \mathrm{~nm}$. As $d$ became smaller, $M_{\mathrm{n}}$ showed large deviation from the $M_{\mathrm{a}}$. However, a large $M$ of $5 \times 10^{5}$ was achieved. The reason for the deviation is because the reduction of the electric field with a decrease in $d$ saturated when the $d$ is too small. Fig. 2 also shows the spatial resolution $\delta$ calculated by $\delta=2 r_{\text {dif }} / M$, where $r_{\text {dif }}$ is the difference of the arrival point between the charged particles with and without initial lateral velocity. The smallest value of $\delta$ reached $3 \mathrm{~nm}$. Improvement of $\delta$ is expected by a smaller aperture that can obtain higher magnification.

\section{$\underline{\text { References: }}$}

[1] O. Nishikawa, M. Kimoto, Appl. Surf. Sci. 76/77 (1994) 424-430.

[2] Y. Gotoh, S. Maeda, and H. Tsuji, Technical Digest of the $30^{\text {th }}$ International Vacuum Nanoelectronics Conference, Regensburg, Germany, July 10-13, 2017, pp. 248-249.

[3] R. G. Wilson and G. R. Brewer, Ion Beams with Applications to Ion implantation, Krieger, Malabar, 1979.

The present study was partially supported by JSPS KAKENHI \#16K13685.

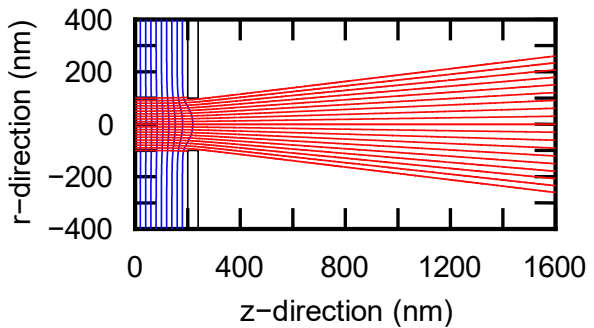

Figure 1. Trajectories of the charged particles with electrostatic divergent lens [2]

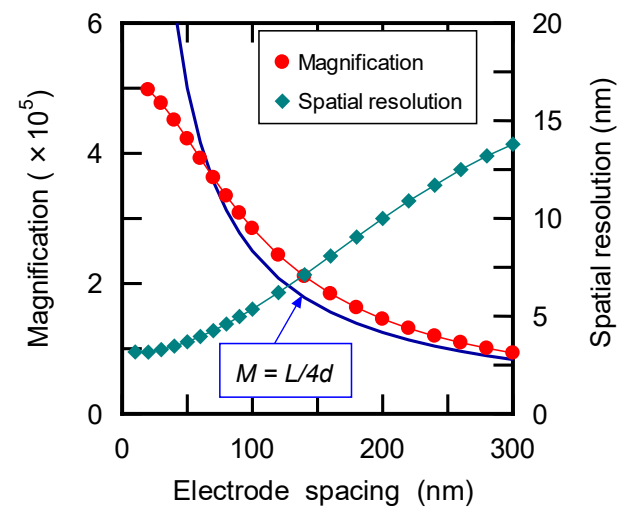

Figure 2. Dependence of the magnification and the spatial resolution on the electrode spacing 


\section{D Imaging of Atom Probe Tip Shapes with Atomic Force Microscopy}

C. Fleischmann ${ }^{1}$, K. Paredis ${ }^{1}$, D. Melkonyan ${ }^{1,2}$, Jonathan Op de Beeck ${ }^{1,2}$, J. Bogdanowicz ${ }^{1}$, R. J. H. Morris ${ }^{1}$, R. Cuduvally ${ }^{1,2}$, W. Vandervorst ${ }^{1,2}$

Corresponding Author: fleischm@imec.be

1. imec, Kapeldreef 75, 3001 Heverlee, Belgium

2. Instituut voor Kern- en Stralingsfysica, KU Leuven, Celestijnenlaan 200D, 3001 Leuven, Belgium.

Accuracy in atom probe (APT) data reconstruction for complex systems undeniably demands knowledge on the real APT tip shape and its evolution. The tip shape maliciously determines the electric field distribution at its apex, and consequently dictates the ion trajectories, i.e. the image formation and magnification in APT. In this context, atomic force microscopy (AFM) has been recently ${ }^{1}$ shown to be a promising alternative to transmission electron microscopy (TEM) ${ }^{2}$ for high resolution, 3D imaging of the APT tip shape. As such, AFM yields direct quantitative height information (topography), suffers less from carbon contamination, while being UHV compatible, relatively cheap and a readily accessible tool.

Relying on three different cases, we will demonstrate that AFM can yield easy and fast access to an APT tip shape. Using a virgin, pre-sharpened silicon microtip, we will allude to the method to overcome the challenge of aligning two very sharp, high aspect ratio tips. The potential of AFM will be subsequently demonstrated on two cases in which the APT tip shows (severe) aberrations from an hemispherical end-shape due to either non-uniform laser light absorption (Figure 1) or the presence of two different materials. Having proven the viability of AFM-based tip shape analysis, our method presents a new and exciting pathway for future tip shape imaging and hybrid-metrology in atom probe applicable to all material systems. The high resolution, quantitative 3D shape information forms a cornerstone for understanding physical mechanisms in laser-assisted APT and may pave the way towards improved data reconstruction algorithms.

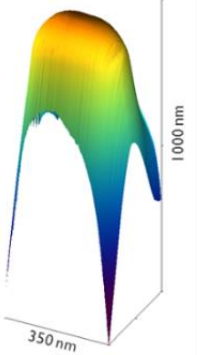

(a)

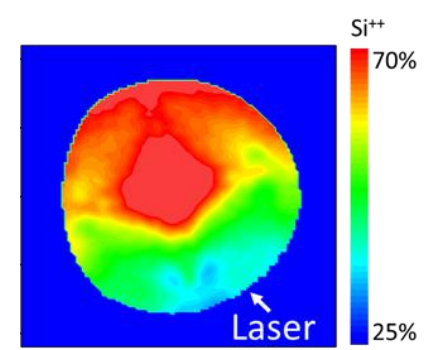

(b)

Figure 1 (a) 3D AFM image and (b) corresponding spatially resolved $\mathrm{Si}^{++}$concentration over the APT tip surface reflecting the difference in field strength originating from the shape asymmetry as seen in (a).

$\underline{\text { References: }}$

[1] C. Fleischmann, K. Paredis, D. Melkonyan, W. Vandervorst, Revealing the 3-dimensional shape of atom probe tips by atomic force microscopy, submitted to Ultramicroscopy (2017)

[2] D. Haley, T. Petersen, S.P. Ringer, G.D.W. Smith, Atom probe trajectory mapping using experimental tip shape measurements, Journal of Microscopy, 244, (2011), 170-180. 


\section{Development of a Superconducting Detector for Atom Probe Tomography}

Joseph Suttle ${ }^{1}$, Thomas F Kelly ${ }^{2}$, and Robert McDermott ${ }^{1}$

Corresponding Author: Suttle@,wisc.edu

1. University of Wisconsin-Madison, Department of Physics, Madison, WI 53703 USA

2. Cameca Instruments, Inc., 5500 Nobel Drive, Madison, WI 53711 USA

Many of the shortcomings of Atom Probe Tomography stem from deficiencies in coupled delay line anode-microchannel plate (MCP) detector. We have developed a superconducting detector with the potential to address these shortcomings. Kinetic energy from field evaporated ions break up Cooper pairs in a current biased superconducting microstrip. This generates a normal zone and an easily measureable pair of voltage pulses. We will report on our ongoing efforts to measure the performance metrics (including detection efficiency and temporal/spatial resolution) of these superconducting detectors in a field ion microscope.

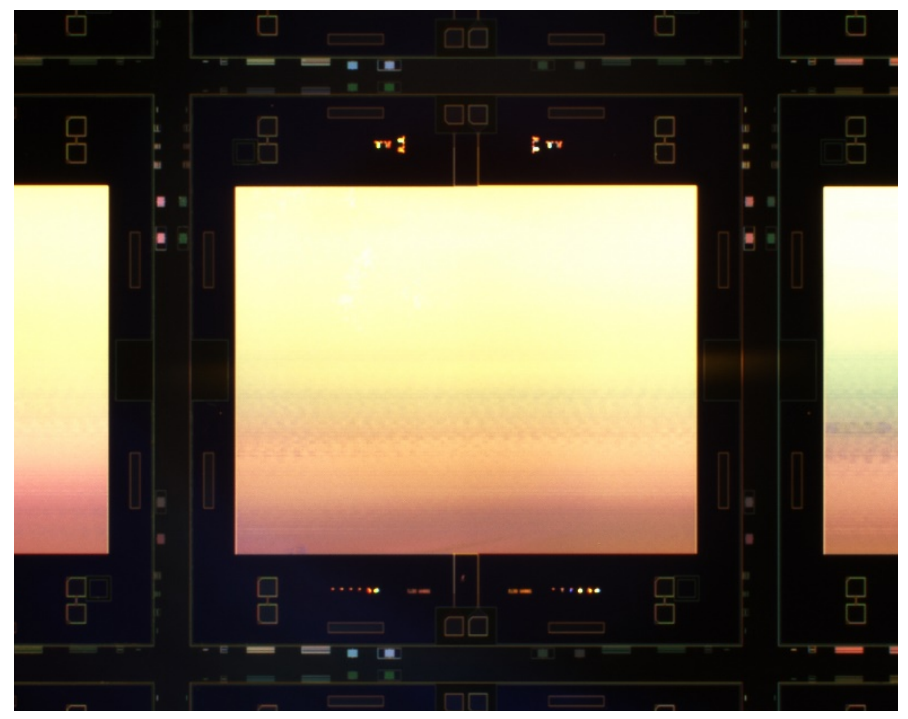

Figure 1: Micrograph of Prototype Detectors 


\section{The Laplace Project@MPIE: modular UHV cryogenic protocols for environmentally-fragile nanostructures}

Leigh Stephenson $^{1}$, Andrew Breen ${ }^{1}$, Isabelle Mouton ${ }^{1}$, Yanhong Chang ${ }^{1}$, Ann-Katrin Rusitzka ${ }^{1}$, Agnieszka Szczpaniak ${ }^{1}$, Uwe Tezins ${ }^{1}$, Andreas Sturm ${ }^{1}$, Thomas Kelly ${ }^{2}$, Dierk Raabe ${ }^{1}$, Baptiste Gault $^{1}$

Corresponding Author: b.gault@mpie.de

1. Max Planck Institute für Eisenforschung, Düsseldorf, Germany.

2. Cameca Instruments, Wisconsin, U.S.A.

Atom probe is the essential characterization tool for chemically-sensitive investigations of a material's nanostructure, but its data is only valid after answering the challenges presented by preparing and preserving a desired state in an atom probe sample. This is important for all experiments, but some materials problems require significantly more isolation from the atmosphere or from room temperature. In collaboration with hardware suppliers, we have developed the necessary instrumentation and the UHV cryogenic transfer protocols.

We use two Ferrovac VSN40S suitcases (now commercially available through Cameca) modified to accept standard Cameca atom probe pucks. Each suitcase typically reaches a level of vacuum in the $10^{-9}-10^{-10}$ mbar range and can be cooled to approx. $-184{ }^{\circ} \mathrm{C}$ with liquid $\mathrm{N}_{2}$. Our two UV laser-assisted atom probes (LEAP 5000XS/XR) are equipped with fast-docking suitcase stations (connection time $\approx 15-20$ minutes) for direct transfers into the buffer chamber of the LEAP. Sample pucks are fitted with an insulating PEEK and a pre-cooled "piggyback" puck both ensure that the transfer from the suitcase into the analysis chamber is both rapid and with very limited undesired warming.

Three other platforms were or are being made to dock with the suitcases. Our xenon-plasma focused-ion beam microscope (FEI Helios) provides us with a unique capability for highthroughput APT sample preparation and, equipped with a solid-state cryo-cooled puck stage, the sample can be milled with less contamination and beam damage. For controlled processing of airsensitive materials and hydrogen gas charging, we developed an environmental reaction chamber (Microscopy Improvements/Cameca) and separate glovebox (Sylatec).

The impact of these protocols will be discussed with reference to various projects at MPIE, including projects considering H/D-charging of various metals, hydrides for energy storage, preparation of hydrated samples and biological samples. 


\title{
High Entropy Alloys to Compositionally Complex Alloys: Relevance of Atom Probe Tomography as a high-throughput Characterization Tool
}

\author{
Konda Gokuldoss, Pradeep ${ }^{1 *}$, Marshal Amalraj ${ }^{2}$, and Budaraju Srinivasa, Murty ${ }^{3}$ \\ Corresponding Author: kgprad@gmail.com; kgprad@iitm.ac.in \\ 1. Materials Chemistry, RWTH Aachen University, Aachen and Germany. \\ 2. Materials Chemistry, RWTH Aachen University, Aachen and Germany. \\ 3*. Indian Institute of Technology Madras, Chennai and India.
}

High entropy alloys (HEA) from its inception has attracted lot of attention owing to the idea of developing single phase solid solutions from multi-principal (usually $\geq 4$ ) constituents with individual concentrations varying between 5-35 at.\% [1]. The interest therefore lies in discovering novel alloys and exploring the physical and mechanical properties arising from multi-component solid solution strengthening. However, the HEA design as of today is very much empirical and hence limited number of single phase solid solution forming systems has been identified. In spite of the fact that the identified single phase solid solutions are metastable in nature, the inherent meta-stability can be utilized to enhance the tensile strength of the HE solid solution without compromising on ductility [2]. In addition, the use of non-equiatomic HEA design has been suggested which consistently delivers single and controlled dual-phase solid solutions over a wide range of compositions [3]. Considering the extensive composition space available for alloy design, combinatorial synthesis of HEAs are also utilized which requires high-throughput characterization techniques for rapid screening and identification of promising alloy compositions that exhibit outstanding properties [4]. In this context the role of atom probe tomography in high-throughput characterization be it,

i) Mechanically alloyed HEA powders and their compacted form [1, 5],

ii) Combinatorial sputtered HEA thin films [4],

iii) Alloys produced by conventional metallurgical casting methods [2, 3]

will be presented with particular emphasis on elemental partitioning, grain boundary segregation and precipitation effects that have contributed immensely for the design of structural and functionally activated HEAs exhibiting competing properties with the conventional materials.

\section{References:}

[1] K. G. Pradeep, N. Wanderka, P. Choi, J. Banhart, B.S. Murty, D. Raabe, Acta Mater. 61 (2013) 46964706.

[2] Z. Li, K. G. Pradeep, Y. Deng, D. Raabe, C. Tasan, Nature. 534 (2016) 227-230.

[3] K. G. Pradeep, C.C. Tasan, M.J. Yao, Y. Deng, H. Springer, D. Raabe, Mater. Sci. Eng. A 648 (2015), 183-192.

[4] A. Marshal, K. G. Pradeep, D. Music, S. Zafferer, P. S. De, J. M. Schneider, J. Alloys and compounds. 691 (2017) 693-689.

[5] M. Vaidya, K. G. Pradeep, B. S. Murty, G. Wilde, S. V. Divinski, Sci. Rep. 7 (2017) 12293. 


\section{Investigation of Clusters in AlxCoCrFeNi High Entropy Alloys}

Talukder Alam ${ }^{1}$, Bharat Gwalani ${ }^{1}$, and Rajarshi Banerjee ${ }^{1}$

Corresponding Author: talukder.alam@unt.edu

1. Department of Materials Science and Engineering, University of North Texas, 3940 N. Elm, Denton, TX 76207, USA.

Solid solution strengthening, within random solid solutions, is a fundamental strengthening mechanism in most alloy systems. While traditionally this strengthening mechanism was largely developed and applied to alloys with one principal component, this notion can be easily extended to high entropy alloys (HEAs). This new class of alloys typically consists of five or more principal components in near equi-atomic proportion. The high configurational entropy in these alloys often results in the stabilization of single concentrated solid solution phases. However, the competing effect of enthalpy of mixing of the constituent principal elements, introduces ordering (or mixing) and clustering (or phase separating) tendencies.

With the increased of $\mathrm{Al}$, sluggish grain growth kinetics and unusually high activation energies for grain growth is demonstrated in $\mathrm{Al}_{\mathrm{x}} \mathrm{CoCrFeNi}(\mathrm{x}=0.1 \& 0.3)$ after solution heat-treatment despite the absence of any secondary precipitates. Atom probe microscopy was utilized to study the clusters in the $\mathrm{Al}_{\mathrm{x}} \mathrm{CoCrFeNi}$. Our study by the in-built tools of IVAS software package indicate that while $\mathrm{Al}_{0.1} \mathrm{CoCrFeNi}$ alloy was found to be homogeneous in a highly cold worked and recrystallized condition, $\mathrm{Al}_{0.3} \mathrm{CoCrFeNi}$ matrix contained Al-rich clusters. These clusters are proposed to act as sites for dislocation pinning, and subsequently provide hindrance to grain growth through 'cluster drag' effect. Their composition and morphology are measured and reported here. The raw data is treated with other custom-built statistical tools to gain more insight into this clustering phenomena.

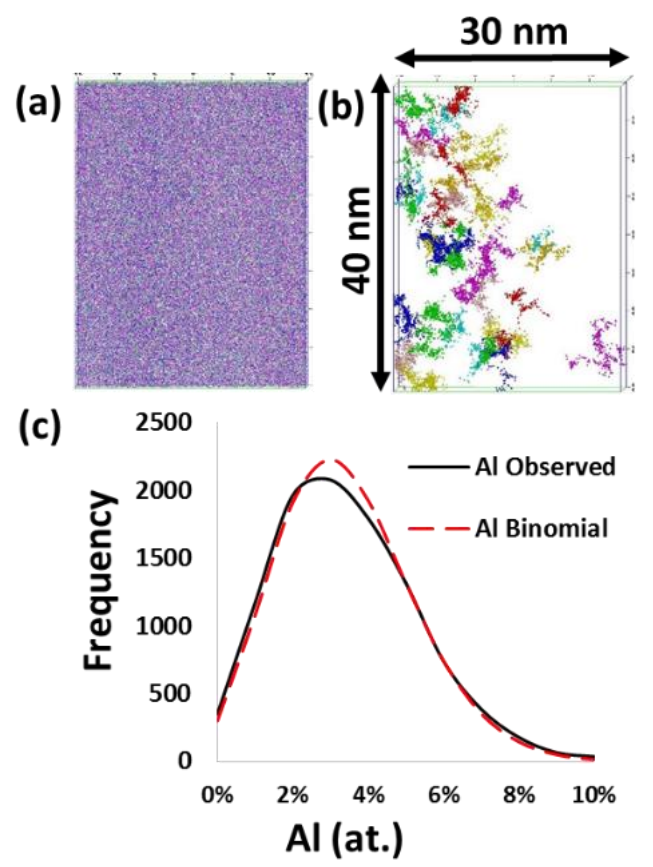

Figure 1 (a) Matrix of $\mathrm{Al}_{0.3} \mathrm{CoCrFeNi}$ (b) Al-rich clusters observed in (a) (c) Frequency distribution of $\mathrm{Al}$ ions depicting a deviation from random or binomial distribution. 


\section{Ordering in Al-containing Refractory High Entropy Alloys Analyzed by Complemental Microstructure Characterization Techniques}

Sascha Seils ${ }^{1,2}$, Hans Chen ${ }^{1}$, Dorothée Vinga Szabó ${ }^{1,2}$, Ian Harding ${ }^{3}$, Sharvan Kumar ${ }^{3}$, Sandra Kauffmann-Weiss ${ }^{4}$, Torben Boll ${ }^{1,2}$, Alexander Kauffmann ${ }^{1}$, and Martin Heilmaier ${ }^{1}$

Corresponding Author: sascha.seils@kit.edu

1. Institute for Applied Materials (IAM), Karlsruhe Institute of Technology (KIT), Karlsruhe, Germany

2. Karlsruhe Nano Micro Facility (KNMF), Karlsruhe Institute of Technology (KIT), EggensteinLeopoldshafen, Germany

3. School of Engineering, Brown University, Providence (RI), USA

4. Institute for Technical Physics (ITEP), Karlsruhe Institute of Technology (KIT), EggensteinLeopoldshafen, Germany

Al-containing refractory high entropy alloys (HEAs) combine metals with high melting points, low density and passivating oxide scales and are prospective for applications as high temperature materials. We present investigations on several derivatives of the $\mathrm{Nb}-\mathrm{Mo}-\mathrm{Cr}-\mathrm{Ti}-\mathrm{Al}$ systems that form single-phase microstructures after arc-melting and subsequent homogenization at $1200{ }^{\circ} \mathrm{C}$ up to $1500^{\circ} \mathrm{C}[1,2]$. Complemental X-ray diffraction (XRD), transmission electron microscopy (TEM) and atom probe tomography (APT) analyses were performed to confirm the chemical homogeneity of the alloys on macroscopic and nanoscopic length scales. In case of XRD analyses, a simple, disordered solid solution is identified, as expected for HEAs. In contrast, TEM selected area diffraction (SAD) reveals evidence for B2 ordering by the appearance of superlattice spots for respective zone axes. Furthermore, TEM bright field (BF) images exhibit thermal anti-phase domain boundaries (APB) which prove a disorder-order phase transformation during cooling. By means of APT, the local composition of the APB was quantified. Thus, elements contributing to the ordering could be identified. These APT results were used to further improve a crystallographic model for Rietveld refinement of the XRD patterns. From this we conclude that certain types and amounts of order cannot be revealed by means of XRD due to partial extinction of the different scattering cross section on each lattice site.
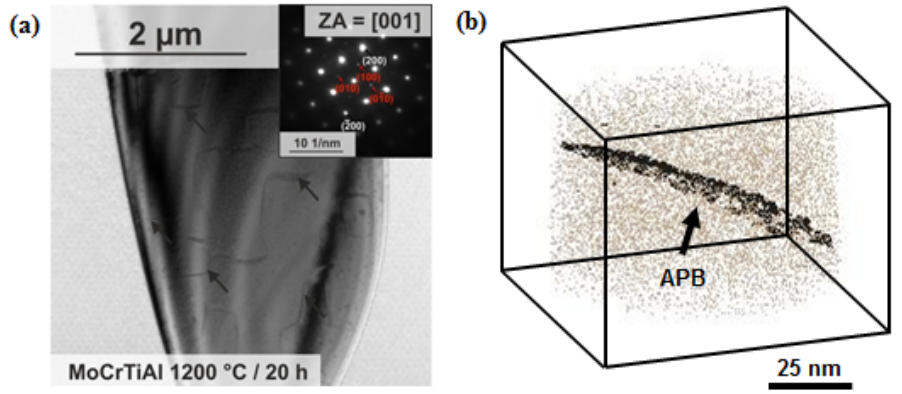

Figure 1 (a) APB of Mo-Cr-Ti-Al in TEM-BF images indicated by arrows. (b) Visualization of the APB in Mo-CrTi-Al by 30 at. $\%$ iso-concentration surfaces in an APT reconstruction showing only $\mathrm{Cr}$ ions.

\section{$\underline{\text { References: }}$}

[1] H. Chen et al., Microstructure and mechanical properties at elevated temperatures of a new Alcontaining refractory high-entropy alloy Nb-Mo-Cr-Ti-Al, Journal of Alloys and Compounds, 661 (2016), 206-215.

[2] H. Chen et al., Contribution of Lattice Distortion to Solid Solution Strengthening in a Series of Refractory High Entropy Alloys, Metallurgical and Materials Transactions A, (2017), 1-10. 


\section{Modifying Transformation Pathways in High Entropy Alloys or Complex Concentrated Alloys via Thermo-Mechanical Processing}

Bharat Gwalani ${ }^{1}$, Stephane Gorsse ${ }^{2}$, Deep Choudhuri ${ }^{1} \&$ R. Banerjee ${ }^{1 *}$

Corresponding Author: bharatgwalani@my.unt.edu

${ }^{1}$ Dept. of Materials Science and Engg., University of North Texas Denton, TX-76207, USA

${ }^{2}$ Bordeaux INP, ENSCBP, 33600 Pessac, France

High-entropy alloys (HEAs) have often been experimentally observed to contain single solid solution phase mainly due to slower kinetics constraining the precipitation of equilibrium second phase. Using $\mathrm{Al}_{0.3} \mathrm{CoCrFeNi}$ as a candidate HEA, it is demonstrated that the thermo-mechanical processing can strongly influence the transformation pathway for isothermal second phase precipitation. A more conventional thermo-mechanical treatment route, involving cold rolling, solutionization in the single fcc phase region, and age hardening at a lower temperature forms a homogeneous distribution of nano-scale $\mathrm{L}_{2}$ (gamma prime like) ordered precipitates within the $f c c$ matrix with discontinuous precipitation of ordered B2 phase on grain boundaries. In contrast, if the age hardening is done on the cold rolled material directly, there is a stark change in the microstructural feature. The pathway for microstructural evolution completely changes with concurrent recrystallization of the matrix $f c c$ grains and precipitation of B2, bcc sigma phases largely at the grain boundaries and nano-clustering within the matrix.

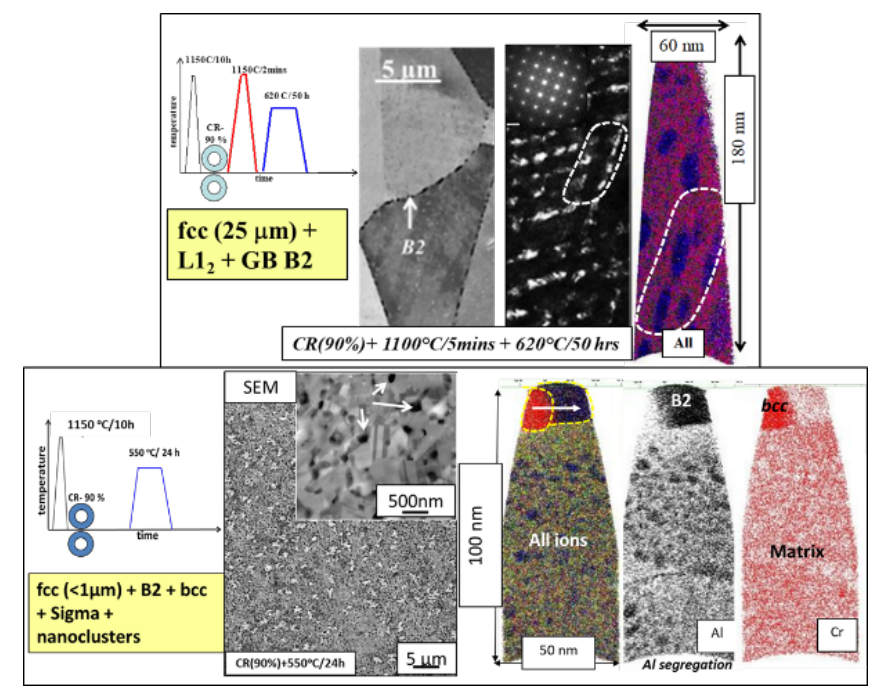

Figure 1 Microstructures in $\mathrm{Al}_{0.3} \mathrm{CoCrFeNi}$ alloy after different thermo-mechanical treatments.

Correlative microscopy technique using transmission electron microscopy and atom probe tomography clearly established the presence, distribution and compositional identify of various phases. The experimental observations have been rationalized via the competition between the thermodynamic driving force and activation barrier for second-phase nucleation in this alloy, coupled with the kinetics of the process. The microstructural variations, resulting from these dramatically different phase transformation pathways, can lead to some rather exceptional mechanical properties that can be varied over a large range even for a single $\mathrm{Al}_{0.3} \mathrm{CoCrFeNi} \mathrm{HEA}$ composition. 


\title{
Effect of Carbon Addition in CrNbTaTiW High Entropy Alloy: An Atom Probe Study
}

\author{
Deodatta Shinde ${ }^{1}$, Mattias Thuvander ${ }^{1}$ Stefan Fritze ${ }^{2}$, Paulius Malinovskis ${ }^{2}$, Erik Lewin ${ }^{2}$, Ulf \\ Jansson $^{2}$ and Krystyna Stiller ${ }^{1}$ \\ Corresponding Author: deodatta.shinde@chalmers.se \\ ${ }^{1}$ Department of Physics, Chalmers University of Technology, SE-412 96 Göteborg, Sweden \\ ${ }^{2}$ Department of Chemistry-Ångström, Uppsala University, SE-751 21 Uppsala, Sweden
}

The quest for new materials with superior physical and mechanical properties is at the heart of the materials science and metallurgy. This search for new and more efficient materials is never ending, eventually leading to new kind of information which is helpful to understand and improve the properties of materials. One such class of material is High Entropy Alloys (HEAs).

HEAs or multi-principal element alloys, is a relatively new concept compared to conventional alloys. HEAs have shown potential in a variety of applications and attractive properties like excellent specific strength, superior mechanical performance at high temperatures, exceptional ductility and fracture toughness at cryogenic temperatures [1, 2]. HEAs are comprised of five or more principal elements, which gives them an advantage of richness in design over conventional alloys where only one or two base elements are used (e.g. Fe in steels, $\mathrm{Ni}$ in Ni-based superalloys). However, there are several unanswered questions when it comes to the superior properties of HEAs and one of them is their microstructure and chemical composition at the atomic scale. Atom Probe Tomography (APT) is a good choice of technique to understand the structure and chemical composition of different elements in HEAs at atomic scale, which has been employed successfully to different HEAs previously [3, 4].

In this work, a DC magnetron sputtered, multi-component, nearly equimolar CrNbTaTiW alloy is studied using APT. The main objective of the work is to understand the microstructural changes at near atomic scale with the addition of carbon in this alloy, which is rarely studied as far as HEAs are concerned. With 8 at.\% $\mathrm{C}$, the $\mathrm{BCC}$ crystalline alloy becomes amorphous and hence challenging to characterize by traditional diffraction (X-ray, electron) and microscopy (TEM) techniques. Interestingly, carbon incorporation has improved the mechanical properties (hardness, elastic modulus) and corrosion resistance (with respect to hyper-duplex stainless steel) of the film. Preliminary APT analyses on CrNbTaTiW-C alloys showed carbidic features (less atomic density) in the 3D reconstructed volume. The detailed analysis and comparison of the data with and without carbon incorporation will also be presented.

\section{$\underline{\text { References: }}$}

1. D.B. Miracle, O.N. Senkov, A critical review of high entropy alloys and related concepts, Acta Materialia 122 (2017) 448

2. Y.F.Ye, Q.Wang, J.Lu, C.T.Liu, Y.Yang, High-entropy alloy: challenges and prospects, Materials Today 19, 6 (2016) 349

3. K.G.Pradeep, N.Wanderka, P.Choi, J.Banhart, B.S.Murty, D.Raabe, Atomic-scale compositional characterization of a nanocrystalline $\mathrm{AlCrCuFeNiZn}$ high-entropy alloy using atom probe tomography, Acta Materialia 61 (2013) 4696

4. K.E. Knipling, J.L. Tharpe, P.K. Liaw, Nanoscale Phase Separation in $\mathrm{Al}_{0.5} \mathrm{CoCrFeNi}(\mathrm{Cu}) \mathrm{High}$ Entropy Alloys as Studied by Atom Probe Tomography, Microsc. Microanal. 23, 1 (2017) 726 


\section{Theoretical Analysis of Dissociation Tracks Observed in TAP}

David Zanuttini ${ }^{1}$, Ivan Blum ${ }^{1}$, Lorenzo Rigutti ${ }^{1}$, Enrico di Russo ${ }^{1}$, François Vurpillot ${ }^{1}$, Julie Douady $^{2}$, Emmanuelle Jacquet ${ }^{2}$, Pierre-Matthieu Anglade ${ }^{2}$, Benoit Gervais ${ }^{2}$

\section{Corresponding Author: gervais@ganil.fr}

1. GPM, UMR CNRS 6634, Université et INSA de Rouen, Avenue de l'Université, BP 12, 76801 Saint Étienne du Rouvray, France

2. CIMAP, UMR CNRS 6252, ENSICAEN, CNRS, CEA/IRAMIS, Université de Caen, Boulevard Henri Becquerel, BP5133, 14070 CAEN cedex 05, France

The emission of single atomic ions in Tomographic Atom Probe (TAP) experiments is often associated to correlated emission leading to multiple events, which can be observed in a correlated event map, where the charge-over-mass ratio of the second fragment is plotted versus the charge-over-mass ratio of the first fragment. In such a map, some specific features appear as correlation tracks, which have been explained as a result of the in-flight dissociation of a parent molecular ion into charged fragments [1]. Though the process is clearly identified, the origin of the in-flight dissociation of molecular dications has only been recently investigated from a theoretical point of view $[2,3]$.

We present here a theoretical analysis of the fragmentation of dicationic dimers for materials often analyzed in TAP experiments: $\mathrm{ZnO}^{2+}, \mathrm{SiO}^{2+}, \mathrm{AlN}^{2+}, \mathrm{GaN}^{2+}$ emitted from corresponding metal-oxide or metal-nitride alloys and also $\mathrm{C}_{2}{ }^{2+}$ emitted from cemented tungsten carbide targets. Our analysis is based on the simulation of the ion dynamics in the field of a paraboloid tip for a model internal energy of the molecule deduced from accurate ab initio calculation of the electronic structure. We show that the spin-orbit coupling between electronic states of different spin symmetries is at the origin of the in-flight dissociation. We shall see however, that the specificity of each molecular dication, and in particular its electronic structure, is essential to analyze the experimental observations, because it conditions the dissociation dynamics and the nature of the dissociating products. Regarding the physical concepts for TAP operation, this dissociation process is a clear indication that excited states are formed during ion emission from the surface.

\section{References:}

[1] D. Saxey, Ultramicroscopy 111, (2011) p. 473.

[2] D. Zanuttini, et al., Phys. Rev. A 95, (2017) p. 061401(R).

[3] D. Zanuttini, et al., J. Chem. Phys. 147, (2017) p. 164301.

[4] We acknowledge the Labex EMC3 for financial support through the AQURATE project. 


\section{Spatial and Compositional biases introduced by Position Sensitive Detection Systems in APT: A Simulation Approach}

C. Bacchi ${ }^{1}$, G. Da Costa ${ }^{1}$, and F. Vurpillot ${ }^{1}$

Corresponding Author: bacchi.christian@ gmail.com

1. Normandie Université, UNIROUEN, INSA Rouen, CNRS, Groupe de Physique des Matériaux, 76000 Rouen, France.

Answering to the question "How reliable is an Atom Probe reconstructed dataset?" is far from being straightforward. From a theoretical point of view, the ultimate metrological performances of this nano-analysis instrument are extremely high: High magnification in 3D (up to the million), high spatial precision $(\sim 0.1 \mathrm{~nm})$, high detection efficiency (up to $80 \%$ of the atoms are collected by the system), equal sensitivity between species. Looking deeper into the data, spatial and compositional biases may degrade APT datasets due to reconstruction distortions, non-optimal analyzing conditions, or intrinsic lack of precision. These fundamental aspects have been studied in details theoretically to anticipate biases through numerical models [1]. However, one of the most important part of the APT operating process still has not been modeled in its entirety; the detection system.

In APT, position and elemental nature of atoms collected are deduced from impact positions and time-of-flights of charged particles emitted from the sample of interest. Position-sensitive detectors used in APT are generally extremely performant when the detector is submitted to individual ion hits, but significant technological bottlenecks have been pointed out when the device is stressed by simultaneous or quasi simultaneous multi-hit impacts $[2,3]$. The quality of the collected data is degraded by the failure of the detection system even in the analysis of standard materials, using perfect analyzing conditions.

This work introduces a systematic study of the impact of APT detection system on material analysis. For this purpose, a simulation tool have been developed to simulate the detection process; from atoms that are field ion emitted to time-of-flight and delay lines time measurements, passing by signals generation on detector outputs. Most of the detection system parameters have been taken into account, such as instrument dead time, timing resolution or even signal threshold. Signals have been reproduced in accordance with their shape, their amplitude distribution and their width distribution. Using this simulation tool, it is possible to evaluate position and composition relative errors caused by the detection system modeled.

\section{References:}

[1] F. Vurpillot, C. Oberdorfer, Modeling Atom Probe Tomography: A review, Ultramicroscopy 159 (2015): 202-216

[2] G. Da Costa, F. Vurpillot, A. Bostel, et al., Design of a delay-line position-sensitive detector with improved performance, Review of Scientific Instruments 76 (1) (2005): 013304

[3] G. Da Costa, et al., Advance in multi-hit detection and quantization in atom probe tomography, Review of Scientific Instruments 83(12) (2012): 123709. 


\section{Evaporation Field and Surface Diffusion on BCC Fe (001) and Cu (001) Under Electric Field Using First-Principles Calculation}

Toshiharu Ohnuma ${ }^{1}$

Corresponding Author: ohnuma@criepi.denken.or.jp

1. Material Science Research Laboratory, Central Research Institute of Electric Power Industry (CRIEPI), 2-6-1, Nagasaka, Yokosuka-shi, Kanagawa-ken, 240-01961 Japan

Atom probe tomography (APT) is a very powerful tool that can observe atomic position and chemical composition of materials three-dimensionally with resolution of sub-nanometer. In APT, atoms are field-evaporated by applying a high electric field to a needle-like sample [1]. Difference of evaporation field, surface diffusion and reconstruction of atoms during field evaporation causes artifacts $[2,3]$.

In recent years, effective screened medium (ESM) method has been developed, and it is possible to calculate efficiently the field evaporation and surface diffusion under applied electric field[4]. In this research, first-principles calculations of evaporation field and surface diffusion of Fe and $\mathrm{Cu}$ on $\mathrm{BCC} \mathrm{Fe} \mathrm{(001)} \mathrm{and} \mathrm{Cu}(001)$ when electric field are applied by ESM method were performed.

The calculation was done using Quantum Espresso package. A combination of the generalized gradient approximation (GGA) for the exchange and correlation energies and density functional theory were employed in this study[5]. We used a slab model based on a 4x4x4 BCC lattice with a total of 128 atoms in the supercell. Vacuum layers of $1.8 \mathrm{~nm}$ or more are formed on both sides in the $\mathrm{z}$ direction with $\mathrm{Fe}(001)$ or $\mathrm{Cu}(001)$.

Nudged Elastic Band (NEB) method was used to calculate the migration energy of surface diffusion by the electric field applied system. In order to calculate with constant electric field in the intermediate configuration of diffusion, it is necessary to control the Fermi level so that the electric field becomes constant (Constant- $\mu$ method), but it was difficult to converge by the conventional method. This can be solved efficiently by ESM method[7] The surface migration calculations of $\mathrm{Fe}$ and $\mathrm{Cu}$ on $\mathrm{BCC} \mathrm{Fe}(001)$ and $\mathrm{Cu}(001)$ were performed. As the electric field increased, the activation energy of diffusion decreased. The surface diffusion activation energies of the electric field of $28 \mathrm{~V} / \mathrm{nm}$ are about $40 \%$ of that of $0 \mathrm{~V} / \mathrm{nm}$ in case of $\mathrm{Fe}$ and $\mathrm{Cu}$ on $\mathrm{Fe}(001)$.

\section{References:}

[1] M. K. Miller and R. G. Forbes, Atom-Probe Tomography: The Local Electrode Atom Probe, Springer (2014).

[2] C. Oberdorfer, G. Schmitz, Microscopy and Microanalysis 17 (2011) 15

[3] M. D. Mulholland and D. N. Seidman, Microscopy and Microanalysis 17 (2011) 950.

[4] M. Otani and O. Sugino, Phys. Rev. B 73, (2006) 115407.

[5] P. Perdew, K. Burke, and M. Ernzerhof, Phys. Rev. Lett. 77, 3865 (1996).

[6] N. Bonnet, T. Morishita, O. Sugino, M. Otani, Phys. Rev. Lett. 109, (2012) 266101 


\title{
Accurate and Efficient Field Evaporation Modelling using Level-set Methods
}

\author{
Charles Fletcher ${ }^{1}$, Michael Moody ${ }^{1}$ and Daniel Haley ${ }^{1}$ \\ Corresponding Author: charles.fletcher@materials.ox.ac.uk \\ 1. Department of Materials, Parks Road, University of Oxford, Oxford, UK.
}

After 20 years the standard approach for APT reconstruction remains based on the pointprojection method [1]. While revolutionary at the time in allowing for near-lattice-level 3D imaging, its assumptions lead to major distortions surrounding anisotropies and inhomogeneous microstructures [1] - often the most interesting properties from a science and engineering perspective. Novel dynamic reconstruction methods have been proposed $[2,3]$ to minimise such distortions with their validity supported by simulation. However, no currently existing algorithm fully connects the problem of reconstruction with the modelling of tip evaporation. Current attempts are limited by either their lack of generality, high computational cost, or numerical stability issues during the reconstruction i.e. atomic and reverse-evaporation models.

Instead of atomic models, we consider the previously introduced level-set method [4] - a technique for accurately tracking the tip surface dynamics throughout the evaporation. Previous models approximated the tip surface evaporation rate, a function of the local electric field, as the mean level-set field curvature. We generalise this model by coupling the level-set to the exact electric field solution across the surface. Our approach implements Boundary Element Methods (BEM) that can efficiently solve the electric field at the surface. Field values in the surrounding space can also be calculated via BEM without an explicit mesh, allowing accurate projection of ions over their entire flight path to the detector. On integration with Electron Tomography data, our model could be applied to perform computationally feasible reconstructions that minimise the distortions currently seen in inhomogeneous materials.
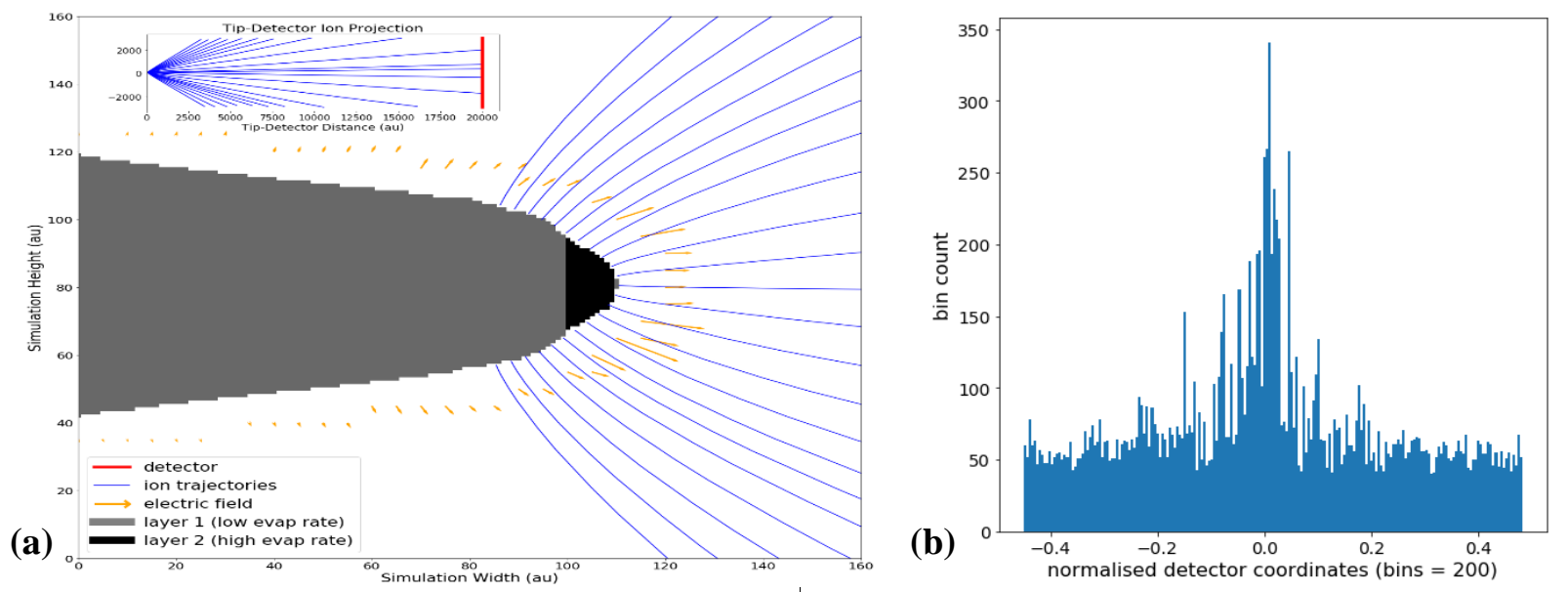

Figure 1 (a) 2D level-set multilayer simulation. (b) Corresponding detector hit map from the projection of 16000 ions.

$\underline{\text { References: }}$

[1] D. J. Larson et al. Atom Probe Tomography Spatial Reconstruction: Status and Directions, Current Opinion in Solid State \& Materials Science, 17 (2013) 236-247

[2] D Beinke et al., Towards an accurate volume reconstruction ..., Ultramicroscopy, 165 (2016) 34-41

[3] N. Rolland et al., A Meshless Algorithm to Model..., Microsc. Microanal. 21 (2015) 1649-1656

[4] D. Haley, M. Moody, G. Smith, Level Set Methods for..., Microsc. Microanal, 19 (2013), 1709-1717 


\title{
Atomistic-Modeling Based Simulation of Field Evaporation Processes
}

\author{
Wolfgang Windl ${ }^{1}{ }$ Christian Oberdorfer ${ }^{1}$, Travis Withrow ${ }^{1}$, Iman Ghamarian ${ }^{2}$, and Emmanuelle \\ Marquis $^{2}$ \\ Corresponding Author: windl.1@,osu.edu \\ 1. Department of Materials Science and Engineering, The Ohio State University, Columbus, \\ USA. \\ 2. Department of Materials Science and Engineering, University of Michigan, Ann Arbor, USA.
}

Atom Probe Tomography (APT) has established itself as an important analysis technique in materials science to explore the three-dimensional (3D) composition of complex nano-scaled samples. However, one of the major factors limiting the impact of atom probe tomography on advancing the understanding of materials microstructures is the lack of a robust theoretical structure justifying the reconstructed images and chemical analyses. The fact that the evaporated structure is only known from the reconstruction and thus depends on the underlying theoretical assumption makes a systematic quantification of chemical and spatial errors and uncertainties in general very hard to impossible. In order to overcome these limitations and develop a more rigorous modeling framework, we have combined the versatile state-of-the-art APT simulation approach introduced in the TAPSIM package [1] with density-functional theory calculations and molecular dynamics (MD) simulations, resulting in a tool that is able to (a) simulate the irregular evaporation sequence from structurally relaxed emitter structures; (b) capture artifacts and features from local variations in evaporation fields [2]; (c) determine alloying effects on evaporation fields which can have a very large effect; and (d) capture dynamic effects and postevaporation relaxation and reconstruction. In this presentation we will apply this approach to a number of elemental solids and alloys with and without microstructural features and discuss observed artifacts that can only be understood when a more complete physical picture is included in the simulation.

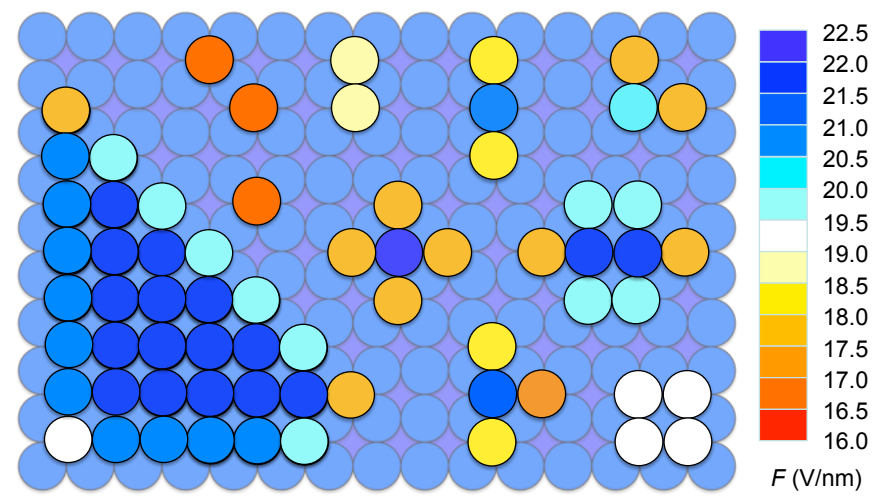

Figure 1: Evaporation fields $F$ for different surface features for the (001) surface of elemental single-crystalline $\mathrm{Al}$, calculated from density functional theory. Evaporation field values scale approximately with the number of bonds, resulting in the probability of an atom to evaporate to not only depend on the local electric field, but also on its zerobarrier evaporation field value.

\section{References:}

[1] C. Oberdorfer, S. M. Eich, and G. Schmitz. "A full- scale simulation approach for atom probe tomography," Ultramicroscopy 128, 55 (2013).

[2] L Yao, T Withrow, OD Restrepo, W Windl, EA Marquis, "Effects of the local structure dependence of evaporation fields on field evaporation behavior," Applied Physics Letters 107, 241602 (2015). 



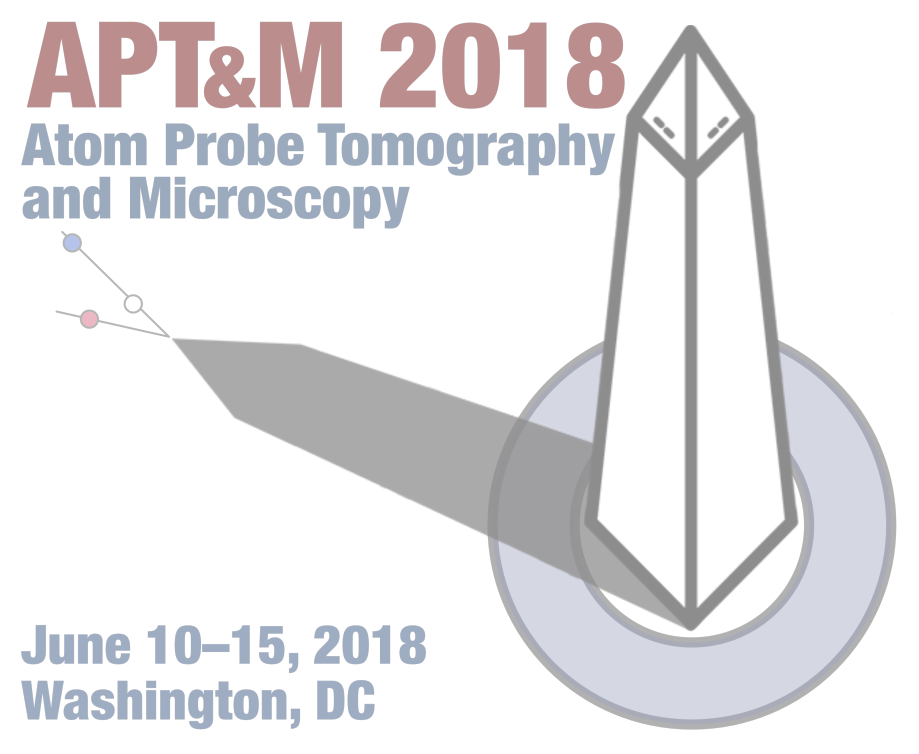

Wednesday Afternoon 

Wednesday Afternoon

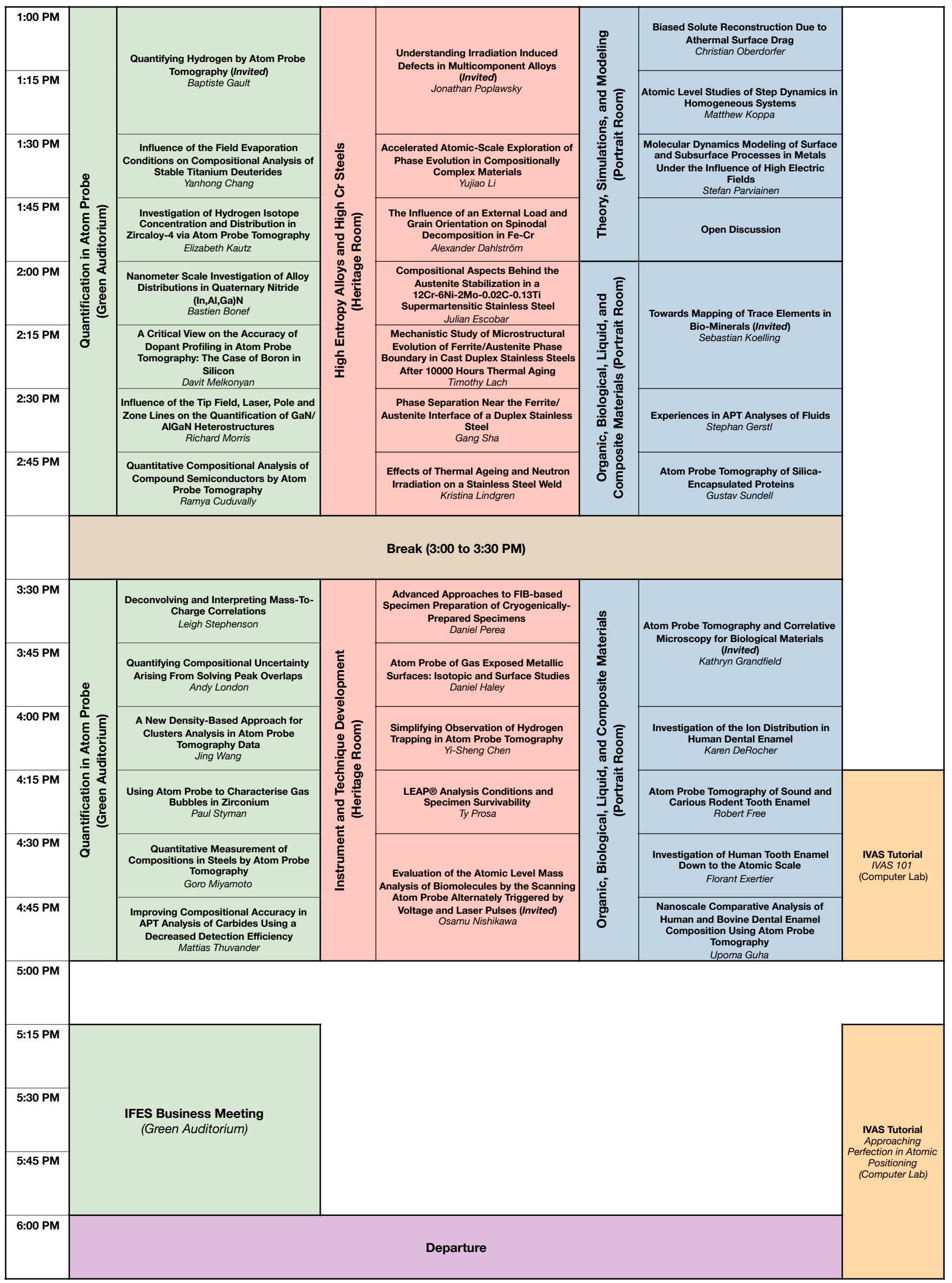





\section{Quantifying Hydrogen by Atom Probe Tomography}

Baptiste Gault ${ }^{1}$, Isabelle Mouton ${ }^{1}$, Yanhong Chang ${ }^{1}$, Andrew J. Breen ${ }^{1}$, Michael Ashton ${ }^{1}$, Siyang Wang $^{2}$, Paraskevas Kontis ${ }^{1}$, Agnieszka Szczepaniak ${ }^{1}$, Michael Herbig ${ }^{1}$, Abigail Ackerman ${ }^{2}$, Felicity Dear $^{2}$, David Dye ${ }^{2}$, Christoph Freysoldt ${ }^{1}$, Jörg Neugebauer ${ }^{1}$, Dierk Raabe ${ }^{1}$, T. Ben Britton $^{2}$, Leigh T. Stephenson ${ }^{1}$

Corresponding Author: b.gault@mpie.de

1. Max-Planck-Institut für Eisenforschung, Düsseldorf, Germany

2. Department of Materials at Imperial College, London, UK.

Observing hydrogen $(\mathrm{H})$ in matter is a formidable challenge as, despite being ubiquitous in nature, it is elusive to scientific scrutiny like no other element. Without nanoscale quantification, it is often simply portrayed as either a blessing or a curse. A blessing certainly in that it is a prime candidate for producing low-carbon emission power. But no less important is the effect of hydrogen embrittlement which has resulted in many catastrophic failures of engineering alloys.

I will discuss some of the hurdles that will need to be overcome in order for atom probe tomography to be fully integrated in a microstructural investigation where precise knowledge of the distribution of $\mathrm{H}$ is crucial to explain the observed physical phenomena. In particular, I will consider issues of quantification of hydrogen and deuterium in stable phases, hydride formation during specimen preparation, and the effect of atom probe running conditions on the quantity of hydrogen detected, providing some reflections on how to mitigate these problems.

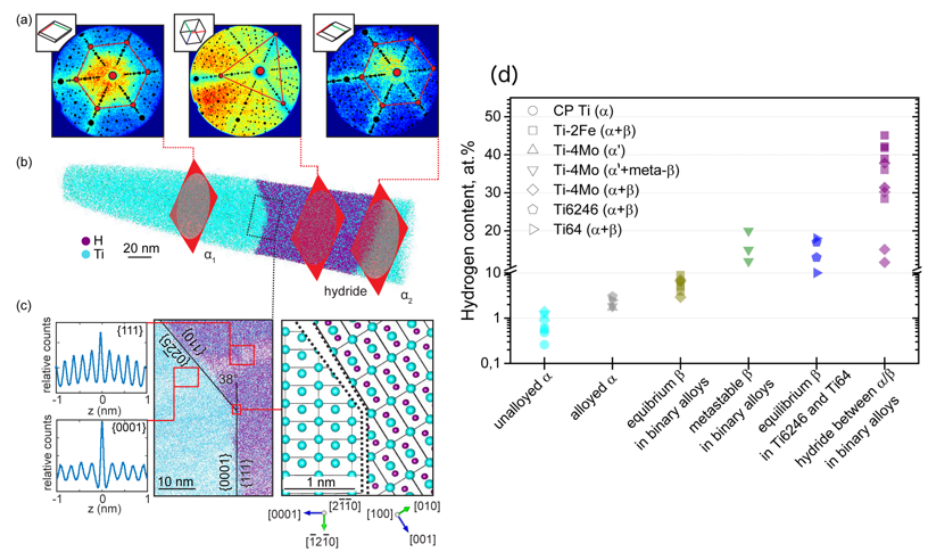

Figure 1 Atom probe crystallography analysis of a hydride precipitated at a $\alpha$ low-angle grain boundary in Ti-2Fe: (a) patterns formed on the detector during the analysis exhibiting the typical symmetries from the local crystalline phase highlighted by the superimposed stereograms; (b) APT reconstruction and (c) spatial distribution maps revealing the presence of atomic planes in the tomographic reconstruction near the $\alpha$-Ti / hydride interface along with model of the faceted $\alpha$ /hydride interface. (d) $\mathrm{H}$ composition in APT datasets depending on the phases detected across all alloys analysed in our study represented by different symbols. $\mathrm{H}$ level in unalloyed $\alpha$, i.e. in $\mathrm{CP}$ Ti and two phase binary model alloys, is $\sim 1$ at.\%. More $\mathrm{H}$ is detected in alloyed $\alpha$ (by $\mathrm{Al}$ in Ti6246 and Ti64, and by Mo in quenched Ti-4Mo with martensite). $\beta$ phases contain much higher amount of $\mathrm{H}$ than $\alpha$ phases in all alloys here. Hydride forms along $\alpha / \beta$ interface in binary alloys or $\alpha$ grain boundaries in $\mathrm{CP} \mathrm{Ti}$, while no hydride observed in commercial Ti alloys, Ti6246 and Ti64. 


\section{Influence of the Field Evaporation Conditions on Compositional Analysis of Stable Titanium Deuterides}

Yanhong Chang ${ }^{1}$, Isabelle Mouton ${ }^{1}$, Leigh Stephenson ${ }^{1}$, Agnieszka Szczpaniak ${ }^{1}$, Guikai Zhang ${ }^{2}$, Dirk Ponge $^{1}$, Dierk Raabe ${ }^{1}$, Baptiste Gault ${ }^{1}$

Corresponding Author: b.gault@mpie.de

1. Max Planck Institute für Eisenforschung, Department Microstructure Physics and Alloy Design, Düsseldorf, Germany.

2. China Academy of Engineering Physics, Institute of Materials, Jiangyou, China.

Atom probe tomography has been increasingly applied to investigate hydrogen embrittlement in metals due to its unique capacity for direct imaging of $\mathrm{H}$ atoms interacting with microstructural features $[1,2]$. However, the observed local changes in $\mathrm{H}$ distribution near or at microstructural features might also be induced by the variations of the local field evaporation conditions, instead of actual H-enrichment or depletion. The aim of this work is to understand the physical mechanisms that lead to such variations with respect to the local field evaporation conditions. As deuterium is often used as a proxy to reveal the $\mathrm{H}$ distribution to avoid or minimize the overlap with residual $\mathrm{H}$ from the vacuum chamber, stable titanium deuterides (approx. 50 at.\% D) were prepared in lieu of hydrides. We performed a systematic study of $\mathrm{H}$ and D composition measurement in pure $\mathrm{Ti}$ and these $\mathrm{Ti}$ - deuterides as a function of the pulse rate, pulse fraction in high-voltage pulsing mode and laser pulse energy. The strong dependence of the residual $\mathrm{H}$ concentration on the pulse rate allows us to distinguish it from the H/D dissolved in materials. We reveal that the detected ion species and H/D composition are strongly dependent on the laser pulse energy, which is interpreted with regards to the global and local field evaporation conditions. Knowing the H/D evaporation behavior under different electric field conditions in stable hydrides or deuterides provides key information to investigate the hydrogen interaction with defects in metals, and further understand the hydrogen embrittlement mechanisms.
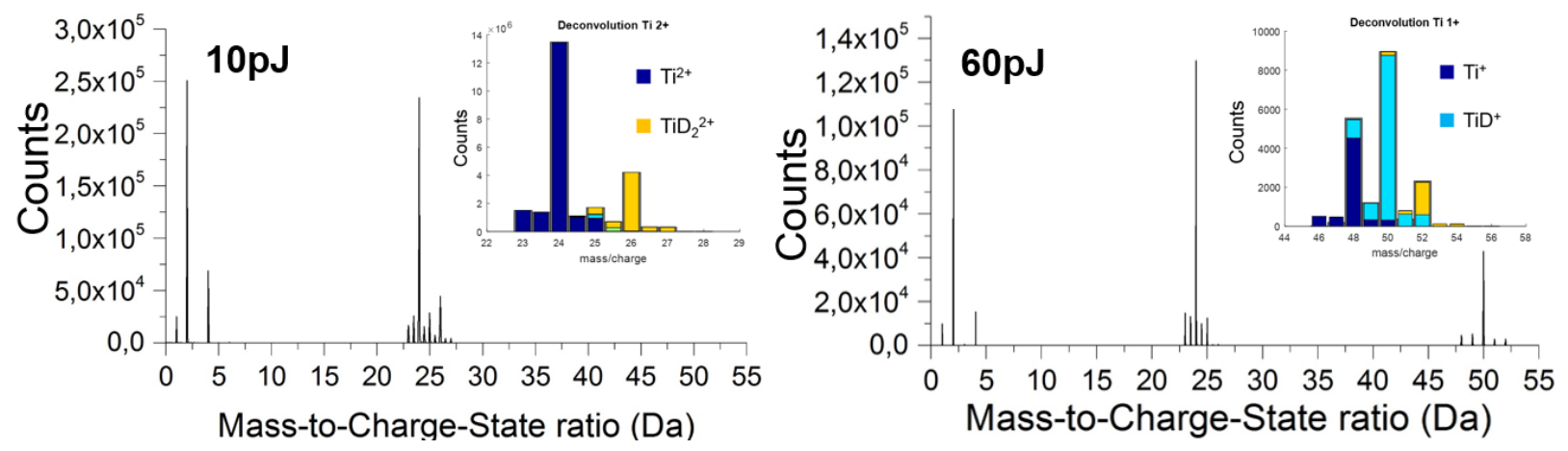

Figure 1: Mass spectra of titanium deuteride with laser pulse energy of (a) 10pJ; (b) 60pJ.

\section{References:}

[1] J. Takahashi, K. Kawakami, T. Tarui, Direct observation of hydrogen-trapping sites in vanadium carbide precipitation steel by atom probe tomography, Scr. Mater. 67 (2012) 213-216.

[2] Y.S. Chen, D. Haley, S.S.A. Gerstl, A.J. London, F. Sweeney, R.A. Wepf, W.M. Rainforth, P.A.J. Bagot, M.P. Moody, Direct observation of individual hydrogen atoms at trapping sites in a ferritic steel, Science (80-. ). 355 (2017) 1196-1199. 


\title{
Investigation of Hydrogen Isotope Concentration and Distribution in Zircaloy-4 via Atom Probe Tomography
}

\author{
Elizabeth Kautz ${ }^{1}$, Bradley Johnson ${ }^{2}$, John Hardy ${ }^{2}$, and Arun Devaraj ${ }^{1}$ \\ Corresponding Author: arun.devaraj@pnnl.gov \\ 1. Physical and Computational Sciences Directorate, Pacific Northwest National Laboratory, \\ Richland, WA 99352, USA \\ 2. Energy and Environment Directorate, Pacific Northwest National Laboratory, Richland, WA \\ 99352, USA
}

Tritium is a radioactive hydrogen isotope $\left({ }^{3} \mathrm{H}\right)$ used in various applications. Since tritium is not naturally abundant, it must be artificially generated and extracted. Tritium Producing Burnable Absorber Rods (TPBARs) are specifically designed to produce and capture tritium when irradiated with neutrons. At the center of each TPBAR, there is a lithium aluminate $\left(\mathrm{LiAlO}_{2}\right)$ ceramic pellet, enriched in ${ }^{6} \mathrm{Li}$, that produces tritium upon neutron irradiation. Tritium is then absorbed by a Zircaloy-4 tube that surrounds the $\mathrm{LiAlO}_{2}$ pellet to preferentially capture the tritium [1]. Currently the amount of tritium absorbed by the Zircaloy-4 getter is not accurately predicted by existing models [2]. In order to improve predictive models and better inform materials processing and design decisions, improved understanding of the mechanisms responsible for hydrogen absorption and distribution in the Zircaloy-4 getter are needed. The overall goal of this work is to measure hydrogen isotopic ratio and spatial distribution in Zircaloy-4 getter before and after exposure to hydrogen and deuterium gases, in order to provide insight into how hydrogen is absorbed in the tritium production process. In this work, comparison of atom probe tomography results from as-received, hydrided, and deuterated Zircaloy-4 samples was performed. The data revealed several overlapping peaks in the mass spectra, and non-uniformity in hydride distribution after exposure to hydrogen and deuterium gases. Additionally, user-selected experimental parameters for pulsed-laser atom probe were studied in order to determine how these parameters impacted hydrogen background, and hydrogen isotope content in Zircaloy-4 samples. The following parameters were tested: laser energies of 80-200 pJ, pulse rates of 125 and $200 \mathrm{kHz}$, and set point temperatures of 40, 50, and $60 \mathrm{~K}$. The work presented here is intended to serve as a baseline for quantification of hydrogen in Zircaloy-4 getter tubes after neutron irradiation.

\section{References:}

[1] A Devaraj, EJ Kautz, EA Vo; B Johnson, DJ Senor, J Hardy; “Atom Probe Tomography for Detection and Quantification of Light Isotopes in TPBAR Components", PNNL-27132, January 2018.

[2] D. J. Senor, "Recommendations for Tritium Science and Technology Research and Development in Support of the Tritium Readiness Campaign, TTP-7-084," PNNL22873, October 2013. 


\section{Nanometer Scale Investigation of Alloy Distributions in Quaternary Nitride (In,Al,Ga)N}

Bastien Bonef ${ }^{1}$, Abdullah Alhassan ${ }^{1}$, Cheyenne Lynsky ${ }^{1}$, Guillaume Lheureux ${ }^{1}$, and James. S. Speck $^{1}$

Corresponding Author: bbonef.prof@,gmail.com

1. Materials Department, University of California, Santa Barbara, CA 93106, USA

Quaternary InAlGaN is a promising alloy which could be used in the next generation nitride based devices such as light emitting diodes (LEDs). Growing it by metal organic vapor phase epitaxy remains a challenge caused by the difficulty to simultaneously incorporate indium and aluminum in the layers. Moreover, the quantification of the III site fraction and the visualization of alloy distribution at the nanometer scale in this material require advanced characterization tool. This is however straightforward using atom probe tomography after considering the limitations of the technique in terms of quantification.

Four InAlGaN samples with grading Al fraction were investigated. The III site fractions are known from Rutherford Backscattering Spectrometry. The samples were evaporated in a LEAP $3000 X$ HR operated in laser-pulse mode. As already reported from III-nitrides [1,2], a high surface field corresponding to a high $\mathrm{Ga}^{++} / \mathrm{Ga}^{+}$ratio is observed around the [0001] axis pole (figure 1(a)). This corresponds to an over-estimation of the Al fraction to the detriment of Ga which is interpreted in terms of preferential evaporation of Ga (figure 1(b)). Indium fraction is not affected by the surface field. The investigation of alloy distribution in the sample is performed away from the high field regions.

Different alloy distributions were observed in the 3D reconstructions of the samples. The first grown $10 \mathrm{~nm}$ of InAlGaN are random alloys according to statistical distribution analysis [3] (I in figure 1(c)). A progressive increase in the $\mathrm{Al}$ and In fraction along the growth direction is observed leading to the formation of V-defects (II in figure 1(c)). A clear segregation of In is observed along the defects facets. Growth conditions are improved to prevent the formation of these defects which allows the use of InAlGaN in nitride-based devices such as LED.
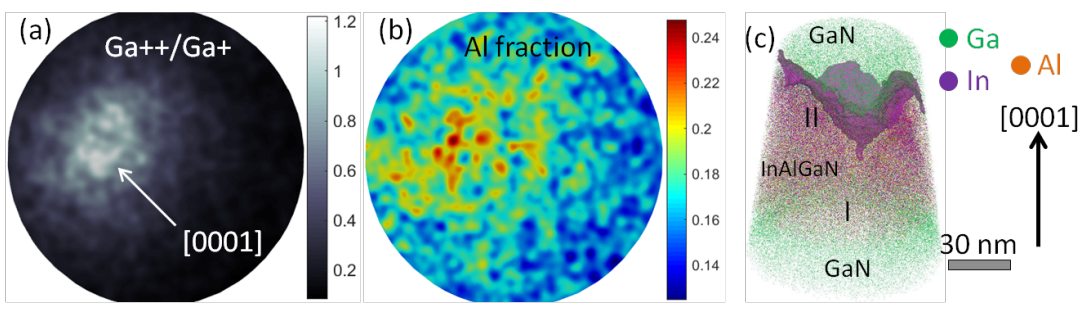

Figure $12 \mathrm{D}$ top views of (a) $\mathrm{Ga}^{++} / \mathrm{Ga}^{+}$charge state ratio at the tip surface and (b) the corresponding $\mathrm{Al}$ fraction. (c) 3D volume of $\operatorname{In}_{22} \mathrm{Al}_{12} \mathrm{Ga}_{66} \mathrm{~N}$ showing an indium iso-surface following a V-defect.

\section{References:}

[1] B. Bonef, et al., J. Appl. Phys. 121, 225701 (2017).

[2] L. Rigutti, et al., J. Appl. Phys. 119, 105704 (2016).

[3] M.P. Moody, et al., Microsc. Res. Tech. 71, 542 (2008). 


\title{
A Critical View on the Accuracy of Dopant Profiling in Atom Probe Tomography: The Case of Boron in Silicon
}

\author{
Davit Melkonyan ${ }^{1,2}$, Claudia Fleischmann², Janusz Bogdanowicz ${ }^{2}$, Richard Morris ${ }^{2}$, Ramya \\ Cuduvally ${ }^{1,2}$, and Wilfried Vandervorst ${ }^{1,2}$ \\ Corresponding author: claudia.fleischmann@imec.be \\ 1. Instituut voor Kern- en Stralingsfysica, KU Leuven, Celestijnenlaan 200D, 3001 Leuven, \\ Belgium \\ 2. Imec, Kapeldreef 75, 3001 Heverlee, Belgium
}

Atom probe tomography (APT) plays a decisive role in the characterization of state-of-the-art 3D semiconductor devices. To meet the required performance, measurement accuracy in both quantification and spatial resolution has to be improved, particularly for 3D dopant profiling. Inaccuracies may originate from physical limitations such as a difference in evaporation field thresholds and laser absorption properties between dopant and matrix atoms. A notorious example is the preferential retention (and migration) of high field materials such as boron on the specimen surface. This degrades the depth and lateral resolution ${ }^{1}$ and reduces the quantification accuracy due to limitations in resolving the correlated (time and space) evaporation events resulting from this retention (multi hit events) ${ }^{2}$. Therefore, B concentrations are often underestimated when measured with APT as compared to their SIMS counterpart ${ }^{2}$.

In this contribution we will focus on the error source being the long ranging transient (as observed in ref [1]) originating from the evaporation field difference between B and Si and the dynamic processes at the surface, i.e. atom migration, dome formation and smoothening. This artifact manifests itself as serious spatial distortions and the severe deviation from stoichiometric emission (and thus correct quantification) leading to long transient tails on steep Boron profiles. As we will show this is linked to the electric field strength at the emitter apex and is only restored by a dynamic enrichment of $B$ at the evaporating surface. At this equilibrium, the effect of the lower B evaporation probability (due to its high evaporation field threshold) is counterbalanced by an increase of potential evaporation sites (due to B-surface enrichment). The width of the transient region, i.e. the number of atoms that need to be evaporated before this equilibrium is reached, depends on the rate of B surface enrichment. Consequently, it scales with the nominal B concentration of the sample. In our experiments several millions of atoms (several $10 \mathrm{~s}$ of nanometers) had to be evaporated to reach equilibrium conditions in a $8.7 \mathrm{E} 19 \mathrm{atoms} / \mathrm{cm}^{3}$ B-doped Si layer. Obviously, this mechanism will deteriorate the leading and trailing edge of a measured depth profile and hence affects both the spatial and quantification accuracy. A mathematical model based on rate equations accounting for $\mathrm{B}$ atom retention and migration has been developed to describe the above discussed observations.

\section{$\underline{\text { References: }}$}

[1] Y. Tu, H. Takamizawa, B. Han, Y. Shimizu, K. Inoue, T. Toyama, F. Yano, A. Nishida, Y. Nagai, Influence of laser power on atom probe tomographic analysis of boron distribution in silicon, Ultramicroscopy, 173 (2017), 58-63.

[2] F. Meisenkothen, E. B. Steel, T. J. Prosa, K. T. Henry, and R. Prakash Kolli, Effects of detector deadtime on quantitative analyses involving boron and multi-hit detection events in atom probe tomography, Ultramicroscopy, 159 (2015), 101-111. 


\section{Influence of the tip field, laser, pole and zone lines on the quantification of GaN/AIGaN heterostructures}

Richard J. H. Morris ${ }^{1}$, Ramya Cuduvally ${ }^{1,2}$, Davit Melkonyan ${ }^{1,2}$, Ming Zhou ${ }^{1}$, Paul van der Heide $^{1}$, Wilfried Vandervorst ${ }^{1,2}$

Corresponding Author: richard.morris@imec.be

1. IMEC, Kapeldreef 75, 3001, Leuven, Belgium.

2. Instituut voor Kern- en Stralingsfysica, KU Leuven, Leuven, Belgium

Quantitative and reliable material characterization can only be realized by understanding how the metrology technique and sample interact. For Atom Probe Tomography (APT), this is non-trivial as the results appear extremely sensitive to the analysis conditions applied. Here we show how the measurement conditions affect the APT results of $\mathrm{GaN}$ and $\mathrm{AlGaN}$, materials under development for power, microwave and optoelectronic devices [1]. For this study, a Cameca LEAP 5000XR and LA-WATAP were used. Bulk $\mathrm{GaN}$ and $\mathrm{Al}_{x} \mathrm{Ga}_{1-x} \mathrm{~N}$ samples of varying $\mathrm{Al}$ composition $(x=0.08,0.44 \& 0.75)$ along with $\mathrm{GaN} / \mathrm{Al}_{x} \mathrm{Ga}_{1-x} \mathrm{~N}$ heterostructures, all grown by metal organic chemical vapor deposition, were analyzed. Within this study base temperatures of $25 \mathrm{~K}$ and $50 \mathrm{~K}$, and laser energies of $15 \mathrm{pJ}-0.005 \mathrm{pJ}$ (LEAP) and 100-10,000 $\mu \mathrm{W}$ (LA-WATAP) were used.

We show that across different instruments and even when using different laser wavelengths, highly reproducible analysis results (figure 1) can be obtained by linking the apparent composition with the average tip field [2]. For $\mathrm{GaN}$, analysis conditions leading to correct stoichiometry can be identified, as predicted in [3] but at variance to [2]. Obviously (not) achieving stoichiometry depends on the different loss mechanisms, such as $\mathrm{N}_{2}$ sublimation, field of evaporation differences between matrix elements and multi hits, and potentially on sample preparation and instrumental differences. When the conditions optimized on the bulk layers were applied to analyze more complex GaN/AlGaN stacks, the Al composition was always overestimated with APT as compared with TEM (EDS) and ERD, although across different samples the same compositional trend is observed. Closer examination of charge state ratios and measured concentrations across the tip apex revealed the impact of the sample crystallography (pole and zone lines) on the tip field and its influence on the $\mathrm{Al}$ composition, at variance with the $\mathrm{Ga}$ and $\mathrm{N}$ distributions, for which the laser direction had a noticeable effect [3]. In conclusion, while accurate average compositional analysis is obtainable, on the nm-scale the sample crystallography and the details of the laser interaction degrade the achievable accuracy leading to apparent concentration variations across the tip apex.

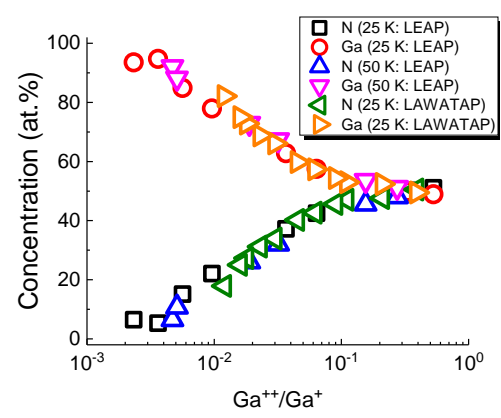

These findings indicate that APT still needs to be improved substantially before it can be used as a true metrology concept.

Figure 1. Measured $G a$ and $N$ concentrations as a function of average tip field $\left(\mathrm{Ga}^{++} / \mathrm{Ga}^{+}\right)$analyzed using $\mathrm{UV}$ and green laser illumination, at two base temperatures (25 $\mathrm{K}$ and $50 \mathrm{~K}$ ) and using two different instruments.

\section{References:}

[1] V. O. Turin \& A. A. Balandin, Electron Lett. 40, 2004, 81.

[2] L. Mancini et al, J. Phys Chem C. 118, 2014, 24136-24151.

[3] D. R. Diercks et al. J Appl. Phys. 114, 2013, 184903. 


\section{Quantitative Compositional Analysis of Compound Semiconductors by Atom Probe Tomography}

R. Cuduvally ${ }^{1,2}$, R. J. H. Morris 2 , J. Bogdanowicz 2 , D. Melkonyan ${ }^{1,2}$, L. Arnoldi ${ }^{3}$, C. Fleischmann ${ }^{2}$, W. Vandervorst ${ }^{1,2}$

Corresponding Author: Ramya.Cuduvally@imec.be

${ }^{1}$ Instituut voor Kern- en Stralingsfysica, KU Leuven, Leuven, Belgium

2. Imec, Kapeldreef 75, 3001, Leuven, Belgium

3. GPM, Universite De Rouen, Avenue de l'Université, 76800 Saint-Étienne-du-Rouvray, France

As III-V materials are explored as a replacement for $\mathrm{Si}$, reliable metrology for stoichiometry, layer thickness and doping is required. While Laser Assisted Atom Probe shows promise in meeting these demands, analysis of III-V based structures is known to suffer from artefacts [1]. For the case of $\mathrm{In}_{\mathrm{x}} \mathrm{Ga}_{1-\mathrm{x}} \mathrm{As}$ (Fig. 1a), the correct concentrations are never reached regardless of the experimental conditions applied. In this work, we report on the phenomena that impact the composition quantification of InGaAs, i.e. As cluster evaporation, As multi-hits and DC evaporation of the low evaporation field species - In and Ga. The As concentration for different fields is always underestimated, but the loss mechanisms at low field (region I) and high field (region II) are different. For the low field, an enhanced As cluster evaporation leads to mass peak overlaps that cannot be deconvoluted for such a mono-isotopic species. The extent of clustering is solely dependent on the field at the tip apex (Fig. 1b) and independent of the laser pulse energy. At high fields, the loss mechanism is dominated by As multi hits together with DC evaporation of the In and Ga. By separating the double hits of As, Ga and In, we conclude (Fig. 1c) that the As pairs dominate. For the separated As pairs at $0.01 \mathrm{pJ}$ with equal and unequal $\mathrm{m} / \mathrm{n}$ ratio, we find a remarkable difference in respective concentrations i.e. $0.76 \%$ and $15.37 \%$. This is partially expected because ions with the same time-of-flight will have a lower probability of being detected. Based on the difference found, an approach for quantifying this loss is being explored. Moreover, our analysis indicates that the composition estimate improves with increasing field because the As multi-hit loss is compensated by an increased $\mathrm{In} / \mathrm{Ga} \mathrm{DC}$ evaporation. Additional studies involving the potential impact of different laser wavelengths and FIB cleaning energies on the quantitative analysis of InGaAs are currently ongoing.
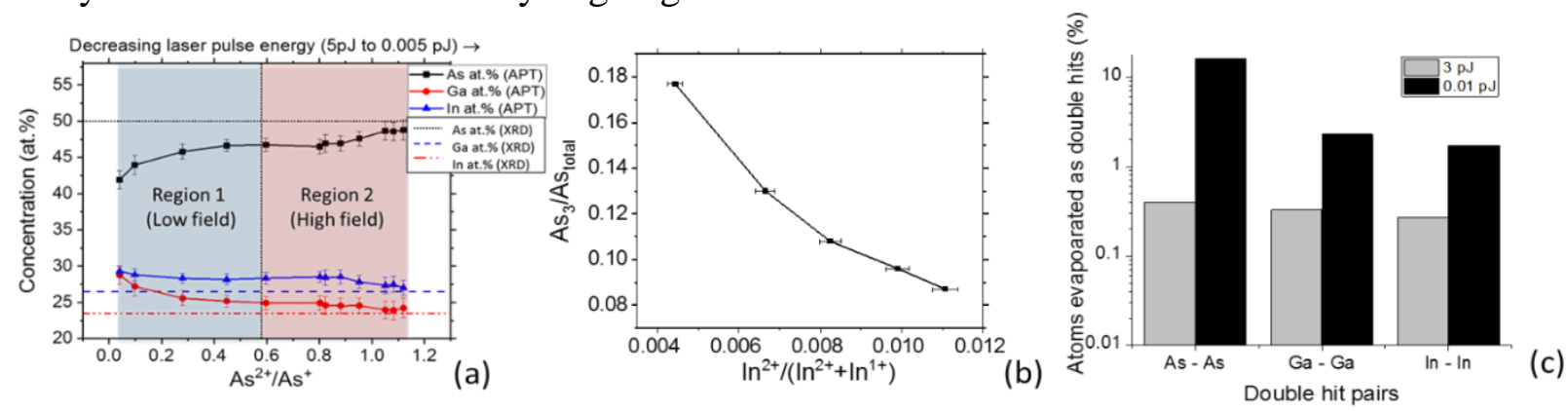

Figure 1 (a) Pulse fraction study on $\operatorname{In}_{0.53}$ GaAs as measured with the LEAP 5000XR at a constant flux (b) $\mathrm{As}_{3}$ concentration as a function of field (c) Double hits of As, Ga and In pairs

\section{$\underline{\text { References: }}$}

[1] L. Mancini, et al. "Composition of wide bandgap semiconductor materials and nanostructures measured by atom probe tomography and its dependence on the surface electric field." The Journal of Physical Chemistry C 118.41 (2014): 24136-24151. 


\section{Deconvolving and Interpreting Mass-To-Charge Correlations}

Leigh Stephenson ${ }^{1}$, Isabelle Mouton ${ }^{1}$, Zirong Peng ${ }^{1}$, Shyam Katnagallu ${ }^{1}$, Mozhdeh Fathidoost ${ }^{1}$, Yanhong Chang ${ }^{1}$, Huan Zhao ${ }^{1}$, Ann-Katrin Rusitzka ${ }^{1}$, Dierk Raabe ${ }^{1}$, and Baptiste Gault ${ }^{1}$

Corresponding Author: 1.stephenson@mpie.de

${ }^{1 .}$ Max Planck Institut für Eisenforschung, Düsseldorf, Germany.

Using sophisticated algorithms to identify and characterize the physical features in three dimensional point clouds of APT data has been a tenacious theme for many research groups. But optimizing these analyses have largely been a snipe hunt - the question "what is the optimal $\mathrm{d}_{\max }$ ?" must be put to bed not because it is unreasonable but because its importance can be overstated. Unsupervised learning procedures have a wide scope beyond this application and they would allow us to explore trends and understand APT data extended to higher dimensional spaces. This is not a new idea [1] but it is one that deserves revival.

The mass-to-charge correlations between multiple detection events have shed light on the often complex phenomenon in APT experiments, including standing field evaporation and the physics of dissociation [2-5]. Instead of focusing on single features within this often complex data, we will demonstrate methods to deconvolve many features at once. Here we demonstrate, amongst other techniques, the application of shared nearest neighbour (SNN) calculations [6] and 2D Kohonen maps [7]. All techniques will be presented in a Python programming environment when convenient.

First, we focus on the atom probe of simple metallic systems, filtering and data mining correlated mass-to-charge data in a dimensionally augmented space combining acquisition data (chiefly from EPOS files) and secondary analysis data (spatial, local field, phase). In this way, we shall identify various phenomena and correlate these with spurious features often seen in the one-dimensional mass-to-charge spectra (humps, high background, tails). In doing so, we better characterize the effects of changing acquisition parameters. We extend this principle to more complex materials (multicomponent alloys, proteins and oxides) to more completely classify every single ion.

\section{$\underline{\text { References: }}$}

[1] S. Broderick, et al., Data mining for isotope discrimination in atom probe tomography, Ultramicroscopy 132, 2013, pp121-128

[2] D. Saxey, Correlated ion analysis and the interpretation of atom probe mass spectra, Ultramicroscopy 111 (6) 2011 pp473-479

[3] D. Zanuttini, et al., Electronic structure and stability of the $\mathrm{SiO} 2+$ dications produced in tomographic atom probe experiments, The Journal of Chemical Physics 2017 147:16

[4] C. Loyola, et al., Impact of extreme electrical fields on charge density distributions in Al3Sc alloy, Journal of Vacuum Science and Technology A: Vacuum, Surfaces and Films 34(6) 061404

[5] Z. Peng, et al., Field evaporation of molecular ions and their dissociation behavior, (to be published 2018)

[6] L. Ertöz, et al., Finding Clusters of Different Sizes, Shapes, and Densities in Noisy, High Dimensional

Data, Proceedings of the 2003 SIAM International Conference on Data Mining

[7] T. Kohonen, Self-Organization and Associative Memory, Springer, Berlin, 1984 


\section{Quantifying Compositional Uncertainty Arising From Solving Peak Overlaps}

Andrew J London ${ }^{1}$

\section{Corresponding Author: andy.london@,ukaea.uk}

1. Culham Centre for Fusion Energy, UKAEA, Abingdon, UK

Summary: Solving peak overlaps improves composition accuracy but introduces uncertainty. A method for quantifying this uncertainty is provided. This can be used to improve composition confidence intervals and for experimental planning.

When ionic species with similar $\mathrm{m} / \mathrm{z}$ values overlap, numerical estimation of the ionic counts from the known natural isotopic abundances can be made [1]. There are many sources of random and systematic error in compositional quantification by atom probe, however, often only the statistical or "counting error" is reported. When two ions overlap they may share more or less signal in the same peaks, see Fe/Cr versus Fe/CO (figures 1a and 1b), this results in more or less uncertainty in the overlap solution. When using maximum likelihood for solving the peak overlap problem [2], an estimate of the standard error of the solution can be made from the curvature of the likelihood function evaluated at the solution. These estimated errors indicate the difficulty of a particular set of overlapping peaks and can displayed as the line thickness in an overlap diagram, e.g. in Figure 1c. While a formal estimate of the standard errors can be made directly from the maximum likelihood solution, once these errors are propagated through subsequent calculations (total composition or converting to weight percent), error propagation becomes non-trivial. Therefore, when calculating the total composition, a bootstrap method is used, where the confidence interval of the composition is estimated numerically. This is of use in planning experiments and more accurately the estimating confidence intervals of compositions in atom probe data.
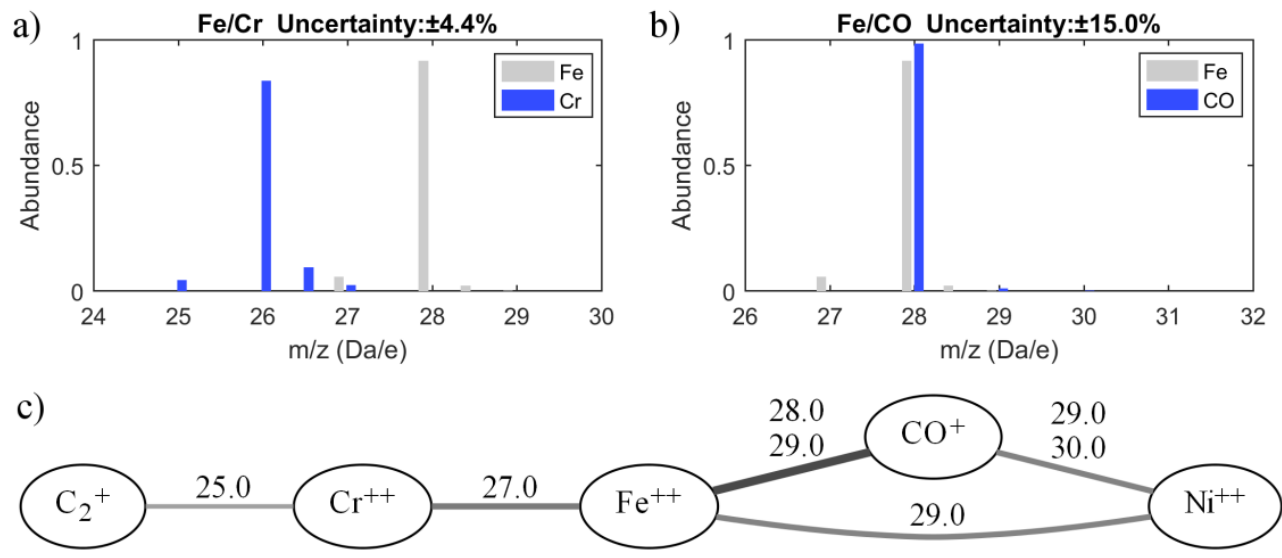

Figure 1 "Easy" $\mathrm{Fe}^{++} / \mathrm{Cr}^{++}$(a) and "hard" $\mathrm{Fe}^{++} / \mathrm{CO}^{+}$(b) overlaps, with associated uncertainty in the solutions: \pm 4.4 or \pm 15 at $\%$ respectively (for 1000 total ions). (c) Overlap diagram showing which species overlap at which $\mathrm{m} / \mathrm{z}$ position. Line thickness indicates the relative uncertainty in solving the overlap, thicker line indicates larger uncertainty.

\section{References:}

[1] M. Miller, et al., Atom Probe Field Ion Microscopy, Oxford Science Publications, Oxford, 1996. [2] A. J. London, et al., Single-Ion Deconvolution of Mass Peak Overlaps for Atom Probe Microscopy, Microscopy and Microanalysis, 23 (2017) 300-306. 


\section{A New Density-based Approach for Clusters Analysis in Atom Probe Tomography Data}

Jing Wang ${ }^{1}$, Nathan Bailey ${ }^{2}$, Peter Hosemann ${ }^{2}$, Daniel K. Schreiber ${ }^{1}$, Mychailo B. Toloczko ${ }^{1}$

Corresponding Author: jing.wang@pnnl.gov

1. Pacific Northwest National Laboratory, Richland, WA, USA

2. University of California, Berkeley, CA, USA

Atom probe tomography (APT) is a powerful technique to characterize nanoscale features in a variety of materials. The current widely used cluster analysis method that is a variant of the DBSCAN algorithm [1] can only extract clusters of the same atomic density. However, clusters of varying atomic density (e.g. YTiO clusters in Fig. 1) exist in APT data obtained from many materials. The current cluster analysis method relies heavily on multiple input parameters, but ideal selection of those parameters is challenging and often times ambiguous. In this study, we present a new cluster analysis method that combines a wellknown algorithm called OPTICS [2] with a cluster extraction algorithm we developed to enable accurate detection of clusters of varying atomic density in APT data. The OPTICS algorithm itself does not extract clusters from data, but instead creates an augmented ordering of the dataset that represents a densitybased clustering structure. The ordering representation contains information that is equivalent to a wide parameter space as in traditional methods such as DBSCAN. Thus retrieval of information on clusters of varying density is possible. With proper estimation based on materials composition, the number of parameters for controlling the outcome of OPTICS can be reduced to one, and other inputs can be estimated based on physical parameters of the material. The effectiveness of this method is demonstrated by application to several small-scale model datasets and a real APT dataset obtained from an oxidedispersion strengthened ferritic alloy specimen. The method's sensitivity with respect to parameter selection is discussed and compared with the current widely used methods.
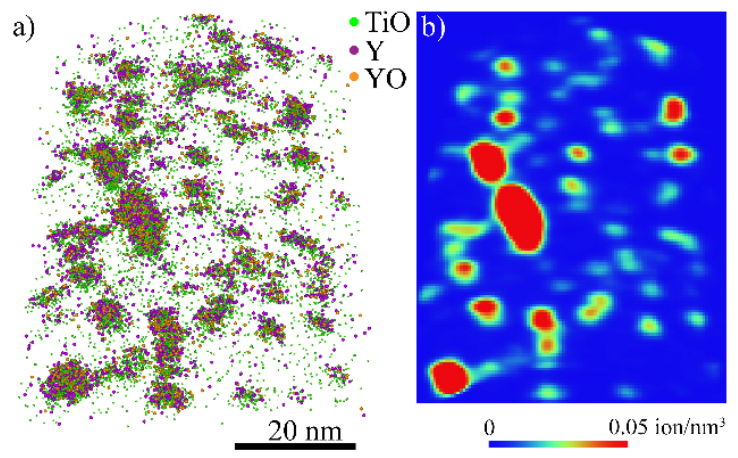

Figure 1 (a) Ion maps of Y, TiO, and YO in an ODS alloy MA957 dataset. (b) the calculated local ion density in IVAS.

\section{References:}

[1] M. Ester, H.-P. Kriegel, J. Sander, X. Xu, A density-based algorithm for discovering clusters in large spatial databases with noise, Kdd, 1996, pp. 226-231.

[2] M. Ankerst, M.M. Breunig, H.-P. Kriegel, J. Sander, OPTICS: ordering points to identify the clustering structure, ACM Sigmod Record, ACM, 1999, pp. 49-60.

[3] This work was sponsored by U.S. Department of Energy, Office of Nuclear Energy, Fuel Cycle R\&D Program. 


\title{
Using Atom Probe to Characterise Gas Bubbles in Zirconium
}

\author{
Paul D Styman ${ }^{1,2}$, James O Douglas ${ }^{2}$ \\ Corresponding Author: paul.d.styman@materials.ox.ac.uk \\ 1. National Nuclear Laboratory, Building D5, Culham Science Centre, Abingdon, Oxfordshire, \\ OX14 3DB, UK. \\ 2. Department of Materials, University of Oxford, Parks road, Oxford, OX13PH, UK.
}

For many years there has been an interest in studying the formation and evolution of rare gas (e.g. helium, krypton and xenon) bubbles in metals e.g. [1-3]. These gases, implanted into metals using ion beams, are highly insoluble in metals and readily form bubbles within the metallic matrix. Several authors have noted that gas bubbles are potential traps for hydrogen e.g. [4]. Hydrogen is known to cause embrittlement of structural materials, including steels and zirconium.

$1 \mathrm{MeV}$ xenon was implanted into zirconium at room temperature to fluences of 3 and $10 \times 10^{15}$ ions $/ \mathrm{cm}^{2}$. Atom Probe Tomography was used to characterize the bubbles. Xenon bubbles were chosen for this work as their freezing point $(\sim 160 \mathrm{~K})$ is higher than the sample analysis temperature $(\sim 50 \mathrm{~K})$. Although the solid bubbles are likely to evaporate rapidly once exposed at the tip apex, it was anticipated that some of the xenon would be field ionized and detected thus enabling a three-dimensional reconstruction of the bubbles to be performed.

Xenon was detected in all datasets, typically appearing as planar features just one atom layer thick. This was consistent with the observations of Estivill [5] since the majority of the xenon in bubbles $(>75 \%)$ was lost to the vacuum. However, it was possible to extract quantitative information on bubble size, volume fraction and number density by analysing the local atomic density of atoms in the reconstructed volume. The planar xenon features were found to correlate with regions of high atomic density, which is consistent with the expected behaviour of a nanoscale cavity. A greater number of larger bubbles were detected in samples implanted to higher dose.

\section{References:}

[1] J. H. Evans, A. J. E. Foreman and R. J. McElroy, Anisotropic diffusion of self-interstitials in zirconium, Journal of Nuclear Materials, vol. 168, pp. 340-342, 1989.

[2] P. D. Edmondson, C. M. Parish, Y. Zhang, A. Hallen and M. Miller, Helium Bubble Distributions in a Nanostructured Ferritic Alloy, Journal of Nuclear Materials, vol. 434, pp. 210-216, 2013.

[3] S Krämer, P. Wells, C. Oberdorfer and R. G. Odette, Correlative TEM and Atom Probe Tomography - A Case Study on Structural Materials for Fusion Reactors, Microscopy and Microanalysis. 23 (Suppl 1), pp. 645-655, 2017.

[4] E. Abramov and D. Eliezer, Trapping of hydrogen in helium-implanted metals, Journal of Materials Science Letters, vol. 7, pp. 108-110, 1988.

[5] R. Estivill, G. Audiot, J.-P. Barnes, A. Grenier and D. Blavette, Preparation and Analysis of Atom Probe Tips by Xenon Focused Ion Beam Milling, Microscopy and Microanalysis, 22, pp. 576-582, 2016. 


\section{Quantitative Measurement of Compositions in Steels by Atom Probe Tomography}

Goro Miyamoto $^{1}$, Tadashi Furuhara ${ }^{1}$

Goro Miyamoto: miyamoto@imr.tohoku.ac.jp

${ }^{1 .}$ Institute for Materials Research, Tohoku University, Sendai, Japan

Carbon (C) is most important element in steels so quantitative measurement of $\mathrm{C}$ by APT is of a great importance although $\mathrm{C}$ content in iron carbide $\left(\mathrm{Fe}_{3} \mathrm{C}\right)$ is known to vary with temperature of APT measurement.Therefore, we have investigated the mechanism of variation in $\mathrm{C}$ content by using $\mathrm{Fe}-\mathrm{C}$ binary alloys with known compositions and developed a method to quantify $\mathrm{C}$ content in APT measurement [1]. This method was also applied to measurements of other alloying elements in ferrous and non-ferrous alloys.

Fe-C binary alloys with known $\mathrm{C}$ contents were measured using LEAP 4000HR under voltage pulse mode. Peaks at 24 and 25 amu were decomposed into $\mathrm{CC}^{+}$and $\mathrm{CCCC}^{2+}$ using an intensity of a peak at $24.5 \mathrm{amu}\left(\mathrm{CCCC}^{2+}\right)$ and natural abundance of $\mathrm{C}$ isotopes.

Squares in Fig. 1 show apparent $\mathrm{C}$ contents after the peak decomposition measured at various temperatures. The apparent $\mathrm{C}$ contents are always higher than nominal ones and increase with lowering temperature in all the alloys. Preferential evaporation / retention also cannot explain the discrepancy because it should be retarded at lower temperature in contrast to the observation. The other possibility is an anomaly in detection of Fe ions. It was also found that the fraction of detected Fe major isotope $(\mathrm{Fe} 56)^{2+}$ is lower than the natural abundance, while the other minor isotopes detected are more than the natural abundance; such deviation increases at lower temperatures. This strongly indicates that a part of Fe ions are lost in detection due to pile-up effect. The number of Fe ions lost by pile-up effect can be estimated from difference between the detected ratio of $\mathrm{Fe}$ isotope ions and natural abundance [1]. Circles in Fig. 1 represent the $\mathrm{C}$ contents of the three alloys corrected by considering Fe ions lost in detection. The corrected $\mathrm{C}$ contents agree well with the nominal ones at all the measurement temperatures.

Lost in detection of matrix ions should result in overestimation not only for $\mathrm{C}$ content but for the other alloying elements in ferrous and non-ferrous alloys. Actually, apparent contents of other substitutional elements in ferrous alloys or in Ti alloy measured by APT are found to be higher than the nominal ones and increase with lowering temperature, in agreement with the case for $\mathrm{C}$ content measurement. It was also found that more accurate contents of those elements can be obtained by applying the same correction method.

\section{References:}

[1] G. Miyamoto et al., Scripta Materialia, 67 (2012), 999.

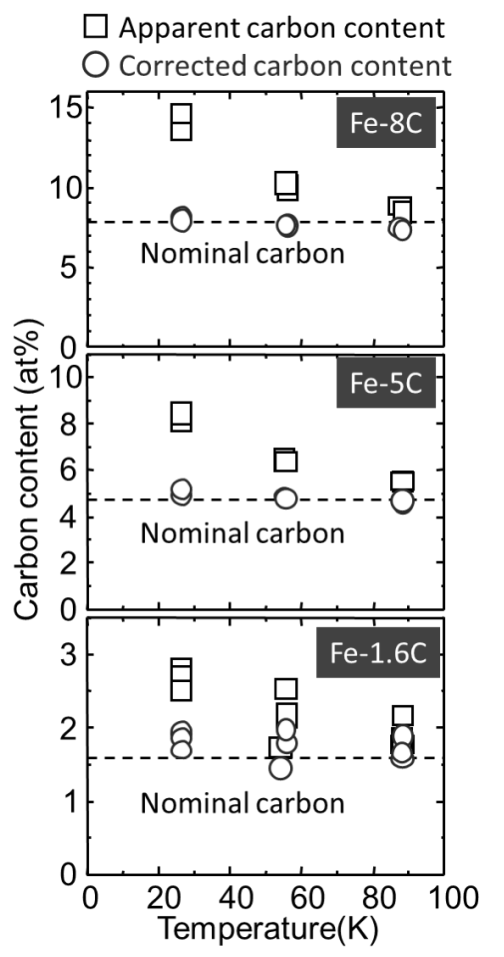

Fig. 1 Apparent and corrected $\mathrm{C}$ contents for the $\mathrm{Fe}-8 \mathrm{C}$, Fe-5C and $\mathrm{Fe}-1.6 \mathrm{C}$ alloys. 


\title{
Improving Compositional Accuracy in APT Analysis of Carbides using a Decreased Detection Efficiency
}

\author{
Mattias Thuvander ${ }^{1}$, Deodatta Shinde ${ }^{1}$ and Krystyna Stiller ${ }^{1}$ \\ Corresponding Author: mattias.thuvander@chalmers.se \\ 1. Department of Physics, Chalmers University of Technology, Goteborg, Sweden.
}

One of the strengths of atom probe tomography (APT) is the ability to measure the composition of small precipitates with good accuracy. In many cases, the accuracy is merely limited by the counting statistics, background noise and possibly mass spectrum overlaps. However, when analyzing carbides, for example in steels, the measured carbon concentration is often lower than the concentration expected from the stoichiometry [1]. The main reason for this carbon deficit is that the field evaporation of ions, particularly carbon ions, from carbides occurs in bursts, with several ions evaporating from the action of a single pulse, resulting in so called multiple events. As the ions in a multiple event often originate from the same region of the sample, they hit a small part of the detector. If then also the mass-to-charge ratios are virtually the same, the detector will not be able to detect (and position) all ions, but the measurement will suffer from detector pile-up. In most cases, detector pile-up will affect carbon more than the metallic elements of the carbide, thus leading to a relative loss of carbon.

In a previous study, we showed that the fraction of multiple events can be significantly decreased by lowering the detection efficiency by inserting a fine mesh grid in the flight path [2]. It was shown that the measured carbon concentration when analyzing bulk WC increased, and got closer to the expected $50 \%$. In the present study, we have applied the same approach to the analysis of small (typically $10 \mathrm{~nm}$ ) carbides (containing Mo, V and $\mathrm{Cr}$ ) in a tool steel. As the carbides are small, the reduced detection efficiency will lead to an increase in the uncertainty due to counting statistics, leading to lower precision. Still, and more importantly, the accuracy is improved when using this method, making it possible to determine the carbide type from the measured carbon concentration.

\section{References:}

[1] M. Thuvander, J. Weidow, J. Angseryd, L.K.L. Falk, F. Liu, M. Sonestedt, K. Stiller and H.-O. Andrén, Quantitative atom probe analysis of carbides, Ultramicroscopy 111 (2011), 604-608.

[2] M. Thuvander, A. Kvist, L.J.S. Johnson, J. Weidow and H.-O. Andrén, Reduction of multiple hits in atom probe tomography, Ultramicroscopy 132 (2013), 81-85. 


\section{Understanding Irradiation Induced Defects in Multicomponent Alloys} Jonathan D. Poplawsky, ${ }^{1}$ Xing Wang, ${ }^{1}$ Wei Guo, ${ }^{1}$ Tengfei Yang, ${ }^{2}$ and Wei-Ying Chen ${ }^{3}$ Corresponding Author: poplawskyjd@ornl.gov

1. Oak Ridge National Laboratory, Oak Ridge, TN, USA.

${ }^{2}$. Department of Nuclear Engineering, University of Tennessee, Knoxville, TN, USA

${ }^{3}$ Nuclear Engineering Department, Argonne National Laboratory, Argonne, IL, USA

The superb mechanical properties of high-entropy alloys (HEAs) and their elevated temperature stability have warranted HEA applications in nuclear systems. Structural evolution and nanoscale compositional changes occur as a function irradiation dose and temperature. An understanding of these phenomena will help to improve the operating temperature and dose range of these materials. Correlative atom probe tomography (APT) and scanning transmission electron microscopy (STEM) has been performed on several multicomponent alloys, including $\mathrm{Al}_{0.1} \mathrm{CoCrFeNi}$, CoCrMnFeNi, and $\mathrm{NiCoCr}$, subject to irradiation at different doses and temperatures to better understand irradiation induced damage in HEAs. For example, a correlative HAADF-STEM image and APT atom map of a NiCoCr alloy irradiated with $200 \mathrm{kV}$ He ions with a maximum dose of $2 \mathrm{dpa}$ and at $500{ }^{\circ} \mathrm{C}$ is shown in Figure $1(\mathrm{a}, \mathrm{b})$. The lighter contrast regions in the HAADF STEM images are due to lower density He bubbles formed in the higher density NiCoCr alloy during irradiation. Density isosurfaces (Figure 1(a)) correlate well with the He bubble regions in the STEM-HAADF image. A proximity histogram (Figure 1(d)) confirms that these regions are enriched in Co and depleted in $\mathrm{Cr}$. The trend of segregation agrees well with the differences in defect diffusion kinetics, in which the migration barrier for $\mathrm{Cr}$ vacancies is the lowest while Co interstitials diffuse the fastest among all three elements. ${ }^{1,2}$
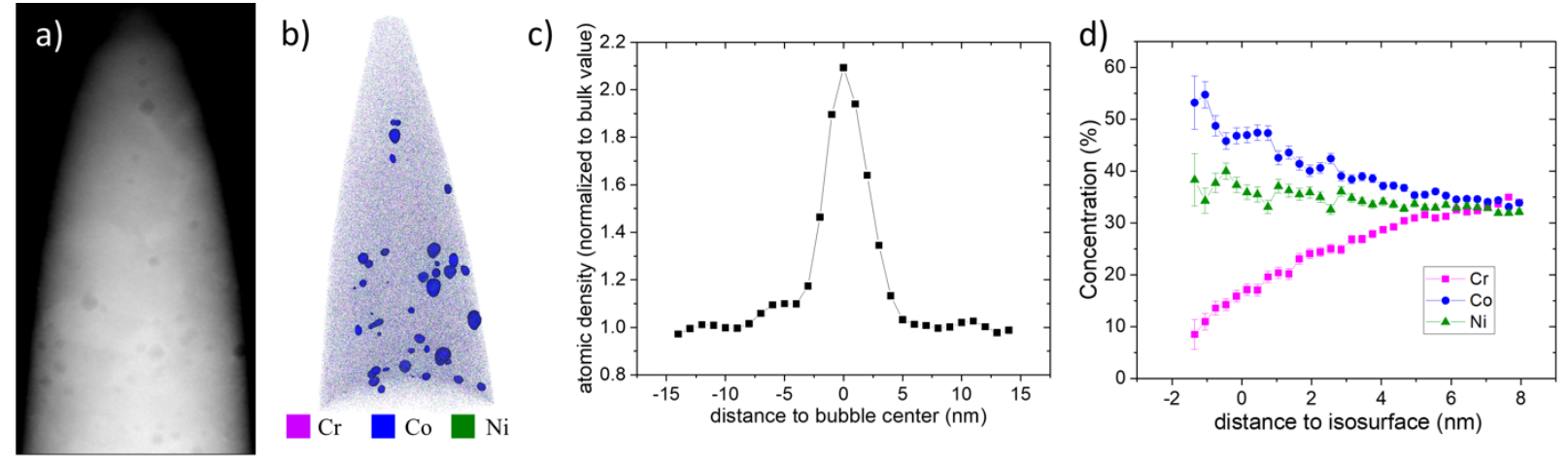

Figure 1 (a) STEM-HAADF image of a He irradiated NiCoCr alloy ready for an APT experiment. (b) APT atom map and isodensity surface. (c) 1DLP across a He bubble showing the density change in the APT dataset. (d) Proximity histogram of the isodensity surfaces.

\section{References:}

[1] S. Zhao, et al. Effect of d electrons on defect properties in $\mathrm{NiCoCr}$ and $\mathrm{NiCoFeCr}$ concentrated solid solution alloys, Phys. Rev. Mater. 13602 (2018) 1-18.

[2] S. Zhao, Y. Osetsky, Y. Zhang, Preferential diffusion in concentrated solid solution alloys: NiFe, $\mathrm{NiCo}$ and NiCoCr, Acta Mater. 128 (2017) 391-399.

[3] Research was conducted at ORNL's Center for Nanophase Materials Sciences (CNMS), which is a U.S. DOE Office of Science User Facility 


\title{
Accelerated Atomic-Scale Exploration of Phase Evolution in Compositionally Complex Materials
}

\author{
Yujiao Li ${ }^{1}$, Alan Savan ${ }^{2}$, Aleksander Kostka ${ }^{1}$, H.S. Stein ${ }^{2}$ and Alfred Ludwig ${ }^{1,2}$ \\ Corresponding Author: alfred.ludwig@rub.de, yujiao.li@rub.de \\ 1. ZGH, Ruhr-University Bochum, Bochum, Germany. \\ 2. Institute for Materials, Ruhr-University Bochum, Bochum, Germany.
}

The design of compositionally complex materials, like multiple principal element alloys, requires knowledge of the complete phase space of the constituents of a multinary system, i.e. which phases form or decompose at different processing conditions. The acquisition of this knowledge is accelerated by a new combinatorial processing platform (CPP) approach [1], which enables: simultaneous synthesis of 36 identical atomic-scale-mixed films by co-deposition of 5 elements on an array of 10-nm-diameter Si tips; rapid phase evolution in the formed nanoscale reactors upon processing; direct atomic-scale analysis of phase evolution after each processing step by atom probe tomography, complemented by transmission electron microscopy. Results from nanocrystalline CrMnFeCoNi show that this alloy is unstable and already decomposes after $1 \mathrm{~h}$ at low temperatures of around $300^{\circ} \mathrm{C}$. The approach is extendible to explore oxidation and corrosion in complex materials on the atomic scale.

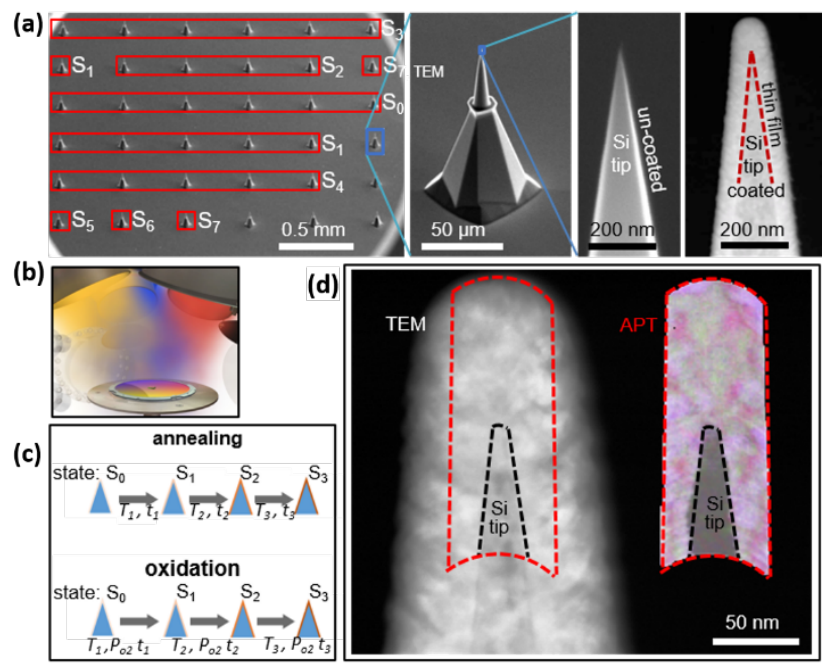

Figure 1 (a) SEM image of a Si tip array. Boxes indicate which tips were used for measurements in each processing state Sx. Middle: Magnified SEM image of an uncoated tip and its sharp end which serves as substrate for the combinatorial deposition of the multinary film. Right: TEM image of a CrMnNiCoFe coated and annealed tip is shown. (b) Schematic of the co-deposition process as applied on different substrates (wafer, single Si tips and TEM-membrane chips): five sputter cathodes deposit simultaneously an atomically mixed film (rotating substrate: homogeneous film; fixed substrate: composition spread). (c) Examples of combinatorial processing using the CPP (a tip is indicated by a blue triangle, the nanoscale coating with orange). (d) TEM of a CrMnFeCoNi film on a Si tip in comparison to an APT analysis.

\section{References:}

[1] Y.J. Li, A. Savan, A. Kostka, H.S. Stein and A. Alfred, Accelerated atomic-scale exploration of phase evolution in compositionally complex materials, Materials Horizons (2018), 86-92. 


\title{
The Influence of an External Load and Grain Orientation on Spinodal Decomposition in $\mathrm{Fe}-\mathrm{Cr}$
}

\author{
Alexander Dahlström ${ }^{1,2}$, Frederic Danoix ${ }^{2}$, Peter Hedström ${ }^{2}$, Joakim Odquvist ${ }^{2}$, Helena Zpolsky $^{1}$ \\ Corresponding Author: alexander.dahlstrom@etu.univ-rouen.fr \\ ${ }^{I}$ Normandie Université, Université de Rouen, INSA Rouen, CNRS, Groupe de Physique des Matériaux, 76000 Rouen, France. \\ ${ }^{2}$ Department of Materials Science and Engineering, KTH (Royal Institute of Technology), SE-100 44 Stockholm, Sweden.
}

$\mathrm{Fe}-\mathrm{Cr}$ alloys serve to great extent as structural materials in their vast variety of applications. Thus the $\mathrm{Fe}-\mathrm{Cr}$ system has been subjected to extensive research as it is the base alloy system of stainless steel [1]. However the understanding of the Fe-Cr system at intermediate temperatures is not conclusive [2]. An issue with $\mathrm{Fe}-\mathrm{Cr}$ alloys at intermediate temperatures in e.g. energy industry were high $\mathrm{Cr}$ grades are used, is the concern of spontaneous de-mixing known as the " $475^{\circ} \mathrm{C}$ embrittlement". It is facilitated by the presence of a wide miscibility gap in the $\mathrm{Fe}-\mathrm{Cr}$ phase diagram. These alloys are often structural components subjected to an external loading during service conditions. Thus fully ferritic Fe-Cr model alloys were stress-aged under elastic tensile loading. Electron backscatter diffraction (EBSD) in combination with Focused Ion Beam (FIB) was used to extract samples in the principal directions of the BCC structure. The modulated nanostructure was analyzed by the means of atom probe tomography (APT). Quantitative 3D characterization of the modulated structure is cumbersome and crystallographic orientation is often neglected. Here the statistical structure factor, pair correlation and cluster erosion methods has been used for short range ordering, long range ordering and precipitate composition analysis respectively. Atomistic 3D modelling based on atomic density function (ADF) theory was used in comparison with experimental result [3]. Utilizing ADF the stress state was extended to tension, compression, pure shear and hydrostatic pressure. Commonly the working hypothesis is that an unstrained isotropic structure is aligned in the elastically soft direction perpendicular to the applied load during stress-ageing [4]. This hypothesis is tested, furthermore the influence of crystallographic orientation and stress state is explored under principal loading conditions.
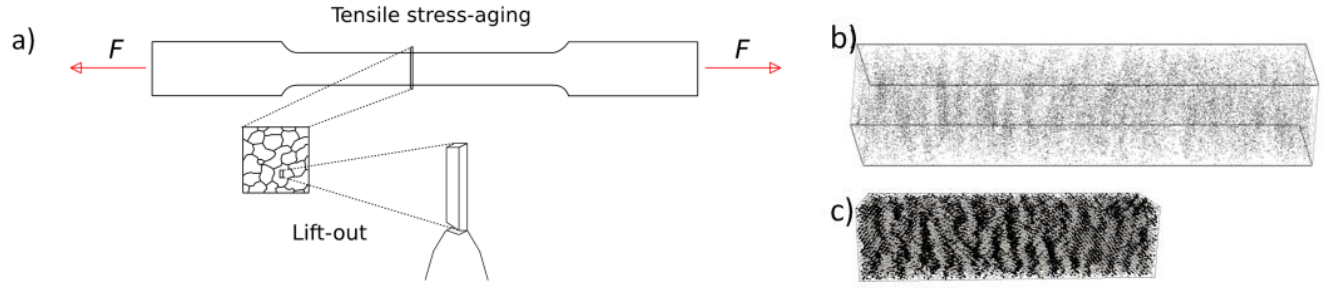

Figure 1, a) Illustration of experimental procedure, b) stress-aged duplex stainless steel APT result of $\mathrm{Cr} 8 \times 8 \times 40 \mathrm{~nm}$ [5], c) ADF modelling of an A-50B alloy with compression constrain $4.8 \times 4.8 \times 19.2 \mathrm{~nm}$.

\section{$\underline{\text { References, }}$}

[1] K.H. Lo, C.H. Shek, and J.K.L. Lai, Mater. Sci. Eng. R Rep., vol. 65, no. 4-6, pp. 39-104, mai 2009.

[2] W. Xiong, P. Hedström, M. Selleby, J. Odqvist, M. Thuvander, and Q. Chen, Calphad, vol. 35, no. 3, pp. 355-366, Sep. 2011.

[3] M. Lavrskyi, H. Zapolsky, and A.G. Khachaturyan, ArXiv14115587 Cond-Mat, Nov. 2014.

[4] Y.-S. Li, H. Zhu, L. Zhang, and X.-L. Cheng, J. Nucl. Mater., vol. 429, no. 1-3, pp. 13-18, Oktober 2012.

[5] J. Zhou, J. Odqvist, M. Thuvander, S. Hertzman, and P. Hedström, Acta Mater., vol. 60, no. 16, pp. 5818-5827, Sep. 2012. 


\title{
Mechanistic Study of Microstructural Evolution of Ferrite/Austenite Phase Boundary in Cast Duplex Stainless Steels after 10000 Hours Thermal Aging
}

\author{
Timothy G. Lach ${ }^{1 *}$, Thak Sang Byun ${ }^{1}$, and Arun Devaraj ${ }^{2}$ \\ ${ }^{1}$ Nuclear Sciences Division, Energy and Environment Directorate, Pacific Northwest National \\ Laboratory, 902 Battelle Blvd. Richland, WA 99352 USA \\ ${ }^{2}$ Physical Sciences Division, Physical and Computational Sciences Directorate, Pacific \\ Northwest National Laboratory, 902 Battelle Blvd. Richland, WA 99352 USA \\ *corresponding author: timothy.lach@pnnl.gov
}

Cast austenitic stainless steels (CASSs) are used for many large components of reactor coolant systems, such as primary coolant piping and pump casings. In this ongoing long-term study, we aim to provide a comprehensive knowledge base for the integrity assessment of CASS components for extended operations at service temperatures of $290-330{ }^{\circ} \mathrm{C}$, with a focus on thermal degradation mechanisms in the cast CF3, CF3M, CF8, and CF8M alloys at reactorrelevant accelerated aging temperatures of $290-400{ }^{\circ} \mathrm{C}$ up to 10000 hours. These alloys, the cast equivalents of the 300-grade stainless steel alloys (304L, 316L, 304, and 316, respectively), develop a duplex austenite/ ferrite duplex structure during casting. While it is known that the ferrite undergoes microstructural changes during thermal aging at moderate temperatures that result in mechanical property degradation and embrittlement, the mechanisms and kinetics of these transformations are not well known to make predictive models for use in long-term service assessments. A quantitative evaluation of the microstructural evolution of the ferrite phase and austenite/ferrite phase boundary after aging was conducted using atom probe tomography combined with analytical scanning transmission electron microscopy. Spinodal decomposition and G-phase precipitation are the primary microstructural changes within the ferrite phase, while at the phase boundary, precipitation of carbides and solute segregation away from the boundary take place. These features, such as those seen in Figure 1 for a phase boundary in a CF3M alloy, among others depend strongly on aging temperature, aging time, and chemical composition of the particular alloys, in particular the concentration of Mo and C. These dependencies will be quantified and discussed thoroughly.

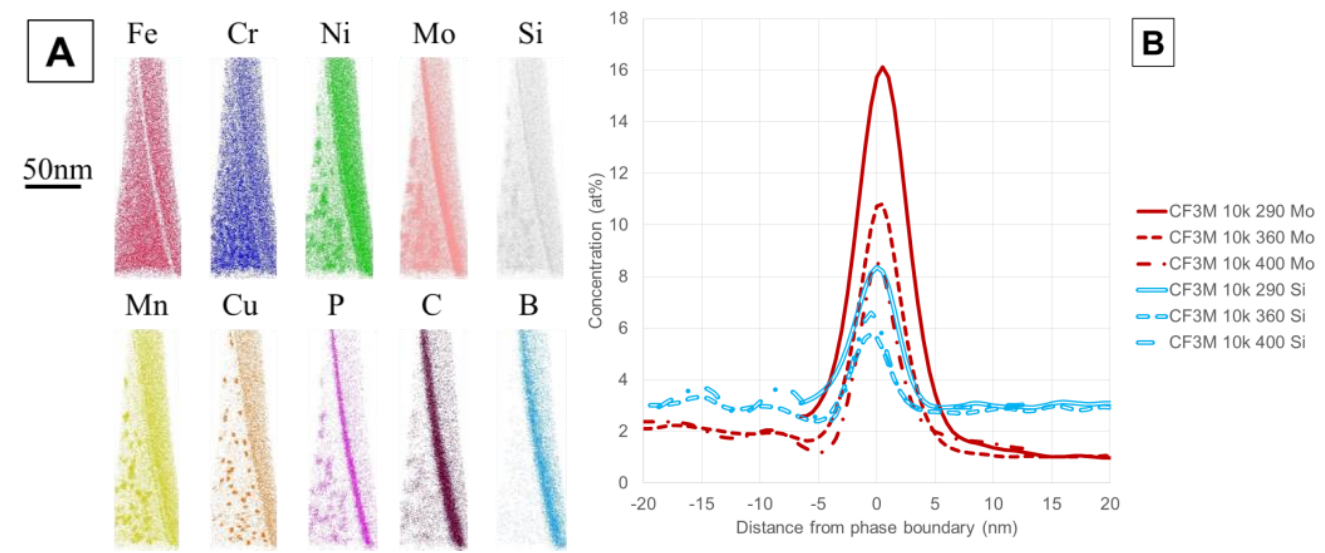

Figure 1: (a) Atomic position of elements in CF3M alloy aged at $400{ }^{\circ} \mathrm{C}$ for 10000 hours. (b) $1 \mathrm{D}$ concentration profile across the phase boundary (ferrite on left, austenite on right) of Mo and $\mathrm{Si}$ aged for 10000 hours at $290^{\circ} \mathrm{C}, 360^{\circ} \mathrm{C}$, and $400^{\circ} \mathrm{C}$. Concentration of Mo and Si, along with other elements, decrease at the phase boundary with increasing aging temperature. 


\section{Phase Separation near the Ferrite/Austenite Interface of a Duplex Stainless Steel}

Gang Sha ${ }^{1}$, Bo Zhang ${ }^{1}$, Shenbao Jin ${ }^{1}$, Shilei $\mathrm{Li}^{2}$, Xitao Wang ${ }^{2}$ and Fei Xue

Gang Sha: gang.sha@njust.edu.cn

1. School of Materials Science and Engineering, Nanjing University of Science and Technology, Nanjing, China.

2. Collaborative Innovation Center of Steel Technology, University of Science and Technology Beijing, Beijing, China.

3. Suzhou Nuclear Power Research Institute, China Guangdong Nuclear Group, Suzhou, China.

Atom probe tomography (APT), emerging as a key micro-analysis technique, has shown important applications in a wide range of advanced materials, including conductive metallic materials, semiconductors and biomaterials etc. The unique quantitative atomistic information about nanostructure and local chemistry of materials revealed by this advanced analysis technique has greatly advanced our understanding about the fundamental science of materials, and provided guidance for design and development of advanced materials.

This contribution will address phase separation near the ferrite/austenite interfaces of a duplex steel during aging at $400^{\circ} \mathrm{C}$ for up to $20,000 \mathrm{~h}$ [1]. FIB has been employed to make APT tip containing the ferrite/austenite interface. APT under UV laser pulsing have been employed successfully to reveal the distribution of elements at the interface. A strongly aging-induced segregation of $\mathrm{Ni}$ at the austenite/ferrite interfaces and the formation of a Ni depletion zone (NDZ) in the ferrite in the vicinity of the interfaces are observed. The unique phase separation behaviors are responsible for thermal embrittlement of the duplex stainless steel.

$\underline{\text { References: }}$

[1] Zhang B, Xue F, Li SL, Wang XT, Liang N, Zhao YH, Sha G*, Acta Mater., (2017)140:388-97 


\section{Effects of Thermal Ageing and Neutron Irradiation on a Stainless Steel Weld}

Kristina Lindgren ${ }^{1}$, Martin Bjurman ${ }^{2,3}$, Pål Efsing ${ }^{3,4}$, Peter Ekström ${ }^{5}$ and Mattias Thuvander ${ }^{1}$

Corresponding Author: kristina.lindgren@chalmers.se

1. Chalmers University of Technology, Department of Physics, Göteborg, Sweden.

2. Studsvik Nuclear AB, Nyköping, Sweden.

3. Royal Institute of Technology (KTH), Department of Solid Mechanics, Stockholm, Sweden.

4. Ringhals AB, Väröbacka, Sweden

5. Swedish Radiation Safety Authority, Solna, Sweden.

The structural material of nuclear power plants might deteriorate during operation due to thermal ageing and irradiation from the reactor core. Possible effects on the materials are an increase in hardness and tensile strength, and a decrease in ductility and fracture toughness. The austenitic welds of the core barrel of the decommissioned José Cabrera pressurised water reactor in Spain, also known as Zorita, containing a few percents of ferrite are no exception. Thermal ageing (at $280-285^{\circ} \mathrm{C}$ ) and neutron irradiation (between 0.15 and 2.0 dpa dependent on position) result in spinodal decomposition and G-phase $\left(\mathrm{Ni}_{16} \mathrm{Si}_{7} \mathrm{Mn}_{6}\right)$ formation in the ferrite [1], and formation of $\mathrm{Ni}_{\mathrm{x}} \mathrm{Si}$ in the austenite, see Figure 1.

In this project, the material was characterised using atom probe tomography. Challenges involved were site-specific specimen preparation in order to analyse the ferrite/austenite interfaces, finding reasonable reconstruction parameters and the inhomogeneous nature of the composition of the welds. Using the radial distribution function to obtain the wavelength and amplitude of the spinodal decomposition [2], it was found that there were no measureable difference in spinodal decomposition of the ferrite between 1 and $2 \mathrm{dpa}$. Also in the $0.15 \mathrm{dpa}$ material there were both G-phase clustering and spinodal decomposition.

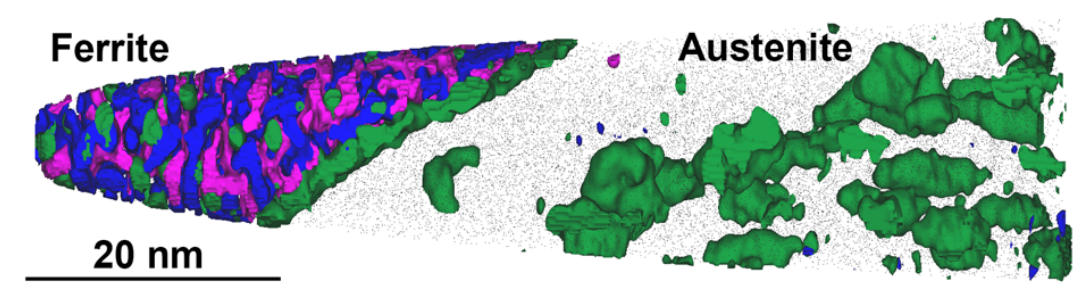

Figure 1 The ferrite/austenite interface in material irradiated to $1 \mathrm{dpa}$. In the ferrite, spinodal decomposition into Cr-rich and Fe-rich volumes is marked, as well as G-phase precipitates. In the austenite, NiSi-particles have formed. Si, Ni and $\mathrm{P}$ have segregated to the ferrite/austenite interface.

\section{References:}

[1] M. Bjurman, K. Lindgren, M. Thuvander, P. Ekström, P. Efsing, Microstructural Evolution of Welded Stainless Steels on Integrated Effect of Thermal Aging and Low Flux Irradiation, in: J.H. Jackson, D.

Paraventi, M. Wright (Eds.) the 18th International Conferece on Environmental Degradation of Materials in Nucler Power Systems - Water Reactors, Springer, 2018, pp. 703-710.

[2] J. Zhou, J. Odqvist, M. Thuvander, P. Hedstrom, Quantitative evaluation of spinodal decomposition in $\mathrm{Fe}-\mathrm{Cr}$ by atom probe tomography and radial distribution function analysis, Microsc Microanal 19(3) (2013) 665-75. 


\title{
Advanced Approaches to FIB-based Specimen Preparation of Cryogenically- prepared Specimens
}

\author{
Daniel E. Perea ${ }^{1}$ and Daniel K. Schreiber ${ }^{2}$ \\ Corresponding Author: daniel.perea@pnnl.gov \\ 1. Environmental \& Molecular Sciences Laboratory, Pacific Northwest National Laboratory, \\ Richland, WA USA. \\ 2. Energy \& Environment Directorate, Pacific Northwest National Laboratory, Richland, WA \\ USA.
}

The utilization of the focused ion beam scanning electron microscope (FIB-SEM) has aided the application of Atom Probe Tomography (APT) to a continually-growing variety of materials, including biological materials [1]. However, the regular application of APT to hydrated interfaces such as those found in biological and environmental materials is lacking in large part due to difficulties in specimen preparation under high and ultra-high vacuum conditions.

Although a FIB-SEM based cryogenic specimen preparation approach offers a rational route to the preparation of such specimens for APT analysis, many practical aspects such as welding and limitations in specimen manipulation become challenging. Here we describe our recent efforts in the development of a site-specific liftout and attachment scheme for cryogenically cooled specimens (Fig. 1). We will also describe a unique multifunctional environmental transfer hub (ETH) to allow controlled cryogenic transfer of specimens for APT analysis [2].
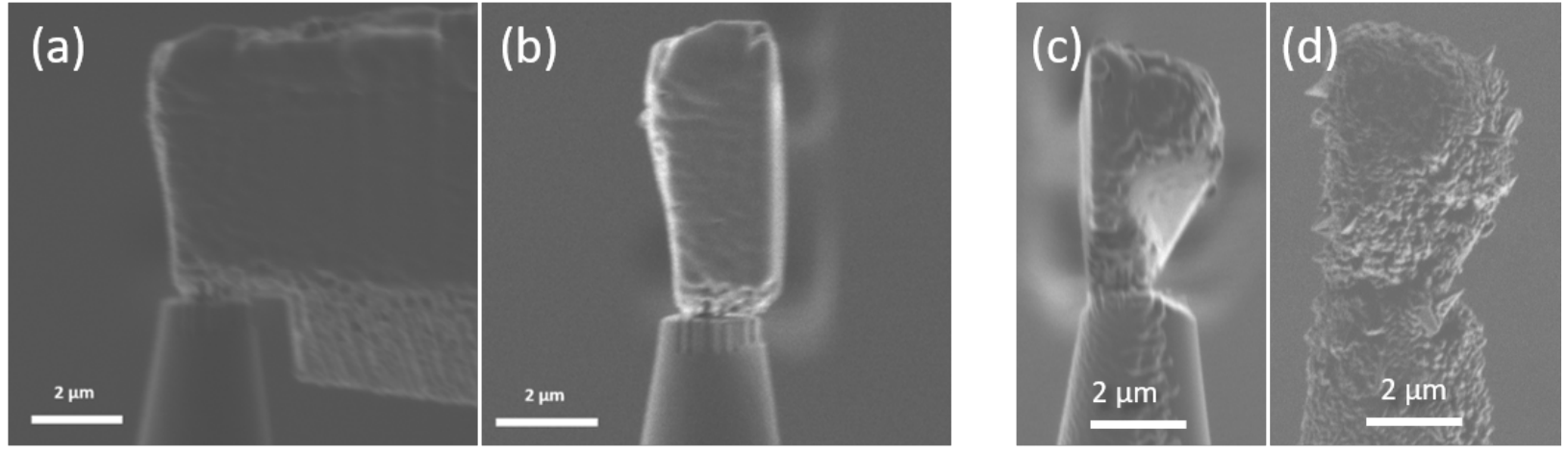

Figure 1 Attachment of a portion of a cryo-cooled liftout using (a-b) redeposition approach and (c-d) notch cut/overcoat of a GIS-delivered gas.

\section{$\underline{\text { References }}$}

[1] D.E. Perea et al. Atom Probe Tomography directly reveals the atomic distribution of phosphorus in resin embedded ferritin, Sci. Rep., 2016, 6:22321

[2] D.E. Perea et al. An environmental transfer hub for multimodal atom probe tomography, Adv. Struct. Chem. Imag., 2017, 3:12

[3] The research was performed at the Environmental Molecular Sciences Laboratory; a national scientific user facility sponsored by the Department of Energy's Office of Biological and Environmental Research located at Pacific Northwest National Laboratory. 


\title{
Atom Probe of Gas Exposed Metallic Surfaces: Isotopic and Surface Studies
}

\author{
D. Haley ${ }^{1}$, P. A.J. Bagot and M. Moody ${ }^{1}$
}

Corresponding Author: daniel.haley@materials.ox.ac.uk

${ }^{1}$. Dept. Materials, University of Oxford, Oxford, United Kingdom

The unambiguous identification of hydrogen within material microstructure via atom probe tomography is challenging, as hydrogen is ubiquitous within both atmospheric and high-vacuum environments. Consequently, to undertake such analyses, isotopically substitutional experiments (e.g. deuterium) are required [1] [2].

We demonstrate a flexible system, or "cell", for the introduction and transfer of samples under high vacuum. This cell allows for to the introduction of controlled gas atmospheres at room or elevated temperatures, and is directly connected to an atom probe for high-vacuum transfer and analysis. We present examples of experiments undertaken exposing specimen to deuterium gas, deuterated water vapour and oxygen, and demonstrate the reliable uptake of gas within these materials.

Based on these experiments, we discuss the implications of hydrogen detection in atom probe, and the limitations for detection in charged materials. We leverage recently developed software to allow for a linear decomposition of chemistry within a material, to provide quantitative bounds on the uptake rates of deuterium within our experiments, when samples are exposed to water and hydrogen environments.
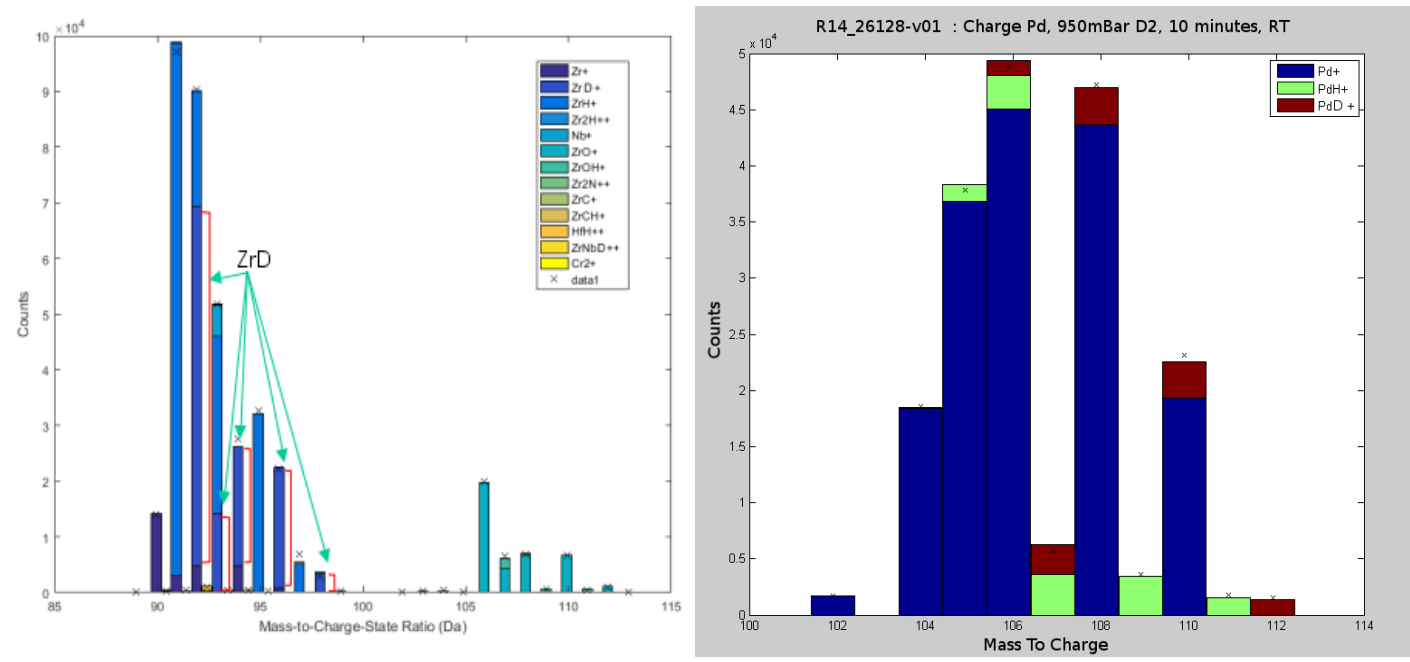

Figure 1 (a) Deuterium charged Zr, showing evaporation as ZrD. (b) D- gas charged Pd, showing decomposition of $\mathrm{PdH}$ and $\mathrm{PdD}$ species, after exposure

\section{References:}

[1] Sundell et al, Direct observation of hydrogen and deuterium in oxide grain boundaries in corroded Zirconium alloys, Corrosion Science, 90 (2015) 1-4

[2] Gemma, R, Analysis of deuterium in V-Fe5at.\% film by atom probe tomography (APT), Journal of Alloys and Compounds, 509 S2 (2011), S872-S876 


\title{
Simplifying Observation of Hydrogen Trapping in Atom Probe Tomography
}

\author{
Yi-Sheng Chen ${ }^{1}$, Daniel Haley ${ }^{1}$, Paul A.J. Bagot ${ }^{1}$, and Michael P. Moody ${ }^{1}$ \\ Corresponding Author: yi-sheng.chen@materials.ox.ac.uk \\ 1. Department of Materials, Oxford University, Oxford, UK
}

The presence of hydrogen within the microstructure of an alloy can lead to a serious reduction of ductility known as hydrogen embrittlement [1]. Some mitigation strategies against this phenomenon have been proposed such as minimizing hydrogen ingress with surface coatings or controlling internal hydrogen diffusion via the introduction of microstructural 'traps', e.g. second phase precipitates [2]. One system of interest in ferritic steels is a microstructure containing finely distributed nano-sized vanadium carbide precipitates, which shows good resistance to hydrogen environments [2]. However, there is a lack of experimental techniques to directly study the interaction between hydrogen and microstructural features, limiting ability to design effective microstructural hydrogen traps.

Atom Probe Tomography (APT) has been demonstrated by Takahashi et al. to have great potential in providing insight into hydrogen trapping [3]. They utilised a specifically modified atom probe instrument, which can provide an in-situ deuterium (D) loading and subsequent cryotransfer capability, to study trapped hydrogen in fine carbides. Charging the sample with D enables the hydrogen loaded in the material to be distinguished from the background hydrogen introduced into the APT analysis. However, there exist only few such modified systems. Hence a simplified experimental configuration to enable the direct observation of hydrogen via APT is proposed here. Following a straightforward electrolytic method to introduce D into APT needle specimens [4], a cryo-transfer protocol (Figure 1) was developed to retain charged D while transferring to the APT vacuum chamber. After electrochemical D loading (Fig. 1-a), the APT steel sample is rapidly loaded into a thermally-insulated atom probe holder, this is then immersed into liquid nitrogen $\left(\mathrm{LN}_{2}\right)$, attached to a pre-cooled cryo-block, and covered (Fig. 1-b). The entire $\mathrm{LN}_{2}$ unit is then moved into a glove box attached to an atom probe, in order to limit the supply of environmental moisture (Fig. 1-c). After loading into the atom probe, samples can be transferred, whilst still remaining at cryogenic temperatures throughout, and hence the charged D can be retained. This protocol requires no modification to commercial atom probe designs and is believed to be able to reasonably reproduce at any other laboratory equipped with an atom probe.

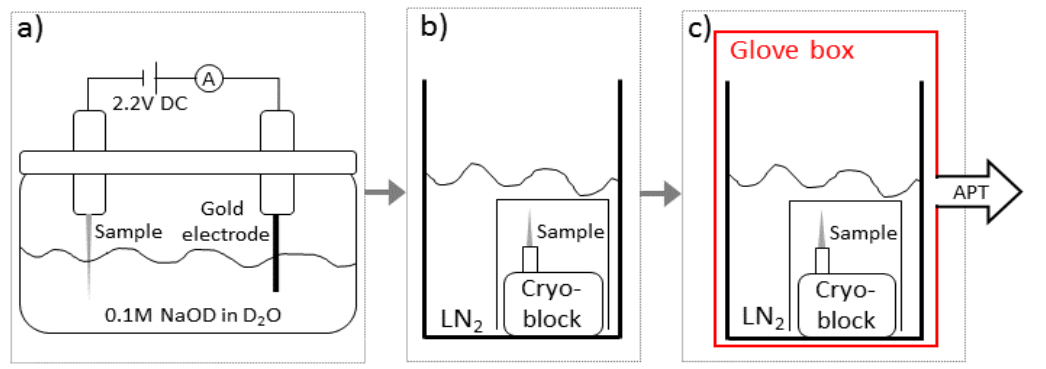

Figure 1 Schematics of the configuration of (a) electrochemical charging of deuterium; (b) and (c) the proposed cold-chain for retaining charged deuterium for APT analysis.

\section{$\underline{\text { References: }}$}

[1] I.M. Robertson, et al., Metall Mater Trans A 46a(2015) 2323-2341.

[2] H. Bhadeshia, Isij Int 56(2016) 24-36.

[3] J. Takahashi, et al., Scripta Mater 67(2012) 213-216.

[4] D. Haley, et al., Int J Hydrogen Energ 39(2014) 12221-12229. 


\section{LEAP ${ }^{\circledR}$ Analysis Conditions and Specimen Survivability}

Ty J. Prosa ${ }^{1}$, Isabelle Martin ${ }^{1}$, and David J. Larson ${ }^{1}$

\section{Corresponding Author: ty.prosa@ametek.com}

${ }^{1 .}$ CAMECA Instruments, Inc., 5470 Nobel Drive, Madison, Wisconsin 53711, USA.

Specimen survivability is a primary concern to those who utilize atom probe tomography (APT) for materials analysis. The state-of-the-art in understanding survivability might best be described as common-sense application of basic physics principles to describe failure mechanisms. For example, APT samples are placed under high tensile stress, so applying a lower force to initiate field evaporation should obviously provide for higher survivabilitycommon sense explanation of first-order analysis yield. To date, the interplay of various conditions (or instrumentation) and how they influence yield (e.g. decreasing the evaporation field or force improves yield), and which factors are more impactful than others has not been substantially studied.

In this presentation, we report on the systematic analysis of a standard sample structure composed of doped and implanted poly-silicon on $12 \mathrm{~nm}$ silicon oxide on a standard $<100>$ silicon substrate [1,2]. More than 250 specimens were manufactured using standard FIB-lift-out techniques and analyzed by LEAP [3] under a variety of conditions to correlate statistically significant survivability trends with analysis conditions and other specimen characteristics. The primary result suggests that while applied field/force plays an obvious role in survivability for this specimen type, the applied field alone does not predict survival. The rate at which ions are extracted from the specimen (both in terms of ions-per-pulse and pulse-frequency) provides similar importance.

Properly interpreting APT data to provide insights into optimizing APT analyses requires linking various aspects of the detected signal with the underlying physics. Besides, temporal, spatial, and chemical information, data can be additionally interpreted to provide new information (i.e. charge-state-ratio (CSR) as a proxy for apex temperature or evaporation field [4]). In this presentation, we will also present CSR data collected from pure silicon and silicon dioxide samples, enabling reliable interpretation of CSR from our compound yield sample. We find that $\mathrm{Si}$ CSRs do not follow the same field relationships in pure $\mathrm{Si}$ versus $\mathrm{SiOx}$.

\section{References:}

[1] A. Portavoce, R. Simola, D. Mangelinck, J. Bernardini, P. Fornara, Dopant Diffusion during Amorphous Silicon Crystallization, Defect and Diffusion Forum. 264 (2007), 33-38.

[2] A. Portavoce, D. Mangelinck, R. Simola, R. Daineche, J. Bernardini, Atom Redistribution during coDoped Amorphous Silicon Crystallization, Defect and Diffusion Forum. 289-292 (2009), 329-337.

[3] D.J. Larson et al., Local Electrode Atom Probe Tomography, Springer, New York, 2013.

[4] D.R. Kingham, The Post-Ionization of Field Evaporated Ions: A Theoretical Explanation of Multiple Charge States, Surf. Sci. 116 (1981), 273.

[5] The authors wish to acknowledge former group members who contributed to this project (S. Strennen, D. Olson), as well as A. Portavoce and D. Mangelinck (IM2NP) for providing the samples material, and R. Martins and J. Goodwin (University of Alabama) for additional TEM characterization. 


\title{
Evaluation of the Atomic Level Mass Analysis of Biomolecules by the Scanning Atom Probe Alternately Triggered by Voltage and Laser Pulses
}

Masahiro Taniguchi ${ }^{1}$, and Osamu Nishikawa ${ }^{2}$

\author{
Corresponding Author: oynishikawa@gmail.com \\ 1. Department of Applied Chemistry, Kanazawa Institute of Technology, Nonoichi, Japan \\ 2. Office of Industry-University Collaboration, Kanazawa Institute of Technol., Nonoichi, Japan
}

Fundamental requirement for the mass analysis of biomolecules is to be quantitative and qualitative. The scanning atom probe (SAP) successfully demonstrated that the obtained mass spectra are quantitative and qualitative $[1,2]$. These mass analyses were proceeded applying positive voltage pulses to a specimen. In order to examine the advantage of laser pulses the SAP alternately triggered by voltage and laser was developed. Figures 1 and 2 show the mass spectra of tryptophan obtained by the alternately triggering SAP [3]. The laser gives a better mass resolution because of its narrower pulse width, 2 ps. Both spectra show the characteristic mass peaks of tryptophan such as $\mathrm{NH}_{2} \mathrm{CH}$ and $\mathrm{COOH}$. However, the unexpected finding is that the mass peaks corresponding to the indoxyl section of tryptophan which is indicated by a red circle in the Figure 1 and 2 were not detected. In order to substantiate the obtained results tryptophan was mass analyzed by the high mass resolution SAP used for other biomolecules. The mass analysis was conducted applying voltage pulses at room temperature because biomolecules are at their natural state and laser beams dissociate the molecules by the irradiate laser beam. The obtained mass spectrum clearly shows the mass peak at $m / n=57$ which corresponds to the doubly charged indoxyl $\left(\mathrm{C}_{8} \mathrm{NH}_{4}\right)^{2+}$. The mass of a fragment of the tryptophan, $\mathrm{OCHCNH}_{2}$, is also 57 . However, left out fragment, $\mathrm{OH}$, are not detected. Accordingly, the mass peak at $\mathrm{m} / \mathrm{n}=57$ could be the doubly charged indoxyl $\left(\mathrm{C}_{8} \mathrm{NH}_{4}\right)^{2+}$.

Mass spectra of the molecules with a benzene ring, tyrosine and phenylalanine, will be presented. Fragments of pyrrolidine of proline and pyrrole of histidine are also presented.

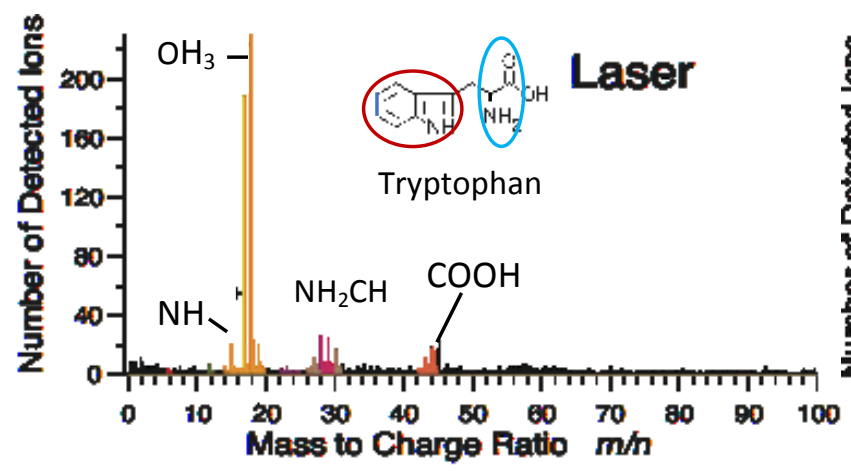

Figure 1. Mass spectrum obtained by triggering laser pulses. Indoxyl and $\mathrm{OCHCNH}_{2}$ fragments are indicated by red and blue loops,

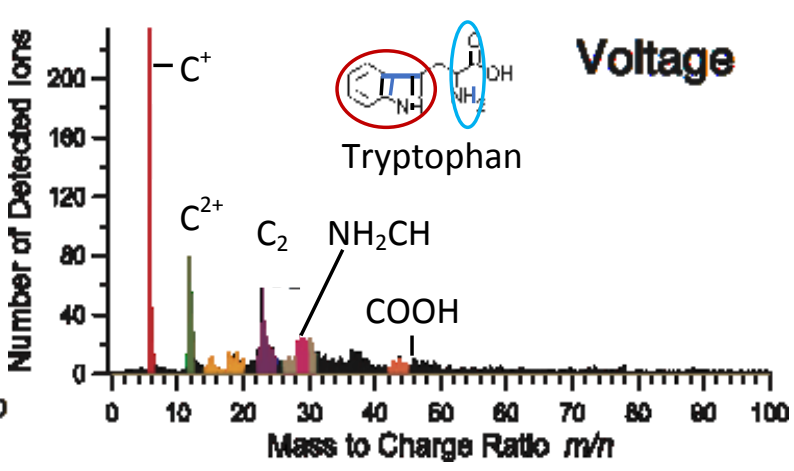

Figure 2. Mass spectrum obtained by triggering voltage pulses. Red and blue loops are indoxyl and $\mathrm{OCHCNH} 2$.

\section{References}

[1] O. Nishikawa and M. Taniguchi, Toward the Atomic Level Mass Analysis of Biomolecules by the Scanning Atom Probe, Micros. Microanal., 23, 336-339 (2016).

[2] O. Nishikawa, M. Taniguchi, and A. Ikai, Atomic level analysis of biomolecules by the scanning atom probe, Appl. Surf. Sci., 256, 1210-1213 (2009).

[3] Masahiro Taniguchi and Osamu Nishikawa. Scanning Atom Probe Analysis Alternately Triggered by Voltage and Laser Pulse, e-J. of Surf. Sci. and Nanotechnol., 14, 69-72 (2016). 


\section{Biased Solute Reconstruction due to Athermal Surface Drag}

Christian Oberdorfer ${ }^{1}$, Travis Withrow ${ }^{1}$, Iman Ghamarian ${ }^{2}$, Li-Jen $\mathrm{Yu}^{2}$, Kevin Fisher ${ }^{2}$, Emmanuelle Marquis ${ }^{2}$, and Wolfgang Windl ${ }^{1}$

Corresponding Author: oberdorfer.4@,osu.edu

1. Department of Materials Science and Engineering, The Ohio State University, Columbus, USA.

2. Department of Materials Science and Engineering, University of Michigan, Ann Arbor, USA.

The atom probe tomography (APT) literature reports many examples of enrichment of solute atoms at densely packed, low-indexed crystallographic poles (fig. a). These findings have generally been explained by directed surface diffusion in a field-gradient that is activated by finite tip temperatures [1]. In order to better understand the mechanisms for such solute movement on the emitter surface, we conducted simulations of the evaporation process with a combined electrostatic and molecular dynamic (MD) approach, or TAPSim-MD. This method allows for atomistic relaxation of the emitter surface before each evaporation event. Simulations of an Al-Cu emitter structure show surprising agreement with experimental observations (fig. b). Detailed analysis of changes on the apex surface during evaporation reveal a net movement of $\mathrm{Cu}$ atoms that follow receding $\{111\}$ terrace ledges toward [111] poles. Confirmed by density functional theory (DFT) calculations, this athermal movement of $\mathrm{Cu}$ atoms is due to the combination of elevated $\mathrm{Cu}$ evaporation field compared to that of $\mathrm{Al}$ and different $\mathrm{Al}-\mathrm{Cu}, \mathrm{Cu}-\mathrm{Cu}$ and $\mathrm{Al}-\mathrm{Al}$ bond strengths. The observed $\mathrm{Cu}$ drag-effect represents a fundamental limitation to the $3 \mathrm{D}$ reconstruction of solute atoms such as $\mathrm{Cu}$ in $\mathrm{Al}$ at low temperatures. While this effect cannot be corrected by adjusting evaporation conditions, it may be limited by increasing temperature (i.e. increased randomization) at which point field-driven diffusion may become the dominant mechanism.
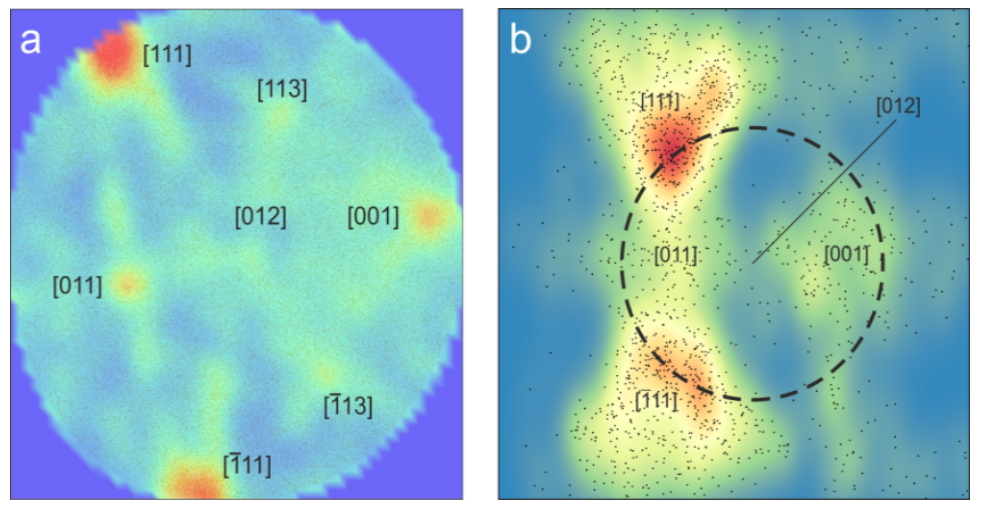

Figure 1: (a) Analysis of $\mathrm{Cu}$ detector hit-density in a Al-96 Cu-4 (at .\%) alloy. Despite the $\mathrm{Cu}$ being in solid solution, enrichment at [111] poles and along low indexed zone lines is seen. (b) The same result obtained from simulated evaporation with the TAPSim-MD approach. Experimen-tal field-of-view is indicated by the inscribed circle.

References:

[1] B. Gault, F. Danoix, K. Hoummada, D. Mangelinck, and H. Leitner; "Impact of directional walk on atom probe analysis"; Ultramicroscopy 113 (2012), 182-191 


\title{
Atomic Level Studies of Step Dynamics in Homogeneous Systems
}

\author{
Matthew Koppa, P. R. Schwoebel, and David Dunlap \\ Corresponding Author: mkoppa@unm.edu \\ Department of Physics and Astronomy, University of New Mexico, Albuquerque, USA.
}

Step dynamics is one of the most important processes governing crystal growth and is therefore of great interest to many technological applications. For example, there are two distinct mechanisms by which a crystal terrace can grow as an adatom diffuses to a step edge, as shown in Figure 1. The adatom may descend either directly as shown in Fig. 1.a or by exchange with a step edge atom as shown in Fig. 1b. Both mechanisms have been observed in heterogenous systems, i.e. adatom-substrate combinations of different elements, using the field ion microscope (FIM) [1]. The FIM can only discern the chemical difference between atoms by relying on a difference in evaporation field and this approach precludes the study of the prototypic case of homogeneous systems.

To investigate step dynamics in homogeneous systems, we are using an atom probe in combination with isotopically enriched material. A number of elements, such as tungsten, have rare, stable isotopes that are available in high purity. By depositing the rare isotope on the substrate, the chemistry, and thus step dynamics, of the homogeneous system is achieved, yet the position of the rare isotope adatom is now identifiable by mass analysis with the atom probe. After the isotope adatom 'descends' at the step edge, the outermost boundary atom is mass analyzed to reveal whether or not exchange occurred. We will present results of our studies to date and what future directions they indicate are worthy of further study.

a)

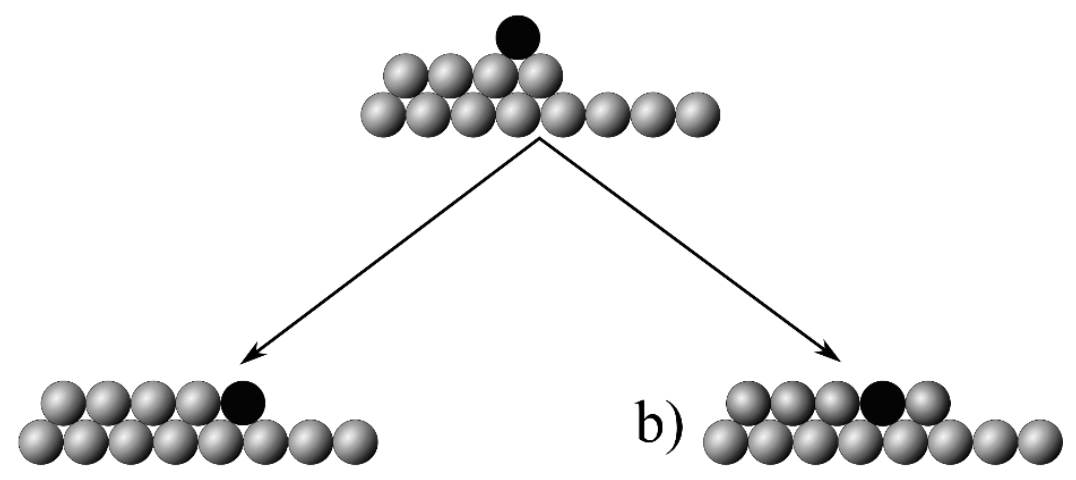

Figure 1 (a) Adatom directly descends over a step edge. (b) Adatom descends by the exchange mechanism

References:

[1] S.C. Wang, G. Ehrlich, Atom Incorporation at Surface Clusters, Physical Review Letters 67 (1991), 2509-2512. 


\title{
Molecular Dynamics Modeling of Surface and Subsurface Processes in Metals Under the Influence of High Electric Fields
}

\author{
S. Parviainen ${ }^{1}$, F. Vurpillot ${ }^{1}$
}

Corresponding Author: stefan.parviainen@univ-rouen.fr

1. Normandie Univ., CNRS, Groupe de Physique des Matériaux, 76000 Rouen, France

Atom Probe Tomography (APT) is an extremely promising technique, with potential to deliver information about the structure and chemical composition of a studied sample in three dimensions with atomic precision. The method relies on the controlled evaporation of individual atoms triggered by an extremely high electric field. While a vast amount of data can be collected in this way, it is not always clear how to interpret it. Even though the data is collected over an extended period of time, the final result is often treated as a static snapshot of the sample, without consideration of some of the dynamic effects that may occur during the measurement itself, such as defect migration.

These kind of effects are minimized by keeping the sample at cryogenic temperatures, which under normal conditions practically eliminates all atomic motion. However, the extremely high electric field present during measurements, tens of $\mathrm{V} / \mathrm{nm}$, reduces migration barriers by inducing strong forces on the atoms in the sample. Thus, a sample may not always remain static, even at the low temperatures used in APT.

In the present work we utilize molecular dynamics simulations to study the motion of ad-atoms on an $\mathrm{W}$ atom probe sample as well as explore some of the possible effects this migration may have, including triggering field evaporation at random points along a pole ledge, and inducing roll-up motion of atoms from lower planes to higher planes. Results show that adatom diffusivity is substantially increased under high electric fields, and increases with increasing field, until the critical evaporation field is reached and evaporation, rather than migration, occurs.

We also study the time-evolution of radiation induced defects in W APT samples in order to determine if the observed defect configuration, in particular defect density and depth, is the result of the initial irradiation, or a result of the imaging process itself. The results show that the mobility of some defect types, such as crowdions, is increased under typical imaging conditions, resulting in defects migrating towards the surface. Thus, the observed depths distribution of defects may be the result of the imaging process itself, rather than the result of the initial radiation.
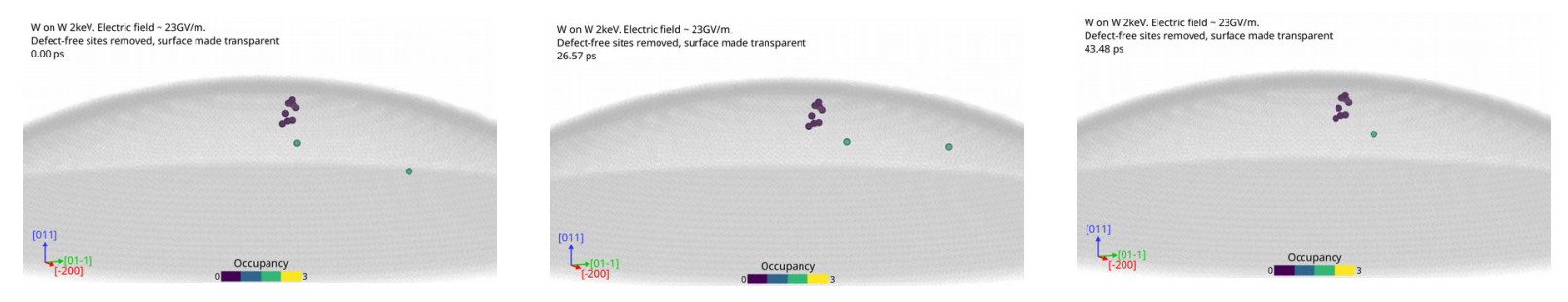

Figure 1: Simulations snaphots showing radiation damage evolution: a stable vacancy cluster and two interstitial atoms, of which one migrates to the surface 


\section{Open Discussion}




\section{Towards Mapping of Trace Elements in Bio-Minerals}

S. Koelling ${ }^{1}$, T. L. Martin ${ }^{2}$, M. Verheijen ${ }^{1,3}$, S. Agarwal ${ }^{4}$, S. Bertazzo ${ }^{5}$, A.J. Fijneman ${ }^{6}$, H. E. King ${ }^{7}$, A. Akiva $^{6}$, P. A. J. Bagot ${ }^{8}$, M. P. Moody ${ }^{8}$, N.A.J.M. Sommerdijk ${ }^{6}$, M.M. Stevens ${ }^{4}$, \& P.M. Koenraad ${ }^{1}$

S. Koelling: s.kolling@tue.nl

${ }^{1}$ Department of Applied Physics, Eindhoven University of Technology, Netherlands

${ }^{2}$ School of Physics, University of Bristol, UK

${ }^{3}$ Philips Innovation Services Eindhoven, Netherlands

${ }^{4}$ Department of Materials, Imperial College London, UK

${ }^{5}$ Department of Medical Physics \& Biomedical Engineering, University College London, UK

${ }^{6}$ Department of Chemical Engineering and Chemistry, Eindhoven University of Technology, Netherlands

${ }^{7}$ Department of Earth Sciences, Utrecht University, Netherlands

${ }^{8}$ Department of Materials, Oxford University, UK

Atom Probe Tomography (APT) has recently been utilized to explore the structure of biological materials like bones [1] or teeth [2]. Unfortunately, the inhomogeneity of the materials and the small volumes imaged in APT make it challenging to generate reproducible analyses and assess the accuracy of the analyses. Here, we will show that applying APT to explore bio-minerals is promising as both the pure mineral and the pure organics can be field evaporated and mass resolutions that allow for the identification of elements below the threshold accessible in EDX or EELS are attainable. However, our analyses on various bio-minerals from mice, sea urchins and humans also show the difficulties arising from analyzing these inhomogeneous materials on such a small scale and highlight the need for 'test materials' that allow to evaluate both the preparation procedures and the measurement protocols utilized to enable APT.

a

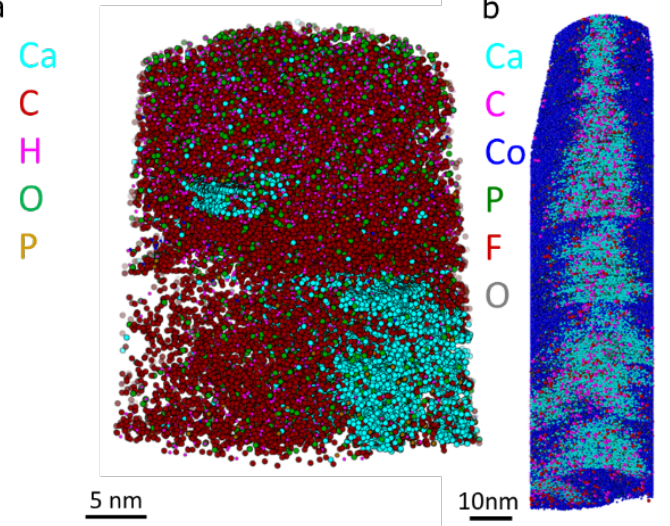

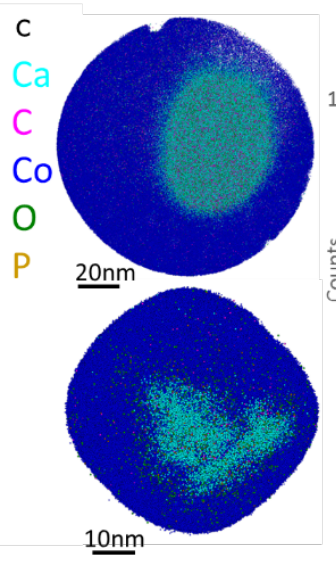

d

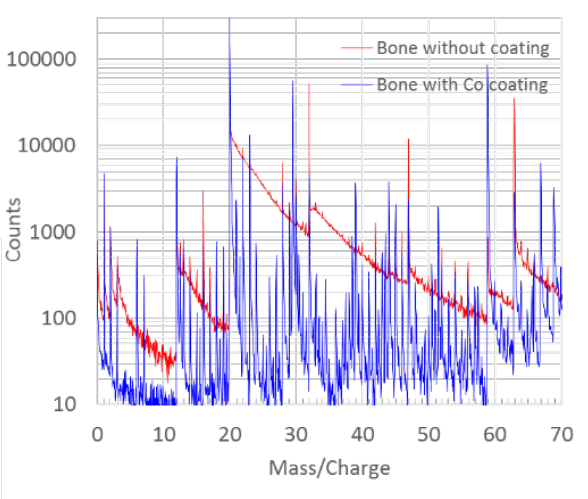

Figure 1: APT analyses of mouse bone $(a, b)$, showing a carbon rich (a) and a mineral rich (b) region embedded in Co. The cross-section perspective (c) reveals that coated APT tips made from bone (c, bottom) via focused ion beam milling do not generally form circular tips as opposed to tips made from artificial mineral crystals (c, top). Mass spectra (d) of APT analysis of mouse bone highlight the need for coating the tips to improve the mass resolution.

\section{References:}

[1] Lyle M. Gordon, Lawrence Tran, and Derk Joester, Atom Probe Tomography of Apatites and BoneType Mineralized Tissues. ACS Nano, 6, 10667 (2012).

[2] Lyle M. Gordon \& Derk Joester, Nanoscale chemical tomography of buried organic-inorganic interfaces in the chiton tooth, Nature, 469, 194 (2011). 


\section{Experiences in APT Analyses of Fluids}

Stephan S.A. Gerstl ${ }^{1,2}$, Robin Schaeublin ${ }^{1,2}$

Corresponding Author: gerstls@ethz.ch

1. Scientific Center for Optical and Electron Microscopy, ETH, Auguste-Piccard-Hof 1, 8093 Zurich, Switzerland.

2. Laboratory of Metal Physics \& Technology, ETH, Vladimir-Prelog-Weg 4, 8093 Zurich, Switzerland.

Characterizing materials at or near the atomic scale, particularly in 3-dimensions, has become synonymous with atom probe tomography - with the commonly accepted definition of 'materials' as being hard and dense in nature. This is demonstrated in that well over $95 \%$ of the atom probes worldwide characterize materials such as metals, semiconductors, oxides, \& minerals (including bio- minerals). After enabling the first vacuum cryogenic transfer of samples into a LEAP platform at ETH Zurich [1,2], multiple analyses of substances with varying degrees of softness have been performed, ranging from viscous syrups to doped aqueous solutions. Now, the correlation to cryo-TEM has been achieved for the first time, on a sample of syrup containing Au nanoparticles.

Furthermore, fluid mass spectra standards were cryogenically arrested, transferred, and analyzed to achieve baselines for data extraction. Results, alongside further developments in cryopreparation methodologies with correlative cryo-TEM observations and evaluation of the partially unknown analysis parameter spaces, will be presented. They show the efficacy of APT's mass spectral analytical abilities and corresponding potential applications of APT to fluids, coupled to TEM observations.

\section{References:}

[1] S.S.A. Gerstl, R. Wepf, Microsc. Microanal. 21, 517 (2015).

[2] Moody M.P., Vella A., Gerstl S.S.A., Bagot P.A.J., MRS Bulletin, 41, pp 40-45 (2016). 


\section{Atom Probe Tomography of Silica-Encapsulated Proteins}

Gustav Sundell ${ }^{1}$, Mats Hulander ${ }^{1}$, Astrid Pihl ${ }^{1}$, and Martin Andersson ${ }^{1}$

Corresponding Author: gustav.sundell@chalmers.se

1. Chalmers University of Technology, Department of Chemistry and Chemical Engineering, Gothenburg, Sweden.

Proteins are the building blocks of life and participate in virtually all processes within living organisms. Protein receptors in the cell membrane govern transport in and out of the cell, and are therefore the target for over $50 \%$ of all modern medicinal drugs [1]. A critical aspect of the field of structural biology is to characterize the structure of proteins in three dimensions. Knowledge of the shape of a e.g. a transmembrane protein can allow for design of new pharmaceutical substances that inhibit virus infections [2].

Traditionally, the functional structure of proteins has been determined using X-ray crystallography or nuclear magnetic resonance (NMR). However, X-ray crystallography can only be applied to proteins in a crystallized state, which restricts its applicability. NMR often relies on excessively complex computer calculations, which limits the technique to the study of small molecules. More recently, development of direct electron detectors has sparked a revolution in cryo electron microscopy for structural biology applications.

Here, we show that atom probe tomography may constitute a complementary method for proteomics, providing both chemical information as well as with 3D structure. We have encapsulated the human antibody Immunoglobulin G (IgG) in a solid silica glass matrix to provide the requisite mechanical stability to allow for atom probe analysis.
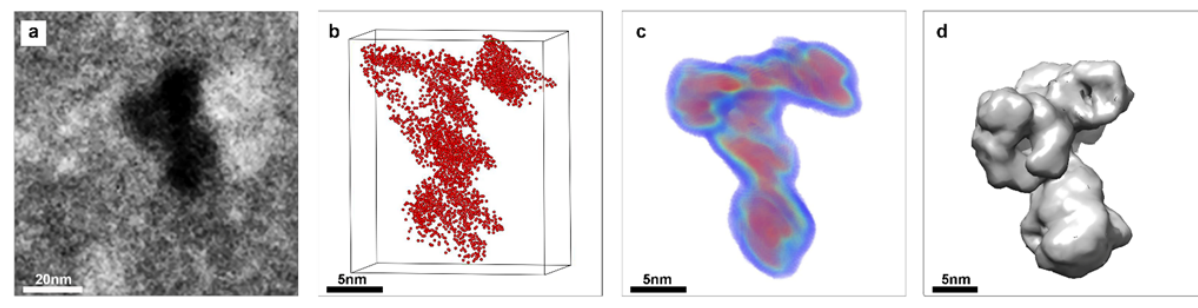

Figure 1 (a) TEM micrograph of a silica-embedded IgG, (b) and (c) atom probe reconstructions of IgG, (d) reference pdb structure of IgG as retrieved from the protein databank.

We present atom probe reconstructions of the antibodies, both in an aggregated and monomer state. We compare the results with known structures retrieved from the protein databank [3]. The challenges and opportunities that are associated with this approach are discussed.

\section{References:}

[1] Overington, J. P., Al-Lazikani, B. \& Hopkins, A. L. How many drug targets are there? Nat Rev Drug Discov 5, 993-996 (2006).

[2] Kielian, M. \& Rey, F. A. Virus membrane-fusion proteins: more than one way to make a hairpin. Nat Rev Micro 4, 67-76 (2006).

[3] Berman, H. M. et al. The protein data bank. Nucleic Acids Res. 28, 235-242 (2000). 


\title{
Atom Probe Tomography and Correlative Microscopy for Biological Materials
}

\author{
Xiaoyue Wang ${ }^{1}$, Bryan E.J. Lee ${ }^{2}$, Brian Langelier ${ }^{3}$, and Kathryn Grandfield ${ }^{1,2}$ \\ Corresponding Author: kgrandfield@mcmaster.ca \\ 1. Department of Materials Science and Engineering, McMaster University, Hamilton, Canada. \\ 2. School of Biomedical Engineering, McMaster University, Hamilton, Canada. \\ 3. Canadian Centre for Electron Microscopy, McMaster University, Hamilton, Canada.
}

Uncovering the mechanisms of biomineralization or biomaterial-tissue interactions is complicated by the complex and 3D hierarchical structure and chemical heterogeneities of many natural biominerals. Elucidating the multi length-scale spatial and chemical structure of bone has the potential to shed light on mineralization mechanisms and lead to improvements in the design of biomaterials for joint replacement and dental applications.

Atom probe tomography (APT), as a microanalysis technique that can provide sub-nm 3D spatial resolution and high elemental sensitivity, is uniquely positioned to provide valuable observations on the structure, formation and attachment of human bone to biomaterials. In our work, we complement APT analysis with other advanced correlative microscopy approaches. This talk will introduce this range of correlative 3D to $4 \mathrm{D}$ techniques to investigate mineralized bone and the bone-implant interface, including on-axis electron tomography, and electron energy loss spectroscopy tomography to reveal structural and chemical heterogeneities in bone and at biointerfaces [1]. These correlative microscopies provide a foundation for understanding the structure and chemical nature of inorganic and organic hierarchical materials.

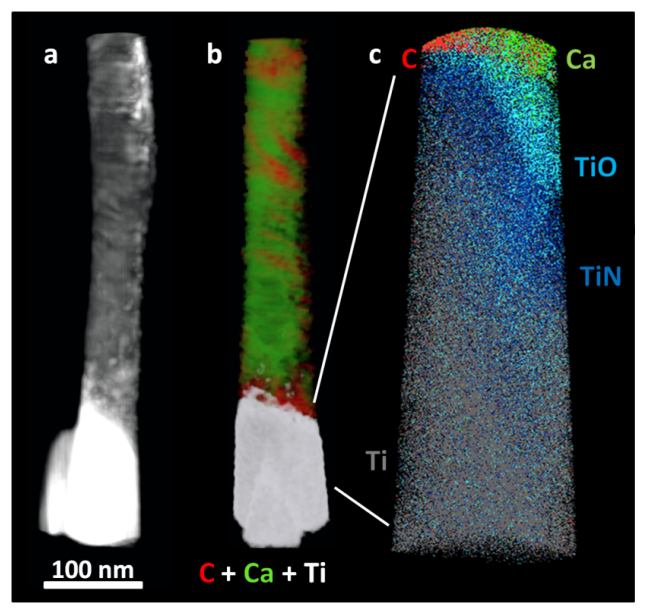

Figure 1. Atom probe tomography (APT) and correlative 4D EELS tomography of a human bone implant interface, including (a) on-axis electron tomography, (b) corresponding EELS tomography, and (c) APT of a small fraction of the same sample at the interface.

References:

[1] B. Langelier, X. Wang, K. Grandfield, Atomic scale chemical tomography of human bone, Scientific Reports, 7 (2017), 39958.

[2] Microscopy was conducted at the Canadian Centre for Electron Microscopy, a center supported by the Natural Sciences and Engineering Research Council of Canada and other government agencies. 


\section{Investigation of the Ion Distribution in Human Dental Enamel}

Karen DeRocher ${ }^{1}$, P. Smeets ${ }^{1}$, M. Cohen ${ }^{2}$, L. Gordon ${ }^{3}$, and D. Joester ${ }^{1}$

Corresponding Author: d-joester@northwestern.edu

${ }^{1}$ Department of Materials Science and Engineering, Northwestern University, Evanston, IL 60208, USA

${ }^{2}$ Johnson and Johnson, New York City, NY 10001, USA

${ }^{3}$ Nano Precision Medical, Emeryville, CA 94608, USA

Dental caries, commonly called tooth decay, is the most prevalent infectious disease in the world, affecting $60-90 \%$ of children worldwide and nearly all adults. The primary target of caries, enamel is composed of woven bundles of hydroxyapatite (OHAp) nanowires. Using a combination of atom probe tomography (APT) and X-ray absorption spectroscopy (XAS), we discovered that, in rodent enamel, nanowires are separated by an amorphous intergranular phase (AIP)[1]. We further established that untreated enamel, in which the AIP is Mg-substituted amorphous calcium phosphate (Mg-ACP), is mechanically weaker and more susceptible to acid dissolution than pigmented enamel, in which the AIP may be comprised of Casubstituted amorphous ferric phosphate (Ca-AFP)[1]. The composition of the AIP thus strongly effects enamel properties. While the crystalline structure of enamel has been studied extensively, little is known about the chemical and structural changes that occur in the interphase region during caries formation, and the role this region plays in dissolution of OHAp crystallites over long periods of time [2].

Here we present preliminary experiments confirming the existence of the intergranular phase reported by La Fontaine et al [3]. We show evidence that the ion distribution inside human enamel crystallites is much more complex than previously observed. Trace elements such as $\mathrm{Mg}, \mathrm{F}$, and $\mathrm{Na}$ are enriched not only between the crystallites, but also within them (Figure 1). In addition, the $\mathrm{Mg}$ and $\mathrm{F}$ distributions show a linear feature in the center of the crystallites, the size and location of which are consistent with the presence of a central dark line (CDL) in enamel observed previously [4], and by our own transmission electron microscopy (TEM) experiments. This complex ion distribution suggests the crystal may experience mechanical strengthening due to residual stresses that result from the relatively high concentration of impurity ions in the OHAp crystal structure. We have developed a preliminary finite element model (FEM) to investigate the magnitude of stress the $\mathrm{Mg}$ distribution may cause inside the crystallite. Looking forward, we will use X-ray nano-diffraction, TEM, and electron diffraction to confirm the results of our FEM.

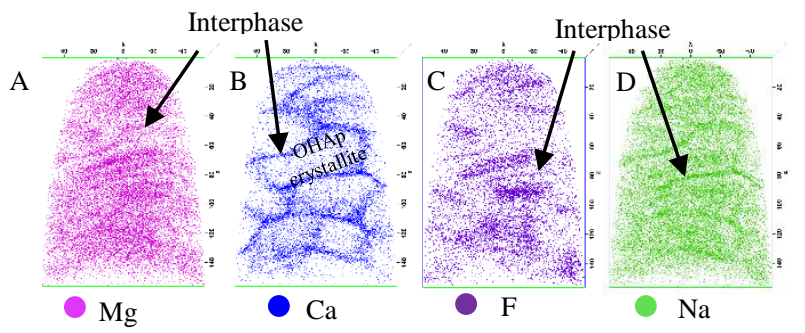

Figure 1: APT reconstructions of fluoridate human enamel showing distribution of minority ions.

References:

[1] Gordon, L. M. et al, Amorphous Intergranular Phases Control the Properties of Rodent Tooth Enamel. Science 2015, 347 (6223), 5.

[2] Robinson, C. et al, The Chemistry of Enamel Caries. Crit. Rev. Oral Bio. \& Med. 2000, 11 (4), 15.

[3] Alexandre La Fontaine et al, Atomic-scale compositional mapping reveals Mg-rich amorphous calcium phospahte in human dental enamel. Sci. Adv. 2016, 2 (9).

[4] Reyes-Gasga, J. et al, Aberration-Corrected Transmission Electron Microscopy Study of the Central

Dark Line Defect in Human Tooth Enamel Crystals. Microscopy and Microanalysis 2016, 22, 9. 


\title{
Atom Probe Tomography of Sound and Carious Rodent Tooth Enamel
}

\author{
Robert Free ${ }^{1}$ and Derk Joester ${ }^{1}$ \\ Corresponding Author: RFree@u.northwestern.edu \\ 1. Northwestern University, Department of Materials Science and Engineering, Evanston, IL, \\ USA
}

Dental caries, one of the world's most prevalent infectious diseases, progresses via the acidic dissolution of tooth enamel. Recent explorations into the nanoscale composition and structure of enamel through atom probe tomography (APT) have demonstrated that the composition of amorphous intergranular phases (AIGPs) between the hydroxylapatite nanowires comprising enamel strongly affect the in vitro acid susceptibility of the overall tissue. [1] Building on these findings, the objective of the current investigation is to explore how the composition of these AIGPs influences and is affected by lesion development in vivo. A rodent caries model has been employed to generate a large pool of early-stage subsurface lesions which are mapped in 3D via X-ray microtomography. [2] Using these maps, lesions are mechanically sectioned and APT tips prepared via FIB-SEM liftout and sharpening. APT reconstructions of volumes taken from sound, mildly demineralized, and intact surface zone enamel reveal some 3-5nm wide regions of magnesium enrichment similar to the AIGPs observed in the enamel of many other species, but with much less order than previously observed (figure 1). Preliminary comparisons between the Mg-rich regions in tips from each enamel location also suggests a decrease in detectable $\mathrm{Mg}$ segregation with increasing severity of demineralization. Applying this approach to a large number of extracted enamel volumes will enable detailed comparisons of the changes to composition within AIGPs during caries, and elucidate how the acid susceptibility of these boundary regions plays a role during caries initiation in vivo.
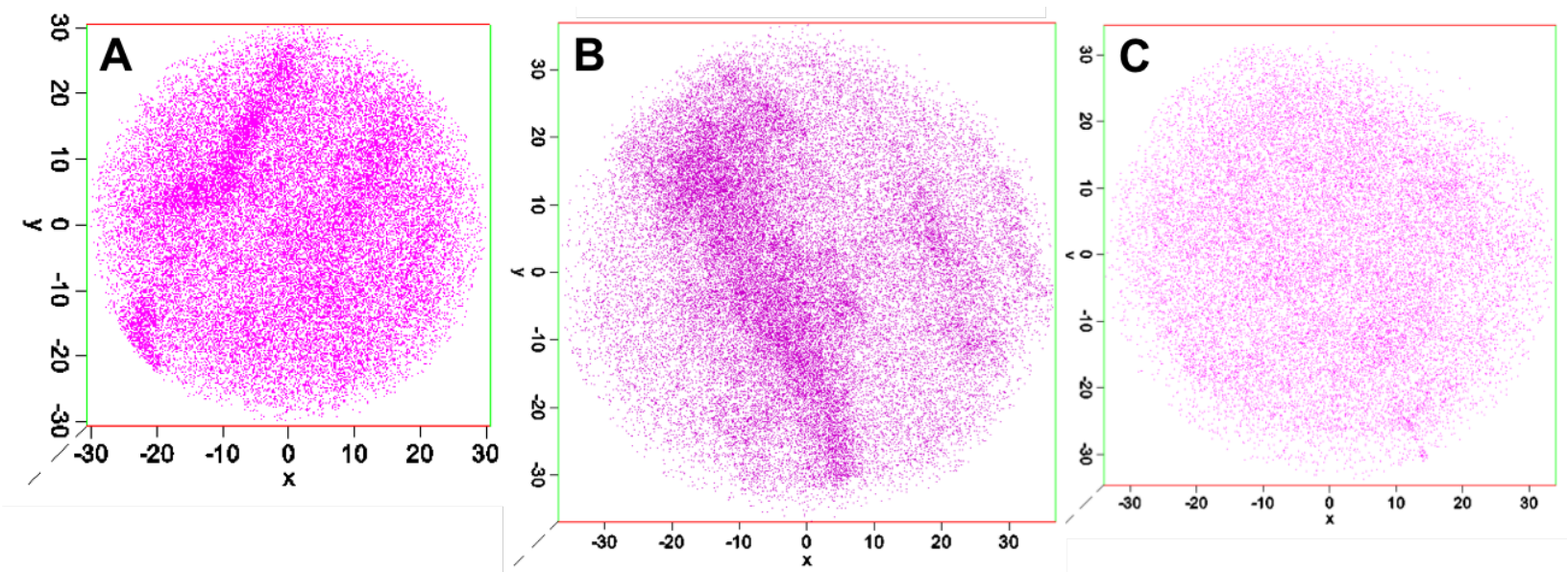

Figure 1. $\mathrm{Mg}^{2+}$ distribution in APT reconstructions of $\mathbf{A}$ ) sound B) intact surface zone, and $\mathbf{C}$ ) mildly demineralized enamel. Scales are in nanometers.

\section{References:}

[1] L. Gordon et al, Science 347 (2015), p. 746.

[2] R. Free et al, J. Synchrotron Rad 24 (2017), p. 1056. 


\section{Investigation of Human Tooth Enamel Down to the Atomic Scale}

Florant Exertier ${ }^{1,2}$, Alexandre La Fontaine ${ }^{1,2}$, Alexander Zavgorodniy ${ }^{3,4}$, and Julie M. Cairney $y^{1,2}$ Corresponding Author: julie.cairney@sydney.edu.au

1. School of Aerospace, Mechanical, Mechatronic Engineering, The University of Sydney, Sydney, Australia

2. Australian Centre for Microscopy and Microanalysis, The University of Sydney, Sydney, Australia

3. Faculty of Dentistry, University of Sydney, Sydney, Australia

4. Institute of Dental Research, Westmead Centre for Oral Health, Sydney, Australia

Human dental enamel, the hardest tissue in the body, plays a vital role in protecting teeth from wear as a result of daily grinding and chewing as well as from chemical attack. It consists of a mineral phase, mainly in the form of highly oriented ribbon-like nanowires of carbonated hydroxyapatite (HAP). It is well established that enamel mechanical strength and fatigue resistance is derived from its hierarchical structure, which consists of periodically-arranged bundles of HAP nanowires. Although recent research has improved our understanding of the HAP crystallization process that leads to this structure, the relationship between microstructure and decay is still not well understood.

The investigation of such structures in human dental enamel requires a wide analysis spectrum from the macroscopic scale down to the atomic scale. Laser-assisted atom probe tomography (APT) was recently used to reveal nano-structures in apatites and rodent tooth enamel, including Mg-rich amorphous calcium phosphate (ACP) nanolayers between the HAP nanowires that make up the enamel, and $\mathrm{Mg}$-rich elongated precipitates and pockets of organic material among the HAP nanowires (1). An earlier study also revealed Fe alongside of $\mathrm{Mg}$ in rodents teeth pigmented enamel ACP nanolayers (2), which was found to render the enamel harder and more resistant to acid attacks. Inspired by this work, we analysed the diffusion of iron in human tooth enamel using an iron-rich solution treatment and its impact on the enamel resistance to acid attack by correlating results from APT and various other microscopy techniques.

\section{References:}

[1] A. La Fontaine, A. Zavgorodniy, H. Liu, R. Zheng, M. Swain, J. Cairney, Atomic-scale compositional mapping reveals Mg-rich amorphous calcium phosphate in human dental enamel, Sci Adv. 2 (2026), e1601145.

[2] L. M. Gordon, M. J. Cohen, K. W. MacRenaris, J. D. Pasteris, T. Seda, D. Joester, Amorphous intergranular phases control the properties of rodent tooth enamel, Science 347 (2015), 746-750. 


\title{
Nanoscale Comparative Analysis of Human and Bovine Dental Enamel Composition Using Atom Probe Tomography
}

\author{
Upoma Guha ${ }^{1}$, Baishakhi Mazumder ${ }^{2}$, and Nirupam Aich $^{3}$ \\ Corresponding Author: guhau@buffalo.edu \\ 1. Department of Restorative Dentistry, School of Dental Medicine, University at Buffalo, The \\ State University of New York, Buffalo, USA. \\ 2. Department of Materials Design and Innovation, University at Buffalo, The State University of \\ New York, Buffalo, USA. \\ 3. Department of Civil, Structural and Environmental Engineering, University at Buffalo, The \\ State University of New York, Buffalo, USA.
}

Bovine teeth have been significantly used as a substitute for human teeth in dental research for the past 30 years. ${ }^{1}$ The easy availability of high quality of bovine teeth along with decreasing availability of human teeth due to recent decline in teeth extraction, quality of the extracted teeth, and ethical issues associated with human based research has led to the rise in this trend.

Although the structural and chemical composition of bulk human and bovine teeth are apparently similar leading to their substitutive usage, understanding of the difference between their nanoscale structure and chemical composition are still lacking - a critical knowledge gap that, when addressed, can render a better understanding and resolution of this controversy. In this study, we aimed to comparatively evaluate the nanoscale chemical composition of human and bovine dental enamel by mapping the distribution of chemical elements and their crystalline structures in both samples using atom probe tomography (APT).

Extracted human and bovine teeth were fixed overnight in $0.2 \mathrm{M}$ sodium cacodylate buffer at $4^{\circ} \mathrm{C}$. The teeth were then placed in Hanks' balanced salt solution for 24 hours at $4^{\circ} \mathrm{C}$ and then dried with compressed air. APT specimen preparation will be followed from a recently published work on human dental enamel. ${ }^{2}$ For APT specimen preparation, enamel samples were cut in smaller dimensions using diamond saw, observed under an optical microscope, tripod-polished, coated with gold, further cut to $4 \mu \mathrm{m}$ wide posts, imaged using Scanning Electron Microscope, and finally cut using a focused ion beam system to prepare the APT needles of 80 to $120 \mathrm{~nm}$ diameter. APT measurements were conducted using Cameca LEAP 5000X HR system. The results of the study indicated towards significant nanoscale differences in the human and bovine teeth, and calls for considerations of these differences while interpreting results from studies that uses bovine teeth instead of human teeth.

References:

[1] G.H. Yassen, J. A. Platt, A. T. Hara, Bovine teeth as substitute for human teeth in dental research: a review of literature, Journal of Oral Science 53 (2011), 273-282.

[2] A. La Fontaine, A. Zavgorodniy, H. Liu, R. Zheng, M. Swain, J. Cairney, Atomic-scale compositional mapping reveals $\mathrm{Mg}$-rich amorphous calcium phosphate in human dental enamel, Science Advances 2 (2016), e1601145. 


\section{IVAS 101 or How to Make the Most Out of Your Dataset Using Your Favorite 3D Reconstruction Wizard?}

Hugues Francois-Saint-Cyr ${ }^{1}$

Corresponding Author: Hugues.FSC@,ametek.com

1. CAMECA Instruments, Inc., 5470 Nobel Drive, Madison, Wisconsin 53711, USA.

Despite the efforts and research deployed, especially for the last 20 years, in terms of specimen preparation and instrumentation to improve Atom Probe Tomography (APT) on materials, the atomic reconstruction in three dimensions (3D) remains the most uncertain step as it relies on many modeling assumptions and individual paths for data processing. This tutorial aims at providing a systematic methodology to treat APT data, hunting down as many clues as possible and exploring many options from the Integrated Visualization and Analysis Software (IVAS), leading to the clearest representation of the reconstruction, even before being able to see the volume in $3 \mathrm{D}$. This tutorial has been specially created for users new to the APT field or willing to accept that their current approach might be either rusty or incomplete. 


\section{Approaching Perfection in Atomic Positioning: From Image Compression and $k$ Factors to Dynamic Considerations}

Alec C. Day ${ }^{1}$ and Hugues Francois-Saint-Cyr ${ }^{2}$

Corresponding Author: alec.day@sydney.edu.au Hugues.FSC@ametek.com

1. The University of Sydney, Australian Centre for Microscopy \& Microanalysis, and School of Aerospace, Mechanical and Mechatronic Engineering, Sydney, NSW 2006, Australia.

2. CAMECA Instruments, Inc., 5470 Nobel Drive, Madison, Wisconsin 53711, USA.

For historical and technological reasons, metallurgists were the first to adopt Atom Probe Tomography (APT) as a routine material characterization technique at the atomic scale. Pure and lightly alloyed metals are known to produce pole patterns on the desorption hit maps, as a result of the ions evaporating from faceted tip shapes. Although aberrations such as holes are observed in a fully dense material, experimental angles and distances between poles are evaluated with respect to the theoretical values to provide an Image Compression Factor (ICF). A precipitatecontaining Al alloy and a steel will be treated using the Pole Indexing and ICF Fit option in IVAS 3.8. An additional step in the data reconstruction will be explored by the research led in APT at the University of Sydney [1], which will account for observed variations in the Image Compression Factor and $\mathrm{k}$ Factor as the tip is being consumed.

[1] Alec Day et al., Novel Crystallographic Framework for Reconstruction in APT, APT\&M 2018. 
Proceedings of Atom Probe Tomography \& Microscopy (APT\&M) 2018, Washington, DC, June 10-15, 2018

NIST SP 2100-03

\section{IFES Business Meeting}

Green Auditorium

5:15 to 6:00 PM 



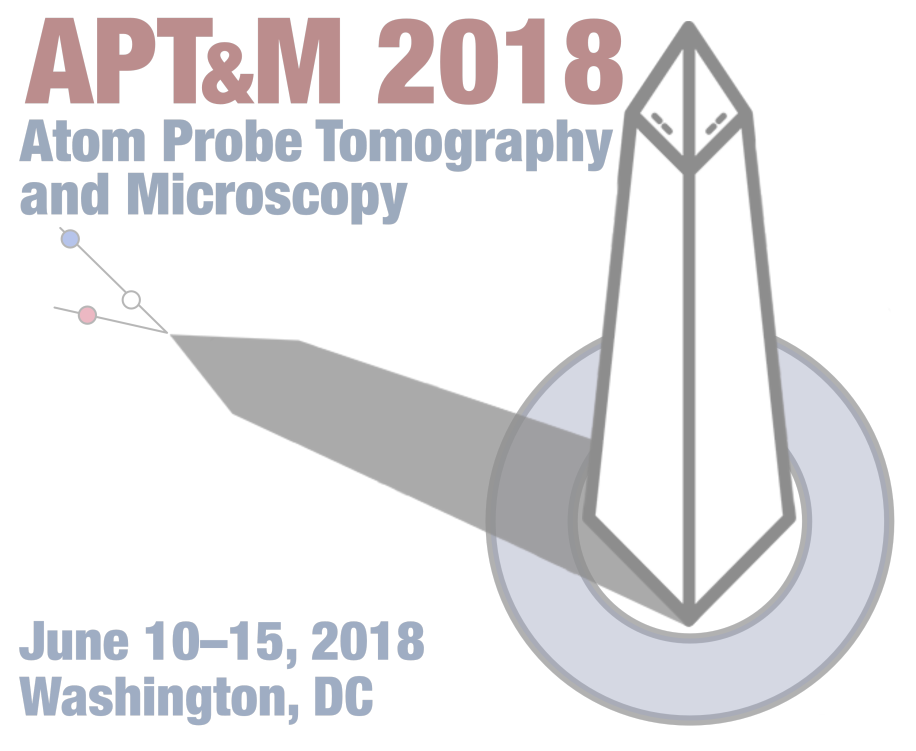

\section{Thursday Morning}





\section{Thursday Morning}

\begin{tabular}{|c|c|c|c|c|c|c|}
\hline 8:45 AM & & & & & & \\
\hline 9:00 AM & \multirow{2}{*}{\multicolumn{6}{|c|}{$\begin{array}{l}\text { Plenary 4: Artificial Intelligence and Machine Learning: An Overview from a National Science Foundation Perspective } \\
\qquad \begin{array}{l}\text { Weng-Keen Wong } \\
\text { (Red Auditorium) }\end{array}\end{array}$}} \\
\hline 9:15 AM & & & & & & \\
\hline 9:30 AM & & & & & & \\
\hline & \multicolumn{6}{|c|}{ Break (9:30 to $10: 00 \mathrm{AM})$} \\
\hline 10:00 AM & \multirow{6}{*}{ 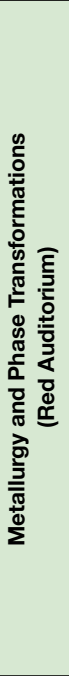 } & Nitrogen and Nitrides in Steels as Seen & $\frac{}{\text { 음 }}$ & $\begin{array}{l}\text { Atomistic Simulations of Metal Surface } \\
\text { Behavior Under High Electric Field }\end{array}$ & \multirow{6}{*}{ 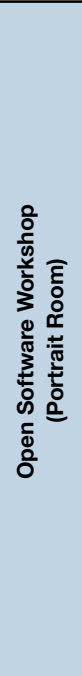 } & $\begin{array}{c}\text { Current Status Of Available Software and } \\
\text { Use Cases } \\
\text { Constantinos Hatzoglou }\end{array}$ \\
\hline 10:15 AM & & Frederic Danoix & $\frac{\hbar}{\delta}$ & $\begin{array}{c}\text { (Invited) } \\
\text { Flyura Djurabekova }\end{array}$ & & $\begin{array}{c}\text { Practical Open-Source Software for APT } \\
\text { Daniel Haley }\end{array}$ \\
\hline 10:30 AM & & $\begin{array}{c}\text { Evolution of Carbide Precipitates in Ti-Mo } \\
\text { Microalloyed Steel } \\
\text { Ross Marceau }\end{array}$ & 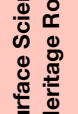 & $\begin{array}{l}\text { Electronic Properties of Adatoms Self- } \\
\text { Diffusing on Metal Surfaces Under } \\
\text { Electric Field } \\
\text { Ekaterina Baibuz }\end{array}$ & & $\begin{array}{c}\text { Characterization Virtual Laboratory: } \\
\text { Sharing Closed-Source Software via a } \\
\text { Cloud-Based Virtual Machine Accessible } \\
\text { with a Web Browser } \\
\text { Anna Ceguerra }\end{array}$ \\
\hline 10:45 AM & & $\begin{array}{l}\text { Atom Probe Compositional Analysis of } \\
\text { Nano-Sized Alloy Carbide in Multiple } \\
\text { Microalloyed Low-Carbon Steels } \\
\text { Yongjie Zhang }\end{array}$ & 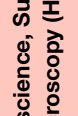 & $\begin{array}{c}\text { Applied Machine Learning in Field lon } \\
\text { Microscopy Aided by Field lon Image } \\
\text { Simulation } \\
\text { Shyam Katnagallu }\end{array}$ & & $\begin{array}{c}\text { Atom-Blend: Using Rendering Engines in } \\
\text { a Common Computer Graphics Package } \\
\text { for High Quality Data Visualization and } \\
\text { Animation } \\
\text { Alec Day }\end{array}$ \\
\hline 11:00 AM & & $\begin{array}{c}\text { Core/Triple Shell Precipitates in Al-Er-Sc- } \\
\text { Zr-(V,Nb,Ta) Alloys } \\
\text { Keith Knipling }\end{array}$ & 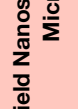 & $\begin{array}{c}\text { Mimicking Electrochemical and } \\
\text { Electrocatalytic Reactions With Atom- } \\
\text { Probe Devices } \\
\text { Norbert Kruse }\end{array}$ & & $\begin{array}{l}\text { CAMECA Demonstration } \\
\text { CAMECA }\end{array}$ \\
\hline \multirow[t]{2}{*}{ 11:15 AM } & & $\begin{array}{c}\text { Atom Probe Characterization of } \\
\text { Strengthening Effects in the Superalloy } \\
718 \\
\text { Felix Theska }\end{array}$ & $\begin{array}{l}\frac{\hbar}{1} \\
\frac{1}{0} \\
\frac{0}{I}\end{array}$ & $\begin{array}{c}\text { A Proposal That Constants Used Widely } \\
\text { in Electron and lon Emission Theory } \\
\text { Should Be Included in International } \\
\text { Listings of Universal Constants } \\
\text { Richard Forbes }\end{array}$ & & Flash Talks \\
\hline & \multicolumn{6}{|c|}{ Lunch / Poster Viewing (11:30 AM to 1:00 PM) } \\
\hline
\end{tabular}





\section{Artificial Intelligence and Machine Learning: An Overview from a National Science Foundation Perspective}

Weng-Keen Wong ${ }^{1}$

Corresponding Author: wwong@,nsf.gov

1. National Science Foundation, Directorate for Computer and Information Science and Engineering, Division of Information and Intelligent Systems, Alexandria, VA, USA.

Artificial Intelligence (AI) is a field of Computer Science that can be informally described as the study of computers and software capable of intelligent behavior. Recently, AI has become ubiquitous, with many applications based on AI affecting different aspects of our everyday lives. This talk will provide an overview of AI with a focus on the area of machine learning, which involves developing algorithms that can learn from data. 


\title{
Nitrogen and Nitrides in Steels as Seen by Atom Probe Tomography
}

\author{
Frederic Danoix ${ }^{1}$ \\ Corresponding Author: frederic.danoix@univ-rouen.fr \\ ${ }^{1 .}$ Normandie University, UNIVROUEN, INSA Rouen, CNRS, Groupe de Physique des \\ Matériaux, UMR 6634, F-76000 Rouen, France
}

Atom probe tomography has been widely used in physical metallurgy, not only because of its ultimate spatial resolution, but also because of its ability to quantify all elements, including light interstitial ones. Clearly because carbon is still the key element in the development of modern steels, for many years APT brings spectacular information regarding its role in the phase transformations leading to final mechanical properties. However, much less attention has been paid to nitrogen, even if this element is becoming more and more important in modern metallurgy. Similarly to carbon, nitrogen is used to modify final mechanical properties through phase transformation modifications, solid solution hardening or nitride precipitation, and its accurate quantification down to the nanometer scale is of great technological importance. In this presentation, we will focus on various aspects of nitrogen metrology in steels. We will show that among all metal-nitrides analyzed so far, only iron nitrides show significant nitrogen depletion, preventing any quantitative measurements. Possible sources of nitrogen loss have been explored, but the situation remains unclear.

One of the main issues in nitrogen metrology in steels is the overlap at $14 \mathrm{Da}$ in mass spectra between nitrogen and silicon. Different options have been used to overcome this problem, including tuning the ${ }^{15} \mathrm{~N} /{ }^{14} \mathrm{~N}$ isotopic ratio, or specific treatment of mass spectra. Analysis of different nanometric nitrides precipitated in steels, including amorphous silicon nitrides, will illustrate the possibilities of such strategies. Among the various illustrations, we will focus on GP-like single atomic layer particles that start the niobium nitride precipitation sequence. They will be shown to nucleate homogeneously in the ferritic matrix, leading to number density of precipitates one or two orders of magnitude larger than in the case of niobium carbides or carbonitrides.

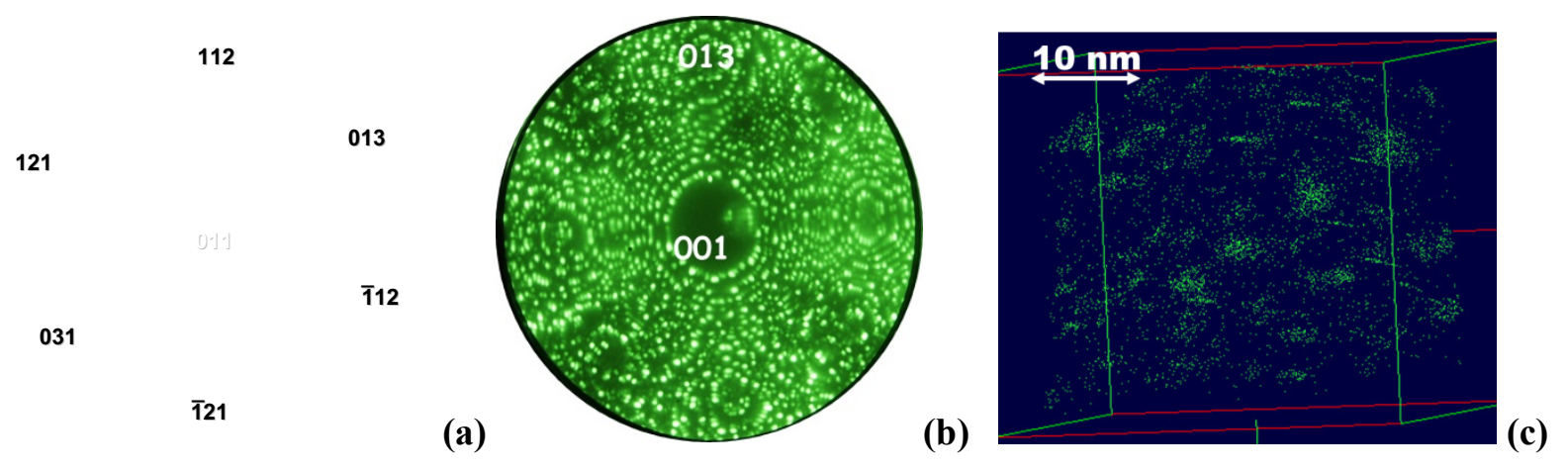

Figure 1 (a) FIM micrograph of GP-like zone niobium nitrides (NbN) seen edge on (white arrow) (b) FIM micrograph of NbN GP-like zone lying on the edge of (001) pole (c) spatial distribution of homogeneously nucleated disc-like niobium nitrides, only two variants are seen simultaneously.

* Contributors are too many to appear individually, but are greatly acknowledged. 


\title{
Evolution of Carbide Precipitates in Ti-Mo Microalloyed Steel
}

\author{
S. Dhara ${ }^{1}$, R.K.W. Marceau ${ }^{1}$, K. Wood $^{2}$, T. Dorin ${ }^{1}$, I.B. Timokhina ${ }^{1}$ and P.D. Hodgson ${ }^{1}$ \\ Corresponding Author: r.marceau@deakin.edu.au \\ 1. Deakin University, Institute for Frontier Materials, Geelong, VIC 3216, Australia. \\ 2. Australian Nuclear Science and Technology Organisation (ANSTO), Kirrawee, NSW 2234, \\ Australia.
}

Interphase precipitation of carbides imparts major strengthening effects in microalloyed steels. The precipitates formed in Ti-Mo bearing steels are particularly known for their fine scale ( 5 $\mathrm{nm}$ in size) and excellent thermal stability, which therefore makes them attractive to the structural automotive industry, however the role of Mo is not clearly understood. In the present work [1], the isothermal evolution of carbide precipitates has been investigated as a function of carbide-forming element concentration ( $\mathrm{Ti}$ and $\mathrm{Mo}$ ) and simulated coiling time at a temperature of $650{ }^{\circ} \mathrm{C}$. Complementary techniques, such as atom probe tomography (APT) and small-angle neutron scattering (SANS) have been used to evaluate the evolution of particle radius, number density, volume fraction and chemical composition, whilst the MC-type crystal structure of the carbides has been confirmed by transmission electron microscopy (TEM).

An APT data analysis procedure for identification of particles has been used to characterise both carbide precipitates (larger particles) and solute clusters (smaller particles). Particles were identified using the maximum separation 'cluster-finding' method after resolving peak overlaps at several locations in the mass spectrum. The cluster-finding algorithm was applied to the data in a two-stage process to properly identify particles having a bimodal size distribution. Furthermore, possible misidentification of matrix atoms (mainly $\mathrm{Fe}$ ) due to the local magnification effect from the difference in field evaporation potential between the matrix and precipitates, has been resolved using an atomic density approach, comparing that measured experimentally using APT to the theoretical density of both the matrix and particles [2].

The possibility of early stage solute clustering and its effect on precipitate formation has also been investigated, where the former are likely precursors to the carbide precipitates observed in the system. In particles having a Guinier radius $>3 \mathrm{~nm}$, the average chemical composition approaches the stable MC carbide stoichiometry with Ti/Mo ratio $\sim 2.5$ and $\mathrm{C} /(\mathrm{Ti}+\mathrm{Mo})$ ratio $\sim 0.55$. We confirm that the precipitates are stable in terms of size over a range of thermomechanical processing conditions, and their sluggish coarsening kinetics are believed to be due to the partial replacement of Ti by Mo in the carbide lattice, which lowers the diffusion rate of $\mathrm{Ti}$ at the particle/matrix interface in the presence of excess $\mathrm{C}$ in the system.

\section{$\underline{\text { References: }}$}

[1]S. Dhara, et al., Precipitation and clustering in a Ti-Mo steel investigated using atom probe tomography and small-angle neutron scattering, Materials Science and Engineering A, accepted (2018).

[2] S. Dhara, et al., Atom probe tomography data analysis procedure for precipitate and cluster identification in a Ti-Mo steel, Data In Brief, under review (2018). 


\title{
Atom Probe Compositional Analysis of Nano-sized Alloy Carbide in Multiple Microalloyed Low-Carbon Steels
}

\author{
Yongjie Zhang $^{1}$, Goro Miyamoto ${ }^{1}$, and Tadashi Furuhara ${ }^{1}$ \\ Corresponding Author: yongjie@imr.tohoku.ac.jp \\ 1. Institute for Materials Research, Tohoku University, Sendai, Japan
}

Introduction The composition of the nano-sized alloy carbide in commercial ferritic steels is extremely important, which determines its dispersion and thus resultant strengthening. Recently, Funakawa et al. reported the increased strength of Ti-added steels by Mo addition and attributed this to the simultaneous precipitation of B1-type (Ti,Mo)C [1], while core-shell structure of $\mathrm{Ti}$ and Mo carbide was also detected by three-dimensional atom probe (3DAP) after isothermal holding [2]. In this study, we focused on the $\mathrm{V}-\mathrm{Ti}$ and $\mathrm{V}-\mathrm{Nb}$ multiple-added low carbon steels, and try to clarify the chemical composition of those alloy carbide through quantitative 3DAP analysis.

Experimental Needle-shaped specimens for 3DAP analysis were prepared from three Fe-0.1C$1.5 \mathrm{Mn}-0.05 \mathrm{Si}$ (mass\%)-based alloys with $0.1 \mathrm{~V}-0.05 \mathrm{Ti}, 0.1 \mathrm{~V}-0.1 \mathrm{Ti}$ and $0.1 \mathrm{~V}-0.1 \mathrm{Nb}$ additions, respectively, which were isothermally transformed from austenite to ferrite at $963 \mathrm{~K}$ and $923 \mathrm{~K}$ for various times. 3DAP measurements were performed at $50 \mathrm{~K}$ with a pulse fraction of $20 \%$ and a pulse rate of $200 \mathrm{kHz}$. Only carbide-forming alloy elements $(\mathrm{V}, \mathrm{Ti}$ and $\mathrm{Nb}$ ) are taken to be the solute atoms for cluster analysis by using the maximum separation method [3].

Results Fig. 1 shows the three-dimensional V and Ti maps of the $0.1 \mathrm{~V}-0.1 \mathrm{Ti}$ alloy isothermally transformed at $923 \mathrm{~K}$ for $60 \mathrm{~s}$, as a typical example. $\mathrm{V}$ and $\mathrm{Ti}$ atoms are clearly enriched into the same regions, indicating the formation of complex precipitation. Fig. 2 shows the typical onedimensional V and Ti concentration profiles of a single cluster formed in the same specimen. Quite low $\mathrm{V}$ and Ti contents in the cluster might be caused by the influence from Fe matrix due to 3DAP artifact. The variations in $\mathrm{V}$ and $\mathrm{Ti}$ contents are synchronized but core-shell structure cannot be identified. Higher atomic ratio of Ti against $\mathrm{V}$ in cluster than the bulk ratio of $\mathrm{V}$ and $\mathrm{Ti}$ addition $(\sim 1.06)$ indicates stronger enrichment of $\mathrm{Ti}$ than $\mathrm{V}$. The homogeneous distribution of carbideforming elements in clusters and stronger enrichment of $\mathrm{Ti}$ or $\mathrm{Nb}$ than $\mathrm{V}$ can also be observed in the other two alloys used in this study.

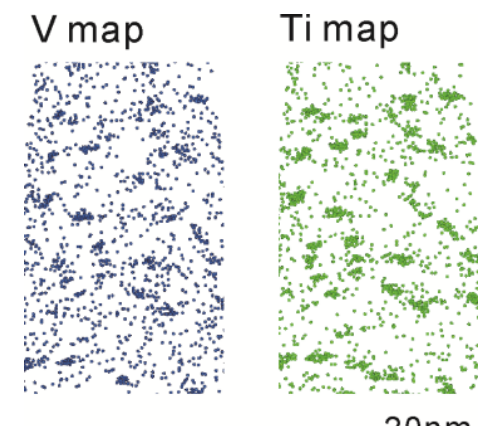

$20 \mathrm{~nm}$

Fig. 1 3D atom maps of 0.1V-0.1Ti alloy transformed at $923 \mathrm{~K}$ for $60 \mathrm{~s}$.

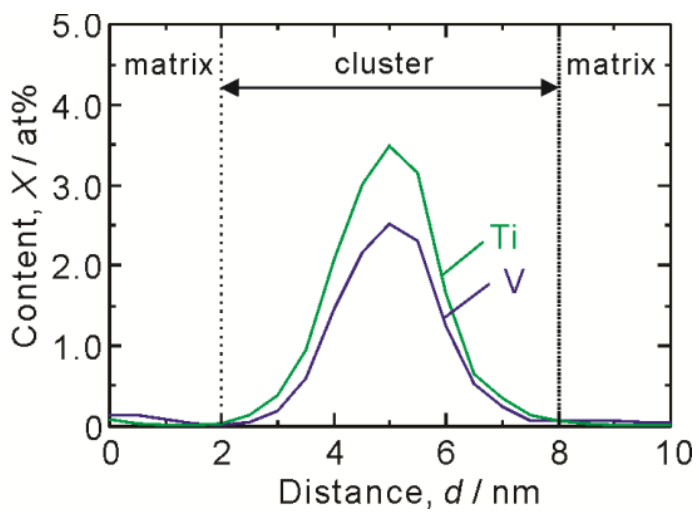

Fig. 2 1D concentration profiles across a single carbide in the transformed specimen in Fig. 1.

\section{Reference}

[1] Y. Funakawa, T. Shinozaki, K. Tomita, T. Yamamoto, E. Maeda, ISIJ Int. 11 (2004) 1945.

[2] J.-B. Seol, S.-H. Na, B. Gault, J.-E. Kim, J.-C. Han, C.-G. Park, D. Raabe, Sci. Rep. 7 (2017) 42547.

[3] D. Vaumousse, A. Cerezo, P.J. Warren, Ultramicros. 95 (2003) 215. 


\title{
Core/Triple Shell Precipitates in Al-Er-Sc-Zr-(V,Nb,Ta) Alloys
}

\author{
Keith E. Knipling ${ }^{1}$ \\ Corresponding Author: keith.knipling@nrl.navy.mil \\ 1. U. S. Naval Research Laboratory, Multifunctional Materials Branch, Washington, DC 20375.
}

Al-Sc alloys are strengthened by nanoscale $\mathrm{Al}_{3} \mathrm{Sc}$ precipitates [1]. By alloying with fasterdiffusing Er and slower-diffusing $\mathrm{Zr}$ additions, complex core/double-shell precipitates are formed, consisting of an Er-enriched core surrounded by a Sc- and Zr-enriched shell [2]. The Erenriched core enhances strength while the Zr-enriched outer shell improves thermal stability. The present study seeks ultimate strength and coarsening resistance by alloying Al-Er-Sc-Zr alloys with Group 5 additions $(\mathrm{M}=\mathrm{V}, \mathrm{Nb}, \mathrm{Ta})$, which are expected to be slower diffusers than $\mathrm{Zr}$ [3]. By sequential nucleation of the constituent solutes we have engineered core/triple shell $\mathrm{Al}_{3}(\mathrm{Er}, \mathrm{Sc}, \mathrm{Zr}, \mathrm{M})$ precipitates. Relationships between the observed mechanical properties and the precipitate sizes and compositions are established using atom-probe tomography throughout the microstructural evolution of the alloys.

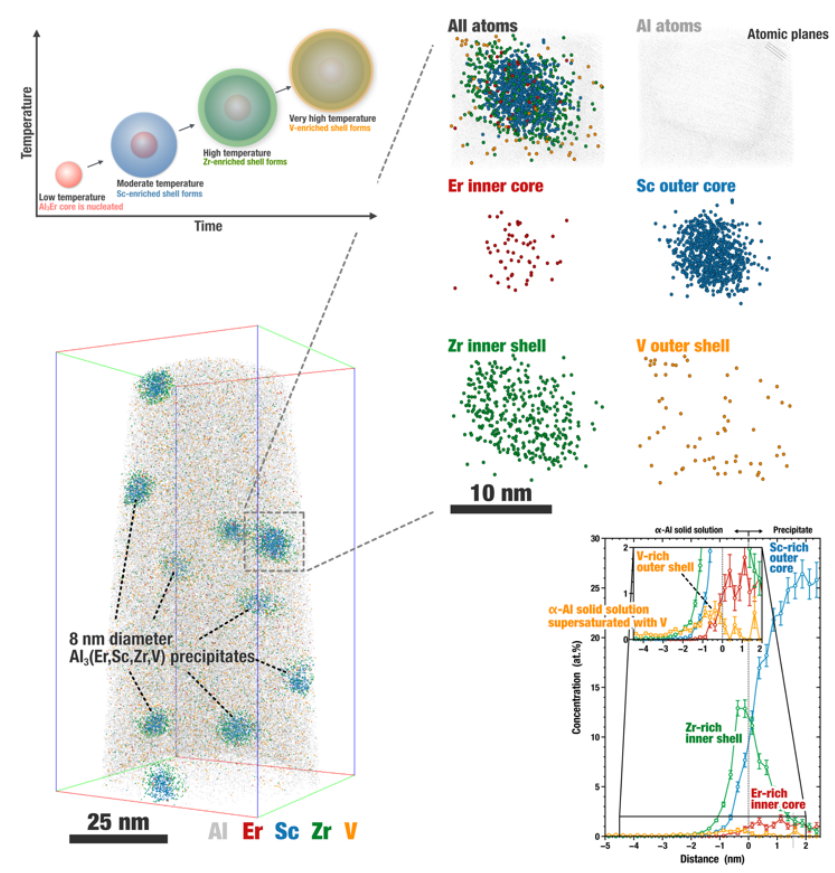

Figure 1 Atom probe reconstruction of an Al-0.004Er-0.042Sc-0.053Zr-0.074V (at.\%) alloy isochronally aged to $425{ }^{\circ} \mathrm{C}$. The core/triple-shell nanostructure of the $\mathrm{Al}_{3}(\mathrm{Er}, \mathrm{Sc}, \mathrm{Zr}, \mathrm{V})$ precipitates is seen in the threedimensional reconstructions and also quantified in the proximity histogram.

\section{References:}

[1] E. Marquis and D. Seidman, Nanoscale Structural Evolution of $\mathrm{Al}_{3} \mathrm{Sc}$ Precipitates in $\mathrm{Al}(\mathrm{Sc})$ Alloys, Acta Materialia 49 (2001), 1909-1919.

[2] C. Booth-Morrison et al., Coarsening resistance at $400{ }^{\circ} \mathrm{C}$ of precipitation-strengthened $\mathrm{Al}-\mathrm{Zr}-\mathrm{Sc}-\mathrm{Er}$ alloys, Acta Materialia 59 (2011), 7029-7042.

[3] K. Knipling et al., Criteria for Developing Castable, Creep-Resistant Aluminum-Based Alloys - A Review, International Journal of Materials Research 97 (2006), 246-265. 


\title{
Atom Probe Characterization of Strengthening Effects in the Superalloy 718
}

\author{
Felix Theska ${ }^{1}$, Simon P. Ringer ${ }^{2}$, Sophie Primig ${ }^{1}$ \\ Corresponding Author: f.theska@unsw.edu.au \\ 1. School of Materials Science \& Engineering, UNSW Sydney, NSW 2052 Australia. \\ 2. School of Aerospace, Mechanical and Mechatronic Engineering, The University of Sydney, \\ NSW 2006, Australia.
}

Ni-based superalloys for aircraft turbine disc applications are precipitation hardened in order to meet demands such as high temperature strength and creep resistance [1]. Atom probe microscopy is an essential tool to reveal processing-microstructure-property relationships in superalloys where a dense structure of nanoscale, ordered precipitates is often found [2]. The present study focusses on superalloy Inconel 718, which is hardened by semi-coherent, ordered $\gamma^{\prime}\left(\mathrm{Ni}_{3}(\mathrm{Al}, \mathrm{Ti})\right)$ and $\gamma$ ' $\left(\mathrm{Ni}_{3}(\mathrm{Nb})\right)$ particles [3]. These precipitates may occur as duplets and triplets with a stacking sequence, dependent on prior processing [4]. The detailed precipitate morphology impacts the resulting mechanical properties. We present reproducible atom probe data reconstruction and analysis particularly suited for precipitation hardened superalloys. While voltage atom probe allows for a more accurate reconstruction, the acquired data volume is often limited. Laser-assisted atom probe provides more statistically significant data as shown in Fig. 1 a). However, the loss of crystallographic information requires the correlation to voltage-mode data sets. Alternatively, a 'mixed-mode' approach can be applied.

Further, an advanced isosurface-method is proposed. Initially, a concentration threshold of $\mathrm{Al}+\mathrm{Ti}$ for the $\gamma^{\prime}$ - and $\mathrm{Nb}$ for the $\gamma$ ' '-particles is set arbitrarily and is then refined. Fig. $1 \mathrm{~b}$ ) shows a cross-sectional concentration profile and its local first derivative. This approach is compared to the proximity histogram method. This enables a study of the precipitate stacking order. Therefore, the $\gamma^{\prime}-\gamma$ ', $\gamma^{\prime}$-matrix and $\gamma^{\prime}$ '-matrix interfaces are quantified as shown in Fig. $1 \mathrm{c}$ ). These developments can be beneficial for improvements in the atom probe based characterization of other precipitation hardened engineering materials such as $\mathrm{Mg}$-, Al- or Fe-based alloys.
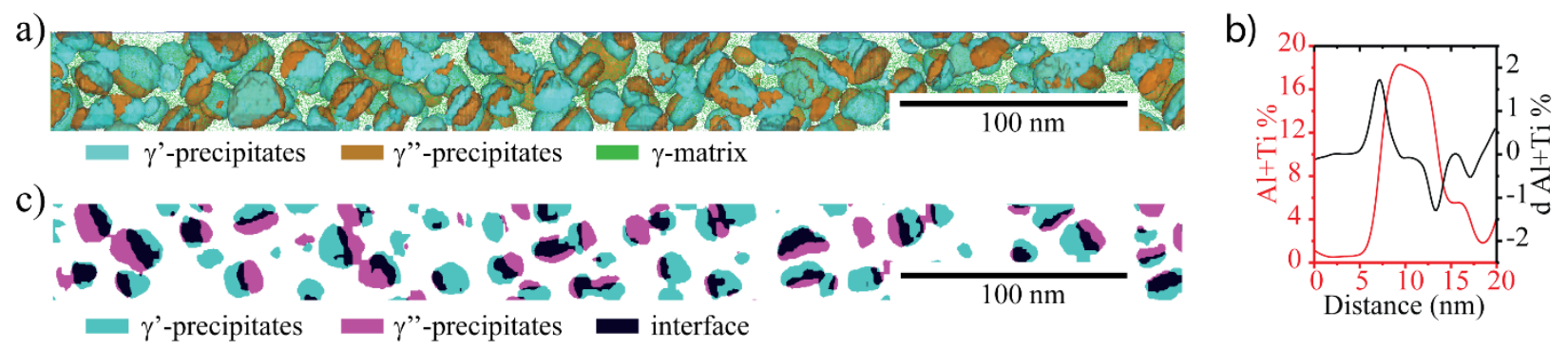

Figure 1 (a) reconstruction of laser-assisted atom probe data (b) cross-sectional concentration profile through a duplet-particle (c) cross-sectional region of interest to identify the particle and matrix interfaces.

\section{References:}

[1] Kozar et al., Strengthening Mechanisms in Polycrystalline Multimodal Nickel-Base Superalloys, Metallurgical and Materials Transactions 40A (2009), 1588-1603.

[2] Gault et al., Atom Probe Microscopy, first ed., Springer, New York, 2008.

[3] Reed, The Superalloys Fundamentals and Applications, first ed., Cambridge University Press, Cambridge, 2006.

[4] Alam et al., Precipitation and clustering in the early stages of ageing in Inconel 718, Materials Science and Engineering A527 (2010), 7770-7774 


\section{Atomistic Simulations of Metal Surface Behavior under High Electric Field}

Flyura Djurabekova, Andreas Kyritsakis, Mihkel Veske, Ekaterina Baibuz, and Ville Jansson Corresponding Author: Flyura.Djurabekova@helsinki.fi

Helsinki Institute of Physics and Department of Physics, University of Helsinki, Finland.

Application of electric fields on metal surfaces may be beneficial and detrimental. In our research, we are looking for the changes on the surface, which may lead to vacuum arcing, known to those who are dealing with the high/voltage electronics as electrical breakdowns. These cause problems in many appliances operating in high electric field, such as the Compact Linear Collider (CLIC), a proposed next-generation particle accelerator in CERN. The breakdown phenomenon is not well understood despite decades of research devoted to investigation of this phenomenon. The complicated nature shows itself differently under different circumstances. Even in such technologically high-level of demand condition as in accelerating structures of future particle colliders, the electric fields near metal surfaces of the structures cannot exceed a certain level, which is well below the theoretical one.

What triggers the surface to break when high electric fields are applied is the focus of the research in our group. Currently we are working on an atom-level theoretical model of surface behavior under high electric fields. The model covers many stages of plasma development and includes different physical processes evolving on different time scales. Our model aims to explain the physical limitation of a metal surface due to electrical breakdowns at the fields well below the critical values known to cause field evaporation of atoms. The core of the model is the atomistic simulations of metal surface features under high electric field.

Currently we develop the simulation tool which concurrently combines the solution of Newtown's equations with the continuous Laplace solver on a mesh built up (and undated regularly) above the

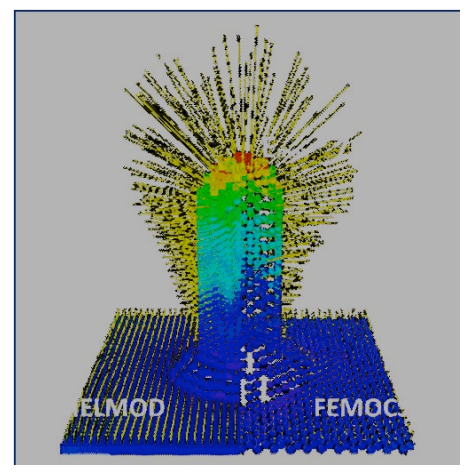

Figure 1 Comparison of HELMOD and FEMOCS field distributions for a surface $\mathrm{Cu}$ tip surface. We upgraded the previously existing Laplace solver (HELMOD [1]) based on the finite difference calculation method. The new approach (FEMOCS [2]) exploits the beneficial flexibility of finite elements instead of fixed mesh of the previous model. The value of the local field found near a surface atom, we translate to the discrete partial charge assigned to the atom and introduce the electric forces due to this charge to the atomistic simulations.

The approach of building up a flexible mesh is, in fact, independent of the main simulation routine. Any simulation, which outputs the simulated structure in Cartesian coordinates of the atoms, can be combined with FEMOCS. The examples of application of the software and interesting insights will be shown and discussed during the presentation.

References:

1. F. Djurabekova, S. Parviainen, et al., Physical Review E 83, (2011), 026704

2. M. Veske, et al. Journal of Computational Physics (2017) 


\section{Electronic Properties of Adatoms Self-diffusing on Metal Surfaces Under Electric Field}

Ekaterina Baibuz $^{1}$, Andreas Kyritsakis ${ }^{1}$, Ville Jansson ${ }^{1}$, and Flyura Djurabekova ${ }^{1}$

Corresponding Author: ekaterina.baibuz@gmail.com

1. University of Helsinki, Helsinki Institute of Physics, Department of Physics, Helsinki, Finland

Understanding the basic phenomena behind the atomic self-diffusion on metal surfaces under the electric field can potentially benefit many applications including atom probe microscopy and accelerator technology.

It has been a common understanding that atomic diffusion is biased towards the sharp features on a surface where local electric field is higher. However, the quantitative relation between the electric field gradient and a change of the migration energy barriers is not yet fully constructed. It was proposed in the 70 s by Tsong et al that the reduction in the energy barrier is related to the change of the surface-induced dipole moment and polarisability of the adatom along the migration path by the electric field. [1]

In this work, we look in detail into the electronic properties of $\mathrm{W}$ and $\mathrm{Cu}$ adatoms self-diffusing on perfect metallic surfaces under electric field by the means of Density Functional Theory. We calculated dipole moments and polarisabilities of adatoms and studied how electronic properties affect the energy barriers under the electric field. We compared the obtained results for both cathode and anode cases.
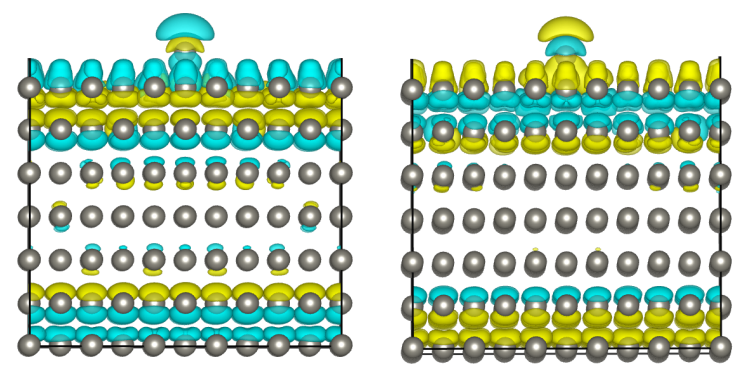

Figure 1 The change of electron density introduced by $1 \mathrm{GV} / \mathrm{m}$ electric field on an anode (right) and a cathode (left) obtained with VASP DFT code. Yellow color correspond to accumulation of electrons due to electric field, blue - to depletion. It could be seen that electrons are pushed by the electric field further to the surface on an anode side and pulled away on a cathode

References:

[1] T. T. Tsong, and G. Kellogg, Direct observation of the directional walk of single adatoms and the adatom polarizability, Physical review B (1975), 12(4), p.1343 


\section{Applied Machine Learning in Field Ion Microscopy Aided by Field Ion Image Simulation}

Shyam Katnagallu ${ }^{1}$, Ali Nematollahi ${ }^{2}$, Blazej Grabowski ${ }^{2}$, Jörg Neugebauer ${ }^{2}$, Dierk Raabe ${ }^{1}$ and Baptiste Gault ${ }^{1}$

Corresponding Author: s.katnagallu@address.com

1. Microstructure physics and alloy design Department, Max-Planck-Institut für Eisenforschung GmbH, Düsseldorf, Germany-40227.

2. Computational materials design Department, Max-Planck-Institut für Eisenforschung GmbH, Düsseldorf, Germany-40227.

Field ion microscopy (FIM) allows to image individual surface atoms by exploiting the ionization of an imaging gas by an intense electric field in the vicinity of a needle-shaped specimen. Widespread use of atomic resolution imaging by FIM has been hampered by a lack of efficient image processing/data extraction tools. We employ advanced data extraction tools for automated detection of atoms and lattice defects for materials characterization. We also show the use of machine learning (ML) approaches for data extraction and develop simple FIM simulations to aid ML by providing a perfectly labelled dataset on which the machine can be trained. The evaporation sequence in these simulations is based on nearest neighbor criterion used in ref [1]. After a brief overview on a new method developed for FIM data extraction we will describe the use of these FIM simulations for a quantitative analysis of distortions in experimental FIM images. These ideas are discussed towards improving the spatial precision of FIM data reconstructions.
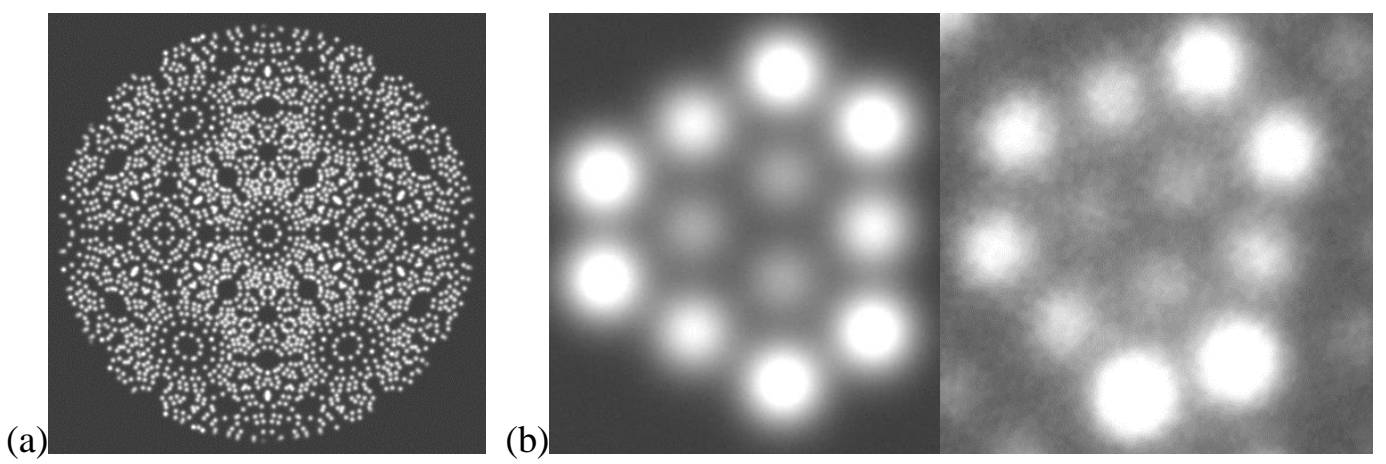

Figure 1 (a) FIM simulation for a tip with $50 \mathrm{~nm}$ radius. (b)Simulation (left) of an outermost (222) bcc plane and the corresponding experimental (right) tungsten (222) plane's FIM image.

\section{References:}

[1] A. J. W. Moore and J. A. Spink, "Field evaporation from tungsten and the bonding of surface atoms", Surf Sci, vol. 12 (1968), pp. 479-496.

[2] Drs. Michal Dagan, Paul Bagot and Prof. Micheal Moody are greatfully acknowledged for providing FIM data on tungsten. 


\title{
Mimicking Electrochemical and Electrocatalytic Reactions with
}

\section{Atom-Probe Devices}

\author{
Norbert Kruse \\ Corresponding Author: Norbert.Kruse@wsu.edu \\ Voiland School of Chemical Engineering and Bioengineering, Washington State University, \\ Pullman, Washington 99164, United States
}

Dynamic imaging of chemical reactions at the solid/gas interface has become possible more than two decades ago through rigorous application of video-FEM/FIM. In some cases simultaneous chemical probing has been achieved to enable a correlation of FEM/FIM pattern sequences and local composition information. While the influence of the electric field interaction with adsorbed dipole species appears to be non-negligible, this influence turned out to be minor for the CO oxidation over Pt metal. Other reactions suffer a strong field influence as will be demonstrated here for the methanol decomposition over Rh and Ru tips. The capacity of 1DAP to provide mechanistic insight by mimicking electrocatalytic conditions in a fuel cell will be highlighted.

As compared to hydrogen, which is the ideal fuel from an electrochemical point of view, the achievable power densities and cell efficiencies for methanol fuel cells are much lower due to poor electrode kinetics. Following on early studies [1,2], we show that the anodic decomposition of methanol in fuel cells leads to $\mathrm{CO}$ and metal-carbonyl formation even at room temperature. 1DAP was conducted by varying the steady electrical field strength up to the onset of field evaporation (figure 1). The results show that for low steady electric fields, the reaction proceeds to completion ( $\Sigma \mathrm{H}_{\mathrm{n}} / \mathrm{CO}=4, \mathrm{n}=2$ for $\mathrm{H}_{2}{ }^{+}$dominating). With increasing steady field, the reaction decelerates. This is evidenced by the formation of large amounts of $\mathrm{CH}_{3}{ }^{+}$and $\mathrm{COH}_{2}{ }^{+}$ intermediates whereby $\mathrm{CH}_{3}{ }^{+}$is formed by field fragmentation of adsorbed methoxy. At the same time, the intensities of the products, $\mathrm{CO}$ and $\Sigma \mathrm{H}_{\mathrm{n}}$ decrease strongly. Field strengths around $2 \mathrm{~V} / \mathrm{nm}$ are critical for the dramatic (negative) change in the reaction rate and the stabilization of

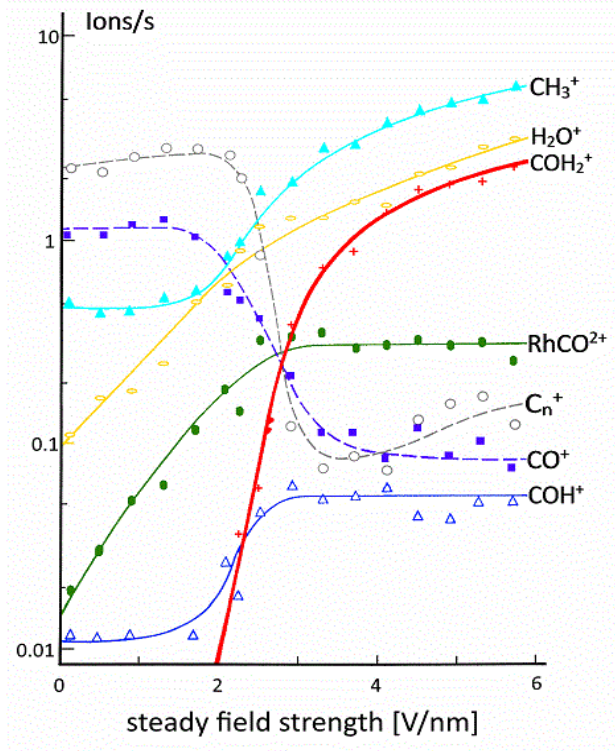
the intermediates. The results clearly demonstrate that the room temperature methanol decomposition during $\mathrm{t}_{\mathrm{R}}=250 \mu \mathrm{s}$ produces adsorbed $\mathrm{CO}$ which acts as a poison in electrocatalytic applications. The poisoning is partly lifted by field strengths above $2 \mathrm{~V} / \mathrm{nm}$.

Figure 1: 1DAP spectra during the ongoing methanol decomposition over $\mathrm{Rh}$ with varying steady electric field at a total field of $\mathrm{F}_{\mathrm{D}}=28 \mathrm{~V} / \mathrm{nm}, \mathrm{T}=298 \mathrm{~K}, \mathrm{p}\left(\mathrm{CH}_{3} \mathrm{OH}\right)=1.3$ $\mathrm{x} 10^{-5} \mathrm{~Pa}$. The reaction time between field pulses is $t_{\mathrm{R}}=250$ $\mu \mathrm{s}$. About 150 atomic sites close to the (001) pole are probed.

\section{References:}

[1] G. K. Chuah et al., J. Catal. 119, 342-353 (1989)

[2] N. Kruse et al., J. Chem. Phys. 91 (1), 577-583 (1989) 


\section{A Proposal That Constants Used Widely in Electron and Ion Emission Theory Should Be Included in International Listings of Universal Constants}

Richard G. Forbes ${ }^{1}$

Corresponding Author: r.forbes@trinity.cantab.net

1. Advanced Technology Institute, University of Surrey, Guildford, Surrey GU2 7XH, UK

Theories of electron and/or ion emission contain a number of "special" universal constants defined in terms of the fundamental scientific constants. Some combinations denoted by these special constants have been regularly used since modern emission theory emerged in 1927/28, although the idea of representing them by a single named symbol came much later.

Given the location of this year's APT\&M meeting, it seems timely to suggest that the more important of these "special constants" should be included in international listings of universal constants, such as that made available on-line by the National Institute of Standards and Technology (NIST). Table 1 below shows some of constants used in field electron emission theory (and the constant $b$ is also used in field ionization theory). It can be seen that several combinations involve fundamental constants and one or both of two "primary universal constants" ( $\mathrm{e}$ and $z_{\mathrm{S}}$ ) associated, respectively, with the Schrödinger equation for electrons and with the statistical mechanical principle that "the electron supply density is constant in energy space, when all relevant states are fully occupied".

The FN constants $a$ and $b$ seem strong candidates for listing, as they are beginning to be regularly used in the literature (though several different notations are in use). The other singlesymbol combinations are not yet widely used, but - due to their intrinsic physical significances-may merit listing at some future point.

Table 1. Some universal constants used in electron and/or ion emission theory. Values are given in the customary units often used, to make certain calculations quick and easy. The symbols $e, m_{\mathrm{e}}, h_{\mathrm{p}}, \hbar$, $k_{\mathrm{B}}$ and $e_{0}$ have their conventional meanings, with $e$ denoting the elementary (positive) charge.

\begin{tabular}{|l|l|l|l|l|l|}
\hline Name & Symbol & Derivation & Expression & Numerical value & Units \\
\hline $\begin{array}{l}\text { Sommerfeld supply } \\
\left.\text { density }{ }^{1}\right)\end{array}$ & $z_{\mathrm{S}}$ & - & $4 \pi m_{\mathrm{e}} / h_{\mathrm{P}}{ }^{3}$ & $1.618311 \times 10^{14}$ & $\mathrm{~A} \mathrm{~m}^{-2} \mathrm{eV}^{-2}$ \\
\hline $\begin{array}{l}\text { Universal theoretical } \\
\text { Richardson constant }\end{array}$ & $A_{\mathrm{R} 0}$ & $z_{\mathrm{S}} k_{\mathrm{B}}{ }^{2}$ & $4 \pi e m_{\mathrm{e}} k_{\mathrm{B}}{ }^{2} / h_{\mathrm{P}}{ }^{3}$ & $1.201735 \times 10^{6}$ & $\mathrm{~A} \mathrm{~m}^{-2} \mathrm{~K}^{-2}$ \\
\hline $\begin{array}{l}\text { Schrödinger equation } \\
\text { constant for electron }\end{array}$ & $\kappa_{\mathrm{e}}$ & - & $\left(2 m_{\mathrm{e}}\right)^{1 / 2} / \hbar$ & 5.123168 & $\mathrm{eV}^{-1 / 2} \mathrm{~nm}^{-1}$ \\
\hline $\begin{array}{l}\text { JWKB constant for } \\
\text { electron }\end{array}$ & $g_{\mathrm{e}}$ & $2 \kappa_{\mathrm{e}}$ & $2\left(2 m_{\mathrm{e}}\right)^{1 / 2} / \hbar$ & 10.24624 & $\mathrm{eV}^{-1 / 2} \mathrm{~nm}^{-1}$ \\
\hline $\begin{array}{l}\text { First Fowler- } \\
\text { Nordheim constant }\end{array}$ & $a$ & $z_{\mathrm{S}}\left(e / 2 \kappa_{\mathrm{e}}\right)^{2}$ & $e^{3 / 8 \pi h_{\mathrm{P}}}$ & 1.541434 & $\mu \mathrm{A} \mathrm{V}^{-2} \mathrm{eV}$ \\
\hline $\begin{array}{l}\text { Second Fowler- } \\
\text { Nordheim constant }\end{array}$ & $b$ & $4 \kappa_{\mathrm{e}} / 3 e$ & $(4 / 3)\left(2 m_{\mathrm{e}}\right)^{1 / 2} / e \hbar$ & 6.830890 & $\mathrm{eV}^{-3 / 2}(\mathrm{~V} / \mathrm{nm})$ \\
\hline Schottky constant & $c_{\mathrm{S}}$ & - & $\left(e^{3} / 4 \pi \varepsilon_{0}\right)^{1 / 2}$ & 1.199985 & $\left.\mathrm{eV}^{1 / 2} / \mathrm{nm}\right)^{-1 / 2}$ \\
\hline
\end{tabular}

$\left.{ }^{1}\right)$ Sommerfeld supply density is the electron current crossing a mathematical plane inside a free-electron metal of large extent, per unit area of the plane, per unit area of energy space, when the relevant electron states are fully occupied.. 
This software workshop, hosted by Karen Henry, focuses on programs, scripts, utilities, and software packages developed by individuals or groups to facilitate data analysis and/or data treatment to advance atomic-scale understanding of materials science processes. The workshop consists of demonstrations by invited presenters, an open forum for users to contribute short, five-minute Flash Talks about a script/utility/program, and an open discussion about the software needs of the community.

Current Status Of Available Software and Use Cases

Constantinos Hatzoglou

10:00 to 10:15 AM

Practical Open-Source Software for APT

Daniel Haley

10:15 to $10: 30 \mathrm{AM}$

Characterization Virtual Laboratory: Sharing Closed-Source Software via a Cloud-Based Virtual Machine Accessible with a Web Browser Anna Ceguerra

10:30 to $10: 45 \mathrm{AM}$

\section{Atom-Blend: Using Rendering Engines in a Common Computer Graphics Package for High Quality Data Visualization and Animation}

Alec Day

10:45 to 11:00 AM

\section{CAMECA Demonstration}

CAMECA

11:00 to $11: 15 \mathrm{AM}$

\section{Flash Talks}

11:15 to $11: 30 \mathrm{AM}$ 


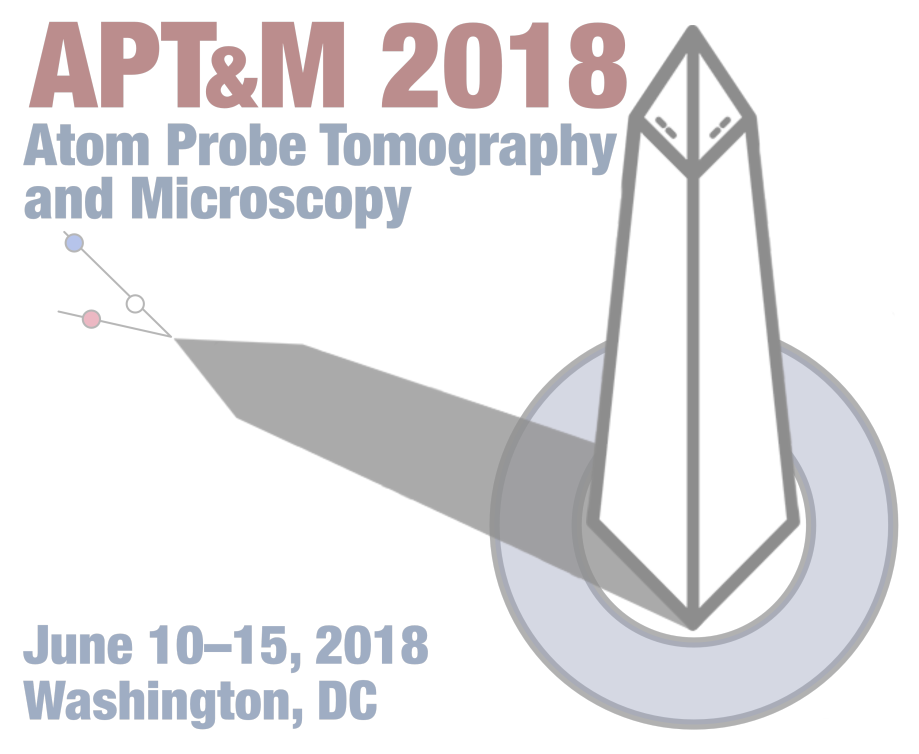

\section{Thursday Afternoon}





\section{Thursday Afternoon}

\begin{tabular}{|c|c|c|c|c|c|c|}
\hline 1:00 PM & \multirow{8}{*}{ 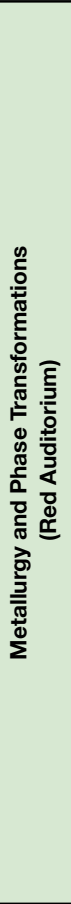 } & $\begin{array}{l}\text { Direct Observation of Niobium } \\
\text { Segregation to Dislocations in Steel } \\
\text { Jun Takahashi }\end{array}$ & 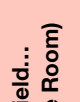 & $\begin{array}{c}\text { Behaviour and Characterisation of } \\
\text { Various Types of Carbon Nanotubes as } \\
\text { Field Emitting Electron Sources } \\
\text { Marwan Mousa }\end{array}$ & \multirow{4}{*}{ 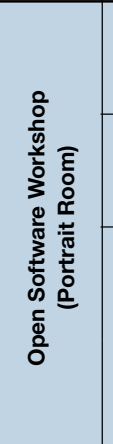 } & Flash Talks \\
\hline 1:15 PM & & \begin{tabular}{|c|} 
Atomic Scale Analysis on Retardation of \\
Recrystallization of Work-Hardened \\
Austenite in V-Added Low Alloy High \\
Carbon Steel \\
Yukiko Kobayashi \\
\end{tabular} & 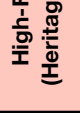 & \begin{tabular}{|c|} 
Electron Field Emission and \\
Spectroscopy of Diamond Nano-Needles \\
Ivan Blum
\end{tabular} & & Flash Talks \\
\hline 1:30 PM & & $\begin{array}{l}\text { Analyzing Boron in 9-12\% Chromium } \\
\text { Steels Using Atom Probe Tomography } \\
\text { Fang Liu }\end{array}$ & \multirow{6}{*}{ 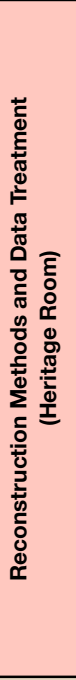 } & $\begin{array}{l}\text { Calibration of Atom Probe Tomography } \\
\text { Reconstructions From Correlation With }\end{array}$ & & \multirow[b]{2}{*}{ Open Forum Discussion } \\
\hline 1:45 PM & & $\begin{array}{l}\text { The Segregation Features at Grain } \\
\text { Boundaries With Various Characters in } \\
\text { Highly Twinned Alloy } 690 \\
\text { Hui Li }\end{array}$ & & $\begin{array}{l}\text { Electron Tomograms or Micrographs } \\
\text { (Invited) } \\
\text { Isabelle Mouton }\end{array}$ & & \\
\hline 2:00 PM & & $\begin{array}{c}\text { APT and STEM Analysis of a Metallic } \\
\text { Nuclear Fuel to Reveal the Influence of } \\
\text { Grain Boundary Segregation on Kinetics } \\
\text { of Discontinuous Precipitation } \\
\text { Arun Devaraj }\end{array}$ & & $\begin{array}{c}\text { Trajectory-Based Atom Probe } \\
\text { Reconstruction With Locally Varying Tip } \\
\text { Shape } \\
\text { Daniel Beinke }\end{array}$ & \multirow{4}{*}{ 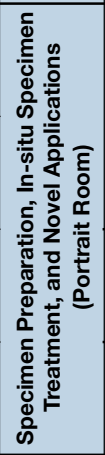 } & \multirow{2}{*}{$\begin{array}{l}\text { Advanced Preparation Methods for } \\
\text { Challenging APT Specimens (Invited) } \\
\text { Dieter Isheim }\end{array}$} \\
\hline 2:15 PM & & $\begin{array}{l}\text { Nanoscale Analysis of lon Irradiated } \\
\text { 14YWTi ODS Steel } \\
\text { Maria Auger }\end{array}$ & & $\begin{array}{l}\text { Making Atom-Probe Reconstruction } \\
\text { Compatible With Newton's Laws } \\
\text { Richard Forbes }\end{array}$ & & \\
\hline 2:30 PM & & $\begin{array}{l}\text { Understanding Behavior and } \\
\text { Performance of Nuclear Fuels via Atom } \\
\text { Probe Tomography } \\
\text { Mukesh Bachhav }\end{array}$ & & $\begin{array}{l}\text { Enhanced Dynamic Reconstruction for } \\
\text { Atom Probe Tomography: A Post Bas } \\
\text { Algorithm } \\
\text { Constantinos Hatzoglou }\end{array}$ & & $\begin{array}{l}\text { Atom Probe Specimen Preparation of } \\
\text { Steels by Transmisssion Kikuchi } \\
\text { Diffraction } \\
\text { Christina Hofer }\end{array}$ \\
\hline \multirow[t]{2}{*}{ 2:45 PM } & & $\begin{array}{c}\text { Atom Probe Tomography as a Nuclear } \\
\text { Forensics Tool - Analysing } \\
\text { Nanoparticulate Material From the } \\
\text { Fukushima Region of Japan } \\
\text { Tomas Martin } \\
\end{array}$ & & $\begin{array}{c}\text { Methodologies for APT Data } \\
\text { Reconstruction Based Upon Correlative } \\
\text { Real Space Imaging and Diffraction With } \\
\text { Electrons } \\
\text { Brian Gorman } \\
\end{array}$ & & $\begin{array}{l}\text { Advanced Sample Preparation } \\
\text { Techniques Using a Laser Ablation } \\
\text { Instrument } \\
\text { Robert Morris }\end{array}$ \\
\hline & \multicolumn{6}{|c|}{ Break (3:00 to 3:30 PM) } \\
\hline 3:30 PM & \multirow{6}{*}{ 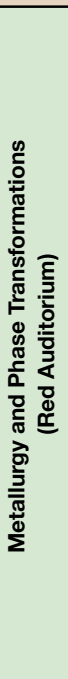 } & $\begin{array}{c}\text { Elemental Partitioning and Site } \\
\text { Occupation of Mo and Cr in Co-Ti Based } \\
\text { Superalloys } \\
\text { Hyeji Im }\end{array}$ & \multirow{6}{*}{ 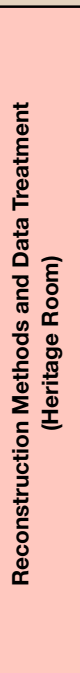 } & $\begin{array}{l}\text { Novel Crystallographic Framework for } \\
\text { Reconstruction in APT } \\
\text { Alec Day }\end{array}$ & \multirow{5}{*}{ 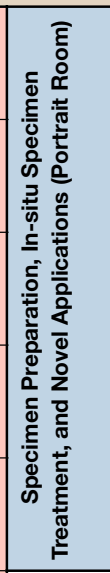 } & $\begin{array}{l}\text { Semi-Automated Specimen Preparation } \\
\text { for Atom Probe Tomography } \\
\text { Michael Schmidt }\end{array}$ \\
\hline 3:45 PM & & $\begin{array}{l}\text { Atom Probe Investigations of the } \\
\text { Miscibility of Cu and Ni } \\
\text { Rüya Duran }\end{array}$ & & $\begin{array}{c}\text { The Atomic Structure of Quasicrystals via } \\
\text { Atom Probe Microscopy } \\
\text { Anna Ceguerra }\end{array}$ & & $\begin{array}{l}\text { A New Method for Mapping the Three- } \\
\text { Dimensional Atomic Distribution Within } \\
\text { Nanoparticles by Atom Probe } \\
\text { Tomography (APT) } \\
\text { Se-Ho Kim }\end{array}$ \\
\hline 4:00 PM & & $\begin{array}{l}\text { Superficial Enrichment in Gold/Silver } \\
\text { Alloys: Study of the Physicoochemical } \\
\text { Influences Using Atom Probe } \\
\text { Tomography } \\
\text { Natalia Gilis }\end{array}$ & & $\begin{array}{c}\text { Applying Principles of Multivariate } \\
\text { Analysis and Geospatial Interpolation } \\
\text { Methods to Atom Probe Tomography } \\
\text { Imaging } \\
\text { Tammy Milillo }\end{array}$ & & $\begin{array}{l}\text { APT Analysis of Pt-Modified } \\
\text { Nanoporous Gold } \\
\text { Brian Langelier }\end{array}$ \\
\hline 4:15 PM & & $\begin{array}{l}\text { Direct Observations of Atomic Hydrogen } \\
\text { and Deuterium Interactions in Zircaloy-4 } \\
\text { Andrew Breen }\end{array}$ & & $\begin{array}{l}\text { From the Mass Spectrum to the Atom } \\
\text { Map: An Alternative Approach to the } \\
\text { Chemical Identification of Elements } \\
\text { François Vurpillot }\end{array}$ & & $\begin{array}{l}\text { In-Situ Crystallization of Metallic Glass in } \\
\text { the Atom Probe } \\
\text { David Diercks }\end{array}$ \\
\hline 4:30 PM & & $\begin{array}{l}\text { Investigating the Effects of Oxygen and } \\
\text { Nitrogen on Titanium Alloys for Turbine } \\
\text { Engine Applications } \\
\text { Hazel Gardner }\end{array}$ & & $\begin{array}{c}\text { Field-Dissociation Dynamics of } \mathbf{P}_{m^{i+}} \\
\text { Molecular lons in High DC Electric Field } \\
\text { Enrico Di Russo }\end{array}$ & & $\begin{array}{l}\text { Atomic Scale Analysis of Magnesium } \\
\text { Oxide Scale } \\
\text { Ingrid McCarroll }\end{array}$ \\
\hline 4:45 PM & & $\begin{array}{l}\text { Atom Probe Characterization of } \\
\text { Thermally Grown Oxides Formed on } \\
\text { Nickel Based Superalloys } \\
\text { Mark Lapington }\end{array}$ & & $\begin{array}{c}\text { Measuring Bond Strength Across } \\
\text { Heterostructure Interfaces Using Atom } \\
\text { Probe Data } \\
\text { Baishakhi Mazumder }\end{array}$ & & \\
\hline \multicolumn{7}{|l|}{ 5:00 PM } \\
\hline \multicolumn{7}{|l|}{ 5:15 PM } \\
\hline 5:30 PM & \multirow{2}{*}{\multicolumn{6}{|c|}{$\begin{array}{c}\text { Departure } \\
\text { (Banquet at 7:00 PM) }\end{array}$}} \\
\hline 5:45 PM & & & & & & \\
\hline 6:00 PM & & & & & & \\
\hline
\end{tabular}





\section{Direct Observation of Niobium Segregation to Dislocations in Steel}

Jun Takahashi ${ }^{1}$, Kazuto Kawakami ${ }^{1}$, Jun-ichi Hamada ${ }^{2}$, and Ken Kimura ${ }^{1}$

Corresponding Author: takahashi.3ct.jun@jp.nssmc.com

1. Nippon Steel \& Sumitomo Metal Corporation, Futtsu-city, Japan

2. Nippon Steel \& Sumikin Stainless Steel Corporation, Hikari-city, Japan

Niobium is one of very important alloying elements in steel because it is effectively used for grain refinement by the retardation of recrystallization in the hot-rolling process [1]. The solute drag effect by solute niobium atoms and the pinning effect by niobium carbonitride precipitates have been proposed for the retardation mechanism of recrystallization. In addition, it is known that niobium in solid solution retards the recovery of dislocations in steel. However, the mechanism has been remained unclear.

We observed the segregation of niobium atoms to dislocations for the first time by atom probe tomography in the niobium added ferritic stainless steel (Fig.1) [2]. The steel has high thermal fatigue resistance and is applied for exhaust manifold in the automotive industry. In the steel, solute carbon and nitrogen were completely scavenged by sufficient amounts of niobium and titanium. The observation results suggest that solute niobium atoms solely have strong attractive interaction with dislocations. We named it niobium-Cottrell atmosphere [3], and proposed the trapping site of niobium atoms at the dislocation and the interaction energy between niobium atom and dislocation through a comparison with segregations to high-angle and low-angle grain boundaries in the steel.

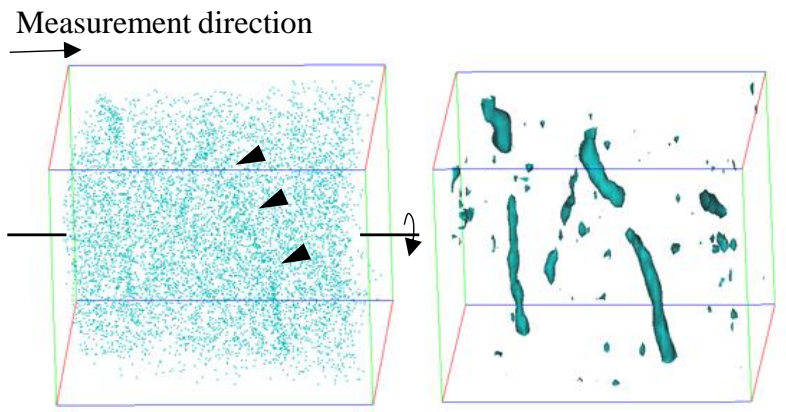

(a)

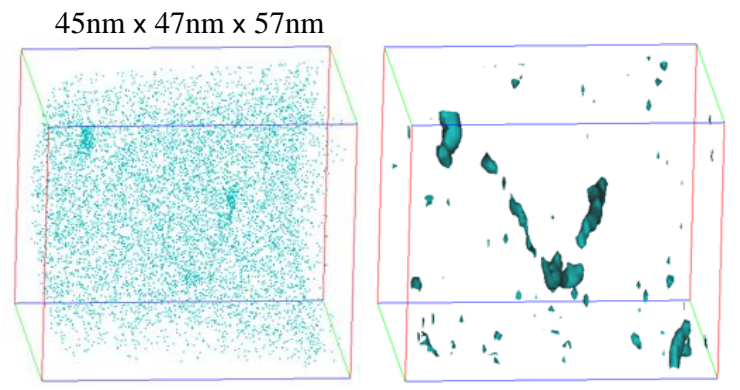

(b)

Figure 1 (a) 3D atom map and 1.2 at.\% isoconcentration surface of niobium atoms in the same volume in a grain of the niobium added stainless steel. (b) Different direction view obtained by rotating around the axis parallel to the measurement direction (the arrow) by about $70^{\circ}$

References:

[1] C. Ouchi, T. Sanpei, T. Okita and I. Kozasu, Microstructural changes of austenite during hot rolling and their effects on transformation kinetics, Hot Deformation of Austenite, ed. by J.B. Ballance, AIME 1976 pp.316-340.

[2] J. Takahashi, K. Kawakami, J. Hamada and K. Kimura, Direct observation of niobium segregation to dislocations in steel, Acta Materialia 107 (2016), 415-422.

[3] A.H. Cottrell and B.A. Bilby, Dislocation theory of yielding and strain ageing of iron, Proc. Phys. Soc. A62 (1948), 49-62. 


\section{Atomic Scale Analysis on Retardation of Recrystallization of Work- Hardened Austenite in V-Added Low Alloy High Carbon Steel}

Yukiko Kobayashi ${ }^{1}$, Manabu Kubota ${ }^{2}$, Jun Takahashi ${ }^{1}$ and Kazuto Kawakami ${ }^{3}$

Corresponding Author: kobayashi.yf6.yukiko@jp.nssmc.com

1. Advanced Technology Research Laboratories, Nippon Steel \& Sumitomo Metal Corporation, Futtsu, Japan.

2. Steel Research Laboratories, Nippon Steel \& Sumitomo Metal Corporation, Amagasaki, Japan.

3. Nippon Steel \& Sumikin Technology Co., Ltd., Futtsu, Japan.

Controlling recrystallization in austenite before phase transformation is an important issue for thermo-mechanical processing of steel. For the retardation mechanism of recrystallization, it is proposed that movement of the interface between recrystallized and un-recrystallized grains is inhibited owing to pinning effect by precipitates or solute drag effect by segregated microalloying elements on the interface [1]. Recently, remarkable retardation of recrystallization of austenite by adding vanadium is reported in high carbon martensitic steel [2]. In this study, the interface between recrystallized/un-recrystallized austenite grains was investigated by APT analysis in order to elucidate the mechanism for the retardation of recrystallization by adding $\mathrm{V}$.

Steel comprising 0.56C-1.5Si-0.7Mn-0.1V(mass\%) was hot-deformed and kept at $800{ }^{\circ} \mathrm{C}$ for various time periods in austenite followed by quenching. Recrystallized and un-recrystallized grains were identified by reconstructed austenite orientation map from a martensite orientation map of EBSD [2,3]. Segregation of V on the interface was observed by APT much later than onset of retardation. On the other hand, the dissolved V content was lower in the recrystallized grains than in the un-recrystallized grains in the early stage of recrystallization. This result indicates that $\mathrm{V}(\mathrm{C}, \mathrm{N})$ precipitates formed and/or grew rapidly on the interface. The pinning effect on the retardation of recrystallization by the $\mathrm{V}(\mathrm{C}, \mathrm{N})$ precipitates will be discussed.
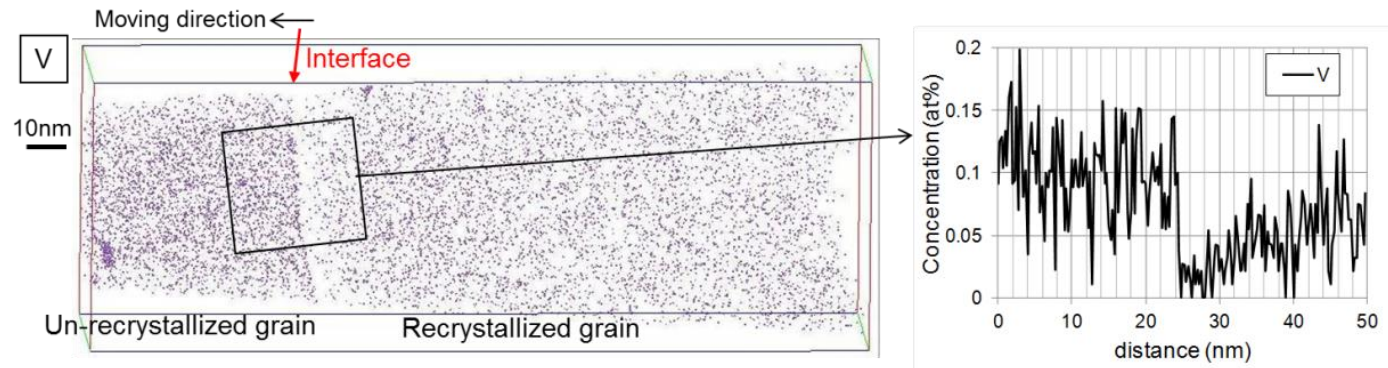

Figure $1 \mathrm{~V}$ atom map of interface between recrystallized and un-recrystallized grains and corresponding $1 \mathrm{D}$ concentration profile near the interface in the early stage of recrystallization $\left(800{ }^{\circ} \mathrm{C}, 100 \mathrm{~s}\right)$.

\section{$\underline{\text { References: }}$}

[1] J.W. Cahn, The inpurity-drag effect in grain boundary motion, Acta Metall. 10 (1962), 789-798.

[2] M. Kubota, K. Ushioda, G. Miyamoto and T. Furuhara, Analysis of recrystallization behavior of hotdeformed austenite reconstructed from electron backscattering diffraction orientation maps of lath martensite, Scr. Mater. 112 (2016), 92-95.

[3] G. Miyamoto, N. Takayama and T. Furuhara, Accurate measurement of the orientation relationship of lathmartensite and bainite by electron backscatter diffraction analysis, Scr. Mater. 60 (2009), 1113-1116. Acknowledgement: The authors are grateful to Prof. G. Miyamoto for the provision of the method of reconstructing the parent austenite orientation map. 


\section{Analyzing Boron in 9-12\% Chromium Steels Using Atom Probe Tomography}

Irina Fedorova ${ }^{1}$, Flemming Bjerg Grumsen ${ }^{1}$, John Hald $^{1}$, Hans-Olof Andrén ${ }^{2}$, Fang Liu ${ }^{3}$

Corresponding Author: fang.liu@,chalmers.se

1. Department of Mechanical Engineering, Technical University of Denmark, 2800 Kgs. Lyngby, Denmark.

2. Department of Physics, Chalmers University of Technology, SE-41296, Gothenburg, Sweden.

${ }^{3 .}$ Department of Industrial and Materials Science, Chalmers University of Technology, SE-41296, Gothenburg, Sweden.

Boron has long been found beneficial to steels. For instance, small addition (usually several tens of ppm) of boron can remarkably improve hardenability of low alloy steels [1], and increase long-term creep resistance of 9-12\% Cr steels [2], which are used for thick section components in steam power plants that generate more than $60 \%$ of electricity worldwide. The improvement in creep resistance was suggested to attribute to boron segregation to grain boundaries during quenching, and the subsequent boron incorporation into certain families of precipitates [3]. However, the detailed mechanisms are not yet fully understood, mainly due to three reasons: Firstly, boron is the fifth lightest element; Secondly, the boron concentration can be rather low, for instance, for grain boundary segregation, the concentration can be well below 1 at.\%; Thirdly, they are often distributed in very small regions, such as boundaries and nano-sized precipitates. Therefore, to gain insights of boron requires ideally quantitative analysis with high sensitivity to B and high spatial resolution, which makes Atom Probe Tomography (APT) a desirable instrument, because of its equally high sensitivity to all elements, and high spatial resolution - potentially down to the atomic level.

However, there are several key challenges to acquire accurate analysis of boron in $9-12 \% \mathrm{Cr}$ steels using APT. First, as mentioned above, boron atoms are often enriched in small and highly site-specific regions, like grain boundaries, which makes specimen preparation for APT demanding. Additionally, boron has a relatively high evaporation field compared to many other elements, such as iron. Furthermore, boron atoms also have the tendency to evaporate together and form multi-hits, which makes accurate quantification difficult [4]. In this paper, we present a novel technique for site-specific APT specimen preparation using a custom-made specimen holder for in-situ Focused Ion Beam/Scanning Electron Microscopy lift-out, combined with imaging using ion channeling contrast and Transmission Kikuchi Diffraction, which enables unambiguous preparation of APT specimen containing specifically selected grain boundaries. Additionally, by comparing the multiple and single hits of metal borides, we also discuss the possible effects of detector dead-time on quantitative analysis of boron in metal borides. Finally, the possible widening of boron distribution profiles over boundaries compared to other elements is particularly analyzed and discussed in light of the evaporation field of the different elements.

\section{$\underline{\text { References: }}$}

[1] N. Tsuji, Y. Matsubara, T. Sakai, Y. Saito, Effect of boron addition on the microstructure of hot-deformed Ti-added interstitial free steel, ISIJ International, Vol. 37 (1997), 797-806.

[2] J. Hald, Microstructure and long-term creep properties of 9-12\% Cr steels, International Journal of Pressure Vessels and Piping, Vol. 85 (2008), 30-37.

[3] F Liu, DHR Fors, A Golpayegani, H-O Andrén and G Wahnström, Effect of Boron on Carbide Coarsening at $873 \mathrm{~K}\left(600{ }^{\circ} \mathrm{C}\right)$ in 9 to 12 pct Chromium Steels, Metall. Mater. Trans. A, Vol. 43 (2012), 4053-4062.

[4] G. Da Costa, H, Wang, S. Duguay, A. Bostel, D. Blavette, B. Deconihout, Advance in multi-hit detection and quantization in atom probe tomography, Review of Scientific Instruments, Vol. 83 (2012), 123709. 


\title{
The Segregation Features at Grain Boundaries with Various Characters in Highly Twinned Alloy 690
}

\author{
Hui $\mathrm{Li}^{1}$, Wenqing Liu ${ }^{1}$ \\ Corresponding Author: huili@,shu.edu.cn
}

1. Key Laboratory for Microstructures, School of Materials Science and Engineering, Shanghai University, Shanghai, P.R. China.

The grain boundary segregation behaviors in Inconel Alloy 690 with high proportional low $\Sigma$ coincidence site lattice (CSL) grain boundaries were studied by atom probe tomography. The Inconel Alloy 690 was solution annealed at $1100{ }^{\circ} \mathrm{C}$ for $15 \mathrm{~min}$, and treated by grain boundary engineering to produce high proportional low $\Sigma$ CSL grain boundaries in the microstructure. And then the samples were aged at $715^{\circ} \mathrm{C}$ for different time to promote grain boundary segregation and carbide precipitation.

The results show that the $\mathrm{C}, \mathrm{B}, \mathrm{Si}$ and $\mathrm{Cr}$ segregate significantly at grain boundary before the carbide precipitation $\left(500{ }^{\circ} \mathrm{C} / 0.5 \mathrm{~h}\right)$. The $\mathrm{C}$ and $\mathrm{Cr}$ co-segregate at the same region in the grain boundary. After the initial nucleation of grain boundary carbide $\left(715^{\circ} \mathrm{C} / 0.5 \mathrm{~h}\right)$, the amount of grain boundary segregation reduced, only a few of $\mathrm{C}$ and $\mathrm{B}$ atoms can be observed at grain boundaries. Most of impurity atoms are segregating in the carbide. None of grain boundary segregation can be observed in the samples aged at $715^{\circ} \mathrm{C}$ for $2 \mathrm{~h}$ and longer time. Furthermore, the results show that the grain boundary characters influence the segregation features significantly, e. g. the $\mathrm{B}$ and $\mathrm{C}$ atoms have higher segregation tendency to the random grain boundary than low $\Sigma$ CSL grain boundaries. The Cr depleted more seriously at the carbide /matrix phase interface than at grain boundary, and more seriously at random grain boundaries than at low $\Sigma$ CSL grain boundaries. Based on the experimental results, the effect of grain boundary characters on the grain boundary composition was discussed.

In additional, the effects of temperature and laser energy on the composition detection of this alloy were investigated. The sample was test at $20 \mathrm{~K}-70 \mathrm{~K}$ and $40 \mathrm{pJ}-80 \mathrm{pJ}$. It shows that the testing parameters influence the detected composition of main elements slightly, such as $\mathrm{Ni}, \mathrm{Cr}$ and Fe. But, the higher temperature and higher laser energy lead to some loss of $\mathrm{C}, \mathrm{B}$ and $\mathrm{Si}$ atoms. If the temperature is lower than $50 \mathrm{~K}$, and the laser energy is lower than $60 \mathrm{pJ}$, the detected composition is similar to the nominal composition of sample without carbide. But, this parameter leads to $\sim 20 \% \mathrm{C}$ atoms loss in the $\mathrm{M}_{23} \mathrm{C}_{6}$ carbide, and some prefer evaporation of $\mathrm{Si}$ atoms in the sample containing carbide. Only the testing temperature lower than $30 \mathrm{~K}$ and laser energy lower than $30 \mathrm{pJ}$ can give the reasonable results in the sample containing carbide. 


\title{
APT and STEM Analysis of a Metallic Nuclear Fuel to Reveal the Influence of Grain Boundary Segregation on Kinetics of Discontinuous Precipitation
}

\author{
Arun Devaraj ${ }^{1}$, Elizabeth Kautz ${ }^{1}$, Libor Kovarik ${ }^{2}$, Saumyadeep Jana ${ }^{3}$, Curt Lavender ${ }^{3}$, and \\ Vineet Joshi ${ }^{3}$ \\ Corresponding Author: arun.devaraj@pnnl.gov \\ 1. Physical and Computational Sciences Directorate, Pacific Northwest National Laboratory, \\ Richland, WA 99352, USA \\ 2. Environmental Molecular Sciences Laboratory, Pacific Northwest National Laboratory, \\ Richland, WA 99352, USA \\ 3. Energy and Environment Directorate, Pacific Northwest National Laboratory, Richland, WA \\ 99352, USA
}

Using detailed aberration-corrected scanning transmission electron microscopy and atom probe tomography analysis of a multicomponent metallic nuclear fuel - uranium-10 wt\% molybdenum (U-10Mo) alloy with several impurities, we demonstrated the ability to change the type of grain boundary complexions, which then controls the subsequent discontinuous precipitation kinetics during sub-eutectoid annealing. A change in grain boundary complexion type from "one characterized by segregation of Mo and impurities at grain boundary" to grain boundaries "with a distinct U2MoSi2C wetting phase precipitate" caused a pronounced reduction in kinetics of subsequent discontinuous precipitation. The broader implication of this work is in highlighting the role of grain boundary segregation in metallic alloys on dictating the fate of grain boundary initiated phase transformations like discontinuous precipitation or cellular transformation. The unique strength of APT in quantifying element segregation to grain boundaries and other interfaces in crucial nuclear fuel materials will be highlighted in addition to its complementary strength with aberration corrected STEM analysis of microstructure, thus contributing to understanding of phase transformation mechanisms in nuclear fuel materials [1,2].

\section{References:}

[1] S. Jana, A. Devaraj, L. Kovarik, B. Arey, L. Sweet, T. Varga, C. Lavender, V. Joshi, Kinetics of Cellular Transformation and Competing Precipitation Mechanisms During Sub-Eutectoid Annealing of U10Mo Alloys, Journal of Alloys and Compounds 723 (2017) 757-771.

[2] A. Devaraj, L. Kovarik, V.V. Joshi, S. Jana, S. Manandhar, B.W. Arey, C.A. Lavender, HighResolution Characterization of UMo Alloy Microstructure, Pacific Northwest National Lab.(PNNL), Richland, WA (United States), 2016. 


\title{
Nanoscale Analysis of Ion Irradiated 14YWTi ODS Steel
}

\author{
M.A. Auger ${ }^{1}$, D.T. Hoelzer², K.G. Field ${ }^{2}$, M.P. Moody ${ }^{1}$ \\ Corresponding Author: maria.auger@materials.ox.ac.uk \\ ${ }^{1}$ Department of Materials, University of Oxford. Parks Road OX1 3PH, Oxford, UK. \\ 2. Oak Ridge National Laboratory, Oak Ridge, TN 37831, Tennessee USA
}

ODS steels have remained as candidate structural materials for nuclear applications over the last decade due to the dispersion of nano-size oxide particles improving their mechanical performance by impeding dislocation motion, enhancing creep resistance and raising the operating temperature. Even though the results currently available indicate the good irradiation resistance of ODS steels, further analysis of different irradiation sources and conditions are still needed.

In this work, the nanoscale microstructure of a 14YWT alloy [1] (SM13 heat) with nominal composition $\mathrm{Fe}-14 \mathrm{Cr}-3 \mathrm{~W}-0.4 \mathrm{Ti}-0.3 \mathrm{Y}_{2} \mathrm{O}_{3}$ (wt. \%) has been characterized by Atom Probe Tomography (APT) before and after irradiation. The irradiation experiment has been performed at CAMS (Center for Accelerator Mass Spectrometry) in LLNL (Lawrence Livermore National Laboratory), CA with $70 \mathrm{MeV} \mathrm{Fe}^{++}$ions $(20 \mathrm{dpa})$ at $450^{\circ} \mathrm{C}$.

APT samples were prepared by the FIB lift-out method and the samples were analyzed using CAMECA LEAP 3000 and 5000 instruments. Fig. 1 shows 3D ion maps of C and Y ions in (a) as-received 14YWT and (b) ion irradiated 14YWT. C segregation to grain boundaries is enhanced by the irradiation, while the nanoparticle dispersion seems to be stable. A detailed cluster analysis before and after irradiation only shows slight differences in the Y-rich nanoparticles regarding their size, number density and composition, so the stability of this alloy to the given irradiation conditions is consistent with previous studies on the radiation tolerance of 14YWT.
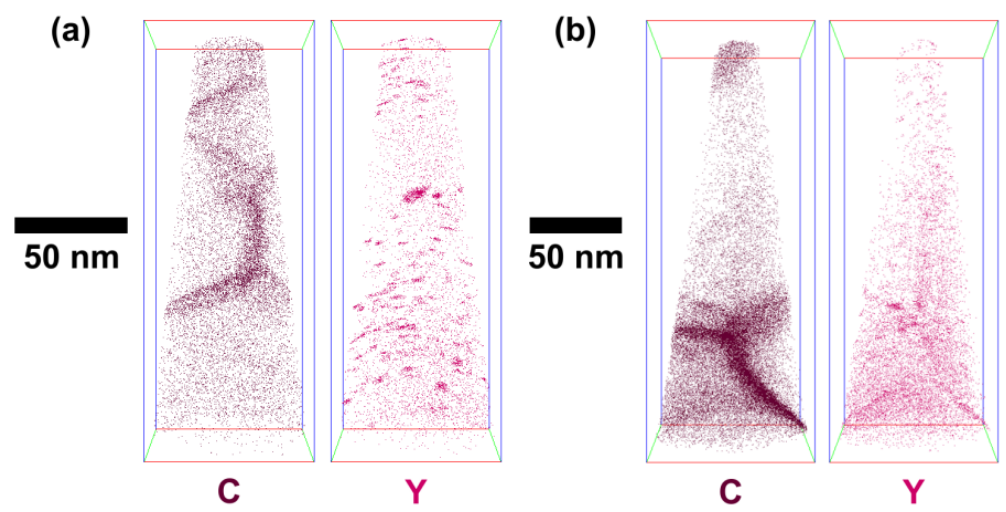

Figure $13 \mathrm{D}$ ion maps of $\mathrm{C}$ and $\mathrm{Y}$ ions in (a) as-received 14YWT and (b) $70 \mathrm{MeV}$ (20 dpa, $\left.450^{\circ} \mathrm{C}\right) \mathrm{Fe}^{++}$irradiated $14 \mathrm{YWT}$.

\section{References:}

[1] D.T. Hoelzer, J. Bentley, M.A. Sokolov, M.K. Miller, G.R. Odette, M.J. Alinger, Influence of particle dispersions on the high-temperature strength of ferritic alloys, J Nucl Mater 367-370 (2007) 166-172.

[2] Funding from US-DOE (DE-NE0000639), and UK-EPSRC (grants EP/P001645/1, EP/L025817/1 and $\mathrm{EP} / \mathrm{M} 022803 / 1)$ is acknowledged. 


\section{Understanding Behavior and Performance of Nuclear Fuels via Atom Probe Tomography}

Mukesh Bachhav $^{1}$, Jian Gan ${ }^{1}$, Brandon Miller ${ }^{1}$, Lingfeng $\mathrm{He}^{1}$, and Dennis Keiser ${ }^{2}$

Corresponding Author: mukesh.bachhav@inl.gov

1. Advanced Characterization Division, Idaho National Laboratory, Idaho Falls, ID 83415, USA

2. Nuclear Fuels and Materials Division, Idaho National Laboratory, Idaho Falls, ID 83415, USA

It is well known that the overall performance of nuclear fuels is affected by microstructural changes during irradiation. One of the main mechanisms that plays a key role in the microstructural changes of fuels is damage associated with the formation and migration of fission products [1]. For instance formation and movement of fission product leads to void formation and grain boundary segregation which can promote integrity loss of the fuel. Also, during irradiation at aggressive reactor conditions, interaction can occur between the fuel particle and matrix in a dispersion fuel that results in development of an interaction layer that is unstable under irradiation, which can contribute to fuel plate failure. Thus, it is important to study the microstructural changes in irradiated fuel in order to understand fission product migration and how they affect the performance of fuels.

Atom Probe Tomography (APT) is employed in current study to elucidate microstructural evolution in fuels to quantify fission products and determine chemistry changes after irradiations. Systematic APT study is carried out on UMo alloy fuel particles [2] coated with a diffusion barrier layer of $\mathrm{ZrN}$ and dispersed in Al matrix. Atom probe analysis on irradiated UMo fuel reveals uniform distribution spherical shaped clusters enriched with fission products such as $\mathrm{Ru}$, $\mathrm{Pd}, \mathrm{Cd}, \mathrm{Ag}, \mathrm{Xe}, \mathrm{Pr}, \mathrm{Nd}$ etc. Quantification and distribution of fission products depended on running conditions of APT instrument (base temperature, pulse rate, detection rate, laser and voltage mode). Our understanding on the microstructural evolution and performance of irradiated fuels will be discussed in this presentation.

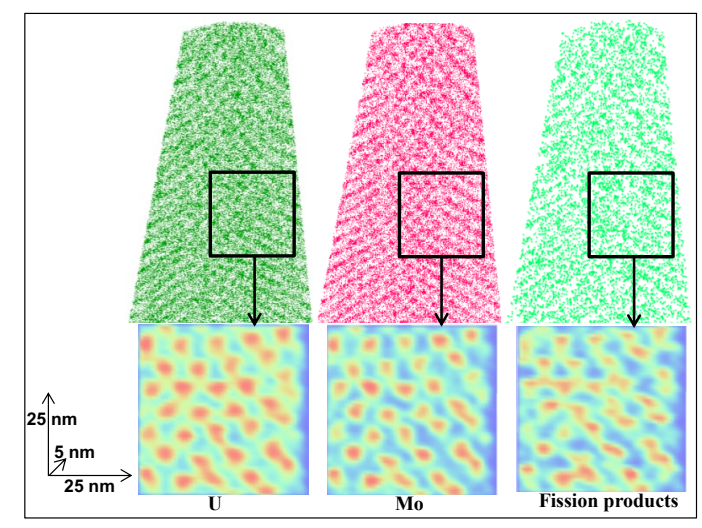

Figure 1: 3D distribution and $2 \mathrm{D}$ density map of $U$, Mo and fission products in irradiated UMo fuel.

\section{References:}

[1] A. Leenaers, S. Van den Berghe, E. Koonen, C. Jarousse, F. Huet, M. Trotabas, M. Boyard, S. Guillot, L. Sannen, M. Verwerft, J. Nucl. Mater. 335 (2004) 39-47.

[2] D. Keiser, E. Perez, T. Wieneck, A. Leenaers, S. Van den Berghe, JNM, 458, (2015), 406-418. 


\title{
Atom Probe Tomography as a Nuclear Forensics tool - Analysing Nanoparticulate Material from the Fukushima Region of Japan
}

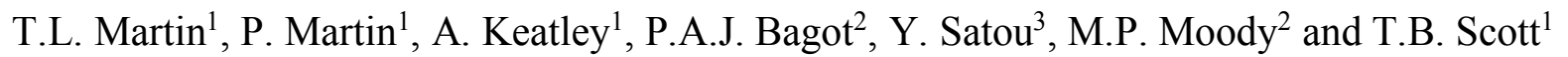 \\ Corresponding Author: tomas.martin@bristol.ac.uk \\ 1. Interface Analysis Centre, School of Physics, University of Bristol, Tyndall Avenue, Bristol \\ BS8 1TH, UK \\ 2. Department of Materials, University of Oxford, Parks Road, Oxford OX1 3PH, UK \\ 3. Collaborative Laboratories for Advanced Decommissioning Science, Japanese Atomic Energy \\ Association, 790-1 Otsuka Motooka, Futabu-gun, Fukushima 979-1151, Japan
}

In addition to its high spatial and chemical resolution, atom probe tomography (APT) can identify divergences from the natural isotopic abundances of elements. This includes the study of relative abundances of parent-daughter nuclei to age geological material [1] or using tracer isotopes in chemical processes, such as actinide corrosion [2]. Another potential field of study is within the rapidly evolving field of nuclear forensics, where isotopic ratios can indicate the radioactive provenance of a material. Identifying a radioactive material's isotopic composition can be vital not only for national nuclear security, operational compliance but also in the remediation of sites of nuclear accidents, such as surrounding the Fukushima Daiichi Nuclear Power Plant (FDNPP) in Japan.

Following the earthquake and tsunami of March 2011, cooling to the reactors on the FDNPP site was lost, resulting in an exothermic reaction of steam with the zirconium cladding in the reactors, releasing significant volumes of hydrogen gas. The ensuing building explosions distributed highly-radioactive particulate across the surrounding Fukushima Prefecture. As each reactor unit was subtly different in its design and at a different stage of the refuelling cycle, fuel material from each core has a characteristic isotopic signature. APT analysis of the isotopic ratios of fallout particulate can identify the reactor unit where each particle originated and so distinguish the edges and overlap of each explosion plume, aiding remediation and understanding of the behaviour of particulate in the plume and environment.

Particulate material ejected from FDNPP was collected from sediment at the edge of the $30 \mathrm{~km}$ exclusion zone. These sub-micron particles were screened using backscattered electron and energy dispersive spectroscopy detectors in a variable-pressure scanning electron microscope to identify those with a high- $Z$ composition [3]. Using a micromanipulator, these were transferred into a focused ion beam and mounted for APT analysis. This paper will present a comparison of the signature of particles from different affected areas around FDNPP with known "core inventory" values. The work will also evaluate the implications of this material on the on-going remediation efforts, in addition to a discussion of the limits of APT in studying the isotopic ratio of actinides.

\section{References:}

[1] J.W. Valley et al, Hadean age for a post-magma-ocean zircon confirmed by atom-probe tomography, Nature Geoscience 7 (2014), 219-223

[2] T.L. Martin et al, Atomic-scale Studies of Uranium Oxidation and Corrosion by Water Vapour, Scientific Reports 6 (2016), 25618

[3] P. Martin et al, In-situ removal and characterisation of uranium-containing particles from sediments surrounding the Fukushima Daiichi Nuclear Power Plant, Spectrochimica Acta Part B: Atomic Spectroscopy 117 (2016) 1-7 


\title{
Elemental Partitioning and Site Occupation of Mo and $\mathrm{Cr}$ in Co-Ti Based Superalloys
}

\author{
Hyeji $\operatorname{Im}^{1}$, Surendra Kumar Makineni ${ }^{2}$, Baptiste Gault ${ }^{2}$, Dierk Raabe ${ }^{2}$, Pyuck-Pa Choi ${ }^{1, *}$
}

*Corresponding Author: p.choi@kaist.ac.kr

1. Department of Materials Science and Engineering, Korea Advanced Institute of Science and Technology (KAIST), 291 Daehak-ro, Yuseong-gu, Daejeon, 34141, Republic of Korea

2. Department of Microstructure Physics and Alloy Design, Max-Planck-Institut für Eisenforschung, Max-Planck-Str. 1, 40237 Düsseldorf, Germany

Co-Ti-based alloys are considered as promising alternatives to the well-known Ni- and Co-Al-Wbased superalloys as they form a thermodynamically stable $\gamma$ (FCC Co solid solution)/ $\gamma^{\prime}\left(\mathrm{Ll}_{2}-\right.$ ordered $\mathrm{Co}_{3} \mathrm{Ti}$ precipitates) microstructure upon ageing [1]. However, the $\gamma^{\prime}$ phase in Co-Ti exhibits only a low volume fraction of about $20 \%$ and a low thermal stability [2].

In order to overcome these drawbacks, we studied the effects of various alloying elements on the properties of Co-Ti alloys. Here we focus on the effects of $\mathrm{Mo}$ and $\mathrm{Cr}$ on the partitioning and siteoccupancy behavior of Co-Ti-Mo, Co-Ti-Cr, and Co-Ti-Mo-Cr alloys. Ti and $\mathrm{Mo}$ are found to strongly partition to $\gamma^{\prime}$, whereas $\mathrm{Co}$ and $\mathrm{Cr}$ partition to $\gamma$. Both $\mathrm{Mo}$ and $\mathrm{Cr}$ are found to increase the volume fraction of $\gamma^{\prime}$ through partial substitution of Ti on its sub-lattice within $\gamma^{\prime}$.

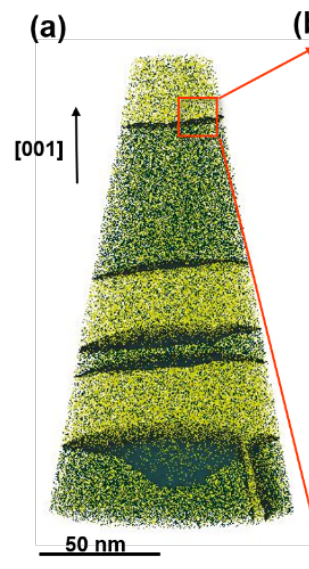

(b)
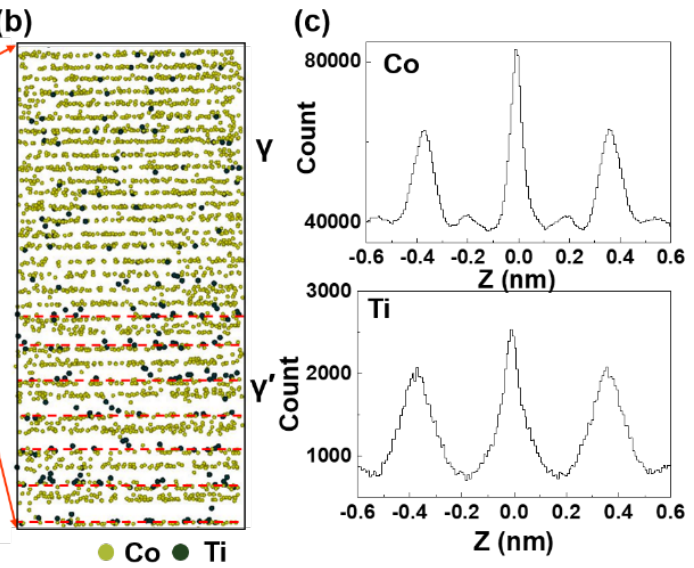

Figure 1 (a) 3D reconstructions of an aged Co-Ti alloy. 12.65 at.\% Ti iso-concentration surfaces highlight the $\gamma / \gamma^{\prime}$ interfaces. (b) (001) lattice planes in $\gamma$ and $\gamma^{\prime}$ phases can be seen. (c) z-spatial distribution maps of Co and Ti in $\gamma^{\prime}$ phase.

\section{References:}

[1] M. Jiang, G. Saren, S.Y. Yang, H.X. Li and S.M. Hao, Phase equilibria in Co-rich region of Co-Ti-Ta system, Trans. Nonferrous Met. Soc. China (English Ed.) 21 (2011), 2391-2395.

[2] C.H. Zenk, S. Neumeier, H.J. Stone and M. Göken, Mechanical properties and lattice misfit of $\gamma / \gamma$ ' strengthened Co-base superalloys in the Co-W-Al-Ti quaternary system, Intermetallics 55 (2014), 28-39. 


\title{
Atom Probe Investigations of the Miscibility of $\mathrm{Cu}$ and $\mathrm{Ni}$
}

\author{
Rüya Duran ${ }^{1}$, Patrick Stender ${ }^{1}$, and Guido Schmitz ${ }^{1}$ \\ Corresponding Author: rueya.duran@mp.imw.uni-stuttgart.de \\ 1. Institute of Material Science, University of Stuttgart, 70569 Stuttgart, Germany
}

Although Copper-Nickel alloys are commonly used in technical applications, their alloying behavior at low temperatures is still controversial. Early thermodynamic analysis of Meijering indicated that the phase diagram may contain a miscibility gap [1]. While direct experimental measurements couldn't be carried out because of the slow kinetics at low temperatures, theoretical and indirect experimental investigations were made $[2,3]$. These lead to the apparent determination of a wide range of different solubility boundaries, but clear evidence is still missing.

In this work, the miscibility gap is determined by a direct experimental method applying Atom Probe Tomography (APT) to thin film structures. The temperature dependence of the segregation in $\mathrm{Cu} / \mathrm{Ni}$ thin films is investigated. To make sure that equilibrium has been reached, different annealing times were tested. To limit the required annealing times, samples consist of a stack of nanometer-sized layers. In this way, every layer can be brought in local (metastable) equilibrium which is way faster than bringing the whole sample in stable equilibrium.

The quantitative evaluation was made by a cluster analysis, which determines the local atomic fraction in coarse-grained analysis cells located around each detected atom. By plotting the abundances of the concentrations, the sample reveals two concentrations of maximum abundance that reflect the existence of a miscibility gap (see Fig.1). The variation of the maxima with temperature delivers the boundary concentrations of the miscibility gap. As an important advantage, this method functions even well after thermal fragmentation of the layer structure.

(a)

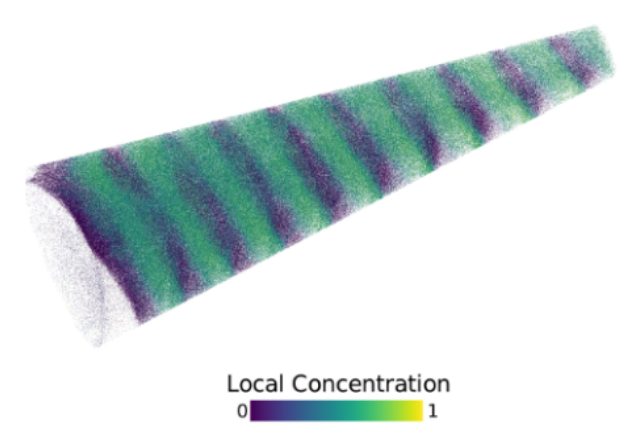

(b)

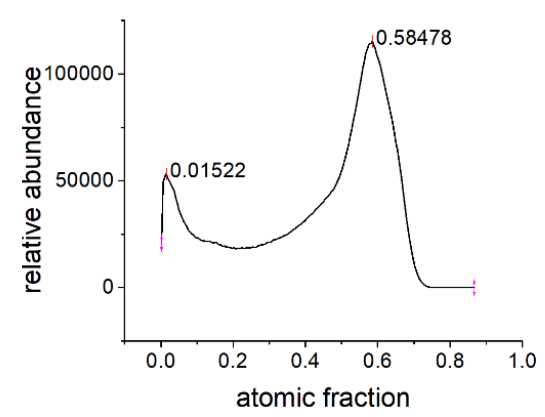

Figure 1 (a) Three-dimensional reconstruction of a multilayered sample annealed at 723K. (b) Cluster concentration histogram of $\mathrm{Cu}$ atoms for the sample in (a)

\section{References:}

[1] J. L. Meijering, Calculation of the nickel-chromium-copper phase diagram from binary data, Acta Metall. 5 (1957), 257

[2] S. Srikanth, K. T. Jacob, Thermodynamic properties of $\mathrm{Cu}-\mathrm{Ni}$ alloys: measurements and assessment, Materials Science and Technology 5 (1989), 427.

[3] J. Tomiska, A. Neckel, Thermodynamics of Solid Cu-Ni Alloys by Knudsen Cell Mass Spectrometry and Re-Calculation of the Phase Diagram, Ber. Bunsenges. Phys. Chem. 88 (1984), 551. 


\title{
Superficial Enrichment in Gold/Silver Alloys: Study of the Physicochemical Influences Using Atom Probe Tomography
}

\author{
N. Gilis ${ }^{1}$, L. Jacobs ${ }^{1}$, S.V. Lambeets ${ }^{1}$, E Genty $^{1}$, C Barroo ${ }^{1,2}$ and T. Visart de Bocarmé ${ }^{1,2}$
}

Corresponding Author: ngilis@ulb.ac.be

${ }^{1}$ Chemical Physics of Materials and Catalysis (CPMCT), Université Libre de Bruxelles, Faculty of Sciences, CP 243, 1050, Brussels, Belgium

${ }^{2}$ Interdisciplinary Centre for Nonlinear Phenomena and Complex Systems (CENOLI), Université libre de Bruxelles (ULB), Faculty of Sciences, Brussels, Belgium

Bimetallic alloys are increasingly used in heterogeneous catalysis. This can be explained by that fact that new features emerge from the alloy that are absent in the parent metal. Synergic effects between the two combined elements creates a more efficient catalyst. One of the most interesting aspect of multicomponent materials in catalysis, is the fact that one could possibly control the catalytic properties of an alloy by controlling the nature and composition of the surface. The gold/silver alloy is an interesting exemplar [1]. Indeed, this alloy combines a high activity and a large selectivity for a broad range of oxidation reaction. Examples have been reported from $\mathrm{CO}$ oxidation at low temperature to more complex oxygen-activated coupling reaction. The silver is crucial to provide the necessary oxygen without leading to a total oxidation [2].

The study of the enrichment phenomenon is conducted by atom probe tomography (APT). This method allows us to establish the chemical composition profile of the alloy with a nanometric lateral resolution and an atomic depth resolution. The atom probe tomography setup is equipped with an in-situ reaction chamber allowing physicochemical treatments with temperature up to $700 \mathrm{~K}$ and pressures up to 1 bar. This overall approach allows us to treat our gold/silver (95/5) samples in condition close to the operating condition of the real catalyst and analyse the evolution of the composition on the surface and the bulk. In this way we aim to establish the physicochemical condition that could allow us to tune the surface composition at will.

Several thermal and chemical treatments were applied on Au-Ag samples to analyze the segregation behavior. Our present research focusses on segregation triggered by high temperatures under UHV conditions, as well as segregation triggered by an oxidizing atmosphere, which in our case is $\mathrm{N}_{2} \mathrm{O}$ and $\mathrm{O}_{2}$. Results show that silver enrichment occurs in the presence of the oxygen rich gas and takes place at lower temperature than with annealing in UHV. These results are in accordance with recent literature [3], [4]. Our results also show different segregation behaviors in the case of annealing or chemically triggered segregation. Indeed, the composition profile of $\mathrm{N}_{2} \mathrm{O}-$ treated sample clearly shows - below the segregated silver layer - a layer which is Ag-depleted, before showing the expected bulk composition. In the case of annealed-samples, the Ag-depleted region is absent. This indicates that $\mathrm{N}_{2} \mathrm{O}$-segregated silver atoms are chemically pumped out from the bulk, just underneath the surface, while annealed-segregated silver atoms probably migrate at the surface from the shank of the tip to the apex part

[1] J. Biener, et al., "Nanoporous Gold: Understanding the Origin of the Reactivity of a 21 st Century Catalyst Made by Pre-Columbian Technology," ACS Catal., vol. 5, no. 11, pp. 6263-6270, 2015.

[2] V. N. M. Rao and N. A. Nielsen, "Process for selective oxidation/dehydrogenation of methanol to formaldehyde using a metal catalyst," European Patent EP0003348 B1, 1979.

[3] G. Guisbiers, et al., "Electrum, the Gold-Silver Alloy, from the Bulk Scale to the Nanoscale: Synthesis, Properties, and Segregation Rules.," ACS Nano, vol. 10, no. 1, pp. 188-98, 2016.

[4] B. Zugic, et al., "Dynamic restructuring drives catalytic activity on nanoporous gold-silver alloy catalysts," Nat. Mater., vol. 1, no. December, pp. 1-8, 2016. 


\section{Direct Observations of Atomic Hydrogen and Deuterium Interactions in Zircaloy-4}

Andrew Breen ${ }^{1}$, Isabelle Mouton ${ }^{1}$, Agnieszka Szczepaniak ${ }^{1}$, Wenjun Lu ${ }^{1}$, Siyang Wang ${ }^{2}$, Paraskevas Kontis ${ }^{1}$, Leigh Stephenson ${ }^{1}$, Yanhong Chang ${ }^{1}$, Christian Liebscher ${ }^{1}$, Ben Britton ${ }^{2}$, Baptiste Gault $^{1}$, Michael Herbig ${ }^{1}$, Dierk Raabe ${ }^{1}$

Corresponding Author: a.breen@mpie.de

1. Max-Planck-Institut für Eisenforschung, Max-Planck-Straße 1, 40237 Düsseldorf, Germany.

2. Department of Materials, Royal School of Mines, Imperial College London, London, SW7

2AZ, UK

The interaction of hydrogen $(\mathrm{H})$ in metals has had significant scientific interest for over a century, particularly in regards to understanding the mechanisms governing hydrogen embrittlement phenomena. For many alloys, the ingress of $\mathrm{H}$, either during fabrication or service, leads to a pronounced reduction in ductility and resultant premature failure. Zirconium alloys are one such example that are susceptible to the formation of hydrides and subsequent embrittlement in aqueous environments. Until recently, the understanding of how $\mathrm{H}$ interacts with metals has been largely based on theoretical modeling as well as indirect experimental observations. Here we show that atom probe tomography (APT) can provide direct 3D observations of the location of $\mathrm{H}$ atoms within metals with sub nm spatial resolution.

In this work, Zircaloy-4 (Zr- $1.5 \% \mathrm{Sn}-0.2 \% \mathrm{Fe}-$ $0.1 \% \mathrm{Cr}$ wt. \%), which has been charged with hydrogen or deuterium (D), is analysed using APT complemented by transmission electron microscopy (TEM) and Electron Backscattered Diffraction (EBSD). Interfacial regions between the parent $\alpha$-Zirconium and hydrides are characterised to help elucidate the mechanisms of hydride formation. The results suggest the presence of several hydride phases such as $\delta\left(\mathrm{ZrH}_{1.66}\right)$ and $\xi\left(\mathrm{Zr}_{2} \mathrm{H}\right)$, with the latter found within a broad and diffuse interfacial region between $\delta$ and the $\alpha$-matrix. Atom probe crystallography methods are used to calibrate the atom probe reconstructions, determine the presence of hydride phases and directly combine crystallographic character of boundaries with segregation behavior of atomic species. The challenges of quantifying $\mathrm{H}$ using APT as well as the implications the technique has for studying $\mathrm{H}$ in metals will be discussed.
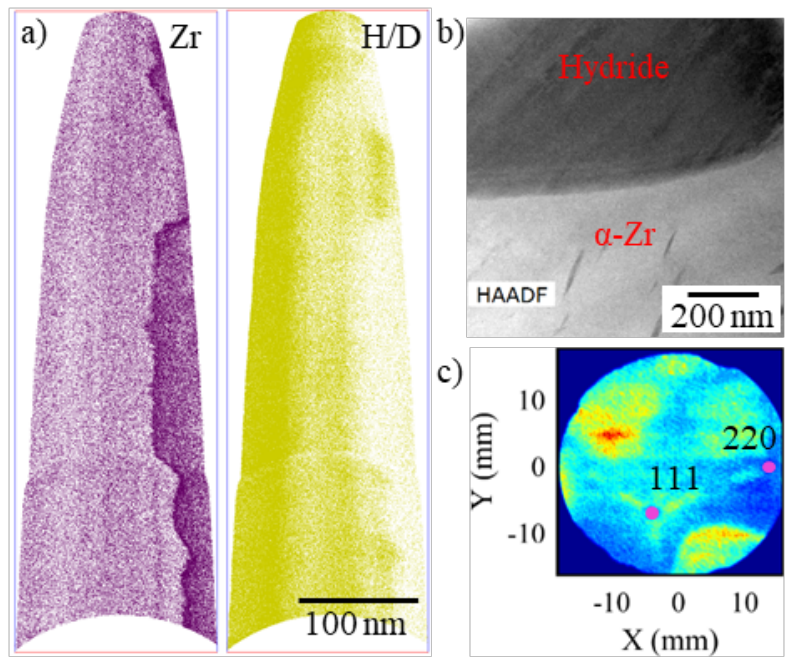

Figure 1 a) atom probe reconstruction of an intergranular Hydride $/ \alpha-\mathrm{Zr}$ interface. b) HAADF TEM image of a similar interface. c) Field desorption map of 1 million atoms showing indexed crystallographic pole and zone line information of a hydride phase 


\title{
Investigating the Effects of Oxygen and Nitrogen on Titanium alloys for Turbine Engine applications
}

\author{
H. M. Gardner ${ }^{1}$, A. Radecka ${ }^{2}$, D. Rugg ${ }^{2}$, D. E. J. Armstrong ${ }^{1}$, M. P. Moody ${ }^{1}$ and P. A. J. Bagot ${ }^{1}$ \\ Corresponding Author: hazel.gardner@materials.ox.ac.uk \\ 1. Department of Materials, University of Oxford, Oxford OX1 3PH, United Kingdom. \\ 2. Rolls-Royce plc, Derby, DE24 8BJ, United Kingdom.
}

Titanium alloys are used in jet engine components owing to their high strength to weight ratio, which helps minimise total engine weight. The effect of oxygen and nitrogen on these alloys is significant and may influence component durability. In particular, these interstitials can degrade mechanical properties of titanium components as a result of environmental exposure during manufacture or service $[1,2]$.

Trace oxygen can cause embrittlement by encouraging formation of nanoscale precipitates of the ordered $\alpha_{2}$ phase $\left(\mathrm{Ti}_{3} \mathrm{Al}\right)$ [3]. TEM and, more recently, APT studies have characterized the onset of formation of these precipitates [4], [5]. However, further work is needed to determine the effect of nitrogen on $\alpha_{2}$ formation, and to compare its behaviour with that of oxygen.

An APT study of the effect of trace oxygen and nitrogen on the microstructure of Ti-7Al and Ti$6 \mathrm{Al}-4 \mathrm{~V}$, respectively, will be presented, including the effects of these elements on $\alpha_{2}$ formation. Microstructural changes observed will be linked to mechanical properties through complementary nanohardness data. The microstructures formed due to trace oxygen and nitrogen will be compared to those found in the oxygen-rich "alpha case" layer (formed in the top $30 \mu \mathrm{m}$ at temperatures above $480^{\circ} \mathrm{C}$ [2]) on an IMI 834 compressor disc. A multi-technique analysis of the alpha case will be presented to correlate oxygen and nitrogen concentration depth profiles with changes in microstructure and hardness.

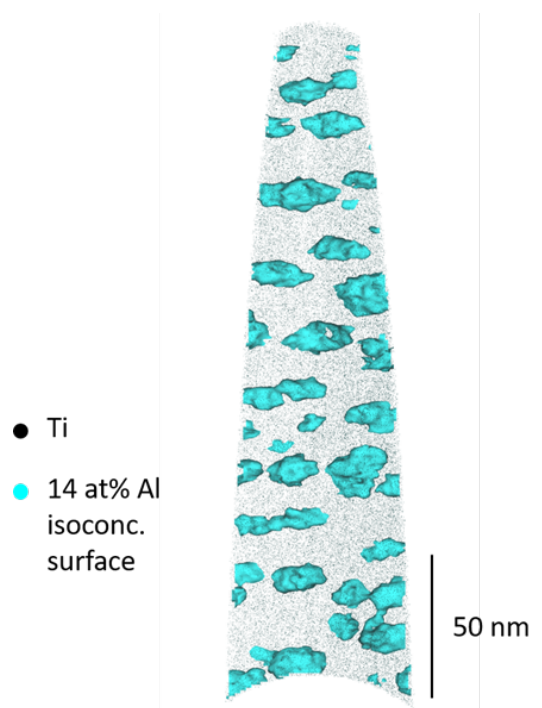

References:

[1] H. R. Ogden and R. I. Jaffee, "The effects of carbon, oxygen and nitrogen on the mechanical properties of titanium and titanium alloys," Titan. Metall. Lab., vol. XXXIII, no. 2, pp. 81-87, 1955.

[2] R. Gaddam, B. Sefer, R. Pederson, and M.-L. Antti, "Study of alpha-case depth in Ti-6Al-2Sn-4Zr-2Mo and Ti-6Al-4V," IOP Conf. Ser. Mater. Sci. Eng., vol. 48, p. 12002, 2013.

[3] J. Y. Lim, "The effect of Oxygen on the structure and mechanical behaviour of aged Ti-8 wt\% Al," Metall. Trans. A, vol. 7, no. 1, pp. 139144, 1976.

[4] H. Liew, G. D. W. Smith, A. Cerezo, and D. J. Larson, "Experimental studies of the phase separation mechanism in Ti-15at\%A1," Mater. Sci. Eng. A, vol. 270, no. 1, pp. 9-13, Sep. 1999.

[5] A. Radecka et al., "Ordering in Titanium Alloys," in Proceedings of the 13th World Conference on Titanium, 2016, no. May, pp. 971-978.

Figure 1 (a) Fig. 1: Slice through APT atom map of 49 day aged Ti-7Al containing 2500 wppm O (nominal). $\alpha_{2}$ precipitates have been highlighted using a blue isoconcentration surface (14 at.\% Al). 


\title{
Atom Probe Characterization of Thermally-Grown Oxides Formed on Nickel Based Superalloys
}

\author{
M.T. Lapington ${ }^{1}$, D.J. Crudden ${ }^{1}$, R.C. Reed ${ }^{1}$, M.P. Moody ${ }^{1}$, P.A.J. Bagot ${ }^{1}$ \\ Corresponding Author: mark.lapington@materials.ox.ac.uk \\ ${ }^{1 .}$ Department of Materials, Parks Road, Oxford, OX1 3PH, United Kingdom
}

In order to withstand the demanding conditions at the heart of modern jet turbine engines, nickel based superalloys need to combine a multitude of properties that often have competing requirements during the alloy design stage. A key element involved in the trade-off between oxidation and strength is titanium, which greatly increases strength but has previously been shown to increase oxidation rates [1]. The mechanism behind this behavior is unresolved, but proposals include a reduction of the activation energy of oxidation due to Ti doping [2], or an increase in ion diffusion rates via cationic vacancy creation [3].

A family of model superalloys has been created specifically to investigate this behavior [4], which are functionally identical except for the systematic substitution of Ti with $\mathrm{Nb}$, which has been shown to have a similar strengthening effect [5]. Thermo-gravimetric analysis performed on these alloys has confirmed that increasing Ti: Nb ratio quickens oxidation kinetics. The oxide scales formed this way were crosssectioned and analyzed using Energy-dispersive X-ray spectroscopy (EDX) to determine the elemental distribution, which found Ti dissolved within the chromia, and in discrete rutile $\left(\mathrm{TiO}_{2}\right)$ particles at the oxide surface.

Nanoscale compositional characterization of chromia scales carried out using Atom Probe Tomography (APT) has revealed that the amount of Ti dissolved in the chromia layer does not correlate with the nominal Ti concentration in the alloy, implying that alteration of bulk ion diffusion rates cannot be the only mechanism at work. High concentrations of Ti and other $\gamma$ ' precipitate-forming elements $(\mathrm{Nb}$ and Ta) have also been located at oxide grain boundaries, which are known to act as fast ion diffusion pathways. The importance of the observed grain boundary segregation with respect to oxidation rates is under further investigation.
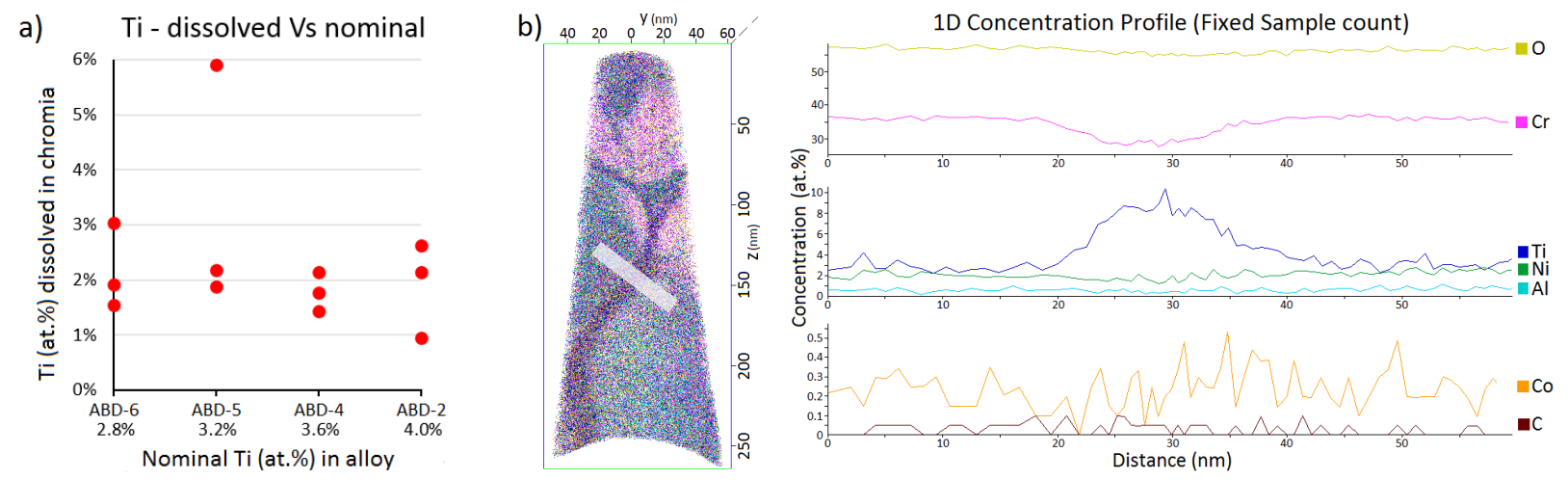

Figure 1 (a) Dissolved titanium concentrations (at.\%) observed in chromia APT datasets for all alloys. (b) APT atom map of chromia oxide including a grain boundary, 1D concentration profile indicating Ti enrichment.

\section{References:}

[1] H. Nagai, M. Okabayashi. Transactions of the Japan Institute of Metals 22 (1981) p691-698.

[2] H. Buscail, S. Perrier, C. Josse. Materials and Corrosion 62 (2011) p416-422.

[3] A. Holt, P. Kofstad. Solid State Ionics 69 (1994) p127-136.

[4] M. Lapington, D. Crudden, R. Reed, M. Moody, P. Bagot. Met. Mat. A (submitted 2017).

[5] D. Crudden, A. Mottura, N. Warnken, B. Raeisinia, R. Reed. Acta Materialia 75 (2014) p356-370. 


\title{
Behaviour and characterisation of various types of carbon nanotubes as field emitting electron sources
}

\author{
Marwan S. Mousa ${ }^{1}$, M-Ali H. AL-Akhras ${ }^{2}$, Samer I. Daradkeh ${ }^{1}$, Ala'a M. Al-Qudah ${ }^{1}$, \\ Hatem Braikat ${ }^{1}$ \\ ${ }^{1}$ Department of Physics Mutah University, Al-Karak, Jordan \\ ${ }^{2}$ Department of Physics, Jordan University of Science and Technology, Irbid, Jordan. \\ Corresponding Author: marwansmousa@yahoo.com
}

Carbon-based nanomaterials have attracted much attention recently, because their unique structure and properties (such as high electrical and thermal conductivity, chemical stability, high aspect ratio and small size [1]) make them perfect candidates for use in many modern scientific and industrial fields.

In our experiments, we studied the field electron emission properties of four types of carbon based nanomaterials: (a) single-walled carbon nanotubes (SWCNTs), produced by a high-pressure carbon monoxide process over Fe particles; (b) multi-walled carbon nanotubes (MWCNTs) Nanocly ${ }^{\mathrm{TM}} \mathrm{NC} 7000$ of carbon purity 90\%; (c) carbon nanotube fibers (CNTFs) PR-1; and (d) nanotubes (CBCNTs) derived from carbon black, type Vulcan XC72. Emitters were prepared by employing a drawing technique using a glass puller, with the CNT material inside a glass tube [2].

A field electron microscope system was used to characterize the emitters [3], via their measured currentvoltage $(I-V)$ characteristics, Fowler-Nordheim (FN) plots, and FEM images. The CBCNT emitters generated linear FN plots, while the FN plots for other types were linear only at low voltage. For CNTF and SWCNT emitters the $I-V$ plots were linear, as illustrated in Fig. 2. FEM images from SWCNTs and CNTFs exhibited a single spot at low voltage, whereas CBCNTs and MWCNTs exhibited a single spot at higher voltages. Image comparisons are shown in Fig. 1.

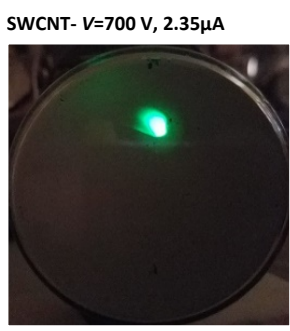

CNTF- $V=1300 \mathrm{~V}, 7.2 \mu \mathrm{A}$

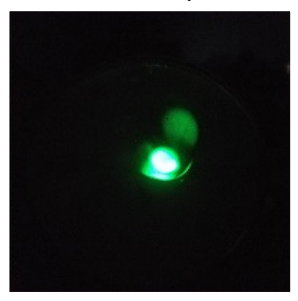

Fig.1. FEM Images

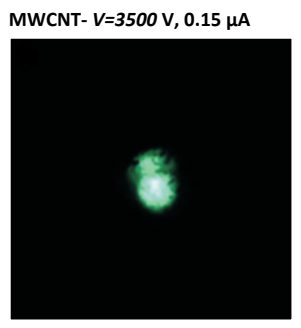

$C B-V=4250 \mathrm{~V}, 0.05 \mu \mathrm{A}$

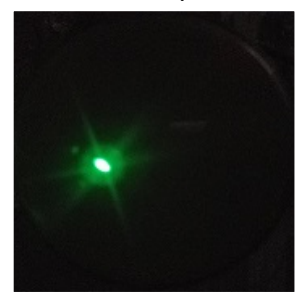

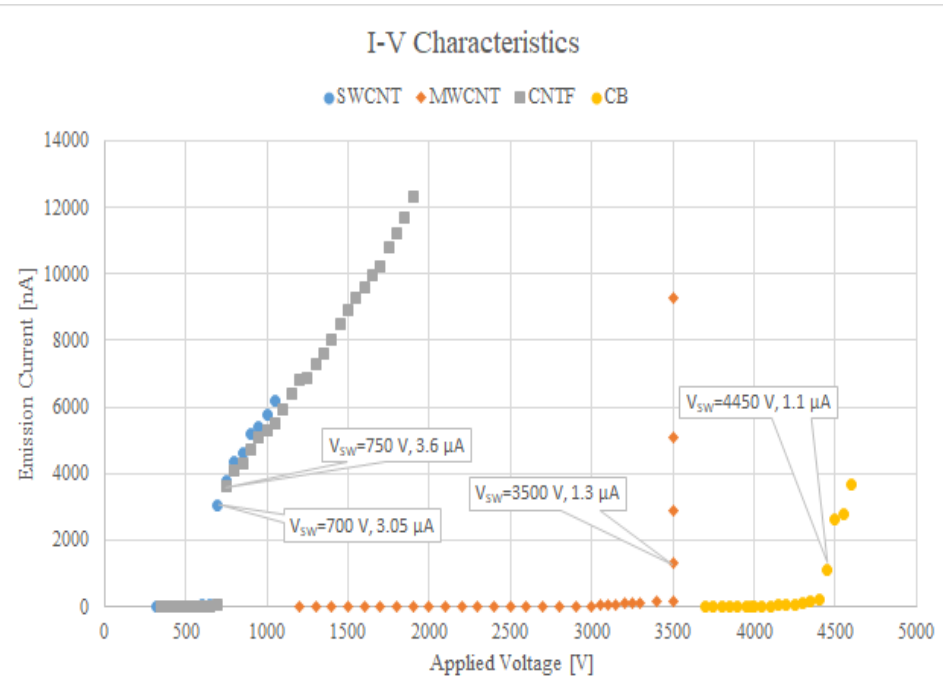

Fig. 2. I-V plots taken from carbon-based-nanomaterial emitters mounted so as to protrude from a Pyrex glass capillary tube

\section{References}

[1] P.M. Ajayan, J.M. Tour, "Nanotube composites", Nature 447 (2007) 1066.

[2] S.I. Daradka, M.S. Mousa, "Switch-on Phenomena and field emission from single-walled carbon nanotubes embedded in glass", Applied Microscopy 47 (2017) 86.

[3] K.O. Mussa, A. Fischer, M.S. Mousa, "Information extraction from FN plots of tungsten microemitters", Ultramicroscopy 132 (2013) 48. 


\section{Electron field emission and spectroscopy of diamond nano-needles}

Ivan Blum ${ }^{1}$, Mario Borz ${ }^{1}$, Olivier Torresin², Julien Mauchain ${ }^{2}$, Benoit Chalopin ${ }^{2}$, Adil

Haboucha $^{3}$, Said Idlahcen ${ }^{3}$, Ammar Hideur ${ }^{3}$, Alexander Obraztsov ${ }^{4,5}$, Angela Vella ${ }^{1}$

Corresponding Author: ivan.blum@univ-rouen.fr

1. GPM - UMR 6634, CNRS-INSA-Université de Rouen, Normandie Université, France.

2. LCAR - UMR 5589, CNRS, Université de Toulouse, France.

3. CORIA - UMR 6614, CNRS-INSA-Université de Rouen, Normandie Université, France.

4. M. V. Lomonosov Moscow State University, Department of Physics, Moscow, Russia.

5. University of Eastern Finland, Joensuu, Finland.

Analysis of low conductivity materials by laser assisted atom probe tomography is often associated with poor data quality, including low mass resolution. One possible explanation is that field evaporation causes a flow of charges through the specimen, which can cause a potential drop and thus variations in time of flight [1] leading to mass resolution worsening. In order to test this hypothesis in a quantitative way, data about the specimens electrical properties are required.

In this work we show that high evaporation rates on diamond nano-needles are also correlated with increases in the ions times of flight (fig. 1.a). In order to study the specimen's electric properties over a wide range of emission currents, we use electron field emission as well as electron spectroscopy. Correlation between emission current and voltage drop provide us with information on the involved conduction mechanisms (fig. 1.b), and laser assisted emission informs us about the emission's response time, and the effect of the illumination area. These results are then compared to the atom probe tomography results and we investigate in which way these electric properties can enable quantitative explanations for the measured mass resolution.
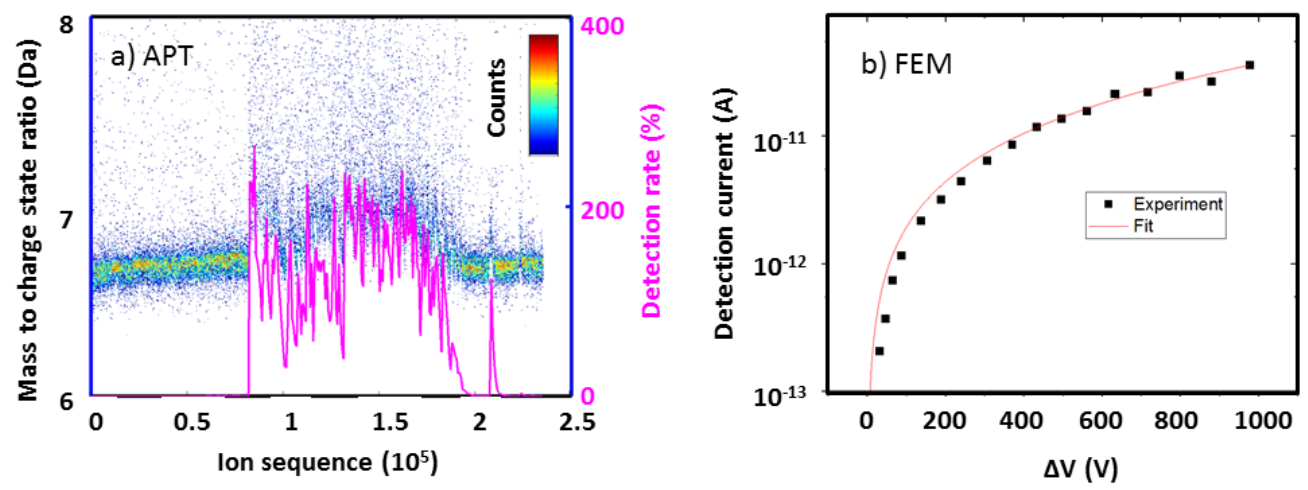

Figure 1 (a) Measured $\mathrm{m} / \mathrm{c}$ ratio for $\mathrm{C}^{2+}$ and detection rate for an analysis of diamond by atom probe tomography and (b) measured emission current as a function of voltage drop in this case without laser illumination, assuming a Poole-Frenkel conduction mechanism yields an accurate fit of the data.

References:

[1] L. Arnoldi, E. Silaeva, A. Gaillard, F. Vurpillot, I. Blum, L. Rigutti, B. Deconihout and A. Vella, Journal of Applied Physics 115 (2014) 203705 


\section{Calibration of atom probe tomography reconstructions from correlation with electron tomograms or micrographs}

I. Mouton ${ }^{1,2}$, S. Katnagallu ${ }^{1}$, T. Schwarz ${ }^{1}$, S. Makineni ${ }^{1}$, T. Printemps ${ }^{2}$, A. Grenier ${ }^{2}$, J.P. Barnes ${ }^{2}$, O. Cojocaru-Mirédin ${ }^{3}$, D. Raabe ${ }^{1}$, B. Gault ${ }^{1}$

Corresponding Author: i.mouton@mpie.de

${ }^{1 .}$ Max-Planck-Institut für Eisenforschung GmbH, Düsseldorf, Germany.

2. Univ. Grenoble Alpes, F-38000 Grenoble, France

CEA, LETI, Minatec Campus, F-38054 Grenoble, France.

3. RWTH Aachen, I. Physikalisches Institut IA, Aachen, Germany

Although the local elemental analysis is not directly influenced by the quality of the reconstruction in atom probe tomography (APT), any structural inferences from APT volumes demand an accurate reconstruction of the point cloud. Current reconstruction methods are based on simple geometrical assumptions that are actually never fulfilled in reality. These algorithms were developed to account for the evolution of the specimen's tip radius of curvature during the analysis, either by making use of the applied voltage and / or directly from the specimen geometry derived from ex-situ observations. To obtain an accurate reconstruction it is necessary to precisely calibrate these reconstruction parameters.

The aim of my work was to achieve automated calibration of reconstruction parameters from electron tomography (ET) and/or electron microscopy (EM) images of the APT specimens. The methods exploit a cross-correlation of microstructural features observed both in APT reconstructions and in the corresponding electron tomogram/micrograph. Figure 1 shows an example of 3D correlation between ET and APT on nanoporous silicon electrochemically filled with nickel so as to enable the APT experiments.

(a) HAADF STEM

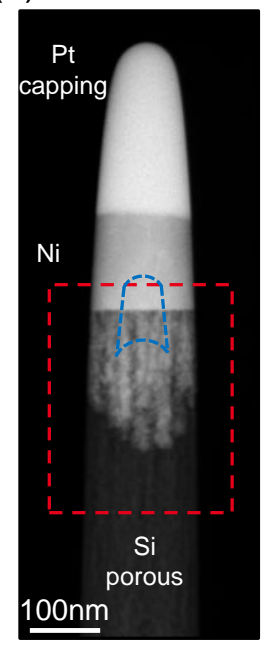

(b) Electron tomography

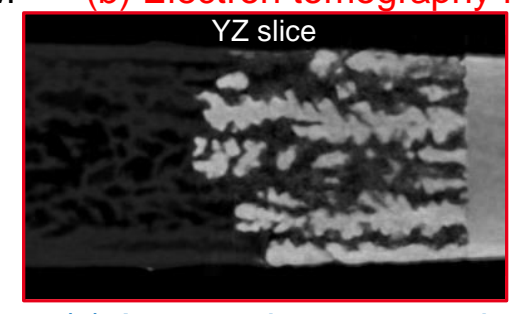

(c) Atom probe tomography reconstruction

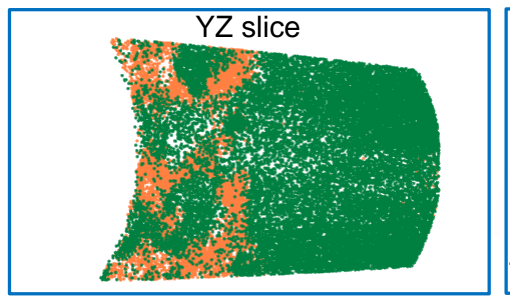

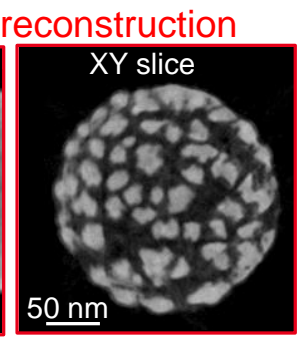

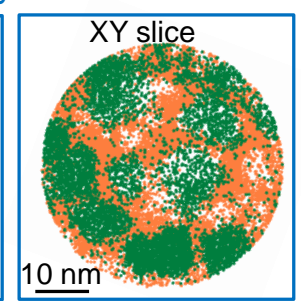

(d) Superposition of APT and electron tomography reconstruction

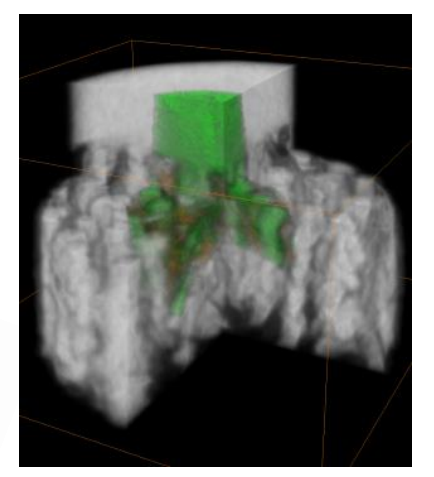

- Si atoms

- Ni atoms

Figure 1 (a) HAADF STEM image of the whole tip with corresponding APT analyzed volume in blue. (b) Slices in $\mathrm{YZ}$ and $\mathrm{XY}$ direction from the electron tomography reconstruction and (c) APT reconstruction. (d) Combined APT and electron tomography reconstruction. 


\section{Trajectory-Based Atom Probe Reconstruction With Locally Varying Tip Shape}

Daniel Beinke ${ }^{1}$, Guido Schmitz ${ }^{1}$

Corresponding Author: daniel.beinke@imw.uni-stuttgart.de

1. Institute of Materials Science, University of Stuttgart, Heisenbergstraße 3, 70569 Stuttgart, Germany.

The standard approach for the reconstruction of atom probe tomography data is based on the assumption that the emitter surface has a spherical shape with a constant curvature radius during field evaporation [1]. This constraint leads to well-known artefacts in the case of inhomogeneous samples containing atom species with different evaporation fields [2].

In our approach, the curvature radius of the tip is allowed to change locally during the reconstruction process. To this end, the emitter surface is divided into small segments, which are lifted upwards individually during reconstruction, according to the locally field evaporated volume. Similar to our earlier work, the order of reconstruction is inversed, which means the last detected ions are reconstructed first. The reconstruction is performed with the help of calculated physically meaningful ion trajectories [3], considering the effect of the varying curvature on the electrical field.

The potential of this technique is demonstrated for different heterogeneous emitter structures.

\section{References:}

[1] P. Bas et al., A general protocol for the reconstruction of 3D atom probe data, Applied Surface Science 87/88 (1995), 298-304.

[2] F. Vurpillot et al., Modeling Image Distortions in 3DAP, Microscopy and Microanalysis 10 (2004), 384-390.

[3] D. Beinke, C. Oberdorfer and G. Schmitz, Towards an accurate volume reconstruction in atom probe tomography, Ultramicroscopy 165 (2016), 34-41. 


\section{Making Atom-Probe Reconstruction Compatible with Newton's Laws}

Richard G. Forbes

Corresponding Author: r.forbes@trinity.cantab.net

Advanced Technology Institute, University of Surrey, Guildford, Surrey GU2 7XH, UK

In atom-probe literature and practice, atom-probe reconstruction is usually based on a projection methodology, using an appropriate inversion formula. I have argued before that we should move to a situation where reconstruction is formally based on charged-particle (CP) optics. This presentation aims to update the argument, and exhibit it to those unfamiliar with it.

In reality, there would be only small changes in the actual outputs, and reconstruction has much bigger problems. However, I believe there would be indirect benefits, as follows.

- The PR advantage of having a reconstruction methodology that is known to be compatible with Newton's Laws, as opposed to one that is known not to be.

- The advantage of having a reconstruction methodology that is clearly intellectually compatible with simulations of ion trajectories based on finite-element or similar methods.

- Better exposure to, and potential integration with, the large amount of material available about the CP optics of field electron emitters.

- Possibly, greater flexibility to consider emitter endforms that are not spherical.

- The possibility of incorporating, into reconstruction arguments, "spot-blurring" arguments that might in some circumstances affect the resolution of reconstruction.

The presentation will summarize the principles of the CP-optics of field emitters, and its application in atom-probe contexts. Topics to be briefly covered will include the following.

- The Hawkes-Kasper optical model [1] of a field ion or electron emitter as a spherical charged-particle emitter (SCPE) followed by a weak converging lens.

- The three types of radial angular magnification, and (equivalently) the three types of (radial) image compression factor.

- The evidence, from a variety of experiments and simulations, that the best working assumption is that Hawkes-Kasper magnification (rather than Helmholtz magnification or small-angle magnification) is nearly constant as a function of angle. [This means that length of arc on a spherical emitter maps into linear distance on a planar detector-in the absence of distortion and other aberrations.]

- The resulting "three-stage ion-optical procedure" for reconstructing lateral position, and its relationship to existing reconstruction formulae.

Reference [2] provides further details of the CP optics of field electron and ion emitters.

I am aware that there have been some improvements in the theory of reconstruction [3] since I first made comments of this kind, and that others have also addressed this problem, but my view is that some further small improvements could usefully be made.

\section{$\underline{\text { References }}$}

[1] P.W. Hawkes and E. Kasper, Principles of Electron Optics (Academic, London, 1996), Vol. 2, Ch.45.

[2] R.G. Forbes, "The theory of bright field electron and field ion emission sources", Chap. 1 in: Nanofabrication using Focused Ion and Electron Beams (Oxford Univ. Press, Oxford, 2012).

[3] D.J. Larson, T. J. Prosa, R.M. Ulfig, B.P. Geiser and T.F. Kelly, Local Electrode Atom Probe Tomography (Springer, New York, 2013), see Appendix C. 


\section{Enhanced Dynamic Reconstruction for Atom Probe Tomography: A Post Bas Algorithm}

Constantinos Hatzoglou ${ }^{1}$, Gérald Da Costa ${ }^{1}$, and Francois Vurpillot ${ }^{1}$

Corresponding Author: constantinos.hatzoglou1@univ-rouen.fr

1. Normandie Université, UNIROUEN, INSA Rouen, CNRS, Groupe de Physique des Matériaux, 76000 Rouen, France.

Experimental artefacts can occur during atom probe tomography (APT) analysis, biasing the observed reconstructed microstructure and the chemical composition measurements. The aim of this study is to override some biases in order to obtain the most congruent reconstruction.

Many studies have focussed on the optimization of atom probe tomography data reconstruction. In this presentation, an enhanced dynamic reconstruction algorithm based on the approach initially developed by Gault et al. in 2011 [1] is proposed. The dynamic reconstruction takes into account the evolution of the reconstruction parameters during the field evaporation of the sample, contrary to the standard reconstruction protocol proposed by Bas et al. in 1995 [2]. Here, the evolution of reconstruction parameters is retrieved by field evaporation simulation. This approach allows a complete tabulation of the parameters evolution as a function of the analysed sample morphology and its initial microstructure. It is showed that this drastically optimizes the spatial accuracy of reconstructed dataset. This will be demonstrated by applying this post-Bas algorithm to single phase materials and to the simple case of A/B layers.

(a)

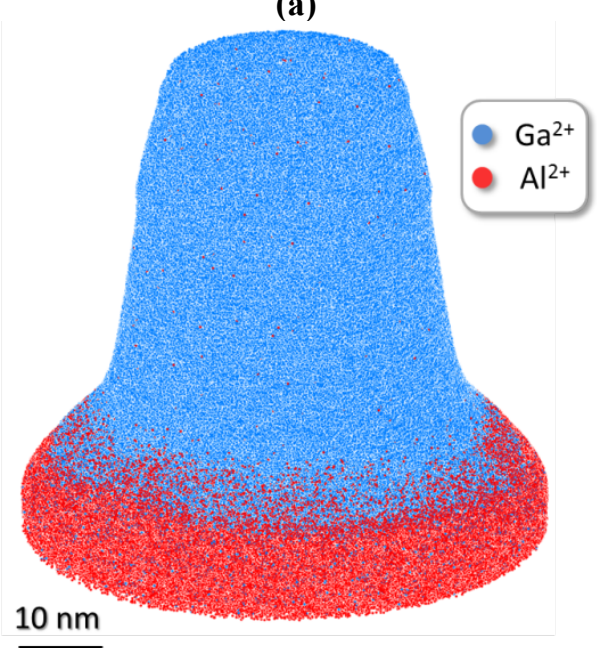

(b)

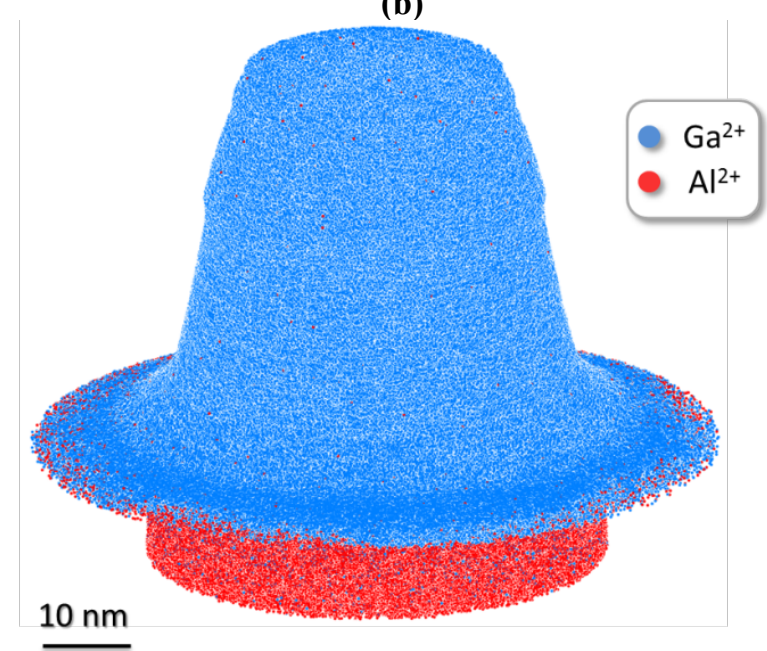

Figure 1: (a) Standard reconstruction and (b) dynamic reconstruction applied to the analyses of the $\mathrm{GaN} / \mathrm{InAlN}$ layer interface.

\section{References:}

[1] B. Gault, Ultramicroscopy. 111 (2011) 1619-1624.

[2] P. Bas, Appl. Surf. Sci. 87 (1995) 298-304. 


\title{
Methodologies for APT Data Reconstruction Based Upon Correlative Real Space Imaging and Diffraction with Electrons
}

\author{
Brian P. Gorman ${ }^{1}$, Anna Ceguerra ${ }^{2}$, Andrew Breen $^{3}$, Julie Cairney ${ }^{2}$, Simon Ringer $^{2}$, David R. Diercks ${ }^{1}$ \\ Corresponding author: bgorman@mines.edu \\ ${ }^{1}$ Colorado School of Mines, Department of Metallurgical and Materials Engineering, 1500 Illinois St., Golden, CO \\ 80401 USA \\ ${ }^{2}$ The University of Sydney, Australian Centre for Microscopy \& Microanalysis, and School of Aerospace, \\ Mechanical and Mechatronic Engineering, NSW 2006, Australia \\ ${ }^{3}$ Max-Planck-Institut für Eisenforschung, Department of Microstructure Physics and Alloy Design, 40237 \\ Düsseldorf, Germany
}

Currently, the most common reconstruction methodologies for atom probe tomography data require knowledge of the material's evaporation field in order to determine the specimen geometry [1]. Unfortunately, this value is not well known for most materials, especially covalent and ionic compounds. With the wide incorporation of laser assisted field evaporation instruments, the temperature dependence of the evaporation field is also needed, as is the specimen temperature during field evaporation. Alternatively, correlative microscopy techniques can be utilized to help confine the evaporated volume as well as many of the other variables required for accurate data reconstruction. However, these variables are still numerous and each contributes to error propagation.

Utilizing correlative characterization techniques during an atom probe experiment introduces the possibility for data reconstruction that does not rely upon knowledge of the evaporation field or specimen temperature, among many other "traditional" reconstruction variables. As detailed recently, in-situ transmission electron microscopy can be used for specimen geometry quantification before and after field evaporation [2]. Such correlative investigations along with electron diffraction allow for atom locations within the evaporated volumes to be quantified a-priori with sub- $\AA$ precision. Correlative TEM and diffraction also allows for quantification of the detection efficiency and ion size, further constraining the reconstruction variables. As a result, only the image compression factor is variable and may be quantified in many cases. Examples of this methodology illustrate the possibility of 3-D atomic resolution. Challenges for future implementation exist, especially in terms of mass spectrum analysis and heterointerfaces.

\section{References:}

[1] F. Vurpillot et al., Ultramicroscopy. 132 (2013) 19.

[2] R. Kirchhofer et al., J. Mat. Res. 30 (08) (2015) 1137. 


\title{
Novel Crystallographic Framework for Reconstruction in APT
}

\author{
Alec C. Day ${ }^{1}$, Anna V. Ceguerra ${ }^{1,2}$, and Simon P. Ringer ${ }^{1}$ \\ Corresponding Author: alec.day@sydney.edu.au \\ 1. The University of Sydney, Australian Centre for Microscopy \& Microanalysis, and School of \\ Aerospace, Mechanical and Mechatronic Engineering, Sydney, NSW 2006, Australia. \\ 2. The University of Sydney, the University of Sydney Nano Institute (Sydney Nano), Sydney, \\ NSW 2006, Australia.
}

The reconstruction process is a critical, yet necessary step to obtain three-dimensional (3D), atomic-scale data from the atom probe microscope. Currently, the standard procedure for reconstruction is based on a geometric simplification of the specimen tip, and the fieldevaporation process, so as to ultimately reduce to at least four required input parameters: evaporation field $F_{e}$ (material specific), detection efficiency $\eta$ (instrument specific), image compression factor (ICF) $\xi$ and the field factor $k_{f}$ (experiment specific). While there have been ongoing efforts to improve the accuracy of the geometric models used for reconstruction (e.g. [13]), the majority of methodologies still require at least those parameters listed above, or their equivalent. As a result, an accurate calibration of these parameters is essential for an accurate reconstruction, and a priori knowledge of the sample and data is required.

Crystallographic features are often used to achieve a calibration of these reconstruction parameters. The information used is usually limited to: 1) distance between observable poles on the detector to calibrate $\xi$, and 2) observed d-spacing at identified crystallographic poles to calibrate $k_{f}$, which also requires knowledge of the material's evaporation field. Furthermore, the calibration is often performed at a discrete 'slice' or section of the overall data-set, thus assuming that the evaporation characteristics and the above parameters do not vary during an experiment. Despite a best attempt at calibration, it is common for such reconstructions to have significant variations in the observed d-spacings and angles between poles throughout the length of the dataset. It is widely recognised that a greater utilisation of the available crystallographic information will enable a more accurate tomographic reconstruction.

In this work, we discuss methods to extract crystallographic information for use in a novel reconstruction framework that does not require the ICF $\xi$ or field factor $k_{f}$ as input parameters. Examples of this method are applied to $\mathrm{Al}$ and $\mathrm{W}$ datasets, and its accuracy is compared to current standard reconstruction methods. Furthermore, we present pathways to perform accurate reconstructions in datasets where minimal crystallographic information appears available.

[1] F. De Geuser, and B. Gault, Reflections on the projection of ions in atom probe tomography, Microscopy and Microanalysis 23.2 (2017), 238-246.

[2] R. Forbes, Case for developing an alternative APT reconstruction algorithm in parallel with IVAS Conference: International Conference on Atom Probe Tomography and Microscopy, Stuttgart, AugustSeptember 2014.

[3] B. Gault, et al., Tomographic Reconstruction in Atom Probe Microscopy: Past, Present... Future?, Microscopy and Microanalysis 15.S2 (2009), 10. 


\section{The Atomic Structure of Quasicrystals via Atom Probe Microscopy}

Anna V. Ceguerra ${ }^{1,2}$ and Simon P. Ringer ${ }^{1}$

Corresponding Author: anna.ceguerra@sydney.edu.au

1. The University of Sydney. Australian Centre for Microscopy \& Microanalysis, and School of Aerospace, Mechanical and Mechatronic Engineering, Sydney, NSW 2006, Australia.

2. The University of Sydney. The University of Sydney Nano Institute (Sydney Nano), Sydney, NSW 2006, Australia.

Quasicrystals have rotational order but no translational periodicity. Their first documented study was published in 1984, which utilised transmission electron microscopy [1]. Since their discovery, several diffraction techniques were used to study the atomic-scale structure of quasicrystals [2-4]. These offer averaged information in the reciprocal space of the bulk crystal.

Atom probe microscopy (APM) includes tomographic and other imaging techniques that provide localised information at the atomic scale in real space. With the chemical information and 3D positions of each atom in a $\sim 100$ million-atom dataset, access to information about the local atomic neighbourhoods becomes available with statistical certainty. These techniques have not been widely applied to the study of stable quasicrystals. Previous work includes field ion microscopy images [5,6], and analysis of metastable quasicrystal phases within amorphous systems or steels [7]. However, given the significant development of APM techniques over the past two decades [8], and in particular the development of atom probe crystallography [9], this work sought to extract localised atomic-scale structural information from quasicrystals.

Here, a data analysis procedure for a stable AlMnPd quasicrystal is reported. We describe the specimen preparation procedure and the atom probe experimental parameters. We define the mathematical formalisms, and the computational tools required to measure the local atomic structure of quasicrystals from atom probe data.

\section{$\underline{\text { References: }}$}

[1] D. Shechtman et al., Metallic Phase with Long-Range Orientational Order and No Translational Symmetry. Physical Review Letters 53 (1984) 1951-4.

[2] K. Hiraga et al., Highly Ordered Icosahedral Quasicrystal of Al-Cu-Fe Alloy Studied by Electron Diffraction and High-Resolution Electron Microscopy. Japanese Journal of Applied Physics 27 (1988)

L951-953.

[3] M. Cornier-Quiquandon et al., Neutron-diffraction study of icosahedral Al-Cu-Fe single quasicrystals. Physical Review B 44 (1991) 2071-2084.

[4] M. Boudard et al., Neutron and x-ray single-crystal study of the AlPdMn icosahedral phase. Journal of Physics: Condensed Matter 4 (1992) 10149-10168.

[5] E.S. Humphreys et al., Characterisation of a rapidly solidified Al-V-Fe alloy. Materials Science and Engineering A 250 (1998) 158-163.

[6] A. Sakai et al., Computer simulation of field-ion images of quasicrystals. Journal de Physique C7 (1086) C7-469 - C7-475

[7] B.S. Murthy et al., Nanoquasicrystalline phase formation in binary Zr-Pd and Zr-Pt alloys. Acta materialia 49 (2001) 3453-3462.

[8] A.V. Ceguerra et al., The rise of computational techniques in atom probe microscopy. Current Opinion in Solid State and Materials Science 17 (2013) 224-235.

[9] B. Gault et al., Atom probe crystallography. Materials Today 15 (2012) 378-386. 


\section{Applying Principles of Multivariate Analysis and Geospatial Interpolation Methods to Atom Probe Tomography Imaging}

Tammy Milillo $^{1}$, Scott Broderick ${ }^{2}$, Krishna Rajan ${ }^{2}$, and Joseph A. Gardella, Jr. ${ }^{1}$

Tammy Milillo: milillo@buffalo.edu

1. State University of New York at Buffalo, Dept. of Chemistry, Buffalo, USA.

2. State University of New York at Buffalo Dept of Materials Design \& Innovation, Buffalo USA

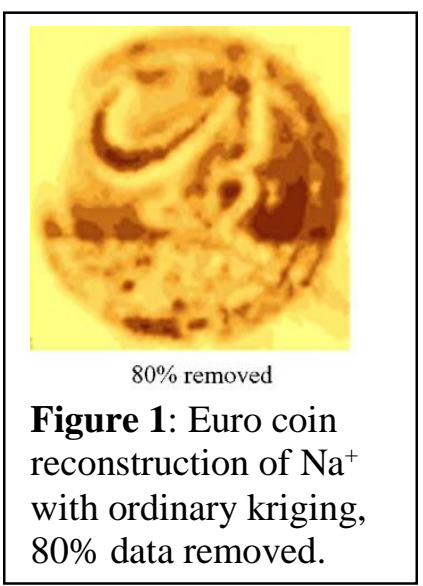

A challenge in atom probe tomography (APT) is defining the interface between phases and tracking the transition between regions of different crystallographic characteristics without bias. This is due to data uncertainty, the atomic detection rate, and user defined parameters in the reconstruction process. This work uses the geostatistical interpolation methods of ordinary kriging and inverse distance weighted (IDW) to rectify these problems, specifically that of missing data in APT imaging. Using geographic location as a variable, it is possible to reconstruct the missing data in an image with interpolation techniques. Previous work $[1,2]$ has shown that these methods were able to accurately estimate concentrations with only $1 \%$ of the original data contained in the image. Fig. 1.

Error can be analyzed for both interpolation methods, allowing for quantifying and analyzing at every pixel in the reconstructed image. The characterization of both image features and noise becomes possible. Validation statistics allow for comparison of different reconstructions of an image for accuracy, feature characterization and identification.

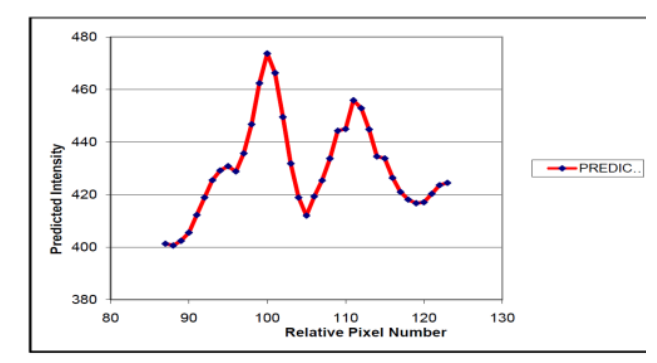

Figure 2: Predicted intensity vs. relative pixel number
By adapting the ASTM depth and spatial resolution standards the spatial resolution at either a chemical or feature edge can be calculated, and fuzzy features at interfaces can be assessed. Fig. 2. We demonstrate this work on a $\mathrm{Cu}-\mathrm{Si}$ system, with oxidation occurring under different processing conditions. This approach will be used to better understand the relationship between processing temperature and time, with the interfacial characteristics. This work will positively impact atom probe tomography: improved reconstruction of the data by more clearly capturing

features such as crystal structure and atomic site occupancy, better assessment of local neighborhood environments, and reducing the user influence in the reconstruction process.

\section{References:}

[1] T. Milillo, J. Gardella Jr.* Spatial Analysis of Time of Flight-Secondary Ion Mass Spectrometric Images by Ordinary Kriging and Inverse Distance Weighted Interpolation Techniques, Anal. Chem. 80, (2008) 4896-4905.

[2] T. Milillo, J. Gardella Jr.* Spatial statistics and interpolation methods for TOF SIMS imaging Ap. Surf. Sci. 252 (2006) 6883-6890.

[3] ASTM E673-90, Depth Profile Resolution Standard. Surf. Interface Anal. 71 (1991) 951. 


\section{From the Mass Spectrum to the Atom Map: An Alternative Approach to the Chemical Identification of Elements}

Francois Vurpillot ${ }^{1}$, Constantinos Hatzoglou ${ }^{1}$, and Frederic Danoix

Corresponding Author: francois.vurpillot@univ-rouen.fr

1. Normandie Université, UNIROUEN, INSA Rouen, CNRS, Groupe de Physique des Matériaux, 76000 Rouen, France.

A broad range of analysing instruments utilizes mass spectrometry to achieve local measurements of composition in a material of interest. The collection of single ions, labelled firstly by the measured mass-over-charge ratio $(\mathrm{m} / \mathrm{z})$, produces a spectrum interpreted as a sum of peaks. The amount of counts under envelops of each identified peak, and the identification of these peaks are used to achieve accurate composition measurements with most of the techniques. Atom probe is particular in this class of instruments. Indeed, we achieve elemental identification of single ions from unique $\mathrm{m} / \mathrm{z}$ measurement of each ion. We give a definitive elemental label to our set of data points organised in a 3D map. The composition measurement is achieved in a second step, by simple counting methods in this post processed dataset. The elemental identification method is the weak-point of the data processing, since for now, it remains completely arbitrary. Note that, efforts exist in the Atom Probe community to solve this issue [1]

When dealing with a single ion, the answer that we wait for is "This ion is a A (or B,C,D ...) atom" in order to label correctly the data point in the dataset. However the relative asked question "what the nature of this data point?" is completely awkward. The only question that can be asked is "what is the probability for this atom to be A, (or B, C, D ...)?".

In this paper, statistical methods, based on expectation-maximization (EM) algorithm, using maximum likelihood or maximum a posteriori (MAP) estimates of parameters have been used to assign elemental probability weights to ions in the dataset [2]. Knowing the answer, with an imposed confidence criteria, a decision can be taken, or even better, the representation of the atom can be adapted. For instance, the radius or the opacity of the displayed atom can be proportional to this probability. Some simple examples showing how this application can improve both atom probe display of atoms and local composition measurements will be presented.

\section{References:}

[1] Andrew J. London, Daniel Haley and Michael P. Moody, Single-Ion Deconvolution of Mass Peak Overlaps for Atom Probe Microscopy, Microscopy and Microanalysis, Volume 23, Issue 2 (Atom Probe Tomography \& Microscopy 2016), April 2017, pp. 300-306

[2] A.P. Dempster, N.M. Laird, D.B. Rubin, Maximum Likelihood from Incomplete Data via the EM Algorithm, J. R. Stat. Soc. Ser. B. 39 (1977) 1-38. 


\section{Field-Dissociation Dynamics of $P_{m}^{i+}$ Molecular Ions in High DC Electric Field}

\section{E. Di Russo ${ }^{1}$, I. Blum ${ }^{1}$, J. Houard ${ }^{1}$, G. Da Costa ${ }^{1}$, D. Blavette ${ }^{1}$ and L. Rigutti ${ }^{1}$.} Corresponding Author: enrico.di-russo1@etu.univ-rouen.fr

1. Normandie Univ., UNIROUEN, Groupe de Physique des Matériaux, 76000 Rouen, France.

Laser-assisted atom probe tomography is a unique technique to the study of molecular ions dissociations occurring during their flight towards the detector [1-3]. In this contribution we present an advanced study of the field-dissociation of $\mathrm{P}_{m}^{i+}(i>1, m>4)$ molecular ions formed during InP atom probe tomography. This material exhibits countless dissociation tracks which can be recognized studying the correlation histogram (fig. a). Atom probe data were analyzed to obtain information as the probability to dissociate $\mathrm{P}_{m}^{i+}$ parent ions the lifetime of such reactions [4]. Moreover, adopting the method recently developed by Blum et al., the effect of Coulomb repulsion during the dissociation of $\mathrm{P}_{2 m}^{2 i+}(i, m>3)$ molecular ions is analyzed (fig. b) [2]. This work opens new perspectives for quantitative studies of dissociation reactions in atom probe tomography.
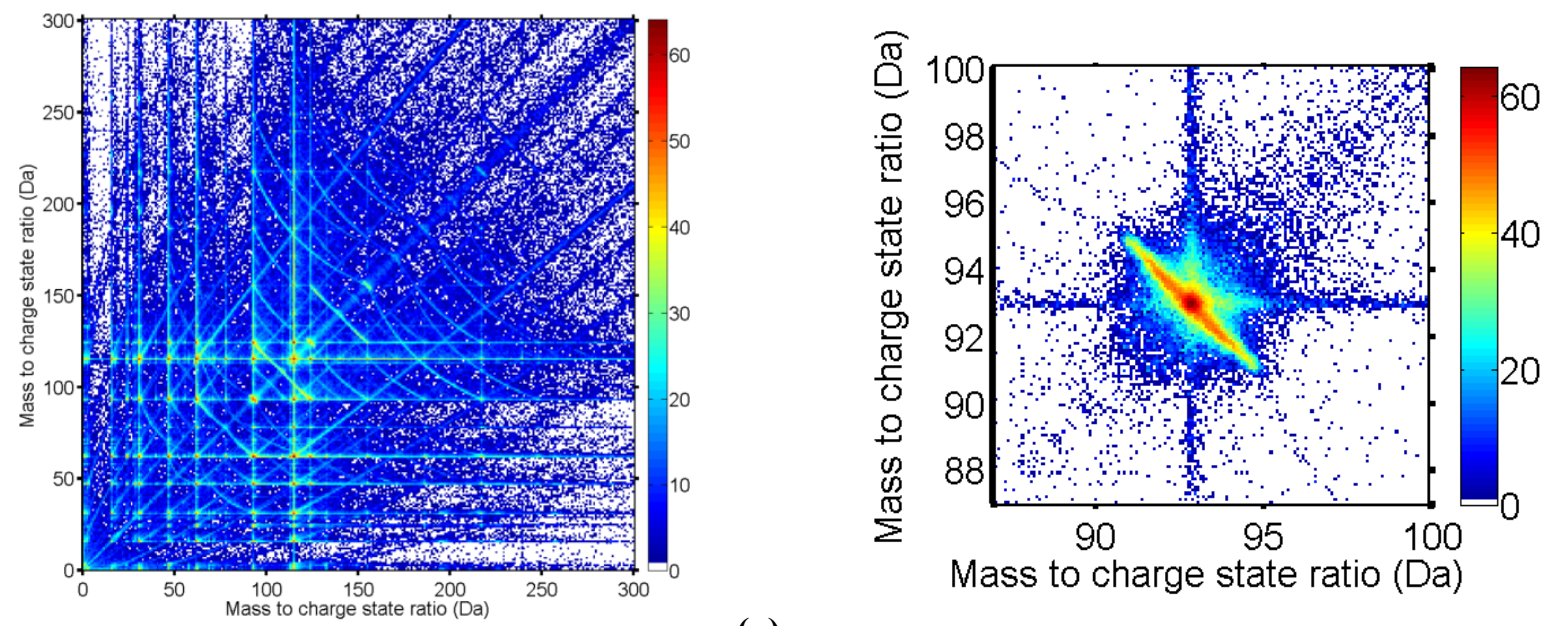

(a)

(b)

Figure (a) Correlation histograms of InP laser-assisted field evaporation. (b) Detail of the correlation histogram reported in (fig. a). The short but intense tracks with negative slope are associated to the dissociation reaction $\mathrm{P}_{6}^{2+} \rightarrow \mathrm{P}_{3}^{+}+\mathrm{P}_{3}^{+}$. The circular red spot at the point $\{93,93\} \mathrm{Da}$ is associated to the correlate evaporation of $\mathrm{P}_{3}^{+}$ions.

References:

[1] D. W. Saxey, Ultramicroscopy 111, 473 (2011).

[2] I. Blum et al., J. Phys. Chem. A 120, 3654 (2016).

[3] B. Gault et al., New J. Phys. 18, 033031 (2016).

[4] E. Di Russo et al., Microscopy and Microanalysis 23.6, 1067 (2017) 


\title{
Measuring Bond Strength Across Heterostructure Interfaces Using Atom Probe Data
}

\author{
O.G. Licata ${ }^{1}$, S.R. Broderick ${ }^{1}$, C.A. Hurni ${ }^{2}$, J.S. Speck², K. Rajan ${ }^{1}$, and B. Mazumder ${ }^{1}$
}

Corresponding Author: baishakh@,buffalo.edu

1. Department of Materials Design and Innovation, University at Buffalo, Buffalo, USA

2. Materials Department, University of California, Santa Barbara, USA

III-Nitride based high electron mobility transistor (HEMT) structures comprising AlN/GaN heterojunctions are of technological interest owing to their contribution in communications. The performance of these devices relies heavily on the quality of this interface. In the present work, $\mathrm{Al}_{\mathrm{x}} \mathrm{Ga}_{1-\mathrm{x}} \mathrm{N} / \mathrm{GaN}$ heterostructures with varying $\mathrm{Al}$ compositions are examined. Detailed compositional and interfacial analysis has been reported previously [1]. Here, we investigate the chemical topography at heterostructure interfaces, in particular, variation in chemistry at the interface with the composition of Al (Figure 1a). Figure $1 \mathrm{~b}$ and 1c show the interfacial ion evaporation map and histogram for double-hit events respectively for the marked $\mathrm{Al}_{\mathrm{x}} \mathrm{Ga}_{1-\mathrm{x}} \mathrm{N}$ layer. The mass-to-charge ratios correspond to chemical identities.

(a)

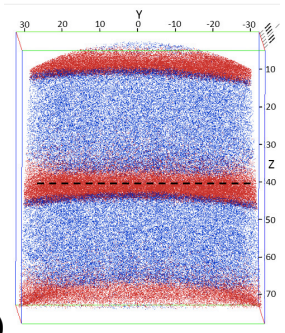

(b)

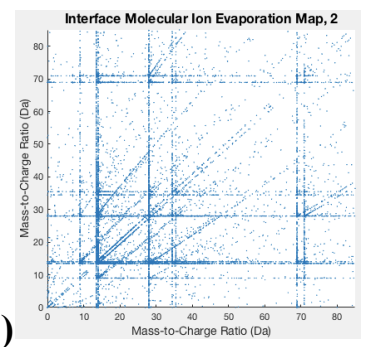

(c)

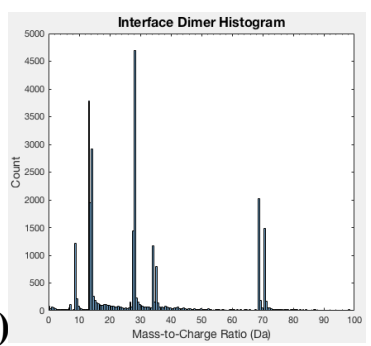

Figure 1 (a) $3 \mathrm{D}$ elemental map of $\mathrm{Al}_{\mathrm{x}} \mathrm{Ga}_{1-\mathrm{x}} \mathrm{N} / \mathrm{GaN}$ with aluminum in red and gallium in blue. (b) Ion evaporation map of upper interface (c) Count of double-hit events from interface, ( $z=34$ to 37.98).

The relative relationships between quantities at the intersection of ion evaporations and the relative chemical compositions (i.e. the ratio between ion evaporation map and elemental composition) indicates the relative bond strength [2]. For example, in the ion evaporation map shown, the amount of Ga-N dimer (intersection of $\mathrm{m} / \mathrm{n}=14$ and $\mathrm{m} / \mathrm{n}=71$ ) is low compared to the bulk ratios. This indicates the higher bond strength in the bulk region, which explains the higher mechanical stability in the bulk region as compared to the interfacial region. Further, within the ion evaporation map shown, the bond strength between Ga-N and Al-N is nearly equivalent, as seen by the ratio of these dimers versus the overall chemistry. From these interpretations, we will present a spectral representation of bond strength as a function of position. This provides an added level of information on bonding and crystal structure not represented through the traditional composition profiles.

\section{$\underline{\text { References: }}$}

[1] B. Mazumder, M. Wong, C. Hurni, Asymmetric interfacial abruptness in N-polar and Ga-polar GaN/AlN/GaN heterostructures, Applied Physics Letters 101 (2012) 091601.

[2] J. Peralta, S.R. Broderick, K. Rajan, Ultramicroscopy 132 (2013), 143-151. 
This software workshop, hosted by Karen Henry, focuses on programs, scripts, utilities, and software packages developed by individuals or groups to facilitate data analysis and/or data treatment to advance atomic-scale understanding of materials science processes. The workshop consists of demonstrations by invited presenters, an open forum for users to contribute short, five-minute Flash Talks about a script/utility/program, and an open discussion about the software needs of the community.

\section{Flash Talks}

1:00 to 1:15 PM

\section{Flash Talks}

$1: 15$ to $1: 30 \mathrm{PM}$

\section{Open Forum Discussion}

1:30 to 2:00 PM 


\title{
Advanced Preparation Methods for Challenging APT Specimens
}

\author{
Dieter Isheim ${ }^{1}$ \\ Corresponding Author: isheim@,northwestern.edu \\ 1. Northwestern University Center for Atom-Probe Tomography, and Department of Materials \\ Science and Engineering, Northwestern University, Evanston, IL, USA.
}

Tip specimens for atom-probe tomography (APT) must conform to very strict criteria regarding geometry and size scale of the tip, in the $100 \mathrm{~nm}$ range. Additionally a number of materials properties are critical for successful APT analysis, including electrical and thermal conductivity, and sufficient mechanical stability of the tip and support structure. Naturally, the sample tip must contain the region of interest (ROI) within the analyzed volume approx. $100 \times 100 \times 100 \mathrm{~nm}^{3}$, and the ROI should remain unaltered by any of the processes utilized during the entire sample preparation sequence. A multitude of methods are available today for streamlined and reproducible tip preparation for a wide variety of sample and material types. The various methods can also be combined in a modular way to streamline preparation. Generally, a sequence of subtractive (cutting, sharpening, etching, electropolishing) and additive (coatings, capping or fixation layers) processes are utilized together with micromanipulation to target and/or re-orient the ROI, and sharpen a tip suitable for APT. Today, perhaps the most versatile tool in the box is focused ion-beam (FIB) milling, e.g. [1], allowing cutting, manipulating, deposition, and tip sharpening in a single instrument. We will here review a number of techniques and preparation strategies for this approach.

APT tips can be prepared relatively easily by FIB from surface layers or thin-film multilayer structures which are topologically directly compatible with the analysis geometry, given suitable capping materials and cutting/sharpening techniques that minimize differential milling. In contrast, materials with characteristic length scales less than $100 \mathrm{~nm}$ in at least two dimensions, or very different milling rates, present the biggest challenges, stemming from the difficulty to cut, manipulate, and target without damaging or destroying the ROI. These materials include some of the most interesting nanostructured materials such as nanowires, semiconductor devices, or small particles with nanometer dimensions. This presentation aims to review current FIBSEM based techniques to prepare APT tips from a variety of materials with nanoscale features structured with zero-dimensional (nanoscale particles), one-dimensional (nanowires), twodimensional (grain boundaries, thin-film multilayers, or heterophase interfaces), or three-dimensional geometries (microelectronic devices, gradient materials, 3D-precipitation structures).

\section{References:}

[1] D.J. Larson, D.T. Foord, A.K. Petford-Long, H. Liew, M.G. Blamire, A. Cerezo, G.D.W. Smith, Ultramicroscopy 79 (1999) 287-293; K. Thompson, D. Lawrence, D.J. Larson, J.D. Olson, T.F. Kelly, B. Gorman, Ultramicroscopy 107 (2007) 131-139; H. Blumtritt, D. Isheim, S. Senz, D.N. Seidman, O. Moutanabbir, Nanotechology 25(2014) 435704.

[2] This work made use of the NUCAPT and NUANCE-EPIC facilities at Northwestern University. The LEAP tomograph at NUCAPT was acquired with grants from the NSF (DMR-0420532) and ONRDURIP (N00014-0400798, -0610539, -0910781, -1712870) programs. NUCAPT and NUANCE received support through the Materials Research Center (NSF DMR-1720139) and the SHyNE Resource (NSF ECCS-1542205), NUCAPT from ISEN at Northwestern University, NUANCE from the International Institute for Nanotechnology (IIN) and the Keck Foundation; and the State of Illinois, through the IIN. 


\title{
Atom Probe Specimen Preparation of Steels by Transmission Kikuchi Diffraction
}

\author{
Christina Hofer ${ }^{1}$, Sandra Ebner ${ }^{1}$, Thomas Kurz², Dominik Brandl ${ }^{3}$, and Ronald Schnitzer ${ }^{1}$ \\ Corresponding Author: christina.hofer@unileoben.ac.at \\ 1. Department of Physical Metallurgy and Materials Testing, Montanuniversität Leoben, Leoben, \\ Austria. \\ 2. voestalpine Stahl GmbH, Linz, Austria. \\ 3. Materials Center Leoben Forschung GmbH, Leoben, Austria.
}

Transmission Kikuchi diffraction (TKD) became an increasingly popular tool to facilitate the sitespecific specimen preparation of atom probe tips in dual-beam FIB/SEM equipped with a conventional EBSD system [1]. Therefore, between the annular milling steps during final shaping of the tip, Kikuchi patterns are recorded from the electron transparent tip in order to get crystallographic information. Thus, specific phases or grain boundaries can be easily prepared within the apex-near region of the needle-shaped specimens. In this study, atom probe tips of a variety of steels were prepared by the combined approach of TKD and FIB milling in order to prepare features of interest and increase the yield of successful APT measurements. For example, it is shown that the retained austenite in advanced high strength steels processed by TRIP-aided bainitic ferrite and quenching and partitioning heat treatments is stabilized by carbon partitioning where the extent of the enrichment depends upon the austenite morphology [2]. Another example from the automotive industry is the investigation of prior austenite grain boundaries in a press hardening steel close to the zinc coating in order to clarify if zinc diffusion takes place during the austenitization treatment [3]. TKD was also applied to high-alloyed steels, for example, in maraging steels, site-specific preparation of austenite was conducted to examine the chemical differences in retained and reverted austenite. While the retained austenite shows a composition close to the nominal composition, the reverted austenite is highly enriched in $\mathrm{Ni}$ and nucleates at the boundaries of the retained austenite or $\mathrm{Cu}$-rich precipitates. TKD not only assists specimen preparation but the crystallographic information further supports the reconstruction and interpretation of the atom probe data.

\section{References:}

[1] K. Babinsky, R. De Kloe, H. Clemens, S. Primig, A novel approach for site-specific atom probe specimen preparation by focused ion beam and transmission electron backscatter diffraction, Ultramicroscopy 144 (2014), 9-18.

[2] C. Hofer, F. Winkelhofer, H. Clemens, S. Primig, Morphology change of retained austenite during austempering of carbide-free bainitic steel, Materials Science \& Engineering A 664 (2016), 236-246.

[3] C. Hofer, T. Kurz, H. Clemens, R. Schnitzer, Atom probe study of prior austenite grain boundaries of zinc-coated press hardened steel, $6^{\text {th }}$ International Conference of Hot Sheet Metal Forming of HighPerformance Steel, Atlanta, 2017, 383-390.

[4] Funding of the Austrian BMVIT in the framework of the program "Production of the future" and the "BMVIT Professorship for Industry" is gratefully acknowledged. 


\title{
Advanced Sample Preparation Techniques Using a Laser Ablation Instrument
}

\author{
Robert A. Morris ${ }^{1}$ and Karen T. Henry ${ }^{1}$ \\ Corresponding Author: robert.morris@unnpp.gov \\ 1. Bechtel Marine Propulsion Corporation, Schenectady, NY, USA
}

A laser ablation system has been used for preparing a variety of specimen geometries, including a specimen substrate carrier able to be used in both a transmission electron microscope (TEM) and an atom probe microscope (APM). The use of a focused laser, which has a spot size of $\sim 8 \mu \mathrm{m}$ and micron-level position accuracy, allows for the preparation of custom grids including the pillar array shown in Figure 1, without the use of chemicals and electropolishing. Using cutting and annular sharpening routines, a 3 pillar array was cut out of a steel substrate and sharpened to a final tip diameter $\sim 2 \mu \mathrm{m}$. By adjusting the laser parameters and laser cut path, a robust base with a sharpened needle shape can be consistently prepared. The limited heat affected region along the cut surface $(\sim 1 \mu \mathrm{m}$ thick) allows for the laser cut pillars to be directly sharpened into atom probe specimens in a focused ion beam (FIB) system or to be used as a sample substrate carrier for traditional FIB lift-out processing.

A significant advantage of the custom laser cut TEM/APM grid shape is that it allows for the grid to be used in a FIB/Scanning electron microscope (for preparation, imaging, and performing microanalysis such as chemical and crystallographic mapping), TEM (for direct imaging of the tip shape, and additional microanalysis), and APM (for field evaporation and three dimensional reconstruction of the tip). The correlative nature of combining several different instruments allows for complementary interpretation of the data. Use of the sample grid also increases efficiency and reduces the likelihood of sample loss/damage during transfers to different sample holders. Another advantage of using the laser system to cut the custom needle grid is the ability to use nearly any thin sheet material for the substrate. These materials can include copper or doped silicon for a conductive APM substrates, or when sharpening the pillar needles into the final dimensions directly, using the actual specimen material.
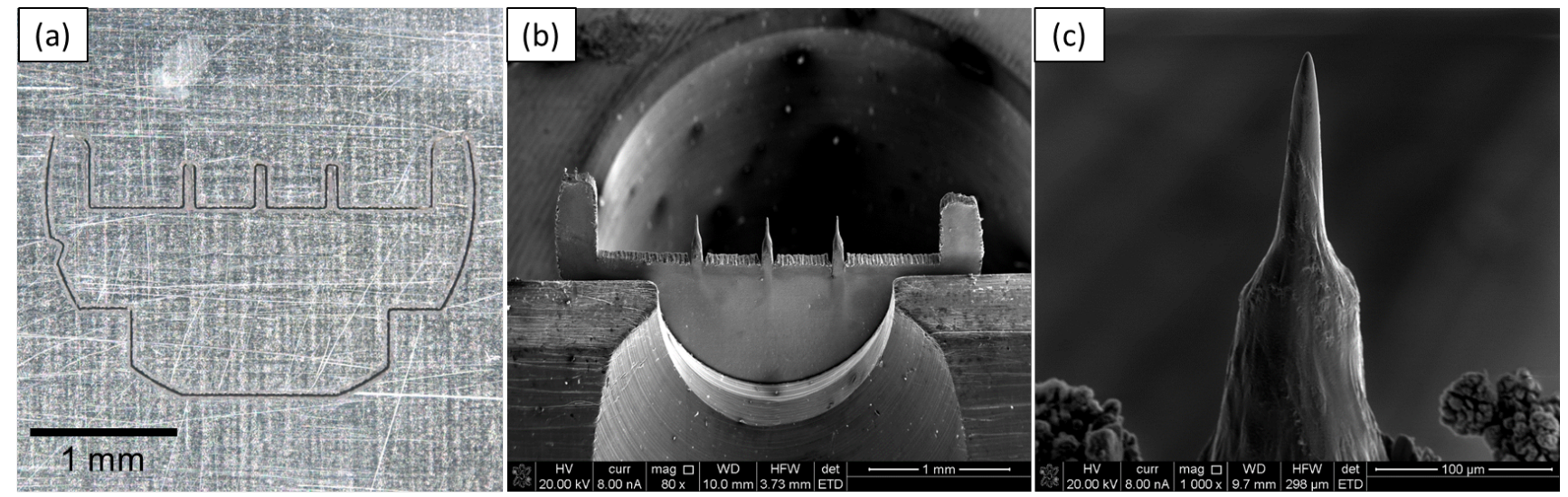

Figure 1: Laser ablation methods to prepare an array of atom probe pillars on a TEM sized specimen holder (a) Initial milling of pillar onto a TEM grid using a thinned sheet of metal. (b) Secondary electron (SE) image of an APM needle array after annular laser milling. (c) Higher magnification SE image of an as-laser cut sharpened needle using a laser ablation system with a tip diameter of $\sim 2 \mu \mathrm{m}$. The as-laser cut tip is ready for sharpening or to be used as a lift out substrate using traditional FIB lift-out processing. 


\title{
Semi-Automated Specimen Preparation for Atom Probe Tomography
}

\author{
Michael B. Schmidt ${ }^{1}$, Nicholas A, Croy ${ }^{1}$, Sang Hoon Lee $^{1}$ and Katherine P. Rice ${ }^{2}$ \\ Corresponding Author: michael.schmidt@,fei.com \\ 1. Thermo Fisher Scientific, Applications Development, Hillsboro, OR 97125, USA. \\ 2. CAMECA Instruments Inc, 5470 Nobel Drive, Madison, WI 53711, USA.
}

Focused ion beam (FIB) based specimen preparation for atom probe tomography (APT) has been used for the past decade to provide a means for targeting a specific region of interest for analysis, and to have a greater degree of control over final tip size and shape [1].

Thermo Fisher Scientific has developed methods to enable customized automated procedures for the atom probe sample preparation process. The recipes are based on the Thermo Scientific ${ }^{\mathrm{TM}}$ iFast Software scripting platform and can easily be modified to control parameters such as unsharpened mount size, weld deposition time, and beam currents used for milling. Herein we describe the methods for fully automated liftout from the sample surface onto microtip post substrates and the semi-automated sample milling into an atom probe ready specimen. Automated and semi-automated sample prep techniques can improve the throughput and consistency of the sample preparation process, leading to more repeatable atom probe results, as well as allowing less-experienced users to prepare specimens without specialized training. We also show atom probe data from auto-prepared samples, such as the B implant data shown in Figure 1c.
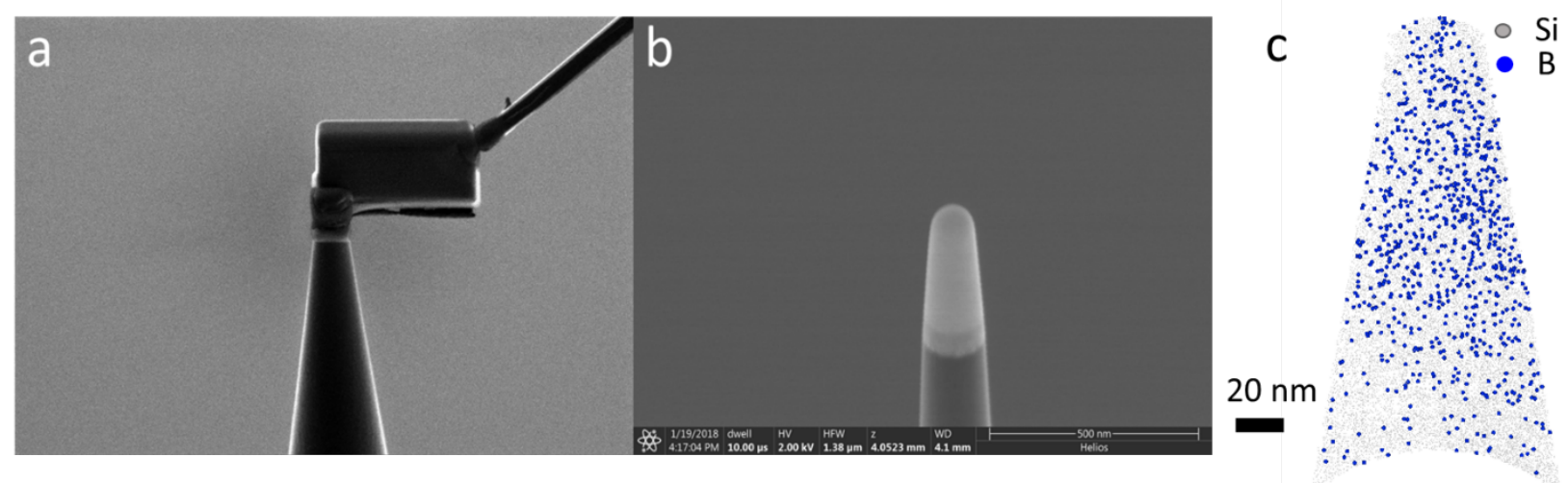

Figure 1 (a) Micromanipulator with wedge welded to microtip coupon post (b) Auto-sharpened sample with Tungsten protective cap prior to low-kV cleaning; (c) $10 \mathrm{~nm}$ slice of APT data obtained from a Bimplanted specimen prepared with auto sample prep techniques.

\section{References:}

[1] Thompson, K., Lawrence, D., Larson, D.J., Olson, J.D., Kelly, T.F., Gorman, B., In situ site-specific specimen preparation for atom probe tomography. Ultramicroscopy 107 (2007), 131-139. 


\section{A New Method for Mapping the Three-Dimensional Atomic Distribution Within Nanoparticles by Atom Probe Tomography (APT)}

Se-Ho Kim ${ }^{1}$, Phil Woong Kang 2 , O Ok Park², Jae-Bok Seol ${ }^{3}$, Jae-Pyoung Ahn ${ }^{4}$, Ji Yeong Lee Pyuck-Pa Choi ${ }^{1}$

\section{Corresponding Author: sehonetkr@kaist.ac.kr}

${ }^{1}$.Department of Materials Science and Engineering, Korea Advanced Institute of Science and Technology 291 Daehak-ro, Yuseong-gu, Daejeon 34141, Republic of Korea

2.Department of Chemical and Biomolecular Engineering, Korea Advanced Institute of Science and Technology 291 Daehak-ro, Yuseong-gu, Daejeon 34141, Republic of Korea

${ }^{3}$ National Institute for Nanomaterials Technology, POSTECH, Pohang 790-784, Republic of Korea

4. Advaned Analysis Center, Korea Institute of Science and Technology, Seoul 136-791, Republic of Korea

Analyzing nanoparticles has been an emerging but also highly challenging field in atom probe tomography (APT) $[1,2]$. In this work, we present a new method for the preparation of APT specimens from metallic nanoparticles of less than $10 \mathrm{~nm}$ in size. This method is based on electrophoresis of nanoparticles on a substrate followed by electroplating of a metallic layer. Transmission electron microscopy (TEM) confirms that particle shape and size are well preserved after these two process steps. APT specimens can be routinely prepared from the deposited nanoparticle/metal films using focused-ion-beam milling (FIB). Correlative TEM/APT analyses on APT specimens and resharpening by FIB prior to APT analyses were found to increase the chances of detecting the nanoparticles within the volume probed by APT. In addition, such correlative analyses help to examine the size and shape of nanoparticles and optimize APT data reconstruction. In addition to the constituent elements of the nanoparticles, we were able to map the distribution of surfactants used during synthesis and deposition of the nanoparticles.

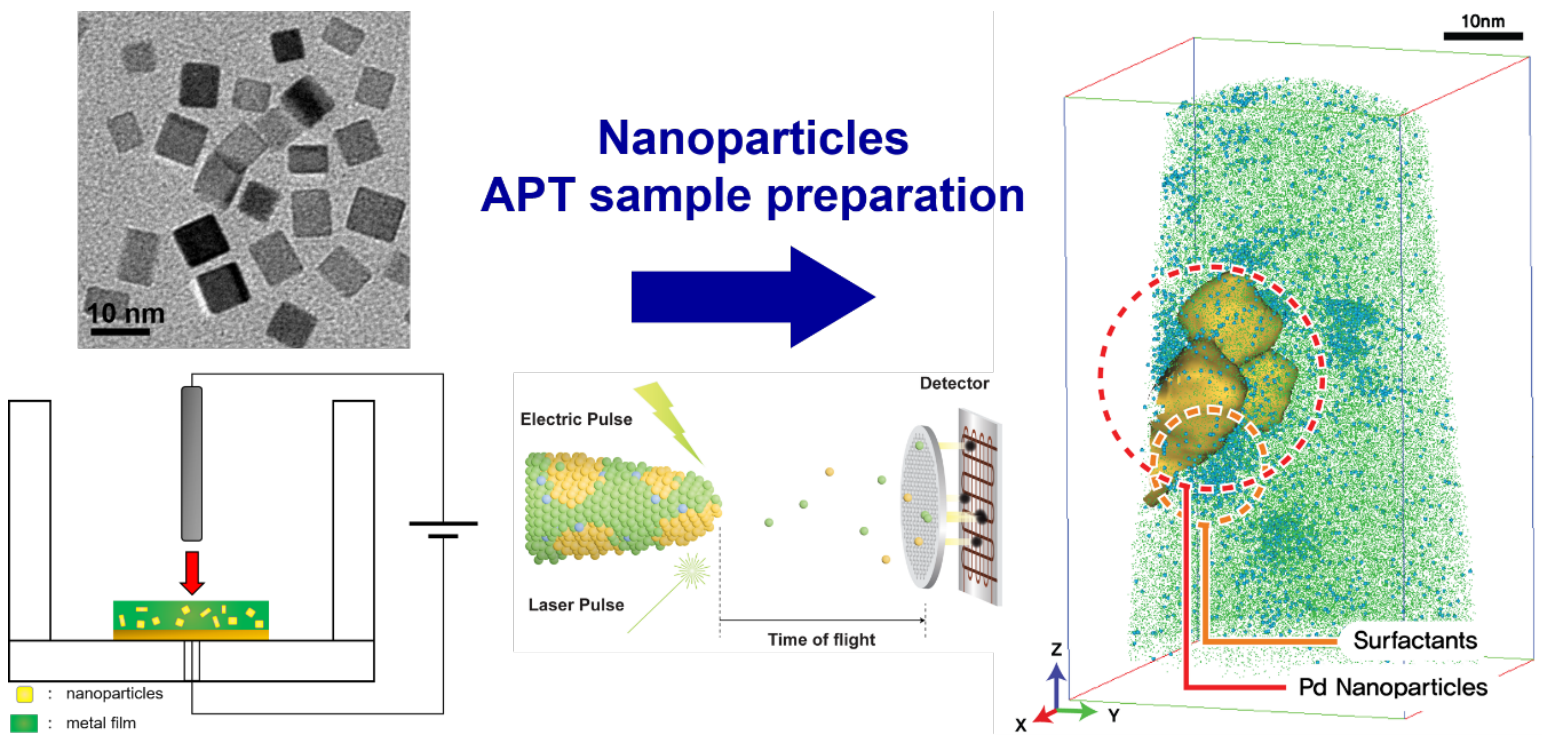

Figure 1. Process for embedding cuboidal Pd nanoparticles in a Ni matrix for APT analysis.

\section{References:}

[1] T. Li et al.., ACS Catalysis 4 (2014) 695.

[2] P. Felfer et al., Ultramicroscopy 159 (2015) 413. 


\title{
APT Analysis of Pt-Modified Nanoporous Gold
}

\author{
Brian Langelier ${ }^{1}$, Ayman A. El-Zoka ${ }^{2}$, Roger C. Newman², Gianluigi A. Botton ${ }^{1}$ \\ Corresponding Author: langelb@mcmaster.ca \\ 1. Department of Materials Science and Engineering, Canadian Centre for Electron Microscopy, \\ McMaster University, 1280 Main St. W., Hamilton, ON, L8S 4M1, Canada. \\ 2. Department of Chemical Engineering and Applied Chemistry, University of Toronto, \\ 200 College Street, Toronto, ON, M5S 3E5, Canada.
}

Nanoporous metals, formed by electrochemical dealloying, possess high surface area to volume ratios vital to applications such as catalysis. Factors such as alloy chemistry allow a tunable porosity; however, understanding the nanoporous structures, and their formation mechanisms, are limited by our ability to characterize these materials. Most analytical methods are hindered by insufficient 3D spatial resolution, or projection effects. APT has the ideal 3D spatial and elemental resolution to characterize nanoporous materials - but to use APT, the pores must be filled to create a consolidated material. Here we employ a method to electrochemically deposit $\mathrm{Cu}$ to fill the open nanoporous structure, and thus make the material suitable for APT analysis [1].

Initial investigations focused on nanoporous gold (NPG) electrochemically dealloyed from Ag$\mathrm{Au}$, and were to successful in yielding APT data of the nanoligaments, revealing Au-rich ligament surfaces and Ag-rich cores [1]. This pore-filling methodology is now expanded to Ag$\mathrm{Au}-\mathrm{Pt}$ ternary alloys, with varying levels of Pt addition, which exhibit finer pore sizes [2]. APT analysis of the Pt-modified NPG yields insights into the action of Pt, which is key to pore size refinement in these materials.

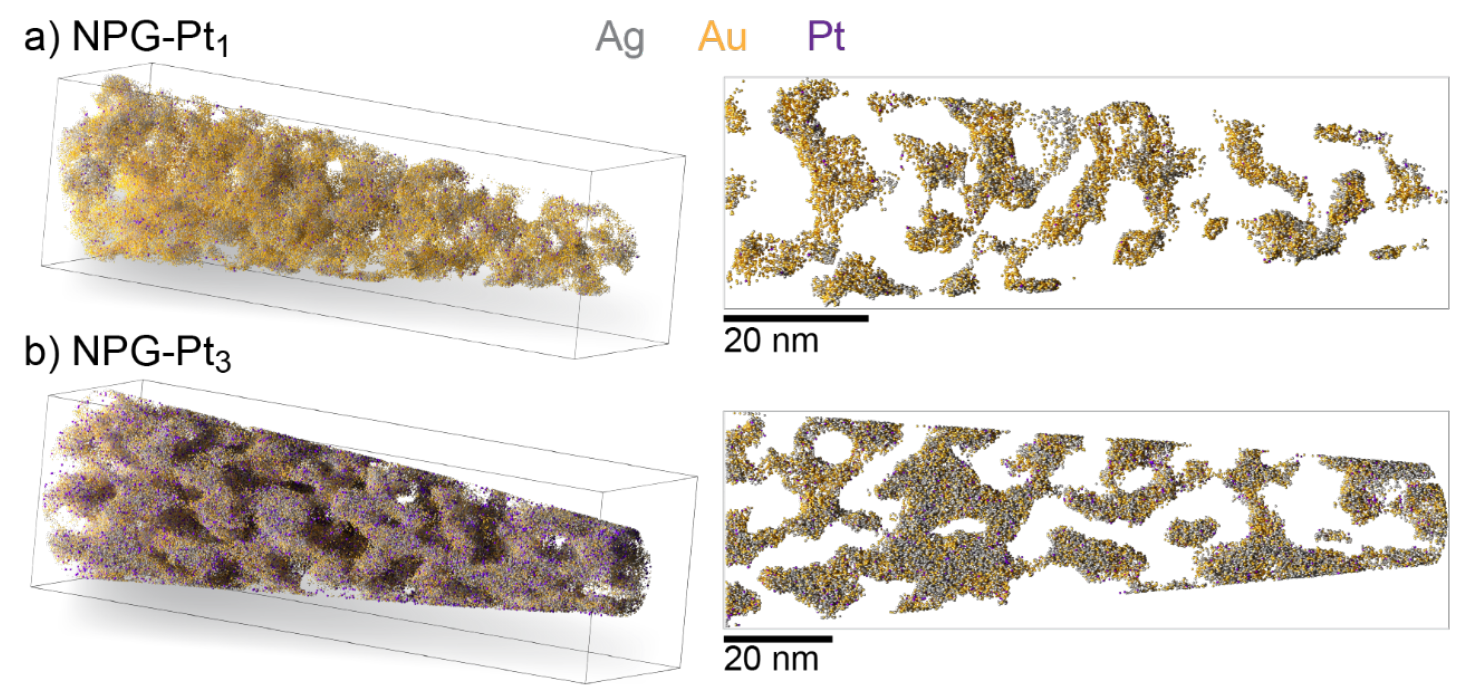

Figure $13 \mathrm{D}$ atom maps of nanoporous gold ligaments, and corresponding $1 \mathrm{~nm}$ thick cross-sections, for bulk alloy content of (a) 1 at.\% Pt (NPG-Pt 1 ) and (b) 3 at.\% Pt (NPG-Pt $\left.{ }_{3}\right)$.

References:

[1] A.A. El-Zoka, B. Langelier, G.A. Botton, R.C. Newman, Materials Characterization 128 (2017) p.269-277.

[2] A.A. El-Zoka, B. Langelier, G.A. Botton, R.C. Newman (2018) submitted. 


\section{In-Situ Crystallization of Metallic Glass in the Atom Probe}

David R. Diercks ${ }^{1}$, Rajesh Jha ${ }^{2}$, Cristian V. Ciobanu², and Aaron P. Stebner ${ }^{2}$

Corresponding Author: ddiercks@mines.edu

1. Department of Metallurgical and Materials Engineering, Colorado School of Mines, Golden, CO USA.

2. Department of Mechanical Engineering, Colorado School of Mines, Golden, CO USA.

Amorphous metals and the thermal processing of them to produce nanocrystalline composites can lead to important magnetic [1] and mechanical [2] properties. For these, control of the final size, composition, and distribution of the phases is of paramount importance. These can be influenced by small changes in initial compositions, temperature, and annealing time. It is also of importance to look at not only the overall crystallization, but also rates of crystal nucleation and growth. Therefore, knowing what occurs at the earliest stages of the crystallization process would shed insight into how to modify the materials and control their properties. However, means of characterizing the early stages of crystallization are challenging because they happen on a time scale of nano to microseconds and a size scale of a few atoms. In-situ synchrotron xray analysis has been used to capture the first seconds of crystallization for such alloys [3], but it provides results averaged on a several micrometer size scale. Atom probe tomography can examine individual clusters and has been applied to analyze such materials, but for samples that were heated ex-situ for 5 to 60 minutes $[4,5]$. Recently it has been demonstrated that dynamic thermal processing can be performed inside a laser-pulsed atom probe instrument [6]. That is, the laser can be used to heat the specimen to induce a diffusive phase transformation as confirmed by electron diffraction and subsequent atom probe analysis. With a laser pulse width of $\sim 10 \mathrm{ps}$ and such a small volume of material, the material is heated then quenched on time scales that are on the order of atomic motion. This is significant in that the earliest stages of crystallization can be captured. Here, the results of the application of this in-situ laser heating method to crystallization of an amorphous Finemet $\left(\mathrm{Fe}_{72.9} \mathrm{Cu}_{1} \mathrm{Nb}_{3.1} \mathrm{Si}_{16.2} \mathrm{~B}_{6.9}\right)$ alloy are presented.

\section{$\underline{\text { References: }}$}

[1] G. Herzer, Modern soft magnets: Amorphous and nanocrystalline materials, Acta Mater. 61 (2013), 718-734.

[2] C.A. Schuh, T.C. Hufnagel, and U. Ramamurty, Mechanical behavior of amorphous alloys, Acta Mater. 55 (2007), 4067-4109.

[3] U. Köster, et al., Nanocrystalline materials by crystallization of metal-metalloid glasses, Mater. Sci. and Engin.: A 133 (1991), 611-615.

[4] K. Hono et al., $\mathrm{Cu}$ clustering and Si partitioning in the early crystallization stage of an $\mathrm{Fe}_{73.5} \mathrm{Si}_{13.5} \mathrm{~B}_{9} \mathrm{Nb}_{3} \mathrm{Cu}_{1}$ amorphous alloy, Acta Mater. 47 (1999), 997-1006.

[5] M. Ohnuma et al., Optimization of the microstructure and properties of Co-substituted $\mathrm{Fe}-\mathrm{Si}-\mathrm{B}-\mathrm{Nb}-$ $\mathrm{Cu}$ nanocrystalline soft magnetic alloys, J. Appl. Phys. 93 (2003), 9186-9194.

[6] R. Kirchhofer, D.R. Diercks, and B.P. Gorman, Near atomic scale quantification of a diffusive phase transformation in $(\mathrm{Zn}, \mathrm{Mg}) \mathrm{O} / \mathrm{Al}_{2} \mathrm{O}_{3}$ using dynamic atom probe tomography, J. Mater. Res. 30 (2015), 1137-1147. 


\section{Atomic Scale Analysis of Magnesium Oxide Scale}

Ingrid McCarroll ${ }^{1,2}$, Daniel Haley ${ }^{3}$, Paul Bagot ${ }^{3}$, Michael Moody ${ }^{3}$, Sebastian Thomas ${ }^{4}$, Nick Birbilis ${ }^{4}$, Julie M. Cairney ${ }^{1,2}$

Corresponding author: ingrid.mccarroll@sydney.edu.au

1. Australian Centre for Microscopy \& Microanalysis, University of Sydney, NSW 2006, Australia

2. School of Aerospace, Mechanical \& Mechatronic Engineering, University of Sydney, NSW 2006, Australia

${ }^{3 .}$ Department of Materials Science, University of Oxford, 16 Parks Rd, Oxford OX1 3PH, UK

${ }^{4 .}$ Department of Materials Science and Engineering, Monash University, Clayton, VIC 3800, Australia

Numerous studies of magnesium $(\mathrm{Mg})$ oxidation have led to a range of theories relating to the cause of Mg's unique corrosion properties, relative to other engineering alloys. Here we analyse $\mathrm{Mg}$ alloys and their dissolution products via atom probe tomography (APT). Controlled oxidation experiments were conducted using an in-situ catalytic reaction chamber, with the results indicating a relationship between the presence of hydrogen in the sample and increased levels of oxidation. These results are in line with previous suggestions that the presence of hydrides in $\mathrm{Mg}$ alloys may play a key role in the oxidation of $\mathrm{Mg}[1,2]$, however, no physical evidence was previously available to support this hypothesis [3]. The current study highlights the capability of APT to provide 3D compositional and spatial information pertaining to the oxidation processes of $\mathrm{Mg}$ alloys.

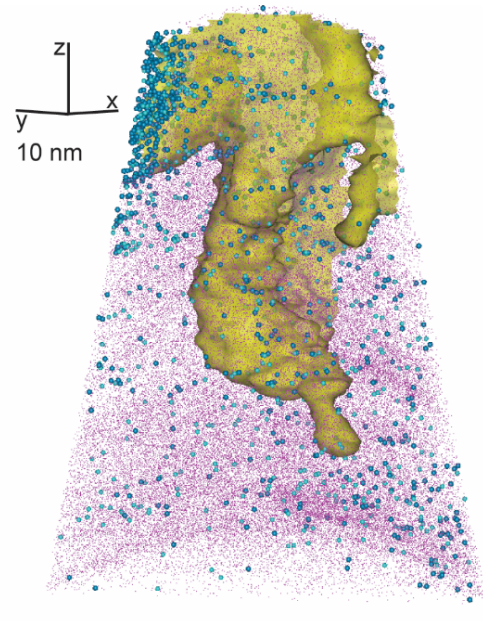

After electropolishing and prior to controlled exposure to $\mathrm{O}_{2}$

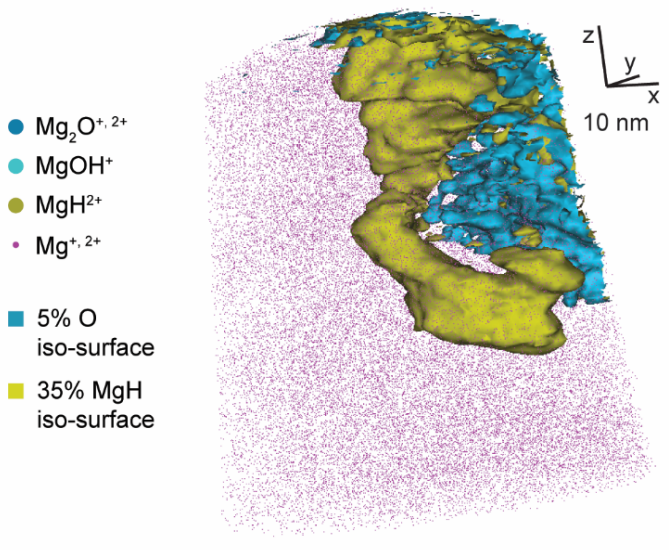

After controlled exposure to $\mathrm{O}_{2}$

Figure 1: Two APT datasets from a single $\mathrm{Mg}-0.1 \mathrm{Ge}(\mathrm{wt} . \%)$ tip, prior to and after exposure to $\mathrm{O}_{2}$.

\section{$\underline{\text { References: }}$}

[1] E. Gulbrandsen, Anodic behaviour of $\mathrm{Mg}$ in $\mathrm{HCO}_{3}{ }^{-} / \mathrm{CO}_{3}{ }^{2-}$ buffer solutions. Quasi-steady measurements, Electrochimica Acta, 37 (1992) 1403-1412.

[2] G.G. Perrault, Potentiostatic study of the magnesium electrode in aqueous solution, Journal of Electroanalytical Chemistry and Interfacial Electrochemistry, 27 (1970) 47-58.

[3] S. Thomas, N.V. Medhekar, G.S. Frankel, N. Birbilis, Corrosion mechanism and hydrogen evolution on Mg, Current Opinion in Solid State and Materials Science, (2014) 10.

[4] Daniel Haley and Michael Moody acknowledge support from EP/L014742/1 


\section{Awards Banquet}

\section{Hilton Hotel, Grand Ballroom}

7:00 to 9:00 PM

The awards banquet wraps up the technical program of APT\&M 2018. Socialize with colleagues over food and drink as the new IFES Fellows, poster award winners, and the Müller Award Competition winner are announced. Sarah Hörst of Johns Hopkins University gives a keynote address on the complex chemical and physical processes that occur in the atmosphere of Saturn's largest moon, Titan. The abstract of this keynote address follows on the next page.

Dr. Sarah Hörst is an assistant professor at Johns Hopkins University in the Department of Earth and Planetary Sciences. Her primary research interest is atmospheric chemistry, particularly the complex organic chemistry occurring in the atmosphere or on the surface of bodies in the solar system. Previously, Dr. Hörst was a National Science Foundation astronomy and astrophysics postdoctoral fellow at the University of Colorado. She earned a B.S. in planetary science and in literature from the California Institute of Technology and her Ph.D. in planetary science from The University of Arizona.

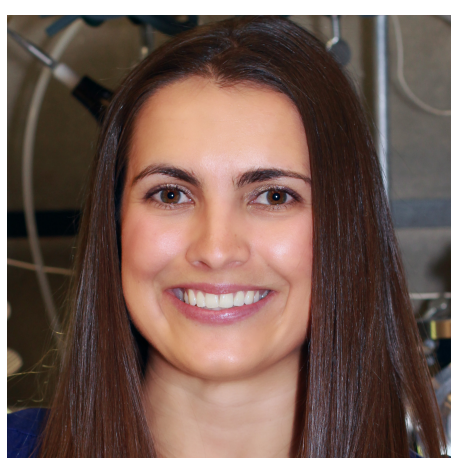




\section{Aromatic, Aliphatic, Enigmatic: The Chemistry of Titan}

\section{Sarah Hörst}

Corresponding Author: sarah.horst@jhu.edu

Johns Hopkins University, Department of Earth and Planetary Sciences, Baltimore, MD 21218.

The extraordinary complexity of Titan's atmospheric chemistry far surpasses that of any other solar system atmosphere. With its thick N2 atmosphere and stable bodies of liquid on its surface, Titan also possesses many physical processes that are similar to those that occur on Earth. The connection between Titan's surface and atmosphere is unique in our solar system; atmospheric chemistry produces materials that are deposited on the surface and subsequently altered by surface-atmosphere interactions such as aeolian and fluvial processes resulting in the formation of extensive dune fields and expansive lakes and seas. Titan's atmosphere is favorable for organic haze formation, which combined with the presence of some oxygen-bearing molecules indicates that Titan's atmosphere may produce molecules of prebiotic interest. The combination of organics and liquid, in the form of water in a subsurface ocean and methane/ethane in the surface lakes and seas, means that Titan may be the ideal place in the solar system to test ideas about habitability, prebiotic chemistry, and the ubiquity and diversity of life in the universe. I will review our current understanding of chemistry on Titan forged from the powerful combination of Earth-based observations, remote sensing and in situ spacecraft measurements, laboratory experiments, and models. I will conclude with some of the questions that remain after Cassini-Huygens. 


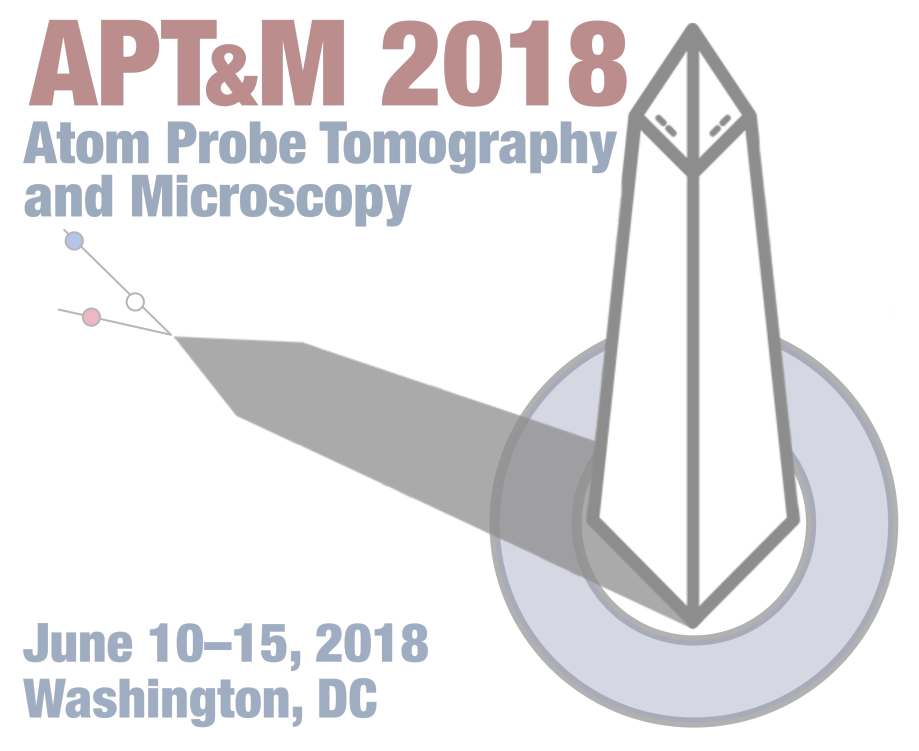

\section{Posters}





\section{Dating Large Impacts with Nanoscale Pb Isotope Clusters in Zircon: A Proof of Concept from the Vredefort Central Uplift}

Arcuri, G. ${ }^{1}$, Moser, D.E. ${ }^{1}$, Reinhard, D.A. ${ }^{2}$, and Langelier, B. ${ }^{3}$

Corresponding Author: garcuri@uwo.ca

1. Department of Earth Sciences, Western University, London, Ontario, Canada

2. CAMECA Instruments, Inc., Madison, Wisconsin, USA

3. Canadian Centre for Electron Microscopy, McMaster University, Hamilton, Ontario, Canada

Improving the accuracy of solar system impact history is a widely-shared goal among planetary science and workers reconstructing the initial conditions of early crustal evolution and pathways to habitability. As one of the most resistant and refractory mineral chronometers zircon has become an attractive tool for recovering impact history. Over the last decade, microstructural research has focused on understanding the reaction of zircon to extreme shock and thermal conditions for the dating of such events; however, when sampled at micron length-scales, mixed $\mathrm{U}-\mathrm{Pb}$ isotope compositions can be produced [e.g., 1] that are difficult to interpret for out-ofcontext samples, such as meteorites. APT work on natural zirconia (baddeleyite) has shown that it is possible to isolate impact-age nanodomains [e.g., 2] but this has not yet been shown in zircon.

Here, we prove this concept with shocked zircon, sampled from the center of Earth's largest known impact, the Vredefort structure in South Africa [1], using U-Pb geochronometry carried out at different length scales. For example, data from multiple APT microptips have been combined to measure 'bulk' concentrations of $\mathrm{U}, \mathrm{Pb}$ and other trace elements across several microns, while sub-mircotip analysis of the same data allows the isolation of discrete nanostructures, and more precise isotopic compositional measurements. These approaches yield unique $\mathrm{U}-\mathrm{Pb}$ and $\mathrm{Pb}-\mathrm{Pb}$ ratios and two distinct $\mathrm{Pb}$ reservoirs at the nanometer scale; $\mathrm{Pb}$-rich clusters and a $\mathrm{Pb}$-depleted homogeneous matrix. A spatial correlation is also observed between clusters of pre-impact, radiogenic $\mathrm{Pb}$, and $\mathrm{Al}$ ions. These results show that primary and impact re-set (e.g., 100\% Pb-loss) domains can exist within microns of one another. This microstructure can only be explained by rapid channelized out-diffusion of $\mathrm{Pb}$ in the complex sequence of crystal strain and melt intrusion unique to central uplift environments of large craters. The migration and preservation of these nanostructural and chemical domains suggest that these incompatible species migrated to, or with, shock-induced defects in the zircon lattice during extreme thermal metamorphism and plastic recovery of the crystal. Some Apollo lunar breccia zircons are known to have similar microstructures and, by secondary ion mass spectrometry, mixed U-Pb compositions. These signatures may prove diagnostic of high-temperature impact environments and present a method by which higher accuracy impact histories can be extracted with APT from planetary zircon.

\section{References:}

[1] D. E. Moser, et al., New zircon shock phenomena and their use for dating and reconstruction of large impact structures revealed by electron nanobeam (EBSD, CL, EDS) and isotopic U- $\mathrm{Pb}$ and $(\mathrm{U}-\mathrm{Th}) / \mathrm{He}$ analysis of the Vredefort dome, Can. J. Earth Sci. 48 (2011), 117-139.

[2] L. White, et al., Atomic-scale age resolution of planetary events, Nature Comm., 8 (2017), 15597. 


\title{
Atom Probe Analysis of Silica Nanospheres in Diamond Anvil Experiments
}

\author{
J. O. Douglas ${ }^{1}$, E.S. Jennings ${ }^{2,3}$, M. P. Moody ${ }^{1}$ and J. Wade ${ }^{4}$
}

Corresponding Author: james.douglas@materials.ox.ac.uk

${ }^{1}$ University of Oxford, Department of Materials, Parks Road, Oxford, OX1 3PH, UK.

2. Bayerisches Geoinstitut, University of Bayreuth, 95440 Bayreuth, Germany.

3. Dept. Earth and Planetary Sciences, Birkbeck, University of London, Malet Street, London WC1E 7HX, UK.

4. University of Oxford, Department of Earth Sciences, South Parks Road, Oxford OX1 3AN, UK.

Laser-heated diamond anvil cell (DAC) experiments are frequently used to examine elemental metal-silicate partitioning at extreme pressure and temperature, with the goal of understanding planetary formation and differentiation processes, and the compositions of metallic planetary cores [1]. Such experiments usually produce a metal 'bleb' which is heterogeneous on the nmscale, containing oxide-rich spheres within an iron-rich metal matrix. The exolved oxides either originate during quench, or are stable during the experiment: these two scenarios have very different implications for the interpretation of metal compositions at experimental conditions. Electron Probe Micro-Analysis (EPMA) is typically used to determine composition in such samples but the analytical interaction volume, usually $\sim 1 \mu \mathrm{m}^{3}$, prevents meaningful qualitative analysis of the nanospheres.

Atom probe tomography carried out on samples extracted from the bleb has determined that the exsolved nanospheres are stoichiometric silica and that the matrix is carbon-bearing iron. A narrow trace-element-enriched interface also exists between the two (Figure 1). The observed lack of clear diffusion gradients may indicate that these features were not exsolved during the rapid quench, meaning that published compositions from integrated analyses of broader regions may not actually reflect equilibrium elemental partitioning: planetary cores may contain less silica than previously thought. However, quench rates in DAC experiments must be known more precisely in order to calculate the expected length-scale of diffusion during quench.

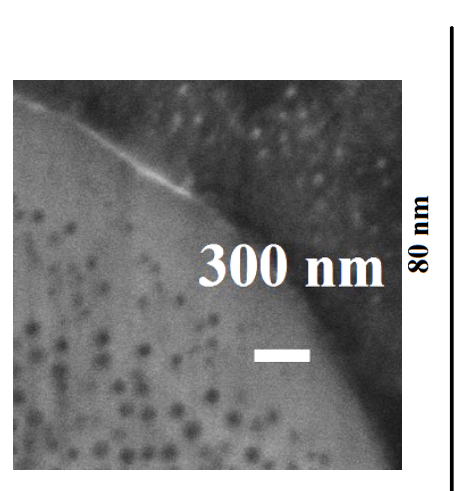

$\underline{\text { References: }}$
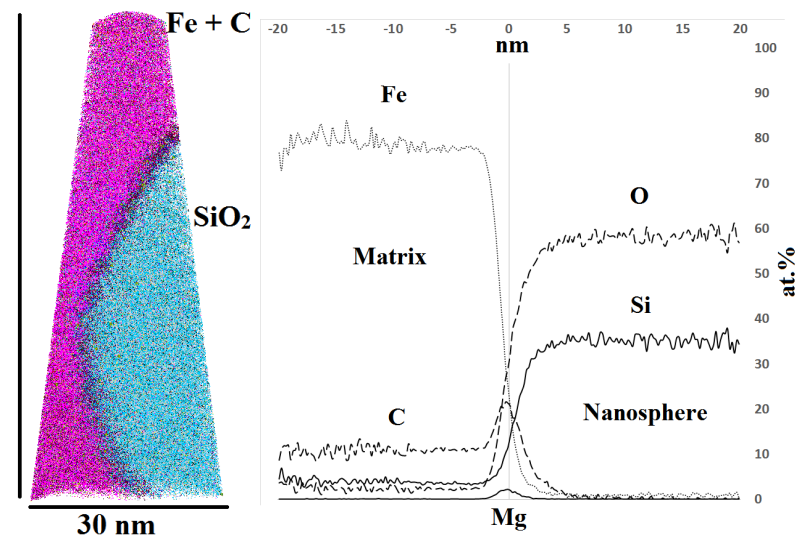

Figure 1: Left - Electron micrograph of dark contrast nanospheres within the metal bleb . Middle - Atom map of interface between matrix and nanosphere. Right Proximity histogram from $10 \%$ Si ions showing change in composition from carbon rich iron in the bleb matrix to silica in the nanosphere.

[1] M. A. Bouhifd and A. P. Jephcoat, The effect of pressure on partitioning of Ni and Co between silicate and iron-rich metal liquids: a diamond-anvil cell study. Earth and Planetary Science Letters 209 (2003), 245-255. 


\title{
Comparison of APT Measurements at Glass Corrosion Fronts From Wet Cryogenically Frozen and Supercritically Dried Samples
}

\author{
Daniel K. Schreiber ${ }^{1}$, Daniel E. Perea ${ }^{2}$, and Joseph V. Ryan ${ }^{1}$ \\ Corresponding Author: daniel.schreiber@pnnl.gov \\ 1. Energy \& Environment Directorate, Pacific Northwest National Laboratory, Richland, WA \\ USA. \\ 2. Environmental \& Molecular Sciences Laboratory, Pacific Northwest National Laboratory, \\ Richland, WA USA.
}

Specimen preparation is one of the most important and challenging aspects of high resolution microscopy. This is particularly apparent when characterizing microstructures resulting from aqueous corrosion where removal of the sample from its corrosive medium can inadvertently change the observed microstructure. While in situ experiments are commonly pursued with electron microscopy techniques to overcome this limitation, it is difficult to imagine in situ aqueous corrosion within the UHV chamber of modern atom probes.

We have prepared APT specimens of glass corrosion resulting from an aqueous environment by two methods: supercritical drying and cryogenic fixation. For supercritically dried samples, routine site-specific focused ion beam (FIB)-based APT specimen preparation is possible. [1] More recently we have demonstrated cryogenic site-specific FIB-based preparation of APT specimens targeting the water/corroded glass interface (Fig. 1). In this talk we compare the resulting APT measurements at the corroded glass / pristine glass interface. The results show that quantitative differences exist depending on the specimen preparation method, particularly in the magnitude and gradient of critical elements across the corrosion front. The impact of these differences on our mechanistic understanding of glass corrosion will be discussed.

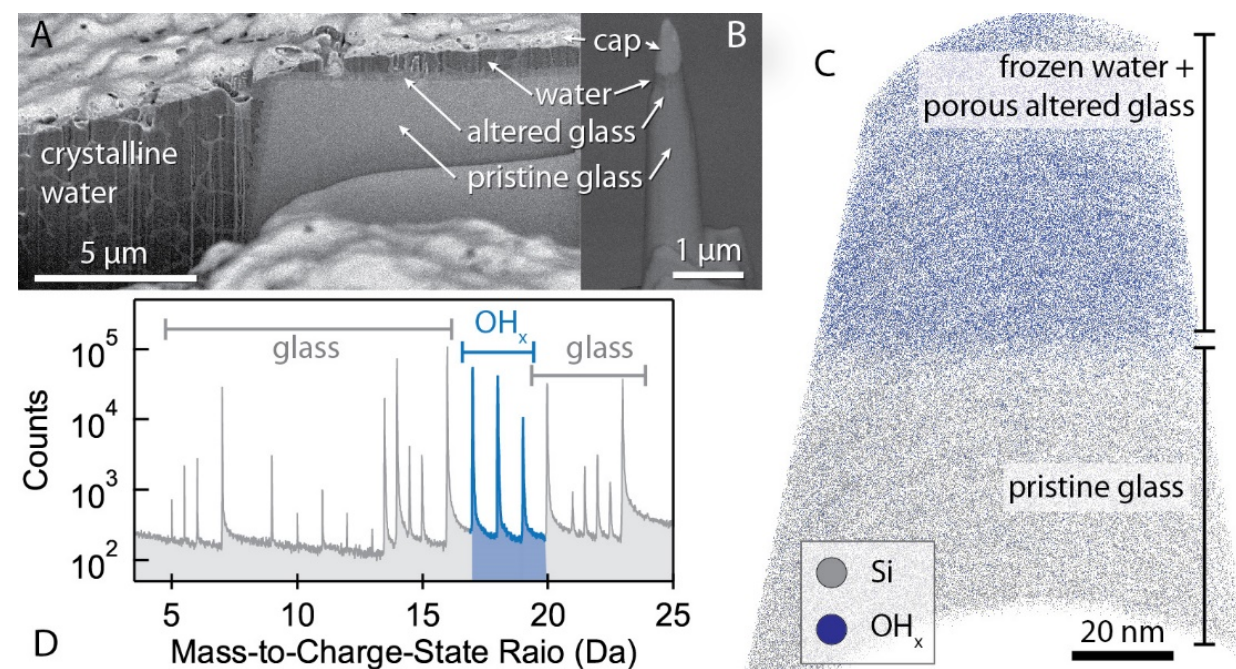

Figure 1 (A, B) SEM images of a corroded glass particle and frozen water. (C, D)

Corresponding APT data of the porous corroded glass + water / pristine glass interface.

References:

[1] K. Thompson, D. Lawrence, D.J. Larson, J.D. Olson, T.F. Kelly, B. Gorman, In situ site-specific specimen preparation for atom probe tomography, Ultramicrosc., 107 (2007) 131-139. 


\section{Analysis of Natural Rutile by Laser-assisted Atom Probe Microscopy}

Rick Verberne $^{1,2}$, David W. Saxey ${ }^{1,2}$, Steven M. Reddy ${ }^{1,2}$, William D.A. Rickard ${ }^{1}$ and Denis Fougerouse ${ }^{1,2}$

Corresponding Author: rick.verberne@postgrad.curtin.edu.au

${ }^{1}$ Geoscience Atom Probe, Advanced Resource Characterisation Facility, John de Laeter Centre, Curtin University, Perth, Australia.

2. School of Earth and Planetary Sciences, Curtin University, Perth, Australia.

Since the introduction of the laser-assisted atom probe, analysis of non-conductive materials, including geological samples, by atom probe microscopy (APM) has become more routine. However, to acquire high quality data the acquisition parameters need to be optimized, both for the material of interest, and for the specific questions to be addressed by the analysis. Here the rutile $\left(\mathrm{TiO}_{2}\right)$ standard 'Windmill Hill Quartzite', which is used for secondary ion mass spectrometry (SIMS) U-Pb dating and laser-ablation inductively coupled plasma mass spectrometry (LA-ICP-MS), was analysed by laser-assisted APM in order to constrain optimal running conditions for rutile. Changes in acquisition parameters such as laser energy and detection rate are evaluated in terms of their effect on background noise, ionisation state, hitmultiplicity and thermal tails. Higher laser energy results in the formation of more complex molecular ions and affects the preferred ionisation state. At lower energies, background noise and hit-multiplicity increase, while the mass spectrum improves, with shorter thermal tails. There also seems to be a correlation between the acquisition voltage and several of these metrics. The effect of the voltage on the data quality remains to be fully understood. The variances in relation to the possible acquisition parameters will be discussed in detail in the context of utilizing rutile within geology. In general, low laser energies $(<80 \mathrm{pJ})$ at low temperatures $(\leq 50 \mathrm{~K})$ give the best results.

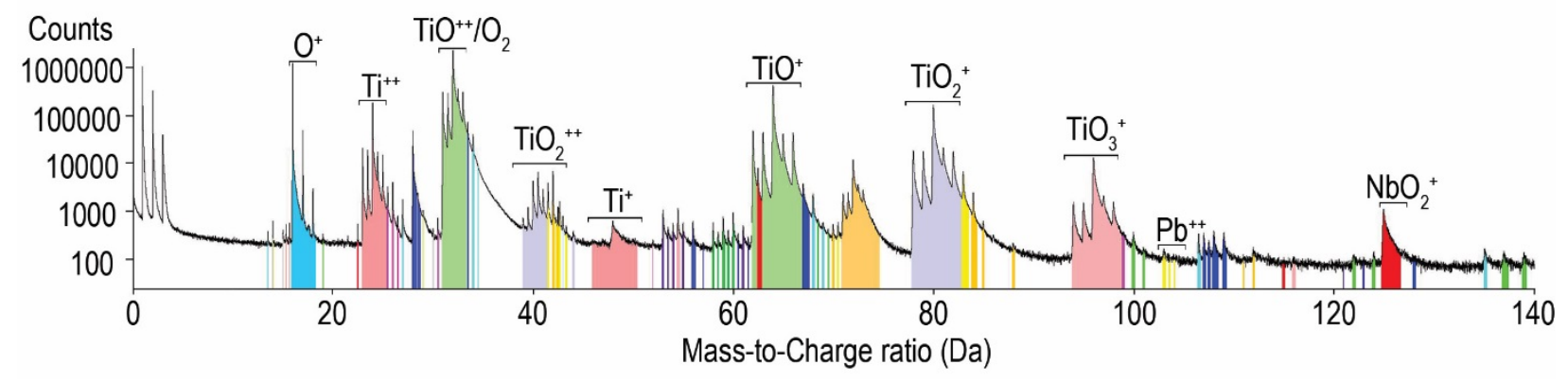

Figure 1 Typical complex mass spectrum for natural rutile as a acquired by atom probe microscopy 


\title{
Mapping Water-filled Inclusions in an Archean Framboidal Pyrite
}

\author{
Daniel E. Perea ${ }^{1}$ and Daniel Gregory ${ }^{2}$ \\ Corresponding Author: daniel.perea@pnnl.gov \\ 1. Environmental \& Molecular Sciences Laboratory, Pacific Northwest National Laboratory, \\ Richland, WA USA. \\ 2. Department of Earth Sciences, University of California, Riverside, CA, USA
}

The formation of sedimentary pyrite is the result of microbial reduction of $\mathrm{SO}_{4}{ }^{2-}$ and subsequent reaction of the resultant $\mathrm{H}_{2} \mathrm{~S}$ with iron. During the formation of pyrite, the incorporation of trace elements (TE) can occur to provide a snapshot of the environment under which it was formed [1]. In an effort to map the composition of TE in sedimentary pyrites, a correlative approach to nanoscale structure/composition mapping was pursued via the application of NanoSIMS, TEM, and APT (Fig. 1). The sample is from the Devonian Leicester Pyrite Member, in New York State. This formation believed to be a sedimentary lag deposit of pyrite formed within the water column during diagenesis. NanoSIMS was used to identify regions of high TE content within and around framboidal pyrite structures and provide a target for nanoscale TEM and APT analysis (Fig. 1a). TEM analysis revealed the presence of a relatively high number of low density regions (Fig. 1b). Correlative APT analysis of the same sample surprisingly revealed these low-density regions to be water filled inclusions (Fig. 1c). Spatially co-located $\mathrm{Na}$ and $\mathrm{Ca}$ suggests the inclusions are ancient sea water.

Figure 1.

(a) NanoSIMS composition mapping to identify a ROI for (b) TEM and (c) APT analysis.
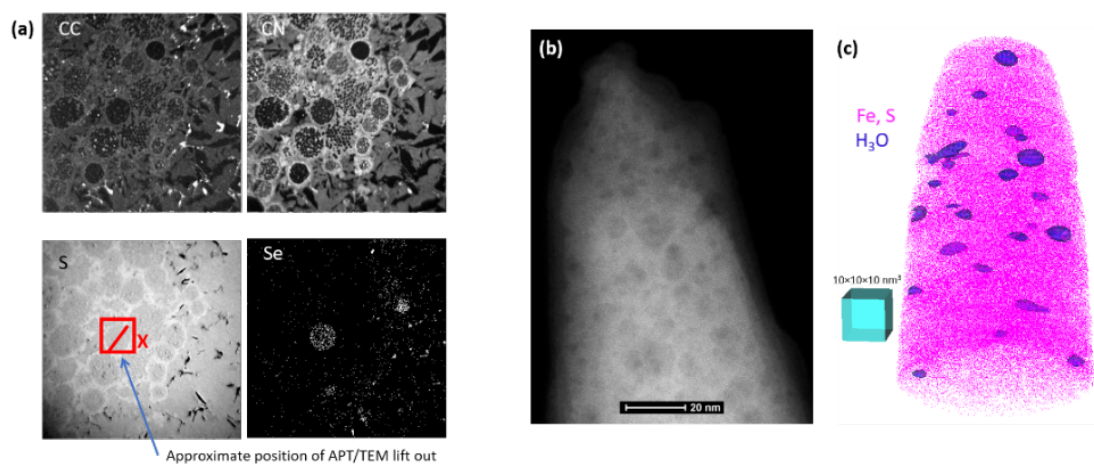

References

[1] Daniel Gregory et al. Comparison of metal enrichment in pyrite framboids from a metalenriched and metal-poor estuary, Am. Mineral., 2014, 99(4): 633-644

[2] The research was performed at the Environmental Molecular Sciences Laboratory; a national scientific user facility sponsored by the Department of Energy's Office of Biological and Environmental Research located at Pacific Northwest National Laboratory. 


\section{Quantitative Analysis of Dopant in Semiconductor Device Using Atom Probe Tomography}

Sung Ho Lee ${ }^{1}$, Seong Pyo Hong ${ }^{1}$, Yong Bum Kim ${ }^{1}$, Eun Kyung Jeong, ${ }^{1}$ Sang Ick Lee ${ }^{1}$, and Joong Jung $\mathrm{Kim}^{1}$

Corresponding Author: sh8270.1ee@samsung.com

${ }^{1 .}$ Manufacturing Engineering Team, Samsung Electronics, Hwaseong city, Republic of Korea.

The atom probe tomography is used to measure elements ratio and impurity concentration in the product of steel and semiconductor industries. Especially, it is a unique tool for getting the dopants concentration on source/drain and channel region of semiconductor chip. Fluctuation of a dopant concentration in there regions leads to serious problems such as degraded device performance and shifts in the electrical factors. Therefore the quantitative measurement of the dopant concentration is critical for the development of semiconductor devices. However, the measurement accuracy of the dopant concentration is greatly influenced by conditions of the atom probe such as laser power and detection rate, etc., making it difficult to obtain reliable data using the atom probe.

In this report, we present a method to get a quantified data from atom probe through measuring the conditions. We were able to find the optimum conditions for the measurement of dopant concentration by comparing with quantified secondary ion mass spectroscopy which has been the reference tool in microelectronics for dopant profiling in semiconductor.

\section{References:}

[1] Didier Blavette and Sebastien Duguay, Atom probe tomography in nanoelectronics, Eur. Phys. J. Appl.Phys. (2014), 10101p1-10101p12.

[2] M.Py, J. P. Barnes, D. Lafond and J. M. Hartmann, Quantitative profiling of SiGe/Si superlattices by time-of-flight secondary ion mass spectrometry, Rapid Communications in Mass Sepctrometry (2011), 629-638.

[3] Koji Inoue, Ajay Kumar Kambham, Dominique Mangelinck, Dan Lawrence and David J. Larson, Atom-probe-tomographic studies on silicon based semiconductor devices, Microscopytoday (2012), 3844. 


\title{
Characterization of a Si FinFET structure and dopants distribution by Atom Probe Tomography
}

\author{
Rong $\mathrm{Hu}^{1}$, Jing Xue ${ }^{1}$, Yanbo Zhang ${ }^{2}$, Huilong $\mathrm{Zhu}^{2}$, Gang Sha ${ }^{1}$ \\ Corresponding Author: gang.sha@,njust.edu.cn \\ 1. Herbert Gleiter Institute of Nanoscience, School of Materials Science and Engineering, \\ Nanjing University of Science and Technology, Nanjing, 210094 China. \\ 2. Institute of Microelectronics of Chinese Academy of Sciences, Beijing 100029 China.
}

The continuous down-scaling and development of advanced semiconductor devices are largely rely on the characterization of their 3D morphology, composition and dopants distribution $[1,2]$. Recently, atom probe tomography has been introduced as a new effective tool into the field [3]. Still due to the complicate structure of the semiconductor devices, many challenges remain to be conquered in order to obtain accurate reconstructions. In this study, we present our recent APT results on a Ni/Pt coated Si finFET structure, which was implanted by $\mathrm{B}, \mathrm{P}$ and As. The sample containing finFET was prepared from two different directions by focused ion beam. When the fin structure was parallel with the sample axis, the difference between the evaporation fields of $\mathrm{Si}$ and $\mathrm{SiO}_{2}$ gave rise to the local magnification of $\mathrm{SiO}_{2}$ and the trajectory aberration, which also resulted in a great difficulty in correctly reconstructing the fin morphology. On the other hand, when the fin structured was perpendicular with the sample axis, such artifacts could be largely avoided. In this way, the morphology characterization and the measurement of dopants distribution can be performed more accurately.

\section{References:}

[1] A.K. Kambham, A. Kumar, M. Gilbert, and W. Vandervorst, Ultramicroscopy 132 (2013), 65-69. [2]. A. Grenier, S. Duguay, J.P. Barnes, R. Serra, G. Haberfehlner, D. Cooper, F. Bertin, S. Barraud, G. Audoit, L. Arnoldi, E. Cadel, A. Chabli, and F. Vurpillot, Ultramicroscopy 136 (2014), 185-192.

[3]. J.P. Barnes, et al, Scripta Materialia 148 (2018), 91-97. 


\section{Increased Detector Efficiency Helps to Reveal New Insights Into Compositional Fluctuations at Defects in $\mathrm{Cu}_{2} \mathrm{ZnSnSe}_{4}$ Thin Films}

Torsten Schwarz, ${ }^{1}$ Alex Redinger, ${ }^{2}$ Susanne Siebentritt, ${ }^{2}$ Baptiste Gault, ${ }^{1}$ Dierk Raabe ${ }^{1}$, and Pyuck-Pa Choi ${ }^{3}$

Corresponding Author: schwarz@mpie.de

1. Max-Planck-Institut für Eisenforschung GmbH, Düsseldorf, Germany.

2. Laboratory for Photovoltaics, University of Luxembourg, Belvaux, Luxembourg.

3. Korea Advanced Institute of Science and Technology, Daejeon, Republic of Korea.

Thin-film solar cells based on the kesterite structured compound semiconductors $\mathrm{Cu}_{2} \mathrm{ZnSnS}(\mathrm{e})_{4}$ $(\mathrm{CZTS}(\mathrm{e}))$ are an emerging novel class of solar cells, which are a promising alternative to $\mathrm{Cu}(\mathrm{In}, \mathrm{Ga}) \mathrm{S}(\mathrm{e})_{2}$ (CIGS) absorbers by reducing material costs. Efficiencies of $>12 \%$ are achieved for CZTSSe based solar cells. This value is still far below the $22.9 \%$ record efficiency of CIGS and further development is needed.

Apart from secondary phases, grain boundaries (GB) and other extended defects, such as stacking faults and dislocations, can also significantly affect the electrical and optical properties of the absorbers since detrimental deep traps within the band gap may be associated with them. Using the LEAP 3000XR tool, we previously detected for CZTSe low temperature precursors that the GBs are Cu-enriched and Sn-depleted, while for the annealed precursor the GBs are decorated by $\mathrm{Na}$ and $\mathrm{K}$ impurities and show (almost) no change in the concentration of the matrix elements [1].

In this study, we investigate CZTSe thin films utilizing the LEAP 5000XS tool. Due to the increased atom detection efficiency of $\approx 80 \%$ and the use of relative concentration fluctuation profiles, we observe a larger variety of compositional fluctuations at defects. Na impurities can also decorate the mainly $\mathrm{Cu}$-enriched GBs in the CZTSe precursor with an average excess of $0.05 \mathrm{at} / \mathrm{nm}^{2}$. For the absorber, the mainly by $\mathrm{Na}$ atoms decorated GBs we also clearly observe fluctuations of the matrix elements, which can vary from one GB to another as shown in Figure 1. We will discuss possible effects of the observed phenomena on the cell performance.

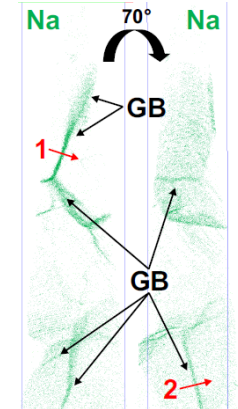

a) $50 \mathrm{~nm}$ b)
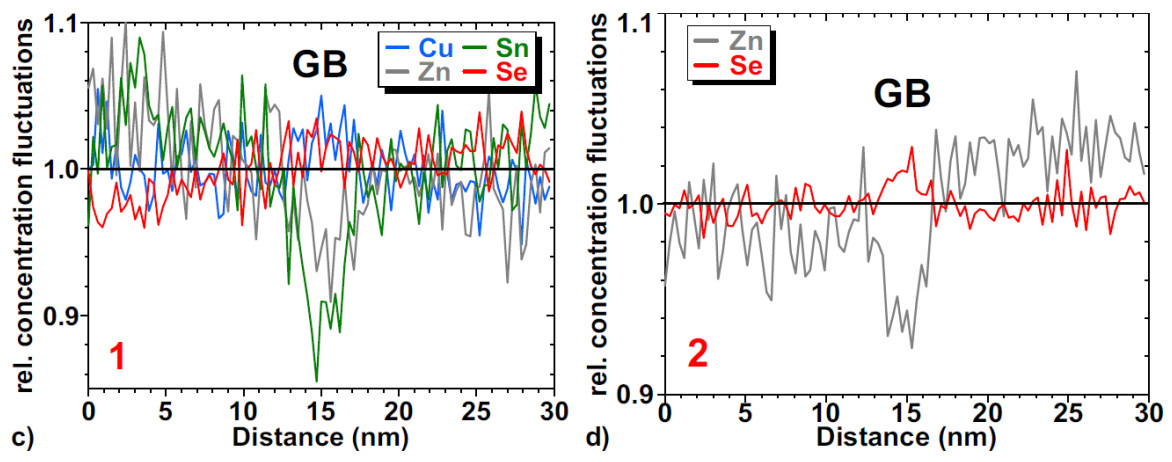

Figure 1 (a)-(b) Na decorated CZTSe GBs. (c)-(d) Relative concentration fluctuation profiles across the GBs 1 and 2 in (a)-(b) revealing small fluctuations of the matrix elements at those GBs.

References:

[1] T. Schwarz, et al., Atom probe tomography study of internal interfaces in $\mathrm{Cu} 2 \mathrm{ZnSnSe} 4$ thin-films, Journal of Applied Physics 118 (2015), 095302. 


\title{
Semiconductor Device Failure Analysis with Atom Probe Tomography
}

\author{
A.D. Giddings ${ }^{1}$, Y. Yang ${ }^{2}$, M.H. Kao ${ }^{1}$, Y.C. Ong ${ }^{1}$, W.S. Hu${ }^{1}$, R.L. Hwang ${ }^{1}$, and J.J. Lee ${ }^{1}$ \\ Corresponding Author: giddings@physics.org \\ 1. 166, Park Ave. II, Hsinchu Science Park, Hsinchu 300-75, TAIWAN \\ 2. 4000, Wen Xiang Road, Songjiang, Shanghai, 201616, CHINA
}

The complex 3D structure and small dimensions of ultimate-scale CMOS transistors provide a unique metrological challenge which makes them a typical target for industrial application of atom probe tomography (APT). However, the majority production of semiconductor devices is based on older technologies employing traditional planar channels and larger feature sizes. This work gives an example of a failure analysis (FA) issue which was analyzed using a combination of metrology techniques in order to demonstrate the impact APT can have in this role.

In this FA issue a series of performance excursions were found in production devices based on a legacy planar transistor architecture featuring poly-Si gates. Electrical measurements found the failed devices all featured a p-MOS transistor with a high threshold voltage, preventing proper gate control. The position of the failed devices was found to be correlated with distance from the $\mathrm{n} / \mathrm{p}$ patterning boundary. Transmission electron microscopy characterization of failed $\mathrm{p}$-MOS devices found unusually large grains in the poly-Si gate. Scanning spreading resistance microscopy and scanning capacitance microscopy (SCM) found the gate had a higher resistance and some grains had a lower carrier concentration. Based on these results, it was hypothesized that possible causes were (i) the large grains were blocking the introduction of the p-type dopant, or (ii) there is lateral diffusion of n-type dopants, which would act as a counter-dopant.

APT measurements were made to compare good devices with a failed p-MOS transistor. In the n-type poly-Si there is significant segregation of dopants to the grain boundaries while in the $\mathrm{p}$ type poly-Si the dopants are more uniformly distributed. In the failed p-MOS transistor ndopants were found to decorate a grain boundary, and one of the grains was found to have elevated levels of this counter dopant, corroborating the SCM results. This indicates that the ndopant is diffusing along the grain boundaries from across the $\mathrm{n} / \mathrm{p}$ boundary into the $\mathrm{p}$-poly gate.

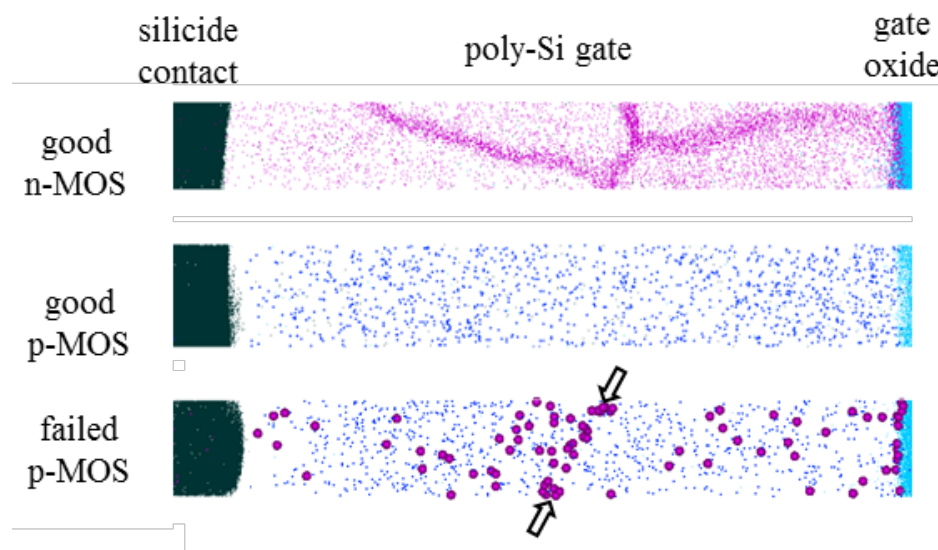

Figure: atom clouds of parts of the good n- and good p-MOS poly gates, and the failed p-MOS. Arrows indicate a region of elevated n-type dopants in the failed p-MOS device, suspected to be a grain boundary. 


\section{Using Atom Probe to Identify Limitations in Photovoltaic Cells}

Mohit Raghuwanshi and Oana Cojocaru-Mirédin

Corresponding Author: raghuwanshi@physik.rwth-aachen.de

RWTH Aachen, I. Physikalisches Institut IA; Sommerfeldstrasse 14, 52074 Aachen, Germany

Solar cells based on $\mathrm{Cu}(\mathrm{In}, \mathrm{Ga}) \mathrm{Se}_{2}$ (CIGS) present efficiency of up to $22.9 \%{ }^{1}$ and is highest under thin film category, this makes CIGS a promising candidate for future solar cell modules. Quite interestingly over the years, its maximum efficiency is achieved in its polycrystalline form, even with its small grain sizes of $2 \mu \mathrm{m}$. Previous studies have shown an important role of all grain boundaries (GBs) ${ }^{2-4}$ towards its output efficiency. Here using electron beam induced current (EBIC) technique we found that most of the GBs are indeed beneficial for the device, however a small percentage $(15 \%)$ of GBs was found detrimental. Hence, in this work we isolate these detrimental GBs and perform correlative EBIC-EBSD and atom probe tomography (APT) on them to identify its type, structure and composition as shown in Figure 1. Results show that composition of detrimental GBs was found very different than previous results obtained on high efficient cells. In this way we can obtain information about 'detrimental' GBs, which could be solved in future by variation in deposition parameters and post deposition treatments.

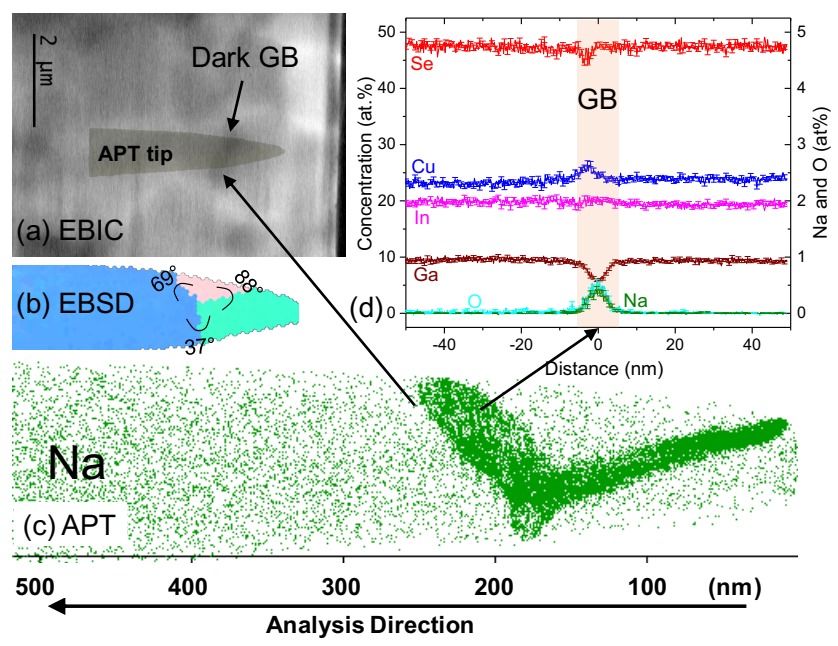

Figure 1 (a) EBIC image on top of CIGS indicating location of detrimental GB and location of APT specimen. (b) EBSD map on top of APT specimen, (c) APT image showing Na atoms segregated along $\mathrm{GB}$, (d) concentration profile of elements across identified detrimental GB

\section{References:}

${ }^{1} \mathrm{https} / /$ www.pv-magazine.com/2017/12/20/solar-frontier-hits-new-thin-film-cell-efficiency-record/

${ }^{2}$ C. Persson and A. Zunger, Phys. Rev. Lett. 91, 266401 (2003).

${ }^{3}$ O. Cojocaru-Mirédin, T. Schwarz, and D. Abou-Ras, Scripta Materialia (2017).

${ }^{4}$ M. Raghuwanshi, E. Cadel, P. Pareige, S. Duguay, F. Couzinie-Devy, L. Arzel, and N. Barreau, Applied Physics Letters 105, 13902 (2014). 


\section{Quantification of Indium Segregation in InAIN Thin-films Grown by Plasma- assisted Molecular Beam Epitaxy}

Alexander Senichev ${ }^{1,2}$, Jonathan Poplawsky ${ }^{3}$, Mohammad Ali Shirazi ${ }^{1,2,4}$, Brandon Dzuba ${ }^{1,2}$, Oana Malis ${ }^{1}$ and Michael J. Manfra ${ }^{1,2,4,5}$

\section{Corresponding Author: senichev@purdue.edu}

${ }^{1}$ Birck Nanotechnology Center, West Lafayette, Indiana 47907, USA

${ }^{2}$ Department of Physics and Astronomy, Purdue University, West Lafayette, Indiana 47907, USA

${ }^{3}$ Center for Nanophase Materials Sciences, Oak Ridge, National Laboratory, 37831, USA

${ }^{4}$ School of Electrical and Computer Engineering, Purdue University, West Lafayette, Indiana 47907, USA

${ }^{5}$ School of Materials Engineering, Purdue University, West Lafayette, Indiana 47907, USA

$\mathrm{In}_{0.17} \mathrm{Al}_{0.83} \mathrm{~N}$ lattice-matched to $\mathrm{GaN}$ in the (0001) plane offering the potential for fabrication of thick strain-free heterostructures necessary for novel light sources based on intersubband optical transitions. However, InAIN is subject to segregation into In-rich regions, deviating strongly from a random alloy distribution. Due to strong differences between its antecedents, AIN and InN, InAlN can form a "honeycomb" structure [1,2]. Current understanding of the growth mechanism controlling segregation remains primitive at best, and importantly, the impact of the residual inhomogeneities on the intersubband optical properties of InAlN/GaN heterostructures remains an outstanding problem [2].

In this work, we systematically investigate alloy inhomogeneity controlled by variation of plasma-assisted molecular beam epitaxy growth conditions using atom probe tomography (APT). Multiple growths are used to study the impact of substrate temperature and III/ $\mathrm{N}$ ratio. We have applied different methods of statistical analysis to reveal trends in indium distribution as a function of growth parameters. In contrast to spinodal decomposition, inhomogeneity of InAlN has a cylindrical symmetry as columnar Al-rich grains surrounded by an In-rich matrix. We present an evolution of the size of Al-rich grains in InAlN films grown at different growth temperatures and discuss the applicability of frequency distribution and autocorrelation function analyses on two-dimensional (2D) and three-dimensional (3D) APT data.
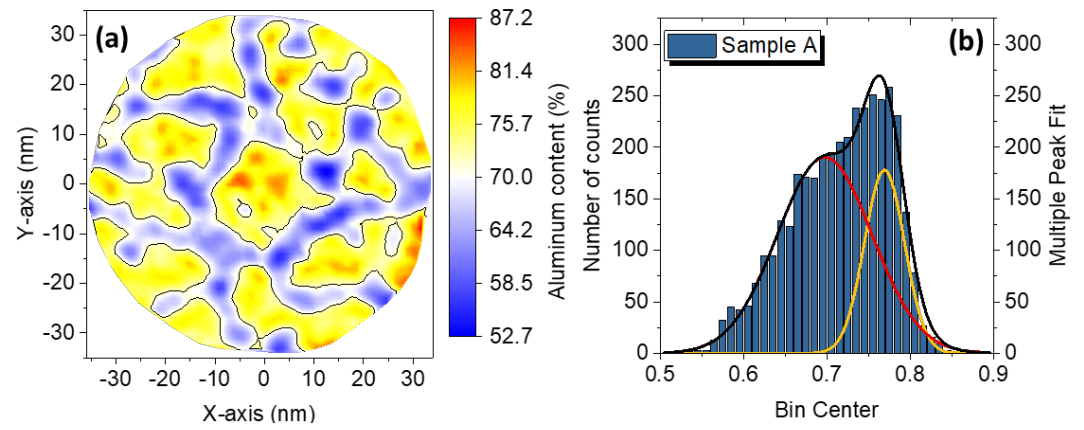

Figure 1 (a) 2D contour plot of Al content in InAlN film c-plane with a $5 \mathrm{~nm}$ thicknes. The contour line indicates the $\mathrm{Al}$ composition at the level of 72 at. \%. (b) Histogram of $\mathrm{Al}$ compositions found in the $2 \mathrm{D}$ contour plot with a double Gaussian shape.

\section{References:}

[1] M. Shirazi-HD et al., Dramatic enhancement of near-infrared intersubband absorption in c-plane AlInN/GaN superlattices, Applied Physics Letters 108, 121108 (2016)

[2] C. Edmunds et al., Comparative study of intersubband absorption in AlGaN/GaN and AlInN/GaN superlattices: Impact of material inhomogeneities, Physical Review B 88, 235306 (2013).

[3] APT was conducted at ORNL's CNMS, which is a U.S. DOE Office of Science User Facility. 


\title{
New Experimental Techniques for Nanoparticle Analysis
}

\author{
Peter Felfer $^{1}$, Kayla Marie Johnson ${ }^{1}$ and Jan Joosten ${ }^{1}$ \\ Corresponding Author: peter.felfer@,fau.de \\ 1. Department of Materials Science, Institute I, Friedrich-Alexander Universität Erlangen- \\ Nürnberg
}

In recent years, the analysis of nanomaterials such as nanoparticles and nanoporous structures has become a focus in the APT community [1,2], driving the need for improved experimental approaches to their analysis. This is stemming from the requirement of having a field emitter with a controlled geometry, able to withstand the stresses imposed on the tip by the high electric fields present. For the analysis of nanoparticles, this demands ways to fixate in a solid or on a tip [3]. If the particles are incorporated into a solid, the pores have to be filled with a material that can be distinguished from the material to analyze, yet has similar field evaporation properties.

In this talk, we will present new experimental ways to produce field emitters for APT analysis of nanoparticles. To enable the analysis of larger metallic nanoparticles, we present a replica method where particles are laid down on a substrate with limited adhesion to metals and coated with a metallic which is stripped from the template (fig. 1 a). This film is then the base for APT analysis of the particles. We will further show a method for the analysis of ceramic particles where atomic layer deposition is used to package them into a thin film.

To analyze very small nanoparticles, we are building a FIM capable of directly transferring particles from an aerosol onto a pre field-evaporated tip in ultra-high vacuum through a differentially pumped spray unit. This instrument will allow us to produce field emitters with single free-standing particles down to the single digit $\mathrm{nm}$ range on the tip apex for analysis.
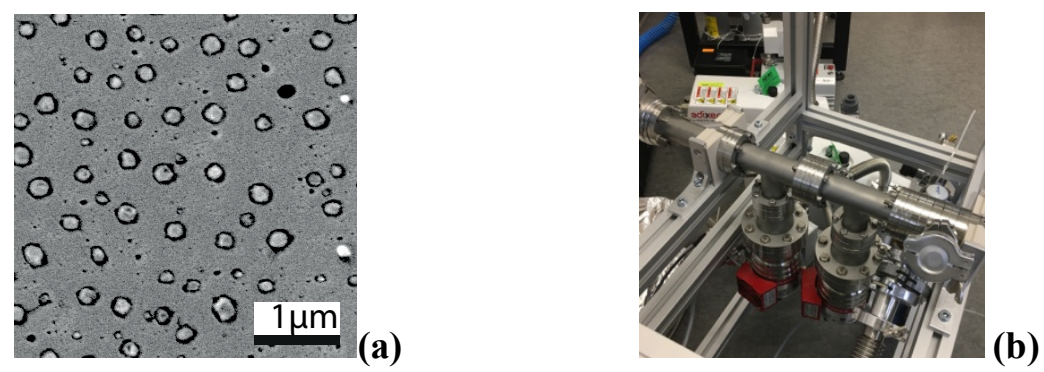

Figure 1 (a) template stripped metallic nanoparticles. (b) Aerosol transfer unit with four pumping stages to be attached to a field ion microscope.

References:

[1] K. Tedsree, et al., Hydrogen production from formic acid decomposition at room temperature using a Ag-Pd core-shell nanocatalyst, Nature Nanotechnology 6 (2011), 302-307.

[2] P. Felfer, et al., Revealing the Distribution of the Atoms within Individual Bimetallic Catalyst Nanoparticles, Angewandte Chemie International Edition 53 (2014), 11190 - 11193.

[3] P. Felfer, et al., New approaches to nanoparticle sample fabrication for atom probe tomography, Ultramicroscopy 159 (2015), 413-419.

[4] The authors would like to acknowledge funding from the German Science Foundation (DFG) 


\title{
New Approaches in Atom Probe Sample Preparation: STEM Imaging and Plasma FIB Milling
}

\author{
Yimeng Chen, Katherine P. Rice, Isabelle Martin and Ty J. Prosa
}

Corresponding Author: Yimeng.Chen@ametek.com

CAMECA Instruments, Inc., 5470 Nobel Drive, Madison, Wisconsin 53711, USA.

In this work, recent sample preparation approaches for atom probe tomography (APT) specimens are presented. Sample preparation is one of the most essential parts of a successful atom probe analysis. Accurately identifying and targeting a specific region of interest (ROI) is key for many atom probe applications. Scanning electron microscopy imaging becomes more challenging when the specimen is milled in the hundreds of nanometers range due the reduction of the electron interaction volume. At this size scale, materials are nearly transparent to $30 \mathrm{kV}$ electrons, which allows STEM imaging which helps to provide greater resolution to accurately target ROIs [1]. The setup of STEM imaging for APT specimens on microtip coupons will be presented and its benefits will be discussed.

A focused ion beam (FIB) based on a Xe plasma-ion-source has the advantage of much higher milling rates compared to conventional Ga-ion FIBs using a $\sim 50$ times higher beam current [2, 3]. In this work, we demonstrate the usage of plasma FIB for "in-place" sample preparation that mills the sample directly from the substrate without requiring liftout. [4]. Figure $1 \mathrm{~b}$ is an example of APT specimen created from a Si wafer substrate. Data quality and $\mathrm{Xe}^{+}$damage will be discussed. This approach is promising particularly for biological samples, where the liftout method is challenging to carry out on cryogenic samples.

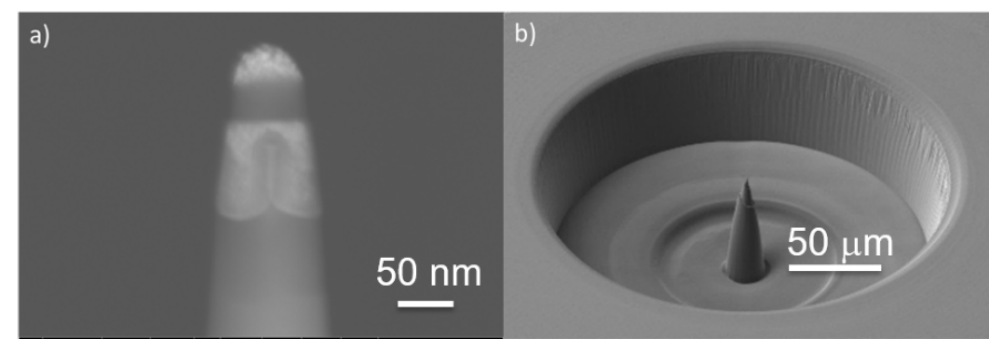

Figure 1 (a) HAADF-STEM image of an APT specimen from a device structure (b) "In-place" APT specimen prepared by plasma FIB.

References:

[1] Stevie, F. A., et al. "Application of focused ion beam lift-out specimen preparation to TEM, SEM, STEM, AES and SIMS analysis." Surface and interface analysis 31.5 (2001): 345-351.

[2] Estivill, Robert, et al. "Preparation and analysis of atom probe tips by xenon focused ion beam milling." Microscopy and Microanalysis 22.3 (2016): 576-582.

[3] Fisher, K., and E. Marquis. "Comparing Plasma-FIB and Ga-FIB Preparation of Atom Probe

Tomography Samples." Microscopy and Microanalysis 22.S3 (2016): 692-693.

[4] Miller, Michael K., and Kaye F. Russell. "Atom probe specimen preparation with a dual beam

SEM/FIB miller." Ultramicroscopy 107.9 (2007): 761-766. 


\section{Implementation of a Commercially Available UHV/Cryogenic Specimen Transfer System for LEAP Microscopes}

Robert M. Ulfig ${ }^{1}$, Peter H. Clifton ${ }^{1}$, Ty J. Prosa ${ }^{1}$, David A. Reinhard ${ }^{1}$, Jeffrey Shepard ${ }^{1}$, D. von Gunten $^{2}$, U. Maier ${ }^{2}$, and Sigrun A. Köster ${ }^{2}$

Corresponding Author: Robert.Ulfig@ametek.com

1. CAMECA Instruments, Inc., 5470 Nobel Drive, Madison, WI 53711 USA.

2. Ferrovac GMBH, Thurgauerstrasse 72, 8050 Zurich Switzerland.

For a wide variety of atom probe specimens, changes related to exposed surfaces, or the bulk temperature history have little or no effect on the goal of the analysis, e.g. characterization of grain chemistry [1]. For other applications a very carefully controlled environment (temperature, pressure, atmosphere, etc.) is critical, for example; rapid oxidizers (e.g. uranium, lithium), surface contamination (e.g. catalysts); characterization of hydrogen content in steels, semiconductors, etc., analysis of materials encased in vitreous ice (e.g. biological), transport between various analysis/treatments (e.g. TEM, FIB-SEM, reaction chambers).

The most challenging specimens are those that need to be kept at cryogenic temperatures after specimen preparation is complete. To date, only a few sites in the world have experimented with cryo transfer into an atom probe. Recently, teams at ETH, Oxford, Pacific Northwest National Laboratory, and the University of Michigan have built or adapted commercial UHV transfer systems with different capabilities [2,3]. Based on demonstrated success, and growing interest in applications that require this capability, a design was completed in a collaboration between the Max Plank Institute for Steel Research, CAMECA Instruments Inc., and Ferrovac GMBH.

The transfer design is based on the mobile UHV Suitcase from Ferrovac. It is customized to transfer samples held in a special LEAP cryo puck that rides on a special carrier puck that is precooled on the LEAP specimen stage (Figure 1). All parts are fully integrated to a modified LEAP 5000 system with specially designed hardware and software. Transfer can be completed in less than one minute, maintaining the sample in true UHV conditions and well below the recrystallization temperature of vitreous ice (near $130 \mathrm{~K}$ ) without contamination (Figure 1C).
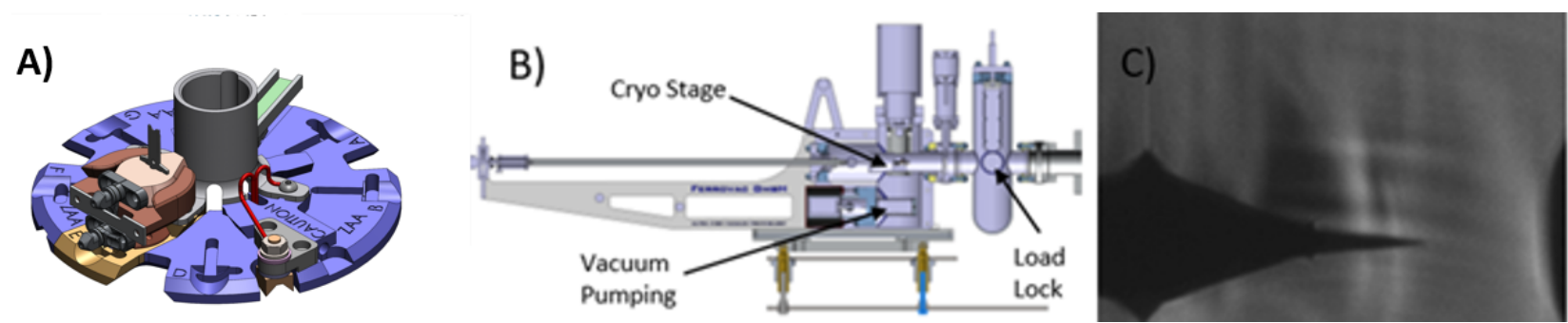

Figure 1 (a) The LEAP cryo puck, in a cryo carrier puck, in a cryo carousel. (b) The Ferrovac UHV-Cryo Suitecase. (c) A specimen after cryo transfer with no visible condensation/contamination.

\section{References:}

[1] D.J. Larson et al., "Local Electrode Atom Probe Tomography", Springer, New York, 2013.

[2] S. Gerstl and R., Wepf, Cryogenic Samples for APT, Microscopy and Microanalysis 21 (S3), (2015).

[3] D.E. Perea et al., An environmental transfer, Advanced Structural Chemical and Imaging 6, (2016). 


\section{Crystallographic Ordering of Ti-Al Alloys in the Presence of $\beta$-Stabilisers and Interstitial Oxygen}

Felicity F. Dear ${ }^{1}$, Paul A.J. Bagot ${ }^{2}$, Hazel Gardner ${ }^{2}$, Anna Radecka ${ }^{3}$, Michael Moody ${ }^{2}$, David Rugg $^{3}$, and David Dye ${ }^{1}$

Corresponding Author: ffd15@ic.ac.uk

1. Department of Materials, Imperial College London, London, U.K.

2. Department of Materials, University of Oxford, Oxford, U.K.

3. Rolls-Royce plc, Derby, U.K.

Mechanistic understanding of plasticity and fatigue in titanium alloys remains incomplete, limiting the management of aero engines to conservative predictions of safe component service times. Crack initiation in high-cycle and cold dwell fatigue modes has been linked to localisation of slip within grains and the resulting high micromechanical stresses [1,2]. This inhomogeneous plasticity is exacerbated by crystallographic ordering of aluminium in the hcp $\alpha$ phase of typical $\alpha+\beta$ alloys, which leads to precipitation of intermetallic $\alpha_{2}\left(\mathrm{Ti}_{3} \mathrm{Al}\right)$ domains [3]. Previous observations have indicated that the kinetics of this phase separation are affected by tertiary elements, such as dilute $\beta$-stabilising vanadium [4]. In this work, an ageing study of a model alloy series is being investigated to better quantify the effects of vanadium, molybdenum and oxygen on $\alpha_{2}$ formation.

Alloys based on Ti-7Al (wt.\%) and containing dilute quantities of vanadium or molybdenum, as well as differing levels of interstitial oxygen, were produced and aged at $550{ }^{\circ} \mathrm{C}$ to promote crystallographic ordering across a 120-day period. Characterisation by dark field TEM has identified trends in growth kinetics, and atom probe tomography provides complementary data to ascertain local compositional variations associated with phase separation. Crystal orientation mapping by EBSD was used to select specific zone axes within microtips. Analysis has revealed the phase segregation of alloying elements, and crystallographic site partitioning of vanadium and molybdenum on the $\alpha_{2}$ lattice.

\section{$\underline{\text { References: }}$}

[1] A.L. Pilchak, Fatigue crack growth rates in alpha titanium: faceted vs. striation growth, Scripta Mater., 68 (2013) 277-280.

[2] F.P.E. Dunne, A. Walker and D. Rugg, A systematic study of hep crystal orientation and morphology effects in polycrystal deformation and fatigue, Proc. R. Soc. A, 463 (2007) 1467-1489.

[3] J.C. Williams, A.W. Sommer and P.P. Tung, The influence of oxygen concentration on the internal stress and dislocation arrangements in $\alpha$ titanium, Metall. Mater. Trans. B, 3 (1972) 2979-2984.

[4] A. Radecka et al., The formation of ordered clusters in Ti-7Al and Ti-6Al-4V, Acta Mater., 112 (2016) 141-149. 


\title{
The Determining Role of $\mathrm{Cu}$ on Precipitation Evolution and Mechanical Properties in Fe-CuNiAIMn Alloy
}

\author{
Qin Shen ${ }^{1}$, Zemin Wang ${ }^{1,2}$, Hui Li1, Wenqing Liu ${ }^{1}$ \\ Corresponding Author: wqliu@staff.shu.edu.cn \\ 1. Institute of Materials, School of Materials Science and Engineering, Shanghai University, \\ Shanghai, China. \\ 2. School of Materials Science and Engineering, Shanghai Institute of Technology, Shanghai, \\ China.
}

The precipitation evolution and mechanical properties in the Fe-0.64CuNiAlMn and Fe1.57CuNiAlMn alloy have been investigated. The precipitation behavior was studied by atom probe tomography (APT). The results showed that content of $\mathrm{Cu}$ had significant influence on the precipitation behavior of $\mathrm{Cu}$-rich and $\mathrm{NiAl}$ nanoparticles such as precipitate size, number density, and distribution. At the early ageing time $(0.1 \mathrm{~h})$, increasing the $\mathrm{Cu}$ content could promote the segregation of $\mathrm{Cu}, \mathrm{Ni}, \mathrm{Al}$ and $\mathrm{Mn}$ in the matrix. Furthermore, $\mathrm{Cu}$ effectively increased precipitate density by increasing the nucleation rate of $\mathrm{Cu}$-rich and $\mathrm{NiAl}$ precipitation, thereby enhancing the hardness and strength of alloy and accelerating the whole process of precipitation hardening. The precipitation sequence of the Fe-0.64CuNiAlMn alloy can be expressed as supersaturated solid solution $\rightarrow \mathrm{NiAl} \rightarrow \mathrm{NiAl}+\mathrm{Cu}$-rich, which is significantly different from the Fe-1.57CuNiAlMn alloy, involving the process of supersaturated solid solution $\rightarrow \mathrm{Cu}$-rich $\rightarrow \mathrm{Cu}$-rich $+\mathrm{NiAl}$. The precipitation mechanisms of $\mathrm{Cu}$-rich/NiAl coprecipitates in two alloys were different, depending on the $\mathrm{Cu} / \mathrm{Ni}$ and $\mathrm{Cu} / \mathrm{Al}$ ratio. 


\title{
Concurrent $(\mathrm{Fe}, \mathrm{Mo})_{2} \mathrm{C}$ Carbides and $\mathrm{Cu}-$ rich Clusters Precipitation in Ferritic Steel Containing Copper During Aging
}

\author{
$\mathrm{Na} \mathrm{Li}^{1}$, Wei Wang ${ }^{1}$, Shijuan Zhu ${ }^{1}$, Bo Wang ${ }^{1}$, and Ziying Zhang ${ }^{1}$ \\ Corresponding Author: wangwei200173@sina.com \\ 1. School of Material Engineering, Shanghai University of Engineering Science, Shanghai \\ 201620 China
}

The concurrent $(\mathrm{Fe}, \mathrm{Mo})_{2} \mathrm{C}$ carbides and $\mathrm{Cu}$-rich clusters precipitation in the ferritic steel containing copper after aging at $400^{\circ} \mathrm{C}$ for $4000 \mathrm{~h}$ have been investigated using transmission electron microscopy (TEM) and atom probe tomography (APT). The results show that spinodal decomposition and an atomic ordering reaction occur in the ferritic steel containing copper. After aging, the retained austenite decomposed by the spinodal mechanism into C-rich and C-lean regions. The $\mathrm{C}$-rich phase transforms into the $(\mathrm{Fe}, \mathrm{Mo})_{2} \mathrm{C}$ carbides via the ordering reaction, while $\mathrm{Cu}$-rich clusters formed in the $\mathrm{C}$-lean regions. The experimental results can be interpreted on the basis of phase separation via spinodal decomposition followed by chemical ordering. The small size of $\mathrm{Cu}$-rich clusters can be attributed to the lower diffusion coefficient of $\mathrm{Cu}$ in the retained austenite.

(a)

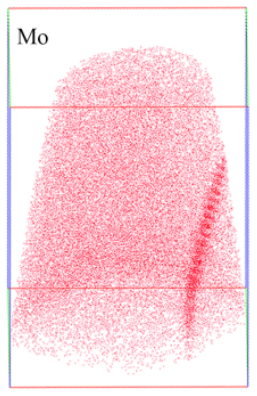

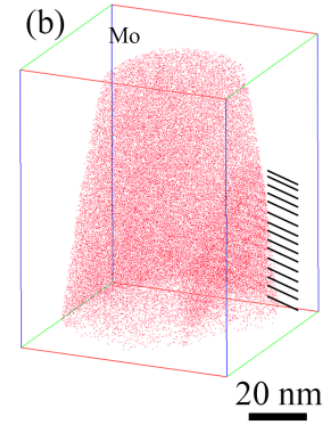

Figure 1 (a) APT map showing the top distribution of molybdenum at a bainitic ferrite boundary for Steel-A aged for $4000 \mathrm{~h}$ at $400^{\circ} \mathrm{C}$; (b) Side view of the bainitic ferrite boundary lateral in (a).

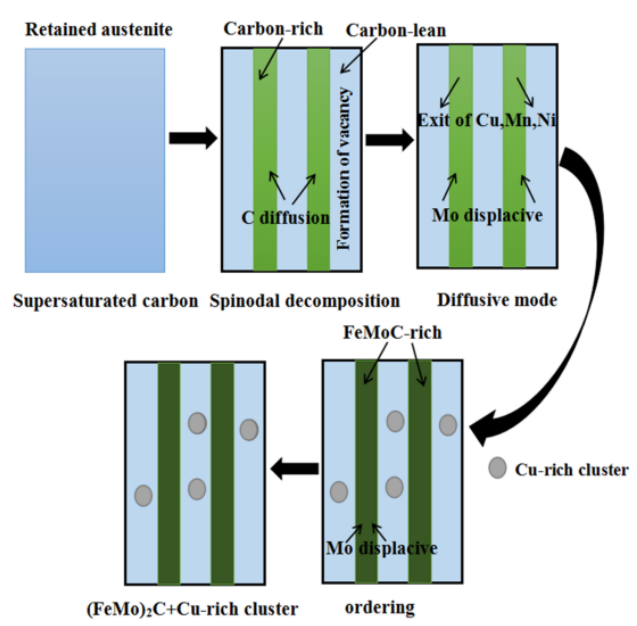

Figure 2 Schematic showing transformation process during aging.

\section{$\underline{\text { References: }}$}

[1] S Pecko, M Petriska, S Sojak and V Slugen. Positron annihilation lifetime study of JRQ and JPA irradiated and annealed reactor pressure vessel steels. Prog Nucl Energ 2016;87:15-19.

[2] A Wagner, et al., Effect of neutron flux on the characteristics of irradiation-induced nanofeatures and hardening in pressure vessel steels. Acta Mater 2016;104:131-42. 


\title{
Quantification of Spinodal Decomposition and G-Phase Precipitation Contributions on Micro-Hardening of Ferrite of Aged Duplex Stainless Steel Owing to Microstructural Characterization Using APT Characterization
}

\author{
R. Badyka ${ }^{1}$, C. Pareige ${ }^{1}$, C. Domain ${ }^{2}$, G. Monnet $^{2}$ and S. Saillet ${ }^{2}$ \\ Corresponding Author: cristelle.pareige@univ-rouen.fr \\ 1. Groupe de Physique des Matériaux, UMR 6634 CNRS, Université de Rouen Normandie et \\ INSA de Rouen Normandie, France \\ 2. EDF R\&D Département Matériaux et Mécanique des Composants, Avenue des Renardières - \\ Ecuelles, F-77818 Moret sur Loing, France.
}

Duplex stainless steels used in primary circuit of $2^{\text {nd }}$ generation nuclear power plant endure thermal ageing at service temperatures $\left(285^{\circ} \mathrm{C}-323^{\circ} \mathrm{C}\right)$. At these temperatures, ageing of Duplex stainless steels (DSS) is attributed to ferrite decomposition via spinodal decomposition into regions enriched in iron $(\alpha)$ and regions enriched in chromium $\left(\alpha^{\prime}\right)$ and precipitation of G-phase particles enriched in $\mathrm{Ni}, \mathrm{Si}, \mathrm{Mn}$ and Mo when present. This leads to an increase in hardness and a decrease in Charpy impact toughness. Whereas this has been known for long, the contribution of both spinodal decomposition and of G-phase precipitation on hardening of the ferrite is still not evaluated. If everybody in the literature agrees on the fact that spinodal decomposition is the main contributor, disagreements exist on the role of G-phase particles: some authors show a minor role of G-phase particles whereas others claim the inverse [1,2]. The aim of this paper is to quantify the relative contribution of spinodal decomposition and G-phase particles on hardness.

This work links microstructural characterization performed using atom probe tomography to micro-hardness of the ferrite for a wide variety of duplex steels (from cast steels with and without Mo to lean steels) aged under different conditions. Owing to the combination of the hardening model developed by Ardell [2] for spinodal decomposition and the one of Monnet [4] for G-phase precipitation, we quantify for the first time the contribution of both mechanism of phase separation on hardening of the ferrite in austeno-ferritic steels. The model developed works whatever the occurrence or not of one of the two phase transformation mechanisms. The micro-hardness increases obtained with the model give an excellent estimation of the experimental values. Owing to this work, the apparent contradiction reported in the literature on the role of the G-phase is clarified and explained. And very importantly, this work shows that conversely to what has been said in the literature for long, G-phase precipitation is the main contributor to hardening in Mo-bearing and Mo-free cast duplex stainless steels at the beginning of the kinetics. Spinodal decomposition becomes the main contributor only at long ageing time.

\section{$\underline{\text { References: }}$}

[1] F. Danoix, et al. , Atom probe and transmission electron microscopy study of reverted duplex stainless steels, Appl. Surf. Sci. 67 (1993) 348-355.

[2] H.M. Chung and O.K. Chopra, Kinetics and Mechanism of Thermal Aging Embrittlement of Duplex Stainless Steels, Argonne National Lab., IL (USA), 1987.

[3] A.J. Ardell, Precipitation hardening, Metall. Trans. A. 16 (1985) 2131-2165.

[4] G. Monnet, Multiscale modeling of precipitation hardening: Application to the $\mathrm{Fe}-\mathrm{Cr}$ alloys, Acta Mater. 95 (2015) 302-311. 


\section{Effects of Ni Addition on the Grain Boundary Strengthening of High Mn Steel Weldments}

Seon-Hyeong $\mathrm{Na}^{1}$, Jae-Bok $\mathrm{Seol}^{2}$ \& Chan-Gyung Park ${ }^{1,2}$

Corresponding Author: cgpark@postech.ac.kr

1. Department of Materials Science and Engineering, POSTECH, Pohang 37673, South Korea.

2. National Institute for Nanomaterials Technology, POSTECH, Pohang 37673, South Korea.

High Mn steels are currently one of the most attractive materials for structural application due to its unique properties1. High Mn steels with a single phase of austenite exhibit both high strength and high toughness due to the twinning induced plasticity, where Mn believed to increase the stability of austenite and stacking fault energy. Although high Mn steels draw an attracting attention because of their excellent properties, however, there are many issues to be solved in the welding process. Especially, improvement of the fracture toughness of the welded zone are needed to be improved significantly.

Most impressive way to improve the toughness is to add $\mathrm{Ni}$ as an austenite stabilizer. It has been reported that $\mathrm{Ni}$ addition can increase significantly the toughness of weldments 2 . However, the cause remains unknown. In the present study, the microstructure of $5 \mathrm{wt} \% \mathrm{Ni}$ added weldment samples were analyzed to understand the effects of Ni addition on the toughness. The direct observation of fracture surface using transmission electron microscopy (TEM) and transmitted Kikuchi diffraction (TKD) found that the deformation behaviors were changed when Ni was added. In addition, atom probe tomography (APT) revealed different segregation phenomenon of the alloying element such as $\mathrm{C}, \mathrm{Mn}$ and Mo in the welded zone. The segregated alloying elements changed the stability of austenite phases and properties of grain boundaries by forming $\mathrm{Mn}$ rich-region. The segregation phenomenon at the welded zone which are related to the grain boundary strengthening will be discussed to understand the improvement of toughness due to $\mathrm{Ni}$ addition.

\section{$\underline{\text { References: }}$}

[1] O. Bouaziz, S. Allain, C.P. Scott, P. Cugy, D. Barbier, High manganese austenitic twinning induced plasticity steels: A review of the microstructure properties relationships, Curr. Opin. Solid State Mater. Sci. 15 (2011) 141.

[2] IW. Han, BK. Lee, JK. Choi, SH. Park and CY. Kang, Microstructure and Mechanical Properties of Cryogenic High-manganese Steel Weld Metal, The Proceedings of 2013 International Offshore and Polar Engineering Conference, International society of offshore and polar engineers, California (2013), pp. 348 


\title{
APT Analysis of the Passive Film Formed on Austenitic Stainless Steels
}

\author{
Jong-Chan Han ${ }^{1}$, Jae-Bok Seol ${ }^{2}$, Shiun Ling ${ }^{3}$, Hyun-Jo Jun ${ }^{3}$, and Chan-Gyung Park $\frac{1,2}{2}$ \\ Corresponding Author: cgpark@postech.ac.kr \\ 1. Department of Materials Science and Engineering, POSTECH, Pohang, South Korea \\ 2. National Institute for Nanotechnology (NINT), POSTECH, Pohang, South Korea \\ 3. ExxonMobil Corporate Strategic Research, Annandale, New jersey, USA
}

Stainless steels (SS) are excellent corrosion resistant materials due to the thin passive film formed in various corrosive environment. Many studies have focused on the corrosion resistance of the passive film depending on the alloying elements, microstructures, and surrounding environments by an electrochemical approach. Since the properties are directly related to the constituents of the passive film, various chemical characterization of passive film has been tried by using AES, XPS, STEM-EDS and STEM-EELS [1,2]. However, the elemental and spatial resolution of the above techniques is not sufficient for the detailed quantitative analysis. For this reason, previous analyses have been difficult to provide the direct evidence about the positive effects of Mo on the corrosion resistance. Also, there have been a lack of research on the effect of $\mathrm{Ni}$, generally used to stabilize austenite phase, on the corrosion resistance.

The present study suggests the utilization of APT to get the direct evidence of the effects of Mo and Ni by characterizing the passive films in atomic scale. The passive films of commercial Niadded SS304L (0.3 wt.\% Mo), and SS316L (2.1 wt.\% Mo) have been analyzed to reveal the effects. The present results exhibited that Mo were present as Mo atoms or Mo-N pairs in steel matrix, which could affect the passive film in the form of dense $\mathrm{MoO}_{2}$ molecular components. This phenomenon was more prominent in SS316L than in SS304L, with the better corrosion resistance of SS316L. Additional focusing on Ni proved that Ni affected the initial formation of the passive film by segregation on the solution-exposed steel surface, which aided the formation of many stable $\mathrm{NiO}$ molecular components near the interface between steel and passive film.

\section{References:}

[1] C.O.A. Olsson, The influence of nitrogen and molybdenum on passive films formed on the austenoferritic stainless steel 2205 studied by AES and XPS, Corr. Sci. 37 (1995) 467-479.

[2] K.N. Oh, S.H. Ahn, K.S. Eom, K.M. Jung and H.S. Kwon, Observation of passive films on Fe-20Cr$x \mathrm{Ni}(x=0,10,20$ wt.\%) alloys using TEM and Cs-corrected STEM-EELS, Corr. Sci. 79 (2014) 34-40. 


\section{Characterizing Solute Hydrogen and Hydrides in Pure and Alloyed Titanium at the Atomic Scale}

Yanhong Chang ${ }^{1}$, Andrew J. Breen ${ }^{1}$, Zahra Tarzimoghadam ${ }^{1}$, Leigh Stephenson ${ }^{1}$, Wenjun Lu ${ }^{1}$, Dirk Ponge $^{1}$, Dierk Raabe ${ }^{1}$, Baptiste Gault ${ }^{1}$

Corresponding Author: b.gault@mpie.de

1. Max Planck Institute für Eisenforschung, Düsseldorf, Germany.

Titanium has a high affinity for hydrogen and is a typical hydride former. Ti-hydride are brittle phases which are likely involved in the premature failure of some Ti-alloys. However, the behavior of $\mathrm{H}$ within materials remains elusive because of the experimental challenge associated to direct imaging of its distribution at near-atomic scale. Here, by using atom probe tomography combined with transmission electron microscopy, we imaged $\mathrm{H}$ as solute in bcc $\beta$-Ti, hydrides and segregated to interfaces in a set of Ti-alloys. The $\mathrm{H}$ is likely introduced during specimen preparation by focused-ion beam. We show that $\mathrm{H}$ composition locally reaches up to approx. 50 at.\% in Ti-hydrides, even though the bulk average $\mathrm{H}$ level was less than 0.1 at.\%. The hydrides were mainly observed to have grown from $\alpha$ grain boundaries and $\alpha / \beta$ interfaces. In cases where no hydrides were imaged, enrichments of up to 30 at.\% $\mathrm{H}$ were observed at interfaces. Besides, the higher $\mathrm{H}$ solubility in $\beta$-Ti than in $\alpha$-Ti was clearly revealed. Direct observation of $\mathrm{H}$ opens opportunities to further the understanding of $\mathrm{H}$ embrittlement mechanisms within materials.
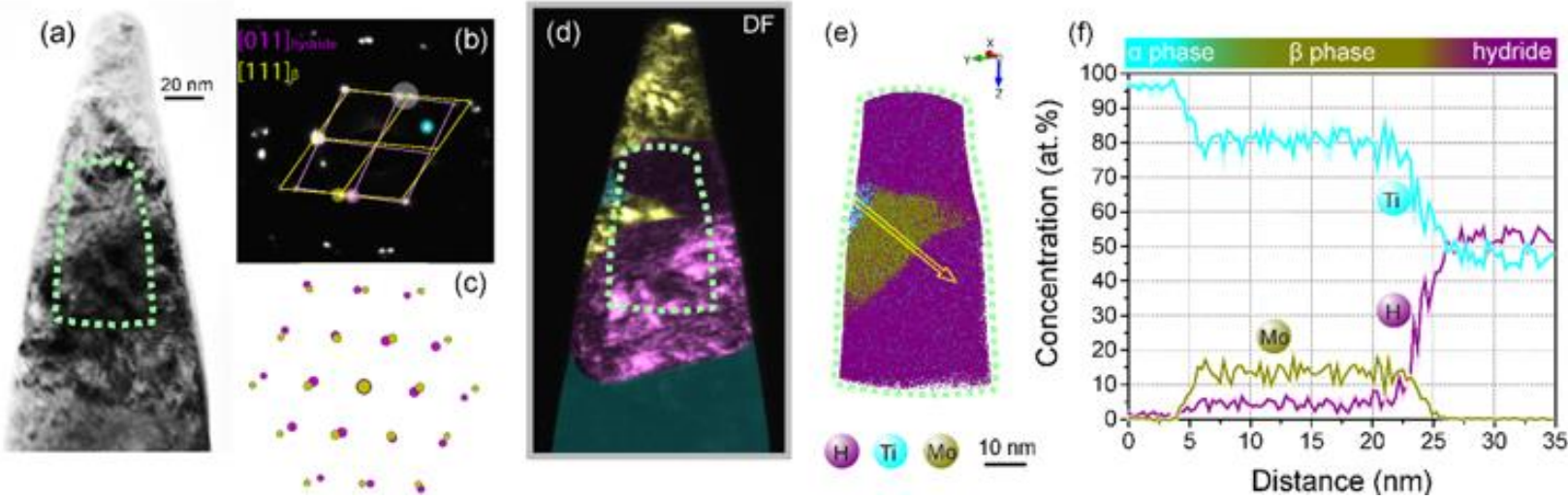

Figure 1: (a) bright-field TEM image of an APT tip from the annealed Ti - 4 wt.\% Mo alloy, (b) selected area diffraction pattern (SADP) indexed as $[\overline{1} 11]_{\beta} / /[011]_{\text {hydride. }}$ (c) Simulated diffraction patterns along the bcc- $\beta$ [111] zone axis parallel to the hydride [011] zone axis. (d) dark-field (DF)-TEM micrographs, where hydride is colored in purple, $\beta$ in yellow and $\alpha$ in cyan. (e) Correlative APT analysis and (f) 1D compositional profile along the yellow arrow in (e) where the errors, represented as line shading, corresponds to the $2 \sigma$ counting errors in each bin of the profile. 


\section{High-Detection-Efficiency APT Analyses of 100 at. ppm Ce Distribution in Heat-Resistant 18-8 Stainless Steel}

Masaya Kozuka ${ }^{1}$, Yasuhiro Aruga ${ }^{1, \#}$, Shigenobu Nanba ${ }^{1}$, Yimeng Chen ${ }^{2}$, Katherine P. Rice ${ }^{2}$ and Ty Prosa ${ }^{2}$

\# Corresponding Author: aruga.yasuhiro@kobelco.com

${ }^{1}$ Materials Research Laboratory, Kobe Steel, Ltd., 1-5-5 Takatsukadai, Nishi-ku, Kobe, Hyogo, 651-2271, JAPAN

${ }^{2}$ CAMECA Instruments, Inc., 5470 Nobel Dr, Madison, WI, 53711, USA

One of the required properties for a heat-resistant 18-8 stainless steel used as boiler tubes in ultrasupercritical (USC) pressure coal-fired boilers is a resistance against exfoliation of an oxidized scale which is formed on inner surface of the boiler tubes to protect a matrix of the boiler tubes against steam oxidation. It is commonly accepted that the resistance against exfoliation of the scale can be improved by adding a small amount of rare-earth elements [1], and recently, Miyamura et al. revealed that doping of 100 at. ppm Ce to the steel suppresses the exfoliation most drastically [2]. However, its mechanism has not established yet due to lack of analytical tool which can be applied to detect such a small amount of Ce concentration and its distribution in Fe-based materials. In this work, we investigated the Ce distribution at the scale / matrix interfaces of an oxidized 18-8 stainless steel with a Ce concentration of $100 \mathrm{ppm}$ by means of conventional FE-EPMA and high-detection-efficiency atom probe tomography (APT; LEAP 5000XS, AMETEK). A $\mu \mathrm{m}$-scale analysis by the FE-EPMA (Figure 1) showed that layered Cr-rich regions spread across the scale / matrix interfaces, meaning a partial formation of a layered $\mathrm{Cr}_{2} \mathrm{O}_{3}$ phase (Cr-rich oxide). It was also observed that there are Fe-rich oxides across the interface, but no Ce signal was detectable due to its low concentration. On the other hand, the high-detection-efficiency APT successfully mapped nanometer-sized Ce-rich region next to the Fe-rich oxide (Figure 2). These results indicate that the doped $100 \mathrm{ppm}$ Ce forms nanometer-sized oxide on the Fe-rich oxide edge after the oxidization treatment. Thus, it is concluded that the high-detection-efficiency APT is a promising analytical tool for detecting 100 ppm Ce distribution in Fe-based materials.

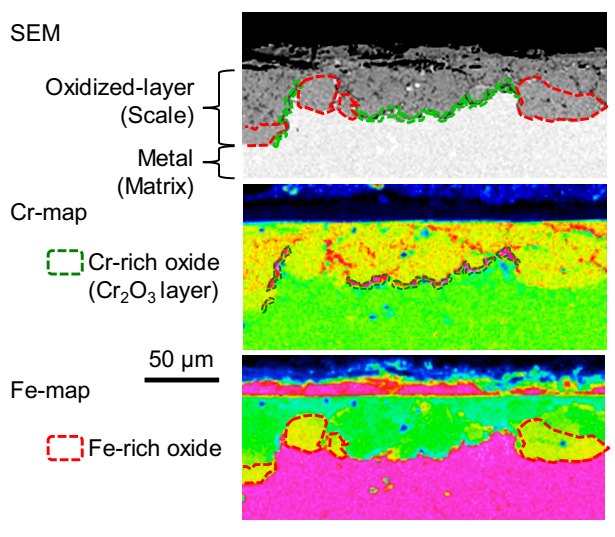

Figure 1 FE-EPMA analyses results.
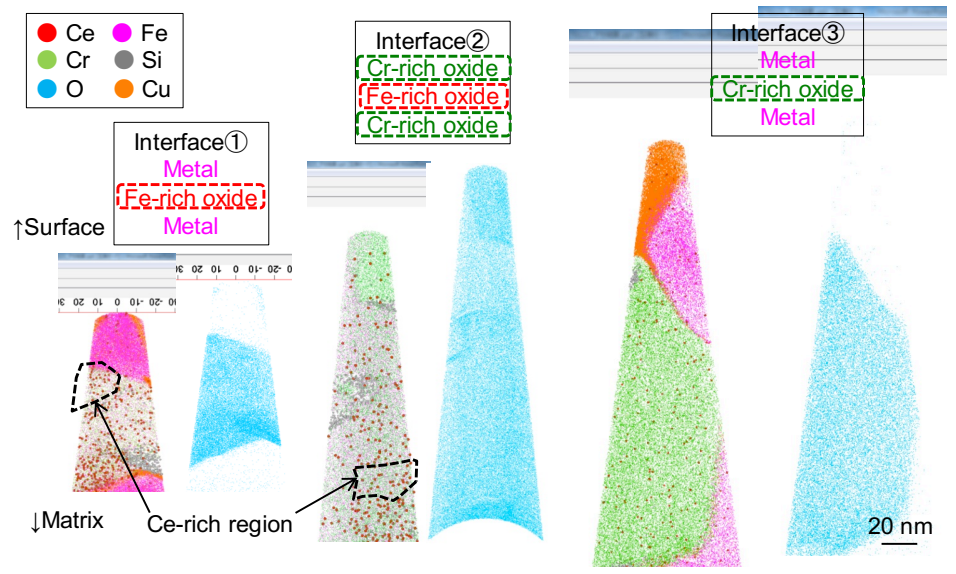

Figure 2 High-detection-efficiency APT analyses results.

\section{References:}

[1] D. P. Whittle and J. Stringer, Phil. Trans. R. Soc. Lond. A 295 (1980), 309-329.

[2] T. Miyamura and S. Nanba, KOBE STEEL ENGINEERING REPORTS 64 (2014), 62-65. 


\section{The Effect of Ni and Mn Content on Precipitation in Ion Irradiated Split Melt Test Steels}

J. Zelenty ${ }^{1}$, N. Almirall ${ }^{2}$, P. Wells ${ }^{2}$, G.R. Odette ${ }^{2}$, J. Douglas ${ }^{1}$, J.M. Hyde ${ }^{1,3}$, M. Moody ${ }^{1}$, G.D.W. Smith $^{1}$, N. Riddle ${ }^{4}$, K. Wilford ${ }^{4}$

Corresponding Author: jonathan.hyde@ materials.ox.ac.uk

${ }^{1 .}$ Department of Materials, University of Oxford, OX1 3PH U.K.

2. Mechanical Engineering Department University of California Santa Barbara, USA

${ }^{3 .}$ National Nuclear Laboratory, Culham, Abingdon, Oxon OX14 3EA, U.K.

4. Rolls-Royce, PO Box 2000, Derby, DE21 7XX, U.K.

A series of split melt test steels with a range of $\mathrm{Ni}$ and $\mathrm{Mn}$ contents (Table 1) have been ionirradiated at $290^{\circ} \mathrm{C}$ with $6.4 \mathrm{MeV} \mathrm{Fe}^{3+}$ ions at DUET in Kyoto, Japan. Atom probe tomography samples were made via focused ion beam (FIB) lift-out from material located 500nm below the surface to avoid regions of high Fe implantation whilst ensuring a sufficiently high dose ( 4dpa) for significant microstructural development to have occurred. APT analyses performed at UCSB of the resulting microstructures showed precipitation of solute clusters containing $\mathrm{Mn}, \mathrm{Ni}$ and $\mathrm{Si}$ in each material condition. The bulk Mn content was found to directly impact the observed $\mathrm{Mn}$ content in the clusters and, in the high Mn steels, the smaller irradiation-induced precipitates were found to contain higher Mn contents than larger precipitates.

In each irradiated sample, a broad range of cluster sizes was observed, characterised by a high number density of very small clusters and, in some cases, evidence of a bimodal distribution which would be consistent with clusters dissolving due to bombardment during irradiation and subsequently re-precipitating to form new, smaller clusters.

Table 1. Compositions (at.\%) of split melt test steels studied.

\begin{tabular}{|l|c|c|c|c|c|c|c|c|}
\hline Sample & $\mathrm{Cu}$ & $\mathrm{Si}$ & $\mathrm{Ni}$ & $\mathrm{Mn}$ & $\mathrm{P}$ & $\mathrm{Cr}$ & $\mathrm{Mo}$ & $\mathrm{C}$ \\
\hline $\mathrm{K}$ & 0.04 & 0.39 & 3.31 & 0.25 & 0.01 & 0.11 & 0.29 & 1.15 \\
\hline $\mathrm{C}$ & 0.04 & 0.40 & 3.31 & 2.02 & 0.01 & 0.11 & 0.29 & 0.92 \\
\hline $\mathrm{G}$ & 0.04 & 0.40 & 1.61 & 2.02 & 0.01 & 0.11 & 0.29 & 0.92 \\
\hline $\mathrm{A}$ & 0.04 & 0.39 & 1.60 & 0.25 & 0.01 & 0.11 & 0.29 & 1.15 \\
\hline $\mathrm{H}$ & 0.04 & 0.39 & 0.19 & 2.02 & 0.01 & 0.11 & 0.29 & 0.92 \\
\hline
\end{tabular}




\title{
Cluster Analysis of a Cast Aluminum Alloy Utilizing an Atom-Probe Tomography
}

\author{
Kyo Jin Hwang ${ }^{1}$, Yimeng Chen ${ }^{2}$, and Yoon-Jun Kim ${ }^{1}$ \\ Corresponding Author: yoonjun@inha.ac.kr \\ 1. Department of Materials Science and Engineering, Inha University, Incheon, Korea \\ 2. Ametek-Cameca Instrument Inc., Madison, USA
}

An A356 cast aluminum alloy ( $\mathrm{Al}-7 \mathrm{wt} . \% \mathrm{Si}-0.3 \mathrm{wt} . \% \mathrm{Mg}$ ), containing approximately 50\% eutectic microstructure. Such a high content of Si with various casting conditions plays a significant role in strengthening of A356 alloy by controlling eutectic morphology and precipitate other intermetallic compounds. Understanding the way to form Si nanoclusters soluble in the $\alpha$-matrix is necessary in order to produce fine eutectic morphology that provides high strength and fatigue resistance to the A356 alloys. Quantification of nanoclusters' size, distribution, and composition gives insight into how one type of atom in the aluminum matrix migrate and attract or repel other types of alloying element atoms. Typical size of nanoclusters is usually smaller than $1 \mathrm{~nm}$ which makes characterization difficult. The threedimensional (3-D) chemical and spatial information can be provided employing an atom probe tomography (APT) which makes it an invaluable analytical method to investigate in a nano- or sub-nano scale.

Nanotips for APT analyses were electropolished applying a DC 30 Volts with an electrolyte containing $10 \%$ perchloric acid in ethanol. Thereafter, due to difficulty in etching high $\mathrm{Si}$ in $\mathrm{Al}$ matrix, a final tip sharpening stage was added utilizing a focused ion beam (FIB, FEI Helios) milling. The final FIB milling was carried out at 2-5 $\mathrm{kV}$. The APT analysis was carried out using the Cameca EIKOS using 0.15 voltage pulse fraction at the specimen base temperature of $50 \mathrm{~K}$. The nanoclusters in the reconstructed nanotips were also identified using the cluster analysis module equipped with the IVAS software package. We found total

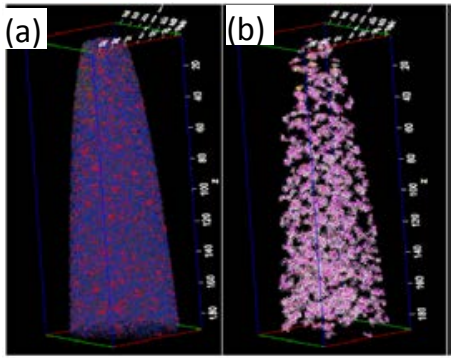

Figure 1 (a) 3-D reconstruction of an A356 alloy ( $\mathrm{Al}$ in blue, $\mathrm{Mg}$ in red, and $\mathrm{Si}$ in gray). (b) Al-Mg-Si nano clusters. $513 \mathrm{Mg}_{2}$ Si clusters in in a 70 x 70 x 190 $\mathrm{nm}^{3}$ dataset of $\alpha$-Al Matrix as shown in Fig. 1 and those average composition is listed in Table 1.

Table 1. Average composition of Al-Mg-Si nanoclusters (unit: at.\%).

\begin{tabular}{lllll}
\hline Si & Mg & O & Ga & Al \\
\hline 63.74 & 34.08 & 1.54 & 0.64 & Bal. \\
\hline
\end{tabular}

\section{References:}

[1] J. Manickaraj, et al., Effect of Incubation Coupled with Artificial Aging in T6 Heat Treatment of A356.2 Aluminum Casting Alloy, International Journal of Metalcasting 5(4) (2011) 17-36.

[2] M. Abdulwahab, et al., Effects of multiple-step thermal ageing treatment on the hardness characteristics of A356.0-type Al-Si-Mg alloy, Mater Design 32(3) (2011) 1159-1166.

[3] M.K. Miller, et al., Review of atom probe FIB-Based specimen preparation methods, Microsc Microanal 13(6) (2007) 428-436.

[4] J.M. Hyde, et al., A sensitivity analysis of the maximum separation method for the characterisation of solute clusters, Ultramicroscopy 111(6) (2011) 440-447.

[5] This research was supported by The Leading Human Resource Training Program of Regional Neo industry through the National Research Foundation of Korea (NRF) funded by the Ministry of Science, ICT and future Planning (NRF-2016H1D5A1910901) 


\title{
Effect of Pre-Aging on the Bake Hardening Response of Al-Mg-Si-Cu-Zn
} Alloy

\author{
M.X. Guo ${ }^{1}$, G.J. Li ${ }^{1}$, Y.D. Zhang ${ }^{2}$, G. Sha ${ }^{2}$, J.S. Zhang ${ }^{1}$, L.Z. Zhuang ${ }^{1}$
}

Corresponding Author: mingxingguo@skl.ustb.edu.cn, gang.sha@,njust.edu.cn

1. State Key Laboratory for Advanced Metals and Materials, University of Science and Technology Beijing, Beijing, 100083, PR China.

2. School of Aerospace, Mechanical \& Mechatronic Engineering, The University of Sydney, NSW 2006, Australia.

To reduce the weight of car body, Al-Mg-Si-Cu alloys have been widely used to produce outer body panels of automobiles due to their favorable high strength-to-weight ratio, corrosion resistance and good formability [1-4]. However, their bake hardening responses still need to be further improved to enhance their dent resistance. In this work, the excellent bake hardening response can be achieved in the Al-Mg-Si-Cu-Zn alloys by the optimized pre-aging treatment. The precipitation behaviors and strengthening of natural aged (T4) and pre-aged (T4P) alloys were investigated by differential scanning calorimetry (DSC), mechanical property measurement, three-dimensional atom probe (3DAP) analysis and theoretical modeling. It is shown that, the pre-aging give a significant effect on the low temperature precipitation process, GP zone dissolution and $\beta^{\prime \prime}$ precipitation, which is further verified by the activation energy of $\beta^{\prime \prime}$ precipitation calculated by a modified Avrami-Johnson-Mehl method. The theoretical models for prediction of precipitation kinetics have been developed in this paper. The improved bake hardening $(\mathrm{BH})$ response of the alloy after pre-aging treatment is mainly attributed to the formation of stable co-clusters, which can serve as nucleus of precipitates or easily transform into precipitates during paint baking treatment.
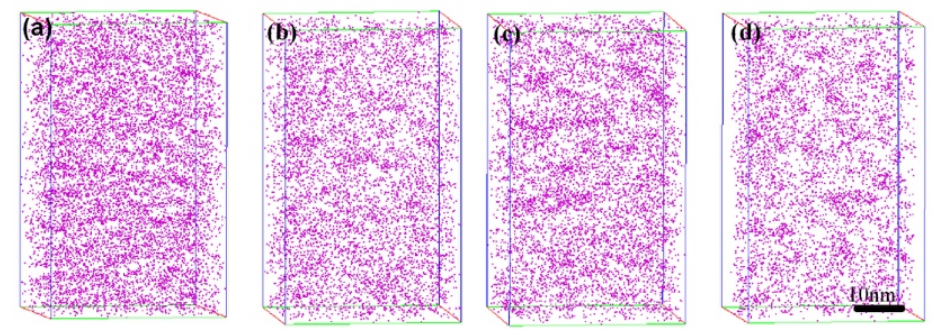

Figure 1 The 3DAP maps of the Al$\mathrm{Mg}-\mathrm{Si}-\mathrm{Cu}-\mathrm{Zn}$ alloy in the different conditions (a) T4 state, (b) T4P state, (c) $\mathrm{T} 4+\mathrm{BH}$ state, $($ d) $\mathrm{T} 4 \mathrm{P}+\mathrm{BH}$ state.

\section{$\underline{\text { References: }}$}

[1] D.J. Chakrabarti, David E. Laughlin, Phase relations and precipitation in Al-Mg-Si alloys with Cu additions, Prog. Mater. Sci. 49(2004) 389-410.

[2] M.X. Guo, G. Sha, L.Y. Cao, W.Q. Liu, J.S. Zhang, L.Z. Zhuang, Enhanced bake-hardening response of an Al-Mg-Si-Cu alloy with Zn addition. Mater. Chem. Phys. 162(2015)15-19.

[3] Y. Birol, Pre-aging to improve bake hardening in a twin-roll cast Al-Mg-Si alloy, Mater. Sci. Eng. A 391(2005) 175-180.

[4] M.X. Guo, Y. Zhang, X.K. Zhang, J.S. Zhang, L.Z. Zhuang, Non-isothermal precipitation behaviors of Al-Mg-Si-Cu alloys with different Zn contents, Mater. Sci. Eng. A, 669(2016) 20-32.

[5] This work was supported by the National Key Research and Development Program of China (2016YFB0300801), the National Natural Science Foundation of China (Nos.51571023, 51301016 and 51571120), and Beijing Natural Science Foundation (No. 2172038). The China Scholarship Council is also gratefully acknowledged for financial support for M.X. Guo. 


\title{
Characterization of Nano-Precipitation in Multiple Component Alloys
}

\author{
Yuan $\mathrm{Wu}^{1}$, Huihui Zhu ${ }^{1}$, Zhifeng Lei ${ }^{1}$, Suihe Jiang ${ }^{1}$, and Zhaoping $\mathrm{Lu}^{1}$ \\ Corresponding Author: wuyuan@ustb.edu.cn \\ 1. State Key Laboratory for Advanced Metals and Materials, Universtiy of Science and \\ Technology, Beijing, China.
}

Increasing simultaneously strength and ductility/toughness is an eternal theme and key point in metals and alloys. However, the tradeoff between strength and ductility/toughness that are often mutually exclusive are determined by the intrinsic dislocation slip-controlled physical nature of plastic deformation, and is a long-term challenge in the field, which severely hinders theoretical development and technical progress. Various approaches have been proposed to address this problem, among which a proper nano-precipitation was proven to be an effective way to increase both strength and ductility by carefully controlling the interface and the characteristics of nanosized precipitates. In this talk, a few cases of nano-precipitation in several representative multi-component alloys, particularly characterization of nanosized precipitates via atom probe tomography will be presented. Evolution of nano-precipitation during deformation and the roles of nano-precipitation in overcoming the strength-ductility tradeoff will be discussed. 


\title{
Nanometer Scale Investigation of Fe Clustering in Half-Heusler Half Metal Candidate $\mathrm{CoTi}_{1-x} \mathrm{Fe}_{x} \mathrm{Sb}$
}

\author{
Bastien Bonef $^{1}$, Sean Harrington ${ }^{1}$, Dan Pennachio ${ }^{1}$, Chris J. Palmstrøm ${ }^{1,2}$, and James S. Speck ${ }^{1}$ \\ Corresponding Author: bbonef.prof@gmail.com \\ 1. Materials Department, University of California, Santa Barbara, CA 93106, USA. \\ 2. Department of Electrical \& Computer Engineering, University of California, Santa Barbara, \\ CA 93106, USA.
}

Half-Heusler half metal candidate CoTi1-xFexSb is a new promising compound due to its diverse electrical and magnetic properties which are intimately linked to the interactions between metallic atoms at the nanometer scale [1]. Atom probe tomography is employed to investigate the presence of nanometer size iron rich domains in $\mathrm{CoTi}_{1-x} \mathrm{Fe}_{x} \mathrm{Sb}$.

$\mathrm{CoTi}_{1-x} \mathrm{Fe}_{x} \mathrm{Sb}$ samples with fraction $x$ of iron of $0.2,0.3$ and 0.5 were investigated. The samples were evaporated in a LEAP 3000X HR operated in voltage-pulse mode with a pulse to base voltage fraction of $25 \%$. The $3 \mathrm{D}$ reconstructions were optimized using spatial desorption maps showing crystallographic poles [2]. Since, atomic planes in the Z direction (figure 1(a)) could be observed, reconstruction parameters (initial tip radius, shank angle and detection efficiency) were optimized to get an expected distance of $2.95 \AA$ between them.

Radial distribution analysis [3] were performed to obtain the self-correlation curves between $\mathrm{Fe}$ atoms (figure 1(b)). Clear iron rich domains were evidenced for $\mathrm{x}=0.2$ and $\mathrm{x}=0.3$ but are no longer observed for $\mathrm{x}=0.5$. Cross and self-correlation curves were also plotted in between $\mathrm{Co}, \mathrm{Ti}$, $\mathrm{Fe}$ and $\mathrm{Sb}$ which did not reveal any other clustering in any samples. These behaviors were confirmed using statistical distribution analysis [4]. Superparamagnetism observed in $\mathrm{x}=0.2$ and $\mathrm{x}=0.3$ samples originates from the presence of Fe rich domains in these samples [1].
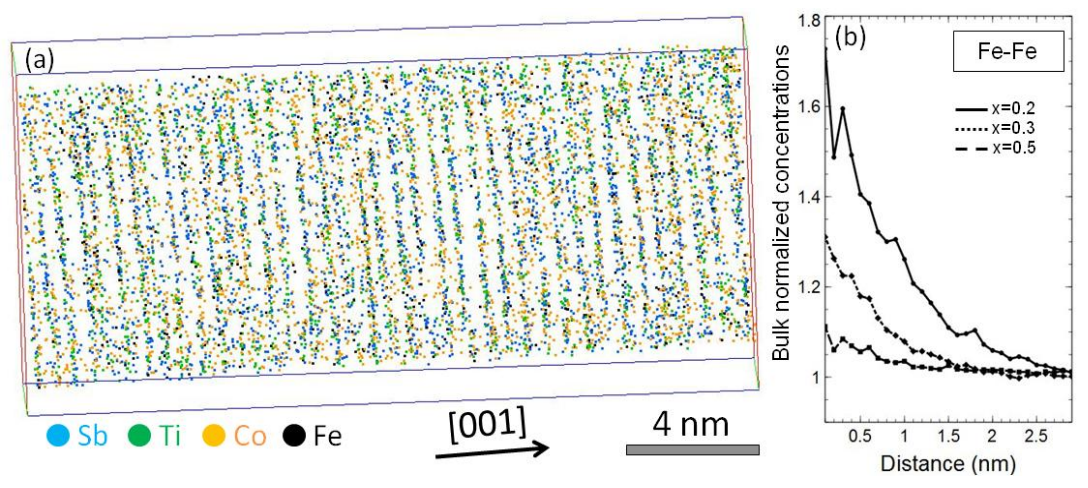

$\underline{\text { References: }}$

[1] S. Harrington et al., Phys. Rev. Mater. 2 (2018), p. 014406.

[2] B. Gault, et al., J. Appl. Phys. 105 (2009), p. 034913.

[3] J. Zhou, J. et al., Microsc. Microanal. 19 (2013), p.665.

[4] M.P. Moody, et al., Microsc. Res. Tech. 71 (2008), p. 542.
Figure 1 (a) APT reconstruction of CoTi0.7Fe0.3Sb showing atomic planes in the $\mathrm{Z}$ direction. (b) Radial distribution analysis showing FeFe cross correlations for fractions of $\mathrm{Fe}$ in the samples of $0.2,0.3$ and 0.5 . 


\section{Atomistic investigation of Niobium precipitation and its effect on recrystallization in steel}

Johannes Webel $^{1}$, Dominik Britz ${ }^{1}$, Jenifer Barrirero ${ }^{1}$, Hardy Mohrbacher ${ }^{2}$, Volker Flaxa ${ }^{3}$, and Frank Mücklich ${ }^{1}$

Corresponding Author: j.webel@mx.uni-saarland.de

1. Saarland University, Department of Functional Materials, Saarbrücken, Germany

2. NiobelCon bvba, Schilde, Belgium, KU Leuven, Dept. MTM, Leuven, Belgium

3. Engineering office and consultant, Salzgitter, Germany

Nanometer-sized niobium precipitates in steel have a huge impact on the mechanical properties by controlling the microstructural grain size and precipitation strengthening [1]. The characterization and quantification of these precipitates is crucial to understand the exact precipitation mechanism. Although niobium precipitation has been extensively studied in the past [2], there are still several open questions, for example, down to which precipitation size the recrystallization is prevented, are there pre-stages of precipitation in the form of chemical fluctuations and how is the precipitation triggered by the local deformation degree.

In the presented work, it is of particular interest to understand how different niobium concentrations affect the initiation of the precipitation during the steel processing and the number and size distribution of the precipitates (Figure 1(a)). Besides, the onset of recrystallization and its retardation were investigated atomistically with the help of atom probe tomography (APT). For this purpose, low-carbon low-alloyed steel samples with different defined concentrations of $\mathrm{Nb}$ (0-0.02-0.04-0.08 mass-\%) were produced and recrystallization curves (Figure 1(b)) were recorded in a thermo-mechanical deformation simulator by doing double-hit compression tests.

(a)

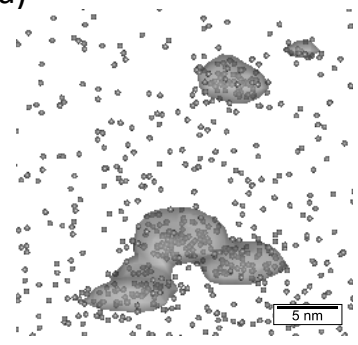

(b)

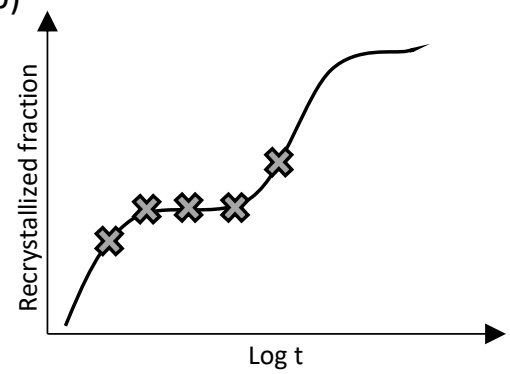

Figure 1 (a) Atom probe reconstruction of $\mathrm{Nb}$ (gray) in steel, with 1\%-iso-concentration surface of $\mathrm{Nb}$ in dark gray. (b) Schematic recrystallization curve of micro-alloyed steel. Crosses schematically mark the states were APT was done.

\section{References:}

[1] S. S. Hansen, J. B. V. Sande, M. Cohen, Niobium carbonitride precipitation and austenite recrystallization in hot-rolled microalloyed steels, Met. Trans. A, 11 (1980), 387-402.

[2] A. J. Deardo, Niobium in modern steels, Int. Mater. Rev., 48 (2003), 371-402. 


\section{Austenitic Nanoclustered ODS Steels for High Temperature Applications}

Sascha Seils ${ }^{1,2}$, Daniel Schliephake ${ }^{1}$, Torben Boll ${ }^{1,2}$, Alexander Kauffmann ${ }^{1}$, Martin Heilmaier ${ }^{1}$

Corresponding Author: sascha.seils@kit.edu

1. Institute for Applied Materials (IAM), Karlsruhe Institute of Technology (KIT), Karlsruhe, Germany

2. Karlsruhe Nano Micro Facility (KNMF), Karlsruhe Institute of Technology (KIT), EggensteinLeopoldshafen, Germany

Oxide dispersion strengthened (ODS) steels are promising materials for applications at temperatures up to $700{ }^{\circ} \mathrm{C}$ in harsh environments as they occur in conventional or fission power plants. Compared to common stainless steels they show an outstanding resistance against swelling under radiation and a superior creep and oxidation resistance [1]. The key to achieve these extraordinary properties are homogenously distributed, nanometer scaled oxide dispersoids (below $4 \mathrm{~nm}$ in size). In our work, we focus on new kinds of austenitic ODS steels expected to exhibit advantageous high temperature properties compared to well-studied ferritic ODS steels. Austenitic ODS steels composed as simple as possible were produced by mechanical alloying and subsequent sintering. Microstructural properties were analyzed in the as-milled powders as well as in the consolidated and annealed material. On the nanoscopic length scale atom probe tomography (APT) showed nanoclusters rich in yttrium, titanium, chromium and oxygen (Figure 1), occurring after every processing step. The APT results were correlated to grain size obtained by orientation imaging. Nanoclusters and austenite grain size of this alloy revealed to be stable even after annealing for $1000 \mathrm{~h}$ at $1000{ }^{\circ} \mathrm{C}$. Thus, we conclude that nanoclusters effectively hinder grain growth by pinning of grain boundaries. The obtained microstructural data was used to verify deformation theories for ODS steels to predict the strength of the material at temperatures up to $800{ }^{\circ} \mathrm{C}$.

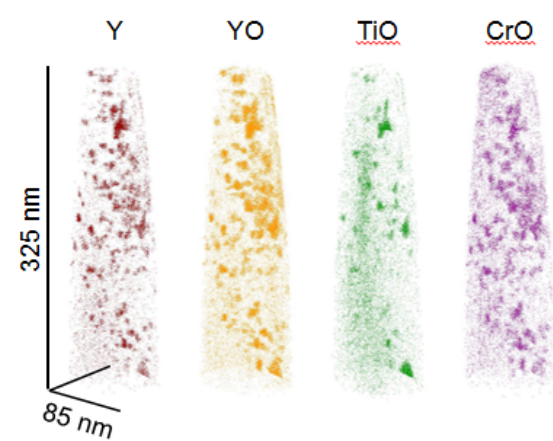

Figure 1. Nanoclusters in Fe-25Cr-20Ni-0.4Ti-0.25 $\mathrm{Y}_{2} \mathrm{O}_{3}$

in the as-consolidated state.

References:

[1] J.H. Schneibel et al., Ultrafine-grained nanocluster-strengthened alloys with unusually high creep strength, Scripta Materialia, 61 (2009), 793-796. 


\title{
Atom probe investigation of a 3D printed $\alpha / \beta$ Ti alloy
}

\author{
K. Eder ${ }^{1}$, X.P. Li ${ }^{2,3}$, G. Ji ${ }^{4}$, L. Yang ${ }^{1}$, S. Dadbakhsh ${ }^{3}$, J. Vleugels ${ }^{5}$, J. Van Humbeeck ${ }^{5}$, J.P. \\ Kruth $^{3}$, J.M. Cairney ${ }^{1}$ \\ Corresponding Author: katja.eder@sydney.edu.au
}

1. Australian Centre for Microscopy and Microanalysis, The University of Sydney, NSW 2006, Australia.

2. School of Mechanical and Manufacturing Engineering, The University of New South Wales, Sydney 2052, Australia.

3. KU Leuven, Department of Mechanical Engineering, Leuven 3001, Belgium.

4. Unité Matériaux et Transformations, CNRS UMR 8207, Université de Lille, Villeneuve d'Ascq 59655, France.

5. KU Leuven, Department of Materials Engineering, Leuven 3001, Belgium.

Titanium alloys are used in a wide range of applications, such as biomedical implants or as building components in aircrafts. This is due to their properties of light weight, high tensile strength and toughness at a wide range of temperatures, as well as good corrosion resistance [1]. However, faster ways of fabricating these alloys need to be explored as demand increases. A way of achieving this is using selective laser melting, a rapid prototyping technique that allows the fast production of complex geometries and custom designs. This is a relatively new way of producing Titanium alloys, which is why the resulting alloy needs to be characterized in more detail to determine if it yields the same properties compared to conventional methods. If secondary particles are added in the fabrication step, the melt pool behavior can change, which in turn will affect the resulting microstructure and properties of the alloy. It is therefore important to know the exact elemental distribution, to tailor these properties, which makes atom probe tomography an ideal analysis tool that can add additional information at the nano-scale.

Here we will present atom probe results from the analysis of a fully dense, crack-free $\alpha / \beta$ titanium alloy that was fabricated using selective laser melting from commercially pure titanium with 1 vol. $\%$ micron-sized $\mathrm{Mo}_{2} \mathrm{C}$ powder. The atom probe results revealed an inhomogeneous distribution of the main elements $(\mathrm{Ti}, \mathrm{Mo}, \mathrm{C})$ and that no location correlation between the $\mathrm{C}$ and Mo occurs.

$\underline{\text { References: }}$

[1] F.H. Froes, Titanium: Physical metallurgy, processing, and applications, ASM International, Materials Park, 2015. 


\title{
Comparing the Effects of Micro-alloy Carbides and Copper Clusters on Hydrogen Trapping in Martensitic Steels
}

\author{
Yu-Chen Lin ${ }^{1}$, Ingrid McCarroll ${ }^{2}$, Julie Cairney ${ }^{2,3}$, Hung-Wei Yen ${ }^{1}$ \\ Corresponding Author: ingrid.mccarroll@sydney.edu.au \\ 1. Department of Materials Science \& Engineering, National Taiwan University, Taipei, 10617 , \\ R.O.C. \\ 2. Australian Centre for Microscopy \& Microanalysis, University of Sydney, NSW 2006, \\ Australia. \\ 3. School of Aerospace, Mechanical \& Mechatronic Engineering, University of Sydney, NSW \\ 2006, Australia.
}

Hydrogen embrittlement can substantially reduce the service lifetime of a material, however, due to the complexities associated with the observation of hydrogen, understanding and controlling for the effects of hydrogen embrittlement is challenging [1]. An alternative method used to understand the distribution of hydrogen within a material, is to replace hydrogen with deuterium. Recent advances in the use of deuterium charging for the preparation of deuterated atom probe tips, has meant that site specific observation of deuterium within steel is now achievable [2].

Presently, a study of copper containing martensitic steels has revealed that the formation of copper precipitates improves the steel's resistance to hydrogen embrittlement. It is therefore deduced that these copper precipitates act as hydrogen trapping sites. Initial atom probe results also reveal the presence of micro-alloyed titanium carbides within the tempered martensitic steel. Using a vacuum transfer system between a deuterium charging station and the atom probe, the present study will provide information as to the capacity of both the micro-alloyed carbides and the copper precipitates to trap deuterium.

a)

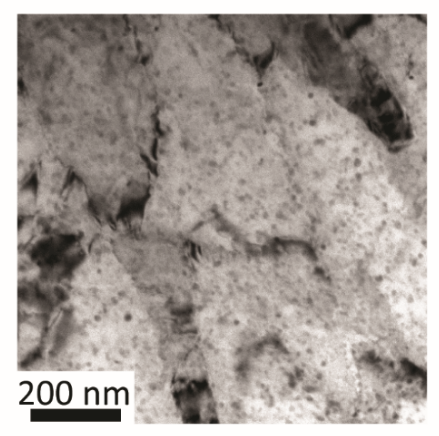

b)

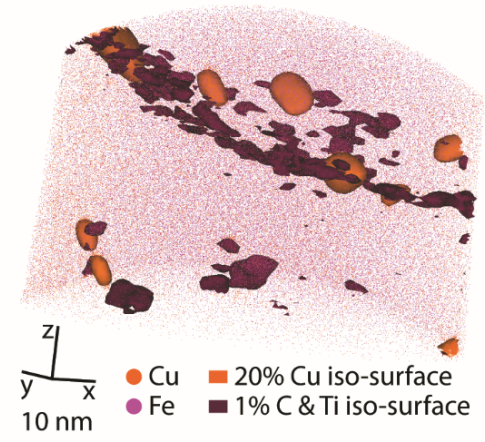

Figure 1 Sample composition: Fe-0.06C-1.5Mn-0.1Si-1.0Cu2.0Ni-0.1Ti-0.2Mo (in wt. \%). a) TEM micrograph of a sample tempered for $60 \mathrm{~min}$ at $950{ }^{\circ} \mathrm{C}$. b) APT dataset from the same sample as in a), showing copper precipitates as well as titanium carbides and segregation of titanium carbide to a grain boundary.

\section{References:}

[1] G. Sundell, M. Thuvander, A.K. Yatim, H. Nordin, H.O. Andrén, Direct observation of hydrogen and deuterium in oxide grain boundaries in corroded Zirconium alloys, Corrosion Science, 90 (2015) 1-4.

[2] Y.-S. Chen, D. Haley, S.S.A. Gerstl, A.J. London, F. Sweeney, R.A. Wepf, W.M. Rainforth, P.A.J. Bagot, M.P. Moody, Direct observation of individual hydrogen atoms at trapping sites in a ferritic steel, Science, 355 (2017) 1196-1199. 


\title{
Atom probe analysis on chemical composition of $\mathrm{TiC}$ precipitates in isothermally-aged ferritic steel
}

\author{
Yukiko Kobayashi ${ }^{1}$, Jun Takahashi ${ }^{1}$, Kazuto Kawakami ${ }^{2}$ and Kazuhiro Hono ${ }^{3}$ \\ Corresponding Author: kobayashi.yf6.yukiko@jp.nssmc.com \\ 1. Nippon Steel \& Sumitomo Metal Corporation, Futtsu, Japan. \\ 2. Nippon Steel \& Sumikin Technology Co., Ltd., Futtsu, Japan. \\ 3. National Institute for Materials Science, Tsukuba, Japan.
}

Fine titanium carbide ( $\mathrm{TiC}$ ) precipitated in ferritic steels is beneficial for particle strengthening for such as high strength steels for automotive. The chemical composition of precipitates is one of important issues for understanding the precipitation kinetics which is crucial for controlling the precipitation state and the properties. The chemical composition of $\mathrm{TiC}$ is also interested since hydrogen trapping site was proposed to be $\mathrm{C}$-vacancies on the surface of coherent $\mathrm{TiC}$ precipitates [1]. According to the Ti-C binary phase diagram, atomic ratio of $\mathrm{C}$ in $\mathrm{TiC}$ (i.e. $\mathrm{C} /(\mathrm{C}+\mathrm{Ti}))$ is $0.4-0.5$. On the other hand, there are various reports on the $\mathrm{C}$ ratio in very small carbides precipitated in steel. Figure 1(a) shows $\mathrm{C}$ ratio of TiC particles in $0.03 \mathrm{C}-0.1 \mathrm{Ti}-0.2 \mathrm{Mn}-$ $3 \mathrm{Al}(\operatorname{mass} \%)$ model steel aged at $580{ }^{\circ} \mathrm{C}$ for various time periods observed by three dimensional atom probe (3DAP) with large angle reflectron [2]. The $\mathrm{C}$ ratio in $\mathrm{TiC}$ particles converged to around 0.4 when TiC particle size increased to $5 \mathrm{~nm}$. However, $\mathrm{C}$ ratio could not be identified in $\sim 1 \mathrm{~nm}$ TiC particles due to large statistical error with $\sim 40 \%$ detection efficiency of 3DAP. Thus, LEAP 5000XS with $~ 80 \%$ detection efficiency was applied for quantitative characterization of small TiC particles by the reduction of the statistical error. Variation in $\mathrm{C}$ ratio in $\mathrm{TiC}$ was decreased with the higher detection efficiency (Figure 1(b)). As a result, it was found that the observed $\mathrm{C}$ ratio of $\mathrm{TiC}$ particle is obviously affected by anomalous concentration of solute $\mathrm{C}$ atoms related to the specific crystallographic direction of ferrite matrix [3]. Particle size dependence of the $\mathrm{C}$ ratio in $\mathrm{TiC}$ will be discussed.

(a) Number of $\mathrm{Ti}$ and $\mathrm{C}$ atoms in each particle (atoms)
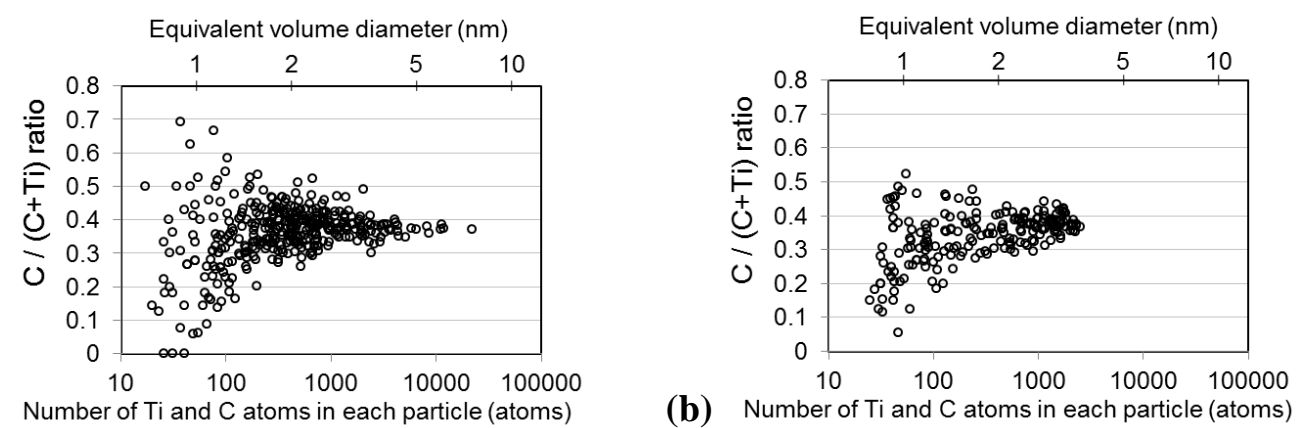

Figure $1 \mathrm{C}$ ratio in each TiC particle measured by (a) 3DAP and (b)LEAP5000XS.

\section{References:}

[1] J. Takahashi, K. Kawakami, Y. Kobayashi, T. Tarui, The first direct observation of hydrogen trapping sites in TiC precipitation-hardening steel through atom probe tomography, Scr. Mater. 63 (2010) 261-264. [2] Y. Kobayashi, J. Takahashi, K. Kawakami, Experimental evaluation of the particle size dependence of the dislocation-particle interaction force in TiC-precipitation-strengthened steel, Scr. Mater. 67 (2012) 854-857.

[3] Y. Kobayashi, J. Takahashi, K. Kawakami, Anomalous distribution in atom map of solute carbon in steel, Ultramicroscopy 111 (2011) 600-603. 


\title{
Interface chemistry between titanium carbide and metal matrix in Al-based composite revealed by atom probe tomography
}

\author{
Shenbao Jin ${ }^{1}$, Yafeng Yang ${ }^{2}$, Qinglong Zhao ${ }^{3}$ and Gang Sha ${ }^{1}$ \\ Corresponding Author: gang.sha@njust.edu.cn \\ 1. School of material science and engineering, Nanjing University of Science and Technology, \\ Nanjing, China. \\ 2. State Key Laboratory of Multiphase Complex Systems, Institute of Process eutectoid \\ Engineering, Chinese Academy of Sciences, Beijing, China. \\ 3. School of material science and engineering, Jilin University, Changchun, China.
}

\begin{abstract}
Morphology control of in-situ synthesized titanium carbide $\left(\mathrm{TiC}_{x}\right)$ crystal in various metal melts is highly desirable because the growth shape of $\mathrm{TiC}_{x}$ greatly changes the mechanical properties of the composites such as toughness, abrasion resistance and fatigue performance. It has been known that the growth shape of $\mathrm{TiC}_{x}$ is determined by a competition of growth rate between different crystallographic directions including $\langle 111\rangle,\langle 110\rangle$ and $\langle 100\rangle$, which is in theory not only influenced by the interface structure between $\mathrm{TiC}_{x}$ and metal matrix (i.e. preferred coherent or quasi-coherent interface) but also by the interface chemistry (i.e. intrinsic stoichiometry $x$ in $\mathrm{TiC}_{x}$ and carbon concentration at the growth interface). However, a direct investigation on $\mathrm{TiC}_{x} /$ metal interface chemistry is quite difficult. In the present paper, atom probe tomography (APT) was used to study the interface chemistry between $\mathrm{TiC}_{x}$ and metal matrix in Al-based composite. $\mathrm{TiC}_{x}$ crystals with octahedron, truncated-octahedron and sphere morphologies are synthesized in Al-based composites through in-situ reaction synthesis in $\mathrm{Al}$ melt at high temperatures. The interface structure between $\mathrm{TiC}_{x}$ and metal matrix is investigated by electron backscattered diffraction (EBSD) and high resolution transmission electron microscopy (HRTEM); while the interface chemistry is studied by APT with FIB-based specimen preparation method. It is found that the stoichiometry of $\mathrm{TiC}_{x}$ and carbon content in $\mathrm{Al}$ matrix vary greatly at different growth stages, corresponding to different growth morphologies of $\mathrm{TiC}_{x}$ crystals. The morphology control mechanism of $\mathrm{TiC}_{x}$ crystal in Al-based composite is then discussed accordingly.
\end{abstract}




\title{
Using atom probe to study the role impurities play on the evolution of the microstructure of nanocrystalline alloys
}

\author{
B.C. Hornbuckle ${ }^{1}$, C.J. Marvel ${ }^{2}$, A. Roberts ${ }^{1}$, T.L Luckenbaugh ${ }^{1}$, M.P. Harmer ${ }^{2}$, and K.A. \\ $\underline{\text { Darling }^{1}}$
}

Corresponding Author: billy.c.hornbuckle.civ@mail.mil

1. Weapons and Materials Research Directorate, U.S. Army Research Laboratory, RDRL-WMMF, Aberdeen Proving Ground, MD 21005, USA.

2. Department of Materials Science and Engineering, Lehigh University, Bethlehem, PA 18015, USA.

While atom probe is becoming a more common tool for material scientists to utilize, there are still areas its application could be tremendously advantageous. One such research area is the field of nanocrystalline alloys. The long, standing goal in the nanocrystalline world is the ability to generate thermally stable alloys; thus allowing for the generation of bulk parts for use in real world applications. There have been a few limited cases where thermally stable alloys have been generated [1,2]. However, these are the exceptions not the norm, yet they have opened the door to understanding the role impurities play on the evolution of the microstructure of these nanocrystalline alloys. The level at which the impurities exists is quite low, and they typically are low atomic number elements to begin. Therefore, atom probe tomography is the best characterization technique to use in identifying the impurities, and their location in the microstructure, i.e. grain boundaries, secondary phases, or grain interiors.

This work looks at both of the thermal stable alloy systems, CuTa and NiW, to identify which impurities are present and their location within the microstructure. The location of the impurities are confirmed with correlated scanning transmission electron microscopy ((S)TEM) to ensure the atom probe data was not affected by evaporation artifacts. These results can then be used to draw conclusions on how to use impurities to researcher's advantage on developing the next generation of thermally stable nanocrystalline alloys.
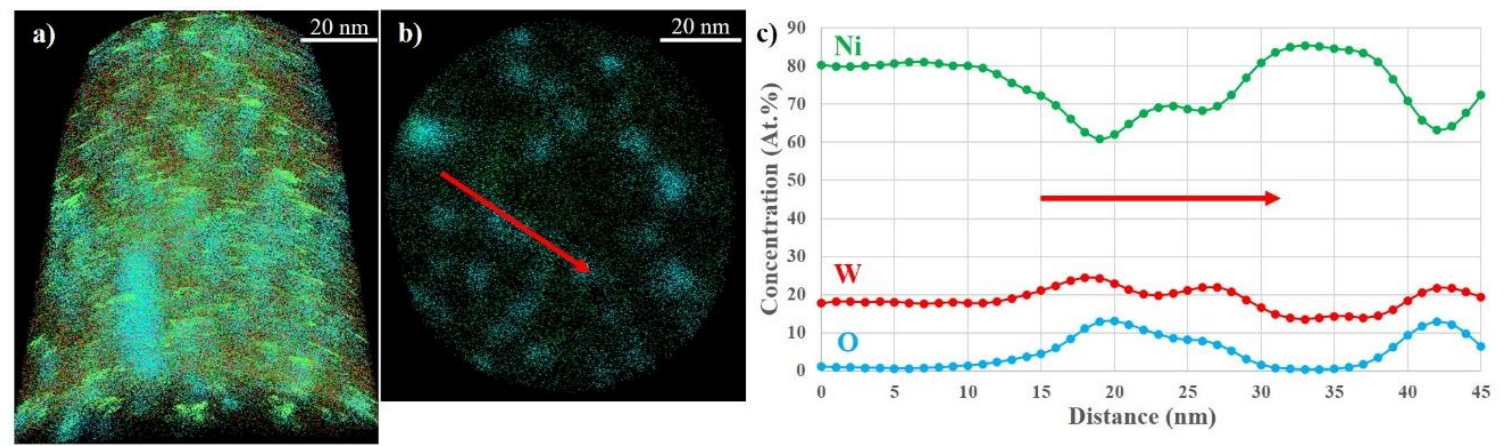

Figure 1 (a) Atom map of electroplated Ni-28W (At.\%). (b) 2D slice displaying Ni and WO atoms showing the grain boundary used to generate 1D profile. (c) 1D profile generated from highlighted grain boundary.

\section{References:}

[1] K.A. Darling, et al., Extreme creep resistance in a microstructurally stable nanocrystalline alloy. Nature 537, no. 7620 (2016): 378.

[2] A.J. Detor and C.A. Schuh. Tailoring and patterning the grain size of nanocrystalline alloys. Acta Materialia 55, no. 1 (2007): 371-379. 


\title{
The Elemental Partition to the Secondary Phases Found in Co-Ni Based Superalloys After Solution and Ageing Heat Treatments
}

\author{
A.M.S. Costa ${ }^{1}$, J.D. Escobar ${ }^{1,2}$, J. P. Oliveira ${ }^{3}$, J.D. Poplawsky ${ }^{4}$, A.J. Ramirez ${ }^{5}$, A. P \\ Tschiptschin 6 , \\ Corresponding Author: alexmatos1980@gmail.com \\ 1. Brazilian Nanotechnology National Laboratory-CNPEM, Brazil \\ 2. College of Mechanical Engineering, University of Campinas - Unicamp, Brazil. \\ 3. New University of Lisbon, Centre of Materials Research (CENIMAT), Portugal. \\ 4. The Center for Nanophase Materials Sciences, Oak Ridge National Laboratory, USA \\ 5. Department of Materials Science and Engineering, The Ohio State University, USA \\ 6. Department of Metallurgical and Materials Engineering, University of São Paulo (USP), Brazil
}

Co-Ni based superalloys hardened by the intermetallic phase $\gamma^{\prime}-\mathrm{Co}_{3}(\mathrm{Al}, \mathrm{W})$ phase and potentially by other secondary phases such as $\mu-\mathrm{Co}_{7} \mathrm{~W}_{6}, \mathrm{Co}_{3} \mathrm{~W}$, Laves, $\beta$-CoAl were evaluated by SEM and APT to understand the elemental partition after being soluted at $1250{ }^{\circ} \mathrm{C}$ for 72 hours followed by ageing at $1000{ }^{\circ} \mathrm{C}$ for 168 hours. The composition of the $\mathrm{Co}-\mathrm{Ni}$ based superalloy (\% at.) was shown in Table 1. As presented in Figure 1 the as cast microstructure consisted of $\gamma$ grains that were decorated with a $\gamma^{\prime}$ precipitates inside them and $\mathrm{MC}$ carbide and $\mu-\mathrm{Co}_{7} \mathrm{~W}_{6}$ phase were found at interdendritic region and grain boundaries. After heat treated at $1250{ }^{\circ} \mathrm{C}$ for 72 hours followed by ageing at $1000{ }^{\circ} \mathrm{C}$ for 168 hours, the Co-Ni superalloy was composed of the $\gamma / \gamma^{\prime}$ matrix and only secondary precipitates of were found. The one-dimensional composition profile measured by APT of ROI showed that $\mu$ phase is rich of in Co and W with important concentrations of $\mathrm{Ni}$ and $\mathrm{Cr}$. The chemical composition of $\gamma^{\prime}$-gamma prime phase was rich in $\mathrm{Ni}$, $\mathrm{Co}$ and Al. Ni replaced Co sites on the ordered structure of $\gamma^{\prime}-\mathrm{Co}_{3}(\mathrm{Al}, \mathrm{W})$ phase and $\mathrm{W}, \mathrm{Cr}$ and $\mathrm{Nb}$ were found in lesser quantities.

Table 1 - Chemical composition of the produced Co-Ni based superalloy.

\begin{tabular}{cccccccccc}
\hline Co & Ni & Al & W & Ta & $\mathbf{C r}$ & $\mathbf{N b}$ & $\mathbf{B}$ & $\mathbf{C}$ & $\begin{array}{c}\boldsymbol{\rho} \\
{\left[\mathbf{g} / \mathbf{c m}^{3}\right]}\end{array}$ \\
\hline Bal. & 40 & 10 & 7.5 & - & 10 & 3 & 0.06 & 0.6 & 8.8 \\
\hline
\end{tabular}

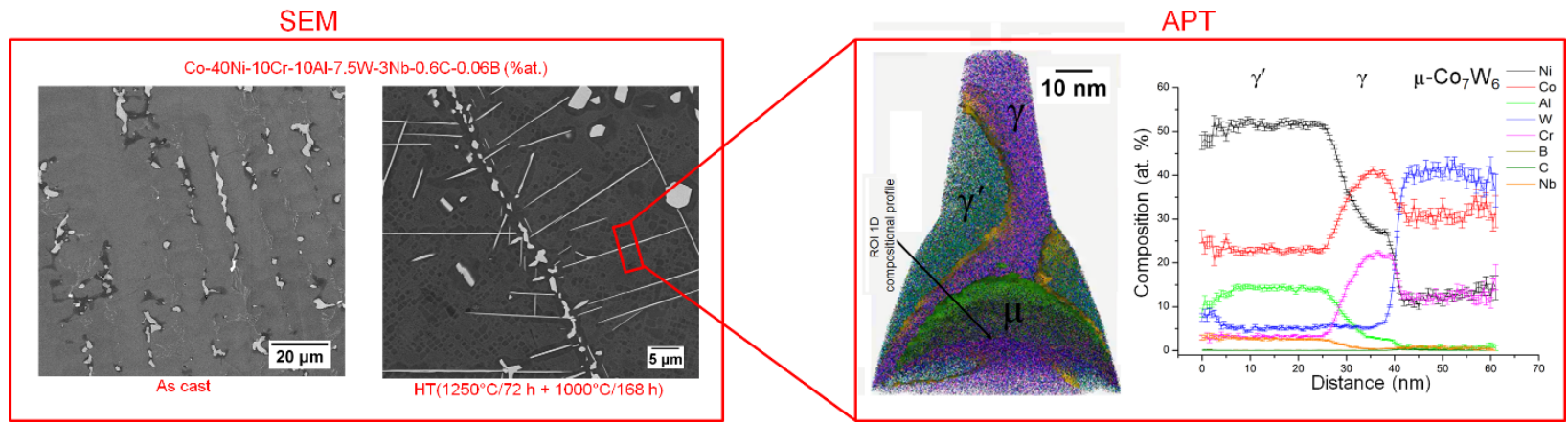

Figure 1 A SEM micrographies of a Co-Ni based superalloy in as cast condition, after solutioned and aged at $1250^{\circ} \mathrm{C} / 72 \mathrm{~h}$ and $1000^{\circ} \mathrm{C} / 168 \mathrm{~h}$ respectively. A ROI (Region of Interest) in the heat treated material was chemically analysed by APT and its one-dimensional chemical profile is presented. 


\section{Nanoscale Phase Separation in $\mathrm{Al}_{0.5} \mathrm{CoCrFeNi}(\mathrm{Cu})$ High Entropy Alloys as Studied by Atom Probe Tomography}

Keith E. Knipling ${ }^{1}$, Joshua L. Tharpe ${ }^{2}$, and Peter K. Liaw ${ }^{2}$

Corresponding Author: keith.knipling@nrl.navy.mil

1. U. S. Naval Research Laboratory, Multifunctional Materials Branch, Washington, DC 20375.

2. The University of Tennessee, Department of Materials Science and Engineering, Knoxville, TN 37996.

High entropy alloys (HEAs) typically contain five or more principal elements in nearly equiatomic proportions [1-3]. Figure 1 shows the as-cast microstructure of an $\mathrm{Al}_{0.5} \mathrm{CoCrFeNi}$ alloy, as observed by scanning electron microscopy (SEM). Dendritic microsegregation results in $\mathrm{Cr}$-, $\mathrm{Fe}$-, and Co-rich dendrites with composition $\mathrm{Cr}_{23.06} \mathrm{Fe}_{22.79} \mathrm{Co}_{22.55} \mathrm{Ni}_{20.66} \mathrm{Al}_{10.94}$ (at.\%) and a face-centered cubic (FCC) crystal structure. The surrounding interdentric region is enriched in $\mathrm{Ni}$ and $\mathrm{Al}$ with composition $\mathrm{Cr}_{23.26} \mathrm{Ni}_{22.01} \mathrm{Co}_{19.64} \mathrm{Fe}_{19.19} \mathrm{Al}_{15.89}$ (at.\%) and is comprised of a disordered body-centered cubic (BCC, A2) phase and an ordered BCC phase (B2) that is formed by spinodal decomposition. These microstructures are investigated in the as-cast state and after thermal annealing, and are correlated to the observed strength, as measured by Vickers microhardness and uniaxial tensile tests.
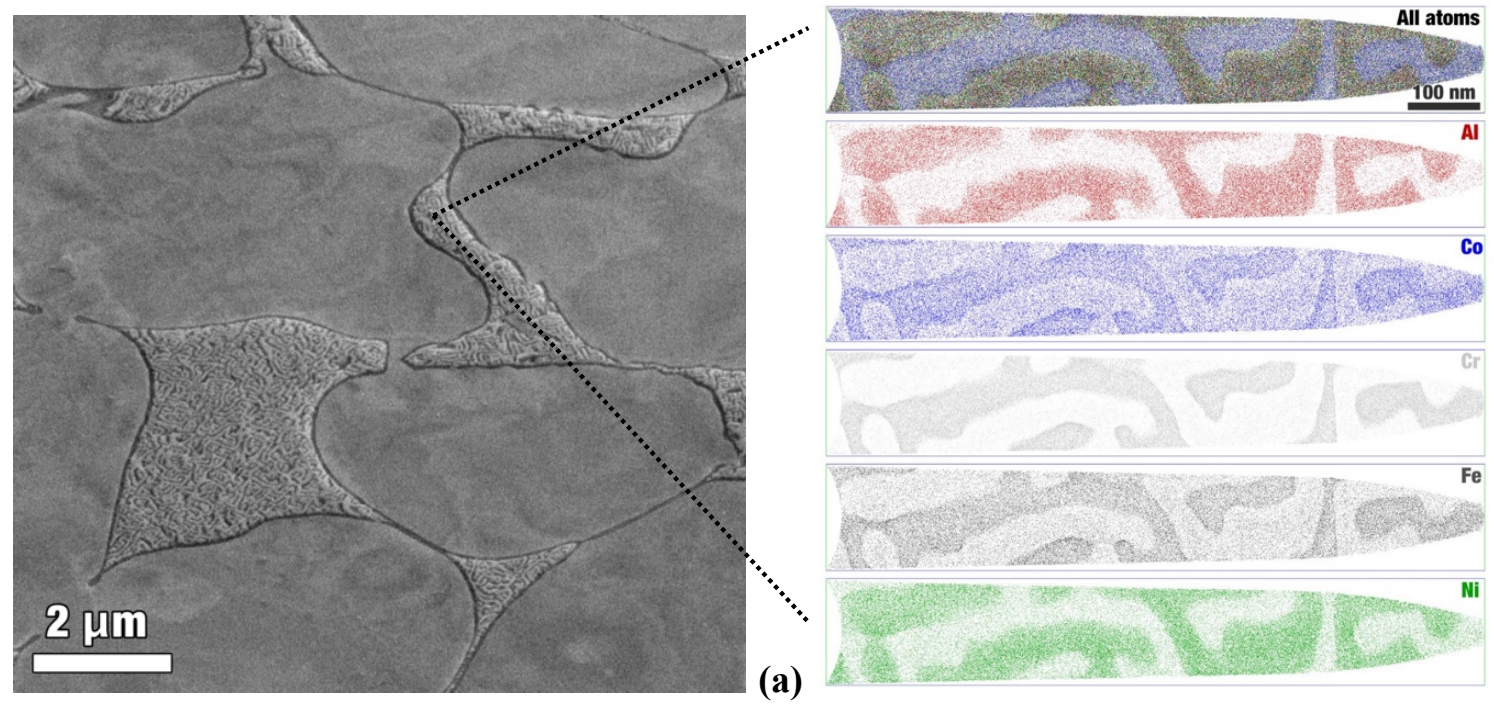

(b)

Figure 1 (a) SEM micrograph of the as-cast $\mathrm{Al}_{0.5} \mathrm{CoCrFeNi}$ alloy investigated. (b) Atom probe tomography (APT) reconstructions displaying the chemical segregation between the spinodally decomposed $\mathrm{BCC}$ and $\mathrm{B} 2$ phases within the interdendritic region.

\section{References:}

[1] J. Yeh, Alloy Design Strategies and Future Trends in High-Entropy Alloys, JOM 65 (2013), 17591771.

[2] J. Yeh, Physical Metallurgy of High-Entropy Alloys, JOM 67 (2015), 2254-2261.

[3] M. Tsai and J Yeh, High-Entropy Alloys: A Critical Review, Materials Research Letters 2 (2014), 107-123. 


\section{Kinetic Simulations of the Austenite Isothermal Growth During Tempering of 12Cr-6Ni-2Mo-0.02C-0.13Ti Supermartensitic Stainless Steel}

J.D. Escobar ${ }^{1,2}$, G. A. Faria ${ }^{3}$, C. A. F. Salvador ${ }^{2}$, J. P. Oliveira ${ }^{4}$, T. Boll ${ }^{5}$ S. Seils ${ }^{5}$, P. R. Mei², A. J. Ramirez ${ }^{3}$.

Corresponding Author: juliandescobar@gmail.com

${ }^{1}$ Brazilian Nanotechnology National Laboratory - CNPEM, Brazil.

${ }^{2}$ College of Mechanical Engineering, University of Campinas - Unicamp, Brazil.

${ }^{3}$ Department of Materials Science and Engineering, The Ohio State University, USA.

${ }^{4}$ Centre of Materials Research (CENIMAT), New University of Lisbon - Portugal.

${ }^{5}$ Institute of Applied Materials, Karlsruhe Institute of Technology. Germany.

The mobility of austenite $(\gamma)$-stabilizing elements, such as $\mathrm{Ni}$ and $\mathrm{Mn}$, in the body-centered-cubic BCC martensitic $\left(\alpha^{\prime}\right)$ matrix towards the $\gamma / \alpha^{\prime}$ interface, as well as the degree of partitioning of these elements into $\gamma$, are fundamental aspects to understand the isothermal growth and stabilization of $\gamma$ during tempering of supermartensitic stainless steels (SMSS) [1,2]. In this work, kinetic modeling of the $\gamma$ isothermal growth was performed using DICTRA $\AA$. The simulation was conducted for a planar geometry having a BCC phase set in all grid points with a constant composition distribution of $\mathrm{Fe}, 12.8 \mathrm{Cr}, 5.6 \mathrm{Ni}, 1.1 \mathrm{Mo}, 0.5 \mathrm{Mn}$ at.\%. At the zero position, an inactive face-centered-cubic FCC phase was introduced. A built-in dislocation assisted diffusion model was used to enhance the mobility, using dislocation densities for $\gamma\left(0.75 \times 10^{14} / \mathrm{m}^{-2}\right)$ and $\alpha^{\prime}\left(2 \times 10^{14} / \mathrm{m}^{-2}\right)$ [3]. The simulations were performed between 580 and $670{ }^{\circ} \mathrm{C}$ during $9000 \mathrm{~s}$. The system width varied between 400 to $175 \mathrm{~nm}$, based on actual STEM measurements of the microstructure after isothermal reversion. The simulated profiles for the distribution of $\mathrm{Ni}$, shown in Figure 1 a), evidenced very good agreement with APT (b) and STEM (c) compositional profiles. The simulated austenite lath sizes matched the experimentally observed ones by STEM (d).
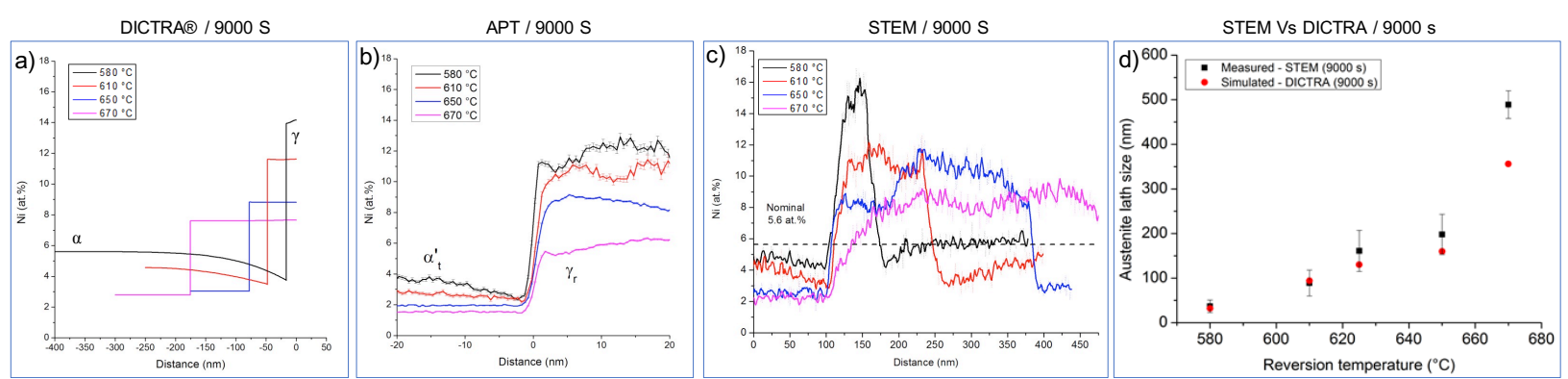

Figure 1. Distribution of Ni after a) DICTRA simulations, b) APT, and c) STEM. The simulated and experimental lath sizes were in good agreement $\mathrm{d}$ ).

\section{References:}

[1] F. Niessen, M. Villa, J. Hald, M. A. J. Somers. Mater. Des., 116 (2017) 8-15.

[2] J. D. Escobar, J.D. Poplawsky, G. A. Faria, J. Rodriguez, J. P. Oliveira, C.A. F. Salvador, P. R. Mei, S. S. Babu, A. J. Ramirez, Mater. Des., 140 (2018) 95-105.

[3] M. Wiessner, E. Gamsjäger, S. van der Zwaag, P. Angerer, Mater. Sci. Eng. A, 682 (2017) 117-125. Authors acknowledge FAPESP (2014/20844-1; 2016/13466-6) for the PhD funding, the Center for Electron Microscopy and Analysis CEMAS, OSU, United States, for the support with TEM experiments, and KNMF Laboratory for Microscopy and Spectroscopy at KIT, Germany, for the support with the APT experiments. 


\title{
Structural Promotion of Potassium in $\mathrm{CoCu}$ Catalysts for $\mathrm{CO}_{2}$ Hydrogenation
}

\author{
Jenny Voss ${ }^{1}$, Daniel E. Perea ${ }^{2}$, Libor Kovarik ${ }^{2}$, Mark Bowden² and Norbert Kruse ${ }^{1,2}$
}

Corresponding Author: norbert.kruse@wsu.edu

1. Voiland School of Chemical Engineering and Bioengineering, Washington State University, Pullman, WA, 99164, USA

2. Environmental Molecular Sciences Laboratory, Pacific Northwest National Laboratory, Richland, WA 99332, USA

We have developed a research program which aims at turning detrimental $\mathrm{CO}_{2}$ into fuel-type hydrocarbons and oxygenates via catalytic hydrogenation. The activity/selectivity performance of this process is strongly dependent on the chemical composition of the catalytic materials employed. We found potassium-promoted $\mathrm{CoCu}$ catalysts tuned the selectivity from that of methane to long chain hydrocarbons and alcohols. In this current contribution, we focus on determining i) how variable amounts of potassium are able to exert this strong influence and ii) how the surface composition of the K-promoted $\mathrm{CoCu}$ catalysts is altered by the reaction.

In previous investigations, Atom Probe Tomography (APT) and Transmission Electron Microscopy (TEM) were used to show CoMnK [1] and CoCuMn [2] catalytic nanoparticles naturally exhibited a Co@Mn and Co@Cu core@shell structure, respectively. For CoCuK catalysts, which are attractive for the catalytic $\mathrm{CO}_{2}$ hydrogenation, no such extended metal-oxide phase was found so far, i.e. as-prepared catalysts are purely metallic. TEM results demonstrate phase-separated cobalt and copper nanoparticles in intimate contact with each other. Such phase separation is in accordance with the poor bulk miscibility of the two metals [3]. The texture of such metallic $\mathrm{Co}-\mathrm{Cu}$ aggregates is strongly influenced by the addition of potassium. For high potassium concentrations, a considerable number of new phases have been observed by microbeam X-ray diffraction. The chemical nature of these phases is presently unknown, but seems to be in relation with the thermal decomposition kinetics of the $\mathrm{CoCuK}$ oxalate precursor.

APT analysis is currently being applied to investigate the influence of potassium loading on the $\mathrm{CoCuK}$ catalyst structure before and after $\mathrm{CO}_{2}$ hydrogenation. To do so, an atmospheric pressure reactor has been mounted allowing the transfer of the samples to the APT chamber without exposing them to air. Preliminary efforts have led to capture of a clean interface between a catalytic $\mathrm{Co}_{3} \mathrm{Cu}_{1}$ nanoparticle before reaction and the surrounding $\mathrm{Pt} / \mathrm{C}$ embedding matrix (Figure 1). Efforts are now being undertaken to capture a full catalyst nanoparticle. References:

[1] J.Voss,Y. Xiang, G. Collinge, D.E. Perea, L. Kovarik, J.S. McEwen and N. Kruse, Characterization of $\mathrm{CoCu}$ - and CoMn-based catalysts for the Fischer Tropsch reaction toward chain-lengthened oxygenates. ACCEPTED: Topics in Catalysis (2018).

[2] Y. Xiang, V. Chitry, P. Liddicoat, J. Cairney, S. Ringer and N. Kruse, Long-Chain Terminal Alcohols through Catalytic CO Hydrogenation, Journal of the American Chemical Society, 135 (2013), 7114-7117.

[3] T. Nishizawa, and K. Ishida, The Co-Cu (Cobalt-Copper) System, Bulletin of Alloy Phase Diagrams, 5 (1984), 161-165.

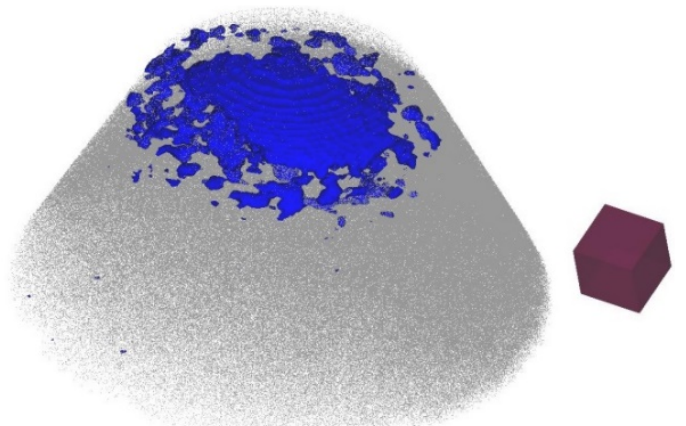

Figure 1. 3-Dimensional distribution of $\mathrm{Pt} / \mathrm{C}$ (grey) and Co (blue). The $\mathrm{Co}_{3} \mathrm{Cu}_{1}$ nanoparticle was embedded in a $\mathrm{Pt} / \mathrm{C}$ matrix deposited in situ using FIB/SEM. Scale cube: $10 \times 10 \times 10 \mathrm{~nm}^{3}$. 


\section{Deviation of Ti-Al-N Composition During APT Analysis of Ti $\mathbf{T}_{0.4} \mathrm{Al}_{0.6} \mathrm{~N}$}

Tim Lehmann ${ }^{1}$, Patrick Stender ${ }^{1}$, Guido Schmitz ${ }^{1}$, Wolfgang Engelhart ${ }^{2}$

Corresponding Author: tim.lehman@mp.imw.uni-stuttgart.de

1. University of Stuttgart, Institute for Materials Science, Chair for Materials Physics, Stuttgart, Germany.

2. Walter AG, Tübingen, Germany.

Atom Probe Tomography (APT) is a powerful technique that allows for a three dimensional chemical analysis of needle-shaped specimens. In order to study decomposition processes in $\mathrm{Ti}_{\mathrm{x}} \mathrm{Al}_{1-\mathrm{x}} \mathrm{N}$-thin films, samples of different composition were produced by HiPIMS. Recent measurements on decomposed $\mathrm{Ti}_{0.4} \mathrm{Al}_{0.6} \mathrm{~N}$-thin films have shown deviations from the expected stoichiometry in the Ti- and Al-rich domains. For a better understanding, pure TiN and AlN powder particles are analyzed as calibration samples by APT. The observed nitrogen contents are compared to the compositions measured by gas extraction and ICP-OES. Appropriate correction factors were derived. By performing the correction, a way more reliable analysis of the measured compositions by APT is achieved.

The powder particles are transferred to $\mathrm{W}$-supports and post-processed via annual milling using a Focused Ion beam (FIB). $\mathrm{Ti}_{0.4} \mathrm{Al}_{0.6} \mathrm{~N}$-films are heat treated in Ar-atmosphere followed by subsequent post processing via FIB-lift-outs and annular milling.
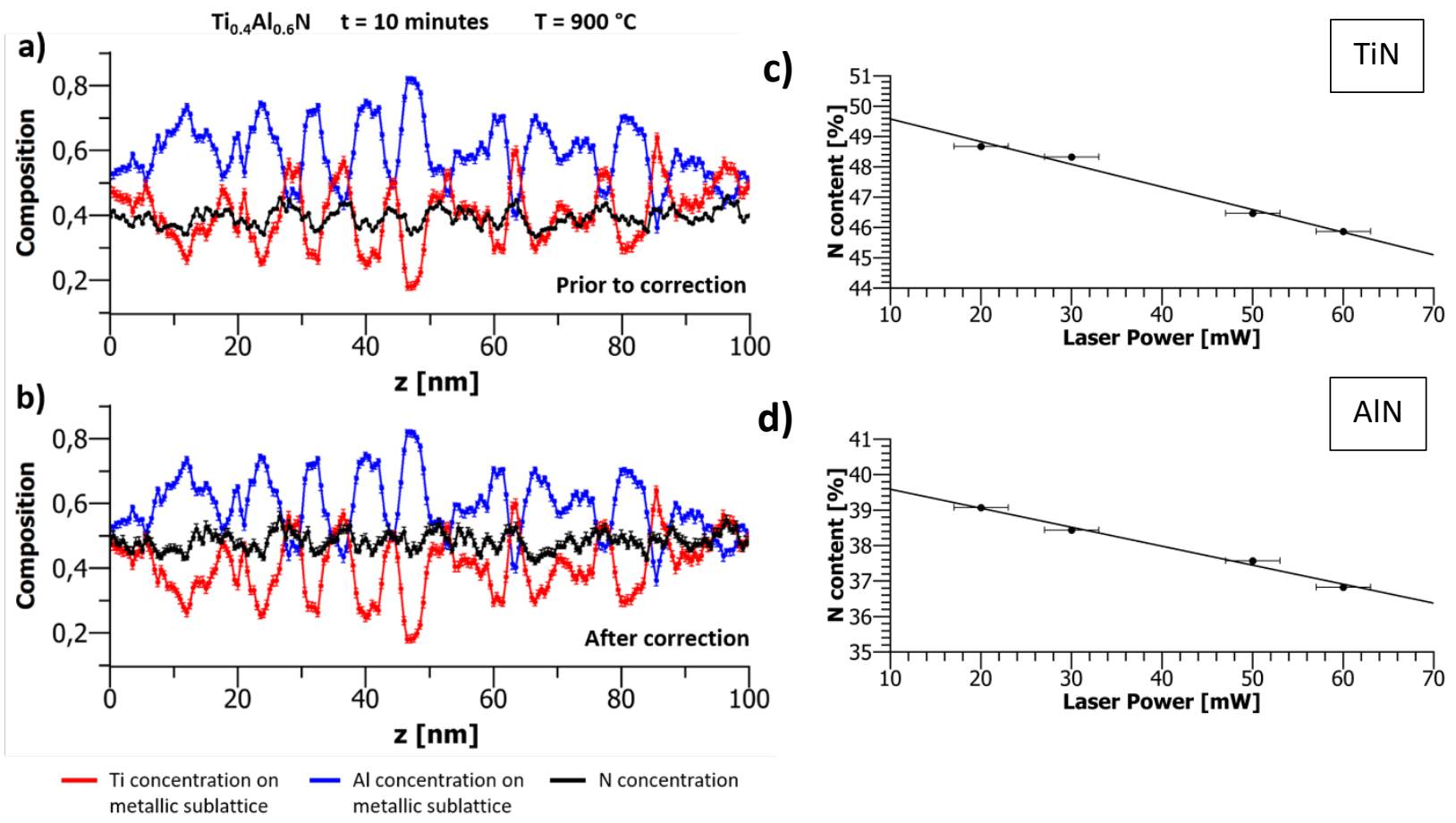

d)

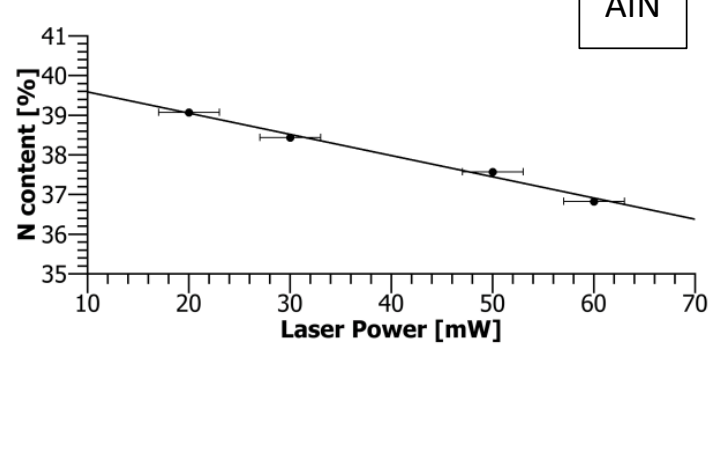

Figure 1: Composition profile of $\mathrm{Ti}_{0.4} \mathrm{Al}_{0.6} \mathrm{~N}$ after inert gas annealing at $900{ }^{\circ} \mathrm{C}$ for 10 minutes. a) prior to correction b) after correction. Measured nitrogen concentration in pure c) TiN and d) AlN as function of laser power. 


\section{APT Study of Boron Doping in FCC Structured High-Entropy Alloys}

Jae Bok Seol ${ }^{1}$, Jae Wung Bae ${ }^{2}$, Jong Chan $\mathrm{Han}^{2}$, Zhiming $\mathrm{Li}^{3}$, Dierk Raabe ${ }^{3}$, and Hyoung Seop $\mathrm{Kim}^{2}$

Corresponding Author: jb_seol@postech.ac.kr

1. National Institute for Nanomaterials Technology, POSTECH, Pohang, South Korea.

2. Department of Materials Science and Engineering, POSTECH, Pohang, South Korea.

3. Max-Planck-Institut für Eisenforschung, Düsseldorf, Germany.

We introduce a new high-entropy alloy (HEA) design approach that is based on compositionally conditioning the grain boundaries instead of the bulk. We found that as little as $30 \mathrm{ppm}$ of boron doping in FCC structured HEAs, more specific in an equiatomic $\mathrm{Fe}_{20} \mathrm{Mn}_{20} \mathrm{Cr}_{20} \mathrm{Co}_{20} \mathrm{Ni}_{20}(\mathrm{at} \%)$ and in a non-equiatomic $\mathrm{Fe}_{40} \mathrm{Mn}_{40} \mathrm{Cr}_{10} \mathrm{Co}_{10}(\mathrm{at} \%)$, improves dramatically their mechanical properties, increasing their yield strength by more than $100 \%$ and ultimate tensile strength by $\sim 40 \%$ at comparable or even better ductility. A combined analysis of transmission electron microscopy with atom probe tomography reveals that solute boron decorates the grain boundaries and acts twofold, through interface strengthening and grain size reduction. Further, boron doping effects on the activation energy for grain growth during recrystallization were estimated.
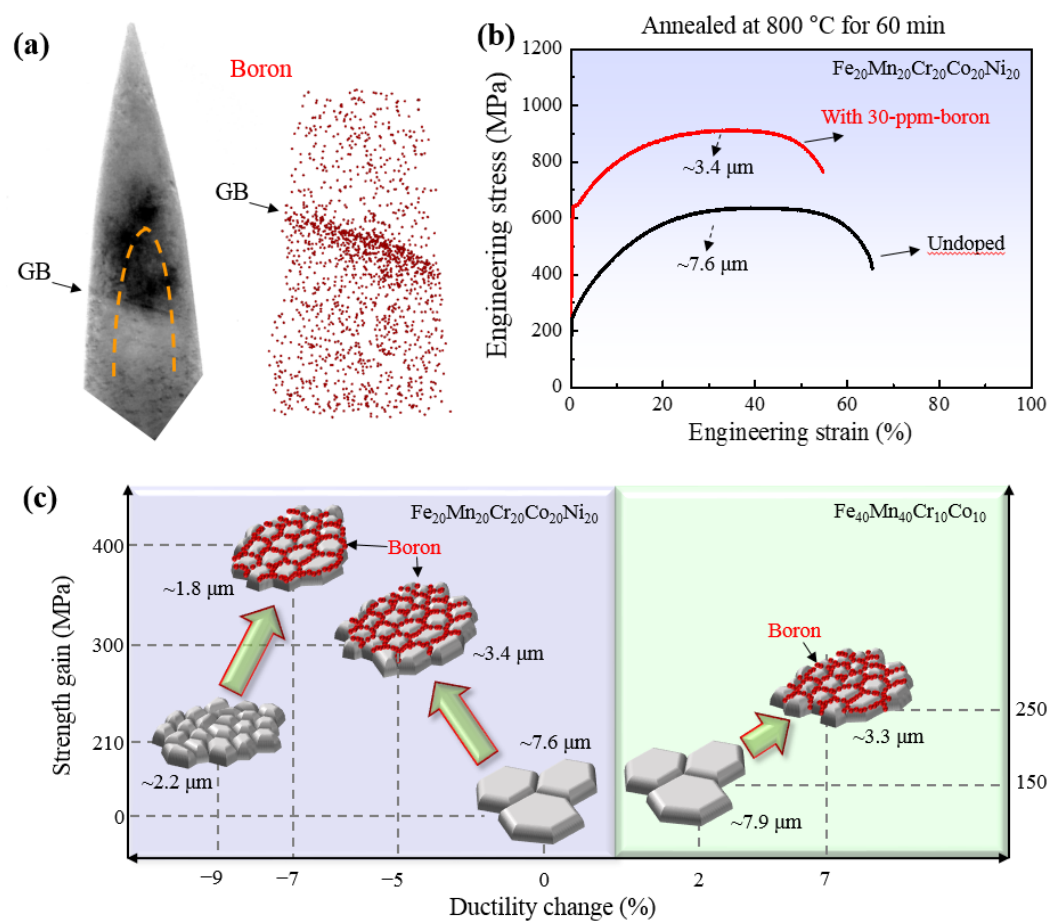

Figure 1 (a) Combined TEM/APT analysis for identification of the exact location of boron in the borondoped $\mathrm{Fe}_{20} \mathrm{Mn}_{20} \mathrm{Cr}_{20} \mathrm{Co}_{20} \mathrm{Ni}_{20}$ HEA. (b) Room-temperature tensile properties of boron-doped and undoped FeMnCrCoNi equiatomic samples. Grain sizes (dotted arrows) of all recrystallized samples are in micrometres. (c) Schematic of the changes of tensile properties due to the grain size stabilization and the boron-enhanced GB cohesion. Reference state is the undoped equiatomic (grain size of $7.6 \mu \mathrm{m}$ ) sample annealed at $800^{\circ} \mathrm{C}$ for $60 \mathrm{~min}$. 


\section{Bulk Metallic Glasses under Atom Probe Microscopy Analysis}

Huma Bilal ${ }^{1,2}$, Bosong $\mathrm{Li}^{3}$, Keita Nomoto ${ }^{1,3}$, Bernd Gludovatz ${ }^{3}$, Simon P. Ringer ${ }^{1,2}$, Jamie J. Kruzic $^{3}$ and Anna Ceguerra ${ }^{1,2}$

Corresponding Author: Huma Bilal, huma.bilal@sydney.edu.au

${ }^{1}$ The University of Sydney. Australian Centre for Microscopy \& Microanalysis, and School of Aerospace, Mechanical and Mechatronic Engineering, Sydney, NSW 2006, Australia.

2 The University of Sydney. The University of Sydney Nano Institute (Sydney Nano), Sydney, NSW 2006, Australia.

${ }^{3}$ School of Mechanical and Manufacturing Engineering, UNSW Sydney, NSW 2052, Australia.

BMGs exhibit a rare combination of strength and toughness that is difficult to achieve by other materials (Fig 1) which makes them favorable for diverse engineering applications like transportation, aerospace, biomedical devices, customised consumer products etc. However, for commercial applications, the reliability of the material and consistency of the targeted properties is very important; here BMGs still lag behind other materials classes making it necessary to improve our understanding of their processing-structure-property relationships. Mainly due to their amorphous nature it is yet not clear how structure links to properties. Recently, it has been suggested that heterogeneous atomic arrangements in the glassy structure control ductility and fracture toughness. However, 3D quantifications of the glassy nanostructures that give good properties have not been obtained yet [1-4].

Without a detailed understanding of the relationships between processing, structure and characteristics of BMGs, it is impossible to achieve improved and repeatable fracture toughness properties. In the discovery of the 3D-structural analysis of BMGs, this poster will present overall contributions of APM and other technologies in this area to date and will additionally highlight limitations and future possibilities using APM techniques.

\section{References:}

[1] B. Gludovatz, S.E. Naleway, R.O. Ritchie, J.J. Kruzic, Sizedependent fracture toughness of bulk metallic glasses, Acta Mater., 70 (2014) 198-207.

[2] B.S. Li, H.S. Shahabi, S. Scudino, J. Eckert, J.J. Kruzic, Designed heterogeneities improve the fracture reliability of a Zr-

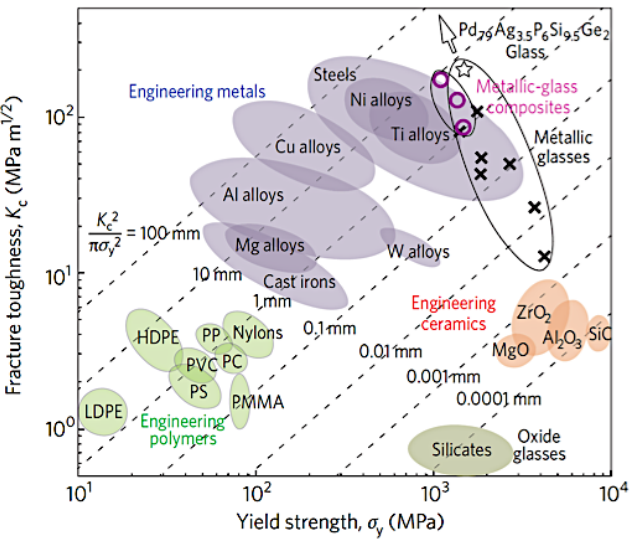

Figure 1:Ashby plot of yield strength vs. fracture toughness showing excellent combinations can be achieved by BMG [5] based bulk metallic glass, Mater. Sci. Eng. A-Struct. Mater. Prop. Microstruct. Process., 646 (2015) 242-8. [3] S.H. Xie, J.J. Kruzic, Cold rolling improves the fracture toughness of a Zr-based bulk metallic glass, J. Alloys Compd., 694 (2017) 1109-20.

[4] B. Gludovatz, D. Granata, K.V.S. Thurston, J.F. Löffler, R.O. Ritchie, On the understanding of the effects of sample size on the variability in fracture toughness of bulk metallic glasses, Acta Mater., 126 (2017) 494-506.

[5] M.D. Demetriou, M.E. Launey, G. Garrett, J.P. Schramm, D.C. Hofmann, W.L. Johnson, R.O. Ritchie, A damage-tolerant glass, Nature Mater., 10 (2011) 123-8. 


\title{
Mapping Carbonates in Enamel and Enamel-Like Carbonated- Hydroxylapatite
}

\author{
Utthara Rameshbabu $^{1}$, Alix Deymier ${ }^{2}$, Jill Pasteris $^{3}$, Derk Joester ${ }^{1}$ \\ Utthara Rameshbabu: utthara@u.northwestern.edu \\ 1. Northwestern University, Materials Sciences and Engineering, Evanston, IL, USA \\ 2. University of Connecticut, Biomedical Engineering, Hartford, CT, USA \\ 3. University of Washington, Department of Earth and Planetary Sciences, St. Louis, MO, USA
}

Dental enamel, a complex naturally-occurring biomineral, makes up the extremely mechanically robust and wear resistant outer layer of teeth. Still, nearly all adults in the world are afflicted by enamel caries, or the acid-demineralization of enamel. To understand and prevent this ubiquitous disease, we must investigate which parts of the enamel structure are especially susceptible to acid dissolution. We do this by probing the structural and chemical characteristics of enamel's underlying microstructure at several length scales.

Enamel is mainly composed of single crystalline hydroxylapatite nanowires, but its solubility and mechanical properties are significantly impacted by trace magnesium, fluoride, and carbonate ion impurities present within and between hydroxylapatite crystallites. Previous work in our group determined the existence of an Mg-enriched amorphous intergranular phase (AIGP) surrounding enamel crystallites using atom-probe tomography (APT). X-ray absorption spectroscopy further suggested the structure of the studied AIGP is analogous to Mg-enriched amorphous calcium phosphate (ACP) which has well-characterized material properties. ${ }^{1}$ Whereas magnesium and fluoride ions have been mapped at sub-micron length scales, carbonate is more difficult to unambiguously detect and quantify on the atomic scale. Another study in our group estimated inorganic carbon concentration in a mouse enamel crystallite by distinguishing it from organic carbon, according to $\mathrm{N}$-containing compound ions in the mass spectrum. ${ }^{2}$ Carbonate remains the most difficult impurity in enamel to quantify and map.

While not straightforward, we believe APT can help deduce the nature of carbonate inclusion in enamel on the crystallite (nanometer) scale, and its effect on enamel properties. We approach this by studying the carbonate signatures of synthetic and biogenic carbonated apatites in addition to enamel. Specifically, we will discuss the sample preparation and APT of 1) carbonated hydroxylapatite nanocrystals synthesized in-vitro, 2) highly mineralized biogenic carbonated apatite in whale rostrum, and 3) human or rat dental enamel.

\section{$\underline{\text { References: }}$}

[1] Gordon LM, Cohen MJ, MacRenaris KW, Pasteris JD, Seda T, Joester D. Amorphous intergranular phases control the properties of rodent tooth enamel. Science.

[2] Gordon LM, Joester D. Mapping residual organics and carbonate at grain boundaries and the amorphous interphase in dental enamel. Frontiers in Physiology. 


\title{
Diamantane Particles in Cryomilled Nanocrystalline Aluminium
}

\author{
Torben Boll ${ }^{1,2}$, Martin Heilmaier ${ }^{1}$, James Earthman ${ }^{3}$
}

Corresponding Author: Torben.Boll@kit.edu

1. Karlsruhe Institute of Technology (KIT), Institute for Applied Materials, Karlsruhe, Germany

2. Karlsruhe Nano Micro Facility, Karlsruhe, Germany

3. University of California, Dept. of Chemical Engineering and Materials Science, Irvine, CA, USA

Diamantane, with the structure formula of $\mathrm{C}_{14} \mathrm{H}_{20}$, exhibits a remarkable chemical and thermal stability in comparison with other carbohydrates. This stability may make Al with embedded diamantane not only a promising material for high strength structural applications [1], [2] but also a good candidate to test atom probe tomography (APT) method modifications aiming for the analysis of biological materials. In particular, diamantane should be an excellent material for APT studies over a large range of different

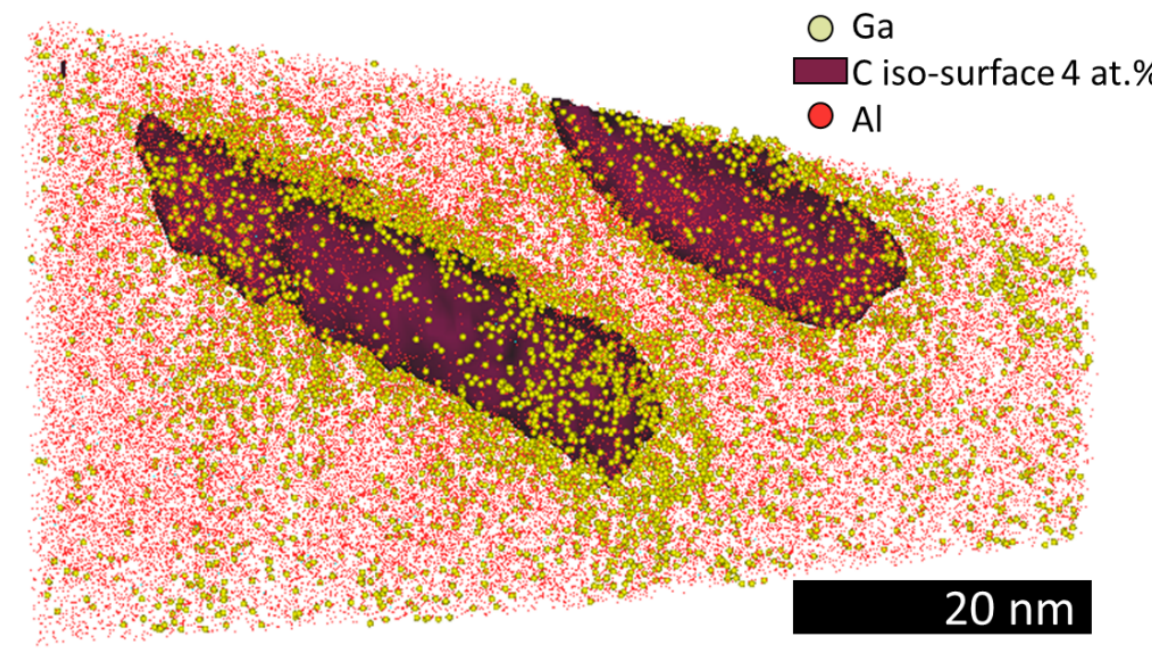

Figure 1 APT of monocrystalline Al with diamantane: The diamantane particles are aligned. Ga ions from the preparation decorate the interface. A grain boundary is not visible. temperatures, laser intensities. It does even allow voltage mode analysis.

Cryomilling of metals is used to reduce the grain size of metals. However, the materials have to be consolidated at high pressures and temperature to obtain a bulk material, causing the grains to grow.

The prepared material was expected to show the typical Hall-Petch behavior and be stronger than the cryomilled material without diamantane due to the smaller grain sizes.

For this study four different samples of cryomilled Al were prepared with (a) no additions (b) diamantane (c) $\mathrm{Mg}$ (d) diamantane $+\mathrm{Mg}$. Differential scanning caliometry (DSC) indirectly shows an influence of diamantane on the grain growth, suggesting that it is located at grain boundaries (GBs). However, to analyze the position of diamantane and the possible effect of $\mathrm{Mg}$, APT is required. While it is difficult to image a non-decorated GB directly (fig. 1), Ga stemming from focused ion beam preparation can be used to identify them. Another path that can also be applied on electrochemically prepared samples is the investigation of the crystallography with spatial distribution maps and Atom Vicinity with crosscorrelation [1], [2] to identify the crystallography of a certain region to indirectly identify a GB.

\section{References:}

[1] T. Boll, Z. Zhu, T. Al-Kassab, and U. Schwingenschlögl, "Atom probe tomography simulations and density functional theory calculations of bonding energies in $\mathrm{Cu}_{3} \mathrm{Au}$.," Microscopy and Microanalysis, vol. 18, no. 5. pp. 964-70, 2012. 


\section{An Analysis of a Unique Prostate Cancer Cell Line Model using 3D Imaging using Atom Probe Tomography}

Michelle R. Friedman ${ }^{1}$, Baishakhi Mazumder ${ }^{2}$, Krishna Rajan ${ }^{3}$, and Alan E. Friedman ${ }^{4}$

Corresponding Author: alanfrie@,buffalo.edu

1. University at Buffalo, School of Engineering and Applied Sciences, Buffalo, NY, USA.

2. University at Buffalo, Department of Materials Design and Innovation, School of Engineering and Applied Sciences, Buffalo, NY, USA.

3. University at Buffalo, Department of Materials Design and Innovation, School of Engineering and Applied Sciences, Buffalo, NY, USA.

4. University at Buffalo, Department of Materials Design and Innovation, School of Engineering and Applied Sciences, Buffalo, NY, USA.

Cancer research struggles with the availability of relevant cellular model systems with adequate physical characterization. Here, we present our unique prostate cancer cell line model system, as characterized using 3D imaging through atom probe tomography (APT). The model system is

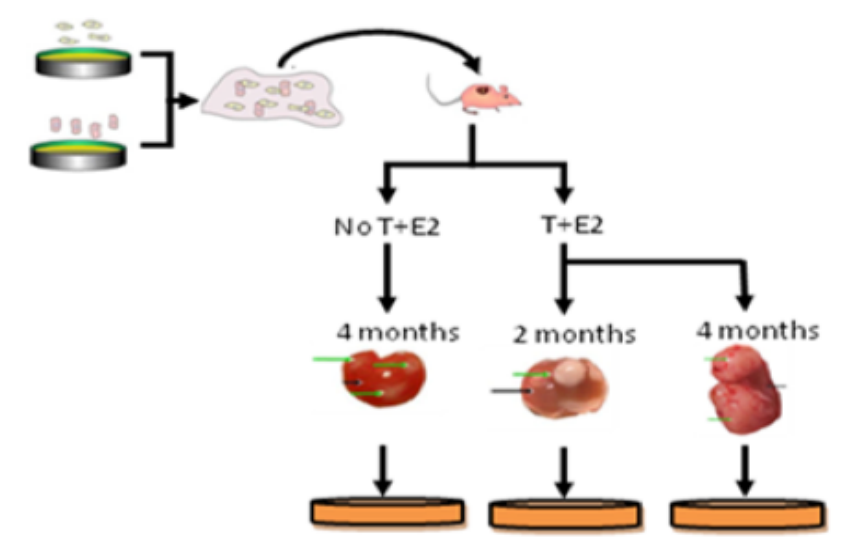

Figure 1. Development of the PHEC cell line model system. PHEC $_{\mathrm{NT}}$ cells (left kidney) remain nontumorigenic even after four months post-kidney zenograph in vivo from athymic mice. The healthy cells originate without hormone treatments, while the cancerous cells come from animals treated with testosterone (T) and estradiol (E2). $\mathrm{PHEC}_{\mathrm{T}}$ (center kidney) are isolated from tumors grown after 2 months in vivo, while $\mathrm{PHEC}_{\mathrm{M}}$ cells are isolated from lymph nodes after 4 months in vivo (right kidney). composed of three isolated cell lines, all called prostate human epithelial cancer (PHEC): non-tumorigenic $\left(\mathrm{PHEC}_{\mathrm{NT}}\right)$, tumorigenic $\left(\mathrm{PHEC}_{\mathrm{T}}\right)$, and metastatic $\left(\mathrm{PHEC}_{\mathrm{M}}\right)$. As the names indicate, these cell lines represent isolated states of human prostate cancer progression. The power of this model system is that the three cell lines not only represent cancer progressing from healthy to metastatic cancer cells, but they are clonal cell lines, indicating that all three originate from the same physiological source. As such, direct comparisons can be made across the three cancer states without concern for normal deviations arising from cell lines arising from different physiological origins. This cancer model has been characterized in the Friedman lab using proteomic and lipidomic methodologies to identify differences in expressed protein concentrations, lipid expression profiles, and protein post-translational modifications. It has also been used as drug testing platform to study the cellular impacts of anti-cancer treatments. In this presentation, we will describe our comparative 3D imaging characterization of the three cell lines. Method development using FIB lift-out and laser-assisted APT will be presented, and results and ramifications for prostate cancer research will be discussed. 


\section{APT measurements of glucose solution tips}

Jonas Ott, Patrick Stender, Guido Schmitz

Corresponding Author: jonas.ott@mp.imw.uni-stuttgart.de

Institute of Materials Science, University of Stuttgart, Stuttgart, Germany

Still after introduction of laser-assisted field evaporation, APT is somewhat restricted in the materials that allow reasonable measurements, which is related to issues such as the ease of sample preparation or the homogeneity of the field desorption. Nearly all atom probe measurements, which were done so far, address well solid materials, only rarely organics. This is mainly reasoned by the difficult often cryogenic preparation of APT tips out of organic liquids.

We developed a preparation protocol for the tip production out of frozen liquids by first tailoring the wetting surface of the support wires. After positioning a liquid droplet to the apex of these supports, tipshaping is performed in the conventional way by FIB milling.

Tips of different purified scientific sugar solutions were measured in a cryo-atom probe. As typical for the field desorption of soft matter, the mass spectra are complex containing large molecular fractions. The comparison of the well-defined initial glucose molecules and the corresponding mass spectrum enables studies of molecular defragmentation in high electric fields. The dependence of the observed molecular fractions on the supplied fields are discussed.
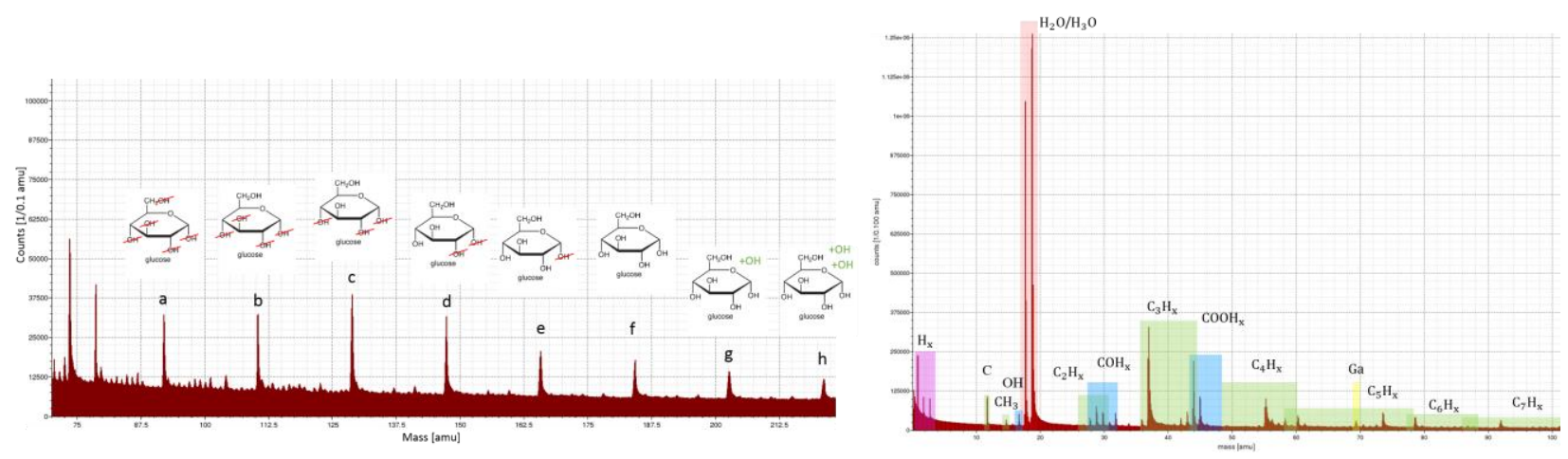


\section{Atomprobe Analysis of Nanoscale Voids in p-type ZnO Nanowires}

Jun Uzuhashi ${ }^{1}$, Ken C. Pradel ${ }^{2}$, Tadakatsu Ohkubo ${ }^{1}$, Naoki Fukata ${ }^{2}$ and Kazuhiro Hono ${ }^{1}$ Corresponding Author: UZUHASHI.Jun@,nims.go.jp

1. Research Center for Magnetic and Spintronic Materials, National Institute for Materials Science, 1-2-1 Sengen, Tsukuba, Ibaraki, 305-0047, Japan.

2. International Center for Materials Nanoarchitectonics, National Institute for Materials Science, 1-1 Namiki, Tsukuba, Ibaraki, 305-0044, Japan.

It was recently discovered that $\mathrm{Sb}$ doping can lead to stable p-type $\mathrm{ZnO}$, but one side effect of the doping is the formation of voids inside the nanowires. In this work, the microstructure of nanoscale voids in $\mathrm{Sb}$ doped p-type $\mathrm{ZnO}$ nanowires was investigated using APT and TEM.

APT specimens of the $\mathrm{ZnO}$ nanowires were prepared using the lift-out method in FEI Helios NanoLab 650 as shown in Figure 1-(a). Specimens were analyzed by laser assisted atomprobe using $400 \mathrm{fs}$ femto-second laser pulses at $100 \mathrm{kHz}$.

In Figure 1-(b), low-density areas can be seen, which correspond to the voids. The Sb atoms are concentrated in the low-density areas as shown in Figure 1-(c). This Sb distribution is consistent with the $\mathrm{Zn}_{7} \mathrm{Sb}_{2} \mathrm{O}_{12}$ phase observed in HAADF-STEM image and Nano-beam electron diffraction (NBD) shown in Figure 1-(d).
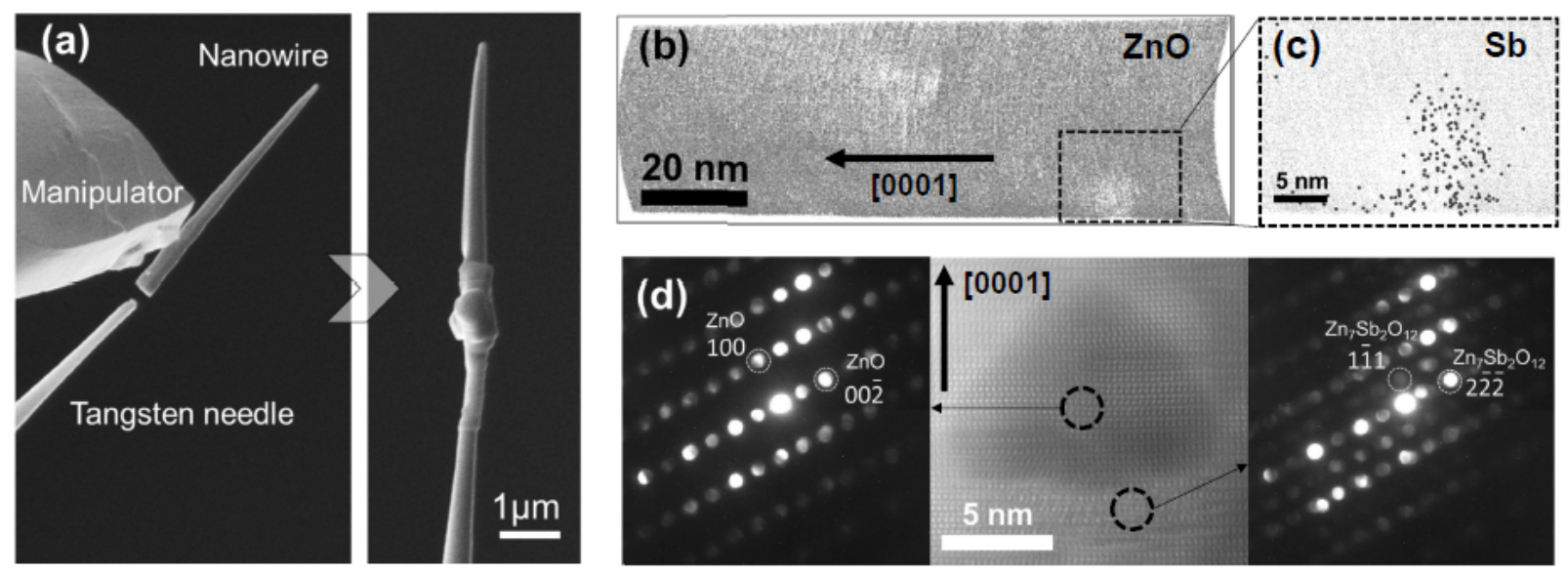

Figure 1 (a) APT specimen preparation. (b) Sliced Zn and O atom map. (c) Zoomed in view of a lowdensity area with the $\mathrm{Sb}$ ions enlarged. (d) HAADF-STEM image of a void and NBD pattern of the void and the phase underneath the void.

References:

[1] Ken C. Pradel, Jun Uzuhashi, Toshiaki Takei, Tadakatsu Ohkubo, Kazuhiro Hono, Naoki Fukata, Investigation of Nanoscale Voids in Sb-doped p-Type ZnO Nanowires, submitted. 


\section{Microstructural investigation of an Fe-Ni meteorite by Complementary APT and 3D Energy Dispersive Spectroscopy}

Joshua F. Einsle ${ }^{1,2}$, Alex Eggeman ${ }^{2}$, Benjamin H. Martineau ${ }^{2}$, Zineb Saghi ${ }^{3}$, Sean M. Collins ${ }^{2}$, Roberts Blukis ${ }^{1}$, Paul A.J. Bagot ${ }^{4}$, Paul Midgley ${ }^{2}$, and Richard J. Harrison ${ }^{1}$

Corresponding Author: paul.bagot@materials.ox.ac.uk

1. University of Cambridge, Department of Earth Sciences, Downing Street, Cambridge, CB2 3EQ, UK

2. University of Cambridge, Department of Materials Science and Metallurgy, Cambridge, CB3 0FS, UK

3. CEA, LETI, MINATEC Campus, F-38054 Grenoble, France

4. University of Oxford, Department of Materials, Oxford, OX1 3PH, UK

Fe-Ni meteorites were formed in the first few million years of our solar system, driven by slow cooling rates $\left(10^{-1}-10^{3}{ }^{\circ} \mathrm{C} /\right.$ million years $)$ that generate a distinctive range of intricate microstructural features. Of current interest are so-called "cloudy zones"; these are nanoscale, ordered Ni-rich (tetrataenite) islands present within an Fe-rich matrix. The island sizes and Ni concentration gradients provide a unique insight into both the thermal and in particular magnetic history of the meteorites. The latter is recorded by the orientation of these ordered tetrataenite islands, which range in sizes from $\sim 10-150 \mathrm{~nm}$ in diameter. Although the structure of such features in recovered meteorites has been intensively studied, uncertainty remains over composition, crystallography, magnetism and how these interrelate. To make progress, highresolution characterization techniques are vital; in this work we report on a detailed multitechnique study of the 3D architecture in a cloudy zone region in a Tazewell IIICD Fe meteorite, combining in particular 3D Electron Dispersion Spectroscopy (3D-EDS) with APT. An in-depth examination of the phase chemistries in the cloudy zone features will be presented, highlighting the formation of the tetrataenite islands in an $\mathrm{Fe}_{3} \mathrm{Ni}$ structure, which has not been seen before in nature. The relative strengths of the different techniques, along with supporting STEM-HAADF and FIB work are also discussed, along with implications for both meteorite formation and for producing novel magnetic materials.

\section{$\mathrm{Fe}$}

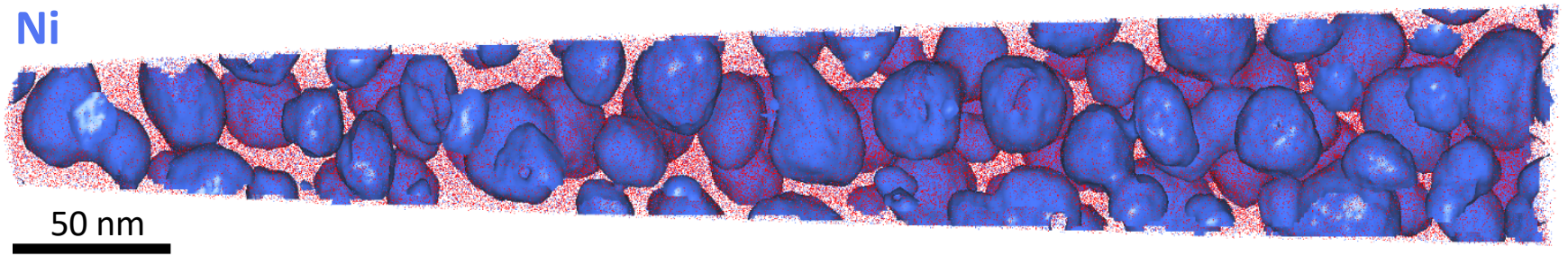

Figure 1 Atom Map of cloudy zone within Fe-Ni meteorite, revealing Ni-rich tetrataenite nanoislands (highlighted by $32.5 \% \mathrm{Ni}$ isoconcentration surface) dispersed inside an Fe-rich matrix. 


\section{Correlative Atom Probe Tomography and In-Situ Scanning Photoelectron Emission Microscopy Applied to Catalytically Active Materials}

Kevin Schweinar ${ }^{1}$, Sebastian Beeg ${ }^{2}$, Philipp Kerger ${ }^{3}$, Caue Correa da Silva ${ }^{3}$, Jing Cao ${ }^{4}$, Matteo Amati $^{5}$, Luca Gregoratti ${ }^{5}$, Patrick Zeller ${ }^{5}$, Dierk Raabe ${ }^{1}$, Robert Schlögl ${ }^{2,4}$, Mark Greiner ${ }^{2}$ and Baptiste Gault ${ }^{1}$

Corresponding Author: k.schweinar@mpie.de

1. Max-Planck-Institut für Eisenforschung GmbH, Microstructure Physics and Alloy Design, Düsseldorf, Germany.

2. Max-Planck-Institut für chemische Energiekonversion, Heterogeneous Reactions, Mülheim

a.d.R., Germany.

3. Max-Planck-Institut für Eisenforschung GmbH, Interface Chemistry and Surface Engineering, Düsseldorf, Germany.

4. Fritz-Haber-Institut, Inorganic Chemistry, Berlin, Germany.

5. Sincrotrone Trieste, Escamicroscopy beamline, Basovizza-Trieste, Italy.

Materials used in many heterogeneous catalytic reactions are exposed to conditions that inevitably lead to microstructural and chemical changes in the bulk and on the surface. To understand their complex interplay and influence on the catalyst's performance, one requires spatially resolved methods that encompass surface and bulk sensitivity on the nanoscale, ideally in-operando.

Here, we combine synchrotron-based in-situ scanning photoelectron microscopy (SPEM) and atom probe tomography (APT) in correlative microscopy experiments. We demonstrate the use of the newly developed near-ambient pressure cell, available at the Escamicroscopy beamline of the Elettra synchrotron facility, to probe the chemical and electronic properties of a catalyst's surface under reaction conditions. Based on the findings from spectroscopy, region of interest are selected for post-reaction APT and further analysis of the microstructure and solute distribution at crystalline defects, including e.g. grain boundaries (GB). In-depth analysis of solute segregation to GBs and related formation of enriched or depleted zones along those in combination with surface-sensitive in-situ spectroscopy helps to understand the heterogeneous surface dynamics on an initially homogeneous material.

We show examples from our pilot study on a polycrystalline, bulk binary $\mathrm{AgCu}$ alloy catalyst in ethylene epoxidation conditions at $450^{\circ} \mathrm{C}$ and $0.1 \mathrm{mbar}$ total pressure. Prior to the reaction, grain boundary character and grain orientation were determined using electron backscatter diffraction (EBSD). Spectroscopy suggests pronounced GB segregation of $\mathrm{Cu}$ and subsequent oxidation under reaction conditions. FIB-lift-outs for APT are prepared on GBs with varying spectroscopic character and different degrees of grain misorientation to investigate solute element distribution across and along those most important microstructural features.

\section{$\underline{\text { References: }}$}

[1] Funding through the International Max-Planck Research School IMPRS-SURMAT is acknowledged. 


\title{
Complementary Studies of Additively Manufactured Stainless Steel Using Transmission Electron Microscopy and Atom Probe Microscopy
}

\author{
Robert A. Morris and Karen T. Henry \\ Corresponding Author: robert.morris@unnpp.gov \\ Bechtel Marine Propulsion Corporation, Schenectady, NY, USA.
}

The ability to manufacture complex three-dimensional components on-demand has become increasingly attractive to the aerospace [1] and medical industries [2]. Additive manufacturing (AM) capabilities such as free-form welding, ultrasonic joining, powder bed fusion, and laser engineered net shaping are a few sub-categories [3] that allow for the layer upon layer build-up of material to form complex geometric features. As with any new process technology, a fundamental understanding of how processing impacts structure and properties is needed before implementation.

Multiple characterization techniques over many length scales are required to build the baseline of properties for AM components. A laser ablation system has allowed for the custom design of sample carriers/grids for the purpose of complementary microscopy studies. The sample carrier shown in Figure 1(a) is easily and readily inserted into a focused ion beam (FIB) system, transmission electron microscope (TEM) or atom probe microscope (APM). In Figure 1(b), a TEM micrograph is shown next to APM concentration maps obtained from the same sample. Pairing these complementary techniques improves visualization of the fine precipitates dispersed within the matrix material and aids in correlating the compositional changes with microstructural features. This presentation will discuss how complementary techniques over several length scales are utilized in the early stages of design to understand how structure and composition play a role in the properties of AM components.

(a)

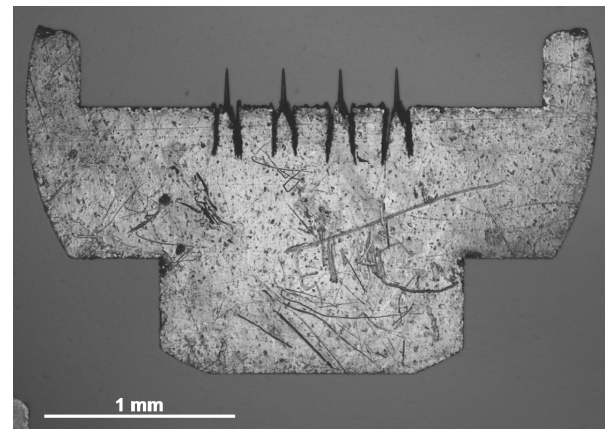

(b)

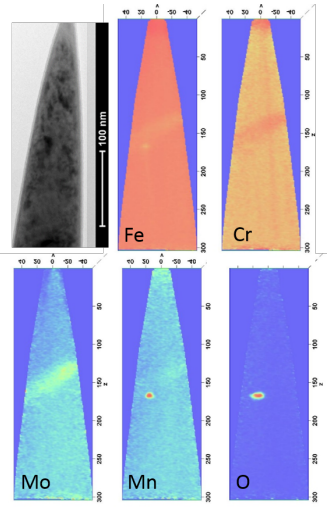

Figure 1 (a) Example of a laser cut sample carrier (b) TEM micrograph along with APM concentration maps for the elements of $\mathrm{Fe}, \mathrm{Cr}$, Mo, $\mathrm{Mn}$, and $\mathrm{O}$.

\section{References:}

[1] L.J. Kumar, C.G. Krishnadas Nair, Current Trends of additive manufacturing in the aerospace industry. In: Advances in 3D printing and additive manufacturing technologies. Spring, Singapore, pp. $39-54$

[2] I. Gibson, D. Rosen, B. Stucker, Medical application for additive manufacturing. In: Additive manufacturing technologies. Springer, Boston MA, pp. 400-414

[3] J.O. Milewski, Additive Manufacturing of Metals, Springer Series in Material Science, Vol 258, Springer, Cham, 2017 


\section{Grain Boundary Segregation in Inconel 718 Superalloy}

Katherine P. Rice ${ }^{1}$, David J. Larson ${ }^{1}$, Yimeng Chen ${ }^{1}$, Niyanth Sridharan ${ }^{2}$ and Suresh Babu ${ }^{2,3}$

Corresponding Author: katherine.rice@ametek.com

1. CAMECA Instruments Inc., 5470 Nobel Dr., Madison WI 53711, USA.

2. Oak Ridge National Laboratory, Oak Ridge, TN, USA.

3. Department of Mechanical, Aerospace, and Biomedical Engineering, University of Tennessee, Knoxville, TN 37996, USA.

Complementary techniques, such as electron backscatter diffraction (EBSD) help inform atom probe tomography (APT) reconstructions, as well as providing additional information that can be used to provide insight into materials science characterization questions. In this work, grain boundary chemistry of solution-annealed Inconel 718 is studied using correlative techniques.

The $\Sigma 3, \Sigma 9$, and $\Sigma 27 a$ grain boundaries in Inconel 718 are identified through large area EBSD mapping and are prepared into needle-shaped atom probe specimens using a focused ion beam. Transmission EBSD was used to accurately locate grain boundaries within $\sim 50 \mathrm{~nm}$ of the tip apex [1]. APT data acquired on EIKOS ${ }^{\mathrm{TM}}$ shows no detectable segregation to the $\Sigma 3$ grain boundary, which is revealed by an increase in atomic density in the APT reconstruction. Boron segregates to the higher energy $\Sigma 9$ and $\Sigma 27 a$ grain boundaries, which are clearly observed. This result shows strong relationship regarding segregation behavior and grain boundary type [2].

The microstructure of additively-manufactured Inconel 718 is more complicated. It is strongly affected but the manufacturing condition. Atom probe data acquired by EIKOS ${ }^{\mathrm{TM}}$ shows the presence of large precipitates in Inconel 718 manufactured by electron beam deposition. Pulsed laser deposition manufacturing leads to a uniform polycrystalline structure [3].

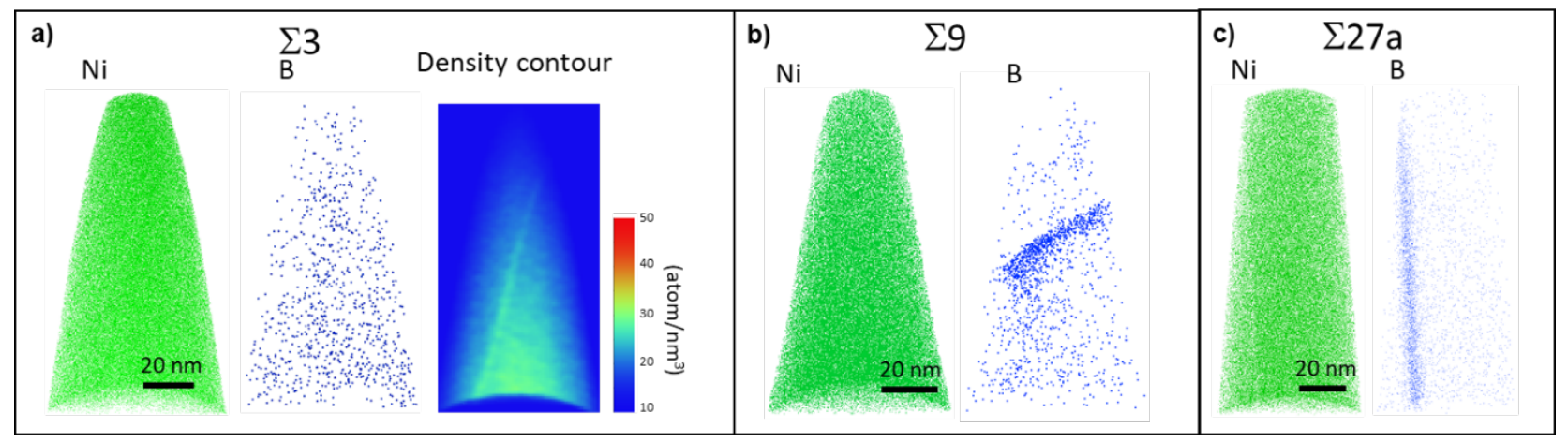

Figure 1 (a) Ni and B ion maps and density contour plot of a $\Sigma 3$ grain boundary in Inconel 718. (b) $\mathrm{Ni}$ and B ion maps of a $\Sigma 9$ grain boundary in Inconel 718, c) Ni and B ion maps of a $\Sigma 27 a$ grain boundary in Inconel 718.

$\underline{\text { References: }}$

[1] Rice, K.P., Chen, Y., Prosa, T.J., Larson, D.J., Implementing Transmission Electron Backscatter Diffraction for Atom Probe Tomography. Microscopy and Microanalysis 22 (2016), 583-588.

[3] Rohrer, G.S., Grain boundary energy anisotropy: a review, J. Mater. Sci. 46 (2011), 5881-5895.

[3] Larson, D.J. et al., Microstructural Investigations in Metals Using Atom Probe Tomography with a Novel Specimen-Electrode Geometry, Journal of Metals (2018), in press. 


\section{Materials Characterization with Atom Probe Tomography and High Resolution Transmission Electron Microscopy}

Francisca Mendez-Martin ${ }^{1}$, Georg Haberfehlner ${ }^{2}$, Mihaela Albu $^{2}$

Corresponding Author: mendez@unileoben.ac.at

1. Chair of Physical Metallurgy and Metallic Materials, Montanuniversitaet Leoben, Leoben, Austria.

2. Graz Centre for Electron Microscopy, Graz, Austria.

By working with atom probe tomography (APT) and doing the data analysis some issues have to be consider: local magnifications, tip geometry, complex evaporation processes, reconstructions artifacts or non-uniform evaporation by multiphase systems with different evaporation fields [1]. Moreover, by combining APT and scanning transmission electron microscopy (STEM) information on chemical composition and crystal structure respectively, can be correlated. In order to overcome these problems and to get the maximal information for the material characterization here we compare the results of the first investigations from different alloys systems by the application of APT together with STEM.

\section{$\underline{\text { References: }}$}

[1] I. Arslan, E. A. Marquis, M. Homer, M. A. Hekmaty and N. C. Bartelt, Towards better 3-D reconstructions by combining electron tomography and atom-probe tomography, Ultramicroscopy 108 (2008), 1579- 1585. 


\section{Correlative Study of the Optical Shaping of a Nano-Scale Tip by Femtosecond Laser Assisted Field Evaporation}

E. Di Russo ${ }^{1}$, J. Houard ${ }^{1}$, V. Langolff ${ }^{1}$, S. Moldovan ${ }^{1}$, L. Rigutti ${ }^{1}$, B. Deconihout ${ }^{1}$, D. Blavette ${ }^{1}$, J. Bogdanowicz ${ }^{2}$ and A. Vella ${ }^{1}$

Corresponding Author: enrico.di-russo1@etu.univ-rouen.fr

1. Normandie Univ., UNIROUEN, Groupe de Physique des Matériaux, 76000 Rouen, France.

2. IMEC, Kapeldreef 75, B-3000 Leuven, Belgium

The interaction between a linearly-polarized femtosecond UV laser and a GaN atom probe tip was investigated [1]. It is shown that both symmetry and local radius of the tip change during the laser-assisted field evaporation of the tip changing the orientation of the laser electric field $F_{\text {laser }}$ (i.e. the angle $\theta$ represented in fig. a). These morphological variations were pointed out performing correlative Scanning Transmission Electron Microscopy (STEM) and Laser-assisted Atom Probe Tomography (La-APT). This advanced approach allows pointing out variation of the tip features following the field evaporation of single atoms [2]. Numerical calculations show the three dimensional thermal response of a GaN tip changing the orientation of $F_{\text {laser }}$ (fig. b) and corroborate the experimental observations [3]. Furthermore, the presented study introduces a new method to reshape nano-tips at the nanometer scale.

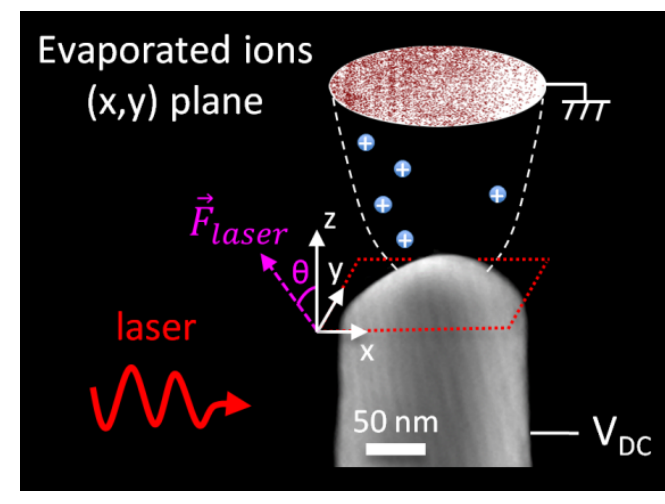

(a)

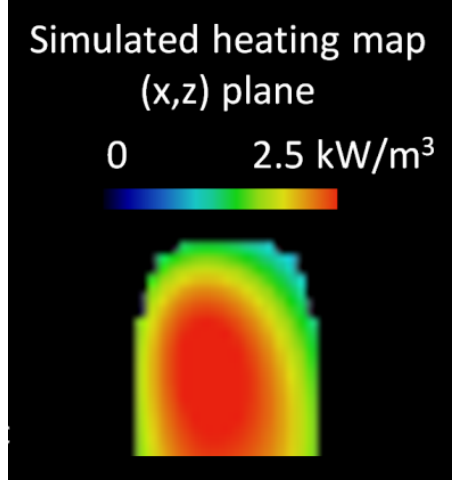

(b)

Figure (a) Schematic representation of La-APT set-up. (b) (x,z) cross section of heating maps taking into account the ballistic motion of carriers for a polarization angle $\theta=90^{\circ}$.

\section{References:}

[1] Gault, B., et al., Rev. Sci. Instrum. 77, 043705 (2006).

[2] S. Koelling et al., J. Appl. Phys. 109, 104909 (2011).

[3] J. Houard et al., Phys. Rev. B 84, 033405 (2011). 


\section{High Resolution Multimodal Compositional Characterization of Nanocrystalline Soft Magnetic Materials}

Trevor Clark ${ }^{1,2}$, Nicole Overman ${ }^{3}$, Xiujuan Jiang ${ }^{3}$, Suveen Mathaudhu², and Arun Devaraj ${ }^{1}$ Corresponding Author: arun.devaraj@pnnl.gov

1. Physical and Computational Sciences Directorate, Pacific Northwest National Laboratory, Richland, WA 99352, USA

2. Mechanical Engineering, University of California, Riverside, Riverside, CA 92501, USA

3. Energy and Environmental Directorate, Pacific Northwest National Laboratory, Richland, WA 99352, USA

The unique coercivity behavior observed in amorphous and nanocrystalline soft magnetic materials can be understood through the random anisotropy model [1] to be dependent on grain size. However, the model fails to address other structure sensitive properties such as permeability, and domain wall motion which can be affected by local anisotropy variations due to compositional variation or defects. Because of the convolution of magnetic and structural information in transmission electron microscopy, understanding of how magnetic domains interact within complex nanostructured bulk samples with many types of defect interactions has proven difficult to probe. Here, an amorphous/nanocrystalline Fe based alloy with a rich variety of defects has been synthesized. Mossbauer studies [2] indicate that solute content influences the number of Fe nearest neighbor and next nearest neighbor interactions which effect local magnetic field and crystal structure. High resolution chemical mapping and correlated structural information is needed to gain insight on fundamental mechanisms of domain-defect interactions. Aberration-corrected scanning transmission electron microscopy, Lorentz transmission electron microscopy and atom probe tomography analysis methods are used to correlate magnetic domain behavior with specific nanoscale defects and features. The broader implication of this work is to make possible the exploration of fundamental physical interactions between nanoscale defects (such as grain boundaries, solute segregation, dislocations and voids) and the saturation magnetization, permeability and coercivity in novel bulk Fe based nanostructured soft- magnets. This knowledge could be harnessed to enable unprecedented improvements in magnetic properties and performance.

\section{References:}

[1] Giselher Herzer, “Modern soft magnets: Amorphous and nanocrystalline materials”, Acta Materialia, 61 (2013) 718-734.

[2] Nicole R. Overman, Xiujuan Jiang, Ravi K. Kukkadapu, Trevor Clark, Timothy J. Roosendaal, Gregory Coffey, Jeffrey E. Shield, Suveen N. Mathaudhu, "Physical and electrical properties of melt-spun Fe-Si (3-8 wt.\%) soft magnetic ribbons”, Materials Characterization 136 (2018) 212-220. 


\title{
Work Function Reduction due to Water Adsorption on Nanostructured Materials Studied by Electron Field Emission
}

\author{
Stefanie Haugg ${ }^{1}$, Christian Henkel ${ }^{1}$, Chris Thomason ${ }^{1}$, Robert Zierold ${ }^{1}$ and Robert H. Blick ${ }^{1}$ \\ Corresponding Author: shaugg@physnet.uni-hamburg.de \\ 1. Center for Hybrid Nanostructures (CHyN), Universität Hamburg, Hamburg, Germany.
}

Nanostructured materials provide a large area for chemical reaction with vaporized water molecules due to their great surface-to-volume ratio. Especially, structural imperfections enable stronger interactions between a water molecule and surface atoms, causing water to be trapped at defect sides of nanostructured samples. Depending on the electronic structure of the bulk material, the water molecule can act as charge donor, causing a negative change in work function. [1]

In this work, the effect of water adsorption on the work function of nanostructured metallic and semiconducting materials was studied with field emission measurements.

We observed a change in onset voltage for electron emission from different materials, comparing the first measurement of a sample, which was stored under ambient conditions before, and a subsequent measurement. It is assumed that desorption of water molecules from the nanostructured surface takes place under the applied electric field during the first measurement and no readsorption occurs before the following examination, since the sample is kept in high vacuum. The observed modifications are dedicated to water molecules affecting the materials electronic structure. Our result for the metal-covered sample is in good agreement with observations made by other scientists, which found a work function reduction of about $60 \%$ due to the interaction of water with metal. [2]
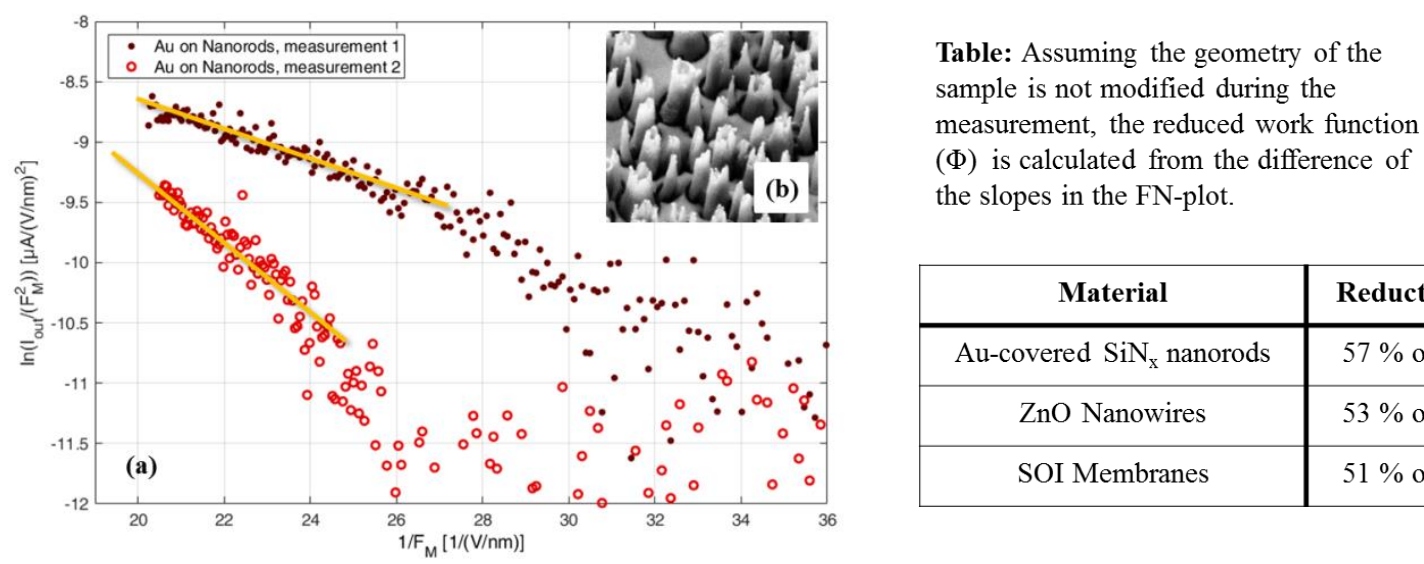

Table: Assuming the geometry of the sample is not modified during the measurement, the reduced work function $(\Phi)$ is calculated from the difference of the slopes in the FN-plot.

\begin{tabular}{|c|c|}
\hline Material & Reduction of $\boldsymbol{\Phi}$ \\
\hline Au-covered $\operatorname{SiN}_{\mathrm{x}}$ nanorods & $57 \%$ of $\Phi_{\text {vacuum }}$ \\
\hline ZnO Nanowires & $53 \%$ of $\Phi_{\text {vacuum }}$ \\
\hline SOI Membranes & $51 \%$ of $\Phi_{\text {vacuum }}$ \\
\hline
\end{tabular}

Figure: (a) Fowler-Nordheim plot of two subsequent measurements with Au-covered silicon nitride nanorods. (b) The inset shows a SEM image of the examined structure.

\section{References:}

[1] P. Thiel, et al., The interaction of water with solid surfaces: Fundamental aspects, Surface Science Reports 7 (1987), 211-385.

[2] F. Musumeci, et al., Influence of water on the work function of certain metals, Chemical Physics Letters 536 (2012), 65-67.

[3] We thank the SFB 986 for financial support. 


\section{Characterization of Multiwall Carbon Nanotubes as a Potential Electron Source}

Marwan S. Mousa ${ }^{1}$, Emad S. Bani Ali ${ }^{1}$, and Mark J. Hagmann ${ }^{2}$

Corresponding Author: marwansmousa@yahoo.com

1. Surface Physics and Materials Technology Lab, Department of Physics, Mu'tah University, AL-Karak, Jordan.

2. University of Utah, Dept. Electrical and Computer Engineering, Salt Lake City, Utah, USA, 84112.

Nanocyl $^{\mathrm{TM}}$ NC 7000 Thin Multiwall Carbon Nanotubes, produced by chemical vapor deposition, were employed in this study. To produce carbon nanotubes (CNT) electron source, the emitters were fabricated using a technique for pulling heated glass capillary tubes into fine points [1,2,3] with its sharp points broken the carbon nanotubes pushed in glass tubes up to the tip making them the protruding tip.

Tapered Pyrex capillary tubes were used to compress multiple multiwall carbon nanotubes when forming a field emitter. This technique does not require the addition of pitch, Epoxy, or other materials which would increase the electrical resistance and thermal resistance and thus lower the maximum current. The distance from the CNT to the tin-oxide coated phosphorus glass anode was standardized at $10 \mathrm{~mm}$. The I-V characteristics were recorded with Fowler-Nordheim plots obtained.

The emission current initially obtained was $0.35 \mu \mathrm{A}$ at applied voltage $2350 \mathrm{~V}$. The field electron emission currents recorded was suddenly increased (switched-on) to the range of $50 \mu \mathrm{A}$ without requiring coatings or binders. Stability measurements for such tip over 40 minutes period showed single and stable concentrated spot on the conductive screen.

Other surface treatment procedures such as thermal treatment at elevated temperatures had little effect on the emission characteristics of this electron source [3].

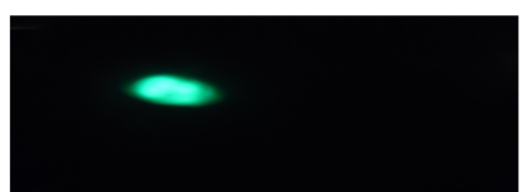

(a)

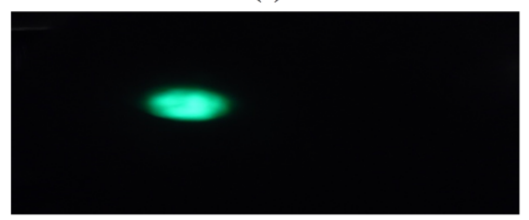

(c)

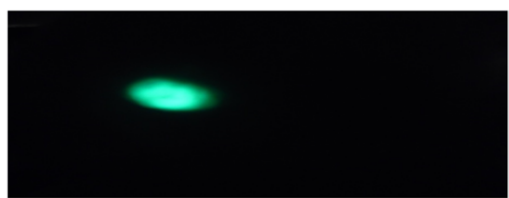

(b)

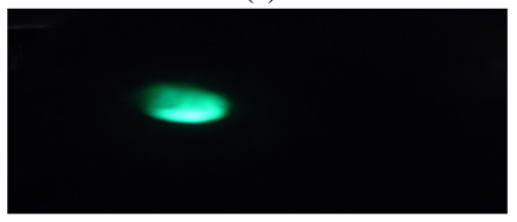

(d)
Figure 1 A sequence of projection images produced of actual size showing temporal stability of the EE for MWCNTs tip (A2) and was recorded at a 10 minutes separation at $V=2350 \mathrm{~V}, I=$ $3.5 \times 10^{-7} \mathrm{~A}$.

$\underline{\text { References: }}$

[1] M.S. Mousa, Surf. Sci. 246, (1996) 79.

[2] M.S. Mousa, Surf. Int. And Analysis 39, (2007) 102.

[3] E.S. Bani Ali, Applied Microscopy, 46, (2016) 244. 


\title{
Field Emission Differences Between Carbon-Black Nanotubes and Single- Walled Carbon Nanotubes, Both Embedded in Glass Tubes
}

\author{
Marwan S. Mousa ${ }^{1}$, Ala'a M. Al-Qudah ${ }^{1}$, Samer I. Daradkeh ${ }^{1}$, Hatem A. Braikat ${ }^{1}$, and
} M-Ali H. Al-Akhras ${ }^{2}$

Corresponding Author: marwansmousa@yahoo.com

1. Surface Physics and Materials Technology Lab, Department of Physics, Mu'tah University, AL-Karak, Jordan.

2. Dept. of Physics, Jordan University of Science and Technology, Irbid, Jordan.

In this study, the field electron emission (FE) properties of carbon-black nanotubes and singlewalled carbon nanotubes (SWCNTs) were studied under ultra-high vacuum conditions. Our carbon black nanotubes were from the Cabot Corporation, USA. Our SWCNTs, from the Carbon Nanotechnology Institution, USA, were produced by catalytic conversion of high-pressure CO over Fe particles.

The carbon black nanotubes and SWCNTs were mounted in tapered Pyrex ${ }^{\mathrm{TM}}$ tubes having an inside diameter of $0.1 \mathrm{~mm}$ and an outside diameter of $1.0 \mathrm{~mm}$, in order to provide physical support for the emitters. The pyrex tubes were then mounted in a traditional field electron microscope (FEM) physical configuration. The study included current-voltage characteristics, Fowler-Nordheim (FN) plots, and FEM images.

All tests were performed were under equivalent conditions. We conclude that the main difference between FE from SWCNTs and FE from carbon-black nanotubes is that the emission current obtained from the SWCNTs is initiated at much lower applied voltage values than that from the carbon black nanotubes. On the other hand, emission-current values from SWCNTs were much lower than those from the carbon-black nanotubes. The carbon-black nanotubes have linear FN plots, whereas those obtained from SWCNTs show deviation from linearity. The FEM images from the carbon-black nanotubes consist of a single image spot, and are generally smaller and brighter than those from SWCNTs. 


\section{A Proposal That Constants Used Widely in Electron and Ion Emission Theory Should Be Included in International Listings of Universal Constants}

Richard G. Forbes ${ }^{1}$

Corresponding Author: r.forbes@trinity.cantab.net

1. Advanced Technology Institute, University of Surrey, Guildford, Surrey GU2 7XH, UK

Theories of electron and/or ion emission contain a number of "special" universal constants defined in terms of the fundamental scientific constants. Some combinations denoted by these special constants have been regularly used since modern emission theory emerged in 1927/28, although the idea of representing them by a single named symbol came much later.

Given the location of this year's APT\&M meeting, it seems timely to suggest that the more important of these "special constants" should be included in international listings of universal constants, such as that made available on-line by the National Institute of Standards and Technology (NIST). Table 1 below shows some of constants used in field electron emission theory (and the constant $b$ is also used in field ionization theory). It can be seen that several combinations involve fundamental constants and one or both of two "primary universal constants" ( $\mathrm{e}$ and $z_{\mathrm{S}}$ ) associated, respectively, with the Schrödinger equation for electrons and with the statistical mechanical principle that "the electron supply density is constant in energy space, when all relevant states are fully occupied".

The FN constants $a$ and $b$ seem strong candidates for listing, as they are beginning to be regularly used in the literature (though several different notations are in use). The other singlesymbol combinations are not yet widely used, but - due to their intrinsic physical significances-may merit listing at some future point.

Table 1. Some universal constants used in electron and/or ion emission theory. Values are given in the customary units often used, to make certain calculations quick and easy. The symbols $e, m_{\mathrm{e}}, h_{\mathrm{p}}, \hbar$, $k_{\mathrm{B}}$ and $e_{0}$ have their conventional meanings, with $e$ denoting the elementary (positive) charge.

\begin{tabular}{|l|l|l|l|l|l|}
\hline Name & Symbol & Derivation & Expression & Numerical value & Units \\
\hline $\begin{array}{l}\text { Sommerfeld supply } \\
\left.\text { density }{ }^{1}\right)\end{array}$ & $z_{\mathrm{S}}$ & - & $4 \pi m_{\mathrm{e}} / h_{\mathrm{P}}{ }^{3}$ & $1.618311 \times 10^{14}$ & $\mathrm{~A} \mathrm{~m}^{-2} \mathrm{eV}^{-2}$ \\
\hline $\begin{array}{l}\text { Universal theoretical } \\
\text { Richardson constant }\end{array}$ & $A_{\mathrm{R} 0}$ & $z_{\mathrm{S}} k_{\mathrm{B}}{ }^{2}$ & $4 \pi e m_{\mathrm{e}} k_{\mathrm{B}}{ }^{2} / h_{\mathrm{P}}{ }^{3}$ & $1.201735 \times 10^{6}$ & $\mathrm{~A} \mathrm{~m}^{-2} \mathrm{~K}^{-2}$ \\
\hline $\begin{array}{l}\text { Schrödinger equation } \\
\text { constant for electron }\end{array}$ & $\kappa_{\mathrm{e}}$ & - & $\left(2 m_{\mathrm{e}}\right)^{1 / 2} / \hbar$ & 5.123168 & $\mathrm{eV}^{-1 / 2} \mathrm{~nm}^{-1}$ \\
\hline $\begin{array}{l}\text { JWKB constant for } \\
\text { electron }\end{array}$ & $g_{\mathrm{e}}$ & $2 \kappa_{\mathrm{e}}$ & $2\left(2 m_{\mathrm{e}}\right)^{1 / 2} / \hbar$ & 10.24624 & $\mathrm{eV}^{-1 / 2} \mathrm{~nm}^{-1}$ \\
\hline $\begin{array}{l}\text { First Fowler- } \\
\text { Nordheim constant }\end{array}$ & $a$ & $z_{\mathrm{S}}\left(e / 2 \kappa_{\mathrm{e}}\right)^{2}$ & $e^{3 / 8 \pi h_{\mathrm{P}}}$ & 1.541434 & $\mu \mathrm{A} \mathrm{V}^{-2} \mathrm{eV}$ \\
\hline $\begin{array}{l}\text { Second Fowler- } \\
\text { Nordheim constant }\end{array}$ & $b$ & $4 \kappa_{\mathrm{e}} / 3 e$ & $(4 / 3)\left(2 m_{\mathrm{e}}\right)^{1 / 2} / e \hbar$ & 6.830890 & $\mathrm{eV}^{-3 / 2}(\mathrm{~V} / \mathrm{nm})$ \\
\hline Schottky constant & $c_{\mathrm{S}}$ & - & $\left(e^{3} / 4 \pi \varepsilon_{0}\right)^{1 / 2}$ & 1.199985 & $\left.\mathrm{eV}^{1 / 2} / \mathrm{nm}\right)^{-1 / 2}$ \\
\hline
\end{tabular}

$\left.{ }^{1}\right)$ Sommerfeld supply density is the electron current crossing a mathematical plane inside a free-electron metal of large extent, per unit area of the plane, per unit area of energy space, when the relevant electron states are fully occupied.. 


\title{
Fowler-Nordheim Plot Shape Associated with Large Series Resistance
}

\author{
Samer I. Daradkesh ${ }^{1}$, Marwan S. Mousa ${ }^{1}$ and Richard G. Forbes ${ }^{2}$, \\ Corresponding Author: r.forbes@surrey,ac,uk \\ ${ }^{1}$ Department of Physics, Mutah University, Al-Karak 61710, Jordan \\ ${ }^{2}$ Advanced Technology Institute, University of Surrey, Guildford, Surrey GU2 7XH, UK
}

Some of us (SID \& MSM) have recently carried out field electron emission (FE) experiments [1] on a carbon nanotube fibre mounted in a glass capillary tube [2]. In the current-voltage $(i-V)$ characteristics, there is a step-like jump in current when the voltage is increased for the first time. However, after turn-on, and in subsequent upwards and downwards sweeps of the measured voltage, the current is found to be a linear function of the voltage. This is unusual, because in FE experiments, the current is usually a distinctly non-linear function of voltage. The experiments presumably indicate that there is a large constant (or nearly constant) ohmic series resistance somewhere in the measurement circuit, with current controlled by this series resistance, rather than by the current-dependent "emission resistance" associated with the FE surface process.

Of particular interest is the shape of the associated Fowler-Nordheim (FN) plot, which initially rises as $1 / V$ decreases but then "turns over", as shown in Fig. 1. In the past, this effect has been termed "saturation", the assumption being that it is associated with a situation in which the ability of the emitter to supply current tends towards a maximum. However, the fact that our $i-V$ characteristics are linear shows that, in at least some cases, this underlying assumption about the interpretation of FN plots is not correct. We suggest that new terminology is needed to describe this kind of behaviour in FN plots: we suggest the term "FN-plot turn-over", and suggest that "FN-plot turn-over may or may not be associated with current saturation".

A recent paper [3] suggests that, amongst several other possibilities, the two main causes of FNplot turn-over could be: (1) series resistance in the measurement circuit, and (2) current (and hence voltage) dependence in the field enhancement factor (FEF). Reference [3] further suggests that, in most cases, the second of these (i.e., FEF-variation) is more likely to be the explanation of observed FN-plot flattening/turn-over. Whilst this may well normally be the case, the results reported here clearly show that there are some cases where FN-plot turn over is due to series resistance. A literature survey has found several other carbon-emitter cases where observed FN plots have shapes broadly similar to those reported here, although there are differences in detail.

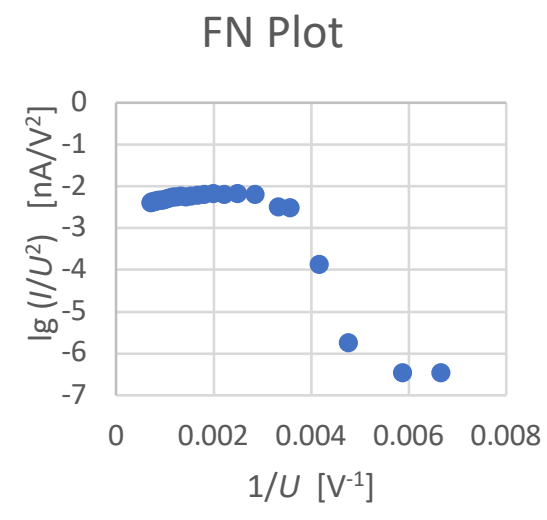

Figure. 1, alongside: Fowler-Nordheim plot taken from a carbon nanotube fibre emitter mounted so as to protrude from a pyrex glass capillary tube. [ $V$ denotes measured voltage, and I measured current.]

\section{References}

[1] M.S. Mousa, M-Ali H. AL-Akhras, \& S.I. Daradkeh, Jordan J. Phys. (accepted for publication).

[2] E.S. Bani Ali \& M.S. Mousa, Appl. Microsc. 46 (2016) 44.

[3] R.G. Forbes, Appl. Phys. Lett. 110 (2017) 133109. 


\title{
Secondary Ion Mass Spectroscopy with ZEISS ORION NanoFab
}

\author{
Soeren Eyhusen ${ }^{1}$, Fouzia Khanom ${ }^{1}$, David Dowsett ${ }^{2}$, and Tom Wirtz ${ }^{3}$ \\ Corresponding Author: soeren.eyhusen@,zeiss.com \\ 1. Carl Zeiss Microscopy, Peabody, MA \\ 2. Luxembourg Ion Optical Nano-Systems Sàrl, L-4362 Luxembourg \\ 3. Luxembourg Institute of Science and Technology, L-4422 Belvaux, Luxembourg.
}

Ion microscopy with helium or neon beams created from a gas field ion source (GFIS) is a powerful technique for many imaging and nanofabrication applications [1]. Due to the small probe size and the high precision of the ion beam, sub-10 nm structures can be routinely fabricated even in very sensitive materials. Additionally, the beam-sample interaction dynamics of helium/neon ion beams offer unique contrast and stunning surface detail at sub $0.5 \mathrm{~nm}$ spatial resolution. However, despite these unique advantages, the analytical capabilities of the instrument are currently limited.

Recently, a Secondary Ion Mass Spectrometer (SIMS) was specifically designed for the ZEISS ORION NanoFab $[2,3]$. SIMS is an extremely powerful analytical technique with excellent sensitivity, high dynamic range, and ability to differentiate between isotopes. In the ORION NanoFab, the neon ion beam is used to generate secondary ions. Other commercially available SIMS instruments typically rely on heavier ions such as Cesium or Oxygen as the primary ion species; however, the relatively large probe size limits the attainable lateral resolution. The neon beam on the other hand has a probe size of less than $2 \mathrm{~nm}$ and the SIMS resolution is therefore only limited by the size of the collision cascade, not the probe size $[3,4]$.

Here, we will show SIMS elemental maps acquired with ZEISS ORION NanoFab with lateral resolutions of less than 20nm. By combining the high resolution imaging capabilities of the instrument with elemental and isotopic maps of the SIMS, multi-modal, correlative data sets can be generated. We will also present application examples such as solar cells, battery materials, and biological tissues.

\section{$\underline{\text { References: }}$}

[1] G. Hlawacek, A. Gölzhäuser, Helium Ion Microscopy, Springer, 2017

[2] T. Wirtz, N. Vanhove, L. Pillatsch, D. Dowsett, S. Sijbrandij, J. Notte, Appl. Phys. Lett. 101 (4) (2012) 041601-1-041601-5

[3] T. Wirtz, D. Dowsett, P. Philipp, Helium Ion Microscopy, edited by G. Hlawacek, A. Gölzhäuser, Springer, 2017

[4] T. Wirtz, P. Philipp, J.-N. Audinot, D. Dowsett, S. Eswara, Nanotechnology 26 (2015) 434001 


\section{Improving APT-AFM Technology: Towards High Resolution 3D APT Tip Shapes}

Jonathan Op de Beeck ${ }^{1,2}$, Claudia Fleischmann ${ }^{2}$, Kristof Paredis $^{2}$, Wilfried Vandervorst ${ }^{1,2}$

Corresponding Author: Jonathan.OpdeBeeck@imec.be

1. KU Leuven, Department of Physics and Astronomy, Celestijnenlaan 200D, B-3001 Leuven, Belgium.

2. IMEC, Kapeldreef 75, 3001 Leuven, Belgium.

The application of laser-assisted atom probe tomography (L-APT) to complex semiconductor structures is hampered by a wide variety of artefacts, therefore a technology to study their root causes is needed. [1] These artefacts, such as image distortions and quantification inaccuracies, are often correlated to the APT tip shape. This drives the need and development of concepts which allow to assess the tip shape. As a result, the imaging of an APT tip by atomic force microscopy (AFM) was pioneered.[2] This innovative technology opens new doorways to increase our understanding of the (laser-assisted) field evaporation of the APT specimen as it has the potential to provide quantitative information in three dimensions with very high resolution.

In this work we will discuss the challenges and solutions when analyzing a microscale APT tip ( $\sim 50 \mathrm{~nm}$ radius) with an even sharper AFM probe, explore its capabilities and limitations and correlate the observed tip shape with the tip-laser interaction and evaporation processes. The intrinsic challenges of this technology are related to obtaining high spatial resolution on a high aspect ratio structure with a curved surface, the resulting tip-sample convolution and possible sample damage. Comparative analysis with scanning electron microscopy and electron tomography will be used to assess the AFM-based metrology of the APT-tip. We will report on the impact of the AFM probe radius and aspect ratio on the obtained resolution, reproducibility and accuracy of the AFM measurements. Furthermore, various AFM-imaging modes (noncontact AFM, scanning tunneling microscopy, PeakForce, tapping, ...) are assessed with respect to their performance in terms of the resolution and potential sample damage. Additionally, the intrinsic link between tip geometry and the charge-state-ratio will be studied by varying laser wavelength and power as this offers an exciting path towards more insight into the underlying physical mechanisms behind L-APT and its artefacts. Targeting the integration in an APTsystem and in-situ measurements during APT operation, we will finally assess the potential impact of the AFM measurements on the evaporation process (surface contamination, damage, loss of resolution, image distortions,...).

\section{$\underline{\text { References: }}$}

[1] J.P. Barnes, A. Grenier, I. Mouton, S. Barraud, G. Audoit, J. Bogdanowicz, C. Fleischmann, D. Melkonyan, W. Vandervorst, S. Duguay, N. Rolland, F. Vurpillot and D. Blavette, Atom probe tomography for advanced nanoelectronic devices: Current status and perspectives, Scripta Materialia in press (2017), 1-7.

[2] C. Fleischmann, K. Paredis, D. Melkonyan, W. Vandervorst, submitted to Ultramicroscopy (2017). 


\section{Cryo-FIB - A Promising Technique to Prevent Undesired Hydrogen Pick- Up During FIB Milling}

Yanhong Chang ${ }^{1}$, Leigh Stephenson ${ }^{1}$, Agnieszka Szczpaniak ${ }^{1}$, Wenjun Lu², Isabelle Mouton ${ }^{1}$, Paraskevas Kontis ${ }^{1}$, Abigail Ackerman ${ }^{3}$, David Dye ${ }^{3}$, Dirk Ponge ${ }^{1}$, Christian Liebscher ${ }^{2}$, Dierk Raabe $^{1}$, Baptiste Gault ${ }^{1}$

Corresponding Author: b.gault@mpie.de

1. Max Planck Institute für Eisenforschung, Department Microstructure Physics and Alloy Design, Düsseldorf, Germany.

2. Max Planck Institute für Eisenforschung, Department Structure and Nano- / Micromechanics of Materials, Düsseldorf, Germany.

3. Department of Materials, Royal School of Mines, Imperial College, Prince Consort Road, London SW7 2BP, United Kingdom.

Mechanical grinding or electro-polishing in water and/or acidic solution of Ti- and Zr-based alloys for specimen preparation is known to cause the formation of hydrides. Focused-ion beam techniques are hence widely used to prepare lamellae for transmission electron microscopy observations and atom probe specimens from those materials. However, besides the common defects (e.g. amorphous surface layer, vacancies, dislocations, etc.) induced by high-energy ion sputtering, it has recently been reported that FIB-milling could possibly trigger undesired hydrides formation during the preparation of TEM lamellae of both alloy families $[1,2]$. It is almost impossible to distinguish them from hydrides formed within the microstructure in service for instance and therefore inspect their structure and composition, which is critical to the service lifetime of engineering parts limited by hydrogen embrittlement.

High $\mathrm{H}$ diffusion rates at FIB operating temperature is poised to play an important role. Our xenon-plasma focused-ion beam microscope (FEI Helios PFIB) equipped with a cryo-stage that is cooled down to $-135^{\circ} \mathrm{C}$. This leads to an improvement in the chamber vacuum quality as well as, and maybe more importantly, to a lower diffusion rate of $\mathrm{H}$, thereby preventing hydride formation during preparation of such specimens.

Phase characterization by TEM observation indicated no hydride in the lamellae and APT further confirmed that no hydride were formed in specimens prepared under cryogenic conditions. Furthermore, solute $\mathrm{H}$ composition in the $\beta$-Ti is also significantly lower than in the specimens prepared at room temperature. Through these measurements, we proved that cryo-FIB is a promising technique to lower hydrogen pick-up and prevent undesired hydride formation induced during FIB milling in hydride-forming materials, such as Ti, Zr.

\section{References:}

[1] R. Ding and I. P. Jones, In situ hydride formation in titanium during focused ion milling. Journal of Electron Microscopy (Tokyo), 60(2011), 1-9.

[2] H. H. Shen, X. T. Zu, B. Chen, C. Q. Huang and K. Sun, Direct observation of hydrogenation and dehydrogenation of a zirconium alloy, Journal of Alloys and Compounds 659 (2016), 23-30. 


\section{A New Grid Design for Fast Correlative APT/TEM Sample Preparation}

Jonathan D. Poplawsky ${ }^{1}$ and Nickolay Lavrik ${ }^{1}$

Corresponding Author: poplawskyjd@ornl.gov

1. Center for Nanophase Materials Sciences, Oak Ridge National Laboratory, Oak Ridge, TN, USA.

Sample preparation for correlative TEM/APT experiments is extremely time consuming, difficult, and intensive. A popular procedure requires electro-polishing a $\mathrm{Cu}$ TEM half-grid followed by annular FIB milling to produce flat-topped posts that are $\sim 2 \mu \mathrm{m}$ in diameter. Recently, Dune Sciences (a small company based in Eugene, Oregon) commercialized a specialized Si grid with four sharp/shapeable mounting fingers (or posts) that can be used to perform correlative TEM/APT experiments on the same needle-shaped sample. These grids have made sample preparation easier, however, they must be FIB milled prior to preparing the sample for APT. Half-grid substrates with $\sim 2 \mu \mathrm{m}$ flat-top posts that are immediately ready for TEM/APT sample preparation have been produced using MEMS based processing.

Secondary electron images of the TEM half grid are shown in Figure 1 showing the shape of the Si microtips integrated onto a $3 \mathrm{~mm}$ TEM half-grid ready for site-selective FIB liftout sample preparation. ${ }^{1}$ The tips hang from the top edge of a $250 \mu \mathrm{m}$ Si grid and come to a point with an approximately $2 \mu \mathrm{m}$ width. The grids are made by depositing a $50 \mathrm{~nm} \mathrm{Al} \mathrm{O}_{3}$ mask on a degeneratively doped Si substrate with a resistivity of $<0.05 \mathrm{Ohm}-\mathrm{cm}$, which is followed by spin coating of a photoresist. Front side lithography in the shape of a Si half-grid is followed by an $\mathrm{A}_{2} \mathrm{O}_{3}$ sputter etch. A deep reactive ion etch of the $\mathrm{Si}$ forms the TEM half-grid shape. The front side lithography step is followed by backside lithography to produce the micropost "pillar" shape, which is followed by backside reactive ion etching (RIE). Plasma-enhanced chemical vapor deposition (PECVD) of the supporting SiOx is conducted before performing the throughwafer etch, and a final HF etch of the SiOx releases the Si chips.

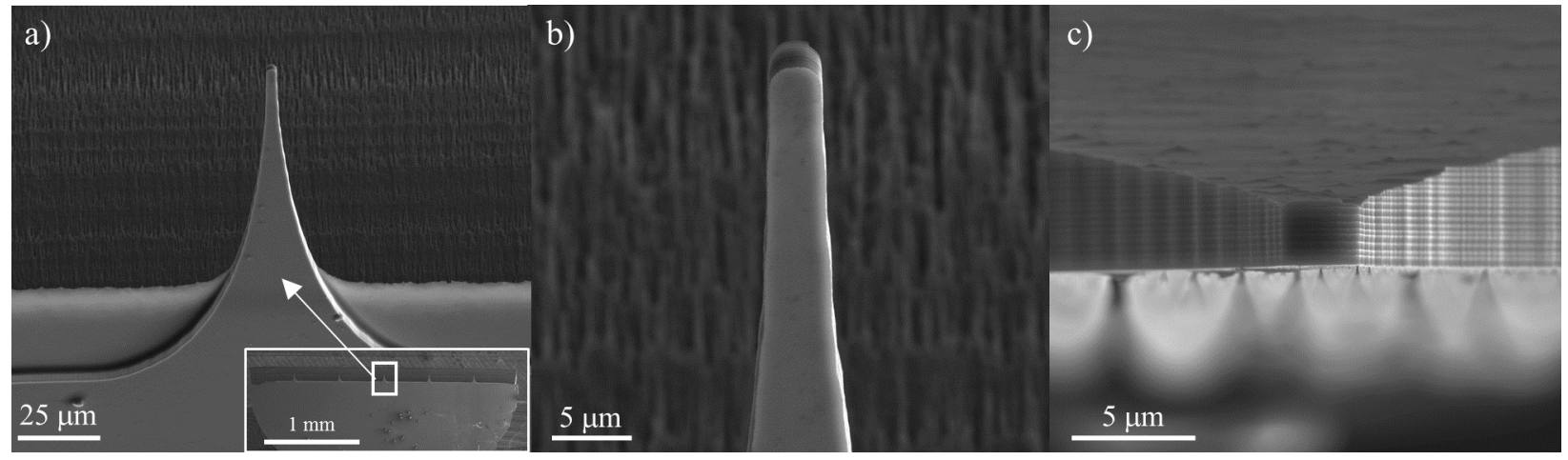

Figure $1(\mathbf{a}, \mathbf{b})$ Magnified view of a Si micropost tilted at 52 degrees with the TEM half grid shown in the inset of (a). (c) A top down view of the Si micropost on the TEM half-grid.

\section{References:}

[1] Thompson, K. et al. In situ site-specific specimen preparation for atom probe tomography. Ultramicroscopy 107 (2007) 131-139.

[2] Research was conducted at ORNL's Center for Nanophase Materials Sciences (CNMS), which is a U.S. DOE Office of Science User Facility. 


\section{Performance of the EIKOS Atom Probe Microscope}

Robert M. Ulfig, Ty J. Prosa, Dan R. Lenz, Joseph H. Bunton, Katherine P. Rice, Yimeng Chen, and David J. Larson

Corresponding Author: Robert.Ulfig@ametek.com

CAMECA $^{\circledR}$ Instruments Inc., 5470 Nobel Dr., Madison WI 53711, USA.

The new EIKOS ${ }^{\mathrm{TM}}$ atom probe design includes an energy compensated flight path in the form of an imaging reflectron, a fiber-based $532 \mathrm{~nm}$ laser, and a counter electrode design that is integrated into the specimen carrier puck. The design impacts spectral performance, field of view and spatial resolution, as well as sample preparation, throughput, a lower cost of ownership.

Both voltage pulsed and laser pulsed spectral performance (mass resolving power) is shown in Table 1 for a variety of materials including Al, Si, W, 316 stainless steel, Inconel 718, and GaN [1]. Example spectra of stainless steel for laser and voltage modes are shown in Figure 1 (a and b), and the spatial resolution, which is similar to a LEAP system, is demonstrated with the spatial distribution map technique in Figure 1 (c) [2]. Performance criteria will be discussed in detail.

Table 1. EIKOS mass resolving power values for various materials (on most populated peak).

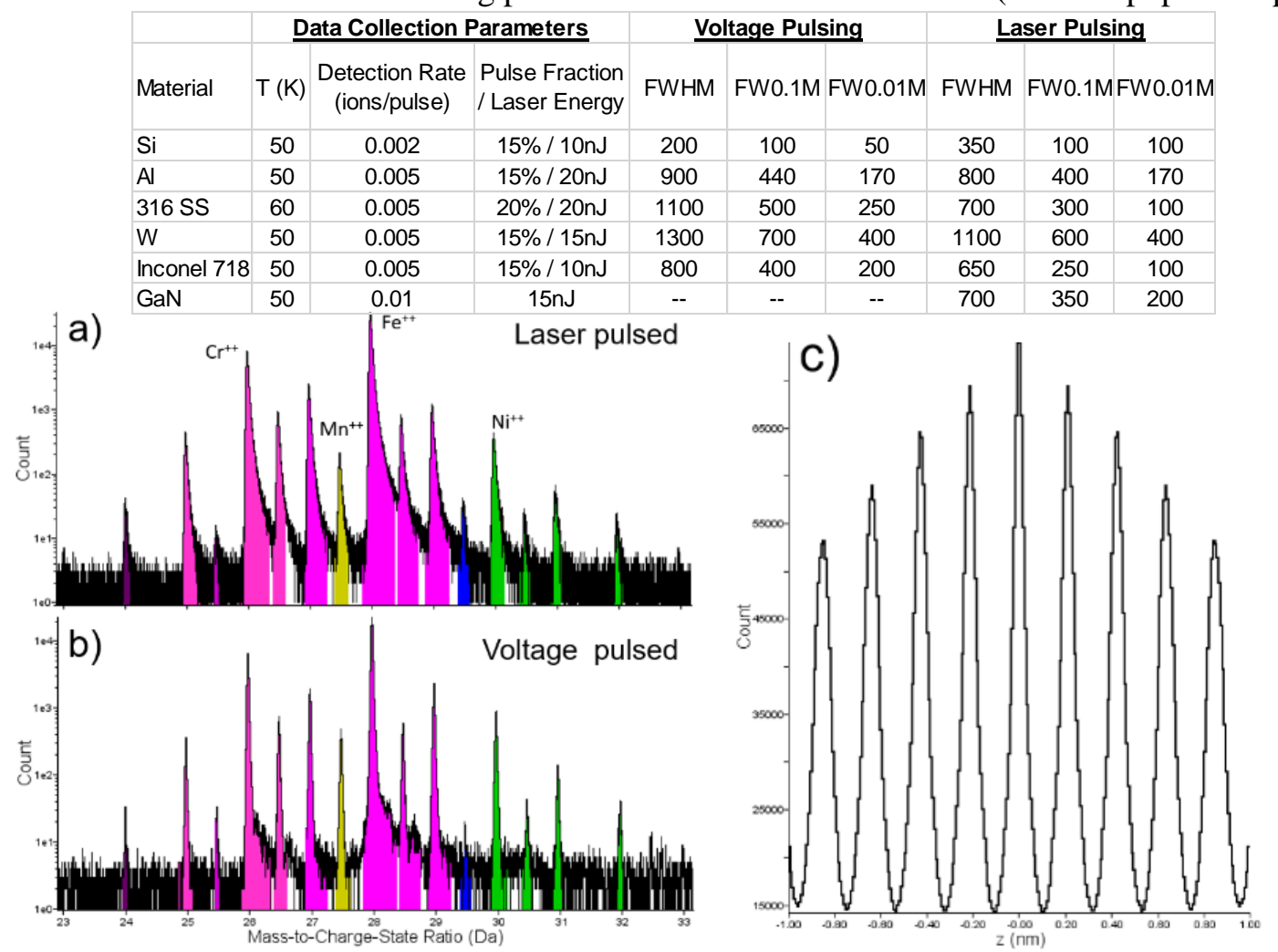

Figure 1 Example mass spectra from the 316 stainless steel in both (a) laser and (b) voltage pulsed modes. (c) A Z-SDM for (002) direction in Tungsten demonstrating the spatial resolution.

\section{$\underline{\text { References: }}$}

[1] D.J. Larson et al., Microstructural Investigations in Metals Using Atom Probe Tomography with a Novel Specimen-Electrode Geometry, Journal of Metals (2018) in press.

[2] B. Geiser et al., Spatial Resolution in Atom Probe Tomography, Micro. Microanal. 13(6) (2007), 43. 


\section{Virtual Scanning Tunneling Microscope for training and teaching}

Mark J. Hagmann ${ }^{1}$, Marwan S. Mousa ${ }^{2}$

Corresponding Author: mark.hagmann@utah.edu

1. University of Utah, Dept. Electrical and Computer Engineering, Salt Lake City, Utah, USA, 84112.

2. Surface Physics and Materials Technology Lab, Department of Physics, Mu'tah University, Al-Karak, 61710, Jordan.

The scanning tunneling microscope (STM) was invented by Gerd Binning and Heinrich Roher at IBM Zurich in 1986 [1]. We have made a LabVIEW virtual instrument (VI) simulating the full operation of an STM as an executable version, not requiring LabVIEW or other software, that is available as a free download on the website www.newpathresearch.com. We hope that this VI will be useful in teaching where there may not be access to an STM, and in training others to use similar instruments. We have used this software to upgrade an in-house STM with a Field Programmable Gate Array to provide deterministic control of multiple parallel functions.

This VI simulates the noise and drift of the instrument during imaging. Several novel methods for feedback control of the tunneling current in addition to the standard proportion-integral (PI) method are included to enable comparison. This work is supported by the National Science Foundation under Grant 164881.

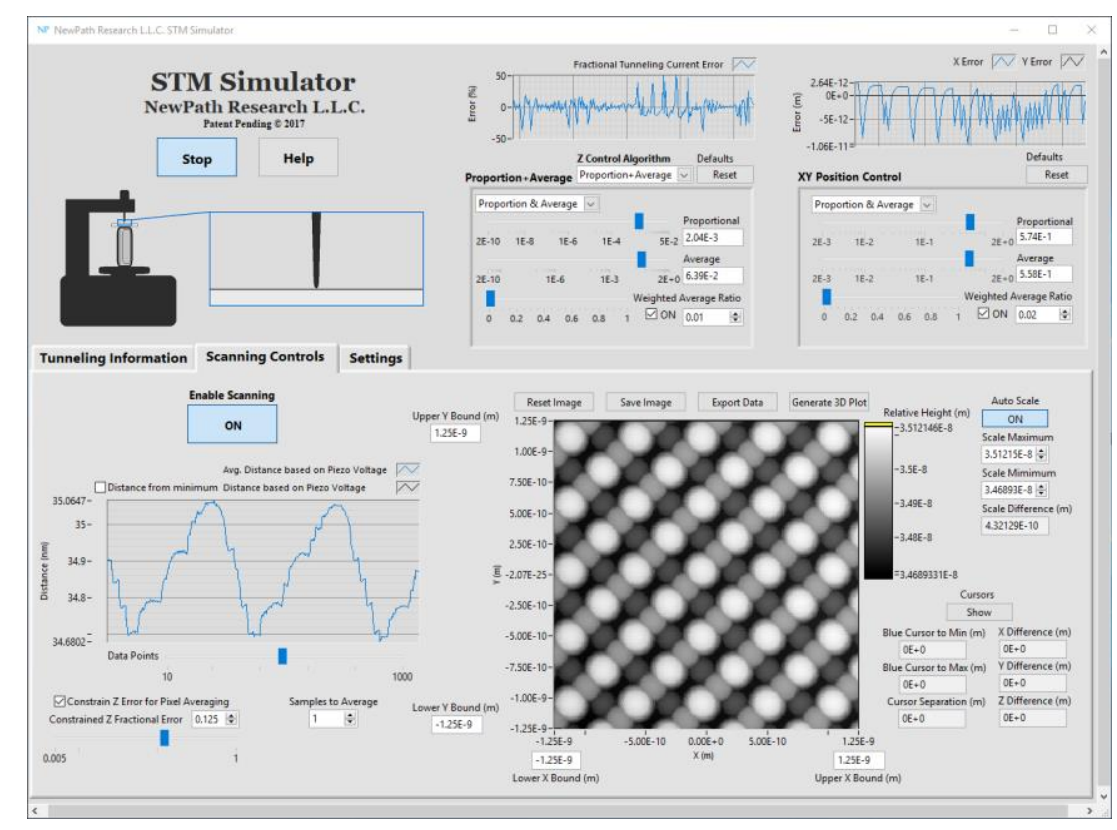

Figure 1 Main display screen of the new STM simulator VI during operation, showing generated image of the Si (100) surface.

\section{References:}

[1] G.Binning and H. Rohrer, IBM J Res Dev 30 (1986) 355-369. 


\section{A New Platform for Atom Probe Analysis: IVAS 4}

David A. Reinhard ${ }^{1}$, Tim Payne ${ }^{1}$, Brian P. Geiser ${ }^{1}$, Geoff Sobering ${ }^{1}$, Ed Oltman ${ }^{1}$, Jerry Mandt ${ }^{1}$, Eric Strennen ${ }^{1}$, David J. Larson ${ }^{1}$, Robert M. Ulfig, ${ }^{1}$ Ty J. Prosa ${ }^{1}$, Isabelle Martin ${ }^{1}$, Katherine P. Rice $^{1}$, Yimeng Chen ${ }^{1}$, Hugues Francois-Saint-Cyr ${ }^{1}$

Corresponding Author: David.Reinhard@ametek.com

1. CAMECA Instruments Inc., Madison USA 53711.

The Integrated Visualization and Analysis Software (IVAS ${ }^{\mathrm{TM}}$ ) from CAMECA $^{\circledR}$ is the most widely used software package for atom probe data analysis with an estimated 1300 users worldwide. After a lifetime of more than 15 years, we are embarking on a complete redevelopment of IVAS. We expect to achieve improved analysis performance as well as shortened feature development time by taking advantage of the latest development tools and platforms.

Objectives of the IVAS 4 project include:

- Database integration - incorporation of information from the entire life of a project from project definition through sample preparation, acquisition, reconstruction, analysis and report generation

- Live calibration and reconstruction - automatic and continuous spectral calibration during acquisition enabling a live $3 \mathrm{D}$ reconstruction

- Spatial ranging - removal of the 1 range file per position file limitation to allow different range definitions to be used for distinct phases

- New file format - replacement of the *.POS file format with an extensible format to allow analyses based on more than the mass-to-charge value of an ion (ex. hitclass)

- Tools for automated analyses - analysis templates for simple analyses and more advanced analysis flowcharts to define procedural analysis chains

- Automatic report generation - analysis results from multiple data sets compiled into a formatted report

- Compatibility with community generated features - ability to call external tools from IVAS and import the results

- Universal hit data export format - standard reconstruction definition to allow users to develop a custom reconstruction algorithm

- Security - user-based permissions to provide secure access to data.

- Performance - much shorter analysis times for the largest data files (threading, GPU acceleration, etc.) 


\section{Extreme Ultraviolet-Assisted Field Ion Evaporation}

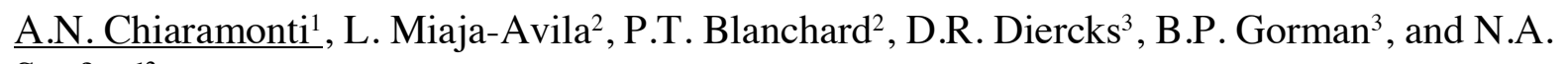
Sanford ${ }^{2}$

Corresponding author: chiaramonti@nist.gov

1. Material Measurement Laboratory, National Institute of Standards and Technology, Boulder, CO USA

2. Physical Measurement Laboratory, National Institute of Standards and Technology, Boulder, CO USA

3. Metallurgical and Materials Engineering, Colorado School of Mines, Golden, CO USA

Laser assisted probe tomography (LAPT) is a powerful tool for materials characterization due to its desirable combination of high spatial resolution and analytical sensitivity. In state of the art LAPT the thermal transient from a near UV laser ( $E \approx 3.5 \mathrm{eV}$ to $3.6 \mathrm{eV} ; \lambda \approx 343 \mathrm{~nm}$ to $355 \mathrm{~nm}$ ) provides the energy to overcome the activation barrier for field ion evaporation. This technique is generally superior to voltage pulsing, which is limited strictly to conductors, and has allowed APT to expand its capability to effectively analyze a wide-range of materials including semiconductors and insulators. However, the thermal process is not without drawbacks. For example, LAPT data quality can be degraded due to thermal tails that limit sensitivity, formation of cluster ions that may have isobaric overlap with elemental species, and undetected neutral species which can adversely influence composition measurements. This is especially true for many ionic and covalent materials and can limit the recovery of bulk stoichiometry or composition to a narrow range of experimental conditions, if at all $[1,2]$.

Ionizing radiation in the extreme ultraviolet (EUV) region of the electromagnetic spectrum $(\approx 10 \mathrm{eV}$ to $\approx 100 \mathrm{eV} ; 124 \mathrm{~nm}$ to $12 \mathrm{~nm})$ may offer a potential athermal field ionization pathway. Dependent on the particular photon energy used, EUV radiation is above the ionization potential of any naturally occurring element and photoionization cross-sections peak in the EUV band across the entire periodic table [3]. EUV is highly absorbed within the first few nm of the sample surface and may also offer a potential in situ method for imaging the evolving specimen shape in real time through simultaneous coherent diffractive imaging or related methods [4].

We will present the instrument design and results from a tunable EUV-APT that uses femtosecond pulsed coherent radiation from phase-matched high harmonic generation in a noble gas cell. Initial experiments using Ar gas $(\mathrm{E}=41.85 \mathrm{eV} ; \lambda=29.6 \mathrm{~nm})$ conclusively demonstrate EUV-assisted field ion evaporation in fused quartz (amorphous $\mathrm{SiO}_{2}$ ). Thermal tails, $\mathrm{Si}^{\mathrm{n}+}, \mathrm{O}^{\mathrm{n}+}$, and $\mathrm{O}_{2}{ }^{\mathrm{n}+}$ peak widths, and the relative number of the cluster ions $\mathrm{SiO}^{\mathrm{n}+}$ and $\mathrm{SiO}_{2}{ }^{\mathrm{n}+}$ are significantly decreased when compared with near UV-LAPT $(\mathrm{E}=3.49 \mathrm{eV} ; \lambda=355 \mathrm{~nm})$ experiments on the same samples and specimens.

[1] Mancini, L. et al. Physical Chemistry C 118 (2014) 24136.

[2] Diercks, D.R. et al. J. Appl. Phys. 114 (2013) 184903.

[3] Yeh, J.-J. and I. Landau. At. Data Nucl. Data Tables 32 (1985) 1.

[4] Gardner, D.F. et al. Nature Photonics 11 (2017) 259. 


\section{Laser-Material Interaction During Atom Probe Tomography of Oxides With Embedded Metal Nanoparticles}

Deodatta Shinde ${ }^{1,3}$, Laurent Arnoldi ${ }^{1}$, Arun Devaraj ${ }^{2}$ and Angela Vella ${ }^{1}$

Corresponding Author: deodatta.shinde@,chalmers.se

${ }^{1}$ Groupe de Physique des Materiaux, Universite et INSA de Rouen - UMR CNRS 6634, France

${ }^{2}$ Physical and Computational Sciences Directorate, Pacific Northwest National Laboratory, USA

Metal nanoparticles embedded in oxide matrix is one of the significant materials systems scientifically as well as technologically. This is due to their various applications in number of fields like medicine, drug delivery, catalysis, plasmonics etc. However, studying such kind of systems in an atom probe context is not an easy task due to the complex interaction of incident laser light and the nanometric tip shaped sample.

Accordingly, in this work, we focused on understanding the fundamental laser-material interaction in the case of oxides with embedded metal nanoparticles through experiments and numerical calculations. By understanding the mechanism of laser absorption, we show that the sources of noise in mass-to-charge spectra are due to the absorption of the laser energy far from the tip apex. We also investigated the role of the sample shape, the laser power, and DC field to determine the conditions to reduce the sources of noise. This work is published in [1] and is also thoroughly discussed in [2]

FDTD

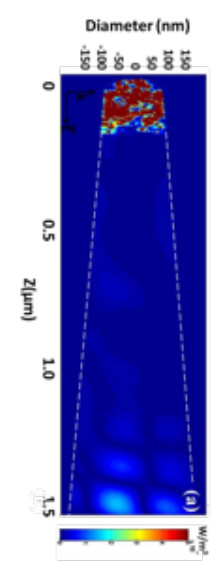

3D Reconstruction

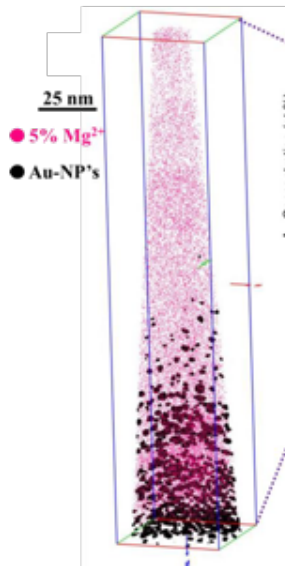

ToF Measurements

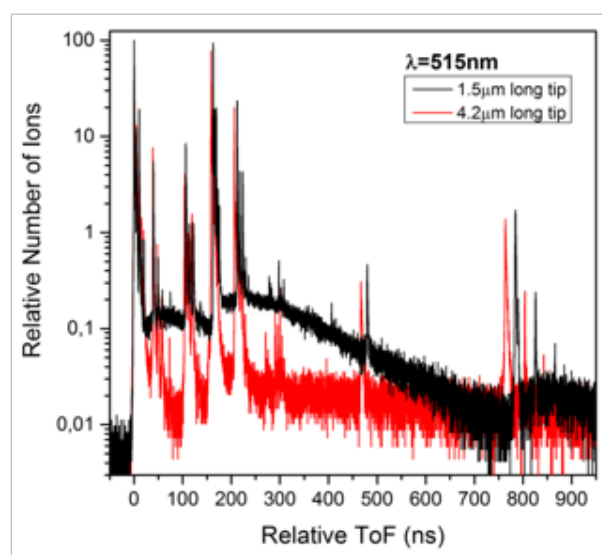

Figure 1 : Understanding laser-matter interaction by correlating the results obtained from numerical calculations (FDTD), atom probe experiments (3D Reconstruction) and studying the evaporation dynamics (ToF measurements)

References:

[1] D. Shinde, L. Arnoldi, A. Devaraj and A. Vella, J. of Appl. Phys. 120 (2016) 164308.

[2] D. Shinde, Laser assisted Atom Probe Tomography as a spectroscopic tool to analyze the materials for energy, Ph.D. Thesis, (January 2016) (https://hal.archives-ouvertes.fr/tel-01380304/)

${ }^{3}$ Current Address: Department of Physics, Chalmers University of Technology, SE-412 96 Göteborg, Sweden 


\section{Standardized APT Tips for Interlaboratory Studies}

M. Dialameh ${ }^{1,2,3,4}$, C. Fleischmann ${ }^{1}$, J. Bogdanowicz ${ }^{1}$, N. De Leo ${ }^{3}$, L. Boarino ${ }^{3}$, W. Vandervorst ${ }^{1,4}$

Corresponding Author: Claudia.Fleischmann@imec.be

1. Imec, Kapeldreef 75, 3001 Heverlee, Belgium

2. Politecnico di Torino, Corso Duca degli Abruzzi, 24, 10129, Turin, Italy

3. Istituto Nazionale di Ricerca Metrologia (INRIM), Strada delle Cacce 91, 10135 Turin, Italy

4. Instituut voor Kern-en Stralingsfysica, KUL, Celestijnenlaan 200D, 3001 Leuven, Belgium

Over the last decade, atom probe tomography (APT) has gone through many technological improvements broadening its applicability to a wide range of materials. Despite this progress, APT when applied to technological relevant applications such as semiconductor devices, still exhibits numerous limitations in terms of the achievable spatial resolution, precision and accuracy [1]. Moreover, uniform analysis procedures and performance assessment are still lacking due to the absence of appropriate standards and reference materials. Finally, the extensive sample preparation using focused ion beam sputtering hampers the widespread assessment of the technique and its properties. As such detailed interlaboratory studies are very scarce as well.

To address the aforementioned limitations and prepare for interlaboratory studies (e.g. under the VAMAS umbrella [2]), we propose a process which allows to prepare multiple APT samples in a reproducible fashion and with minimized tip to tip variations in view of tip radius and shank angle. The APT tips will be exploited in the preliminary studies preparing for interlaboratory studies where we will address the uncertainty budget in APT analysis. Such standardized tip shape is also extremely helpful in fundamental studies (e.g. tip-laser interactions) as they allow to eliminate variability contributions in ion-beam damage, retained Ga dose and distribution, tip geometry, heat transfer (shank angle), etc. In addition, the suggested sample geometry allows to maximize the field of view and could enable the employment of the APT sample sidewalls and the known tip radius as a reference during data reconstruction, which constrains the reconstruction parameters and hence improves accuracy in the atom position.

Relying on finite element simulations, we have refined the sample geometry, i.e. a nanowire on top of cylindrical micro-post, and the dimension (diameter and height) to maximize the electric field enhancement at the tip apex and the field of view, as proposed in reference [3]. The sample preparation is based on full wafer scale processing using standard lithography and etching techniques and thus allows for high throughput sample fabrication. As initial test vehicle, we have optimized this procedure for the preparation of APT samples from a boron-doped ( 1E20 atoms $\left./ \mathrm{cm}^{3}\right)$, epitaxially grown $\mathrm{SiGe}(25 \% \mathrm{Ge})$ layer $(100 \mathrm{~nm})$ on a $300 \mathrm{~mm} \mathrm{Si}(100)$ wafer. The complementary characterization of the same (or similar) wafer using different (reference-free) analysis techniques to extract layer thickness, composition and dopant concentration, together with the uniformity of the different APT samples, will form a cornerstone to launch an interlaboratory study on APT analysis.

\section{References:}

[1] Larson, D. J., et al. Toward atom probe tomography of microelectronic devices, Journal of Physics: Conference Series 326 (2011), 012030.

[2] http://www.vamas.org

[3] Du, S., et al. Full tip imaging in atom probe tomography, Ultramicroscopy 124 (2013), 96-101. 


\section{Quantification of APT Physical Limitations on Chemical Composition of Precipitates in Fe-Cr Alloys}

Cristelle Pareige ${ }^{1}$, Constantinos Hatzoglou ${ }^{1}$, Gerald Da Costa ${ }^{1}$, Philippe Pareige ${ }^{1}$, M. Roussel ${ }^{1}$, Bertrand Radiguet ${ }^{1}$

Corresponding Author: cristelle.pareige@univ-rouen.fr

1. Normandie Univ, UNIROUEN, INSA Rouen, CNRS, Groupe de Physique des Matériaux, 76000 Rouen, France.

Among the different nano-features at the origin of embrittlement of Fe-Cr alloys and steels, $\alpha^{\prime}$ precipitates play a major role in steels with $\mathrm{Cr}$ content higher than about $10 \%$. If Atom Probe Tomography (APT) is recognized as an efficient characterisation technique for characterizing $\alpha^{\prime}$ precipitates, discussion remains on its ability to measure the actual composition of small particles. At the origin of this discussion, two APT limitations: its lateral resolution and local magnification effects due to the difference in field evaporation between the matrix and the particles. In this work, the impact of these two limitations are quantified for the first time using numerical approaches [1-4] and analytical models [5]. Each of these effects has been quantified both separately then in combination on $\alpha^{\prime}$ particles $1 \mathrm{~nm}$ in radius of different composition

\section{References:}

[1] J.M. Hyde, G. DaCosta, C. Hatzoglou, H. Weekes, B. Radiguet, P.D. Styman, F. Vurpillot, C. Pareige, A. Etienne, G. Bonny, N. Castin, L. Malerba, P. Pareige, Analysis of Radiation Damage in Light Water Reactors: Comparison of Cluster Analysis Methods for the Analysis of Atom Probe Data, Microsc. Microanal. 23 (2017) 366-375. doi:10.1017/S1431927616012678.

[2] D. Blavette, F. Vurpillot, P. Pareige, A. Menand, A model accounting for spatial overlaps in 3D atomprobe microscopy, Ultramicroscopy. 89 (2001) 145-153.

[3] F. Vurpillot, M. Grubber, S. Dugay, E. Cadel, B. Deconihout, Modeling artifacts in the analysis of test semiconductor structures in atom probe tomography, AIP Conf Proc. Eds. Secula, Erik M.; Seiler, David G.; Khosla, Rajinder P.; Herr, Dan; Garner, C.Michael; McDonald, Robert; Diebold, Alain C., Albany (New York) (2009) 175-180.

[4] M. Gruber, F. Vurpillot, A. Bostel, B. Deconihout, Field evaporation: A kinetic Monte Carlo approach on the influence of temperature, Surf. Sci. 605 (2011) 2025-2031. doi:10.1016/j.susc.2011.07.022.

[5] C. Hatzoglou, B. Radiguet, P. Pareige, Experimental artefacts occurring during atom probe tomography analysis of oxide nanoparticles in metallic matrix: Quantification and correction, J. Nucl. Mater. 492 (2017) 279-291. doi:10.1016/j.jnucmat.2017.05.008. 


\section{Quantification of Halogen in Si-substrate for Laser-Assisted APT}

Tomokazu Sasaki ${ }^{1}$, Kazuya Toda $^{1}$, Naomi Arai ${ }^{1}$, Yutaro Hori ${ }^{1}$, Norihito Mayama ${ }^{1}$, and Yutaka Fukushima ${ }^{1}$

Corresponding Author: tomokazu.sasaki@nanoanalysis.co.jp

1. Physical Analysis Technology Center, Toshiba Nanoanalysis Corporation, Yokohama, Japan.

Dry-etching gas, wet-etching liquid and ion implantation species applied for silicon-based semiconductor wafer processing often contain halogen such as fluorine and chlorine. However, it is well-known that those halogens in the residue in semiconductor devices sometimes deteriorate their electric properties. Therefore analysis of halogen is considered to be very important to understand causes of failures and optimize dry-etching, wet-etching and the others processing. Generally TEM/EDS method is available for analysis of halogen in device structures, which provides elemental mapping images with detection limit of about $5 \mathrm{E}^{19}$ atoms $/ \mathrm{cm}^{3}$. APT is also believed to have a potential to analyze halogen in semiconductor devices three-dimensionally with better detection limit than TEM/EDS.

Recently it has been reported that APT quantitative performance of boron, carbon, arsenic and phosphorus in Si were studied. [1-4] However, APT quantitative performance of halogen in Si still remain unknown and should be studied. Especially APT measurement detects positive ions electric-field-evaporated from a needle-like specimen, although halogen atoms seem difficult to be positive ions due to their large electronegativity. In this case, their peak positions in the mass spectrum may be affected by the feature, which results in complicating their peak identification.

In this study, Si-substrates ion implanted by fluorine and chlorine were prepared and measured with laser-assisted APT (LEAP4000XSi). Then, APT quantitative performance of halogen was evaluated with comparing SIMS measurements.

As a result, it was found that molecular ions such as $\mathrm{SiF}^{+}$and $\mathrm{SiCl}^{+}$were detected while singular ions such as $\mathrm{F}+$ and $\mathrm{Cl}+$ were under detection limit. The measurement conditions of laser pulse energy and specimen temperature were $30 \mathrm{pJ}$ and $70 \mathrm{~K}$, respectively. In this case, profiles of halogen in APT analysis showed good agreement with ones in SIMS analysis. This result indicated that APT had high quantitative performance for halogen in Si.

We performed the similar experiments on sodium and argon. Sodium was often cared about in semiconductor devices, and argon was generally used in semiconductor processing. However, sodium is known as a mobile ion and argon has very low boiling temperature, and these features may affect APT quantitative performance of them. In our presentation, we will also discuss APT quantitative performance of these elements.

References:

[1] P.A. Ronsheim, M. Hatzistergos, and S.Jin, J. Vac. Sci. Technol. B 28, C1E1 (2010)

[2] M. Ngamo, S. Duguay, F. Cristiano, K. Daoud-Ketata, and P. Pareige, J. Appl. Phys. 105, 104904(2009)

[3]T. Sasaki, S. Ishimura, M. Kato, H. Akutsu, M. Tomita, T. Kinno, and H. Uchida, presented at $19^{\text {th }}$ Int. Conf. Secondary Ion Mass Spectrometry (SIMS-19), 2013

[4]T. Kinno, T. Sasaki, M. Tomita and T Ohkubo, Jpn. J. Appl. Phys. 56, 116601(2017) 


\section{Correcting the Boron Concentration for the Detection Losses Through Multi Hit Events.}

D. Melkonyan ${ }^{1,2}$, C. Fleischmann², J. Bogdanowicz², R.J.H. Morris ${ }^{2}$, R. Cuduvally ${ }^{1,2}$ and W. Vandervorst ${ }^{1,2}$

Corresponding author: claudia.fleischmann@imec.be

1. Instituut voor Kern- en Stralingsfysica, KU Leuven, Celestijnenlaan 200D, 3001 Leuven, Belgium

2. Imec, Kapeldreef 75, 3001 Heverlee, Belgium

The 3D dopant distribution determines the performance of the state of the art semiconductor devices, hence accurate 3D mapping of these low concentration solutes is critical for driving the development of high performance devices. Currently, APT is the only technique that can resolve the $3 \mathrm{D}$ dopant distribution with up to 1 part per million sensitivities. However, in many cases the technique suffers from measurement artefacts significantly impairing the data accuracy.

In this contribution we will discuss the multi hit defectivity and means to correct them for Boron, which is a notorious case where APT underestimates the actual concentration, whereby the error is seen to increase with dopant concentration [1-2]. The underestimate finds its origin in the high evaporation field of B, causing the B atoms to preferentially retain at the emitter apex during field evaporation of the surrounding Si atoms. This leads to the evaporation of the B atoms as atomic bursts [2] and its presence within multi hit events. The limited capacity of APT to resolve the multi hit events of B-B pairs (and consequently their rejection) leads to the B underestimate. We show that the $B$ loss due to multi hit events is around $\sim 10 \%$ for the $8.7 \times 10^{19}$ at $/ \mathrm{cm}^{3}$ dopant concentration. Based on a detailed analysis of the different B-B double hit pairs, it can be concluded that the ${ }^{10} \mathrm{~B}-{ }^{11} \mathrm{~B}$ pairs do not induce ion pile up and thus are recorded accurately, while the ${ }^{10} \mathrm{~B}-{ }^{10} \mathrm{~B}$ and ${ }^{11} \mathrm{~B}-{ }^{11} \mathrm{~B}$ are greatly underestimated. Based on the isotopic abundances, we can calculate the probability of forming double hits $\left({ }^{10} \mathrm{~B}-{ }^{11} \mathrm{~B},{ }^{10} \mathrm{~B}-{ }^{10} \mathrm{~B},{ }^{11} \mathrm{~B}-{ }^{11} \mathrm{~B}\right)$, and thus reconstruct the expected amount of the ${ }^{10} \mathrm{~B}-{ }^{10} \mathrm{~B}$ and ${ }^{11} \mathrm{~B}-{ }^{11} \mathrm{~B}$ pairs, using the accurately detected amount of ${ }^{10} \mathrm{~B}-{ }^{11} \mathrm{~B}$ pairs. As such we find that due to the formation of ${ }^{11} \mathrm{~B}-{ }^{11} \mathrm{~B}$ and ${ }^{10} \mathrm{~B}-{ }^{10} \mathrm{~B}$ double hit pairs, the $\mathrm{B}$ concentration is underestimated by $4.6 \%$ and $0.3 \%$, respectively. The minor loss due to ${ }^{10} \mathrm{~B}-{ }^{10} \mathrm{~B}$ pairs originates from the low abundance of ${ }^{10} \mathrm{~B}(20 \%)$ isotope and thus low probability $(4 \%)$ of ${ }^{10} \mathrm{~B}-{ }^{10} \mathrm{~B}$ formation. As a result, a more accurate $\mathrm{B}$ concentration can be achieved by using only the detected ${ }^{10} \mathrm{~B}$ isotope at high concentrations, whereas at low concentrations the ${ }^{11} \mathrm{~B}$ can still be used due to the low formation probability at low concentrations of the ${ }^{11} \mathrm{~B}-{ }^{11} \mathrm{~B}$ pairs. A comparison APT-SIMS demonstrates the accuracy of this approach.

\section{References:}

[1] F. Meisenkothen, E. B. Steel, T. J. Prosa, K. T. Henry, and R. Prakash Kolli, "Effects of detector dead-time on quantitative analyses involving boron and multi-hit detection events in atom probe tomography," Ultramicroscopy, vol. 159, pp. 101-111, Dec. 2015.

[2] G. D. Costa, H. Wang, S. Duguay, A. Bostel, D. Blavette, and B. Deconihout, "Advance in multi-hit detection and quantization in atom probe tomography," Rev. Sci. Instrum., vol. 83, no. 12, p. 123709, Dec. 2012. 


\title{
Study on Quantitative Analysis of Carbon and Nitrogen in Stoichiometric Cementite and Roaldite by Atom Probe Tomography
}

\author{
J. Takahashi ${ }^{1}$, K. Kawakami ${ }^{2}$ and Y. Kobayashi ${ }^{1}$ \\ Corresponding Author: takahashi.3ct.jun@jp.nssmc.com \\ ${ }^{1 .}$ Nippon Steel \& Sumitomo Metal Corporation, Futtsu-city, Japan. \\ 2. Nippon Steel \& Sumitomo Metal Technology, Futtsu-city, Japan
}

Quantitative analysis performances of interstitial atoms in steel, namely, $\mathrm{C}$ and $\mathrm{N}$ were investigated using cementite $\left(\theta-\mathrm{Fe}_{3} \mathrm{C}: 25 \mathrm{at} . \% \mathrm{C}\right)$ in pearlite steel [1] and roaldite $\left(\gamma-\mathrm{Fe}_{4} \mathrm{~N}\right.$ : $20 \mathrm{at} . \% \mathrm{~N}$ ) in Braunite steel in pulsed voltage and pulsed laser atom probes. Temperature and pulse fraction dependences of apparent $\mathrm{C}$ and $\mathrm{N}$ concentrations were measured under various conditions. Good coincidence with $25 \mathrm{at} . \% \mathrm{C}$ in cementite was obtained in pulsed voltage atom probe by considering the appropriate assignment of $\mathrm{C}$ molecular ions [1] and the detection-loss of $\mathrm{Fe}^{2+}$ ions [2]. Pulsed laser atom probe showed better analysis performance of $\mathrm{C}$ due to reduction in the detection-loss. In contrast, significant lack of apparent concentration of $\mathrm{N}$ in roaldite was observed in both atom probes, and high pulse energy resulted in much lower $\mathrm{N}$ concentration in pulsed laser atom probe (Fig.1). It implied that a large peak of ${ }^{56} \mathrm{Fe}^{2+}$ hinders a peak of ${ }^{14} \mathrm{~N}_{2}{ }^{+}$at $28 \mathrm{Da}$ in mass spectrum and thus the decrement in apparent concentration of $\mathrm{N}$ corresponds to the amount of $\mathrm{N}_{2}{ }^{+}$. However, it seemed difficult to quantitatively explain the decrement of $\mathrm{N}$ even using the assumption [3]. We discuss the cause of the missing $\mathrm{N}$. On the other hand, the pulse fraction dependence in pulsed voltage atom probe indicated that $\mathrm{C}$ in cementite hardly showed preferential evaporation and retention, whereas $\mathrm{N}$ in roaldite clearly showed preferential retention. The difference in field evaporation between $\mathrm{C}$ and $\mathrm{N}$ was explained by the bonding forms of $\mathrm{Fe}-\mathrm{C}$ and $\mathrm{Fe}-\mathrm{N}$ in each precipitate.

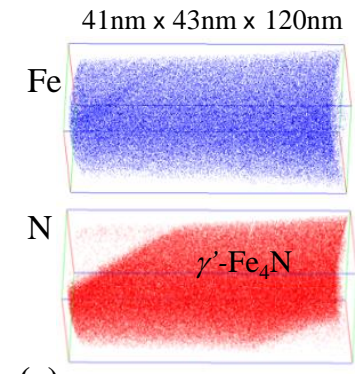

(a)

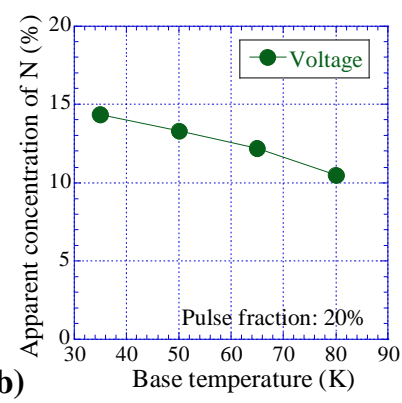

(b)

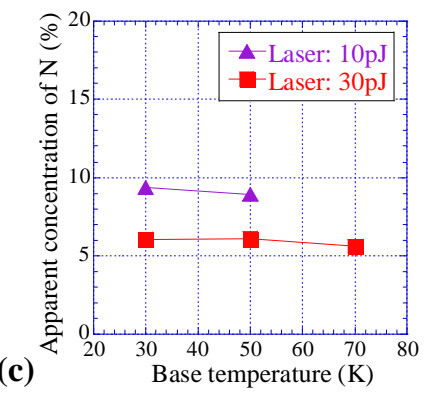

Figure 1. (a) 3D elemental maps of the Braunite steel. Apparent concentrations of $\mathrm{N}$ in the roaldite $(\gamma$ $\mathrm{Fe}_{4} \mathrm{~N}$ ) precipitate by (b) pulsed voltage and (c) pulsed laser atom probes as a function of base temperature.

\section{References:}

[1] J. Takahashi, K. Kawakami and K. Kobayashi, Quantitative analysis of carbon content in cementite in steel by atom probe tomography, Ultramicroscopy 111 (2011),1233-1238.

[2] G. Miyamoto, K. Shinbo and T. Furuhara, Quantitative measurement of carbon content in Fe-C binary alloys by atom probe tomography, Scripta Mater. 67 (2012), 999-1002.

[3] B. Langelier, H.P. Van Landeghem, G.A. Botton and H.S. Zurob, Interface segregation and nitrogen measurement in Fe-Mn-N steel by atom probe tomography, Microsc. Microanal. 23 (2017), 385-395.

Acknowledgement: The authors are grateful to Prof. N. Nakada for provision of the Braunite steel. 


\title{
Field-Dependent Measurement of GaAs Composition by Atom Probe Tomography
}

\author{
E. Di Russo ${ }^{1}$, I. Blum ${ }^{1}$, J. Houard ${ }^{1}$, G. Da Costa $^{1}$, D. Blavette ${ }^{1}$, and L. Rigutti ${ }^{1}$
}

Corresponding Author: enrico.di-russo1@etu.univ-rouen.fr

1. Normandie Univ, UNIROUEN, INSA Rouen, CNRS, Groupe de Physique des Matériaux, 76000 Rouen.

The composition measured by laser-assisted atom probe tomography in GaAs may be inaccurate depending on the experimental conditions used [1,2]. In this work, we assess the role of the static $\mathrm{DC}$ field and of the impinging laser energy $\left(\mathrm{E}_{\text {las }}\right)$ on such compositional bias, finding that the former has a primary influence, while the latter has a weaker one in the interval of parameters explored $[3,4]$. The measured atomic fraction of Ga may thus vary from 0.55 at low field conditions to 0.35 at high field (Fig. 1 a). These results have been interpreted in terms of preferential evaporation of $\mathrm{Ga}$ at high field, while the deficit of As is most likely explained by the formation of neutral As complexes either by direct ejection or upon dissociation of larger clusters after evaporation. In fact, the evolution of the abundances of different As molecular ions reveals that large As-molecular ions can be formed only at low fields, while $\mathrm{E}_{\text {las }}$ does not significantly affect the As clustering [4]. Moreover, high electric fields can also lead to the field dissociation of the As-clusters formed on tip surface (Fig. 1 b). The study of multiple detection events is consistent with this scenario.

(a)

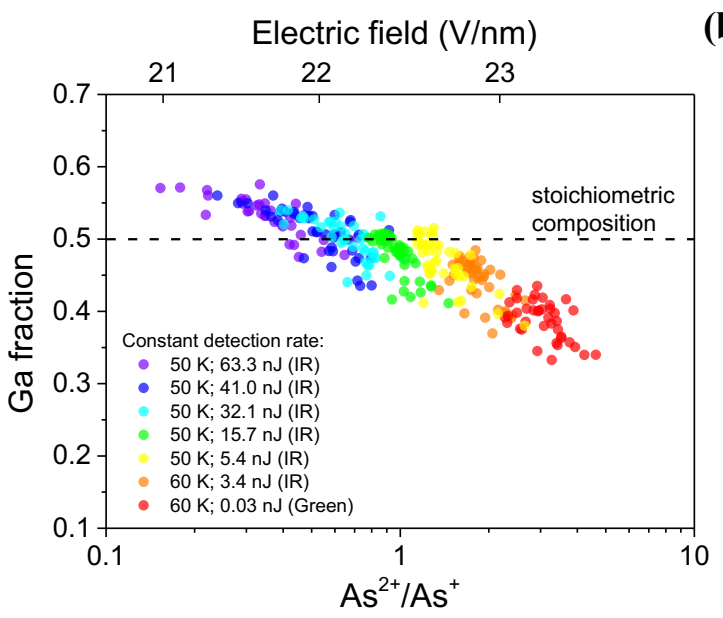

(b)

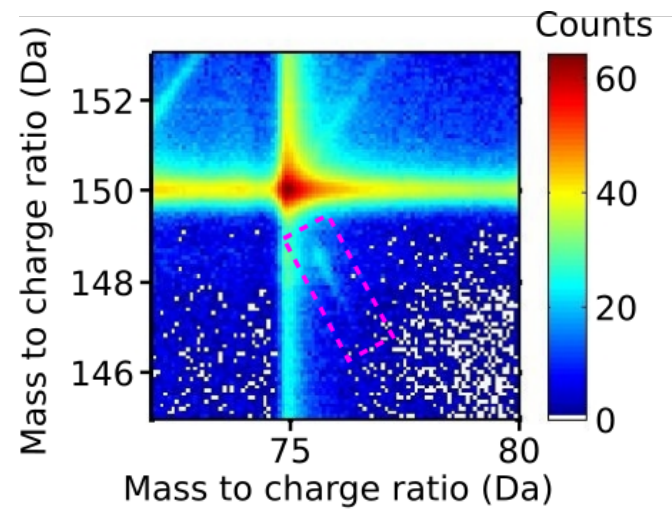

Figure 1 (a) Locally-resolved composition measured in GaAs for constant detection rate measurements plotted as a function of the local ratio $\mathrm{As}^{2+} / \mathrm{As}^{+}$. (b) Detail of correlation histograms associated to the dissociation process $\mathrm{As}_{3}^{2+} \rightarrow \mathrm{As}_{2}^{+}+\mathrm{As}^{+}$. The dataset contains $\sim 19$ million of multiple events.

References:

[1] B. P. Gorman et al., Photovoltaic Specialists Conference (PVSC), 37th IEEE (2011).

[2] A. Cerezo et al., Journal of Microscopy 141, Pt.2, 155 (1986).

[3] L. Mancini et al., J. Phys. Chem. C, 118, 24136 (2014).

[4] E. Di Russo et al, Microscopy and Microanalysis 23.6, 1067 (2017). 


\title{
Analysis of Field Evaporated Ions from Transition Metal Nitrides
}

\author{
Chikasa Nishimura $^{1}$, Yuki Haneji ${ }^{1}$, Hiroshi Tsuji ${ }^{1}$, and $\underline{\text { Yasuhito Gotoh }}^{1}$ \\ Corresponding Author: gotoh.yasuhito.5w@kyoto-u.ac.jp \\ 1. Department of Electric Science and Engineering, Kyoto University, Kyoto, Japan.
}

While atom probe (AP) can now analyze various materials, the accuracy in composition of compounds remains questionable, especially for the compounds including light elements such as nitrogen $(\mathrm{N})$ and oxygen [1-3]. For quantitative analysis in AP, it is important to understand the field evaporation mechanism of light elements. In this study, nature of field evaporation of $\mathrm{N}$ ions were investigated with compounds that contain nitrogen. Nitrides of group IV transition metal, i.e. titanium (Ti), zirconium ( $\mathrm{Zr}$ ) and hafnium (Hf) nitrides were chosen as the samples because of their good electrical conductivities. The nitride tips were prepared by sputter-deposition of a nitride thin film on an etched tungsten (W) tip. Field-evaporated ions were mass-analyzed by a time of flight (TOF) mass spectrometer. In the TOF measurements, a static voltage was applied to the tip to investigate field-evaporated ions under the condition where the secondary phenomena induced by a sudden change of field or temperature on the surface were suppressed. Measurements were done with three fixed voltages without controlling the evaporation rate. Tables 1 and 2 show the results of the observation of field-evaporated ions of $\mathrm{TiN}$ and $\mathrm{ZrN}$, respectively. For TiN and $\mathrm{ZrN}$, both atomic metal ions and molecular ions were detected. TiN and $\mathrm{ZrN}$ tended to evaporate in the form of a triple-charged molecular ions. For $\mathrm{ZrN}, \mathrm{Zr}^{2+}, \mathrm{Zr}^{3+}, \mathrm{ZrN}^{3+}$ and $(\mathrm{ZrN})_{2}{ }^{3+}$ were observed at a lower dc voltage. Applying a higher voltage, $\mathrm{N}^{+}$ions were detected in addition to these ions. These results suggest that the evaporation field of $\mathrm{N}^{+}$is higher than those of $\mathrm{Zr}^{3+}$ and $(\mathrm{ZrN})_{2}{ }^{3+}$. For $\mathrm{HfN}$, no ions could be detected. The evaporation fields of the ions from the transition metal nitrides were preliminary estimated with the empirically obtained work function [3], the bond energy [4] and the results of molecular orbital calculation. Some results of TOF measurements were explained by these calculation.

Table 1 Observed ions from TiN tip

\begin{tabular}{|c|c:c|c|}
\hline \multirow{4}{*}{ TiN-tip } & \multicolumn{3}{|c|}{ Applied Voltage } \\
\cline { 2 - 4 } & $\mathbf{6 . 0 ~ k V}$ & $\mathbf{1 0 . 0} \mathbf{~ k V}$ & $\mathbf{1 4 . 0 ~ k V}$ \\
\cline { 2 - 4 } & $\mathrm{TiN}^{3+}$ & $\mathrm{TiN}^{3+}$ & \\
\cline { 2 - 4 } & $\mathrm{TiN}^{2+}$ & $\mathrm{TiN}^{2+}$ & \\
\cline { 2 - 4 } & $\left(\mathrm{TiN}_{2}{ }^{3+}\right.$ & $\left(\mathrm{TiN}_{2}{ }^{3+}\right.$ & \\
\cline { 2 - 4 } & $\mathrm{Ti}_{3}{ }^{2+}$ & & \\
\cline { 2 - 4 } & $\left(\mathrm{TiN}_{4}{ }^{3+}\right.$ & $(\mathrm{TiN})_{4}{ }^{3+}$ & \\
\hline
\end{tabular}

Table 2 Observed ions from ZrN tip

\begin{tabular}{|c|c|c|c|}
\hline \multirow{4}{*}{ ZrN-tip } & \multicolumn{3}{|c|}{ Applied Voltage } \\
\cline { 2 - 4 } & $\mathbf{1 2 . 0} \mathbf{~ k V}$ & $\mathbf{1 4 . 0} \mathbf{~ k V}$ & $\mathbf{1 5 . 0 ~ k V}$ \\
\cline { 2 - 4 } & & & $\mathrm{N}^{+}$ \\
\cline { 2 - 4 } & & $\mathrm{Zr}^{3+}$ & $\mathrm{Zr}^{3+}$ \\
\cline { 2 - 4 } & $\mathrm{ZrN}^{3+}$ & $\mathrm{ZrN}^{3+}$ & \\
\cline { 2 - 4 } & $\mathrm{Zr}^{2+}$ & $\mathrm{Zr}^{2+}$ & $\mathrm{Zr}^{2+}$ \\
\cline { 2 - 4 } & & $(\mathrm{ZrN})_{2}{ }^{3+}$ & $(\mathrm{ZrN})_{2}{ }^{3+}$ \\
\hline
\end{tabular}

$\underline{\text { References: }}$

[1] J. R. Riley, et al., ACS Nano 6 (2012) 3898-3906.

[2] R. Agrawal et al., J. Phys. Chem. C 115 (2011) 17688-17694.

[3] R. Fujii et al., Vacuum 80 (2008) 832-385.

[4] H. O. Pierson, Handbook of Refractory Carbides and Nitrides: Properties, Characteristics, Processing, and Applications, Noyes, Westwood, 1996. 


\section{Correction of APT Chemical Composition Measurements Biases in Nano- Particles: A Chemical Composition Correction Model}

Constantinos Hatzoglou ${ }^{1}$, Bertrand Radiguet ${ }^{1}$, and Philippe Pareige ${ }^{1}$

Corresponding Author: constantinos.hatzoglou1@univ-rouen.fr

1. Normandie Univ, UNIROUEN, INSA Rouen, CNRS, Groupe de Physique des Matériaux, 76000 Rouen, France.

Since the advent of atom probe tomography (APT), it is possible to study materials at the nanoscale and in three dimensions. However, analysis of nano-objects whose size is close to the technique resolution brings it to its limits, especially if they are embedded in a matrix with a different evaporation field. In this case, biases may occur. These artefacts are quiet well known from the APT community [1-3]. Focusing on nano-particles, it is observed that these artefacts can skew there apparent reconstructed morphology and the chemical composition measurements [4]. On this last point, there is no correction model suitable for all kind of particles.

In this presentation, an analytical model developed to quantify bias in chemical composition measurements and to provide the reliable chemical composition of particles is described. After the description of this chemical composition correction (CCC) model and a discussion of its limitations, it is faced to field evaporation simulation to validate it. Subsequently, the application of this model will be presented in various examples of nano-particles in different systems.

\section{References:}

[1] B. Gault, M.P. Moody, J.M. Cairney, S.P. Ringer, Atom Probe Microscopy, 2012th ed., Springer, 2012.

[2] W. Lefebvre, F. Vurpillot, X. Sauvage, Atom probe tomography: put theory into practice, Elsevier, Boston, MA, 2016.

[3] M.K. Miller, Atom Probe Tomography: Analysis at the Atomic Level, Springer US, 2000.

[4] C. Hatzoglou, B. Radiguet, P. Pareige, Experimental artefacts occurring during atom probe tomography analysis of oxide nanoparticles in metallic matrix: Quantification and correction, J. Nucl. Mater. 492 (2017) 279-291. doi:10.1016/j.jnucmat.2017.05.008. 


\section{Discrimination of Ions Through Machine Learning of Atom Probe Data}

Scott Broderick ${ }^{1}$, Bhargavea Urala Kota ${ }^{2}$, Tianmu Zhang ${ }^{1}$, Ramachandran Subramanian², Srirangaraj Setlur ${ }^{2}$, Venu Govindaraju ${ }^{2}$, Krishna Rajan ${ }^{1}$

Corresponding Author: Scott Broderick, scottbro@buffalo.edu

1. Department of Materials Design and Innovation, University at Buffalo, Buffalo, NY USA.

2. Department of Computer Science and Engineering, University at Buffalo, Buffalo, NY USA.

We present a new approach based on clustering algorithms for discriminating isotopes which have overlapping mass-to-charge ratios $(\mathrm{m} / \mathrm{n})$ in an atom probe tomography (APT) reconstruction. This addresses a significant challenge as in many cases (for example, $\mathrm{Si}^{2+} / \mathrm{N}^{+}$) the individual ions collected cannot be assigned their chemistries. For these cases, even with significant improvements in the chemical resolution of the detectors, additional analysis and information in the reconstruction is required. We previously developed an approach which captured the kinetic energy behavior of the atoms through a multi-tier data dimensionality reduction analysis, allowing us to discriminate the isotopes [1]. This required utilizing more of the data available from an experiment (such as instrument parameters, evaporation physics, and tip geometry), thus accounting for the complexity of the evaporation physics which extends beyond solely time-of-flight (TOF) and ion mass. The current work builds on this prior approach by performing an alternate machine learning analysis which provides a higher level of isotope discrimination.

As a test case, we analyzed an Al-Mg-Sc sample, which has overlapping isotopes in the TOF spectrum (Figure 1), however the isotopes are separated enough to use machine learning algorithms and test the disambiguation of the spectrum into different isotopes. We have experimented with several unsupervised clustering algorithms and Gaussian mixture models (GMM) to cluster each row of the APT data into groups corresponding to a single isotope. Since the ground truth (true isotope identity for each row) is unknown, we evaluate our algorithms by measuring the distribution of isotopes obtained and comparing them to natural isotope distributions. The results of the approach as applied to nitride systems will be presented [2].
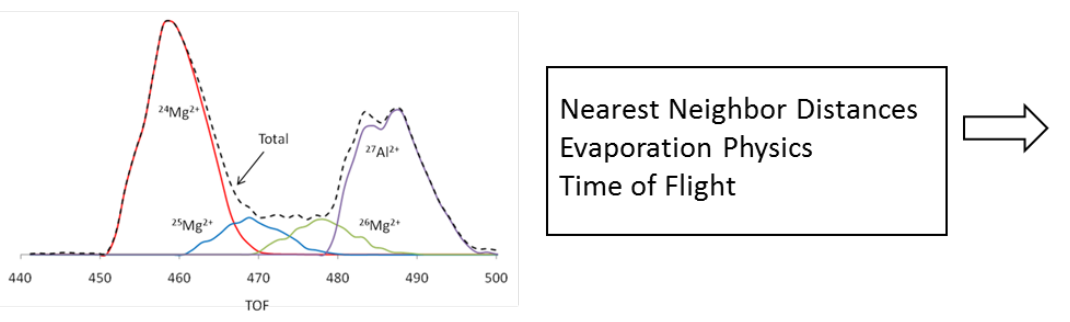

Correct Label of Element
\begin{tabular}{|c|c|c|}
\hline Entry & Position & $\underline{\text { Label }}$ \\
23 & $\mathrm{x}, \mathrm{y}, \mathrm{z}$ & $\mathrm{Al}$ \\
27 & $\cdot$ & $\mathrm{Al}$ \\
31 & $\cdot$ & $\mathrm{Mg}$ \\
48 & $\cdot$ & $\mathrm{Al}$ \\
52 & $\cdot$ & $\mathrm{Sc}$ \\
\hline
\end{tabular}

Figure 1 The logic of this work. As an example, the isotopes of $\mathrm{Mg}$ and $\mathrm{Al}$ overlap when plotted versus TOF. By incorporating the APT data with clustering and GMM algorithms, we are able to correctly assign the atom label.

\section{References:}

[1] S.R. Broderick, A. Bryden, S.K. Suram, K. Rajan, Data mining for isotope discrimination in atom probe tomograph, Ultramicroscopy 132 (2013) 121-128.

[2] We acknowledge support from NSF DIBBs project award number ARI 1640867. 


\title{
A High Multiple Hits Correction Factor for Atom Probe Tomography
}

\author{
D.J. Larson ${ }^{1}$, T.J. Prosa ${ }^{1}$, E. Oltman ${ }^{1}$, D.A. Reinhard ${ }^{1}$, B.P. Geiser ${ }^{1}$ and R.M. Ulfig ${ }^{1}$ \\ ${ }^{1}$ CAMECA Instruments Inc., 5470 Nobel Drive, Madison, WI 53711 USA
}

Microchannel plate transducer amplifiers, used to detect ions in atom probe tomography (APT), have a reduced detection efficiency for multiple ion impacts (i.e. multiple ions that impact the detector within a very small period in time and space). These effects are typically referred to as "multiple hits" [1,2], and may cause "ion pile up" [3,4]. For the technologically relevant case of boron in silicon [4-6], this can be a significant problem. Evidence of this issue is seen in secondary ion mass spectrometry (SIMS) versus APT depth profiles that have been corrected using known standard values for the NIST standard reference material SRM-2137. Although several factors may contribute to this discrepancy, it is believed that multiple ion events at high boron concentration underlie this issue [4,7], as prior work shows boron concentrations lower than excepted, particularly for highly doped samples [4]. The shape of boron APT profiles often matches well with SIMS data (Fig.1), but the absolute APT concentration can be lower than the SIMS data. This difference appears to scale with boron concentration, suggesting a possible direct dependence on multiple events. The current work uses a boron implanted sample described previously [8] to develop a simple correction factor similar to the relative-sensitivity factor (RSF) typically used to calibrate SIMS data [9]). This correction factor, based upon the assumption that boron deficiency is a function of boron multiple hits, is a simple scaling factor easily applied to any previously calculated boron concentration using APT. An example result is shown in Fig. 2 for the NIST SRM-2137. In addition, corrections derived directly from the multiple hits detected within APT data itself are being explored.
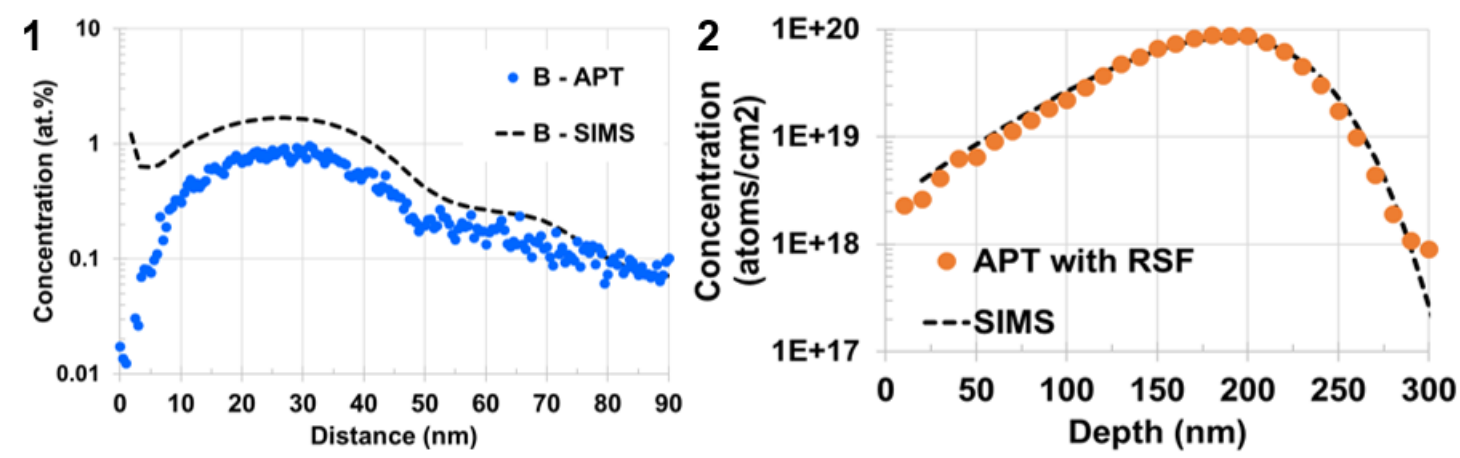

References:

[1] O Jagutzki et al., IEEE Trans. Nucl. Sci. 49 (2002), p. 2477

[2] G Da Costa et al., Rev. Sci. Instrum. 76 (2005), p. 013304-1

[3] MK Miller, GDW. Smith, Atom Probe Microanalysis: Principles and Applications to Materials

Problems, (Materials Research Society, Pittsburgh) (1989).

[4] G Da Costa et al., Rev. Sci. Instrum. 83 (2012), p. 123709.

[5] P Ronsheim et al., Appl. Surf. Sci. 255 (2008), p. 1547

[6] D Blavette et al., Mater. Sci. Eng. A. 7 (2011), p. 012004-1

[7] T Philippe et al., Ultramicroscopy. 109 (2009), p. 1304

[8] DJ Larson et al., Microsc. Microanal. 20 (2014), p. 2088

[9] F. Stevie, Secondary Ion Mass Spectrometry: Applications for Depth Profiling and Surface Characterization, Momentum Press, 2015. 


\section{Quantitative Aspects of PLAD Sidewall Doping Characterization by SIMS and APT}

Dimitry Kouzminov $^{1}$, James Cournoyer ${ }^{1}$, Harish Muthuraman ${ }^{1}$, Somchintana Norasetthekul ${ }^{1}$, Qi $\mathrm{Gao}^{1}$

Corresponding Author: Dimitry_Kouzminov@amat.com

1.Applied Materials Inc., Applications Lab, 35 Dory Road, Gloucester, MA 01930, USA.

Fin sidewall doping by plasma implantation (PLAD) is a focus of the current study. Immersion plasma implantation is considered to be an attractive alternative to beamline implantation due to its ability to achieve sidewall doping of 3D structures. Unlike planar transistors, 3D devices require junction characterization both normally to the wafer surface and across sidewall. SIMS (1.5D SIMS) has been demonstrated as a reliable process monitor allowing to measure total implant dose into the sidewall. At the same time, Atom Probe Tomography (APT), unlike any other technique, allows to measure dopant distribution in a single device with detection limits as low as $5 \mathrm{E} 18 \mathrm{~cm}^{-3}$. Both 1.5D SIMS and APT require additional process steps, i.e. trench backfill, to perform analysis. Due to its nature, plasma implants result in high dopant concentration at the surface, and therefore may be altered by trench backfill step. In this paper, we present a study of Fin doping by AsH3 and B2H6 plasma, using both SIMS, 1.5D SIMS and APT as complimentary techniques. Certain artifacts affecting dopant quantification, which we encountered in this study and attempted to resolve, are discussed in this paper. 


\section{Measuring the Spatial Accuracy of an Atom Probe Tomography Reconstruction}

Anna V. Ceguerra ${ }^{1,2}$, Alec C. Day ${ }^{1}$, and Simon P. Ringer ${ }^{1}$

Corresponding Author: anna.ceguerra@sydney.edu.au

1. The University of Sydney. Australian Centre for Microscopy \& Microanalysis, and School of Aerospace, Mechanical and Mechatronic Engineering, Sydney, NSW 2006, Australia.

2. The University of Sydney. The University of Sydney Nano Institute (Sydney Nano), Sydney, NSW 2006, Australia.

In the two decades that have followed the introduction of the 3D back projection algorithm [1], there have been a number of approaches proposed for the atom probe tomographic reconstruction [e.g. 2]. Each approach addresses a unique problem in the original reconstruction protocol. However, the results of these various reconstruction approaches are not straightforward to compare. Spatial distribution maps [3,4], for example, are not appropriate because they convolve information about the suitability of the instrumental settings such as pulse fraction, laser energy, and temperature for an individual experiment. In fact, we suggest that there is no common metric for assessment of the spatial accuracy of a particular tomographic reconstruction.

In this contribution, we address this issue and propose a standard measurement for the spatial accuracy of a tomographic reconstruction. Our definition exploits atom probe crystallography and is based on observable structural features in the data. We demonstrate this approach so as to enable a quantitative assessment of the accuracy of a number of different approaches to the tomographic reconstruction and we do this across a number of different atom probe data sets. The definition for spatial accuracy of the tomographic reconstruction as proposed here is not limited to crystallographic measurements. It may be extended to incorporate the definition of other known features such as grain boundary interfaces, second phase precipitates with known orientation relationships, twins, or the topology of structures within the data set.

\section{$\underline{\text { References: }}$}

[1] P. Bas et al., A general protocol for the reconstruction of 3D atom probe data. Applied Surface Science 87-88 (1995) 298-304.

[2] F. Vurpillot et al., Reconstructing atom probe data: A review. Ultramicroscopy 132 (2013) 19-30.

[3] B.P. Geiser et al., Spatial Distribution Maps for Atom Probe Tomography. Microscopy and Microanalysis 13 (2007) 437-447.

[4] B. Gault et al., Spatial Resolution in Atom Probe Tomography. Microscopy and Microanalysis 16 (2010) 99-110. 


\section{Automated Calibration Of Atom Probe Reconstructions Using GPU Based Fourier Analysis in a Python Workbench}

Shyam Katnagallu ${ }^{1}$, Giuseppe Di Bernardo ${ }^{2}$, Leigh Stephenson ${ }^{1}$, Markus Rampp ${ }^{2}$, Frédéric De Geuser $^{3}$, Dierk Raabe ${ }^{1}$ and Baptiste Gault ${ }^{1}$

Corresponding Author: s.katnagallu@mpie.de

${ }^{1 .}$ Microstructure physics and alloy design Department, Max-Planck-Institut für Eisenforschung GmbH, Dusseldorf, Germany-40227.

2. HPC Application Support \& Scientific Visualization Group, Max Planck Computing and Data Facility, Garching and Germany-85748.

3. Univ. Grenoble Alpes, CNRS, Grenoble INP, SIMAP, 38000 Grenoble, France

The ability of atom probe tomography (APT) to deliver three dimensional atomic scale chemical information together with the crystallographic/structural knowledge (in some cases) makes it an invaluable technique for material characterization. For any meaningful crystallographic or structural information to be extracted, the reconstructions must be precisely calibrated. Calibration of APT volumes usually involves evaluating reconstruction parameters for a particular run. Evaluation of these parameters usually take advantage of the partial crystallographic information available within an APT dataset. In this work, we developed a python-based workbench for easy calibrations of the aforementioned parameters, leading to an automated calibration and reconstruction routine based on Fourier analysis of the reconstruction. A discrete Fourier transform on a 3D volume typically containing $10^{8}$ atoms is an $\mathrm{O}\left(\mathrm{N}^{2}\right)$ operation and for an evaluation at $10^{6}$ reciprocal points this equates to $10^{15}$ floating point operations (FLOP). The modern graphical processing units (GPU) are capable of performing $10^{13} \mathrm{FLOP} / \mathrm{s}$ which reduces the calculation time to a matter of minutes. These discrete Fourier transformations are well suited for GPUs as they are efficient at parallelization. Computation of X-ray scattering maps using a python library PYNX on GPUs is already shown to be efficient[1]. We extend the approach to APT volumes and propose an automated calibration routine for volumes in this work.
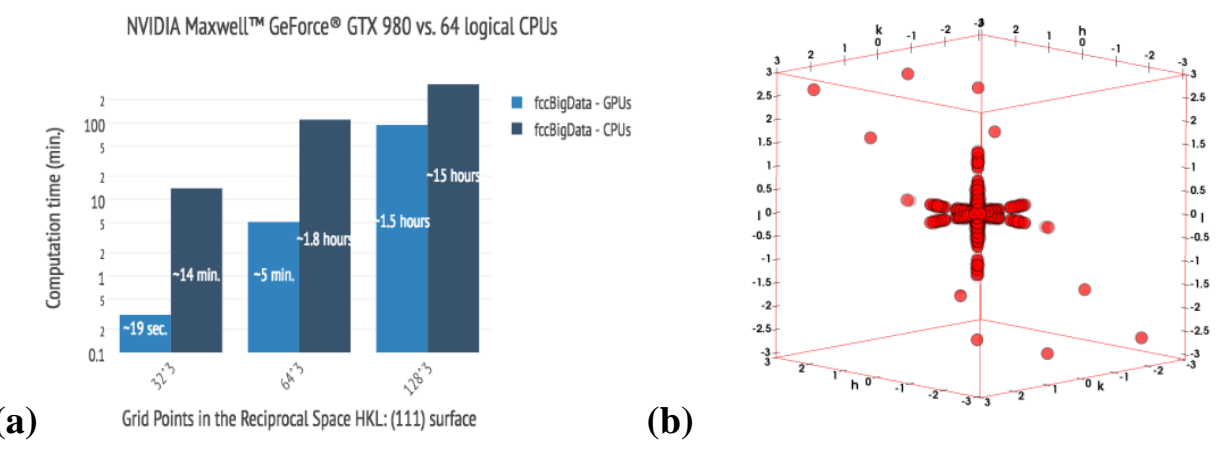

(b)

Figure 1 (a) Calculation speed comparison between a GPU and 64 CPUs for a pure Aluminum APT dataset containing $10^{8}$ atoms. (b) Fourier transform of the same dataset visualized in Paraview ${ }^{\mathrm{TM}}$.

$\underline{\text { References: }}$

[1] V. Favre-Nicolin, J. Coraux, M.-I. Richard, and H. Renevier, "Fast computing of scattering maps of nanostructures using graphical processing units," 2011. 


\title{
EPOSA - User-Friendly Software for Expanded Atom Probe Analysis
}

\author{
Jens Keutgen ${ }^{1}$, Oana Cojocaru-Mirédin ${ }^{1}$, and Matthias Wuttig ${ }^{1}$ \\ Corresponding Author: keutgen@physik.rwth-aachen.de \\ 1. I. Physikalisches Institut (IA) RWTH Aachen, 52056 Aachen, Germany.
}

As the atom probe community expands, more people have the need for specialized analysis methods for their experiments that are not covered by IVAS. Although scripts written in Matlab or Python make these analyses possible they fall short when an interactive manipulation of the dataset or analysis parameters is necessary for a good understanding. To counter these problems, I developed an easy to use Graphical User Interface (GUI) that imports EPOS files and gives the user simple options to select and manipulate the data. By building a strong base code for the GUI and the data selection it is now possible to rapidly expand the analysis methods as needed.

In our group, this software was mainly used for investigating multiple events in phase change materials and analyzing the buffer/absorber interface of CIGS solar cells. This allowed us to get a deeper understanding of the connection between multiple events and the bonding mechanisms in phase change materials [1]. In case of the interface analysis, we faced problems of overlapping peaks at the interface between buffer and absorber (see Fig. 1b) that resulted in inaccurate proximity histograms (Fig. 1a). For this I developed an algorithm that creates an isosurfaces and performs a peak decomposition for every bin of the proximity histogram without much external input from the user. Thus, it is possible to perform the analysis for any kind of interface with overlapping peaks. With these features already present it is now necessary to showcase the software to a broader audience for feedback and to expand the available analysis tools inside the atom probe community.
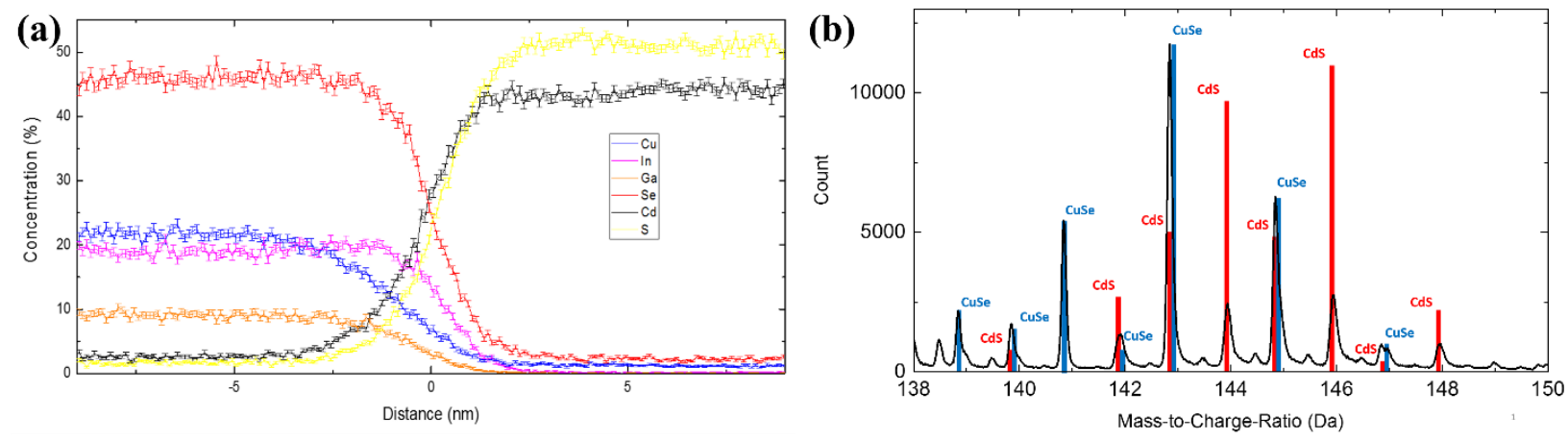

Figure 1 (a) Overestimation of certain elements inside the proximity histogram caused by overlapping peaks. (b) Example of overlapping peaks. Both CdS and CuSe show peaks in the range of 140 Da to 148 Da. Decomposition for these peaks inside the proximity histogram is necessary for a correct representation of the data.

$\underline{\text { References: }}$

[1] Min Zhu, Oana Cojocaru-Mirédin, Matthias Wuttig, Unique bond breaking in crystalline phase change materials and the quest for metavalent bonding, Submitted to Advanced Materials (2017) 


\title{
Self-Consistent Reconstructions Utilizing Electron Microscopy
}

\author{
David R. Diercks ${ }^{1}$ and Brian P. Gorman ${ }^{1}$ \\ Corresponding Author: ddiercks@mines.edu \\ 1. Department of Metallurgical and Materials Engineering, Colorado School of Mines, Golden, \\ CO USA.
}

Atom probe tomography (APT) reconstructions can provide valuable information on nm-scale compositional variations within materials, such as dopant distributions [1] and space-charge potentials at grain boundaries [2]. As such, the spatial accuracy of the reconstruction is of primary importance in producing valid results for many analyses. The most commonly used reconstruction methods are all based on point-projection models [3-6] where a set of userspecified parameters is necessary. Sometimes the validity of a particular reconstruction and the values used can be assessed from features observed during field evaporation or within the reconstructed volume, such as direct measurement of the image compression factor (ICF) from the detector histogram [7] or the flatness and spacings of crystalline planes [4,8]. For materials without these built-in calibrations the solution is under-constrained - there are more parameters than means of assessing their validity. In these cases, determining what reconstruction inputs to use is not straightforward. The implications of this can be quite significant; APT analysis of a National Institute of Standards and Technology Standard Reference Material showed that uncertainties in the measured implant dose were dominated by reconstruction errors [8]. Here we demonstrate that imaging of the tip before and after APT analysis provides additional constraints for generating reliable, self-consistent reconstructions. While such approaches for promoting accuracy of APT reconstructions have been outlined [9], this work explores the influence of particular variables in detail and focuses on metrics for assessing the consistency between input/known parameters and the resulting reconstructions. It is found that there is not one unique solution; a series of geometrically equivalent solutions can be created by adjusting the detection efficiency and ICF proportionally. If either of these values can be independently determined, then a reconstruction with unique values results. Concepts in this methodology are general and should be straightforward to incorporate into reconstruction protocols.

\section{References:}

[1] K. Thompson et al, Imaging of Arsenic Cottrell Atmospheres Around Silicon Defects by ThreeDimensional Atom Probe Tomography, Science 317 (2007), 1370.

[2] D.R. Diercks et al., Three-dimensional quantification of composition and electrostatic potential at individual grain boundaries in doped ceria, J. Mater. Chem. A 4 (2016), 5167-5175.

[3] D. Blavette, et al., Direction et distance d'analyse à la sonde atomique, Rev. Phys. Appl. 17 (1982), 435-440.

[4] P. Bas et al., A general protocol for the reconstruction of 3D atom probe data, Appl. Surf. Sci. 87 (1995), 298-304.

[5] B. Gault et al., Atom probe microscopy, Springer 2012.

[6] D.J. Larson et al., Local Electrode Atom Probe Tomography: A User's Guide, Springer 2013.

[7] B. Gault, et al., Advances in the calibration of atom probe tomographic reconstruction, J.Appl. Phys. 105 (2009), 034913.

[8] T.J. Prosa et al., Analysis of implanted silicon dopant profiles, Ultramicroscopy 132 (2013), 179-185.

[9] T.J. Prosa et al., Approaches for Promoting Accurate Atom Probe Reconstruction, Microscopy and Microanalysis 22(S3) (2016), 664-665. 


\section{A Precise Method for Analysis of Elemental Distribution Inside Solute Clusters}

Takumi Kitayama ${ }^{1}$, Masaya Kozuka ${ }^{2}$, Yasuhiro Aruga ${ }^{2}$, Chikara Ichihara $^{1}$

Corresponding Author: kitayama.takumi@kobelco.com

${ }^{1}$ Applied Physics Research Laboratory, Kobe Steel, Ltd., Kobe, Japan

${ }^{2}$ Materials Research Laboratory, Kobe Steel, Ltd., Kobe, Japan

Solute clusters are formed by substituting at the most tens of solute atoms in matrix lattice points. They strongly influence on mechanical property of metals depending on its number density, size, compositional elements, etc. The atom probe tomography (APT) is widely known as a cluster analysis tool. Nevertheless, cluster analyzing on IVAS ${ }^{\mathrm{TM}}$, standard software for APT data analysis nowadays, is not always sufficient especially for evaluating elemental distribution inside solute clusters. Even though ellipsoidal clusters with various sizes are dispersed, the software cannot illustrate the internal elemental distribution profile of the clusters directly. Besides, calculating statistical errors of each profile data point always costs an enormous time. These limitations make it difficult to obtain highly reliable knowledge for applying to material development. For example, Aruga et al. reported that $\mathrm{Mg} / \mathrm{Si}$ ratio varies from the central region of Mg-Si clusters toward the outside in an aged Al-Mg-Si alloy by means of APT. Herein they could not notice the reliability of the result sufficiently.

In this study we developed a code which can directly illustrate an average concentration profile inside a cluster even in the case of including various sizes of ellipsoidal clusters. The profile can be with respect to absolute distance and includes errors in each data point. The developed code was verified its reliability by analyzing a cluster model which has inhomogeneous elemental distribution. Clusters are fitted with ellipsoids using variance-covariance matrix and each ellipsoid has three main axes. Solute atoms in the clusters are identified by the coordinates with respect to the main-axes. These axes are normalized to the specific axis by solute standard deviations on each axis. This is equivalent to transforming the ellipsoid into a sphere. Thereby, the structure inside the cluster is represented only by the radius of the sphere in the real cluster scale. When averaging elemental distribution, statistical errors are also calculated and thus the reliability can be evaluated. This is especially important when we consider the central region of clusters, i.e. fewer atoms exist. We will demonstrate and discuss the result of reliability verification on the day.

\section{$\underline{\text { References: }}$}

[1] Y. Aruga et al., Scr. Mater. 116 (2016) 82. 


\section{Reconstruction Solution Envelopes for Improved Morphological Confidence}

Andrew P. Proudian ${ }^{1}$, Matthew B. Jaskot ${ }^{2}$, David R. Diercks ${ }^{2,3}$, Brian P. Gorman ${ }^{2,3}$, and Jeramy D. Zimmerman ${ }^{1,2}$

Corresponding Author: jdzimmer@mines.edu

1. Department of Physics, Colorado School of Mines (CSM), Golden, CO USA

2. Materials Science Program, CSM, Golden, CO USA

3. Department of Materials and Metallurgical Engineering, CSM, Golden, CO USA

Extracting morphological information from an atom probe sample requires a high degree of confidence in the reconstruction. Traditionally, this has been done by using the crystal structure of the material being imaged as a internal fiducial. However, this only works on systems with a high degree of crystallinity. Others have pursued correlative TEM imaging of the sample before (and after) atom probe analysis to bound the reconstruction.[1] This has broader applicability, but excludes those systems that are sensitive to electron radiation. Even with these methods the quality of the reconstruction is limited by the lower $x-y$ resolution of the instrument.

Furthermore, bounding the reconstruction still leaves a number of solutions that satisfy this constraint.

Recently, atom probe tomography of molecular organic systems has been pursued to better understand their morphology.[2] Unfortunately, these materials tend to have a low degree of crystallinity and degrade under electron radiation, preventing both methods for confident reconstruction. Thus, a new approach is needed.

We have developed a reconstruction method that provides a solution envelope, increasing confidence in the conclusions drawn from the data regardless of the exact solution. We mitigate the impact of the lower $\mathrm{x}-\mathrm{y}$ resolution on analysis by shifting points in $\mathrm{x} \& \mathrm{y}$. This adjustment is determined by solving the Assignment and Exact Cover Problems for slices of data in detector space. The resultant solution sets can then be used to determine the robustness of the morphological signal as compared to a null hypothesis of random labeling. This process can also constrain the reconstruction to maintain density and minimum particle spacing.

We compare this method with common methods of reconstruction for two types of samples: (1) inorganic TEM imaged samples, demonstrating reconstructions at least as accurate as standard methods; (2) organic materials showing higher reconstruction accuracy.

$\underline{\text { References: }}$

[1] Gorman et al., Mater. Sci. Technol., 24, 682-688 (2008).

[2] Proudian et al., Nano Lett., 16 (10), 6086-6091 (2016). 


\title{
Seeing Is Not Always Believing: The Influence of Isosurface Selection in Quantitative Outputs From Proximity Histograms
}

\author{
Dallin J. Barton ${ }^{1}$, B. Chad Hornbuckle ${ }^{2}$, Kristopher A. Darling ${ }^{2}$ and Gregory B. Thompson ${ }^{1}$
}

Corresponding Author: dbarton@crimson.ua.edu

1. University of Alabama, Tuscaloosa, AL USA.

2. Army Research Laboratory, Aberdeen, MD USA.

A common procedure for atom probe compositional analysis is the proximity histogram or proxigram. The proxigram was developed by Hellman et al. [1] to find the average chemical compositional profile across curved surfaces. This profile and corresponding compositional information is dependent on the selected isoconcentration value. Thus, the selection of this value to create the 'isosurface' is paramount in delineating the compositional information generated inside and outside this surface, with those values relative to the selected isoconcentration. This poster provides a complementary study to previous work by Hornbuckle et al. [2] who reported a procedure to identify the isosurface concentration values in a high solute, low partitioning system. Unlike that work, this study involves a low solute, high partitioning system where phase separation is easily visualized. Using an oxide dispersion strengthened alloy - $91 \mathrm{Fe}-8 \mathrm{Ni}-1 \mathrm{Zr}$, we demonstrate how errors come about by quantitatively comparing the particle sizes, number density, interparticle spacing, and compositional information between the precipitated oxides. Fig. 1(a) reveals how the number density changes (with Fig, 1(b) the corresponding compositional changes) with alterations of the $\mathrm{Zr}$ isosurface values. Fig. 1(c) is a nearest neighbor (NN) cluster analysis along with three different $\mathrm{Zr}$ isosurfaces reconstructions. Using the Hornbuckle et al. analysis, the isosurface near $11 \% \mathrm{Zr}$ was found to be the correct selection; however, the isosurface reconstruction, Fig. 1(b), visually does not appear to agree with the cluster analysis. If one used the $1 \% \mathrm{Zr}$, though visually appearing to match the NN method, a nearly one-order of magnitude inaccuracy in the number density would be found with similar compositional errors. This poster will demonstrate how qualitative visual identifications for isosurfaces can yield such errors, with a procedure to avoid such issues in data analysis.

(a)

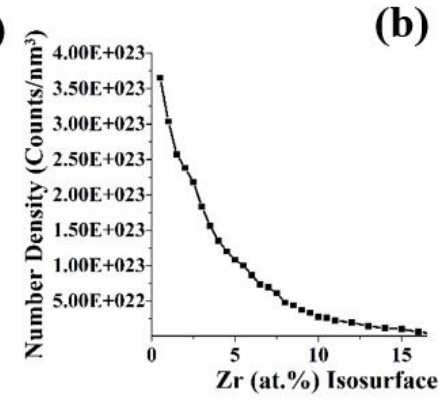

(b)

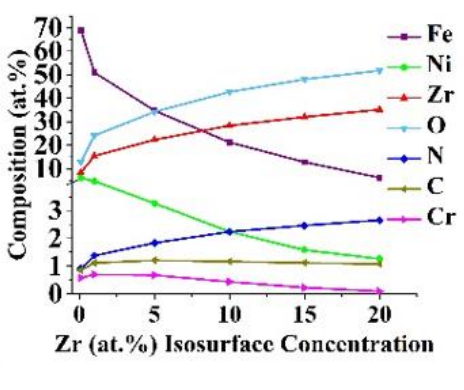

(c)

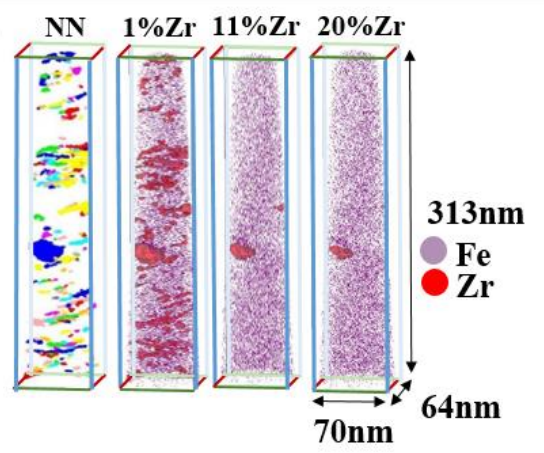

Figure 1 (a) Number density (b) compositional profiles (c) NN cluster and Zr isosurface reconstructions

References:

[1] O. Hellman et al., Microscopy and Microanalysis (2000), 439-444.

[2] B.C. Hornbuckle et al., Ultramicroscopy (2015), 346-353.

[3] Funding for this research is through ARO-W911NF-15-2-0050 


\title{
An Open-Source, Graphical Software Package for Computer-Assisted Species Assignment in Atom Probe Tomography Compositional Analysis
}

\author{
Paul Blanchard ${ }^{1}$, Ann Chiaramonti-Debay ${ }^{1}$, Norman Sanford ${ }^{1}$, and Sarah Barili ${ }^{1,2}$ \\ Corresponding Author: paul.blanchard@,nist.gov \\ 1. National Institute of Standards and Technology (NIST), Boulder, CO, USA. \\ 2. University of Colorado, Department of Computer Science, Boulder, CO, USA.
}

Identifying the ionic species associated with detection events in atom probe tomography (APT) data is a challenging task. Due to the formation of molecular ions, even relatively simple materials often yield complicated mass spectra. The manual ranging of such spectra is laborious and prone to error [1]. In this work, we demonstrate an open-source software tool with a graphical user interface to assist with species assignment in APT analysis. Features include: (1) An automated $m / q$-to-formula calculator, which creates an interactive and sortable table of plausible ionic species matching a given $\mathrm{m} / \mathrm{q}$ peak position. The calculator permits placing limits on the size and complexity of the molecules considered. (2) Tools to assign ionic species to all or a fraction of each $\mathrm{m} / \mathrm{q}$ peak. These tools can also be used to decompose overlapping peaks based on isotopic abundances. (3) An interactive multi-hit mass correlation histogram [2]. When a user selects a molecule, its calculated dissociation track can be shown as an overlay. Additional features will be discussed at the meeting. By combining automated calculations with user interactivity, this software can improve both the speed and accuracy of APT compositional analysis.

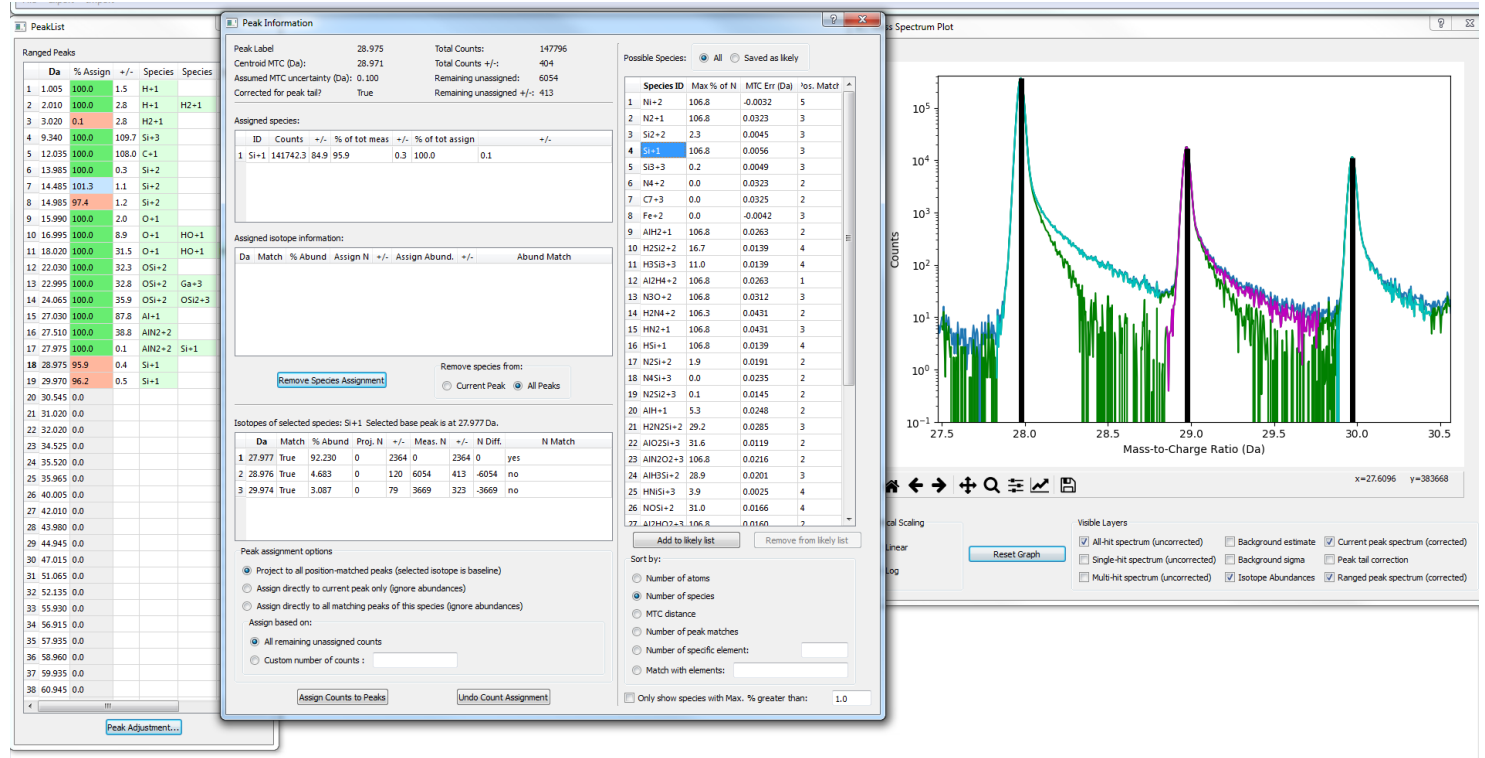

Figure 1. Software main user interface: list of peaks (left), sortable table of possible species for the currently-selected peak (center), and mass spectrum with isotope overlay (right).

References:

[1] D. Haley et al., Guided mass spectrum labelling in atom probe tomography, Ultramicroscopy 159 (2015), 338-345.

[2] D. W. Saxey, Correlated ion analysis and the interpretation of atom probe mass spectra,

Ultramicroscopy 111 (2011), 473-479. 


\section{Detector Space Fourier Analysis for Atom Probe}

Sean Jones ${ }^{1}$, Brian Gorman ${ }^{1}$

Sean Jones: seanjones@mines.edu

Brian Gorman: bgorman@mines.edu

1. Colorado School of Mines, Material Science, Golden, CO and USA

Utilizing trends in the detector space of an Atom Probe data set, techniques used in aberration corrected TEM may be adapted, with the goal of increasing reconstruction fidelity. Because the sample is in three dimensions while the image is in two-dimensional detector space, it is beneficial to bin detector data with a third dimension. Choosing this additional dimension to be time of detection (td), one can obtain data reflecting the surface of the sample for any period.

Fourier Brightfield analysis of the td segregated slices yields values corresponding to the topologically convoluted electric field term interacting with the specimen as well as other ion trajectory aberration inducing effects. For a sample with a uniform evaporation potential, the Brightfield surface is proportional to the convoluted electric field. For a sample with locally changing evaporation potentials, this surface can be used to locate these interfaces and correct for them. Figure 1 (a) shows a Brightfield surface of a heterogeneous interface. Varying the Brightfield aperture size allows for variable resolution limits.

Furthermore, Darkfield analysis can be used to quantify aberrations, tip shape irregularities, and evolution of the evaporating surface. AC term Fourier analysis was used to calculate time dependent spherical and chromatic aberrations Figure 1 (b). Through these methods, ion trajectory aberrations can be quantified, which may lead to more accurate reconstructions. For example, these dynamic aberration variables can serve to replace and add to the static image compression term currently used in APT reconstruction. While this method is demonstrated as a standalone processing step, it can aid in cross correlative techniques by dramatically simplifying orientation problems and providing the convoluted electric field for deconvolution purposes.

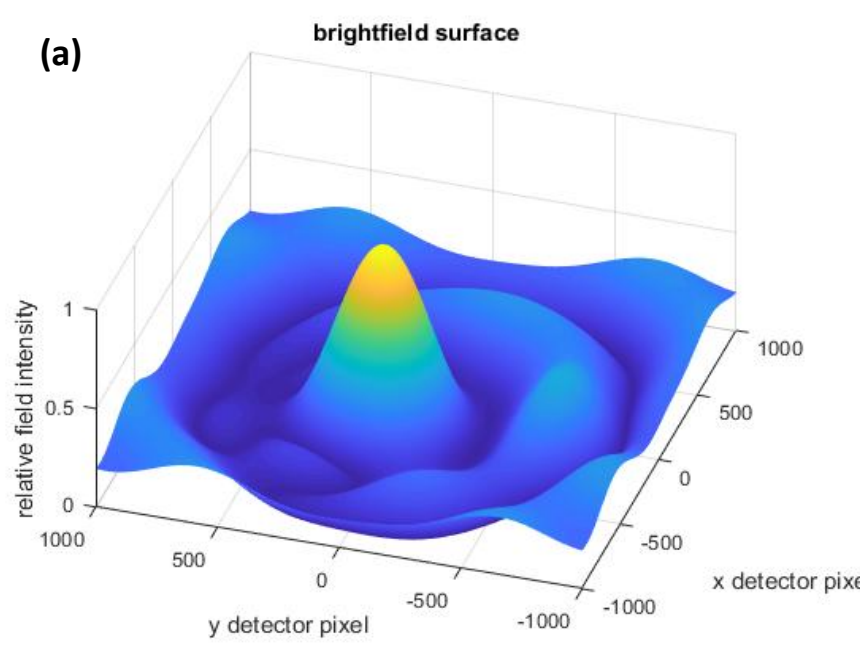

(b)

Figure 1 (a) A Brightfield surface showing tip deformation due to a heterogeneous interface.

(b) calculated spherical aberration as a function of td. At each td step, spherical aberration was calculated in the $\mathrm{x}$ and $\mathrm{y}$ directions. Close agreement supports radial symmetry expected in spherical aberrations.

Note the sudden drop in spherical aberration caused by the interfacial deformation seen in (a) 


\section{Direct Determination of Misorientation and Grain Boundary Parameters From APT Projections}

Leigh Stephenson ${ }^{1}$, Shyam Katnagallu ${ }^{1}$, Huan Zhao ${ }^{1}$, Anna Ceguerra ${ }^{2}$, Andrew Breen ${ }^{1}$, Simon Ringer $^{2}$, Dierk Raabe ${ }^{1}$, Baptiste Gault ${ }^{1}$

Corresponding Author: 1.stephenson@mpie.de

${ }^{1}$ Max Planck Institut für Eisenforschung, Düsseldorf, Germany.

2.The University of Sydney, Australian Centre for Microscopy \& Microanalysis, and School of Aerospace, Mechanical and Mechatronic Engineering, Australia.

When compared to electron diffraction techniques, atom probe is not a traditional method for crystallographic microanalysis. That said, atom probe's chemical sensitivity and near-atomic resolution has encouraged many researchers to investigate its applicability in exploring intergranular architecture on the nanoscale [1-5]. These efforts have yielded results unattainable by other microscopies and have stimulated further endeavours to refine the accuracy, precision and application of atom probe crystallography.

The observation of crystallographic artefacts (poles and zone lines) within the detector histogram allows for the exploration of projection models. Should these artefacts not be evident, data mining methods can be used to elucidate crystallographic signal [6,7]. We will briefly present the mathematical calculations for determining a model's image compression parameters using the spherical law of cosines (small angle, standard, Helmholtz and Hawkes-Kasper) and present a method for determining a bespoke projection without reliance on a model.

From this crystallographic projection information, we calculate an individual grain's orientation with respect to coordinate axes; if this is performed for two grains, we represent their misorientation using an easily calculated angle-axis representation. Finally, given the measured projection model and the grain orientations, we demonstrate how the detector trace of an interface (between grains or phases) can provide sufficient information to calculate the grain boundary normal locally. We demonstrate this for defined aluminium bi-crystals and for a 7xxx aluminium alloy. The novelty herein is that all calculations are performed without the need for a tomographic reconstruction of the detected ions - only the field evaporation image is required.

\section{References:}

[1] P. Liddicoat, et al., Nanostructural hierarchy increases the strength of aluminium alloys, Nature Communications 1, Article number: 63 (2010)

[2] L. Yao, et al., The anatomy of grain boundaries: Their structure and atomic-level solute distribution, Scripta Materialia 69(8) (2013) 622-625

[3] P. Felfer, et al., Applying computational geometry techniques for advanced feature analysis in atom probe data, Ultramicroscopy 132 (2013) 100-106

[4] Chen, Y. Schuh, C., Grain boundary networks in nanocrystalline alloys from atom probe tomography quantization and autocorrelation mapping, Physica Status Solidi a-Applications and Materials Science 212(10) (2015) pp 2302-2308

[5] Breen, A., et al., Correlating Atom Probe Crystallographic Measurements with Transmission Kikuchi Diffraction Data, Atom Probe Tomography \& Microscopy 23 (2) (2016) pp. 279-290

[6] L. Yao, A filtering method to reveal crystalline patterns from atom probe microscopy desorption maps, MethodsX 3 (2016) pp 268-273

[7] Nathan Wallace et al. ${ }^{2}$ for their APT\&M2016 poster on this subject. A paper is soon to follow. 


\section{Atom Probe Learning Module on MyScope}

Anna V. Ceguerra ${ }^{1,2}$, Ingrid McCarroll ${ }^{1,2}$, Alex La Fontaine ${ }^{1}$, and Julie M. Cairney ${ }^{1,2}$

Corresponding Author: anna.ceguerra@sydney.edu.au

1. The University of Sydney. Australian Centre for Microscopy \& Microanalysis, and School of Aerospace, Mechanical and Mechatronic Engineering, Sydney, NSW 2006, Australia.

2. Microscopy Australia (formerly known as the Australian Microscopy \& Microanalysis Research Facility, or AMMRF).

Microscopy Australia provides a web-based learning module called MyScope [1]. MyScope contains several modules separated by microscopy technique, including scanning electron microscopy, transmission electron microscopy, and X-ray diffraction. It is produced with new users in mind but is also utilised by experienced microscopists who want to refresh their knowledge. MyScope was first made available online in 2010, and in 2017 alone it had been accessed by over 130,000 new users worldwide.

In the next iteration of MyScope, there will be an Atom Probe Tomography (APT) module. The module content will include a basic guide to APT, figures illustrating APT concepts, and a simulator to simulate an atom probe experiment with different experimental parameters. The module is being released as a preview during this APT\&M 2018 meeting.

\section{References:}

[1] http://www.ammrf.org.au/myscope/ 


\section{Density Functional Theory for Ion Identity in Mass Spectra}

C. Lynch ${ }^{1}$, M. Moody ${ }^{1}$ and D. Haley ${ }^{1}$

Corresponding Author: daniel.haley@materials.ox.ac.uk

1. Dept. Materials, University of Oxford, Oxford, United Kingdom

APT is a characterisation technique, in which the atomic identity of the evaporated species is not directly measured, but rather inferred from time-of-flight. This identification of atomic species from the mass spectrum underpins the $3 \mathrm{D}$ reconstruction, and all subsequent analysis in an atom probe experiment. Incorrect identification of species has been cited as a leading source of compositional error. The identification of the evaporated ionic species is often difficult, particularly where species can evaporate as molecules. In such cases, often complex isotope patterns are observed, which are difficult to interpret, and for which there are numerous plausible combinations of species. This makes reliable identification of the composition of some peaks in the mass spectrum challenging, and creates ambiguities in APT analyses.

It has been shown by several authors[1][2] that molecular species within the high electric field environment of the APT experiment can be studied effectively using Density Functional Theory (DFT). We present a DFT framework to restrict candidate molecular species feasibly contributing to peaks in a mass spectrum by demonstrating that certain combinations cannot occur within an atom probe experiment. We provide a method and framework to predict the formation of bimolecular species detected within an APT experiment, and thus enhance the accuracy of the interpretation of the mass-to-charge spectrum. This enables higher confidence and improved reporting of compositional measurements within an atom probe experiment, and combines theoretical modelling with experimental observation in a practical manner. Integration of this stability prediction data into APT peak identification (APTTools) software is demonstrated.

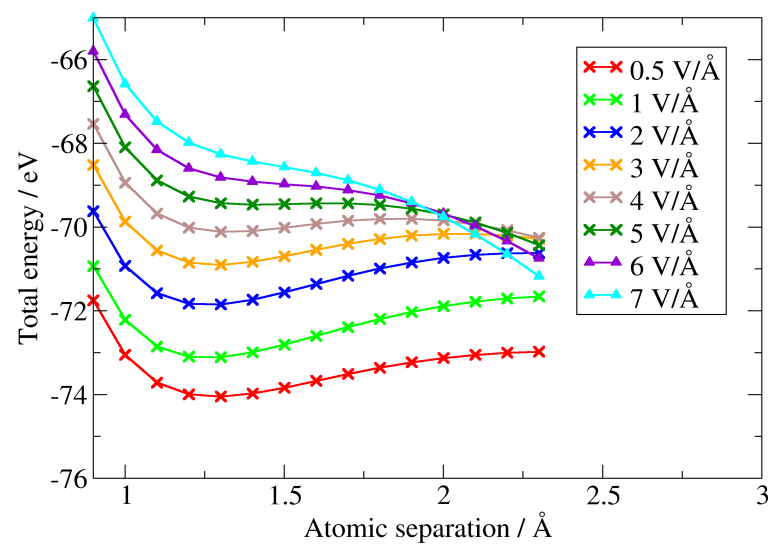

Figure 1. DFT calculations for $\mathrm{BH}^{2+}$ in a vacuum, under increasing applied electrostatic field gradient.

\section{References:}

[1] E.P. Silaeva, M. Karahka, H.J. Kreuzer, Current Opinion in Solid State and Materials Science, 17, (2013), 211-216

[2] I. Blum, D. Zanuttini, L. Rigutti, F. Vurpillot, J. Douady, E. Jacquet, P-M. Anglade, B. Gervais, A. Vella, A. Gaillard, Microscopy and Microanalysis, 22 S3, (2016), 662-663 


\section{A layer-by-layer reconstruction method including field of view effects, missing atoms and laser effects}

Yu-Ting Ling ${ }^{*}$, Janusz Bogdanowicz $^{1}$, Claudia Fleischmann ${ }^{1}$, and Wilfried Vandervorst ${ }^{1,2}$

*Corresponding Author: Yu.Ting.Ling@imec.be

${ }^{1}$ Imec vzw, Kapeldreef 75, B-3001 Leuven, Belgium

${ }^{2}$ Instituut voor Kern- en Stralingsfysica, KU Leuven, Belgium

Atom probe tomography is a powerful technique that reveals the three-dimensional atom distribution of a sample. A well controlled evaporation process and a suitable reconstruction algorithm make it possible to achieve an atomic scaled resolution of an emitter tip. The conventional reconstruction method back projects measured atoms from a detector to the emitter surface by assuming a perfect hemispherical tip radius and a straight flight from the tip to the detector. However, when applying this to an experimental data the reconstruction is hampered by the missing atoms as induced by the limited of field-of-view (FOV) and detection efficiency. Moreover, when reconstructing semiconductor tips evaporated with Laser illumination one must address the non-hemispherical and asymmetric tip evolution and its impact on local magnification and trajectories [1].

Here we developed a layer-by-layer reconstruction method which addresses these limitations by including an estimate of the real number of atoms evaporated through concurrent evaporation simulations. An empirical evaporation rates model derived from published data [2] and a Laser pulsing model were implemented into the full-dimensional simulator TAPSim [3]. To mimic the effect of the laser, we also introduce thermal gradients within the sample which address the effect of the local heating of the tip apex. Simulations of the tip shape evolution can now reproduce the (a)symmetrical tip shapes as observed under laser assisted evaporation.

Our reconstruction approach relies on predicting the point of origin of the detected atoms by matching its detection point with the predictions based on the simulated (laser assisted) evaporation process of the last simulated layer. The non-detected atoms (FOV and detector efficiency) are added based on counting the pulse numbers required to evaporate one layer. This concept was demonstrated by reconstructing silicon atoms of several simulated evaporation sequences whereby the effects of FOV and detector efficiency are included by removing atoms from the data set. The presented model showed significant improvement in spatial accuracy after reconstruction which not only recovered the complete tip but also removed the density variations due to poles on hit maps. The new evaporation model decouples field and thermal evaporation behaviors and benefit to the new reconstruction method for symmetric and asymmetric tips.

\section{References:}

[1] S. Koelling, N. Innocenti, A. Schulze, M. Gilbert, A. K. Kambham and W. Vandervorst, In-situ observation of non-hemispherical tip shape formation during laser-assisted atom probe tomography, J. Appl. Phys. 109 (2011), 104909.

[2] K. Thompson, J. Sebastian and S. Gerstl, Observations of Si field evaporation, Ultramicroscopy 107 (2007), 124-130.

[3] C. Oberdorfer, S. M. Eich and G. Schmitz, A full-scale simulation approach for atom probe tomography, Ultramicroscopy 128 (2013), 55-67. 


\title{
Building a Library of Simulated Atom Probe Data for Different Crystal Structures and Pillar Orientations Using TAPSim
}

\author{
Markus Kühbach $^{1}$, Andrew Breen ${ }^{1}$, Michael Herbig ${ }^{1}$, Baptiste Gault ${ }^{1}$, Dierk Raabe ${ }^{1}$ \\ Corresponding Author: m.kuehbach@mpie.de \\ 1. Max-Planck-Institut für Eisenforschung GmbH, \\ Max-Planck-Straße 1, 40237 Düsseldorf, Germany.
}

TAPSim is an open source software package for modeling the process of field evaporation in atom probe experiments. Developed by Oberdorfer et al. ${ }^{[1]}$, it utilizes explicit electrostatic field computations in combination with a Monte Carlo scheme for predicting the evaporation sequence of a user-defined tip emitter structure and computing the corresponding ion trajectories. With these capabilities, it is a useful tool for improving the understanding of the field evaporation process, facilitating the development of the tomographic reconstruction and the interpretation of field evaporation artefacts. However, the iterative solving of fields renders each simulation numerically costly and requires substantial data-mining. In effect, practically studies have not yet been routinely conducted.

In this presentation we summarize the process of building an open source library of simulated field desorption maps for differently oriented synthetic pillars of the face-centered cubic (FCC), body-centered-cubic (BCC), and hexagonal-close-packed (HCP) crystal structures. Specifically, a total of 101 single-crystalline pillars each were evaporated. Their lattices were oriented randomly to sample economically the individual stereographic triangles. Such data is intended to facilitate the interpretation of low density zone lines and poles that are observed on detector hit maps during atom probe experiments. The datasets and corresponding tools will be made publically available to the APT community in an effort to provide better access to simulated atom probe datasets.
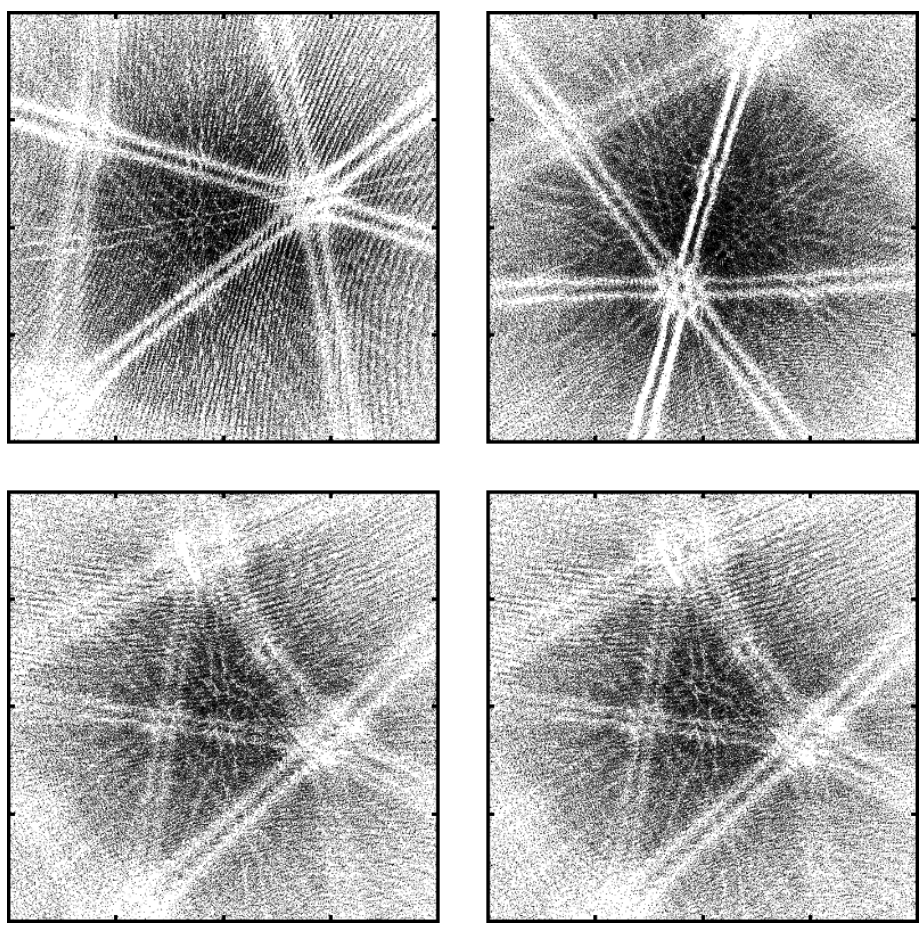

Figure 1 Field desorption map for synthetic Al - FCC (top left), $\mathrm{W}$ - BCC (top right), Mg - HCP (bottom left), and Zr - HCP (bottom right) pillars, whose crystal lattices were rotated by the same random orientation.

\section{References:}

[1] Christian Oberdorfer, Sebastian Manuel Eich, Guido Schmitz, A full-scale simulation approach for atom probe tomography, Ultramicroscopy 128 (2013), 55-67 


\title{
Comment on the Competing Influences of Field-Energy and PPI Terms on the Activation Energy for Partial-Ion Migration at Highly Charged Surfaces
}

\author{
Richard G. Forbes ${ }^{1}$ \\ Corresponding Author: r.forbes@trinity.cantab.net \\ 1. Advanced Technology Institute, University of Surrey, Guildford, Surrey GU2 7XH, UK
}

In accordance with Gauss' theorem, the surface atoms at highly charged metal surfaces need to be treated as partial ions [1]. The behavior of such ions, when there is a high-voltage generator between the emitter and the counter-electrode, is governed by the so-called electrical Gibbs function $G^{\text {el }}[1]$, first explicitly identified (but not under this name) in Ref. [2].

In simple non-atomic theories, expressions for $G^{\mathrm{el}}$ (and for a small change $\delta G^{\mathrm{el}}$ in $G^{\mathrm{el}}$ ) have terms that relate to (a) the emitter bulk, (b) the zero-field surface free energy per unit area (or "surface tension"), and (c) the electrostatic field-energy associated with the electrical capacitance between the emitter and the counter-electrode. In simple applications, changes in the bulk term are neglected. The play-off between the "surface tension" and "field-energy terms" leads [2] to such effects as the formation of so-called Taylor cones (first observed by Gray in 1732). Effects of this type show clearly that the field-energy/field-stress/capacitance term is real and important.

In atomic-level theories, an additional field-squared $\left(F^{2}\right)$ term appears, the so-called "PPI" term [1]. This is associated with the field-induced polarization and partial ionization of the surface atoms, as modified by effects due to mutual depolarization.

It is widely thought that applying a high field increases surface-atom bonding energy from its zero-field value $\Lambda^{0}$ to $\Lambda^{F}=\Lambda^{0}+1 / 2 c F^{2}$, where $c$ is usually taken as constant. Originally, this $F^{2}$ term and were thought to be related to PPI effects, but later a good match was found between $c$-values for rhodium derived from (a) appearance-energy experiments, and (b) field-energybased theory, which gives $c=\varepsilon_{0} \Omega$, where $\Omega$ is the atomic volume of the evaporating atom and $\varepsilon_{0}$ is the electric constant. Probably the reason that the PPI term does not come into play is that the removal of a kink-site atom leaves the PPI state of the surface much the same as it was before.

In high-field surface migration, a partial ion first has to escape from its local bonding site, and it is known that the application of high fields to the emitter surface must reduce the activation energy for escape. This presumably happens because the escaping partial ion is more exposed to the field at the saddle point than at the bonding site. Until recently, the author had assumed that this was another field-energy effect. However, recent high-field quantum-mechanical calculations by Jannson and collaborators [3], based on a PPI-type formulation of the underlying physics, yield good agreement with old measurements by Tsong and by Kellogg on diffusion activation energies in high electrostatic fields. It seems that the underlying physical reason for this must be that, unlike the field evaporation situation, the PPI environments associated with the bonding site and saddle point are significantly different. Hence the PPI term is dominant.

\section{References}

[1] M.K. Miller and R.G. Forbes, Atom Probe Tomography: The Local Electrode Atom Probe (Springer, New York, 2014).

[2] N.N. Ljepojevic and R.G. Forbes, Proc. Roy. Soc. Lond. A 450 (1995) 177.

[3] V. Jansson, E. Baibu, A. Kyritsakis and F. Djurabekova, arXiv:1709.04694 . 



\section{Author Index}

Aboulfadl, Hisham, 100

Ackerman, Abigail, 131, 291

Adams, Thomas, 93

Agarwal, Shweta, 160

Ahn, Jae-Pyoung, 223

Aich, Nirupam, 167

Akiva, Anat, 160

AL-Akhras, M-Ali, 205, 286

Al-Qudah, Ala'a, 205, 286

Alam, Talukder, 117

Albu, Mihaela, 281

Aleev, Andrey, 110

Alhassan, Abdullah, 134

Almirall, Nathan, 253

Amalraj, Marshal, 116

Amati, Matteo, 278

Amichi, Lynda, 97

An, Xianghai, 44

Andersson, Martin, 162

Andrén, Hans-Olof, 193

Anglade, Pierre-Matthieu, 121

Arai, Naomi, 300

Arcuri, Gabriel, 231

Arey, Bruce, 57

Armstrong, David, 203

Arnoldi, Laurent, 66, 68, 137, 297

Aruga, Yasuhiro, 252, 313

Ashton, Michael, 109, 131

Auger, Maria, 94, 196

Babu, Suresh, 280

Bacchi, Christian, 122

Bachhav, Mukesh, 197

Badyka, R., 248

Bae, Jae Wung, 270

Bagot, Paul, 62, 85, 92, 152, 153, 160, 198, 203, 204, 226, 245, 277

Baibuz, Ekaterina, 181, 182

Bailey, Nathan, 140

Bakr, Abu, 92

Balla, Irdi, 111

Banerjee, Rajarshi, 117, 119

Bani Ali, Emad, 285

Barili, Sarah, 316

Barnes, Jean Paul, 207

Barrirero, Jenifer, 258

Barroo, Cédric, 201

Barton, Dallin, 315
Beeg, Sebastian, 278

Beinke, Daniel, 208

Benedix, Gretchen, 54

Bertagni, Angela, 58

Bertazzo, Sergio, 160

Bhadharla, Pranav, 56

Bian, Ming-Zhe, 88

Bilal, Huma, 271

Birbilis, Nick, 226

Bjurman, Martin, 150

Blanchard, Paul, 63, 296, 316

Blavette, Didier, 42, 216, 282, 303

Blick, Robert, 284

Blukis, Roberts, 277

Blum, Ivan, 42, 59, 66, 68, 121, 206, 216, 303

Boarino, Luca, 298

Bogdanowicz, Janusz, 99, 113, 135, $137,282,298,301,321$

Boll, Torben, 74, 118, 147, 259, 267, 273

Bonef, Bastien, 134, 257

Borz, Mario, 67, 68, 206

Bos, Jan-Willem, 62

Botton, Gianluigi, 224

Bougerol, Catherine, 97

Bourrat, Xavier, 61

Bowden, Mark, 268

Boyce, Brad, 84

Brabant, Paul, 93

Bracco, Jacquelyn, 58

Braikat, Hatem, 205, 286

Brandl, Dominik, 220

Breen, Andrew, 115, 131, 202, 211, $251,318,322$

Britton, T. Ben, 131, 202

Britz, Dominik, 258

Broderick, Scott, 214, 217, 306

Bruemmer, Stephen, 87

Bunton, Joseph, 293

Burgess, Tim, 95

Burke, M. Grace, 85

Burton, George, 75

Byun, Thak-Sang, 148

Bäcke, Olof, 74

Cairney, Julie, 166, 211, 226, 260, 261,319

Cao, Jing, 278
Carruthers, Alexander, 85

Castro, Celia, 59

Ceguerra, Anna, 211-213, 271, $309,318,319$

Chabanais, Florian, 59

Chae, Byeong-Gyu, 64, 76

Chalopin, Benoit, 206

Chang, Yanhong, 115, 131, 132, $138,202,251,291$

Chauveau, Jean-Michel, 42

Chen, Chien-Chun, 35

Chen, Hans, 118

Chen, Hansheng, 95

Chen, Wei-Ying, 144

Chen, Yi-Sheng, 153

Chen, Yimeng, 243, 252, 254, 280 , 293, 295

Cherevko, Serhiy, 80

Chiaramonti, Ann, 63, 296, 316

Cho, Ju-Young, 65

Choi, Pyuck-Pa, 199, 223, 238

Choudhuri, Deep, 119

Chow, Lee, 60

Chung, Keith, 93

Ciobanu, Cristian, 225

Clark, Trevor, 283

Clarke, Blythe, 84

Cliff, John, 57

Clifton, Peter, 244

Cohen, Michael, 164

Cojocaru-Mirédin, Oana, 65, 81, 101, 207, 240, 311

Collins, Sean, 277

Cooper, David, 97

Cormier, Jonathan, 89

Costa, Alex, 265

Cournoyer, James, 308

Croy, Nicholas, 222

Crudden, David, 204

Cuduvally, Ramya, 113, 135-137, 301

Cui, Xiangyuan, 95

Da Costa, Gérald, 42, 122, 210, 216, 299, 303

da Silva, Alisson Kwiatkowski, 43

da Silva, Caue Correa, 278

Dadbakhsh, Sasan, 260

Dahlström, Alexander, 146 
Daly, Luke, 62

Danoix, Frédéric, 146, 176, 215

Daradkeh, Samer, 205, 286

Daradkesh, Samer, 288

Darling, James, 55

Darling, Kristopher, 264, 315

Day, Alec, 169, 212, 309

De Geuser, Frédéric, 310

de Kloe, René, 61

De Leo, Natascia, 298

De Mierry, Philippe, 97

Dear, Felicity, 131, 245

Deconihout, Bernard, 282

Dehm, Gerhard, 83

DeRocher, Karen, 164

Devaraj, Arun, 46, 133, 148, 195 , 283, 297

Deymier, Alix, 272

Dhara, Sharmistha, 177

Di Bernardo, Giuseppe, 310

Di Russo, Enrico, 42, 121, 216, 282, 303

Dialameh, Masoud, 298

Diercks, David, 63, 75, 96, 102, 211, 225, 296, 312, 314

Djurabekova, Flyura, 181, 182

Domain, Christophe, 82, 248

Dorin, Thomas, 177

Douady, Julie, 121

Douglas, James, 85, 92, 141, 232, 253

Dowsett, David, 289

Dronskowski, Richard, 65

Du, Sichao, 95

Duewer, David, 77

Dunlap, David, 157

Duran, Rüya, 200

Dye, David, 131, 245, 291

Dzuba, Brandon, 241

Earthman, James, 273

Ebner, Sandra, 220

Eder, Katja, 260

Edoff, Marika, 100

Efsing, Pål, 150

Eggeman, Alex, 277

Einsle, Joshua, 277

Eisenbach, Markus, 35

Ekström, Peter, 150

El-Zoka, Ayman, 224

Engelhart, Wolfgang, 269

Ercius, Peter, 35

Escobar, Julian, 147, 265, 267
Etienne, Auriane, 66

Exertier, Florant, 166

Eyhusen, Soeren, 289

Faria, Guilherme, 147, 267

Fathidoost, Mozhdeh, 138

Fedorova, Irina, 193

Felfer, Peter, 80, 242

Field, Kevin, 196

Fijneman, Remco, 160

Fisher, Kevin, 156

Fisher, Louise, 54

Flaxa, Volker, 258

Fleischmann, Claudia, 99, 113, 135, 137, 290, 298, 301, 321

Fletcher, Charles, 124

Floss, Christine, 56

Forbes, Richard, 185, 209, 287, 288,323

Fougerouse, Denis, 36, 54, 234

Francois-Saint-Cyr, Hugues, 61, 103, 104, 168, 169, 295

Free, Robert, 165

Freysoldt, Christoph, 109, 131

Friedman, Alan, 274

Friedman, Michelle, 274

Fritze, Stefan, 120

Fujishin, Helena-Nikolai, 96, 102

Fukata, Naoki, 276

Fukushima, Yutaka, 300

Furuhara, Tadashi, 142, 178

Gan, Jian, 197

Gao, Qi, 308

Gao, Qiang, 95

Gardella, Joseph, 214

Gardner, Hazel, 203, 245

Gault, Baptiste, 43, 80, 81, 89, 115, $131,132,138,183,199$, 202, 207, 238, 251, 278, 291, 310, 318, 322

Geiger, Simon, 80

Geiser, Brian, 28, 295, 307

Genty, Eric, 201

Gerard, Angela, 87

Gerstl, Stephan, 161

Gervais, Benoit, 121

Ghamarian, Iman, 125, 156

Gibouin, David, 61

Giddings, A. Devin, 239

Gilis, Natalia, 201

Gludovatz, Bernd, 271

Gordon, Lyle, 164
Gorman, Brian, 63, 75, 96, 102, 211, 296, 312, 314, 317

Gorsse, Stephane, 119

Gotoh, Yasuhito, 112, 304

Govindaraju, Venu, 306

Grabowski, Blazej, 183

Grandfield, Kathryn, 163

Gregoratti, Luca, 278

Gregory, Daniel, 235

Greiner, Mark, 278

Grenier, Adeline, 97, 207

Grumsen, Flemming Bjerg, 193

Guha, Upoma, 167

Guillermier, Christelle, 61

Guo, Mingxing, 255

Guo, Wei, 144

Gwalani, Bharat, 117, 119

Haberfehlner, Georg, 281

Haboucha, Adil, 206

Hagmann, Mark, 285, 294

Hald, John, 193

Haley, Daniel, 124, 152, 153, 226, 320

Halpin, John, 62

Hamada, Jun-ichi, 191

Han, Bin, 91

Han, Jong Chan, 250, 270

Haneji, Yuki, 304

Harding, Ian, 118

Hardy, John, 133

Hariharan, Avinash, 86

Harmer, Martin, 264

Harrington, Sean, 257

Harrison, Richard, 277

Hatzoglou, Constantinos, 43, 210, 215, 299, 305

Haugg, Stefanie, 284

He, Lingfeng, 197

Hedström, Peter, 146

Heilmaier, Martin, 118, 259, 273

Henkel, Christian, 284

Henry, Karen, 221, 279

Herbig, Michael, 83, 131, 202, 322

Hideur, Ammar, 206

Higgins, Steven, 58

Hodgson, Peter, 177

Hoelzer, David, 196

Hofer, Christina, 220

Hollermann, Henning, 101

Hong, Seong Pyo, 236

Hono, Kazuhiro, 88, 262, 276

Hori, Yutaro, 300 
Proceedings of Atom Probe Tomography \& Microscopy (APT\&M) 2018, Washington, DC, June 10-15, 2018 NIST SP 2100-03

Hornbuckle, B. Chad, 264, 315

Hosemann, Peter, 140

Houard, Jonathan, 42, 66, 67, 216, 282,303

Hough, Rob, 54

Hoummada, Khalid, 60

$\mathrm{Hu}$, Rong, 73, 237

Hu, Wei-Shan, 239

Hugues, Maxime, 42

Hulander, Mats, 162

Hurni, Christophe, 217

Hwang, Kyo Jin, 254

Hwang, Ruey-Lian, 239

Hyde, Jonathan, 253

Hörst, Sarah, 227

Ichihara, Chikara, 313

Idlahcen, Said, 206

Ievlev, Anton, 58

Im, Hyeji, 199

Inoue, Koji, 91

Isheim, Dieter, 56, 219

Ismagilov, Rinat, 66

Jacobs, Luc, 201

Jacquet, Emmanuelle, 121

Jagadish, Chennupati, 95

Jana, Saumyadeep, 195

Jansson, Ulf, 120

Jansson, Ville, 181, 182

Jaskot, Matthew, 96, 102, 314

Jennings, Eleanor, 232

Jeong, Eun Kyung, 236

Jha, Rajesh, 225

Ji, Gang, 260

Jiang, Suihe, 256

Jiang, Xiujuan, 283

Jin, Shenbao, 44, 149, 263

Jindra, Sarah, 58

Joester, Derk, 164, 165, 272

Johnson, Bradley, 133

Johnson, Kayla, 242

Jones, Eric, 93

Jones, Sean, 317

Joosten, Jan, 242

Joshi, Vineet, 195

Jouneau, Pierre-Henri, 97

Julien, François Henry, 42

Jägle, Eric, 86

Kamado, Shigeharu, 88

Kang, Phil Woong, 223

Kao, Ming-Hong, 239

Kapteyn, Henry, 53
Kasian, Olga, 80

Katnagallu, Shyam, 138, 183, 207, 310,318

Kauffmann, Alexander, 118, 259

Kauffmann-Weiss, Sandra, 118

Kautz, Elizabeth, 133, 195

Kawabe, Nozomu, 88

Kawakami, Kazuto, 191, 192, 262, 302

Keatley, Anya, 198

Keiser, Dennis, 197

Keller, Jan, 100

Kelly, Thomas, 114, 115

Kent, Paul, 35

Kerger, Philipp, 278

Keutgen, Jens, 65, 311

Khan, Mansoor, 95

Khanom, Fouzia, 289

Kilburn, Matt, 54

Kim, Hyoung Seop, 270

Kim, Joong Jung, 236

Kim, Se-Ho, 223

Kim, Yong Bum, 236

Kim, Yoon Jun, 254

Kimura, Ken, 191

King, Helen, 160

Kirkland, Chris, 54

Kitayama, Takumi, 313

Knight, Thomas, 93

Knipling, Keith, 179, 266

Kobayashi, Yukiko, 192, 262, 302

Koelling, Sebastian, 160

Koenraad, Paul, 160

Kontis, Paraskevas, 89, 131, 202, 291

Koppa, Matthew, 157

Kostka, Aleksander, 145

Kota, Bhargavea Urala, 306

Kouzminov, Dimitry, 308

Kovarik, Libor, 195, 268

Kozuka, Masaya, 252, 313

Kruse, Norbert, 45, 184, 268

Kruska, Karen, 87

Kruth, Jean-Pierre, 260

Kruzic, Jamie, 271

Kubota, Manabu, 192

Kumar, Sharvan, 118

Kurz, Thomas, 220

Kwak, Chang-Min, 64, 76

Kyritsakis, Andreas, 181, 182

Köster, Sigrun, 244

Kühbach, Markus, 322

Küpers, Michael, 65
La Fontaine, Alexandre, 166, 319

Lach, Timothy, 148

Lambeets, Sten, 45, 201

Langelier, Brian, 55, 163, 224, 231

Langolff, Vivien, 282

Lapington, Mark, 204

Larsen, Jes, 100

Larson, David, 61, 154, 280, 293, 295, 307

Lavender, Curt, 195

Lavrik, Nickolay, 292

Lee, Bryan, 163

Lee, Jang Jung, 239

Lee, Ji Yeong, 223

Lee, Sang Hoon, 222

Lee, Sang Ick, 236

Lee, Sung Ho, 236

Lefebvre, Williams, 59

Lehmann, Tim, 269

Lei, Zhifeng, 256

Lenz, Dan, 293

Leonard, Donavan, 93

Lewin, Erik, 120

Lewis, Josiah, 56

Lheureux, Guillaume, 134

Li, Gaojie, 255

Li, Hui, 194, 246

Li, Jiehua, 44

$\mathrm{Li}, \mathrm{Na}, 247$

Li, Shilei, 149

Li, Tong, 80

Li, Xiaopeng, 260

Li, Yujiao, 145

Li, Zhiming, 270

Liao, Xiaozhou, 44

Liaw, Peter, 266

Licata, Olivia, 217

Liebscher, Christian, 83, 202, 291

Lin, Yu-Chen, 261

Lindgren, Kristina, 150

Ling, Yu-Ting, 321

Liu, Bosong, 271

Liu, Fang, 193

Liu, Hui, 95

Liu, Jia, 57

Liu, Jizi, 73

Liu, Wenqing, 194, 246

Lomuscio, Alberto, 81

London, Andrew, 139

Lorenz, Matthias, 58

Lu, Wenjun, 83, 202, 251, 291

Lu, Yifeng, 43

Lu, Zhaoping, 256 
Luckenbaugh, Tom, 264

Ludwig, Alfred, 145

Lukyanchuk, Anton, 110

Lynch, Charlotte, 320

Lynsky, Cheyenne, 134

Müller, Erwin, 21

MacLaren, Donald, 62

Maeda, Saba, 112

Maier, Urs, 244

Makineni, Surendra, 89, 199, 207

Malinovskis, Paulius, 120

Malis, Oana, 241

Malykhin, S. A., 67

Mandt, Jerry, 295

Manfra, Michael, 241

Marceau, Ross, 83, 177

Marquis, Emmanuelle, 125, 156

Martin, Andy, 104

Martin, Isabelle, 61, 154, 243, 295

Martin, Peter, 198

Martin, Tomas, 160, 198

Martineau, Benjamin, 277

Martins, George, 92

Marvel, Chris, 264

Mathaudhu, Suveen, 283

Mauchain, Julien, 206

Mayama, Norihito, 300

Mayrhofer, Karl, 80

Mazumder, Baishakhi, 98, 167, 217, 274

McCarroll, Ingrid, 226, 261

McDermott, Robert, 114

Mei, Paulo, 147, 267

Melkonyan, Davit, 99, 113, 135137,301

Melmed, Allan, 15

Mendez-Martin, Francisca, 281

Meng, Ying Shirley, 46

Mercurio, Kevin, 93

Miaja-Avila, Luis, 63, 296

Miao, Jianwei, 35

Midgley, Paul, 277

Milillo, Tammy, 214

Miller, Brandon, 197

Mio, Antonio, 65, 101

Mishra, Arpit, 109

Miyamoto, Goro, 142, 178

Mohrbacher, Hardy, 258

Moldovan, Simona, 42, 282

Monnet, G., 248

Moody, Michael, 62, 85, 92, 94, 124, 152, 153, 160, 196,
198, 203, 204, 226, 232, $245,253,320$

More, Karren, 58

Morris, Richard, 99, 113, 135-137, 301

Morris, Robert, 221, 279

Morsdorf, Lutz, 83

Moser, Desmond, 55, 231

Mousa, Marwan, 205, 285, 286, 288, 294

Mouton, Isabelle, 97, 115, 131, 132, 138, 202, 207, 291

Murnane, Margaret, 53

Murphy, John, 92

Murty, Budaraju Srinivasa, 116

Muthuraman, Harish, 308

Mücklich, Frank, 258

Na, Seon-Hyeong, 249

Nagai, Yasuyoshi, 91

Nakata, Taiki, 88

Nanba, Shigenobu, 252

Nematollahi, Ali, 183

Neugebauer, Jörg, 109, 131, 183

Newman, Roger, 224

Nishikawa, Osamu, 155

Nishimura, Chikasa, 304

Nomoto, Keita, 271

Norasetthekul, Somchintana, 308

Normand, Antoine, 59, 67

Nowell, Matthew, 61

Nylese, Tara, 61

Oberdorfer, Christian, 125, 156

Obraztsov, Alexander, 66-68, 206

Odette, G. Robert, 253

Odquvist, Joakim, 146

Ohkubo, Tadakatsu, 276

Ohnuma, Toshiharu, 123

Oliveira, Joao, 147, 265, 267

Olszta, Matthew, 87

Oltman, Ed, 295, 307

Ong, Yi Ching, 239

Op de Beeck, Jonathan, 113, 290

Ophus, Colin, 35

Ott, Jonas, 111, 275

Overman, Nicole, 283

Palmstrøm, Chris, 257

Panitz, John, 21, 41

Paredis, Kristof, 99, 113, 290

Pareige, Cristelle, 248, 299

Pareige, Philippe, 82, 299, 305

Parikh, Pritesh, 46
Park, Chan-Gyung, 64, 76, 249, 250

Park, O Ok, 223

Park, Seong Yong, 76

Parviainen, Stefan, 158

Pasteris, Jill, 272

Payne, Tim, 295

Pearce, Mark, 54

Peng, Zirong, 43, 138

Pennachio, Dan, 257

Perea, Daniel, 45, 57, 151, 233, 235, 268

Pihl, Astrid, 162

Pint, Bruce, 74

Platzer-Björkman, Charlotte, 100

Ponge, Dirk, 43, 132, 251, 291

Poplawsky, Jonathan, 58, 93, 98, 144, 241, 265, 292

Portavoce, Alain, 60

Pradeep, Konda Gokuldoss, 116

Pradel, Ken, 276

Primig, Sophie, 180

Printemps, Tony, 207

Prosa, Ty, 61, 154, 243, 244, 252, 293, 295, 307

Proudian, Andrew, 96, 102, 314

Pryor, Alan, 35

Qu, Jiangtao, 95

Quiambao, Kathleen, 87

Raabe, Dierk, 43, 80, 81, 83, 86, 89, $115,131,132,138,183$, 199, 202, 207, 238, 251, 270, 278, 291, 310, 318, 322

Radecka, Anna, 203, 245

Radiguet, Bertrand, 82, 299, 305

Raghuwanshi, Mohit, 240

Rajan, Krishna, 214, 217, 274, 306

Rameshbabu, Utthara, 272

Ramirez, Antonio, 147, 265, 267

Rampp, Markus, 310

Raznitsyn, Oleg, 110

Reddy, Steven, 36, 54, 234

Redinger, Alex, 238

Reed, Roger, 204

Reinhard, David, 55, 231, 244, 295 , 307

Rice, Katherine, 222, 243, 252, 280, 293, 295

Rickard, William, 36, 54, 234

Riddle, Nick, 253

Rigutti, Lorenzo, 42, 59, 67, 121, 216, 282, 303 
Proceedings of Atom Probe Tomography \& Microscopy (APT\&M) 2018, Washington, DC, June 10-15, 2018 NIST SP 2100-03

Ringer, Simon, 95, 180, 211-213, $271,309,318$

Risse, Jeroen, 86

Roberts, Anthony, 264

Rogozhkin, Sergey, 110

Rosso, Kevin, 57

Roussel, M, 299

Rugg, David, 203, 245

Rusitzka, Ann-Katrin, 115, 138

Ryan, Joseph, 233

Sabirianov, Renat, 35

Saghi, Zineb, 277

Saillet, S., 248

Salvador, Camilo, 147, 267

Sanford, Norman, 63, 296, 316

Sasaki, Taisuke, 88

Sasaki, Tomokazu, 300

Satou, Yukihiko, 198

Sattari, Mohammad, 100

Savan, Alan, 145

Saxey, David, 36, 54, 234

Schaeublin, Robin, 161

Scheu, Christina, 80

Schliephake, Daniel, 259

Schlögl, Robert, 278

Schmidt, Michael, 222

Schmitz, Guido, 111, 200, 208, 269, 275

Schnitzer, Ronald, 220

Schreiber, Daniel, 57, 87, 140, 151, 233

Schwarz, Torsten, 81, 207, 238

Schweinar, Kevin, 278

Schwoebel, Paul, 157

Scott, John Henry, 90

Scott, Mary, 35

Scott, Thomas, 198

Scully, John, 87

Seidel, Jan, 94

Seidman, David, 56

Seils, Sascha, 118, 147, 259, 267

Senichev, Alexander, 241

Seo, Jung-Hun, 98

Seol, Jae-Bok, 64, 76, 223, 249, 250, 270

Setlur, Srirangaraj, 306

Sha, Gang, 44, 73, 149, 237, 255, 263

Sharma, Pankaj, 94

Shea, Patrick, 93

Shen, Qin, 246

Shen, Yang, 82

Shepard, Jeffrey, 244
Shimizu, Yasuo, 91

Shinde, Deodatta, 120, 143, 297

Shirazi, Mohammad Ali, 241

Shirley, Ying, 46

Shutov, Anton, 110

Siebentritt, Susanne, 81, 238

Silaeva, Elena, 67

Silva Santisteban, Tomas, 27

Smeets, Paul, 164

Smith, George, 253

Sobering, Geoff, 295

Sommerdijk, Nico, 160

Song, Jay Hyok, 76

Speck, James, 134, 217, 257

Spies, Maria, 66

Sridharan, Niyanth, 280

Stack, Andrew, 58

Stebner, Aaron, 225

Stechmann, Guillaume, 81

Stein, Helge, 145

Steinhauser, Matthew, 61

Stender, Patrick, 111, 200, 269, 275

Stephenson, Leigh, 115, 131, 132, 138, 202, 251, 291, 310, 318

Stevens, Molly, 160

Stiller, Krystyna, 74, 120, 143

Strennen, Eric, 295

Sturm, Andreas, 115

Styman, Paul, 141

Subramanian, Ramachandran, 306

Sun, Fan, 35

Sundell, Gustav, 162

Suttle, Joseph, 114

Szabó, Dorothée Vinga, 118

Szczepaniak, Agnieszka, 115, 131, 132, 202, 291

Tait, Kim, 55

Takahashi, Jun, 191, 192, 262, 302

Tan, Hark Hoe, 95

Taniguchi, Masahiro, 155

Tarzimoghadam, Zahra, 251

Taylor, Sandra, 57

Tchernycheva, Maria, 42

Tezins, Uwe, 115

Tharpe, Joshua, 266

Theis, Wolfgang, 35

Theska, Felix, 180

Thomas, Sebastian, 226

Thomason, Chris, 284

Thompson, Gregory, 47, 84, 315

Thuvander, Mattias, 120, 143, 150
Timokhina, Ilana, 177

Toda, Kazuya, 300

Todeschini, Patrick, 82

Toloczko, Mychailo, 140

Torresin, Olivier, 206

Tschiptschin, André, 265

Tsuji, Hiroshi, 304

Tu, Yuan, 91

Ulfig, Robert, 244, 293, 295, 307

Unocic, Kinga, 74

Uzuhashi, Jun, 276

Vaidya, Mayur, 116

van der Heide, Paul, 99, 136

Van Humbeeck, Jan, 260

Vandervorst, Wilfried, 99, 113, 135-137, 290, 298, 301, 321

Vella, Angela, 66-68, 206, 282, 297

Vennéguès, Philippe, 97

Venturi, Linda, 67

Verberne, Rick, 234

Verheijen, Marcel, 160

Veske, Mihkel, 181

Vincent, Galen, 96, 102

Visart de Bocarmé, Thierry, 45, 201

Vleugels, Jozef, 260

von Gunten, David, 244

Voss, Jenny, 268

Vurpillot, François, 43, 59, 121, $122,158,210,215$

Wade, Jon, 232

Wang, Bo, 247

Wang, Changhong, 95

Wang, Jing, 140

Wang, Siyang, 131, 202

Wang, Wei, 247

Wang, Weichao, 95

Wang, Xiaoyue, 163

Wang, Xing, 144

Wang, Xitao, 149

Wang, Zemin, 246

Webel, Johannes, 258

Weber, Juliane, 58

Wells, Peter, 253

White, Lee, 55

Wilford, Keith, 253

Wille, Guillaume, 61

Windl, Wolfgang, 125, 156

Wirtz, Tom, 289

Withrow, Travis, 125, 156

Wong, Weng-Keen, 175 
Wood, Kathleen, 177

Wu, Li, 35

Wu, Yuan, 256

Wuerz, Roland, 81

Wuttig, Matthias, 65, 101, 311

Xu, Rui, 35

Xue, Fei, 149

Xue, Jing, 44, 237

Yang, Limei, 260

Yang, Tengfei, 144

Yang, Yafeng, 263

Yang, Yong, 239

Yang, Yongsoo, 35

Yen, Hung-Wei, 261

Yoshida, Yu, 88
Yu, Li-Jen, 156

Yu, Yuan, 65

Zanuttini, David, 121

Zavgorodniy, Alexander, 166

Zelenty, Jennifer, 253

Zeller, Patrick, 278

Zeng, Hao, 35

Zhang, Bo, 149

Zhang, Guikai, 132

Zhang, Jishan, 255

Zhang, Leifeng, 82

Zhang, Siyuan, 80

Zhang, Tianmu, 306

Zhang, Yanbo, 237

Zhang, Yidong, 255

Zhang, Yongjie, 178
Zhang, Ziying, 247

Zhao, Huan, 138, 318

Zhao, Qinglong, 263

Zheng, Rongkun, 95

Zhou, Jihan, 35

Zhou, Ming, 136

Zhou, Qi, 73

Zhou, Xuyang, 47, 84

Zhu, Huihui, 256

Zhu, Huilong, 237

Zhu, Min, 65

Zhu, Shijuan, 247

Zhuang, Linzhong, 255

Zierold, Robert, 284

Zimmerman, Jeramy, 96, 102, 314

Zpolsky, Helena, 146 


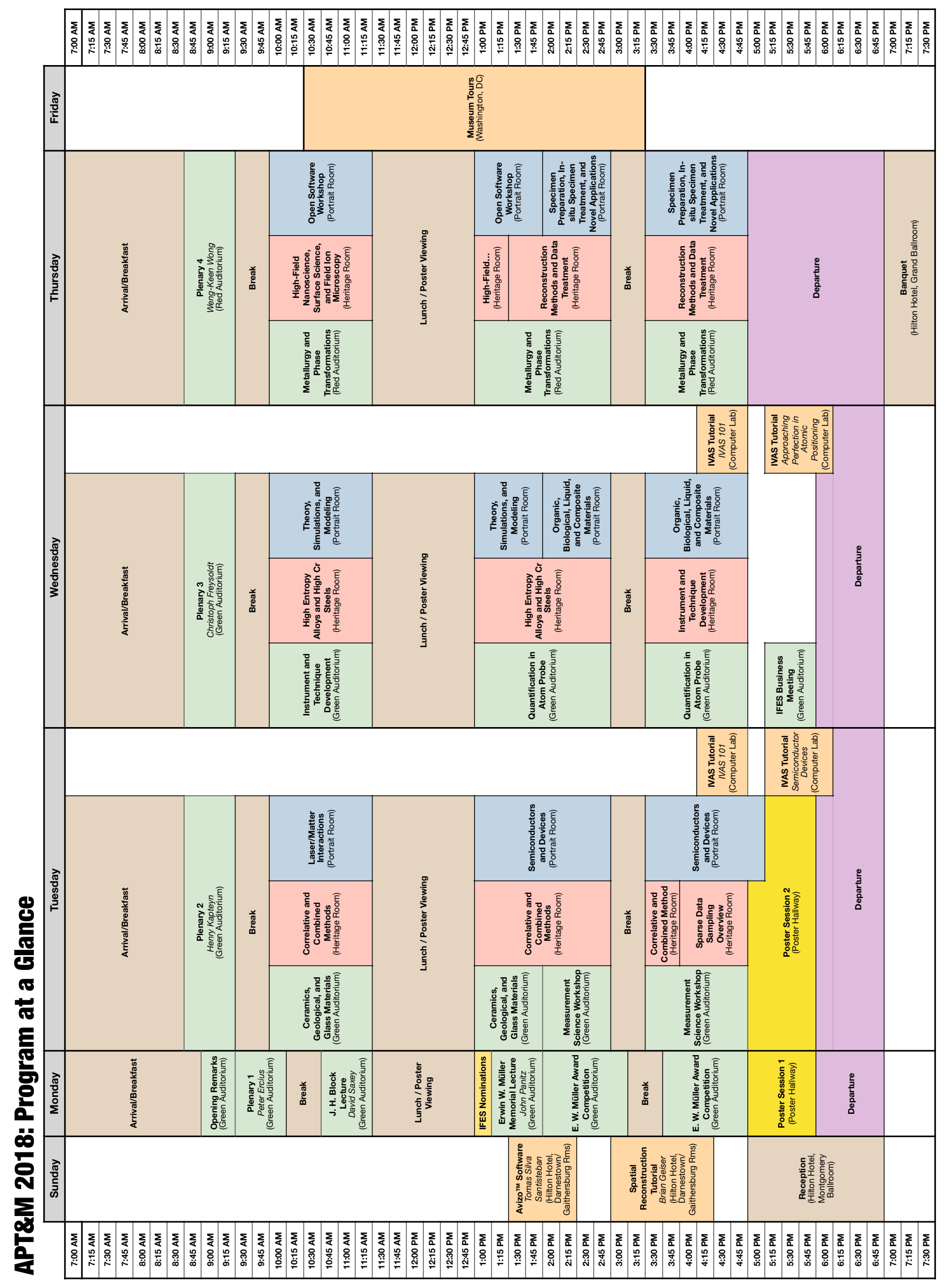

